\title{
Urban-Suburban Migration in the United States, 1955-2000
}

by

\author{
Todd K. Gardner \\ U.S. Census Bureau
}

\begin{abstract}
CES 16-08
February, 2016

The research program of the Center for Economic Studies (CES) produces a wide range of economic analyses to improve the statistical programs of the U.S. Census Bureau. Many of these analyses take the form of CES research papers. The papers have not undergone the review accorded Census Bureau publications and no endorsement should be inferred. Any opinions and conclusions expressed herein are those of the author(s) and do not necessarily represent the views of the U.S. Census Bureau. All results have been reviewed to ensure that no confidential information is disclosed. Republication in whole or part must be cleared with the authors.

To obtain information about the series, see www.census.gov/ces or contact Fariha Kamal, Editor, Discussion Papers, U.S. Census Bureau, Center for Economic Studies 2K132B, 4600 Silver Hill Road, Washington, DC 20233, CES.Papers.List@census.gov. To subscribe to the series, please click here.
\end{abstract}




\begin{abstract}
This study uses census microdata from 1960 to 2010 to look at the rates of suburbanization in the 100 largest metro areas. Looking at the racial and ethnic composition of the population, and then further breaking down these groups by income, it's clear that more affluent people were more likely to move to the suburbs. Also, the White non-Hispanic population has long been the most suburbanized group. A majority of the White population lived in suburbs by 1960 in the 100 largest metro areas, while most of the Black non-Hispanic population lived in urban core areas as late as 2000. The Hispanic and Asian populations went from majority urban to majority suburban during this period.
\end{abstract}

Keyword: suburbanization, race, ethnicity

\footnotetext{
${ }^{*}$ This work is released to inform interested parties of ongoing research and to encourage discussion of work in progress. Any views or opinions expressed in the paper are the authors' own and do not necessarily reflect the views or opinions of the U.S. Census Bureau. Please direct correspondence to Todd Gardner, U.S. Census Bureau, Center for Economic Studies, 4600 Silver Hill Rd., Washington, DC 20233, or via email at Todd.K.Gardner@census.gov.
} 


\section{Introduction}

This study uses census microdata from 1960 to 2000 to look at the urban-suburban migration in the 100 largest metro areas during this period. The population is divided into five racial/ethnic groups: non-Hispanic whites, non-Hispanic blacks, Hispanics, Asians, and other races. These groups are then further divided based on household income.

\section{Methodology}

Income - For this study, households are categorized by income using the poverty measure for income earned in the year preceding each decennial census. The migration figures, then, are limited to only those households in the poverty universe. Households are divided into three categories:

- Low income - Those below the poverty threshold

- Medium Income - Income above the poverty threshold but less than three times the poverty threshold

- High Income - More than three times the poverty threshold

Racial/Ethnic Categories - The resident population and the composition of the local government workforce uses the following categories:

- Hispanic - Hispanic ethnicity (see note below for how this was determined in each year) of any race. In this study Hispanic ethnicity was determined first and treated as a mutually exclusive category with the race groups.

- White Non-Hispanic - White in 1960, 1970, 1980 and 1990, and White alone in the 2000 census, and not of Hispanic origin.

- Black Non-Hispanic - Black, African American, or Negro in 1960, 1970, 1980 and 1990, and Black alone in the 2000 census, and not of Hispanic origin.

- Asian - For the 1960 census the only Asian nationalities explicitly listed on the questionnaire were Japanese, Chinese and Filipino. In addition to these categories, Korean was also included on the 1970 census questionnaire. The list also included Vietnamese and Asian Indian on the 1980 and 1990 census questionnaires. On all forms a box was provided to specify another race category. The box on the 1960, 1970 and 1980 forms was for any "other" category. On the 1990 questionnaire a separate box was labeled "Other API", which stood for "Asian or Pacific Islander", and a separate entry field was given for "Other Asian" on the Census 2000 form. This study uses the categories as given in each census, even though fewer national groups were listed on the early census forms.

- Other - All other race categories not covered above, and all mulitirace categories in the 2000 census.

Hispanic Ethnicity - Since 1980 the decennial census has included questions on Hispanic origin. In the 1970 census, the Census Bureau experimented with a few different variables to establish Hispanic ethnicity, notably "Puerto Rican Stock," "Spanish Origin or Descent" and "Spanish 
Surname", all of which are used to establish Hispanic ethnicity for 1970 in this study. The "Spanish Surname" variable is included in the 1960 and 1970 data, although it is only coded for residents of California, Texas, Arizona, New Mexico and Colorado. In this study Hispanic ethnicity was established for 1960 by using a combination of birthplace, parental birthplace, language spoken at home, and Spanish surname.

Metropolitan areas and central cities - This study uses the current metropolitan statistical area definitions (as published by the Office of Management and Budget in February, 2013) of the 100 most populous metro areas as of the 2010 census. The February, 2013, definitions use counties as the basic geographic unit, and these county definitions are applied to all years of data used in this study. This study also tabulates figures for "traditional central cities," which are those places that were designated as central cities of metro areas no later than 1960 for those areas classified as metropolitan by 1960. A few of the 100 largest metro areas did not achieve metropolitan status until 1970 or 1980 . For these areas, this study considers the cities of that were designated when the metro area was first delineated as traditional central cities. These include San Buenaventura (Ventura), CA, Edinburg, TX, McAllen, TX, Boise City, ID, Santa Rosa, CA, and Modesto, CA, which were the original central cities in metro areas initially defined in 1970, and Poughkeepsie, NY, Sarasota, FL, Bradenton, FL, Lakeland, FL, Melbourne, FL, and Titusville, FL, which were the original central cities in metro areas initially defined in 1980.

\section{Results}

Looking at the 100 largest metro areas as they are currently defined, census data from the 1960, 1970, 1980, 1990 and 2000 censuses reveal continuous suburban growth in the United States. Using historical central city designations (that is, cities classified as "central cities" at the start of this era) to distinguish between urban and suburban territory, the 100 largest metropolitan areas were already majority suburban in 1960 , with 56.6 percent of the population residing outside of the central cities. By 1970 the suburban population had risen above 60 percent, and by 2000 almost 70 percent of the population of large metropolitan areas lived outside of central cities. The decline in central city residence among the White non-Hispanic population (hereafter referred to as "White") was particularly dramatic, dropping from 39.1 percent of the population in 1960 to 21.3 percent in 2000. Though minority populations have grown more rapidly than the White population, Whites constituted a majority of the metropolitan population in all regions throughout this period.

The Black non-Hispanic population (hereafter referred to as "Black") was the most urban population throughout this period, as 71.1 percent of the Black population lived in central cities in 1960. Even though the black population was also suburbanizing, a majority of the Black metropolitan population (56.1 percent) lived in central cities in 2000 . The South was home to nearly half of the Black metropolitan population, while the Hispanic metropolitan population, despite rapid growth in all regions, remained concentrated (43.7 percent) in the West at the end of the twentieth century. The Hispanic population living in the 100 largest metropolitan areas numbered just over 4 million in 1960 but increased to over 28 million by 2000. During that time the Hispanic population went from majority urban to majority suburban, as the population living outside central cities went from 37.5 percent in 1960 to 56.5 percent in 2000. 
Each census from 1960 to 2000 asked respondents to identify where they lived five years before that census. Comparing information about where respondents were living at the time of each census with where they had lived five years before reveals that migration from central cities to suburban areas increased every decade following the 1950s. That is, the percentage of people reporting that they lived in a central city five years before the census and then moved to a suburban area in the same metropolitan area by the time they filled out their census form increased from 10.3 percent in the period from 1955 to 1960 , to 12.8 percent from 1965 to 1970, and then steadily increased to 15.0 percent by the 2000 census. Of course, people were also moving in the other direction, from suburbs to central cities, but thus migration was smaller. Although the percentage of the people moving from suburbs to central cities rose from 2.1 percent during the period from 1955 to 1960 , to 3.6 percent from 1965 to 1970 , the rate fell thereafter to 3.1 percent by the 2000 census. The urban-suburban net migration rate compares the number of people moving from central cities to suburbs with those moving in the opposite direction. The urban-suburban net migration rate (migrants from suburbs to central cities minus migrants from central cities to suburbs per 1000 central city residents) remained fairly consistent throughout this period. The net migration rate for the general population from 1955 to 1960 was -69.4 per 1000 central city residents. That is, more people were moving from central cities to the suburbs than in the opposite direction, which results in a negative number. The outward migration even slightly increased to -74.0 by the 2000 census.

Between 1955 and 1960 Whites by far had the highest rates of suburbanization in the United States. This was the case in every region, although Hispanics had a higher urban-suburban net migration (-75.7) than Whites (-61.3) in the West. Of course, the Hispanic population was relatively small at this time. White suburbanization in the 1950 s was occurring the most rapidly in the Midwest, particularly in industrial centers, such as Cleveland, St. Louis and Detroit. In the following decade, white suburbanization accelerated in the South, as well as in Sunbelt cities, such as Las Vegas and Miami. Through the rest of the twentieth century, the highest rates of suburbanization among Whites continued to be in the Sunbelt, though the suburbanization of the White population continued in almost all of the 100 largest metro areas. In later decades suburbanization rates among the non-White population, particularly Hispanics and Asians, increased but White urban-suburban net migration was consistently higher than all other groups.

The suburbanization of the Black population changed dramatically in the second half of the twentieth century. Between 1955 and 1960, the flows of Black migrants between cities and suburbs were almost equal. In fact, in large metro areas in the South, more Blacks moved from suburbs to central cities than the other way around. This was the case in the Midwest in the following decade, as Black rates of suburbanization remained low. By the 1970s, however, the Black population began to moving out to the suburbs at increasing rates, although the Black urban-suburban net migration rate was substantially lower than among Whites in all regions except the West. The highest rates of Black suburbanization were in the same Sunbelt metro areas that saw rapid suburbanization of the White population in the later decades of the late-twentieth century, particularly Miami, Las Vegas and Orlando.

Even more striking are the varying rates of suburbanization by income. Using the poverty threshold to divide the population into low-income households (those living in poverty), middle-income households (not in poverty but less than three times the poverty threshold), and 
high-income households (incomes at least three times the poverty threshold), it's clear that suburbanization has been strongly correlated with income throughout the last half of the twentieth century. In every decade the lowest urban-suburban net migration rates are among the poorest population, and the highest rates of suburbanization are among the wealthiest households. This pattern is evident in every region of the United States, particularly in the Northeast and Midwest, and persisted until the end of the twentieth century. The South and West also follow this pattern, but by the end of the twentieth century, low-income households in the South and the West have somewhat higher rates of suburbanization than their counterparts in the Northeast and Midwest and show less disparity with more affluent households, particularly in the West.

The suburbanization of the low-income Black population has lagged far behind the general population. Between 1955 and 1960 slightly more low-income Blacks moved into central cities from suburbs than moved in the opposite direction. This "reverse" net migration actually increased during the following decade, from 0.3 to 6.5 per 1000 Black central city residents. In the 1970 s, however, the low-income Black population began to suburbanize, though at modest rates compared to other groups. Affluent Whites, by contrast, have consistently been the most rapidly suburbanizing population. From 1955 to 1960 the urban-suburban net migration rate of affluent Whites was -98.9 , and by the end of the $20^{\text {th }}$ century the urban-suburban net migration rate among Whites had risen to -103.5. In later decades the suburbanization rates of affluent Hispanics and Asians had risen to levels comparable to the affluent White population. Though affluent Blacks were suburbanizing faster than less affluent Blacks, the urban-suburban net migration rate among affluent Blacks continued to trail affluent households in other groups.

\section{Conclusion}

In the second half of the twentieth century, the suburban population in the 100 largest metropolitan areas grew rapidly. Much of this growth was from people moving from central cities to areas outside the urban core. Though all groups contributed to suburban population growth, affluent people were more likely to migrate from central cities to suburbs than households with lower incomes. A greater share of the White non-Hispanic population lived in suburbs throughout this period, and the Black non-Hispanic population was the most concentrated in urban core areas. Other than the low-income Black population, which had a net in-migration to central cities in the 1950s and 1960s, people of all groups migrated at greater rates to the suburbs than to central cities. The low-income black population showed this same pattern beginning in the 1970s. Hispanics and Asians, particularly those with high incomes, migrated rapidly to the suburbs in the later decades of the twentieth century, and their numbers approached those of the White population by the end of the century. All racial/ethnic groups other than Blacks were majority suburban by 2000. 
Urban and Suburban Residence, 1955-1995, and 5-year migration

\section{The United States}

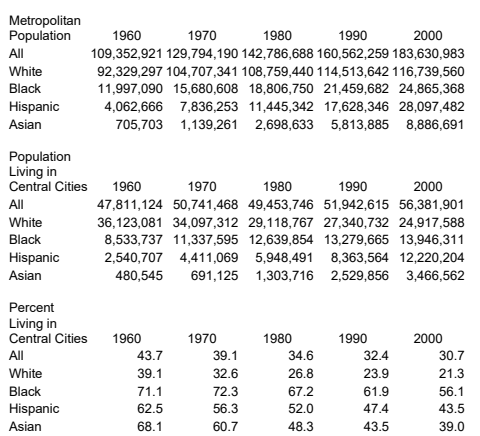

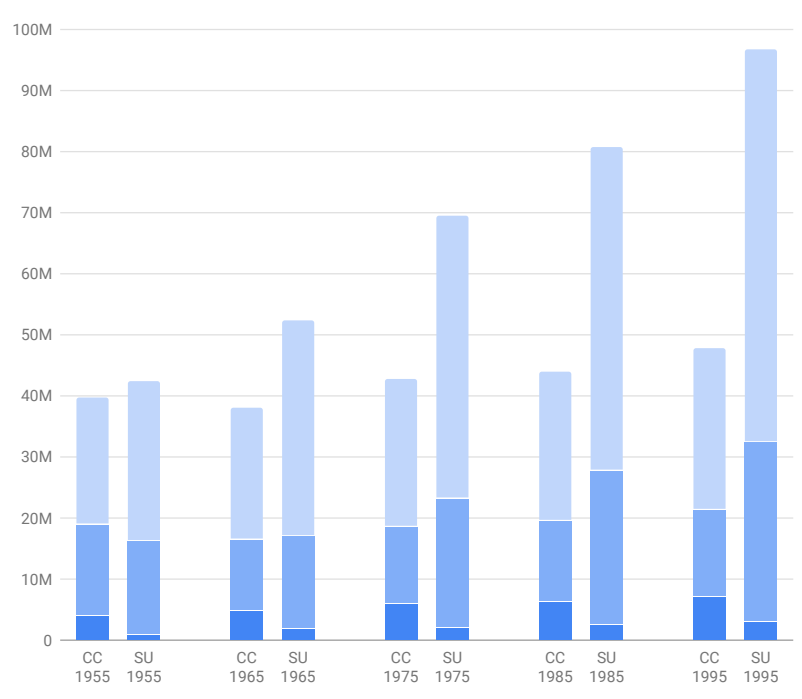

Central City (CC)/Suburban (SU) Residence in Year
Migrants from central city to suburbs or suburbs to central city in next five years

Migrants within central city or within suburban area in next five years

Nonmovers (lived in the same house five years later)
Urban-Suburban Net Migration Rate by Race

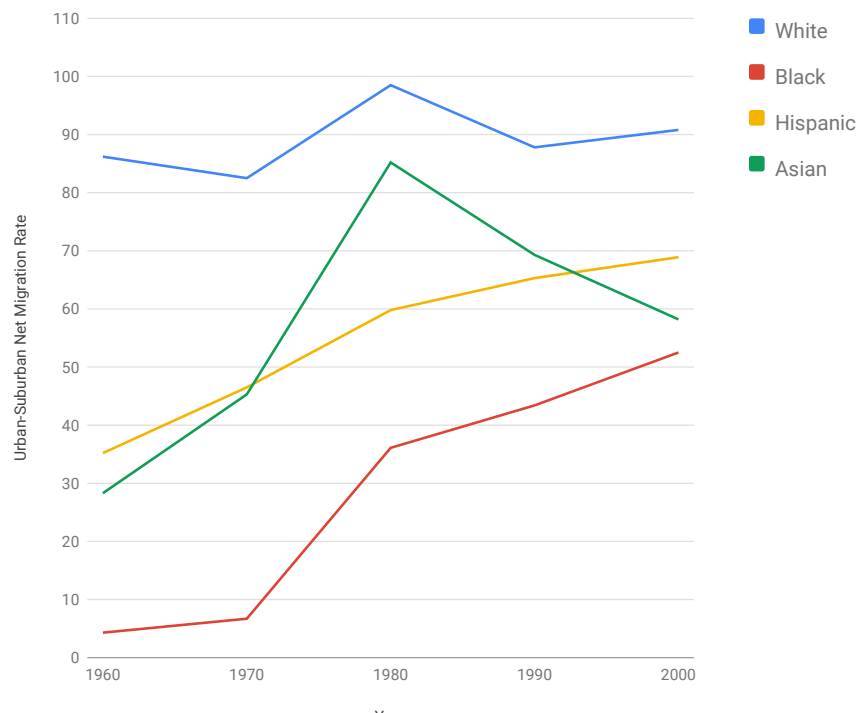

Low-Income Persons by Race

Middle-Income Persons by Race
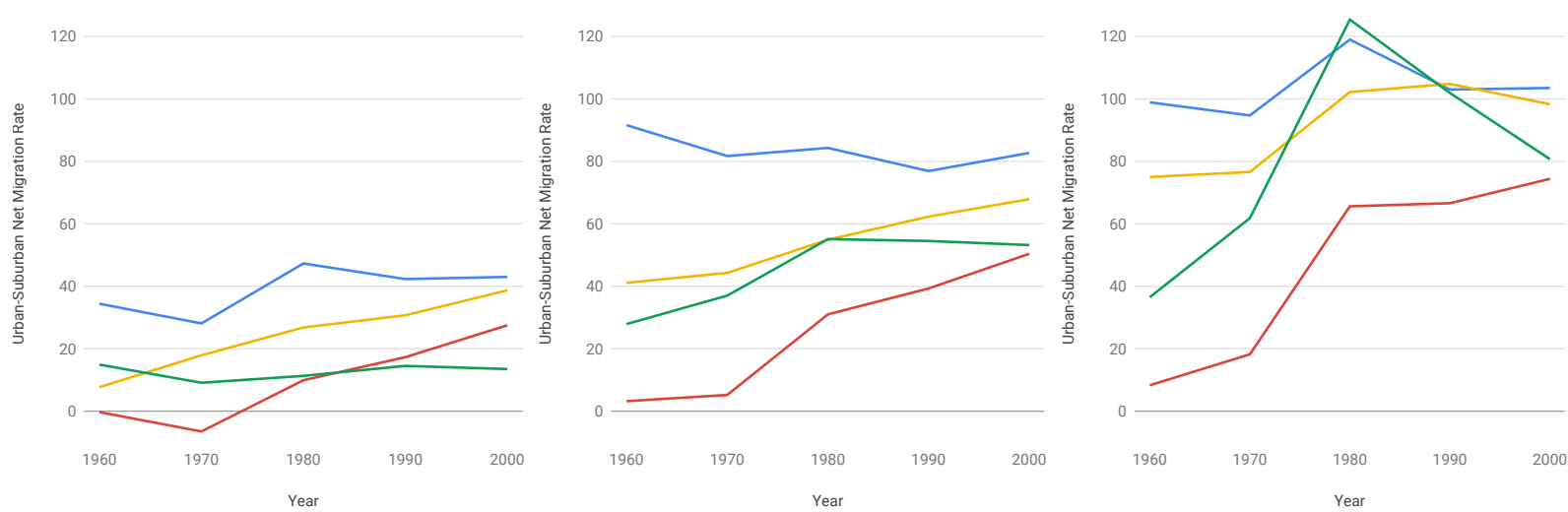

Year

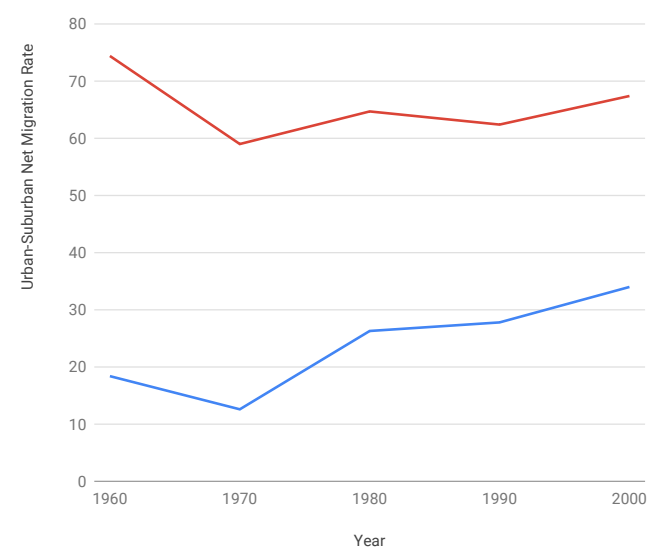

High-Income Persons by Race
Low Income

- Middle Income

High Income
White

- Black

Hispanic

- Asian 
Urban and Suburban Residence, 1955-1995, and 5-year migration

$25 \mathrm{M}$

\section{The Northeast Region}

$\begin{array}{llllll}\text { Metropolitan } & 1960 & 1970 & 1980 & 1990 & 2000 \\ \text { Population } & 1900 & \end{array}$

$\begin{array}{ll}\text { All } & 36,241,08940,086,56839,387,44240,551,25542,879,506 \\ \text { White } & 32,199,28533,895,78931,698,86630,707,08629,469,543\end{array}$

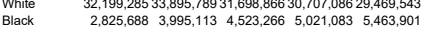

$\begin{array}{llllll}\text { Hispanic } & 1,103,915 & 1,948,541 & 2,501,225 & 3,457,933 & 4,932,242\end{array}$

Asian $\quad \begin{array}{lllll}75,920 & 181,718 & 516,447 & 1,207,073 & 1,987,625\end{array}$

Population
Living in

$\begin{array}{llllll}\text { Living in } & & & & & \\ \text { Central Cities } & 1960 & 1970 & 1980 & 1990 & 2000\end{array}$

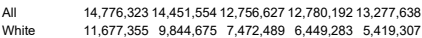

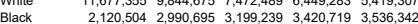

$\begin{array}{lrrrrr}\text { Hispanic } & 903,318 & 1,460,995 & 1,728,616 & 2,213,838 & 2,867,567 \\ \text { Asian } & 55,391 & 122,294 & 282,348 & 621,640 & 971,724\end{array}$

$\begin{array}{lccccc}\begin{array}{l}\text { Percent } \\ \text { Living in }\end{array} & & & & & \\ \text { Central Cities } & 1960 & 1970 & 1980 & 1990 & 2000 \\ \text { All } & 40.8 & 36.1 & 32.4 & 31.5 & 31.0\end{array}$

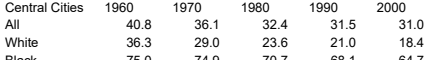

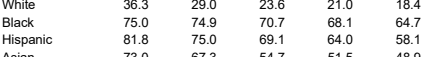

$20 \mathrm{M}$

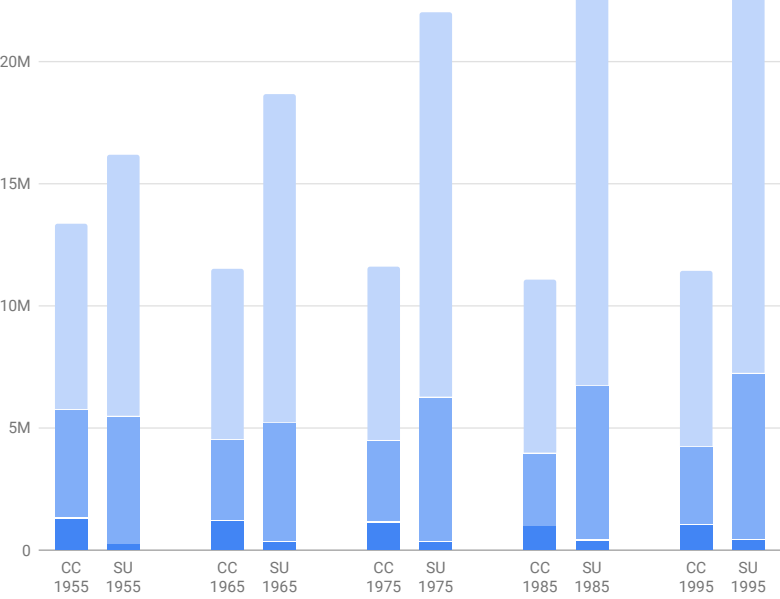

Central City (CC)/Suburban (SU) Residence in Year
Migrants from central city to suburbs or - suburbs to central city in next five years

Migrants within central city or within

suburban area in next five years

Nonmovers (lived in the same house five years later)
Urban-Suburban Net Migration Rate by Race

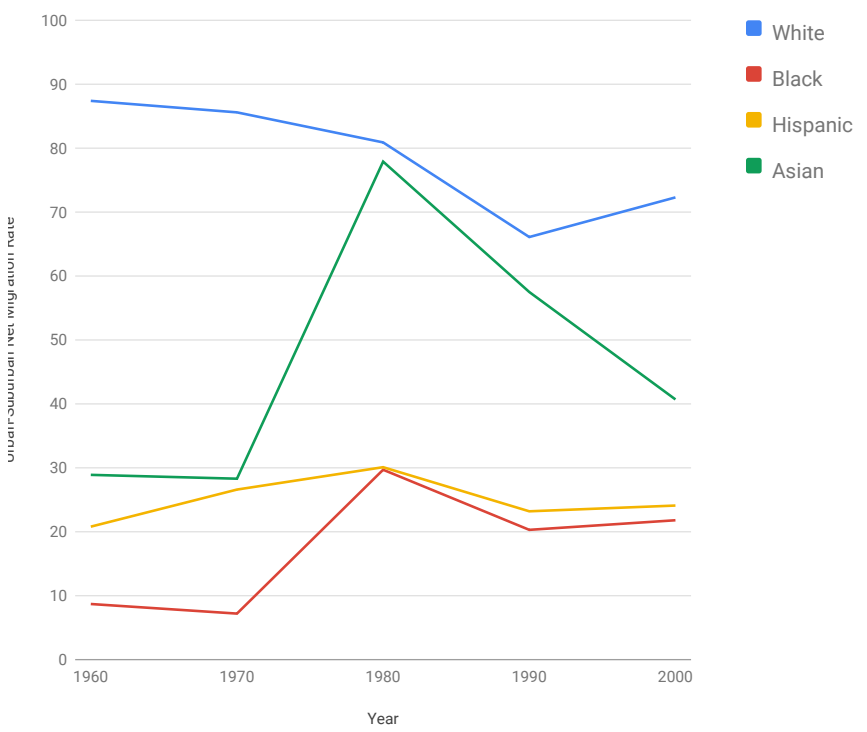

Low-Income Persons by Race

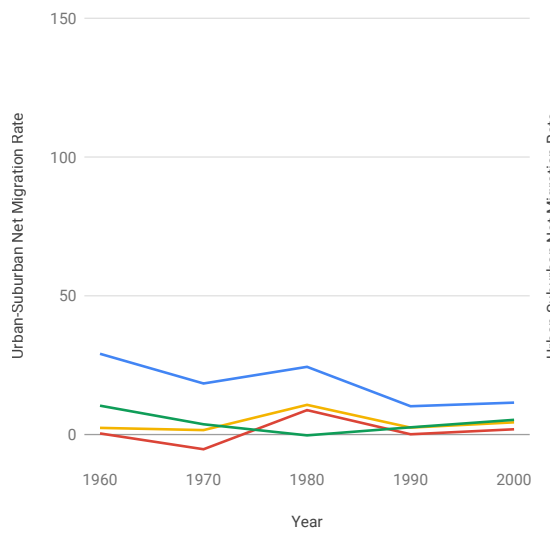

150

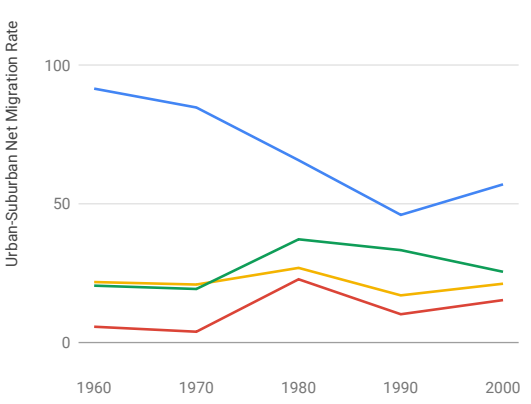

Year
Urban-Suburban Net Migration Rate by Income

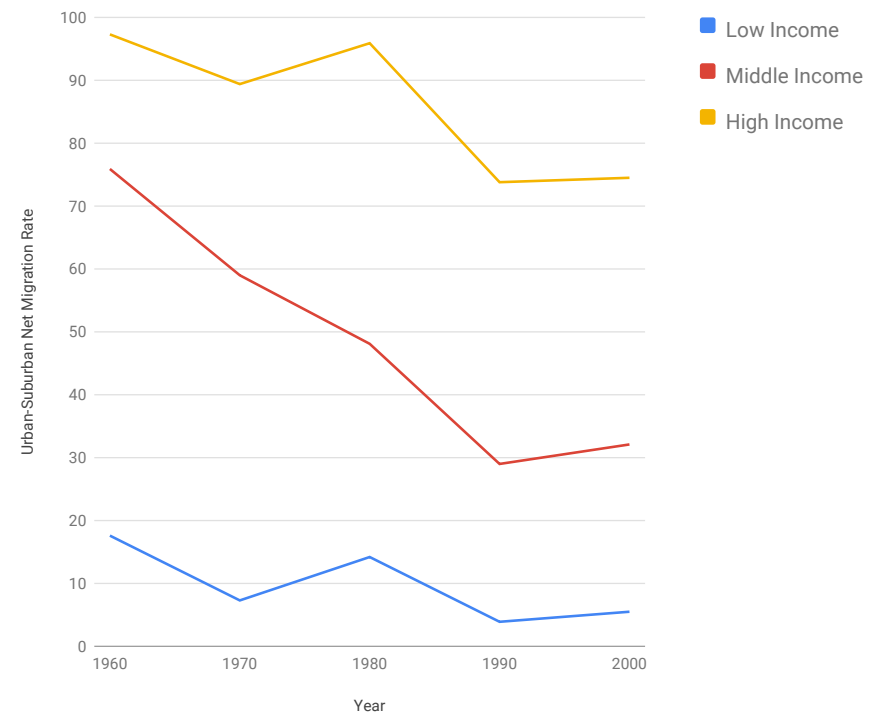

High-Income Persons by Race

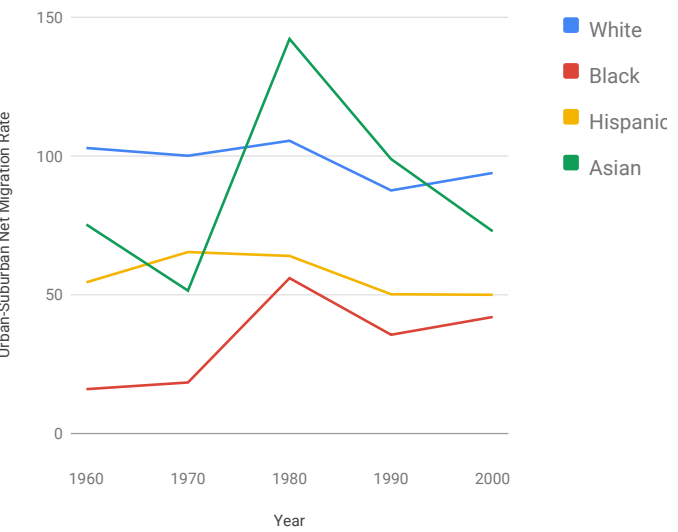


The Midwest Region Urban and Suburban Residence, 1955-1995, and 5-year migration

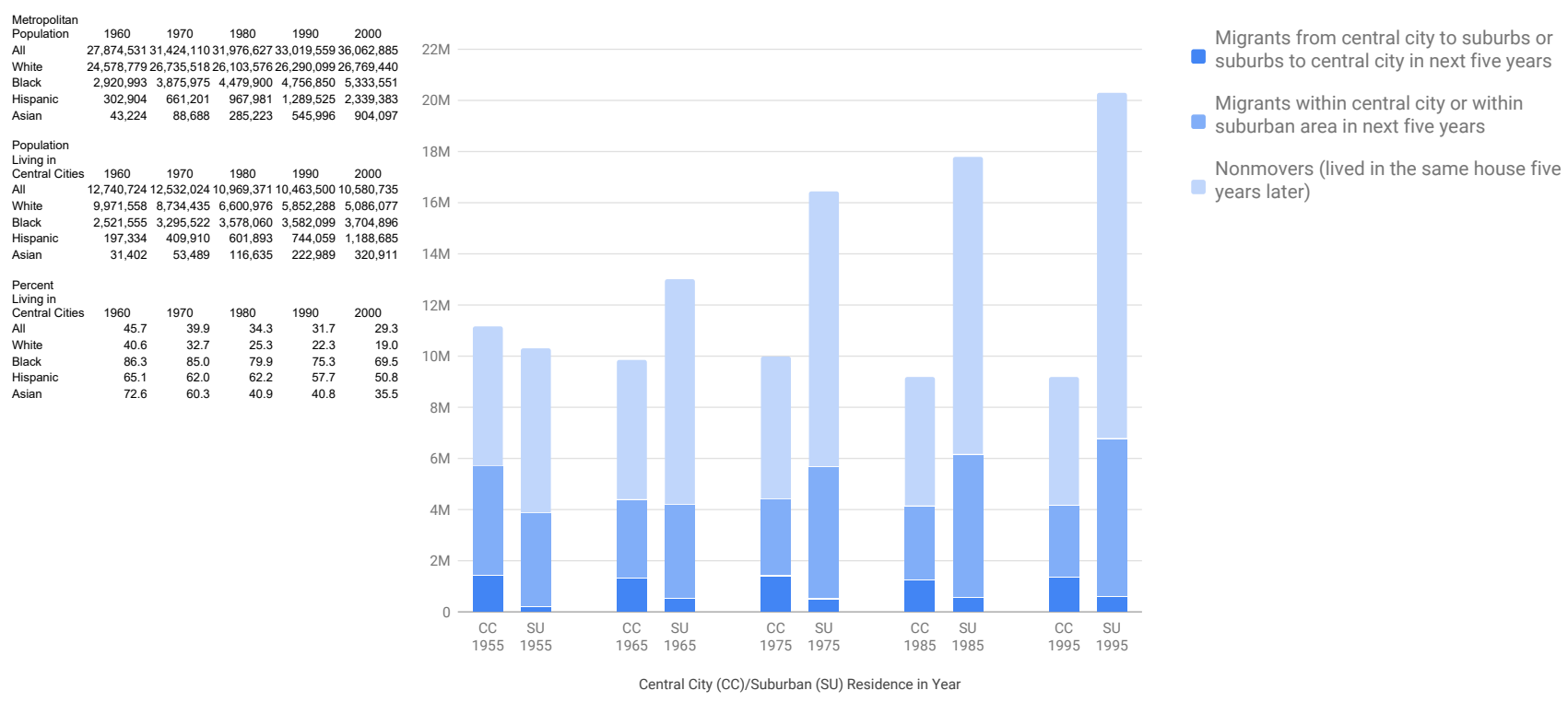

Urban-Suburban Net Migration Rate by Race
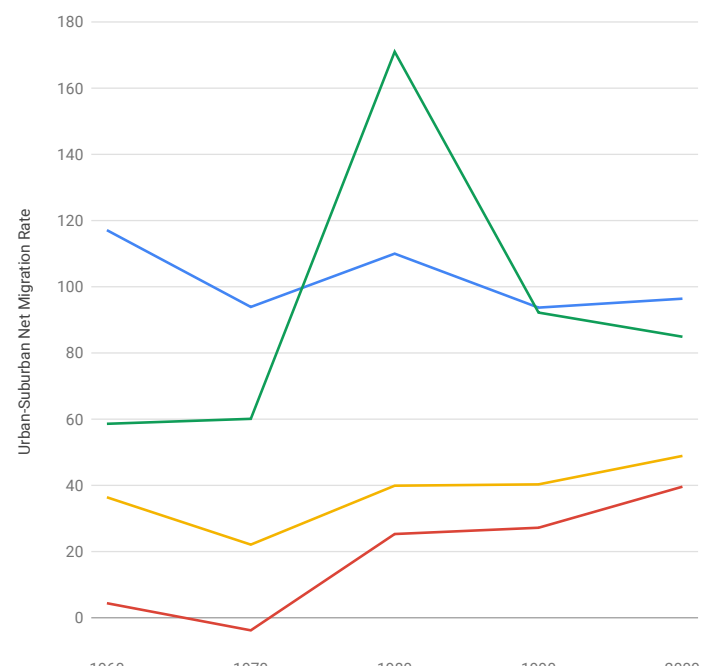

1960

Year
White

Black

Hispanic

- Asian

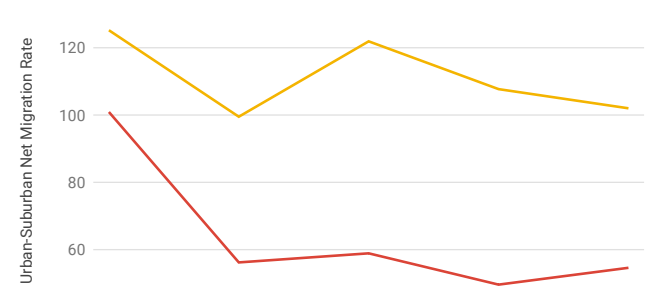

40

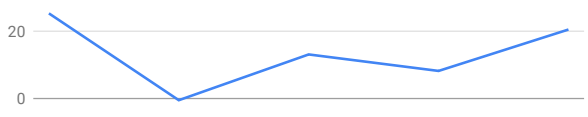

1960
2000

Year
Low-Income Persons by Race

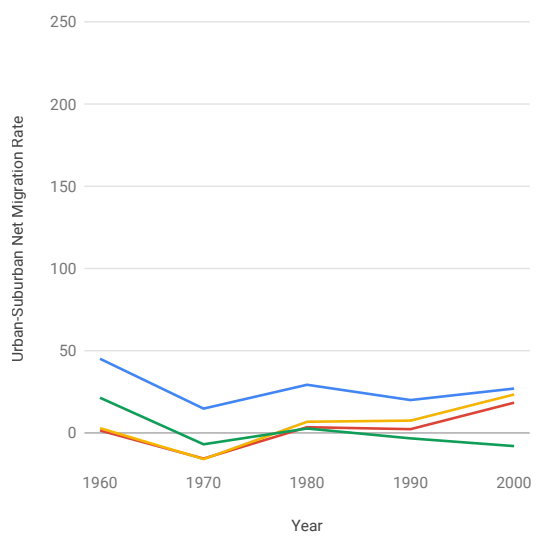

Middle-Income Persons by Race

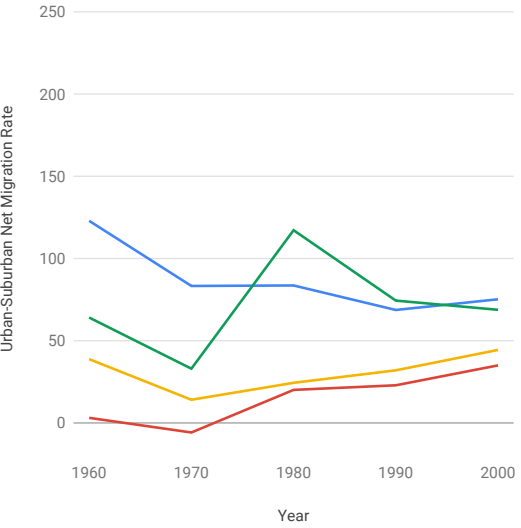

High-Income Persons by Race

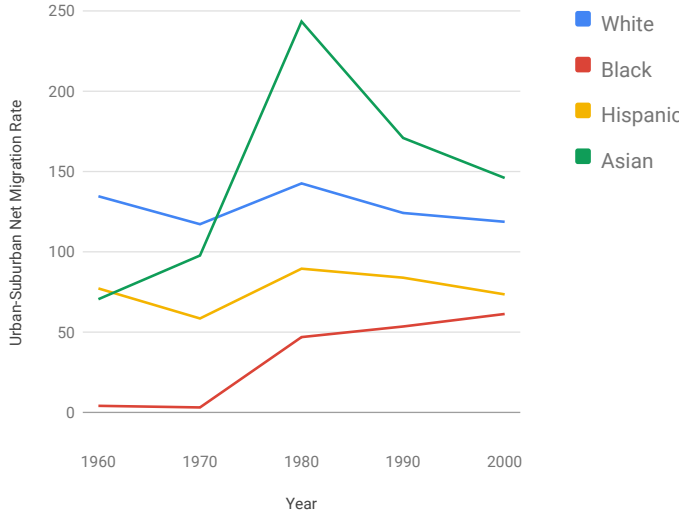


The South Region

Urban and Suburban Residence, 1955-1995, and 5-year migration

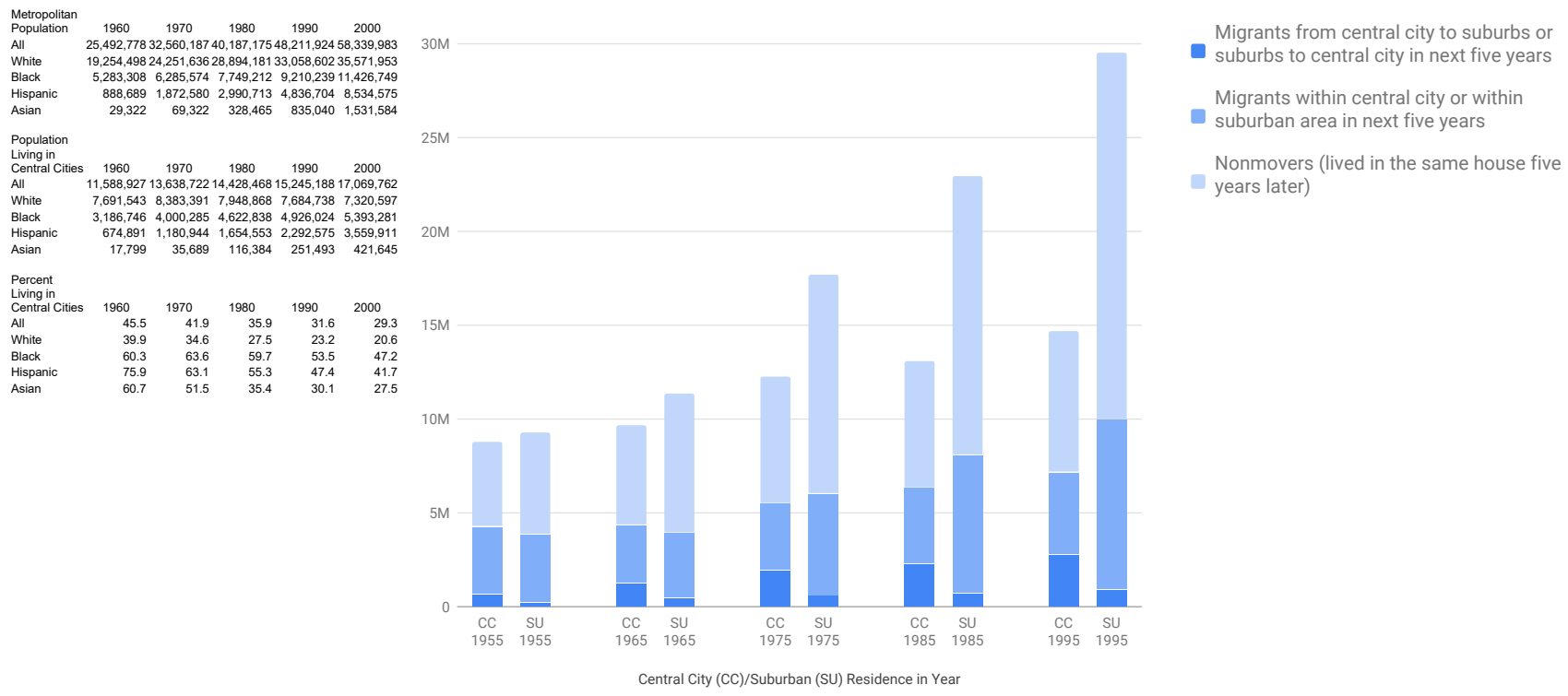

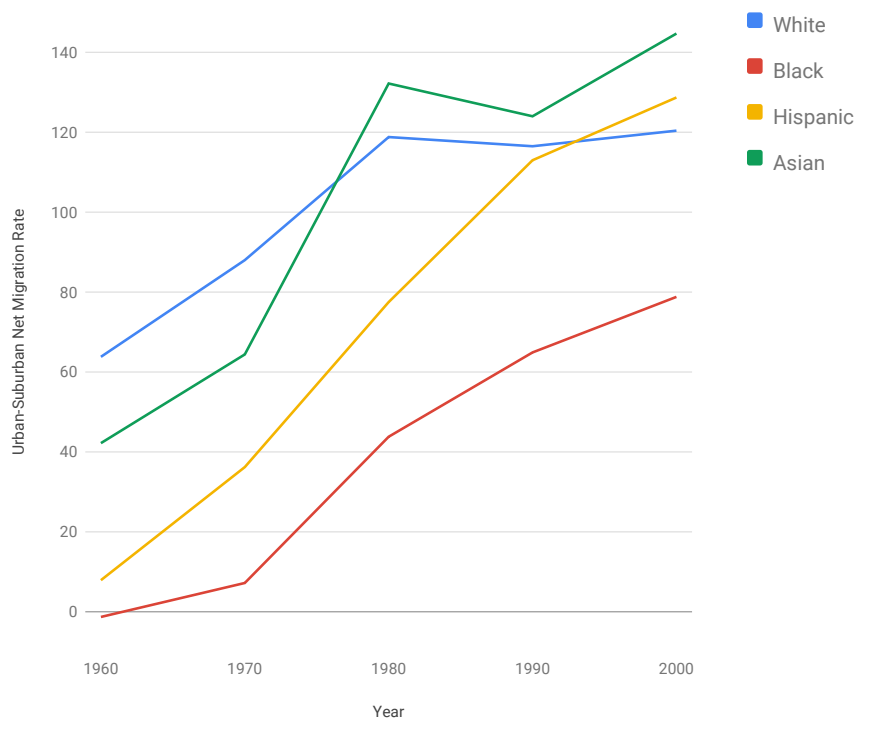

Low-Income Persons by Race

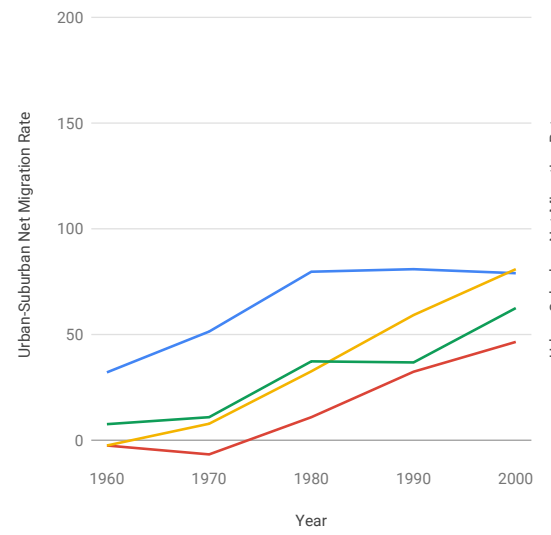

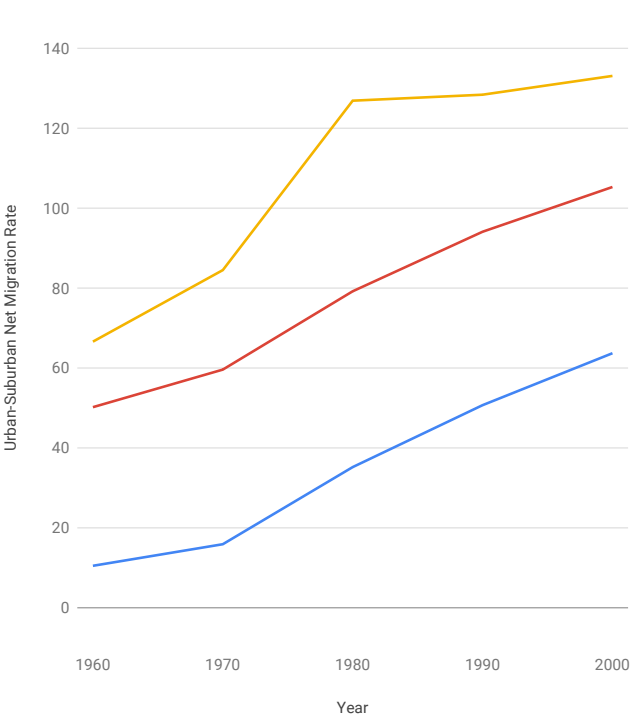

Low Income

- Middle Income

High Income

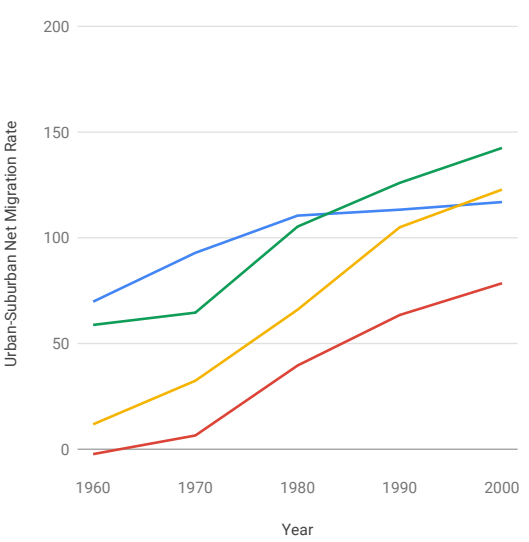

Year
High-Income Persons by Race

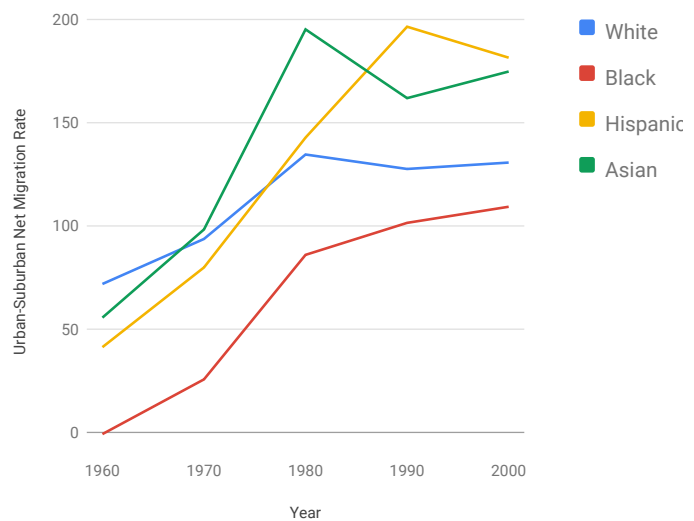


The West Region

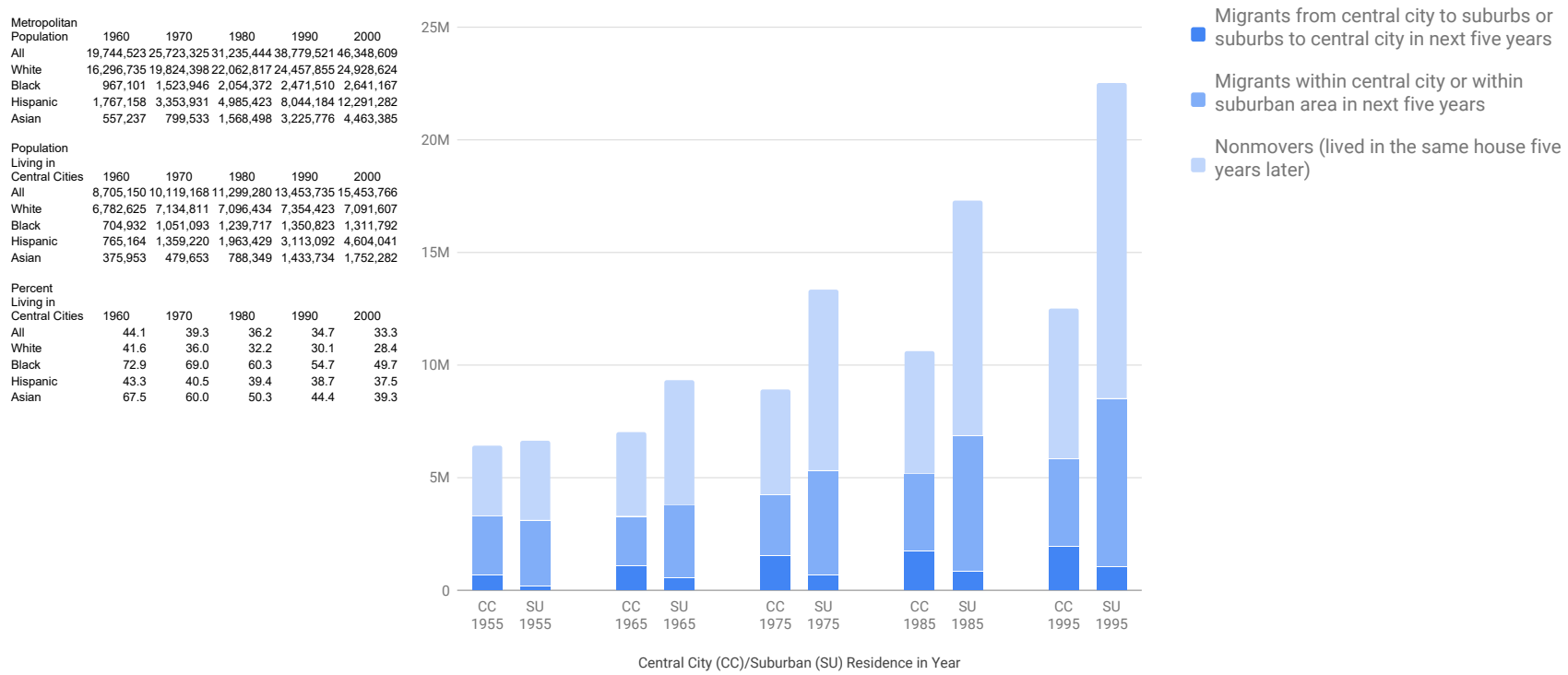

Urban-Suburban Net Migration Rate by Race

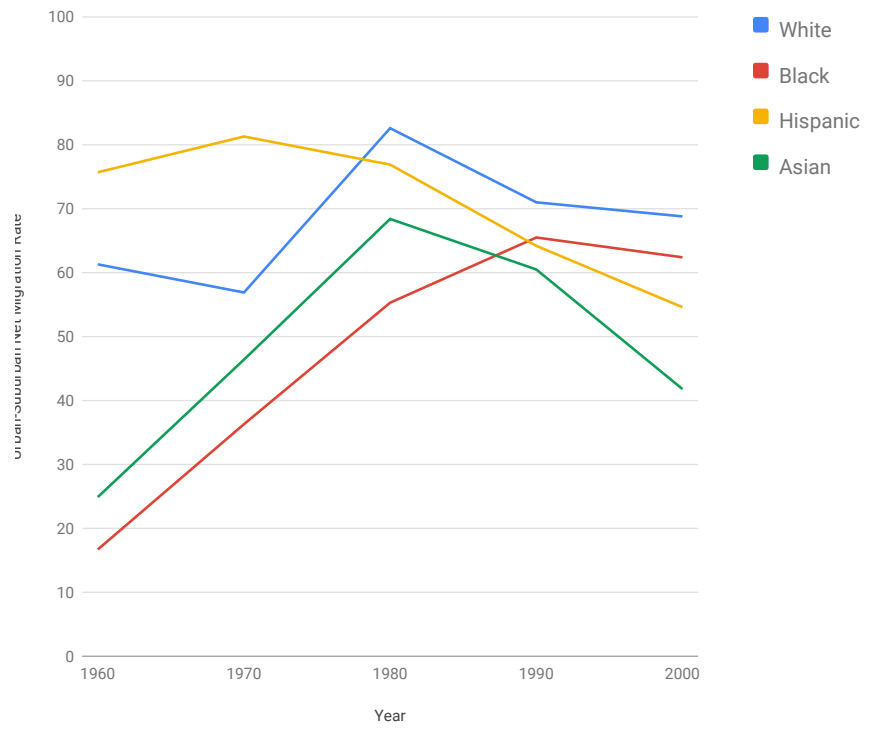

Low-Income Persons by Race

Middle-Income Persons by Race
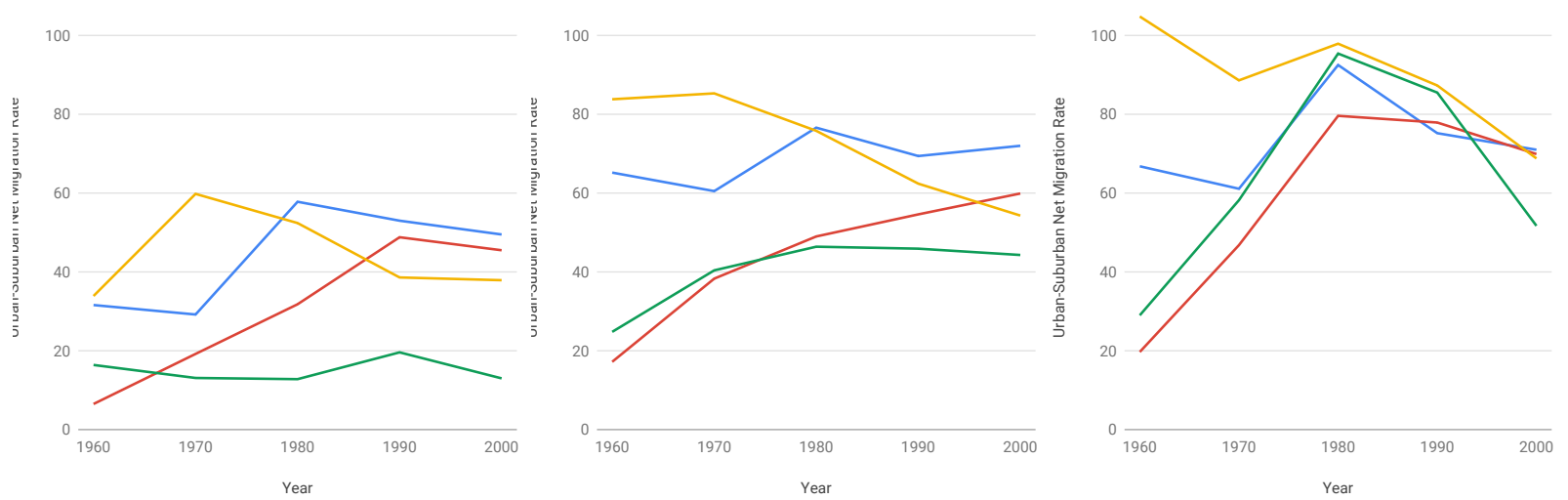

White

- Black

Hispanic

- Asian 
Urban and Suburban Residence, 1955-1995, and 5-year migration

$400 \mathrm{~K}$

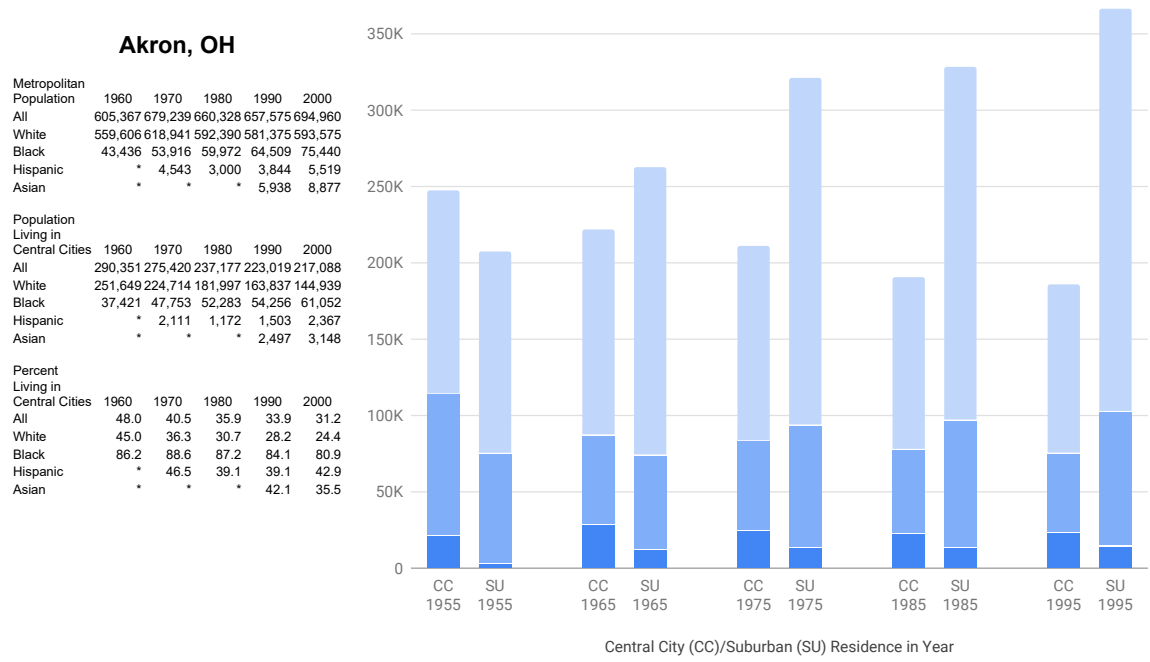

Migrants from central city to suburbs or suburbs to central city in next five years

Migrants within central city or within suburban area in next five years

Nonmovers (lived in the same house five
years later)
Urban-Suburban Net Migration Rate by Race

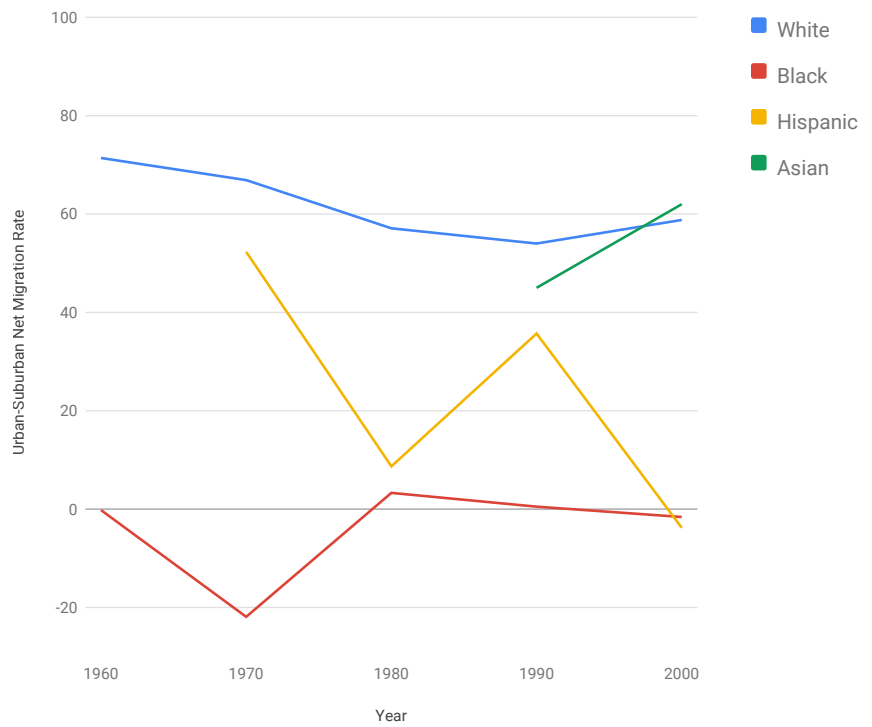

Low-Income Persons by Race

Middle-Income Persons by Race
Urban-Suburban Net Migration Rate by Income
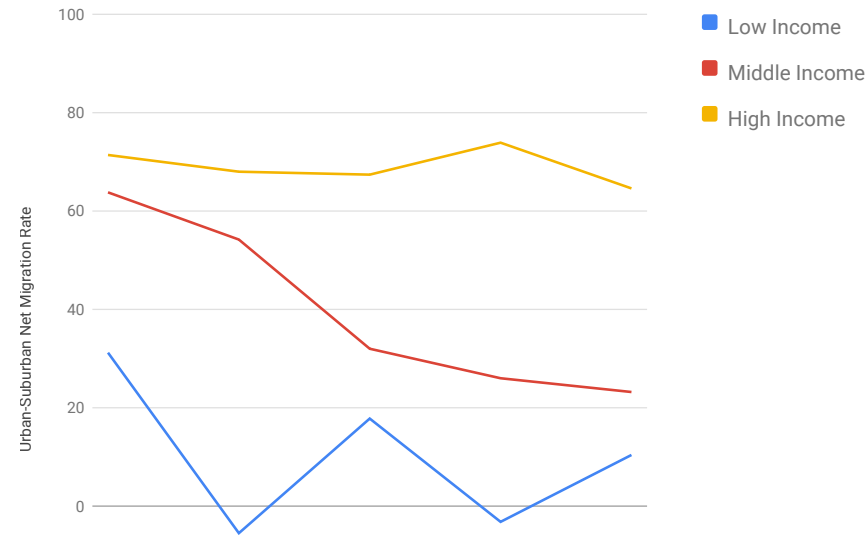

$-20$

$\begin{array}{lllll}1960 & 1970 & 1980 & 1990 & 2000\end{array}$

Year

High-Income Persons by Race

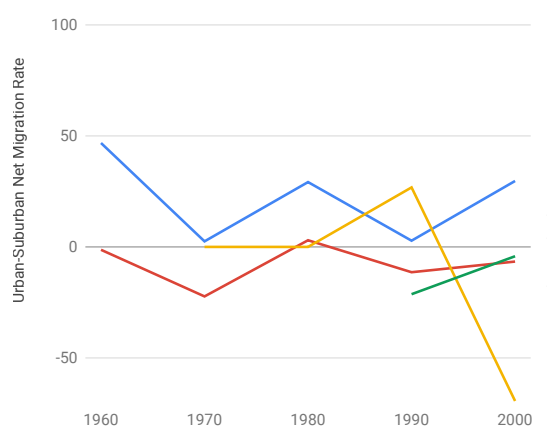

Year
100

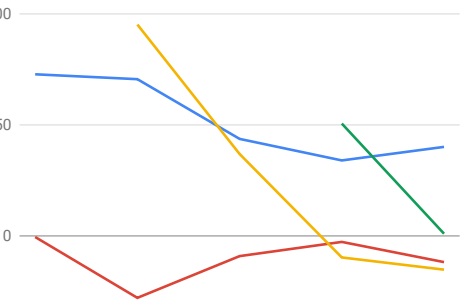

$1960 \quad 1970 \quad 1980$

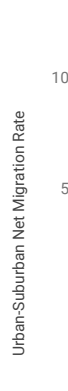

$-50$
White

- Black

Hispanic

- Asian 
Urban and Suburban Residence, 1955-1995, and 5-year migration

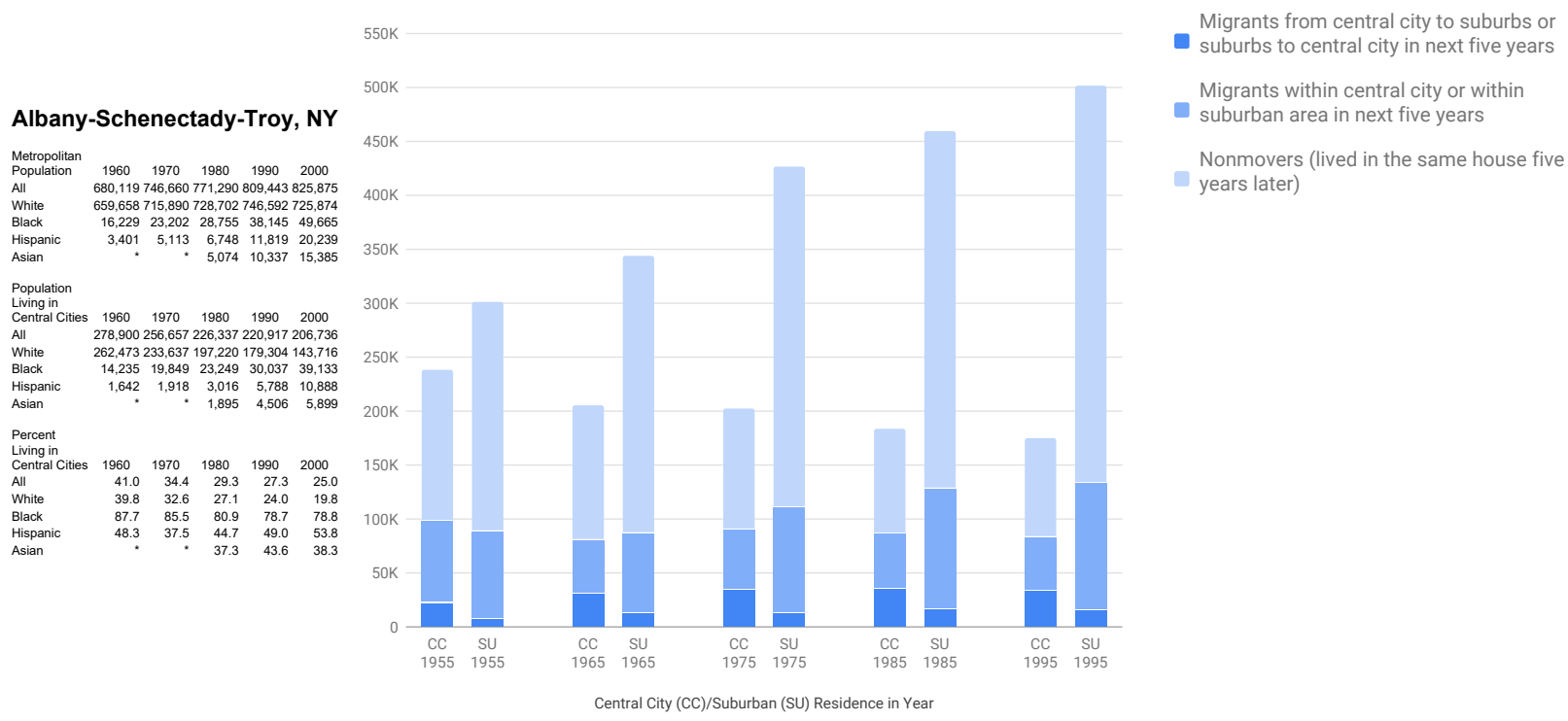

Urban-Suburban Net Migration Rate by Race

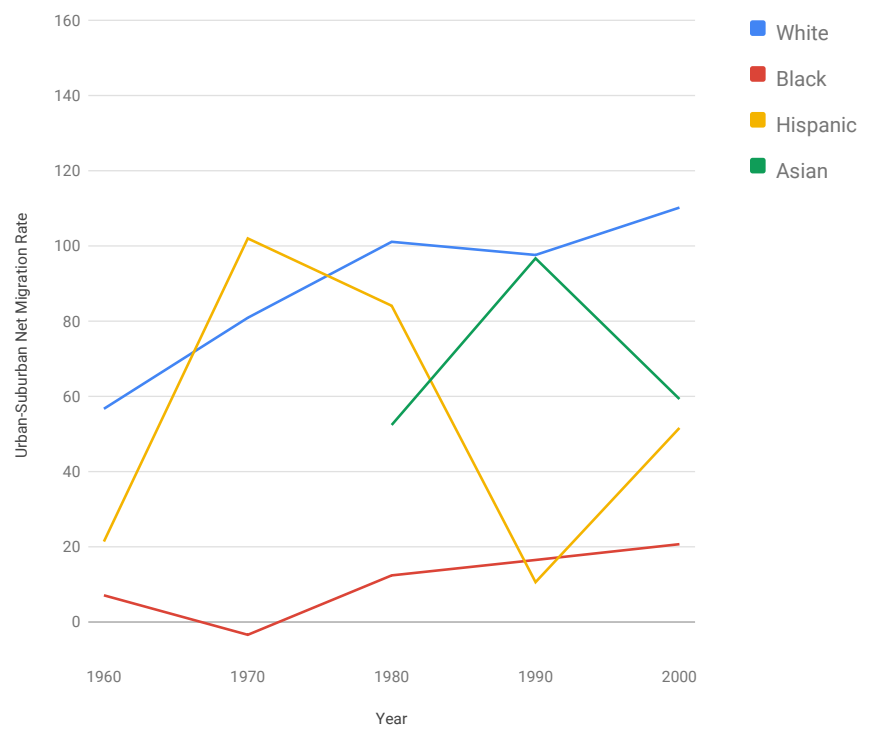

Low-Income Persons by Race

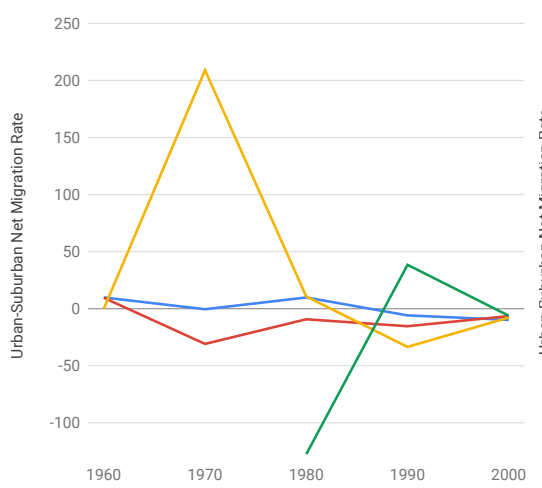

Year
Urban-Suburban Net Migration Rate by Income

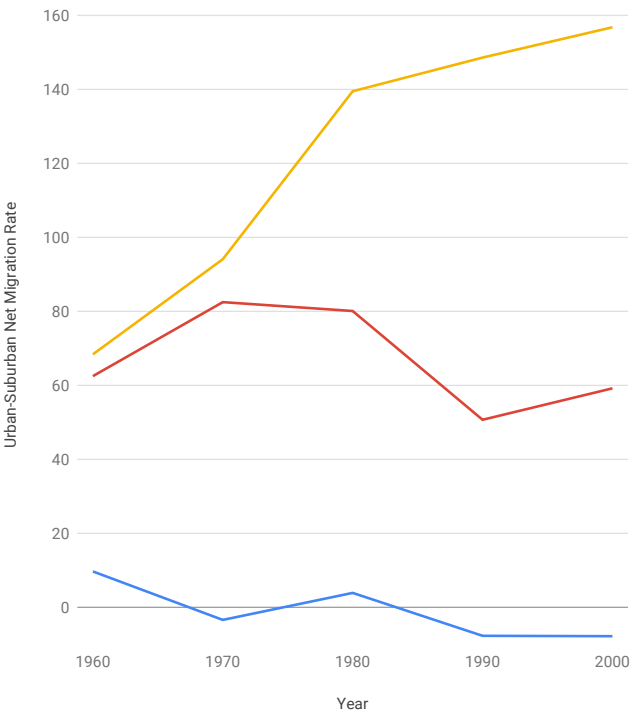

Low Income

- Middle Income

High Income
Middle-Income Persons by Race

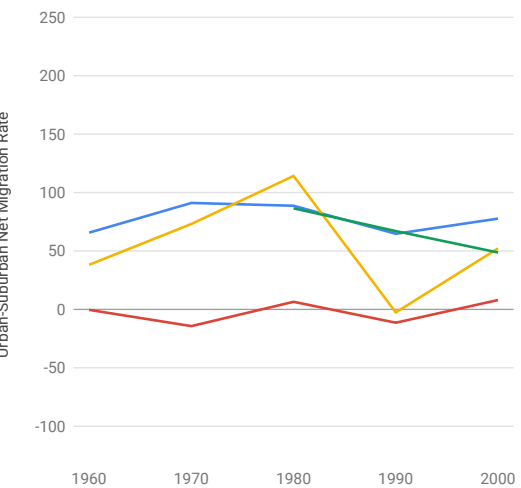

Year
High-Income Persons by Race

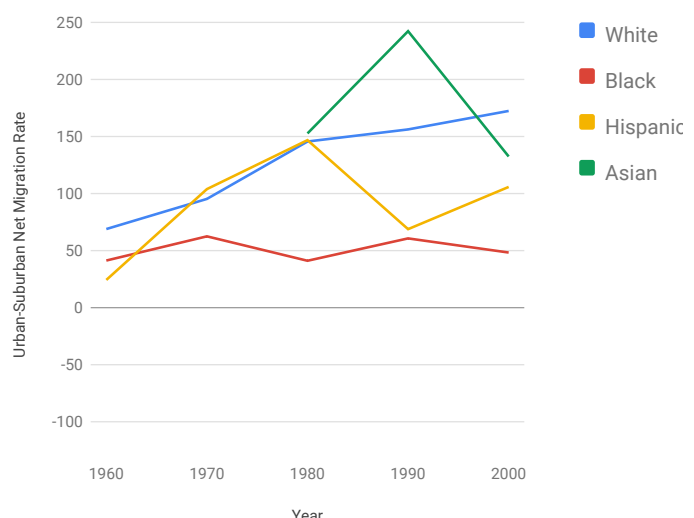


Albuquerque, NM

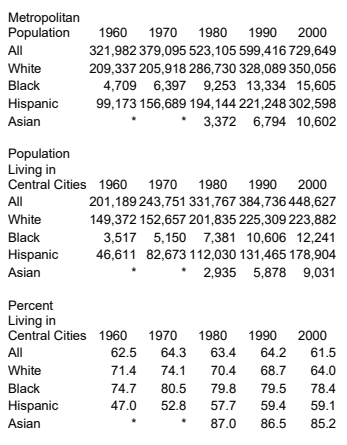

Urban and Suburban Residence, 1955-1995, and 5-year migration

$400 \mathrm{~K}$ $350 k$

$300 \mathrm{~K}$ 250

$$
200 \mathrm{~K}
$$

Urban-Suburban Net Migration Rate by Race

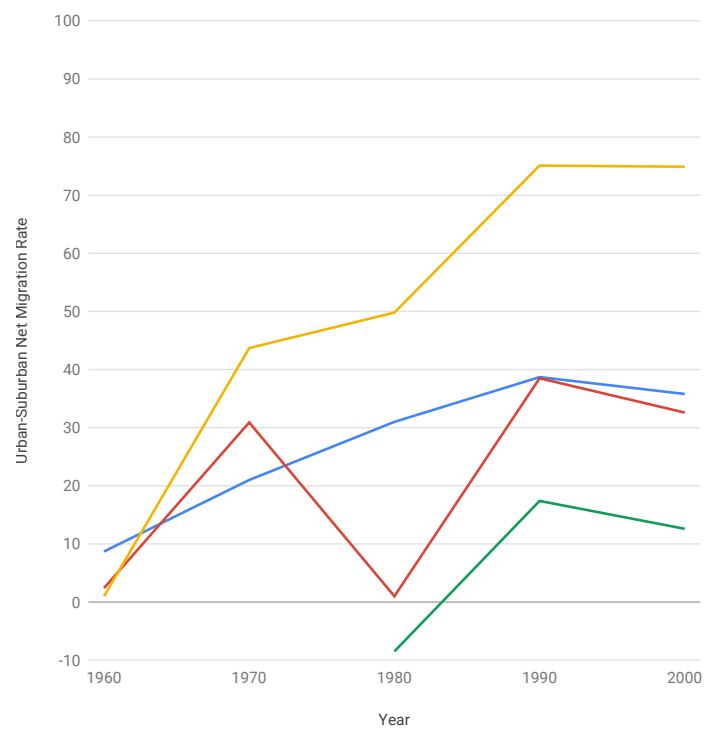

Urban-Suburban Net Migration Rate by Income

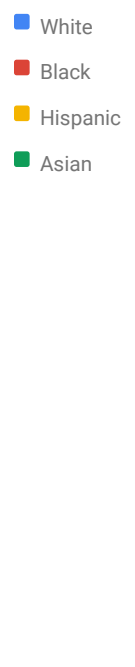

100

90

80
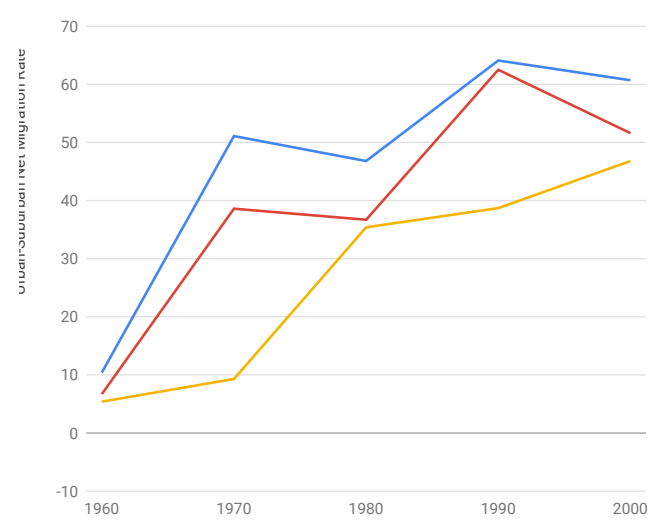

Year
Migrants from central city to suburbs or - suburbs to central city in next five years

Migrants within central city or within

suburban area in next five years

Nonmovers (lived in the same house five

years later)
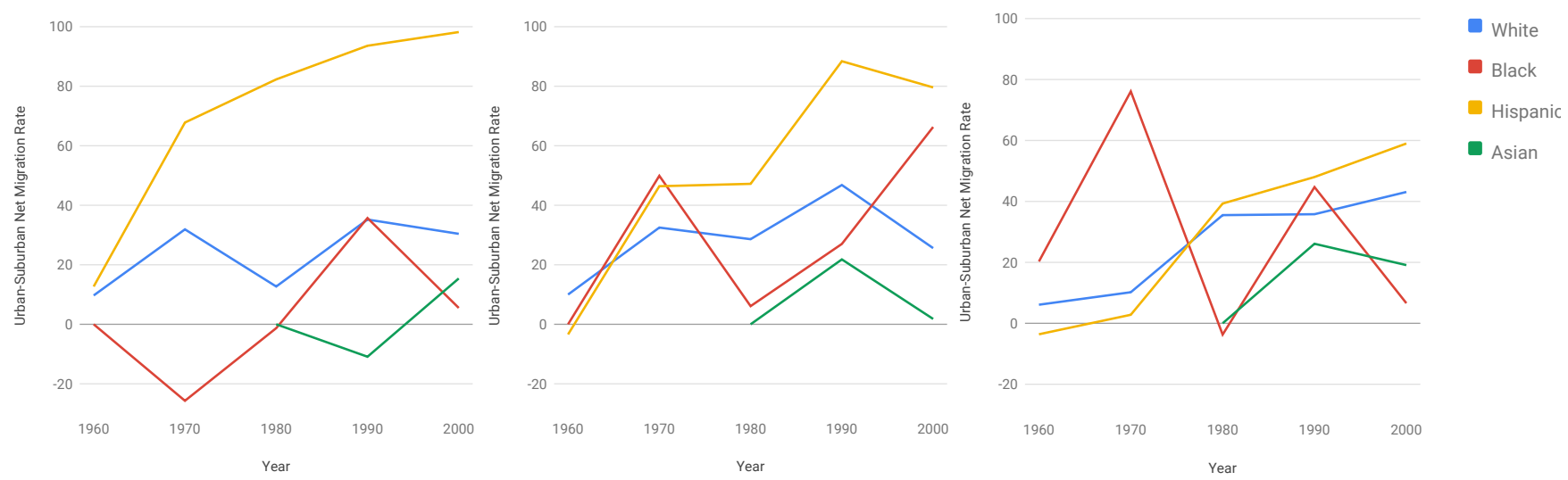
Allentown-Bethlehem-Easton, PA-NJ Urban and Suburban Residence, 1955-1995, and 5-year migration

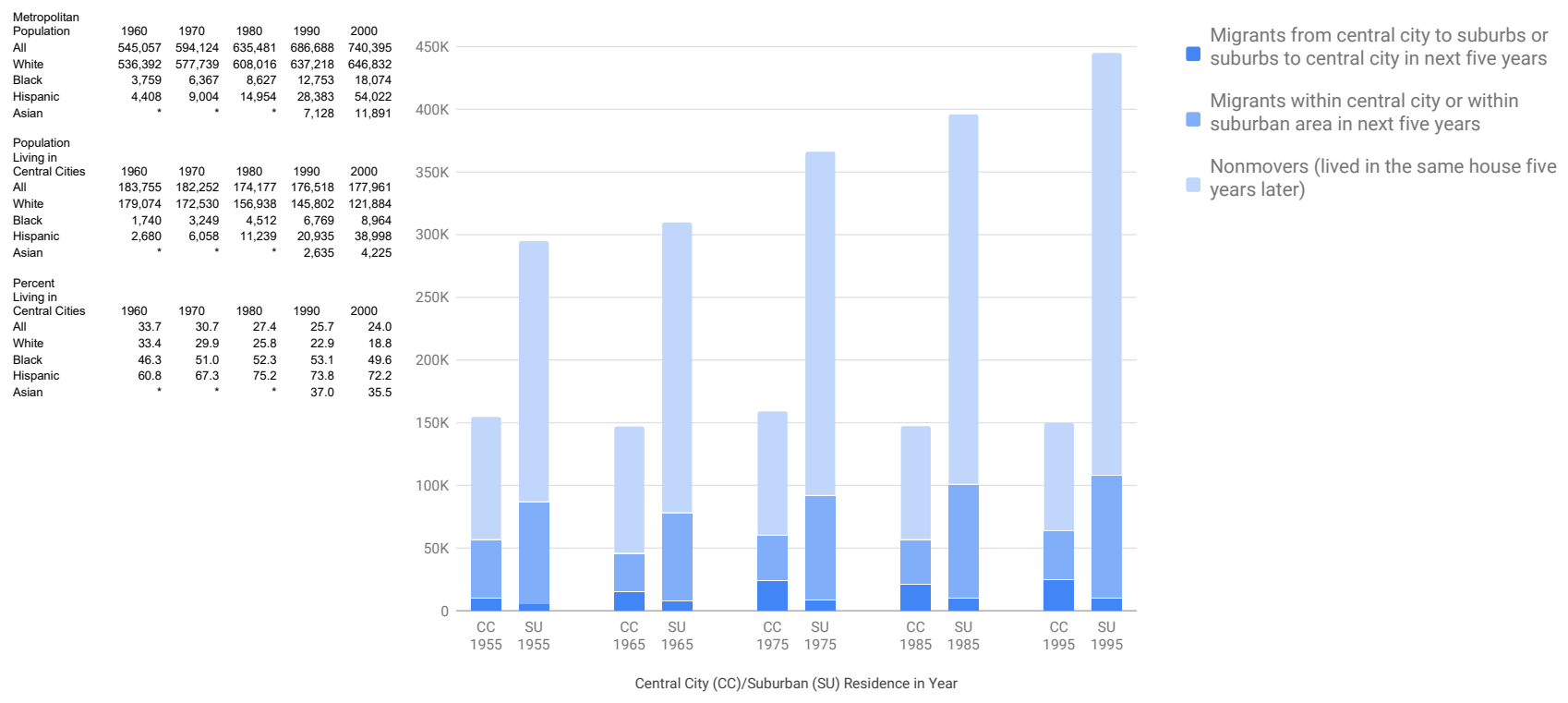

Urban-Suburban Net Migration Rate by Race

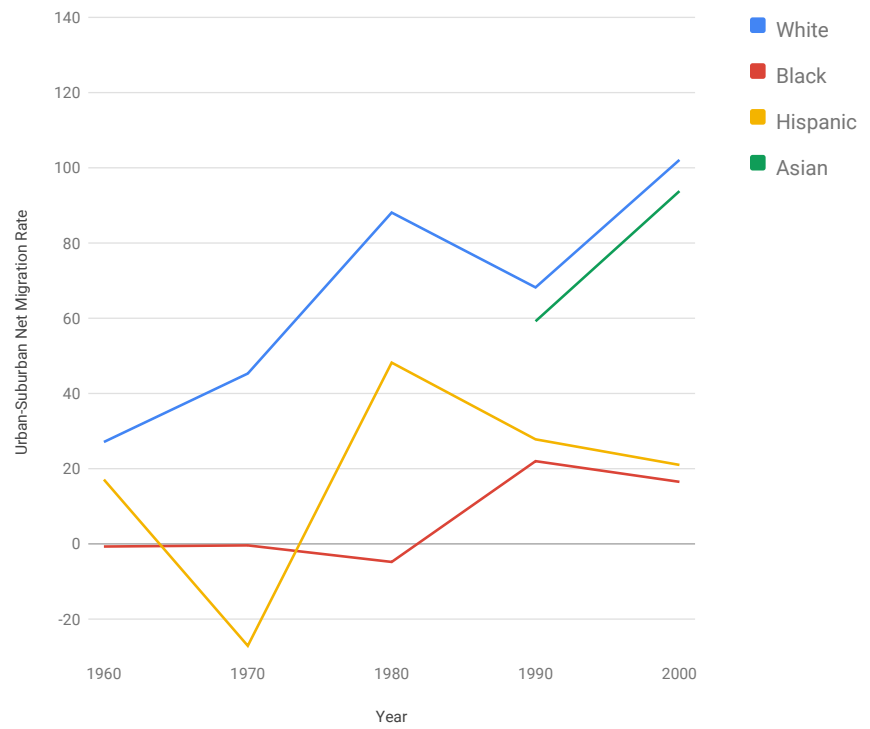

Low-Income Persons by Race

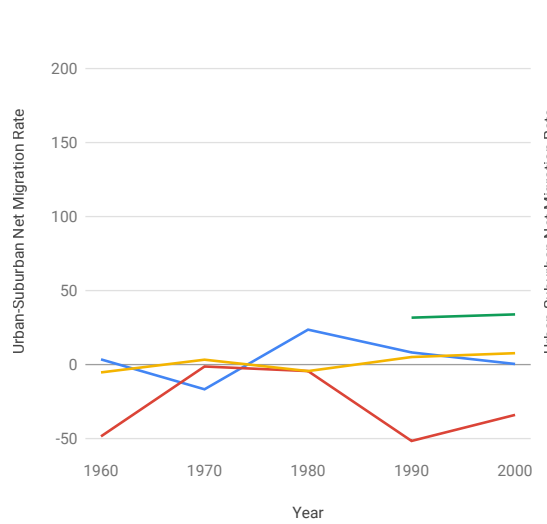

Middle-Income Persons by Race
Urban-Suburban Net Migration Rate by Income

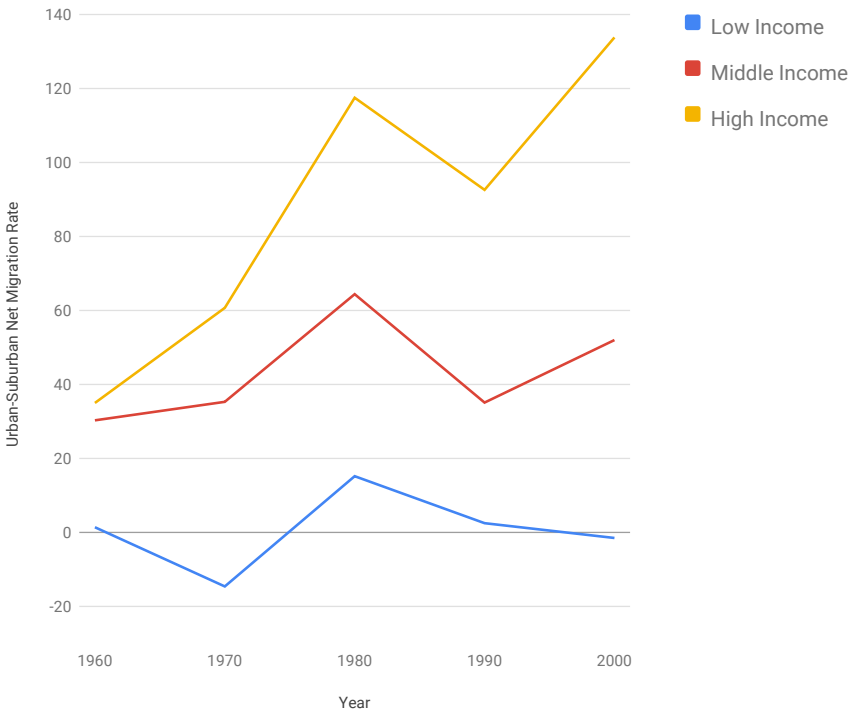

High-Income Persons by Race

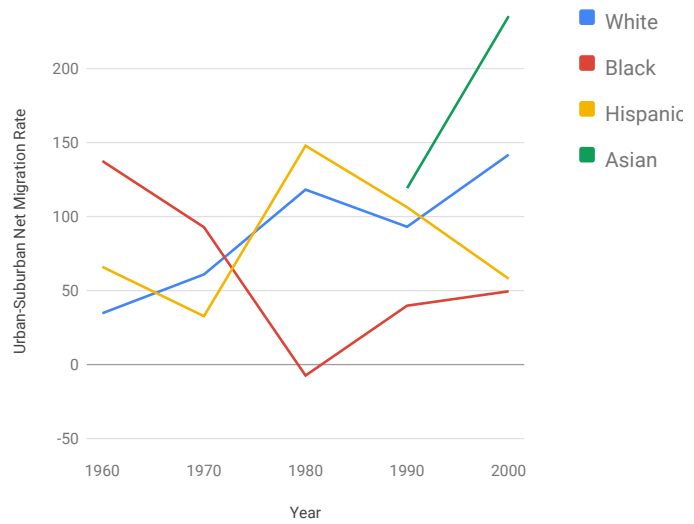




\section{Atlanta, GA}

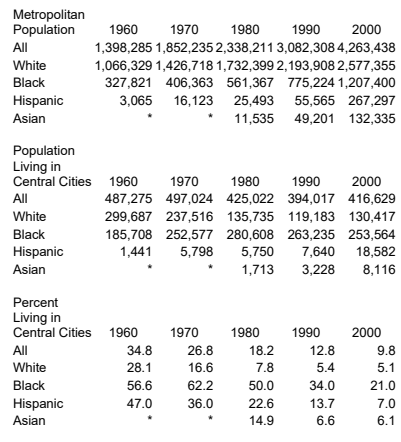

Urban and Suburban Residence, 1955-1995, and 5-year migration

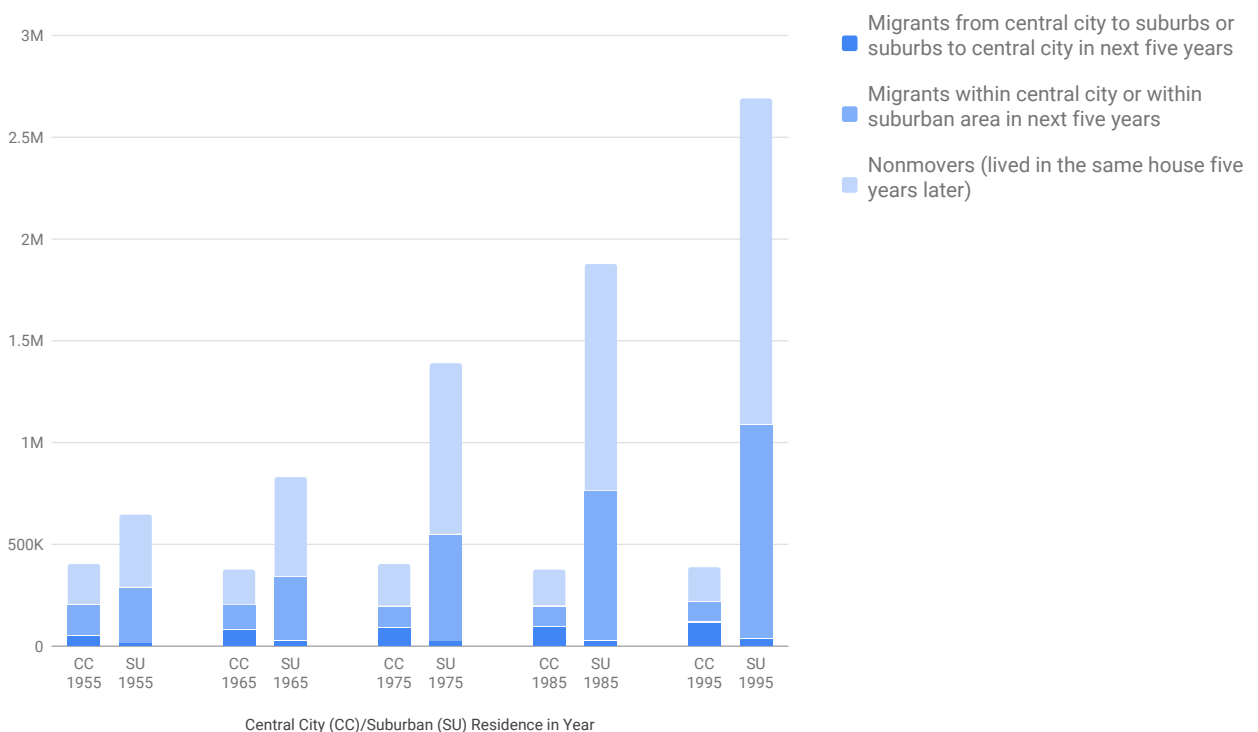

Urban-Suburban Net Migration Rate by Income

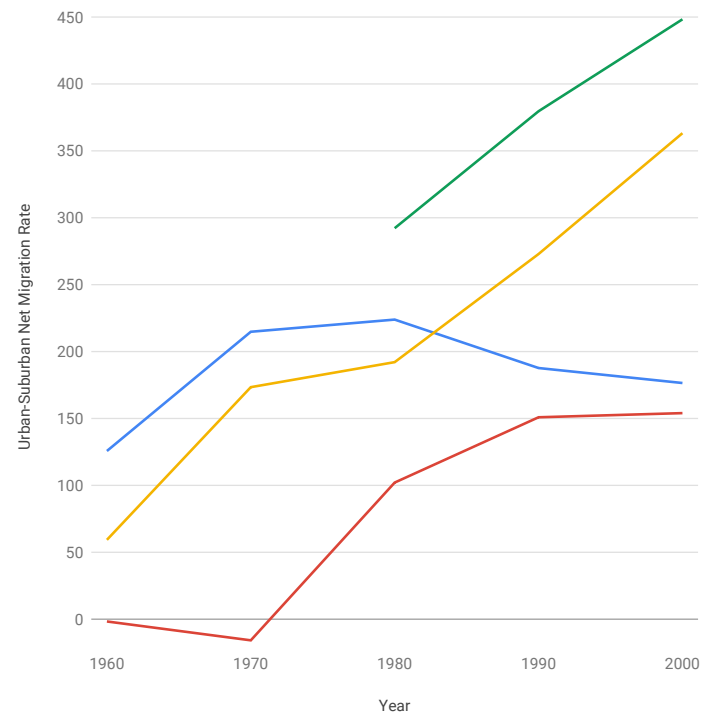

Low-Income Persons by Race
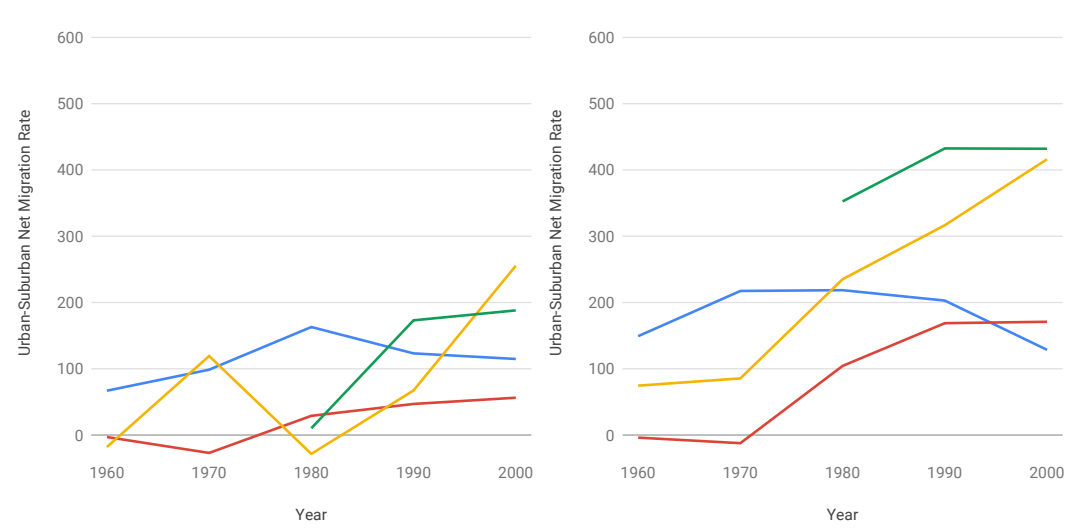

White

Black

Hispanic

- Asian

$$
450
$$$$
400
$$

350
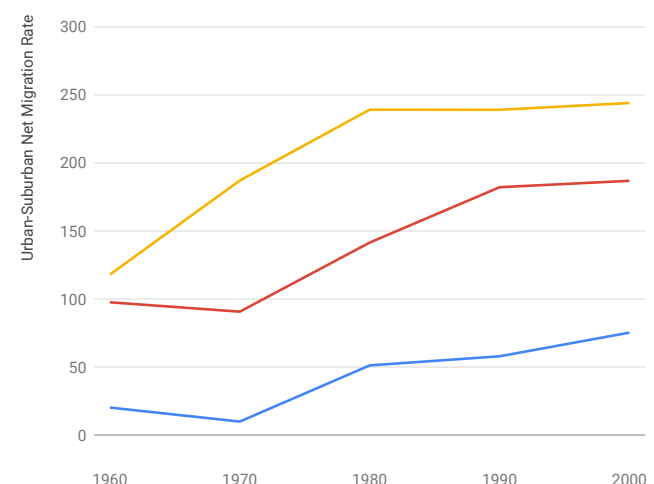

Year
Middle-Income Persons by Race
Low Income

Middle Income

High Income
High-Income Persons by Race

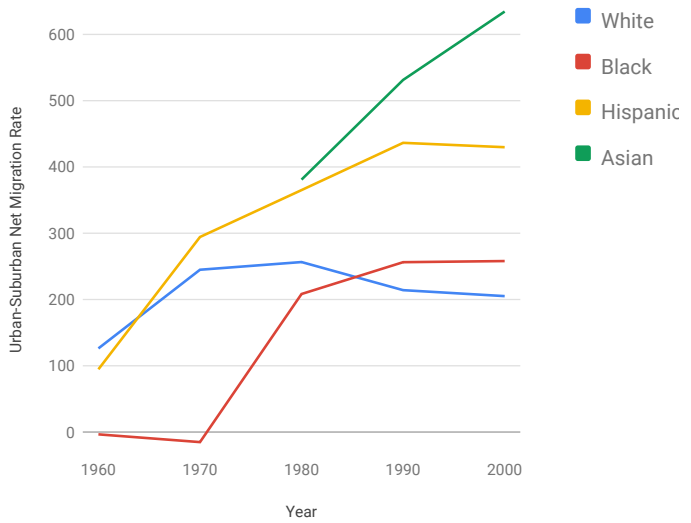


Urban and Suburban Residence, 1955-1995, and 5-year migration

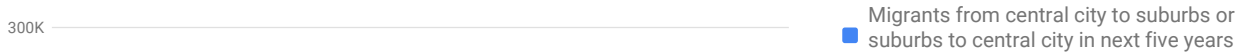

Augusta, GA

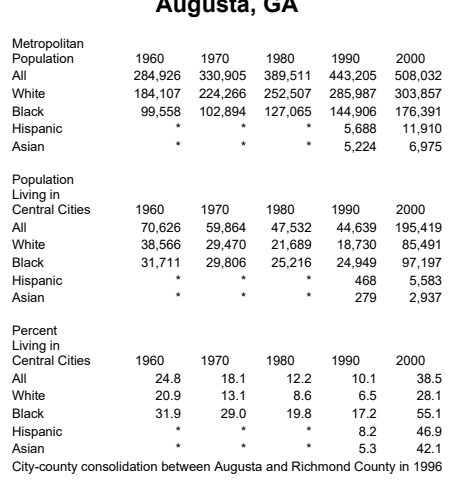

Urban-Suburban Net Migration Rate by Race

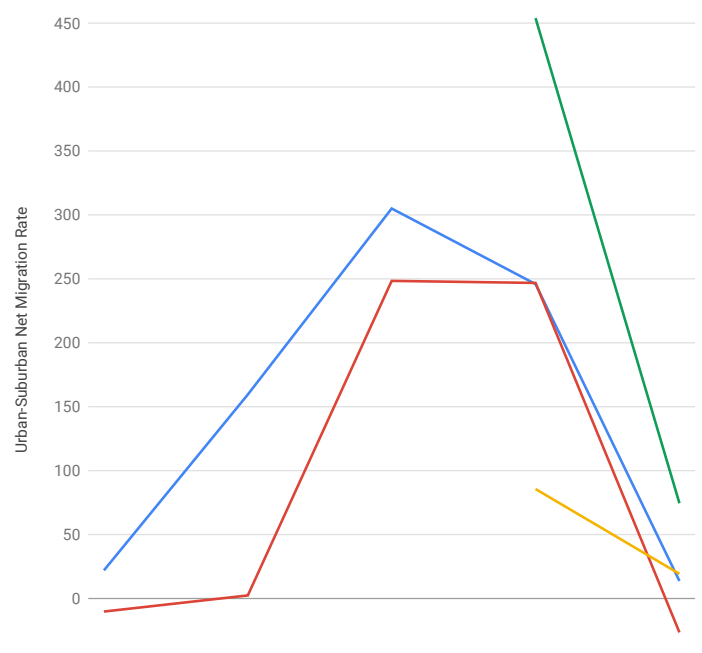

1960
1980

Year

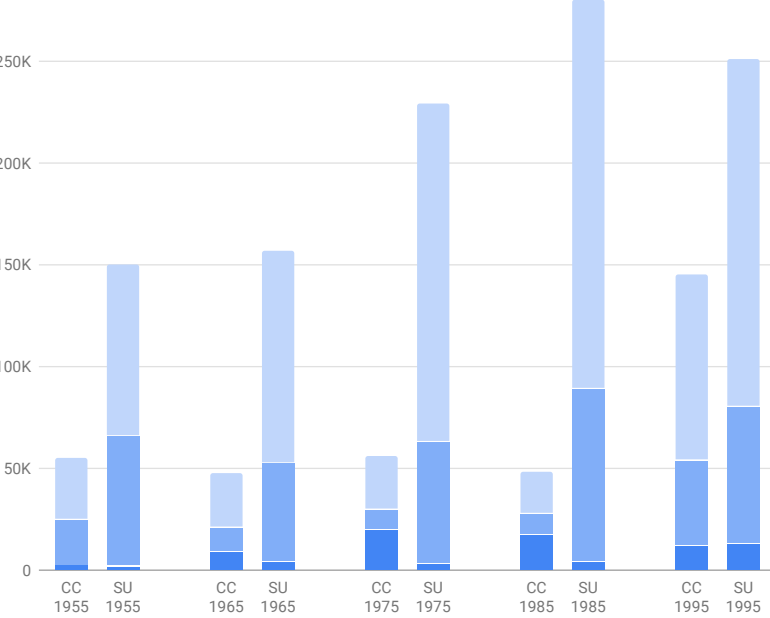

Migrants within central city or within

- suburban area in next five years

Nonmovers (lived in the same house five years later)

Central City (CC)/Suburban (SU) Residence in Year

Urban-Suburban Net Migration Rate by Income

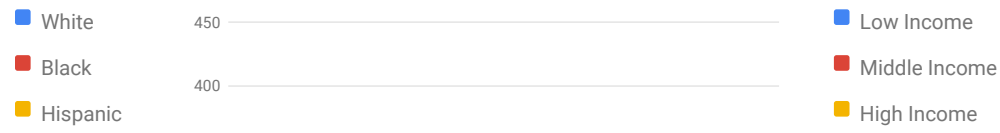

- Asian

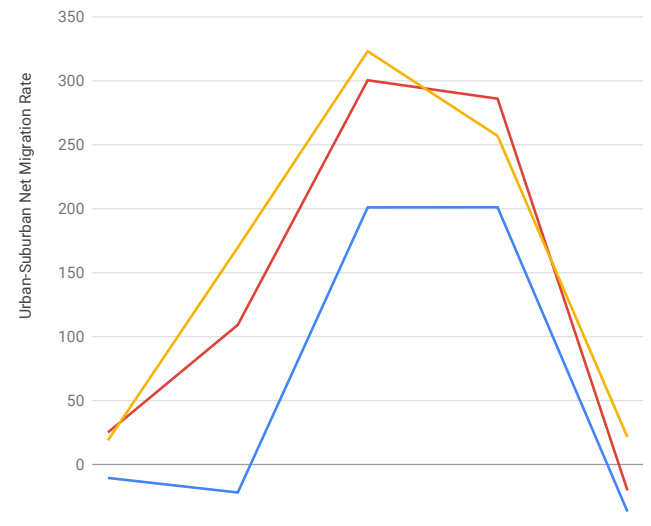

1960

1970

Year

High-Income Persons by Race

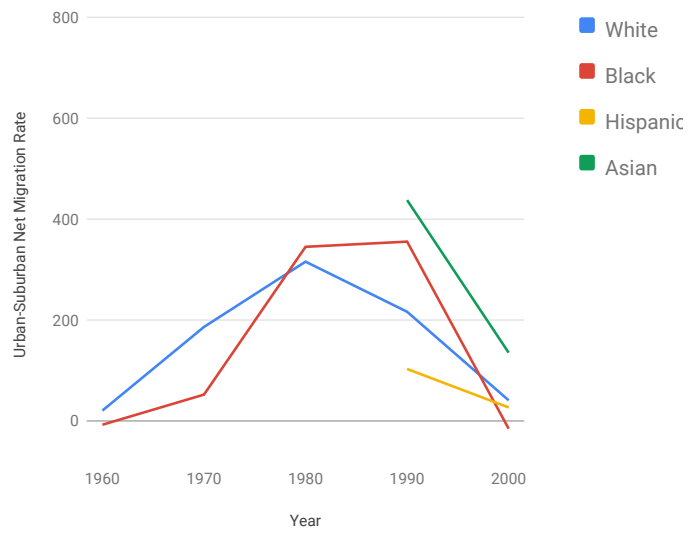


Urban and Suburban Residence, 1955-1995, and 5-year migration

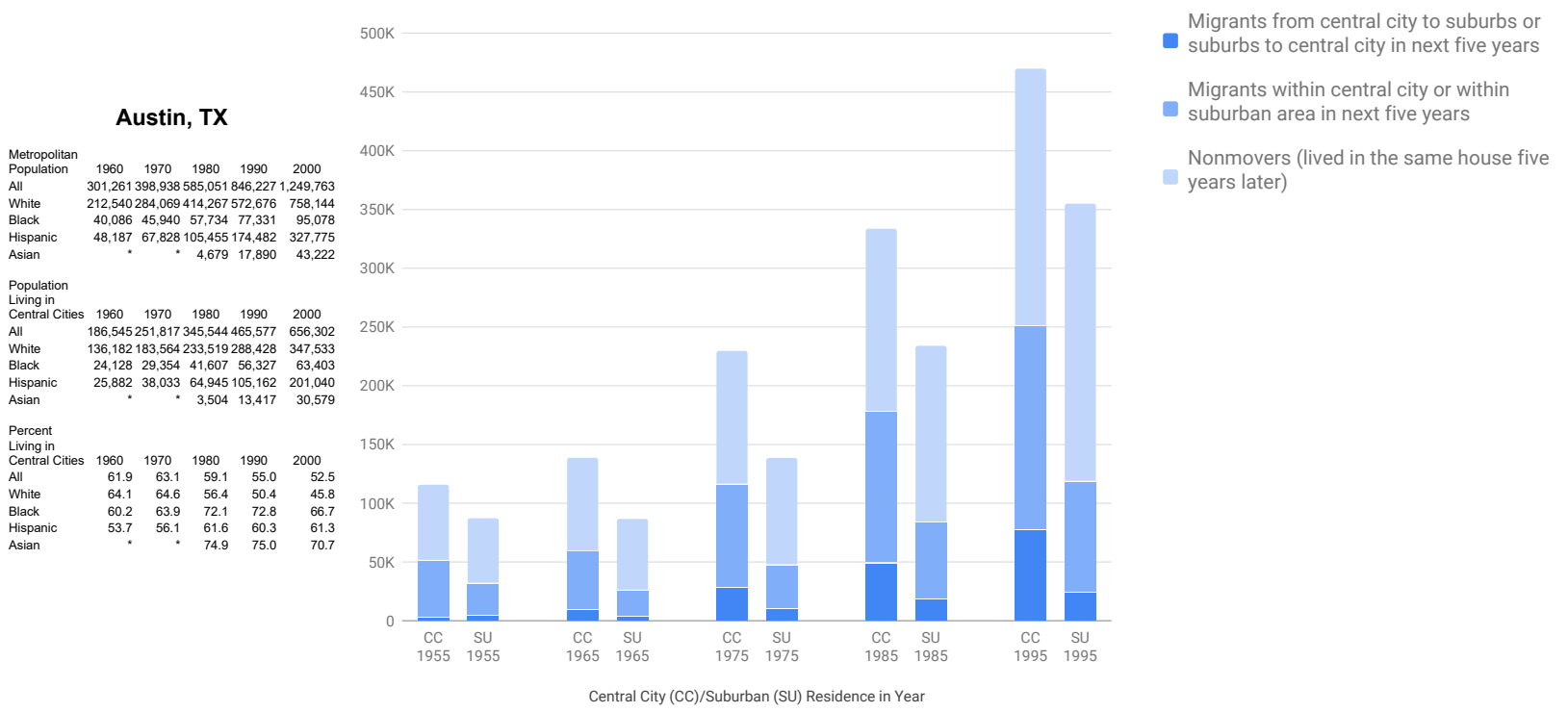

Urban-Suburban Net Migration Rate by Race

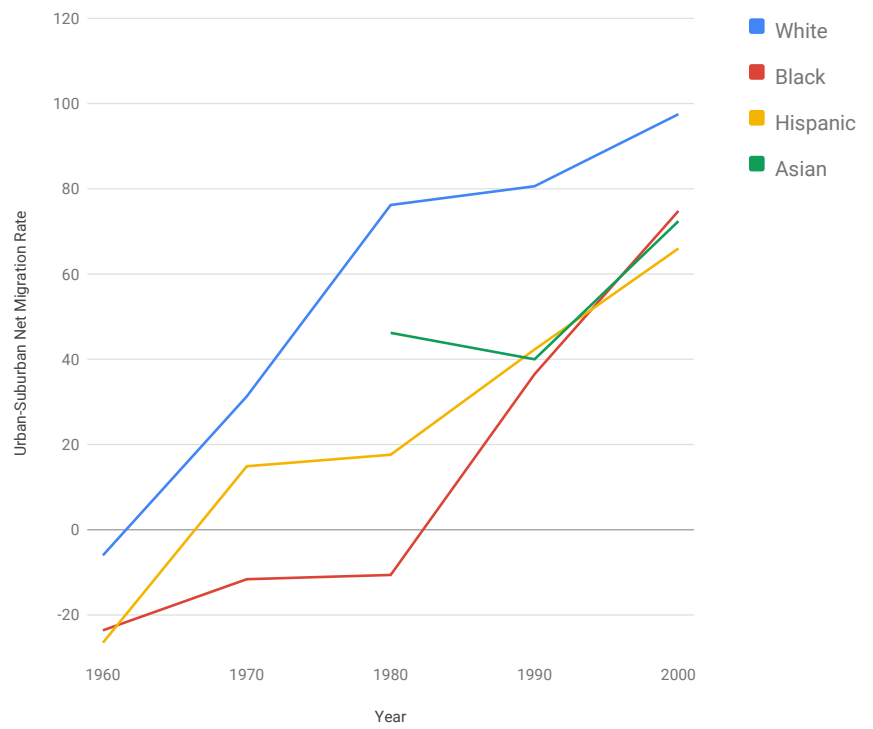

Low-Income Persons by Race

Middle-Income Persons by Race
Urban-Suburban Net Migration Rate by Income

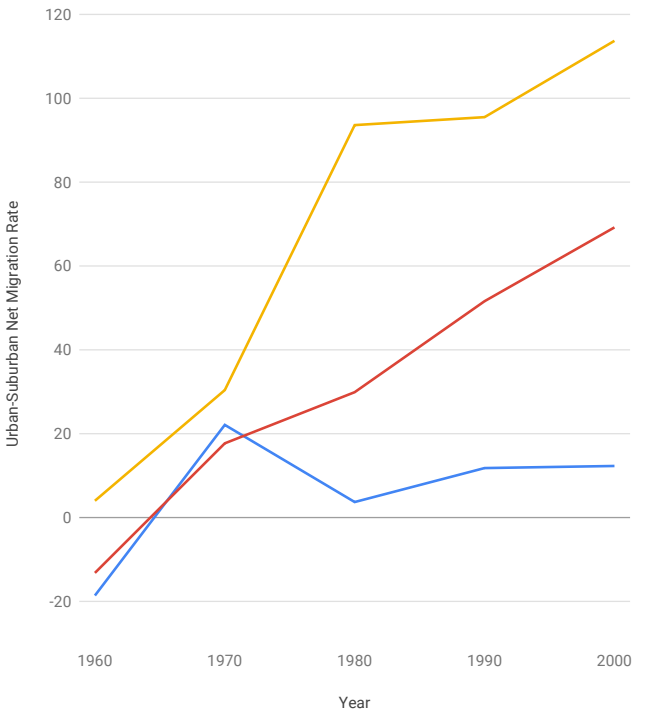

High-Income Persons by Race

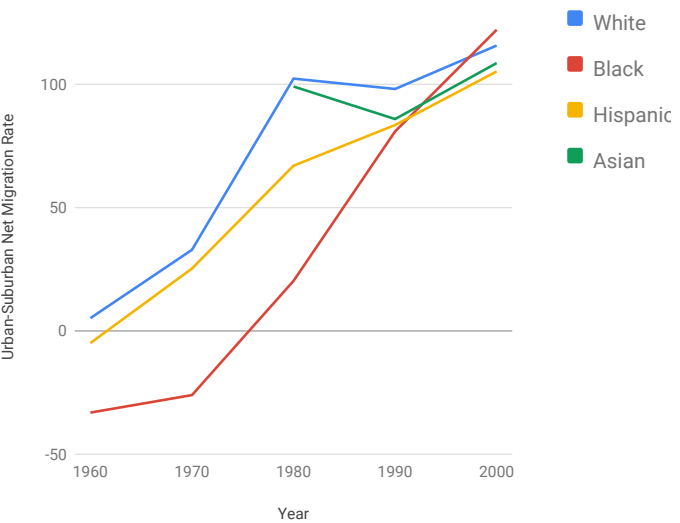


Bakersfield, CA

Urban and Suburban Residence, 1955-1995, and 5-year migration

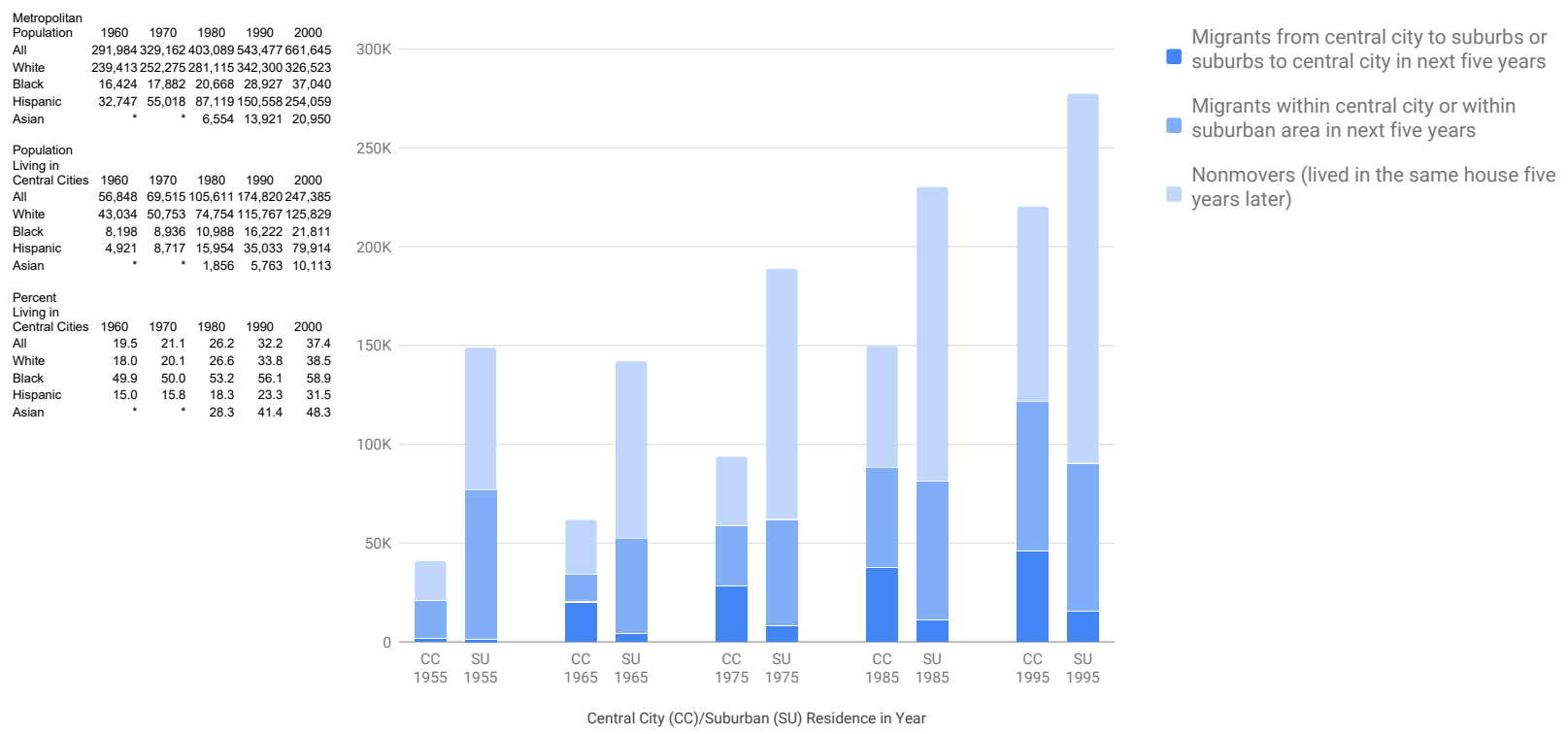

Urban-Suburban Net Migration Rate by Race

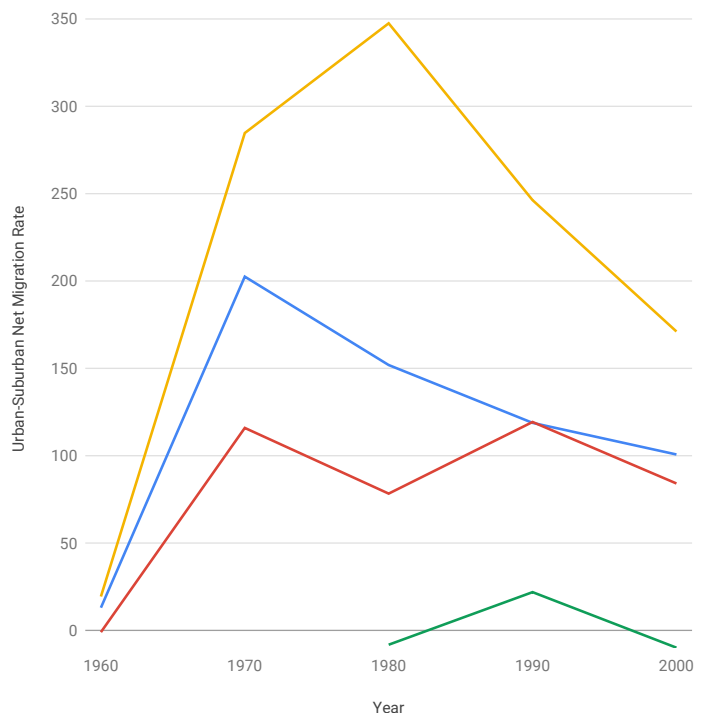

Low-Income Persons by Race

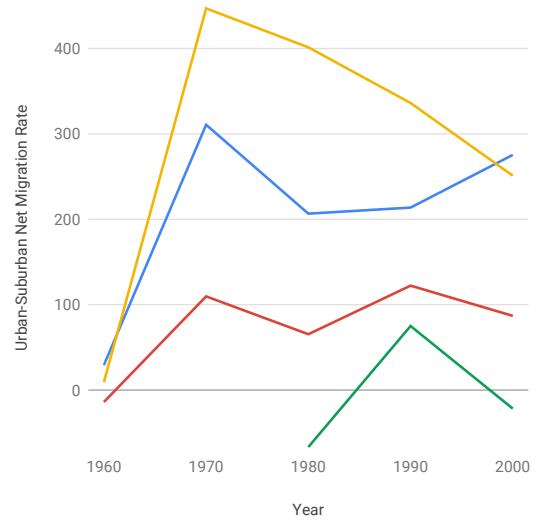

Middle-Income Persons by Race

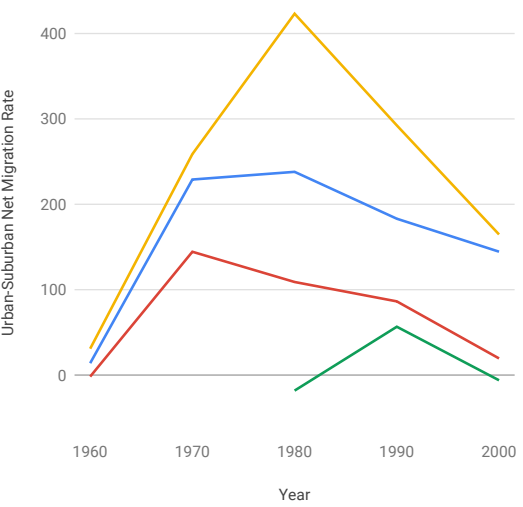

400
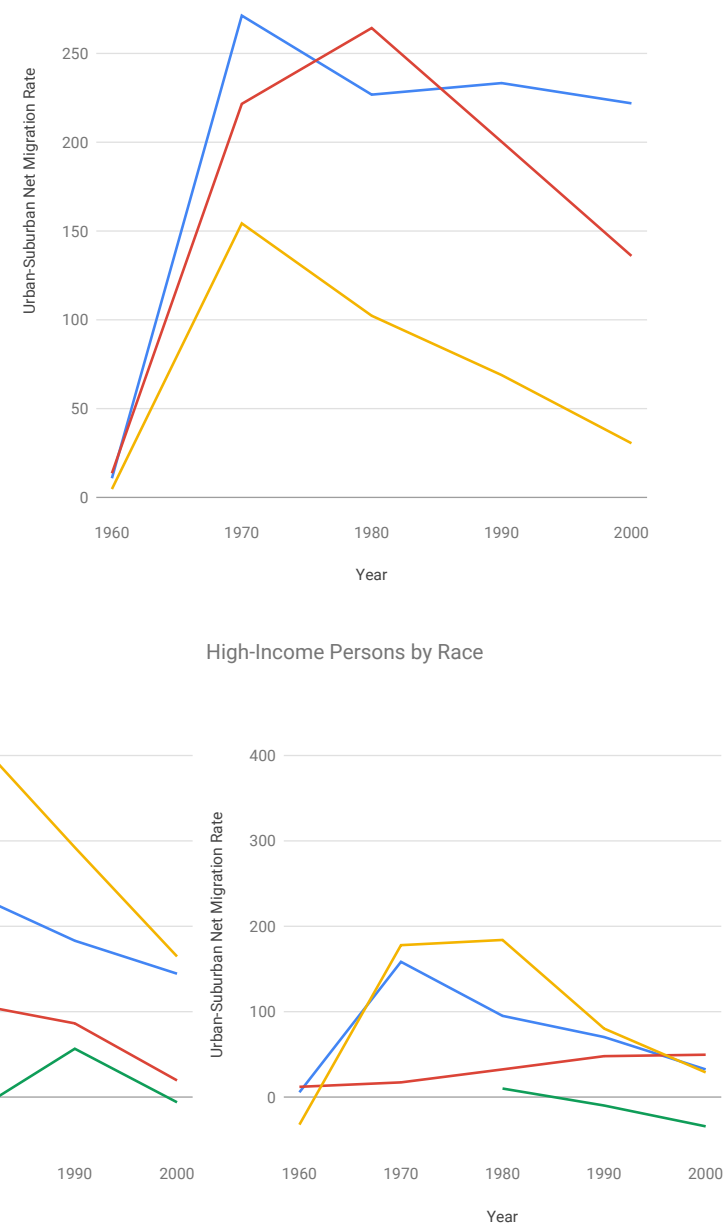

High-Income Persons by Race
Low Income

- Middle Income

High Income

White

- Black

Hispanic

- Asian 
Baltimore, MD

Urban and Suburban Residence, 1955-1995, and 5-year migration

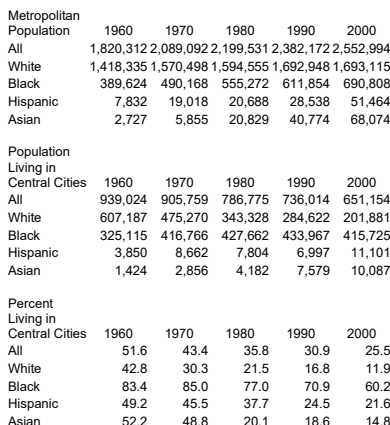

$1.6 \mathrm{M}$

$1 \mathrm{M}$

$$
\begin{array}{ll} 
& \\
9 & 800 \mathrm{~K} \\
2 & \\
2 . & \\
8 & 600 \mathrm{~K}
\end{array}
$$

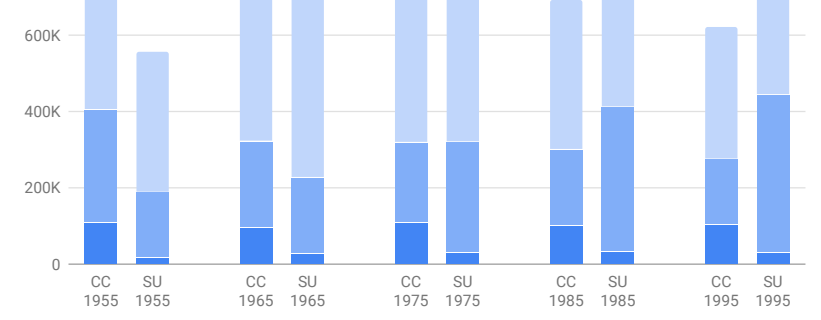

Central City (CC)/Suburban (SU) Residence in Year

\author{
Migrants from central city to suburbs or \\ suburbs to central city in next five years \\ Migrants within central city or within \\ suburban area in next five years \\ Nonmovers (lived in the same house five \\ years later)
}

Urban-Suburban Net Migration Rate by Race

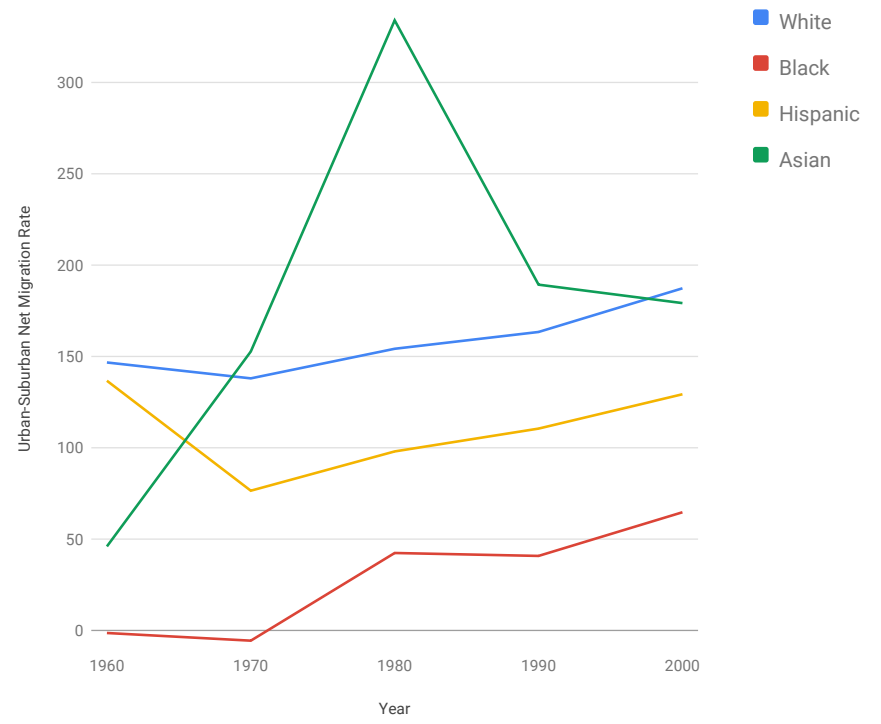

Low-Income Persons by Race
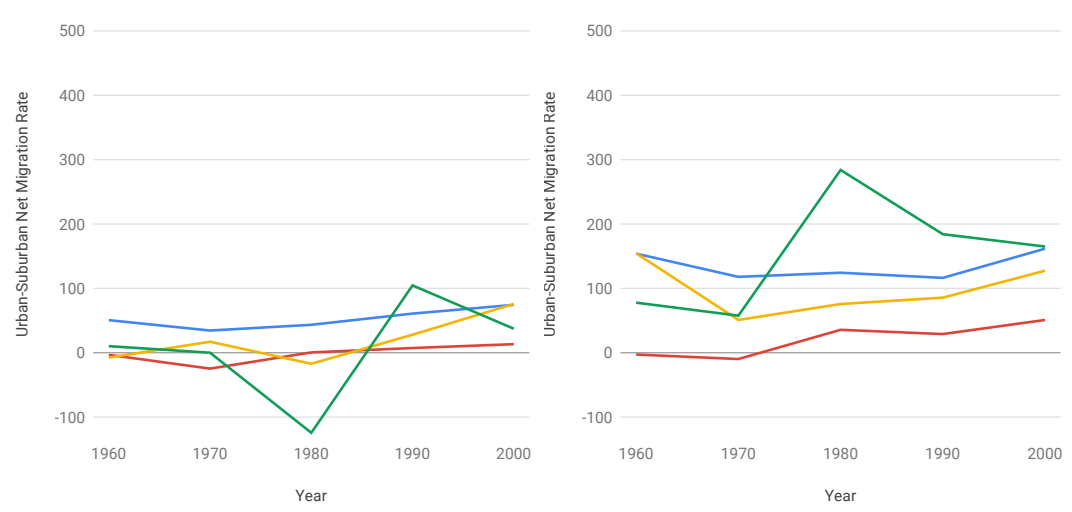

Year
Urban-Suburban Net Migration Rate by Income
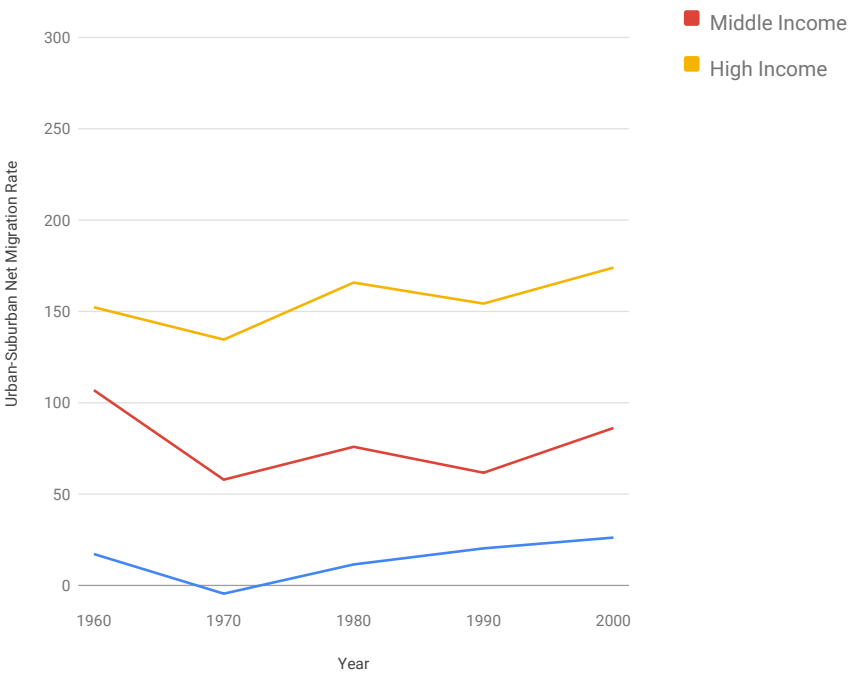

High-Income Persons by Race

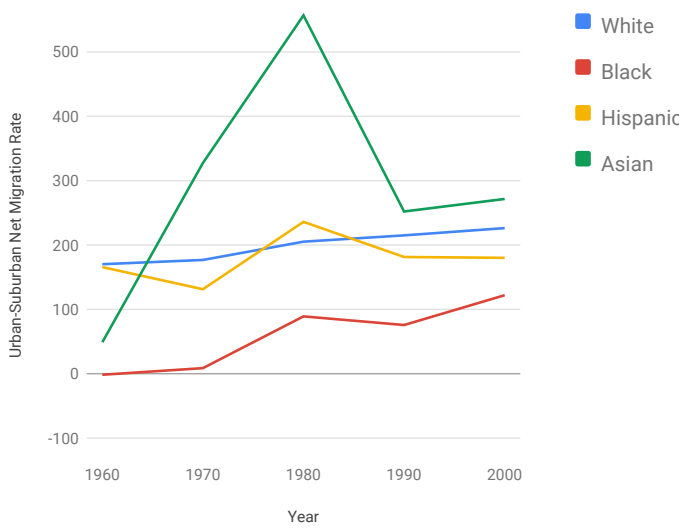




\section{Baton Rouge, LA}

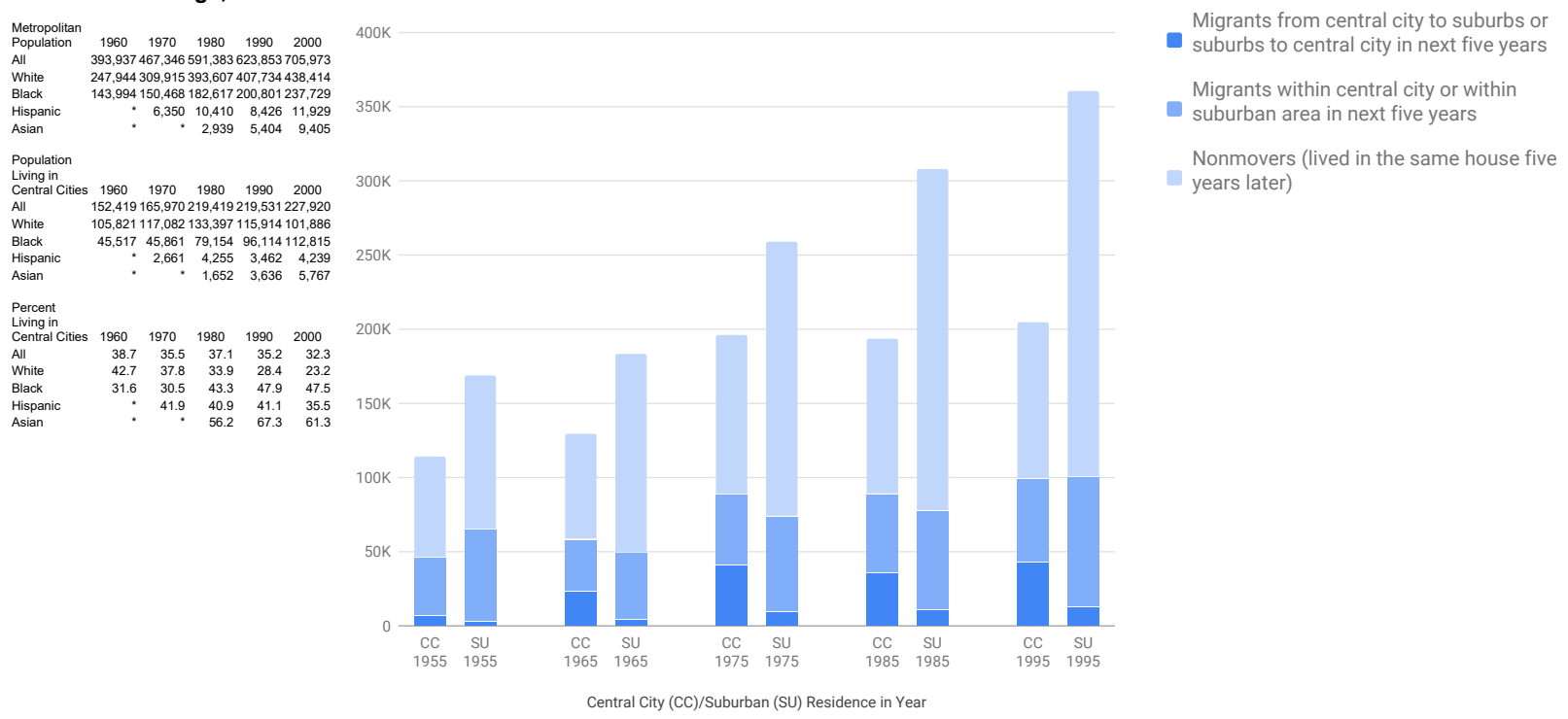

Urban-Suburban Net Migration Rate by Race

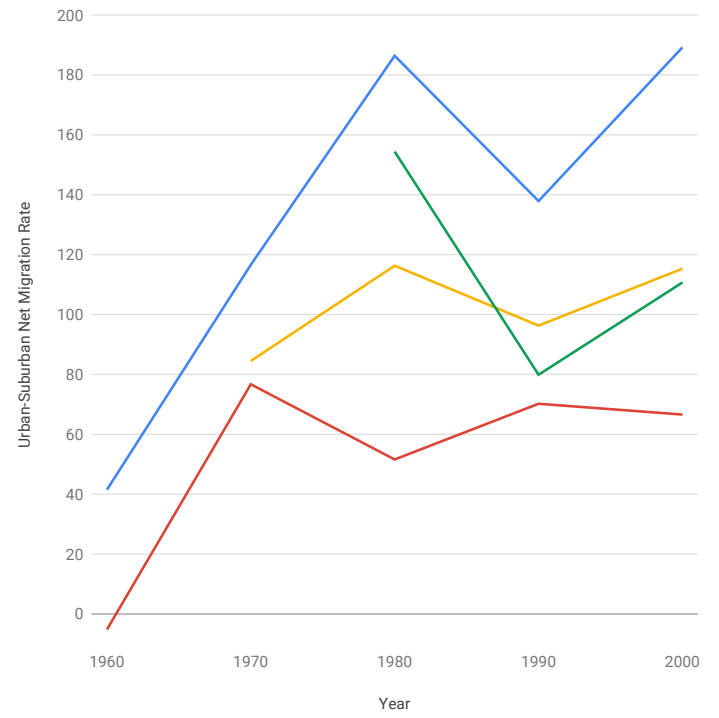

Low-Income Persons by Race

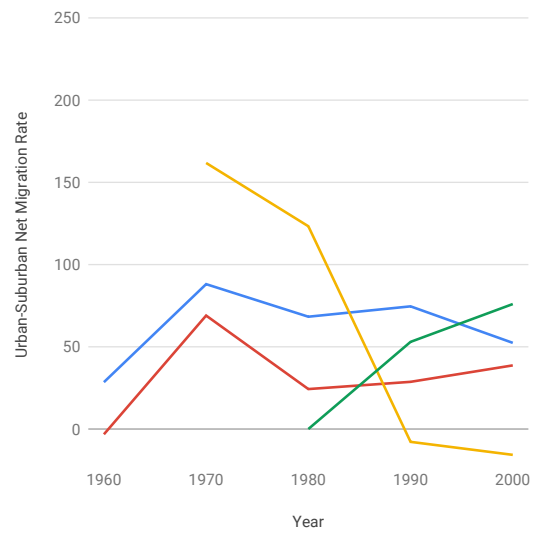

Middle-Income Persons by Race
Urban-Suburban Net Migration Rate by Income
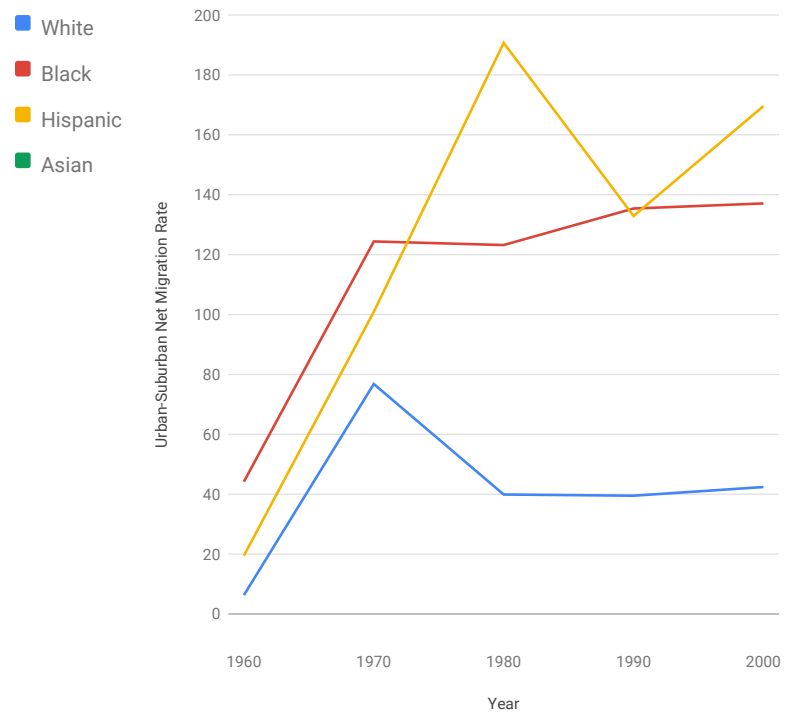

- Low Income

Middle Income

- High Income
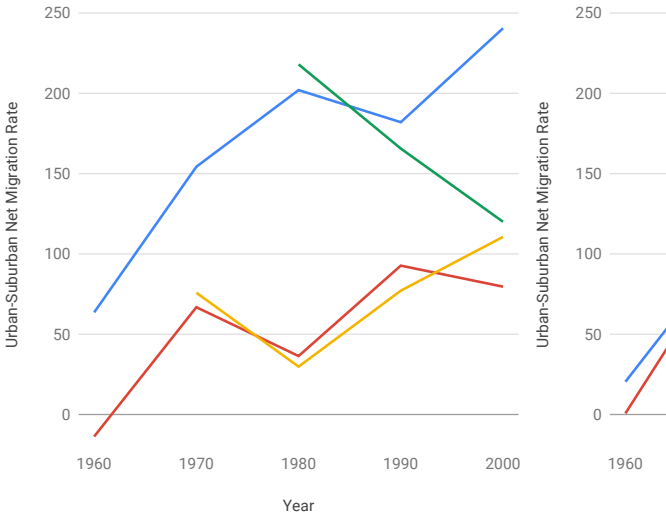

$1960 \quad 1970$
White

- Black

Hispanic

- Asian 
Urban and Suburban Residence, 1955-1995, and 5-year migration

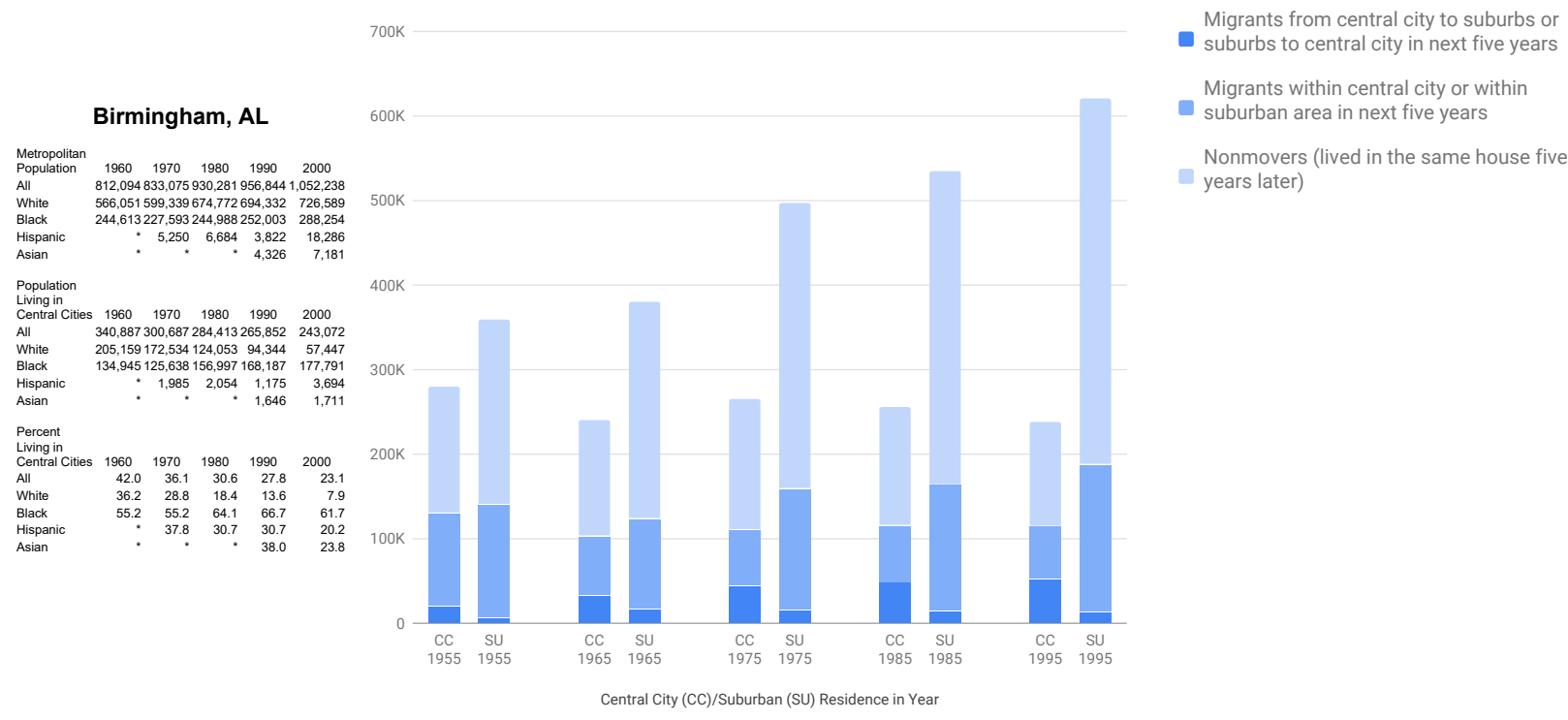

Urban-Suburban Net Migration Rate by Race

Urban-Suburban Net Migration Rate by Income

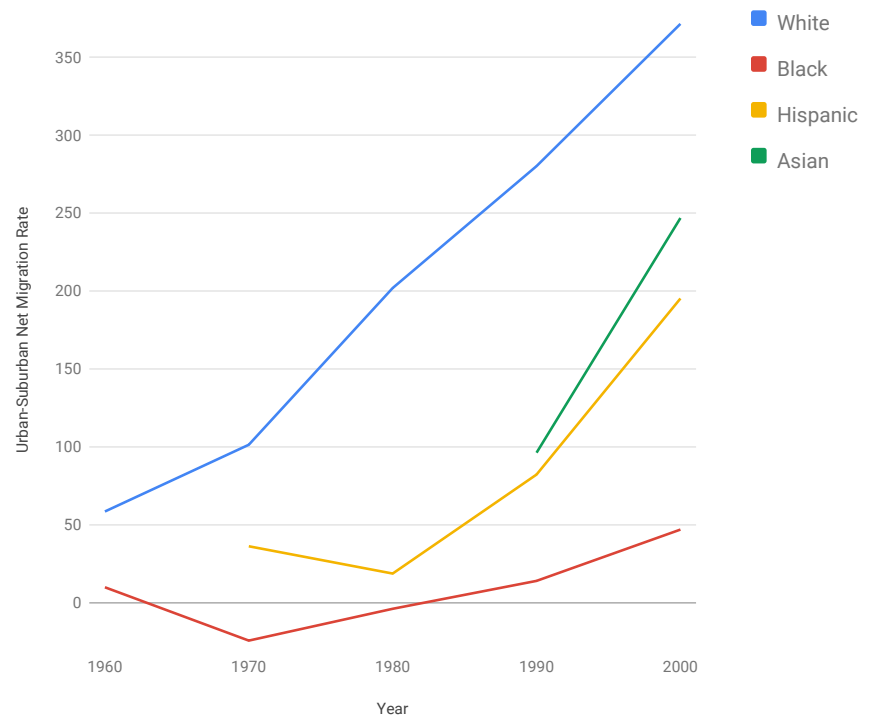

Low-Income Persons by Race

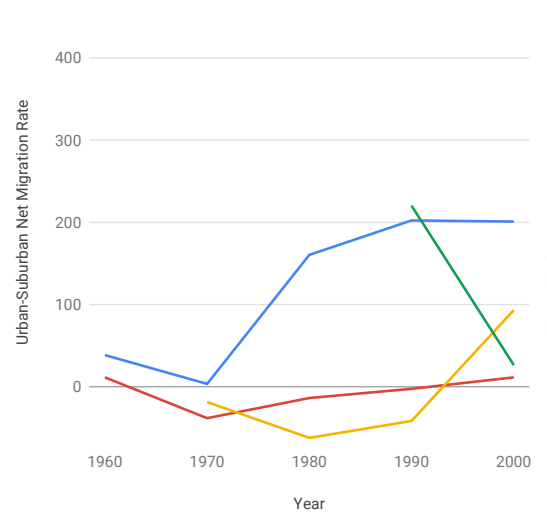

Year
Middle-Income Persons by Race

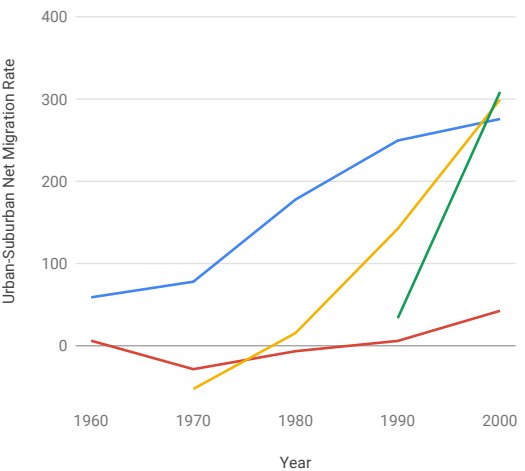

Year

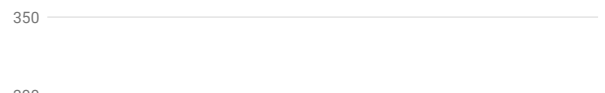

300

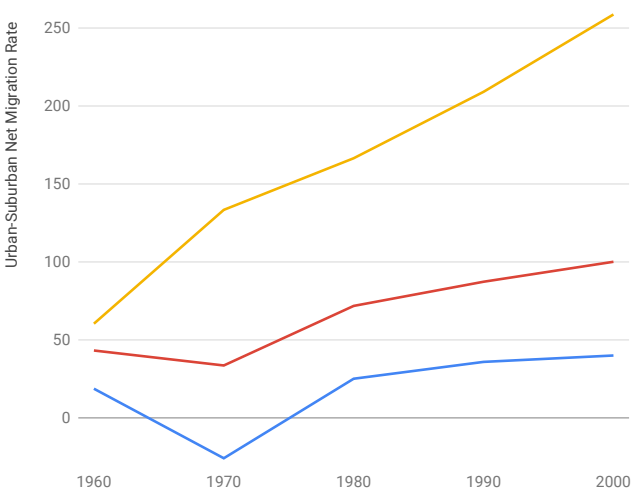

Year
- Low Income

- Middle Income

High Income

High-Income Persons by Race

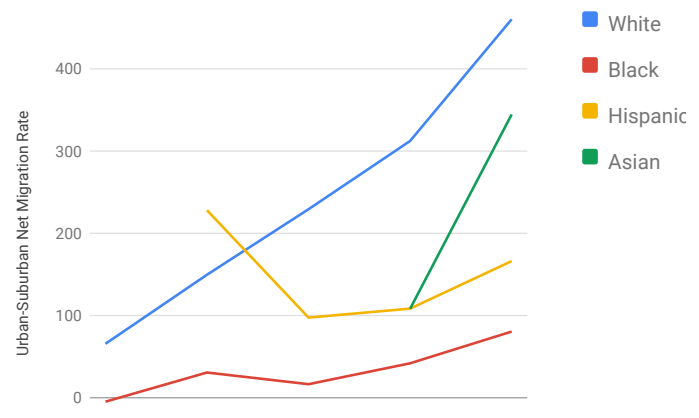




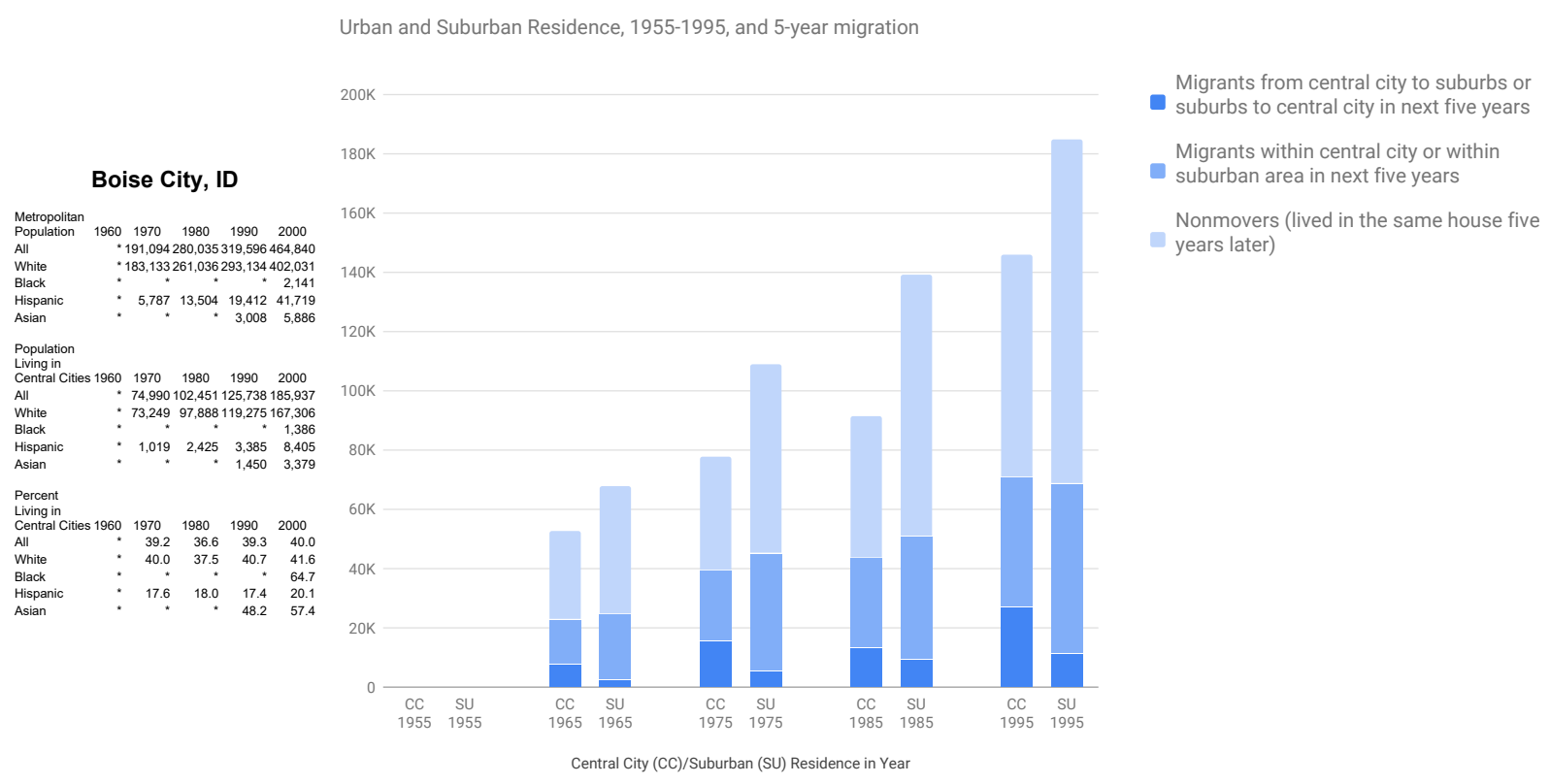

Urban-Suburban Net Migration Rate by Race

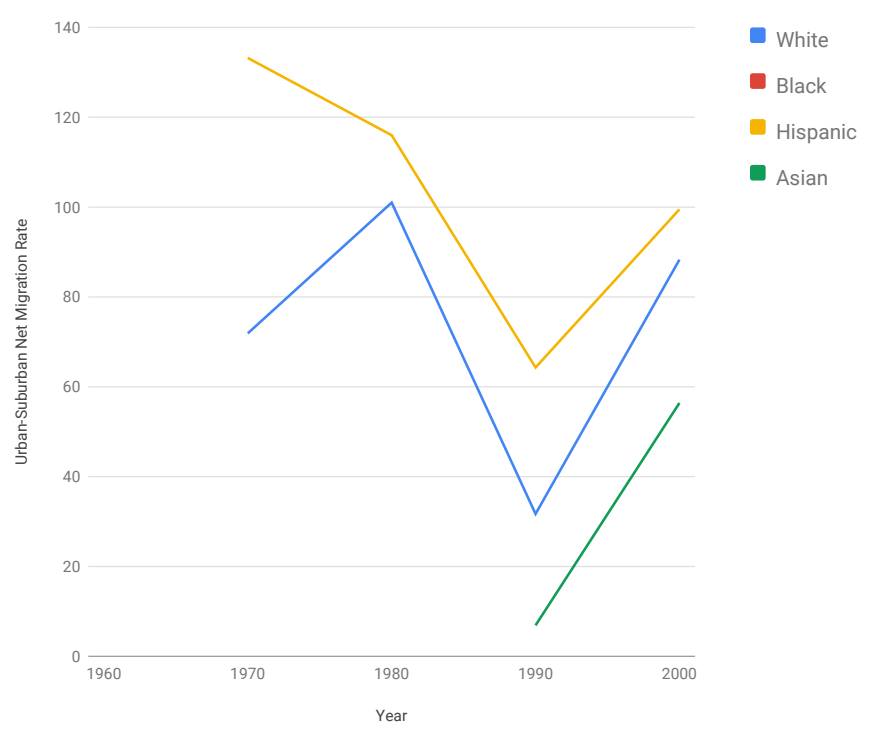

Low-Income Persons by Race

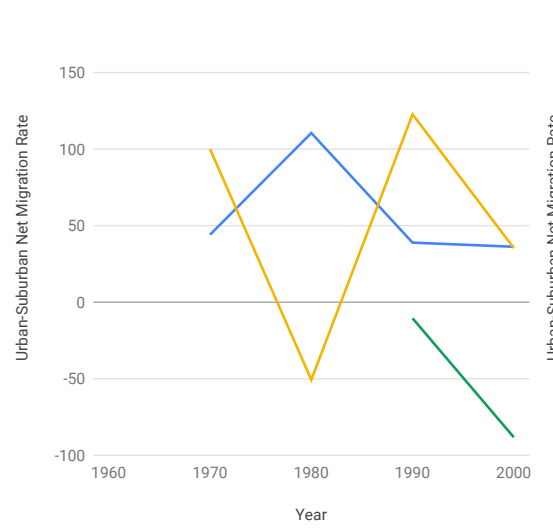

Middle-Income Persons by Race
Urban-Suburban Net Migration Rate by Income

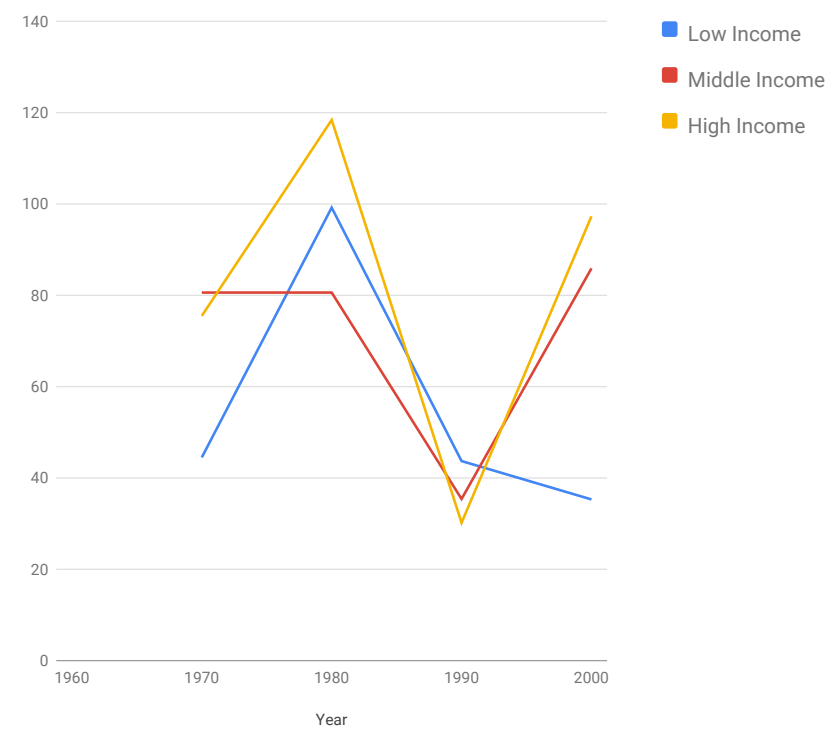

High-Income Persons by Race

Black

Hispanic

- Asian 


\section{Boston, MA-NH}

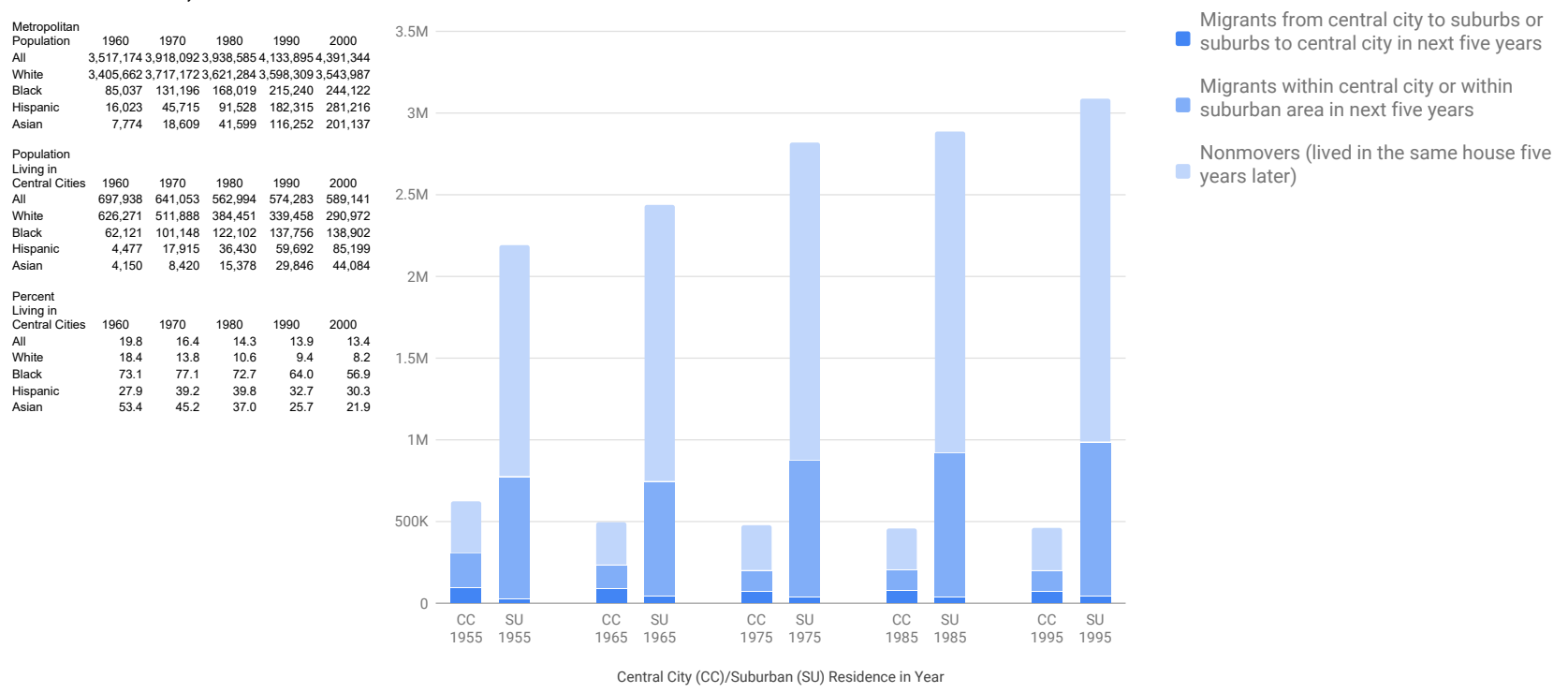

Urban-Suburban Net Migration Rate by Race

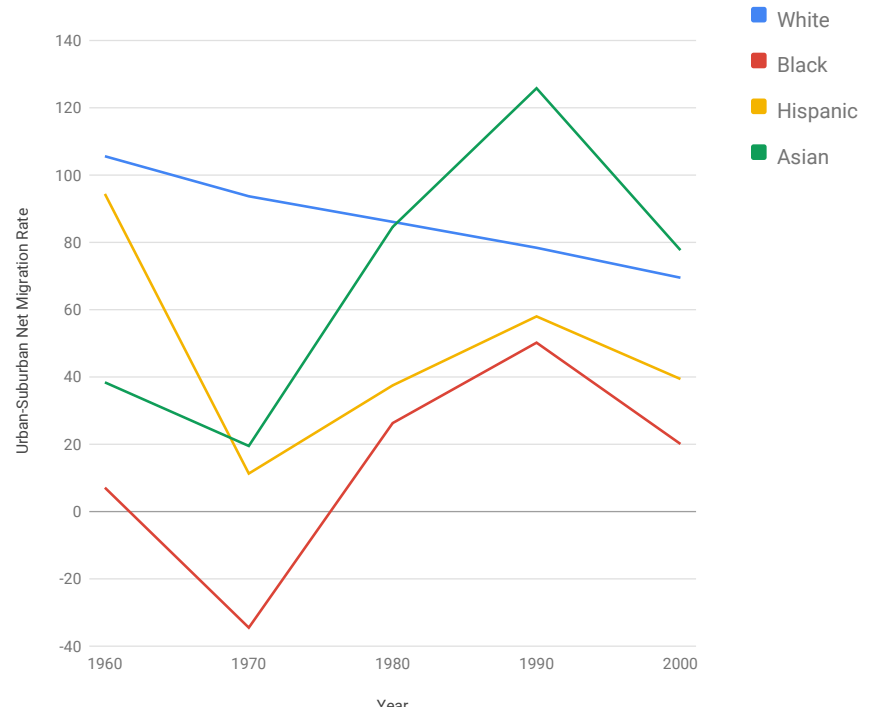

Low-Income Persons by Race

Middle-Income Persons by Race
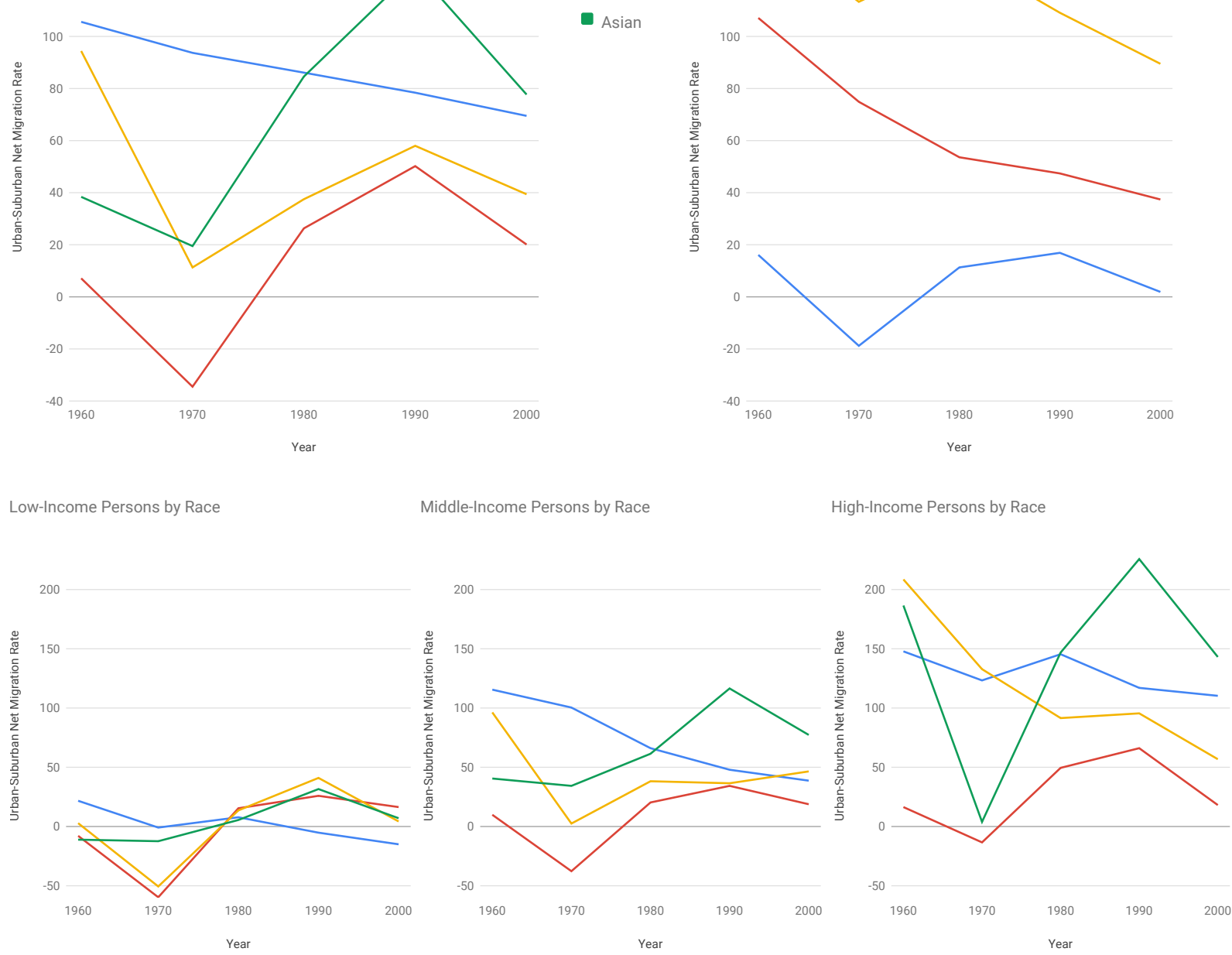

Low Income

- Middle Income

High Income

White

- Black

Hispanic

Asian 
Bridgeport-Stamford-Norwalk, CT

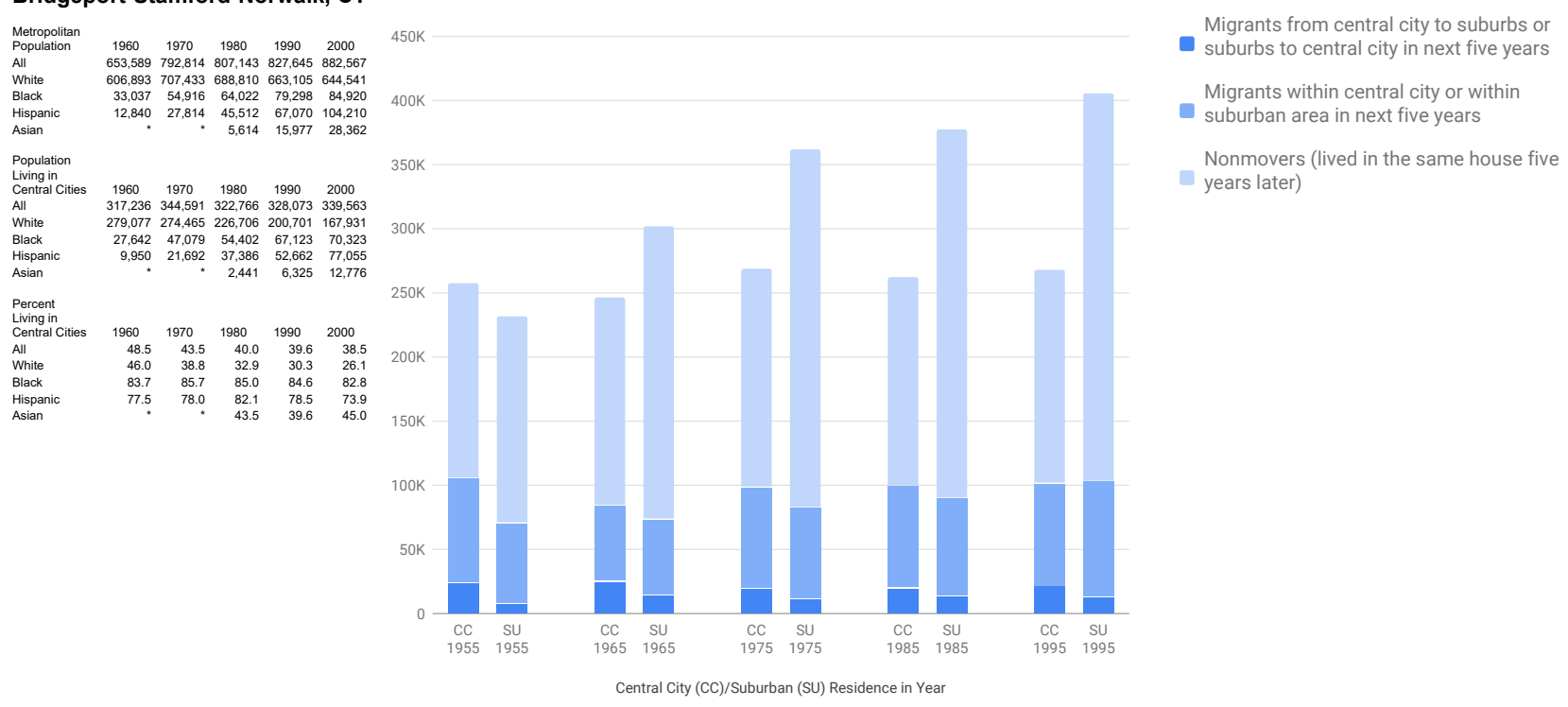

Urban-Suburban Net Migration Rate by Race

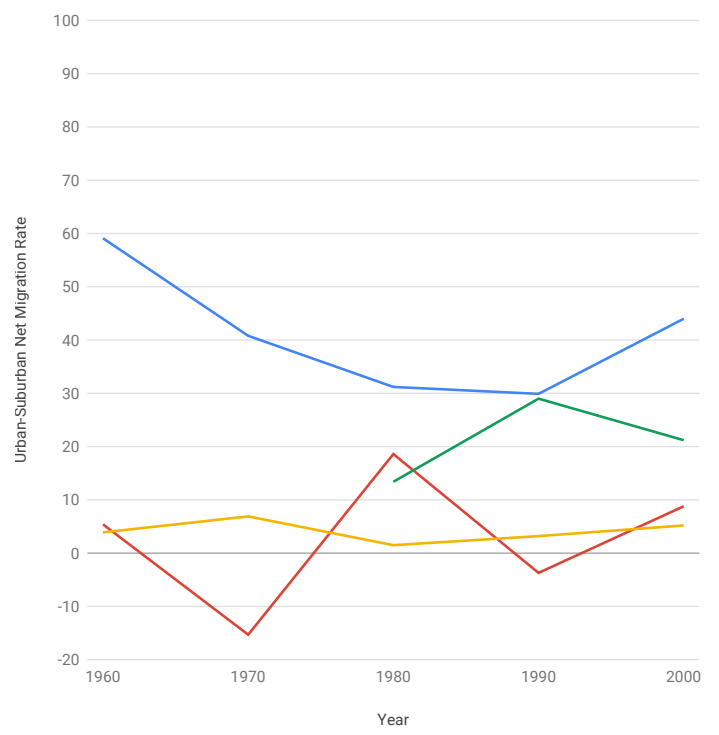

Low-Income Persons by Race

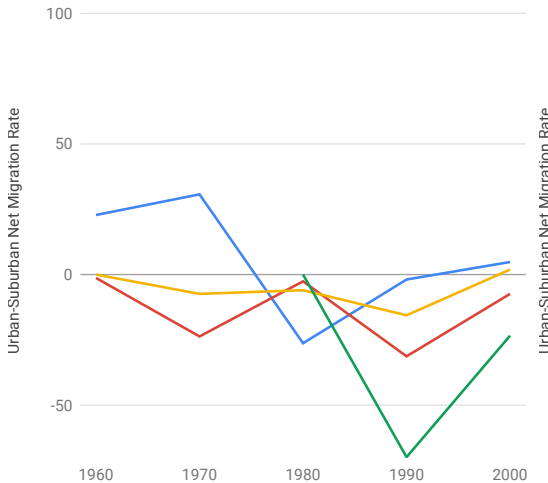

Year
Middle-Income Persons by Race

100

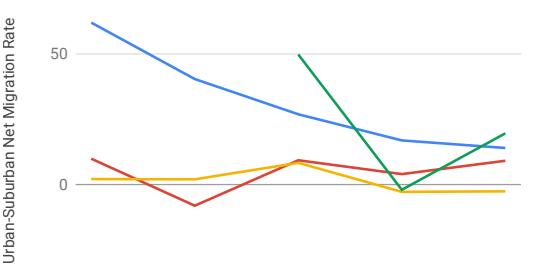

$-50$
Urban-Suburban Net Migration Rate by Income
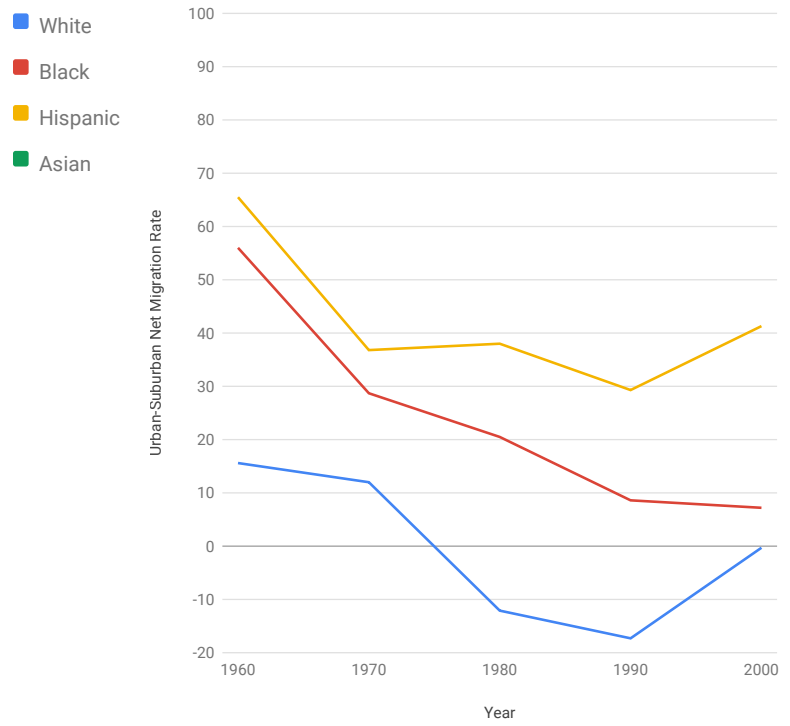

High-Income Persons by Race

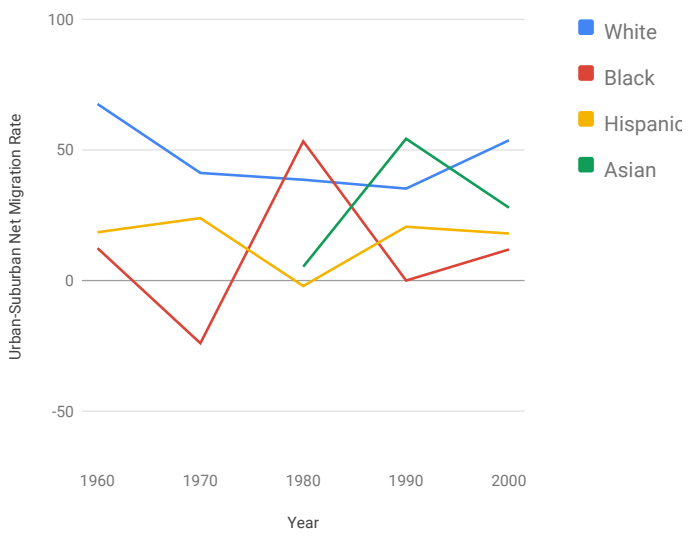




\section{Buffalo, NY}

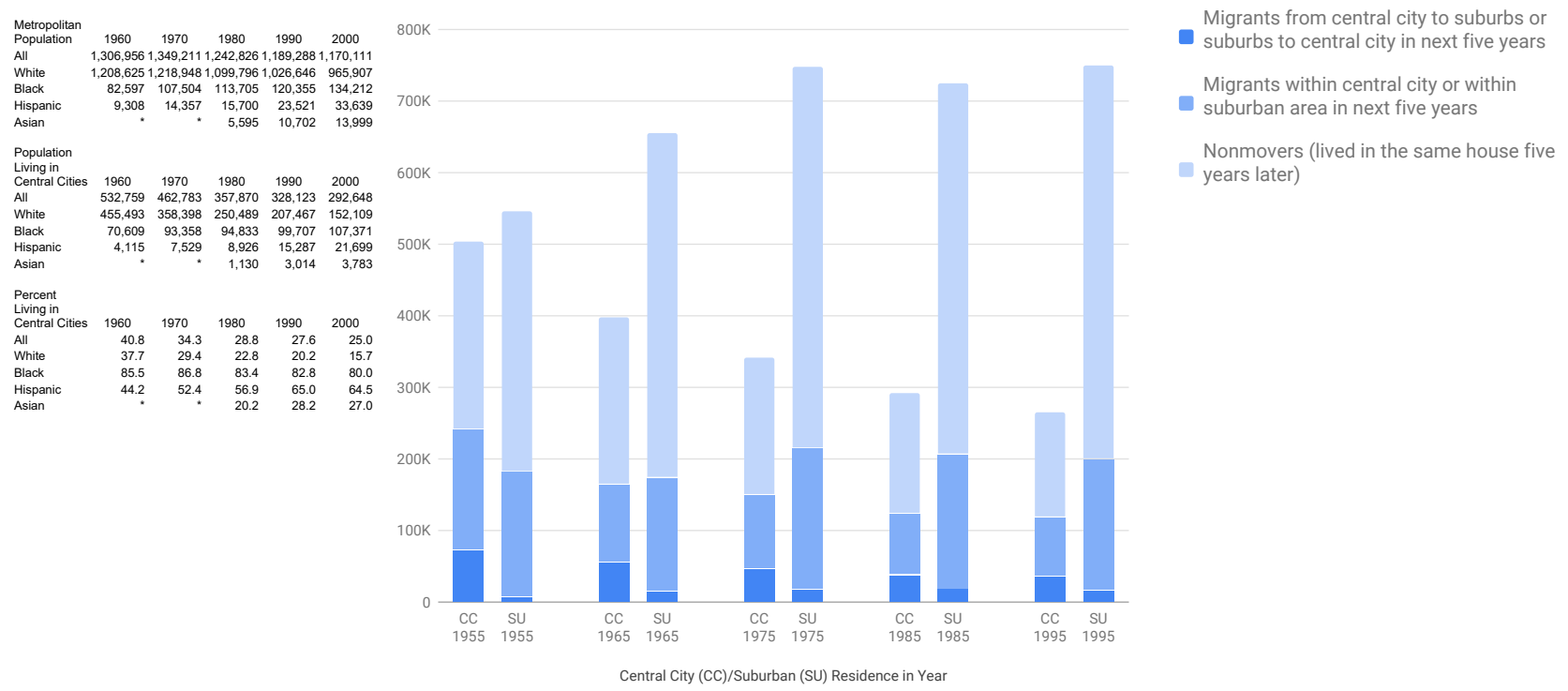

Urban-Suburban Net Migration Rate by Race

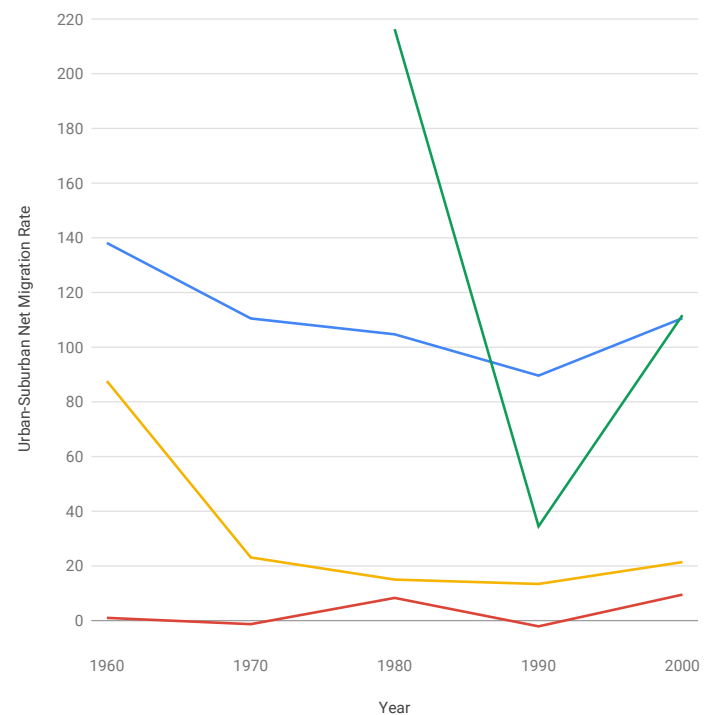

Low-Income Persons by Race

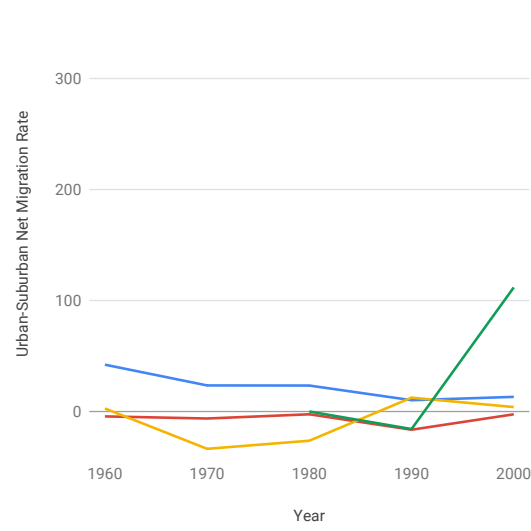

Middle-Income Persons by Race

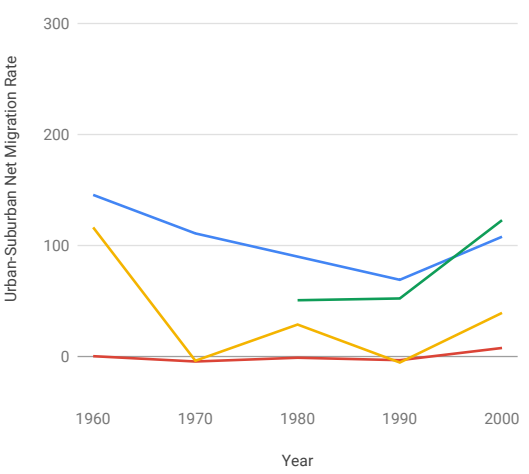

White

Black

Hispanic

- Asian

Urban-Suburban Net Migration Rate by Income
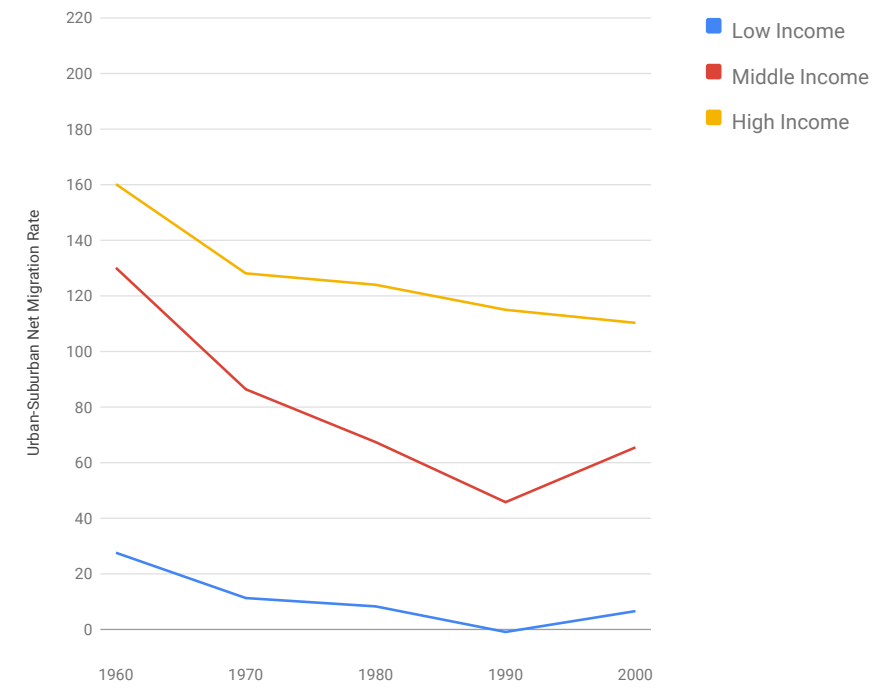

High-Income Persons by Race

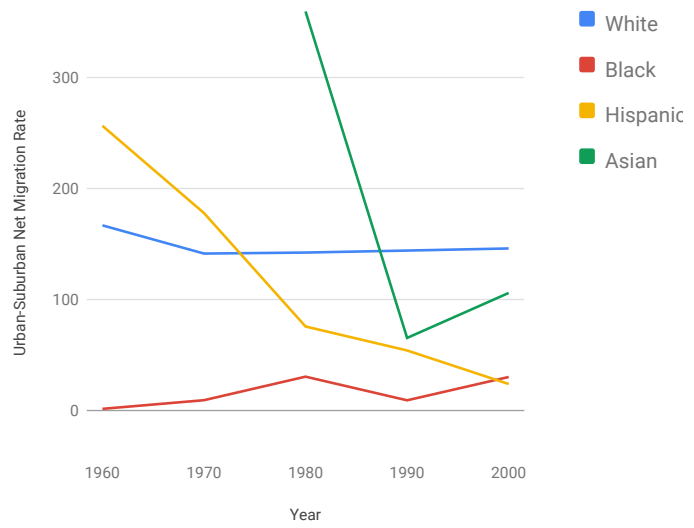


Urban and Suburban Residence, 1955-1995, and 5-year migration $250 \mathrm{~K}-\begin{aligned} & \text { Migrants from central city to suburbs or } \\ & \text { suburbs to central city in next five years }\end{aligned}$

Migrants within central city or within

- suburban area in next five years

Nonmovers (lived in the same house five years later)

Metropolitan

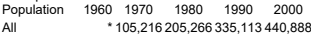

White $\quad: 90,986182,430296,163361,259$
Black

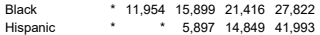

Asian * * "

Population
Living in

$\begin{array}{lllll}\text { Living in } & & & & \\ \text { Central Cities } 1960 & 1970 & 1980 & 1990 & 2000 \\ \text { All } & 27,351 & 36,638 & 45,206 & 48,046\end{array}$

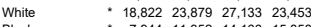

$\begin{array}{llllll}\text { Black } \quad 7,944 & 11,352 & 14,166 & 15,850\end{array}$

$\begin{array}{llllll}\text { Hispanic : " } & 1,244 & 3,283 & 6,836 & 10\end{array}$

Percent
Living in

$\begin{array}{lllll}\text { Living in } & 1980 \\ \text { Central Cities } 1960 & 1970 & 1980 & 1990 & 2000\end{array}$

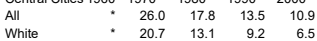

$\begin{array}{llrrrr}\text { Black } & \text { : } & 66.5 & 71.4 & 66.1 & 57.0 \\ \text { Hispanic } & \vdots & : & 21.1 & 22.1 & 16.3\end{array}$

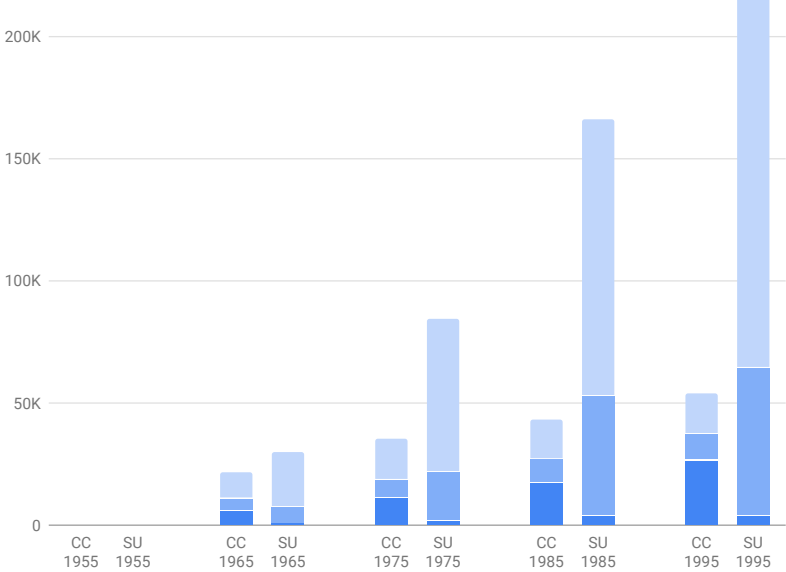

Central City (CC)/Suburban (SU) Residence in Year

Urban-Suburban Net Migration Rate by Race
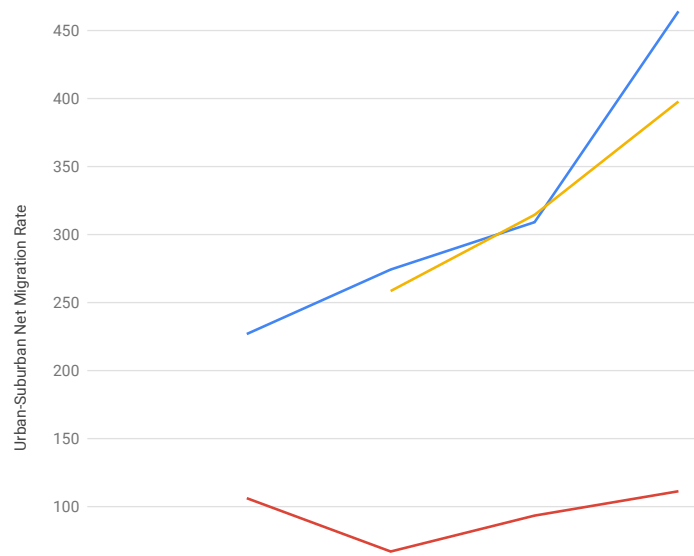

50

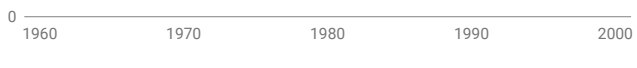

Year

Low-Income Persons by Race
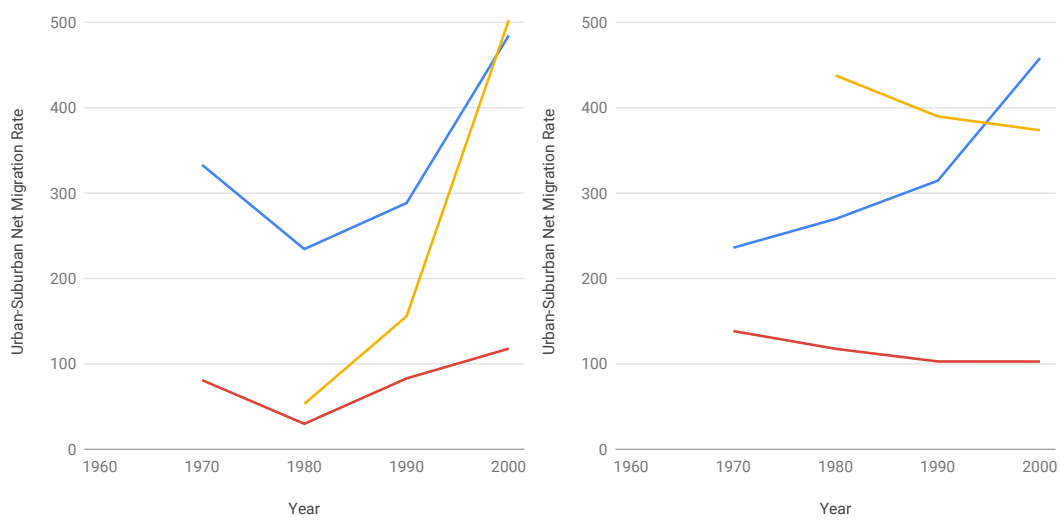

Year
Urban-Suburban Net Migration Rate by Income
White

- Black

Hispanic

- Asian

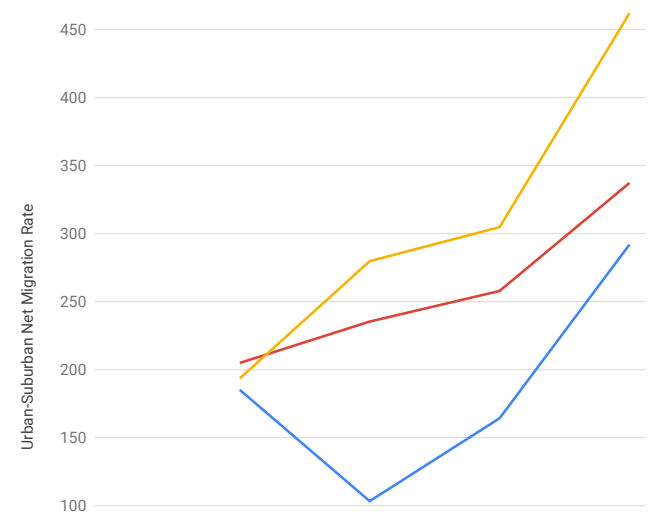

50

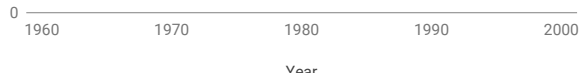

Year
- Low Income

- Middle Income

- High Income
High-Income Persons by Race

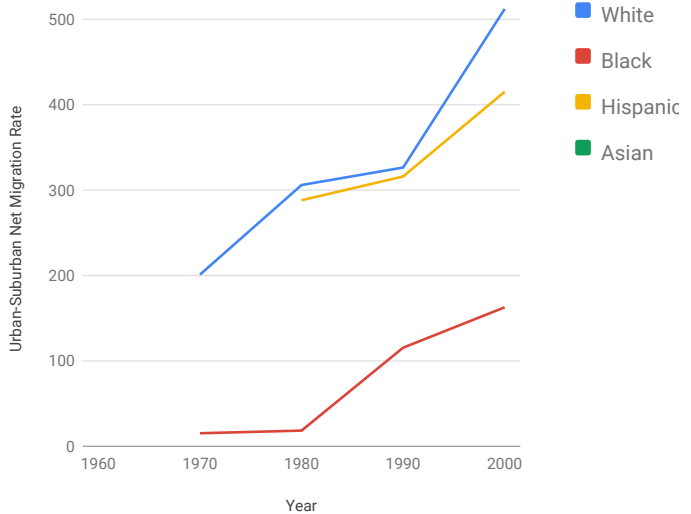


Urban and Suburban Residence, 1955-1995, and 5-year migration

$350 \mathrm{~K}$

Charleston, SC

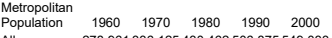

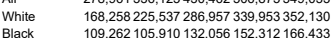

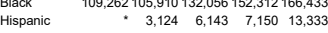

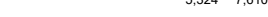

Population
Living in

$\begin{array}{llllll}\text { Living in } & 1960 & 1970 & 1980 & 1990 & 2000\end{array}$

$\begin{array}{llllll}\text { All } & 65,925 & 66,934 & 69,510 & 80,414 & 96,086 \\ \text { White } & 32,133 & 36,341 & 36,319 & 45,740 & 60,048\end{array}$

Black $\quad 33,47930,134 \quad 32,00933,424,31,981$

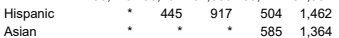

Percent
Living in

$\begin{array}{llllll}\text { Living in } & & & & & \\ \text { Central Cities } & 1960 & 1970 & 1980 & 1990 & 2000\end{array}$

$\begin{array}{lllllr}\text { All } & 23.6 & 19.9 & 16.1 & 15.9 & 17.5 \\ \text { White } & 19.1 & 16.1 & 12.7 & 13.5 & 17.1 \\ & & 3.6 & & & \end{array}$

\begin{tabular}{lrrrrrr} 
Black & 30.6 & 28.5 & 24.2 & 21.9 & 19.2 \\
\hline & 14.2 & 14.9 & 7.0 & 110
\end{tabular}

\section{$300 \mathrm{~K}$ \\ $200 \mathrm{k}$ \\ $150 \mathrm{~K}$}

$100 \mathrm{~K}$

$50 \mathrm{~K}$

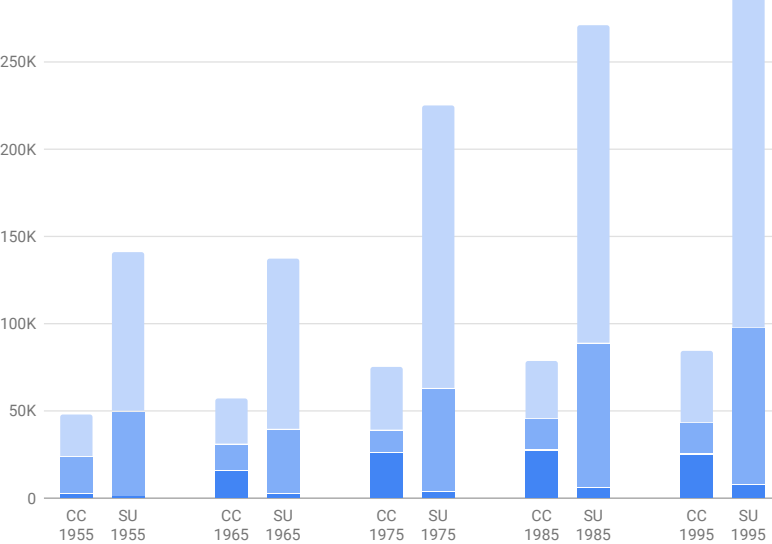

Migrants from central city to suburbs or - suburbs to central city in next five years

Migrants within central city or within

suburban area in next five years

Nonmovers (lived in the same house five

years later)
Urban-Suburban Net Migration Rate by Race

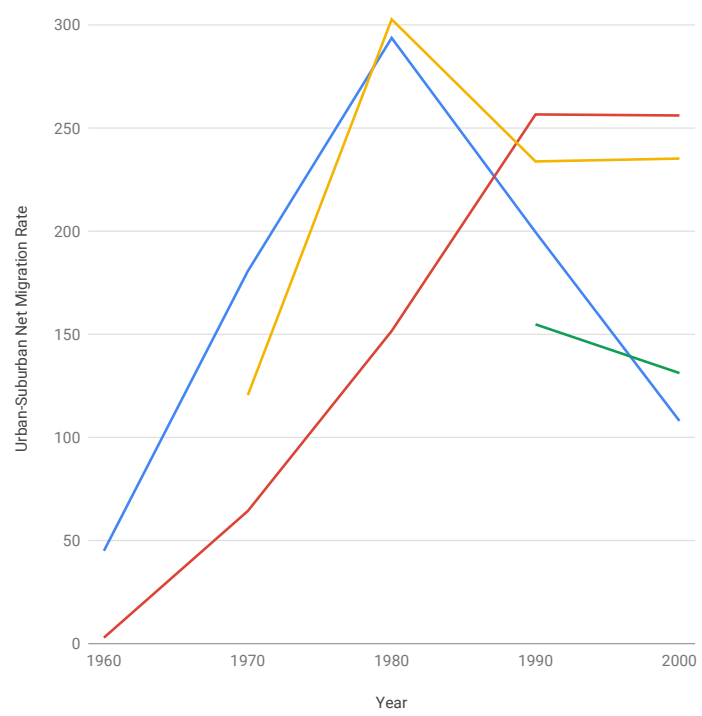

Low-Income Persons by Race

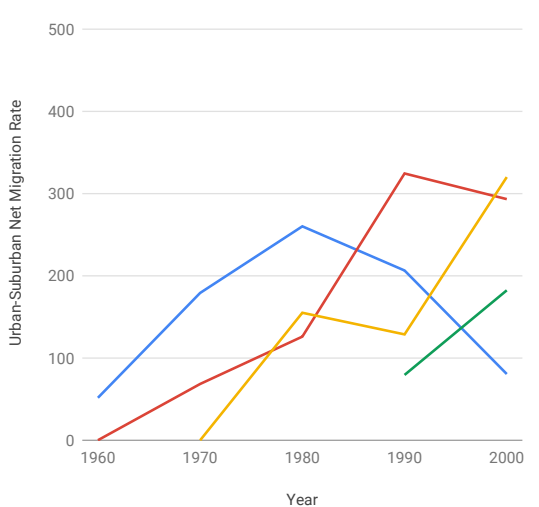

500

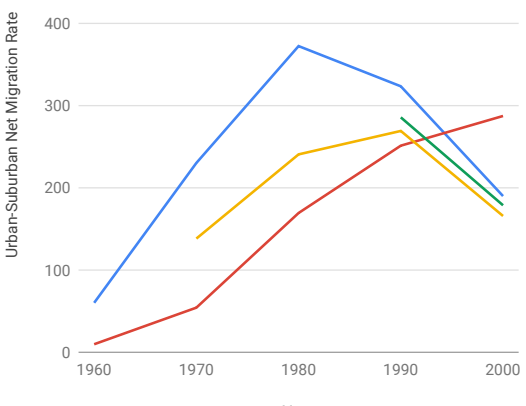

Year
Urban-Suburban Net Migration Rate by Income
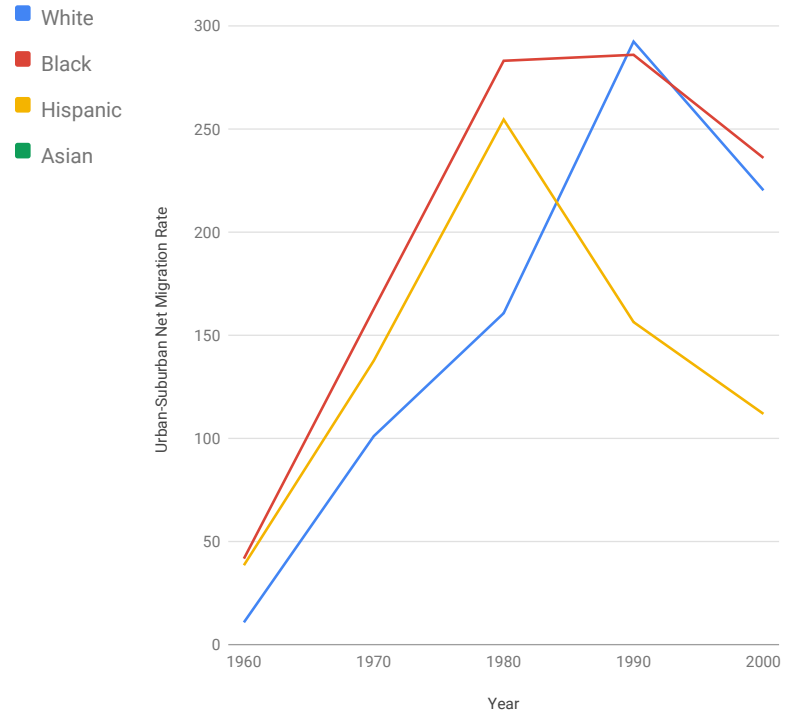

- Low Income

- Middle Income

- High Income 


\section{Charlotte, NC-SC}

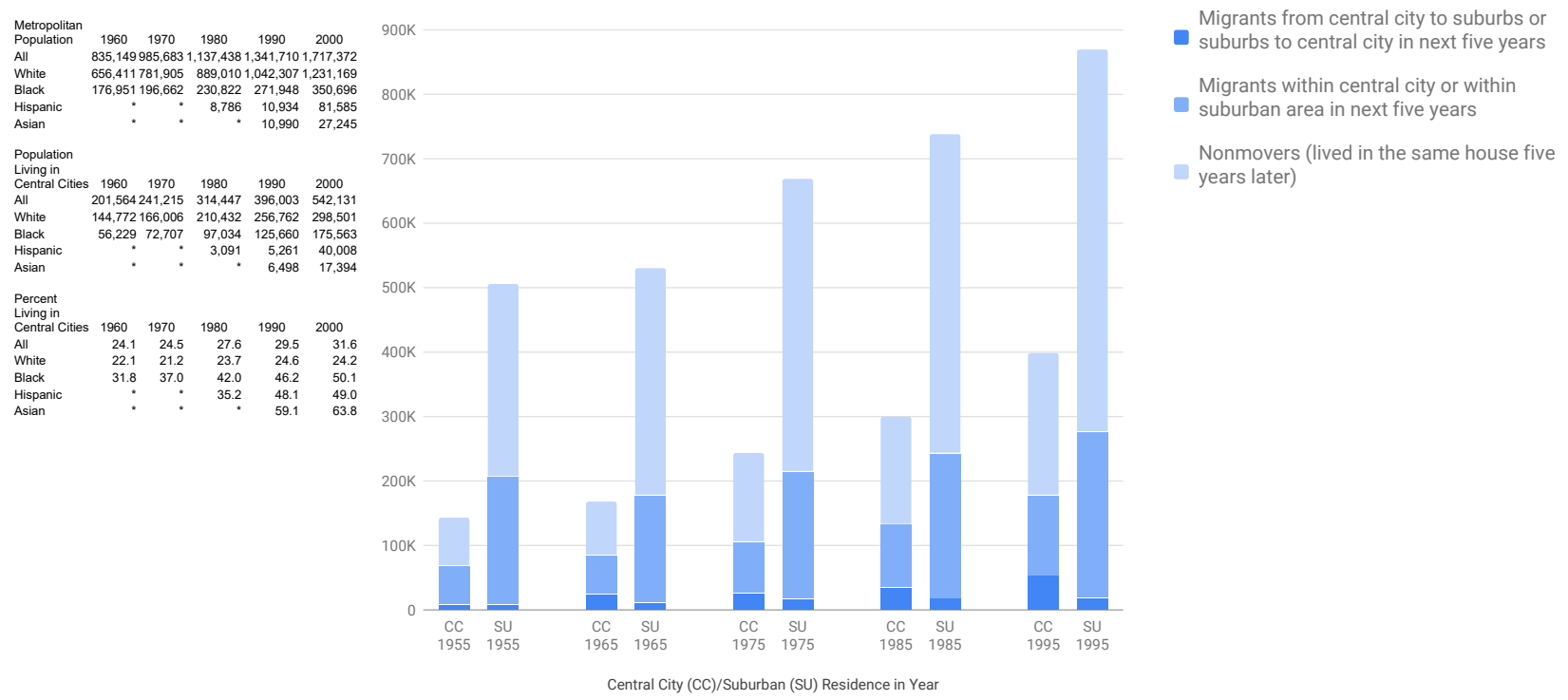

Urban-Suburban Net Migration Rate by Race

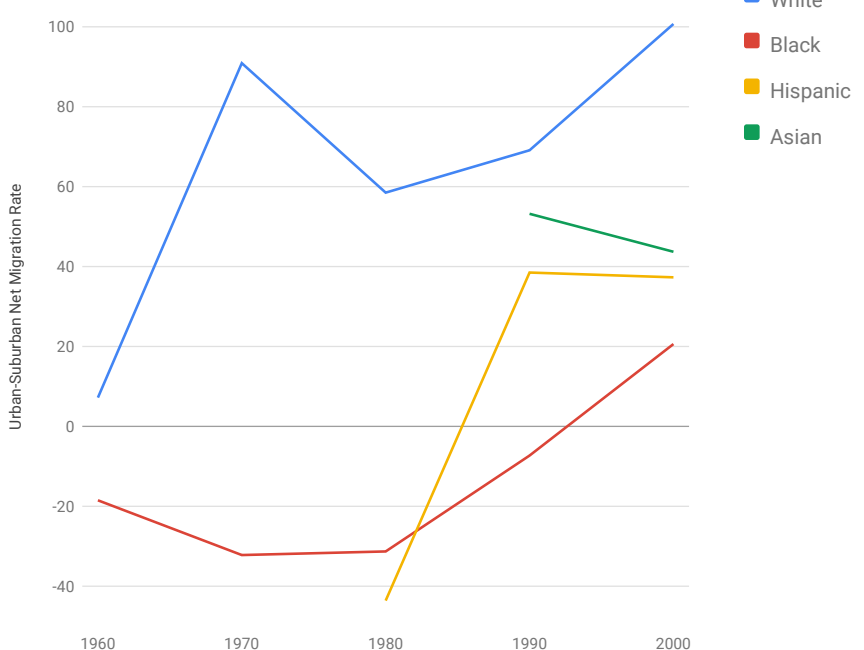

Year
Middle-Income Persons by Race

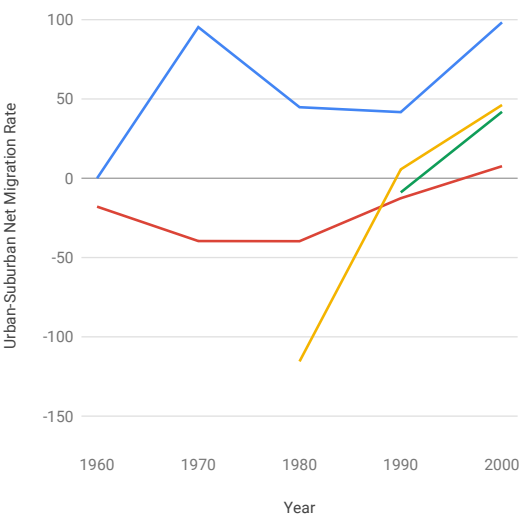

Year
Urban-Suburban Net Migration Rate by Income

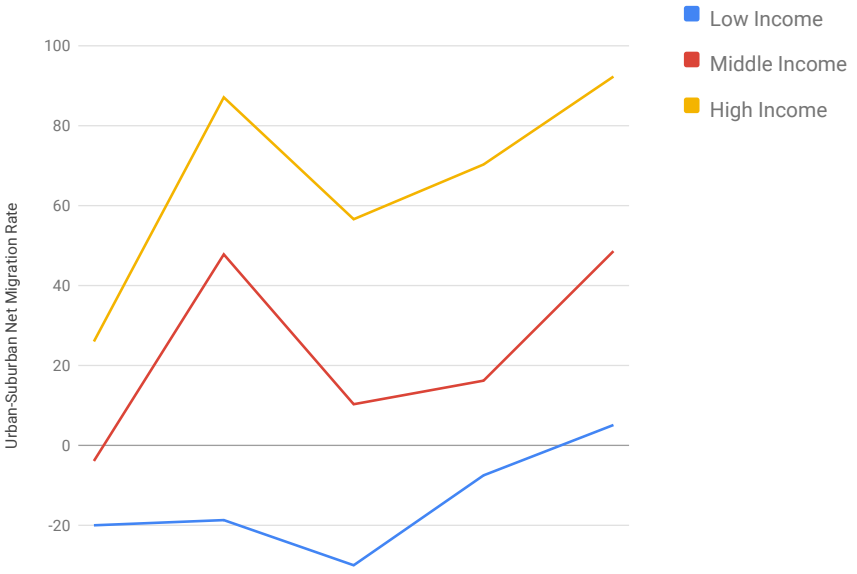

$-40$

$\begin{array}{lllll}1960 & 1970 & 1980 & 1990 & 2000\end{array}$

Year
Low-Income Persons by Race

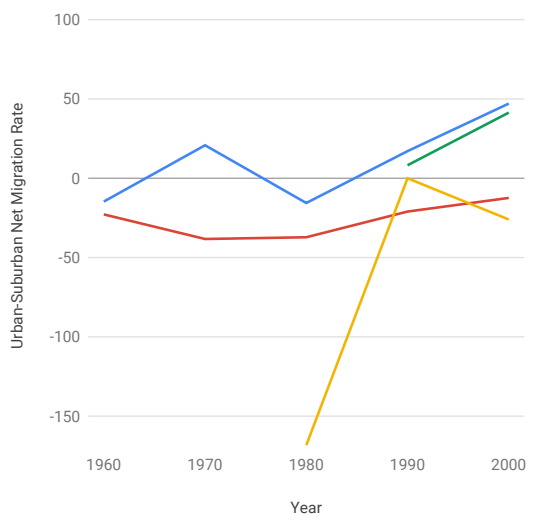

High-Income Persons by Race

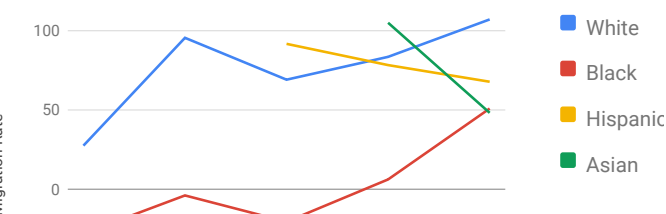




\section{Chattanooga, TN-GA}

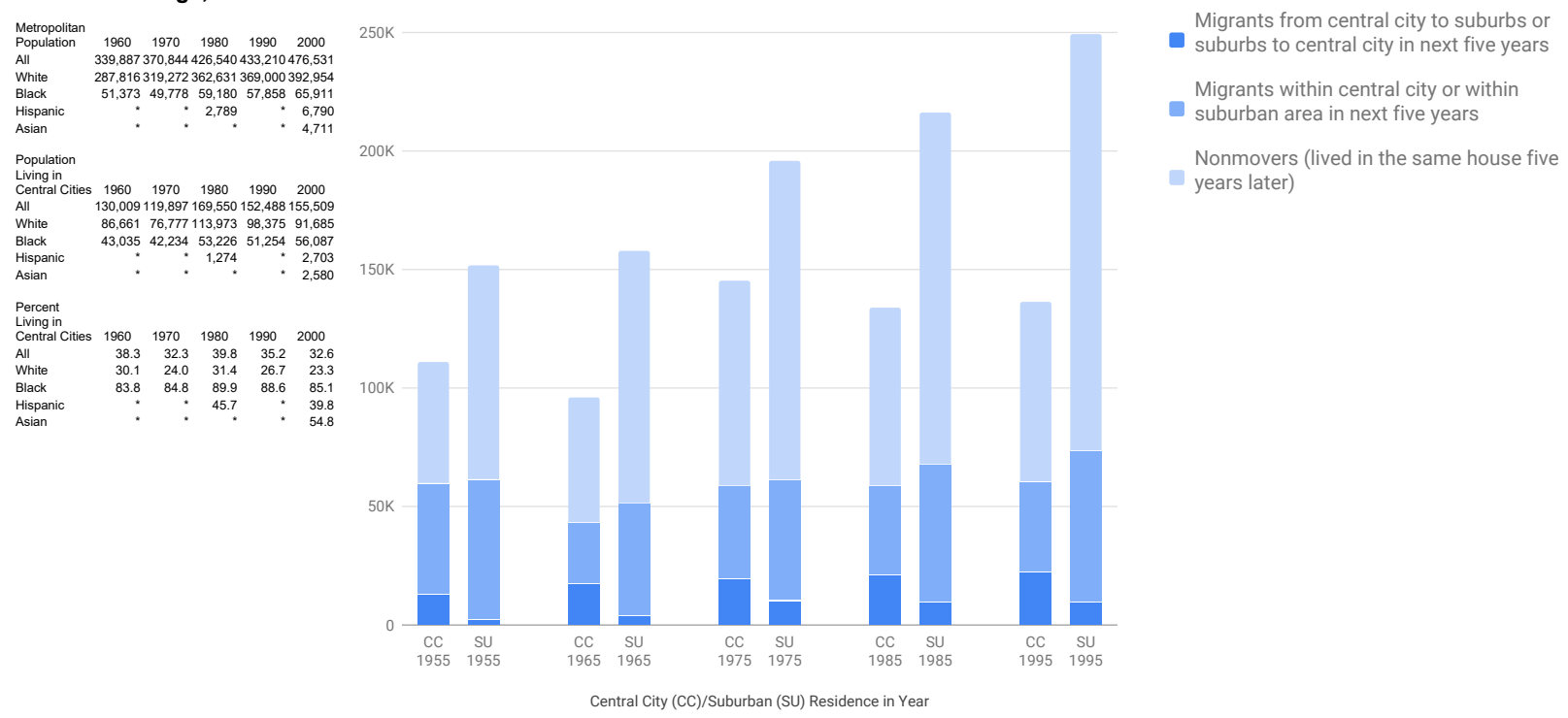

Urban-Suburban Net Migration Rate by Race

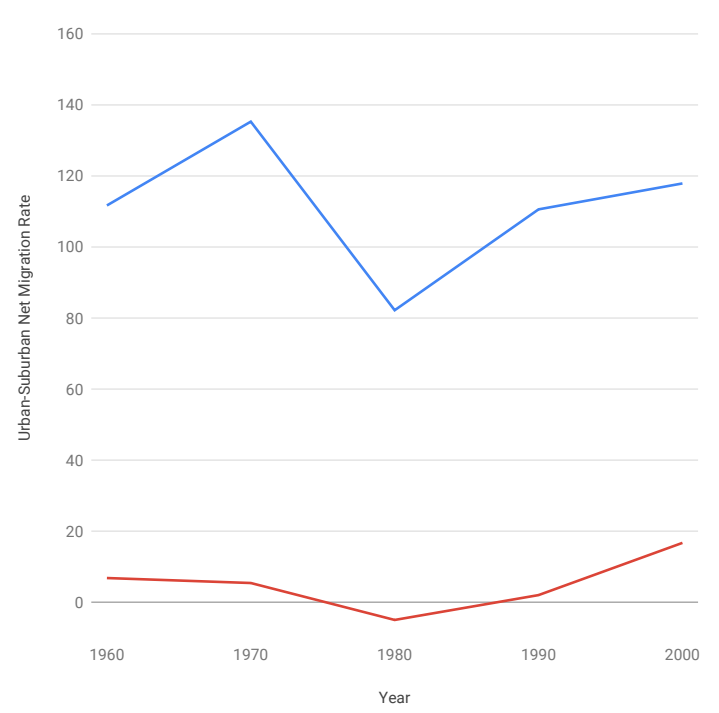

Low-Income Persons by Race

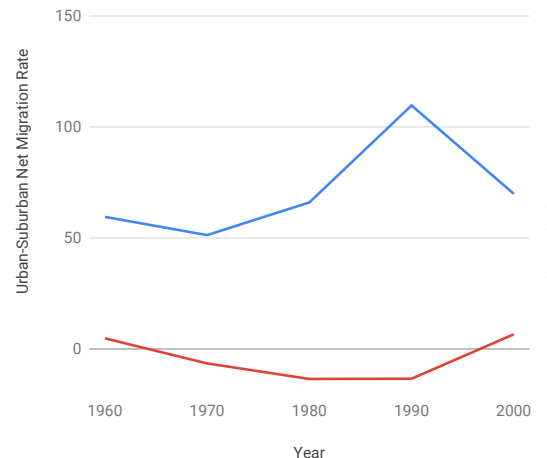

Year
Middle-Income Persons by Race
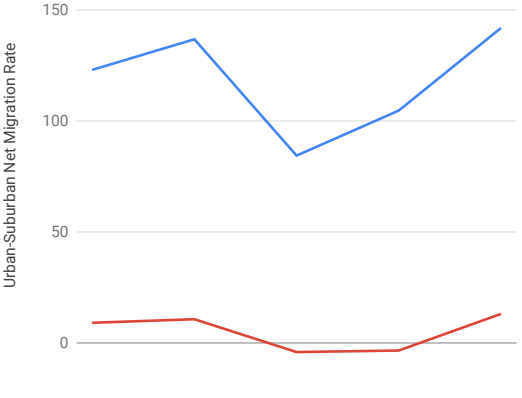

Year
Urban-Suburban Net Migration Rate by Income
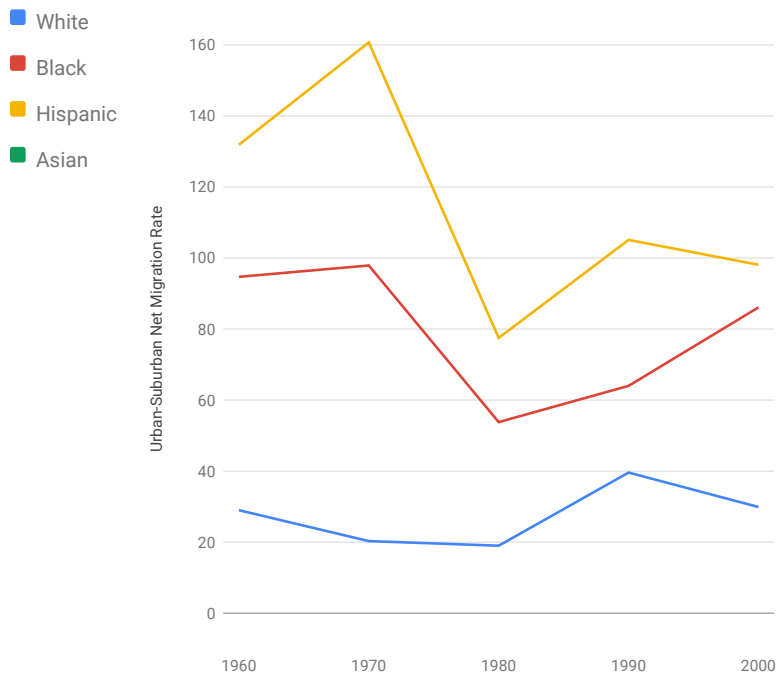

High-Income Persons by Race

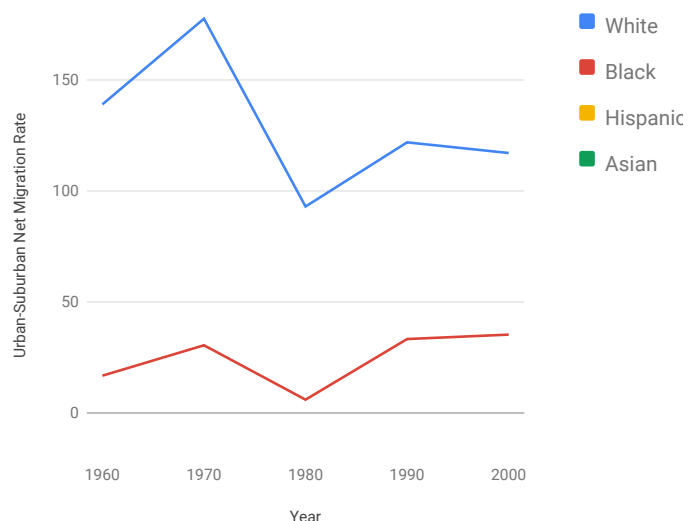




\section{Chicago, IL-IN-WI}

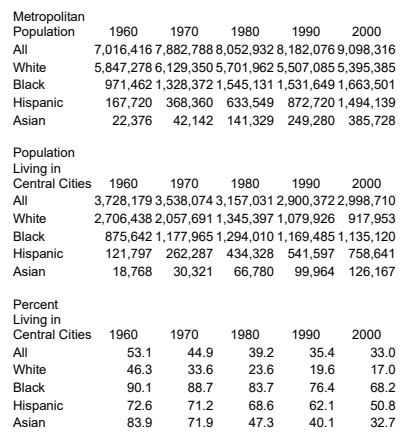

Urban and Suburban Residence, 1955-1995, and 5-year migration

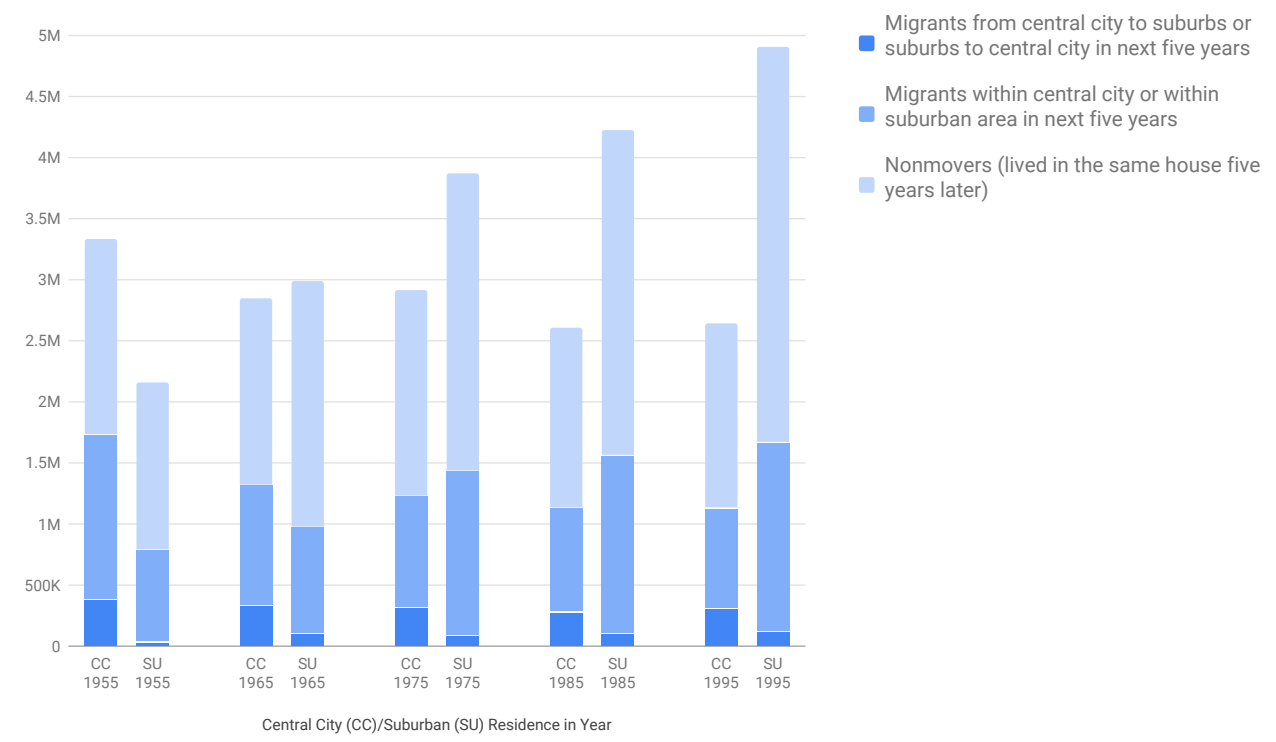

Urban-Suburban Net Migration Rate by Race

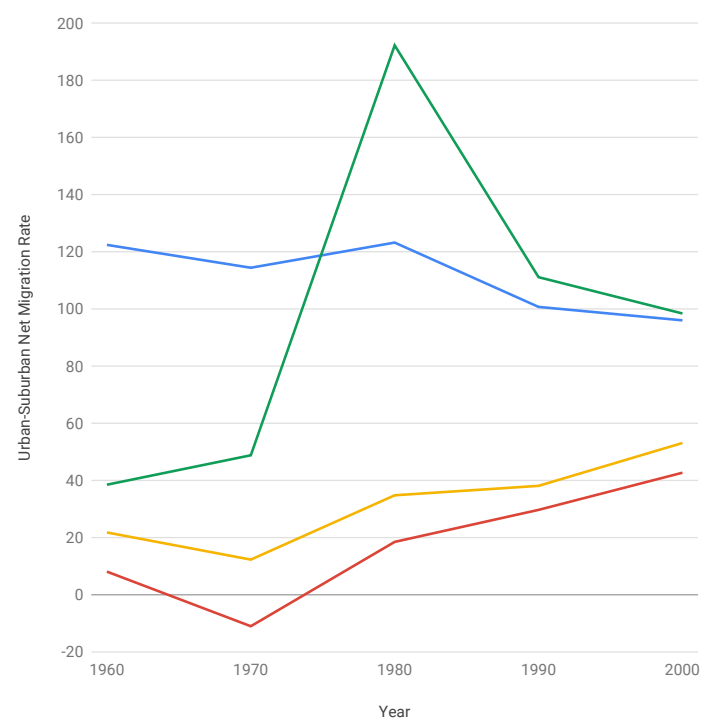

Low-Income Persons by Race

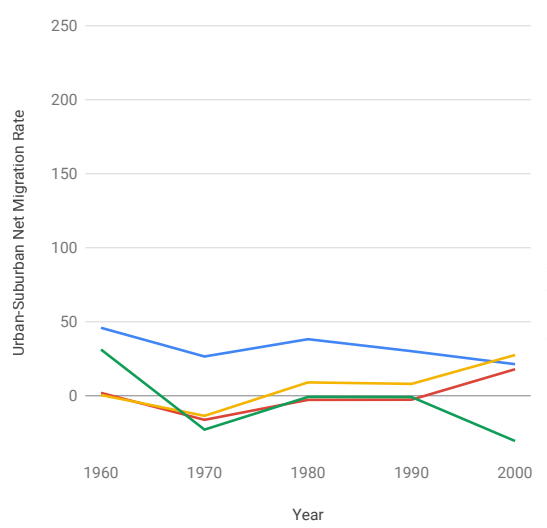

Middle-Income Persons by Race
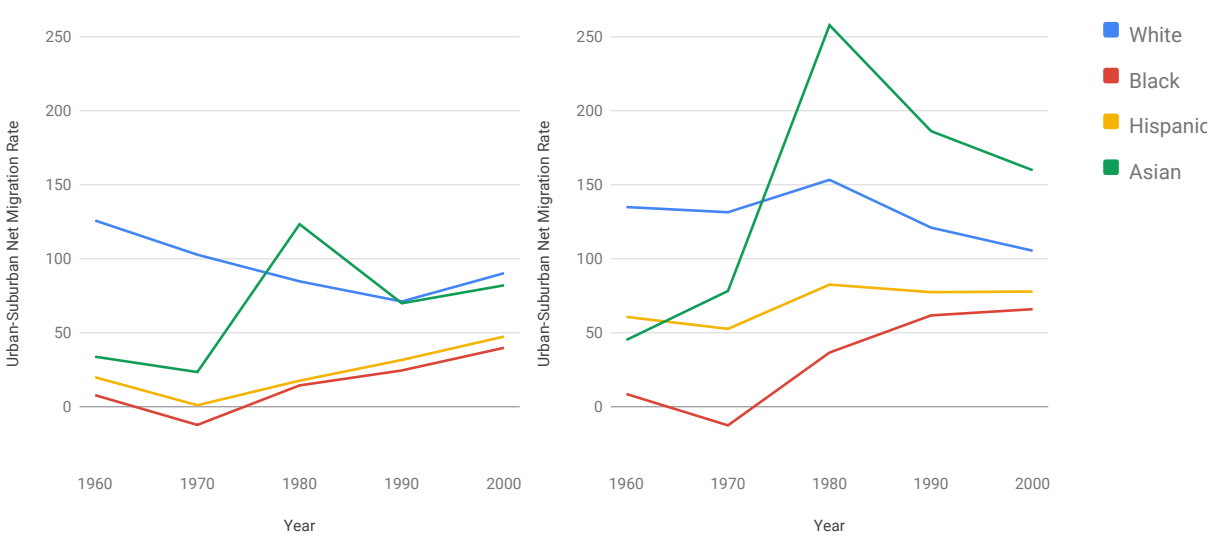

White

- Black

Hispanic

- Asian

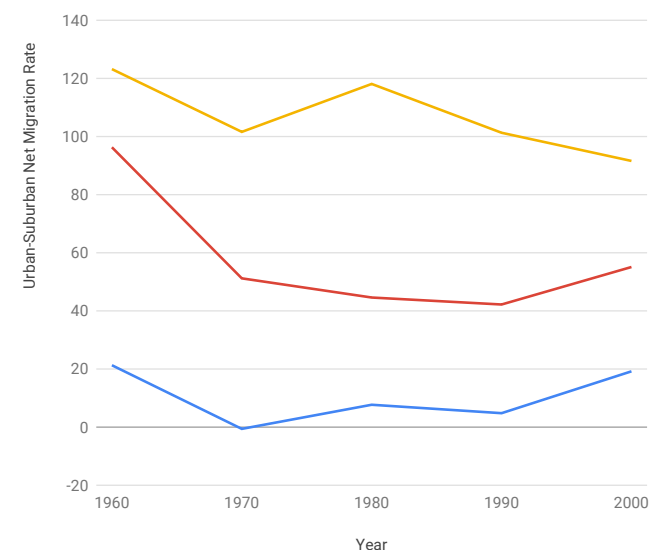

Low Income

- Middle Income

- High Income
High-Income Persons by Race

Year 
Urban and Suburban Residence, 1955-1995, and 5-year migration

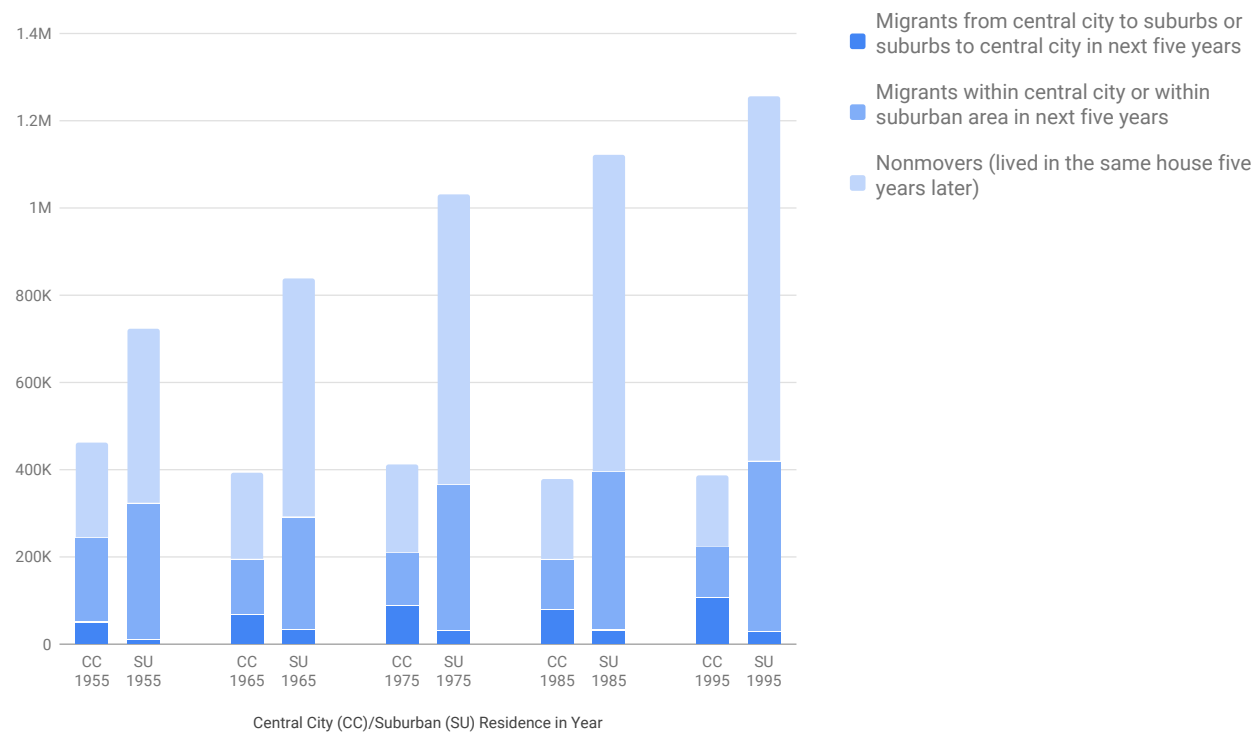

Urban-Suburban Net Migration Rate by Race

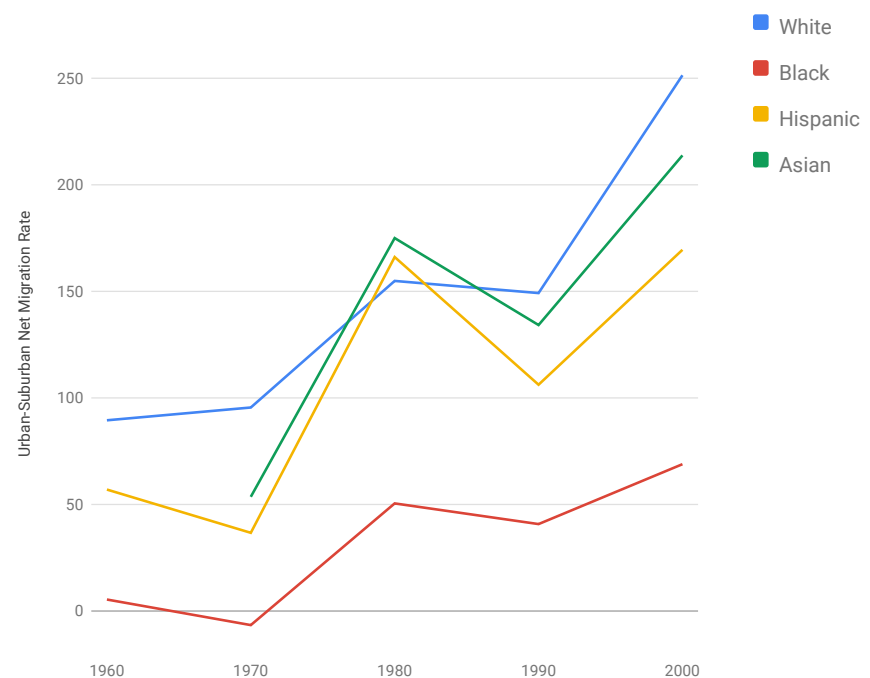

Year
Middle-Income Persons by Race
Urban-Suburban Net Migration Rate by Income

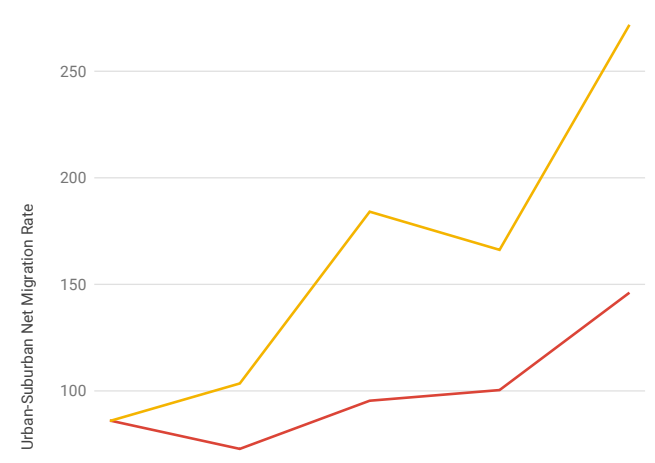

- Low Income

Middle Income

High Income

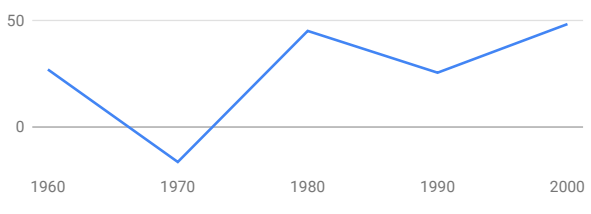

Year

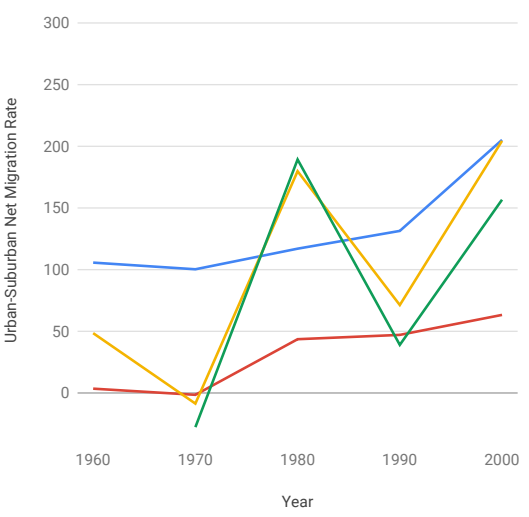

Year

High-Income Persons by Race
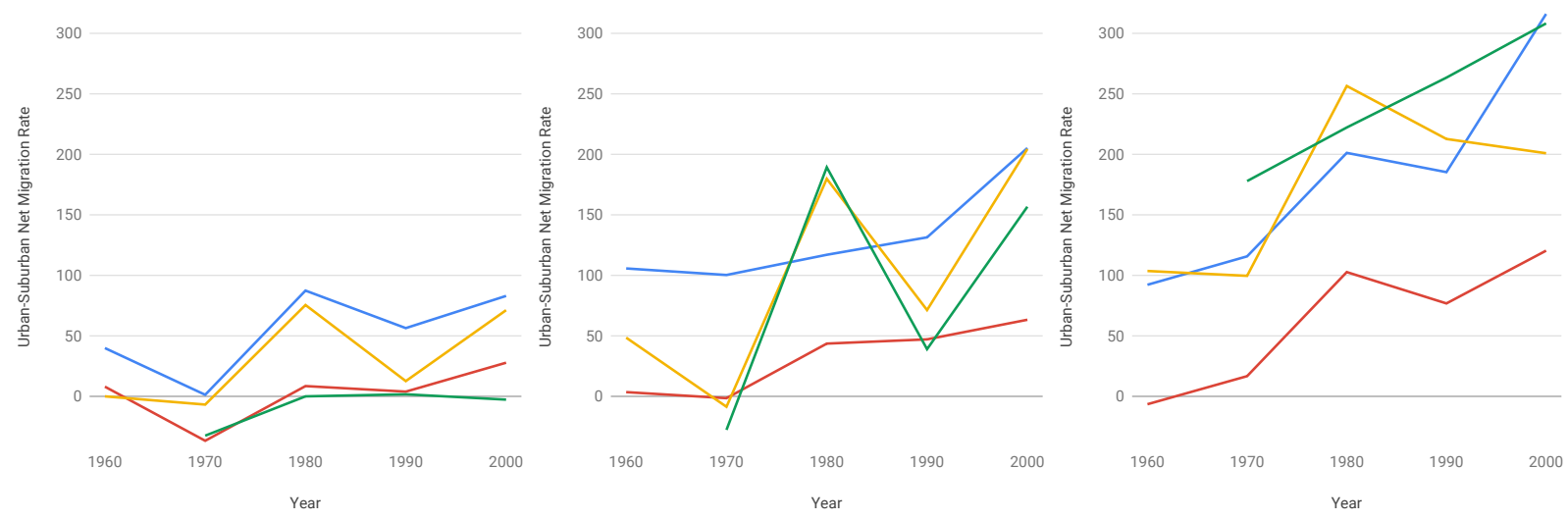

White

- Black

- Hispanic

- Asian 
Urban and Suburban Residence, 1955-1995, and 5-year migration

$1.4 \mathrm{M}$

Cleveland, $\mathrm{OH}$

$\begin{array}{lrllll}\text { Metropolitan } & 1960 & 1970 & 1980 & 1990 & 2000 \\ \text { Population } & 1960 \\ \text { All } & 2,126,9832,321,0372,173,7342,102,2482,148,143\end{array}$

$\begin{array}{ll}\text { All } & 2,126,9832,321,0372,173,7342,102,2482,148,143 \\ \text { White } & 1,834,9021,932,6251,752,7731,653,4831,602,583\end{array}$

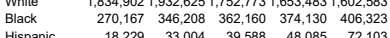

$\begin{array}{lrrrrr}\text { Hispanic } & 18,229 & 33,004 & 39,588 & 48,085 & 72,103 \\ \text { Asian } & 2,468 & 6,023 & 14,218 & 21,036 & 31,131\end{array}$

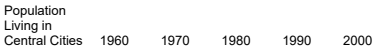

$\begin{array}{llllll}\text { Central Cities } & 1960 & 1970 & 1980 & 1990 & 2000 \\ \text { All } & 919,832 & 804,693 & 631,360 & 562,362 & 534275\end{array}$

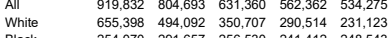

$\begin{array}{lllll}254,070 & 291,657 & 256,530 & 241,412 & 248,543\end{array}$

$\begin{array}{lrrrrr}\text { Hispanic } & 8,196 & 15,103 & 18,572 & 23,446 & 35,950 \\ \text { Asian } & 1,399 & 2,100 & 3,273 & 5,005 & 6,949\end{array}$

$\begin{array}{lllllll}\text { Percent } & & & & & \\ \text { Living in } \\ \text { Central Cities } & 1960 & 1970 & 1980 & 1990 & 2000 & 400 K\end{array}$

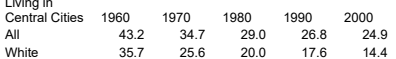

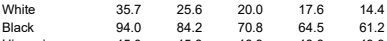

$\begin{array}{lllllll}\text { Hispanic } & 94.0 & 84.2 & 70.8 & 64.5 & 61.2 & \\ \text { Asian } & 45.0 & 45.8 & 46.9 & 48.8 & 49.9 & 200 \mathrm{~K} \\ & 56.7 & 34.9 & 23.0 & 23.8 & 22.3 & \end{array}$

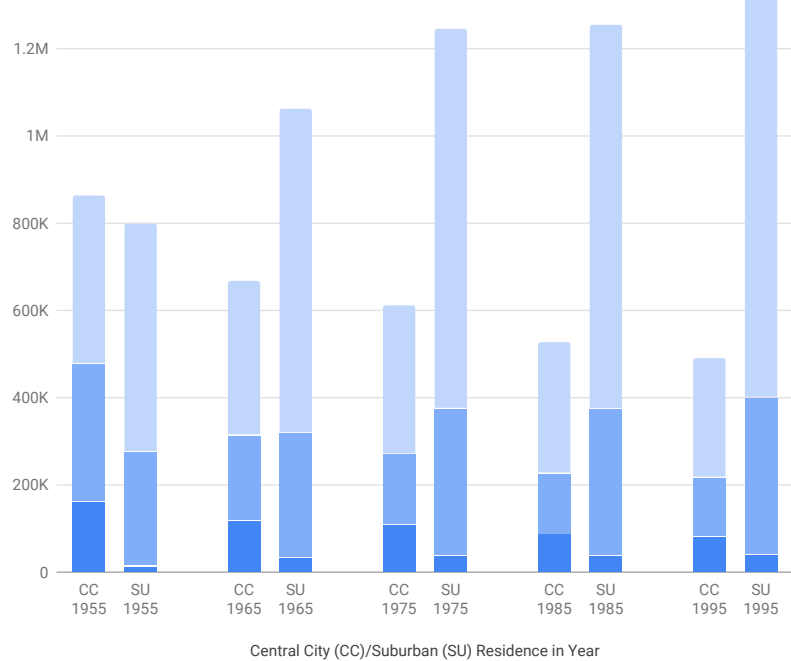

Migrants from central city to suburbs or suburbs to central city in next five years

Migrants within central city or within suburban area in next five years

Nonmovers (lived in the same house five

years later)
Urban-Suburban Net Migration Rate by Race

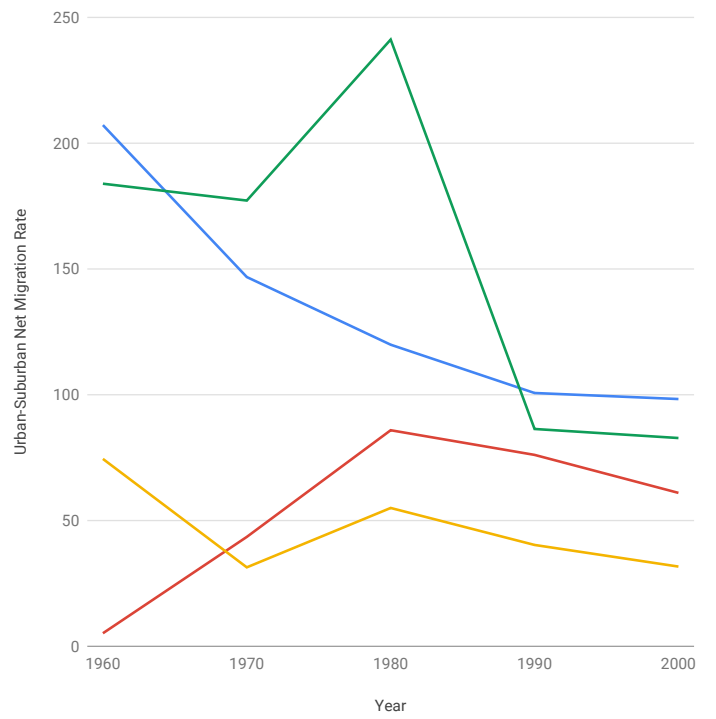

Low-Income Persons by Race

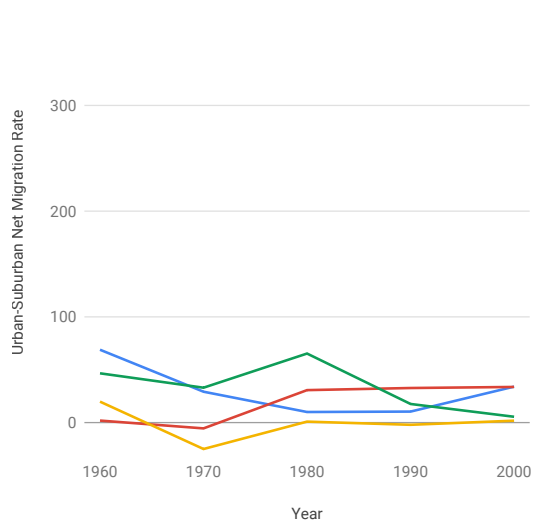

Middle-Income Persons by Race

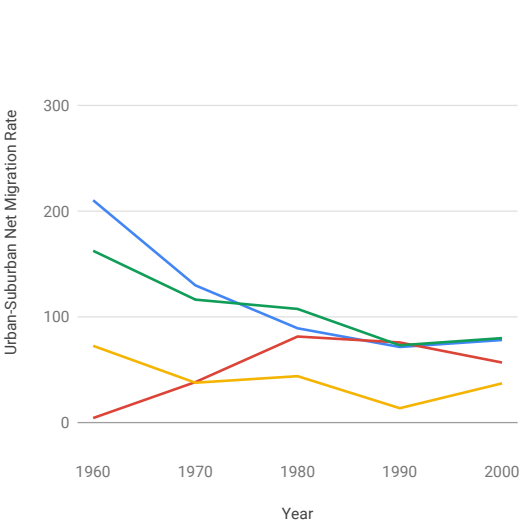

Urban-Suburban Net Migration Rate by Income

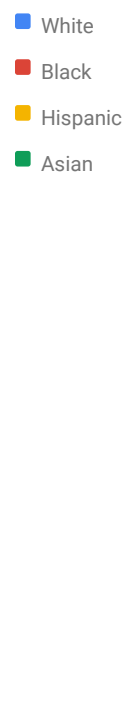

250
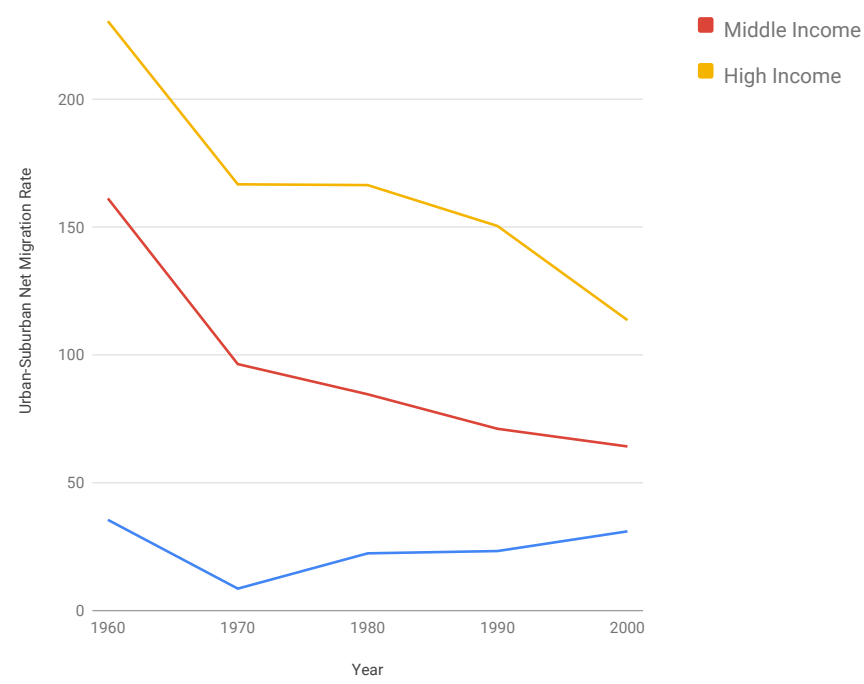

High-Income Persons by Race

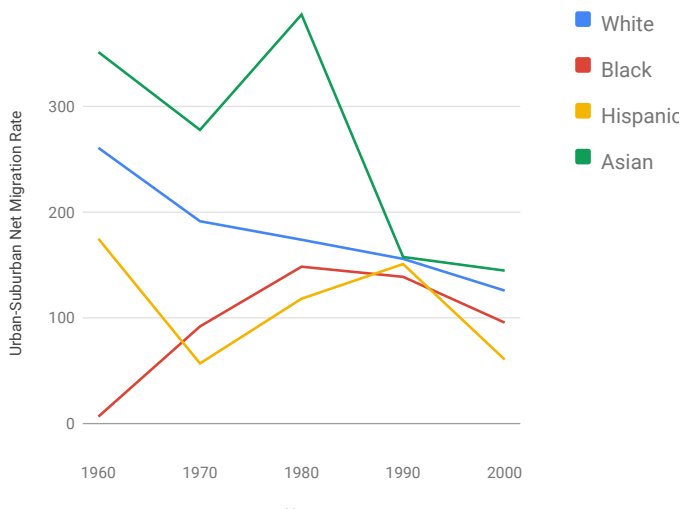


Urban and Suburban Residence, 1955-1995, and 5-year migration

\section{Colorado Springs, CO}

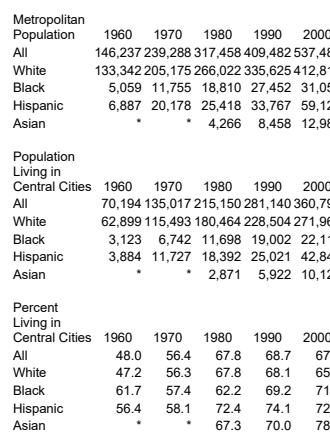

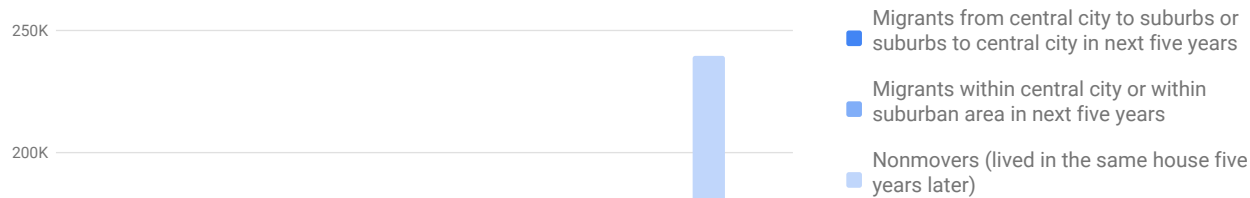

Migrants from central city to suburbs o - suburbs to central city in next five years

Migrants within central city or within

suburban area in next five years

Nonmovers (lived in the same house five years later)

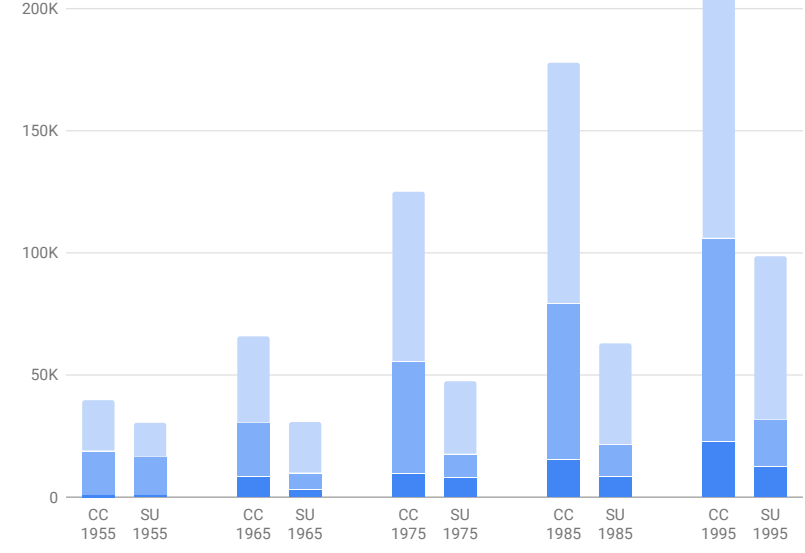

Central City (CC)/Suburban (SU) Residence in Yea

Urban-Suburban Net Migration Rate by Race

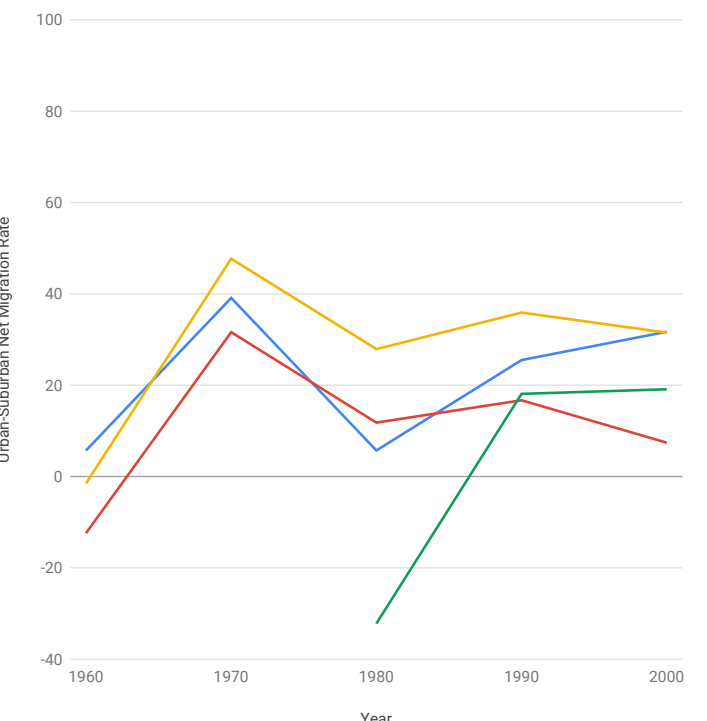

Low-Income Persons by Race

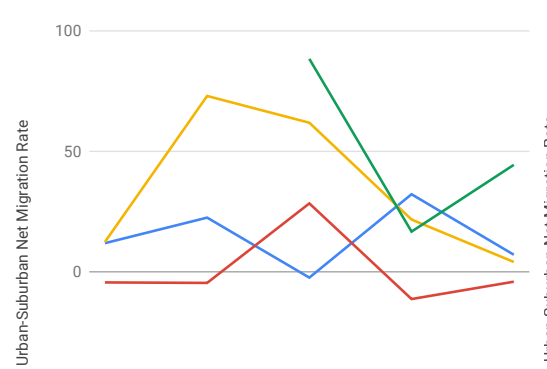

$-50$

1960
White

Black

Hispanic

- Asian

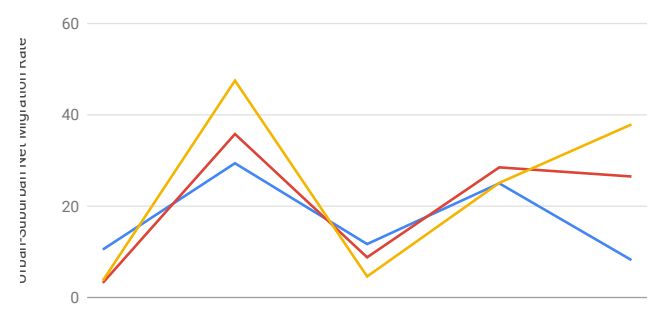

$-20$

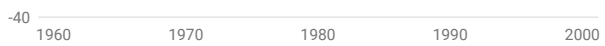

Year
Low Income

- Middle Income

- High Income
Middle-Income Persons by Race

100

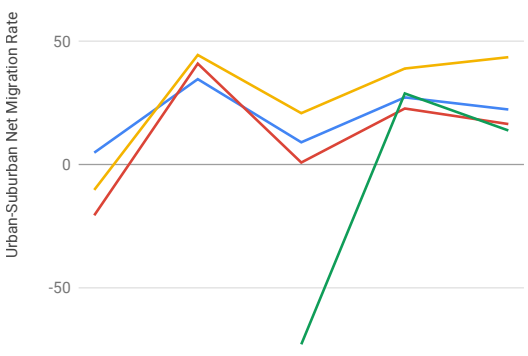

Year
100

White

- Black

Hispanic

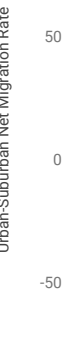

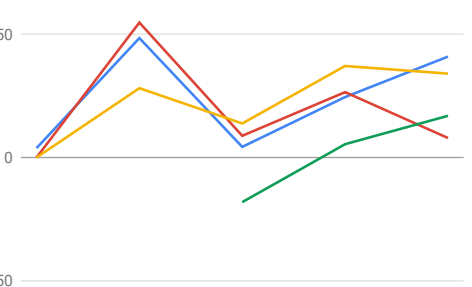

- Asian 
Urban and Suburban Residence, 1955-1995, and 5-year migration

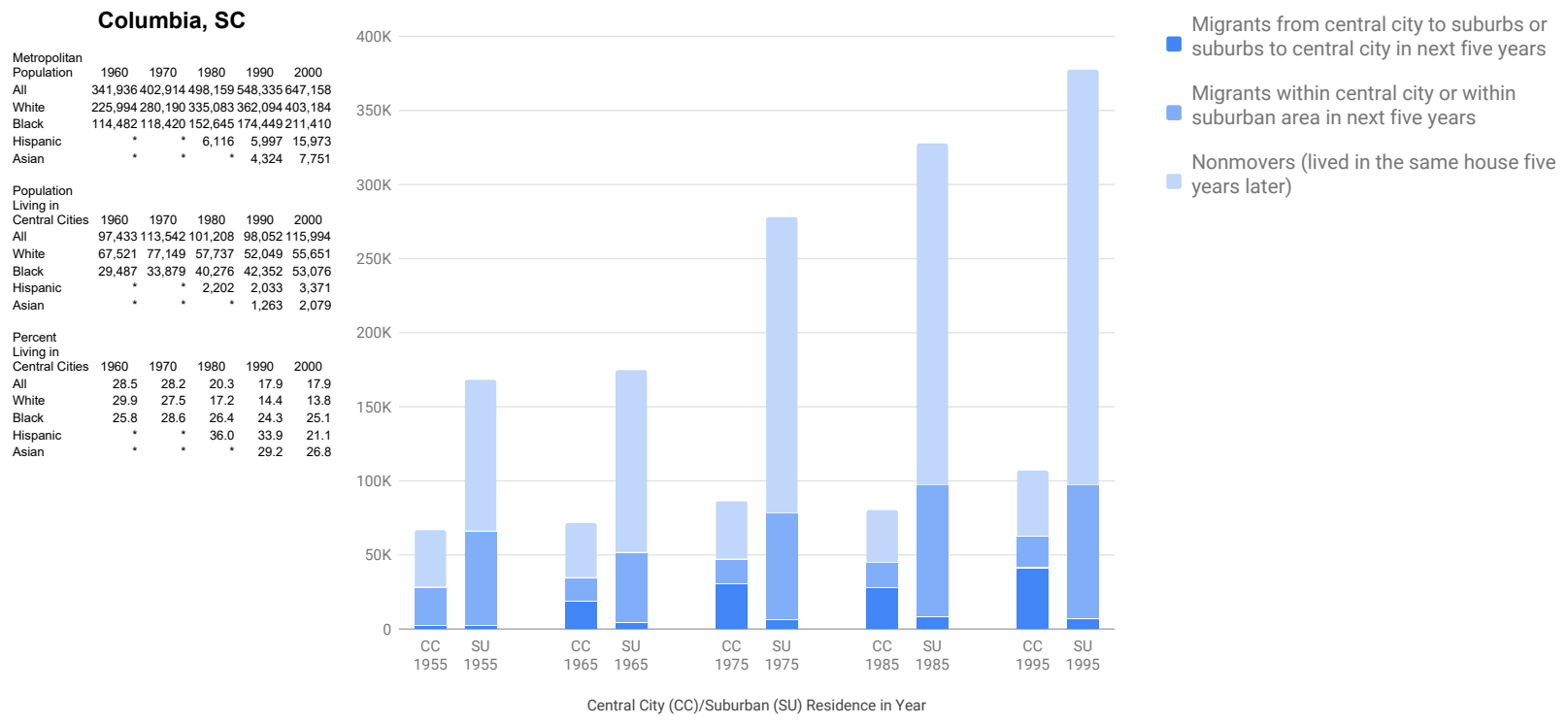

Urban-Suburban Net Migration Rate by Race

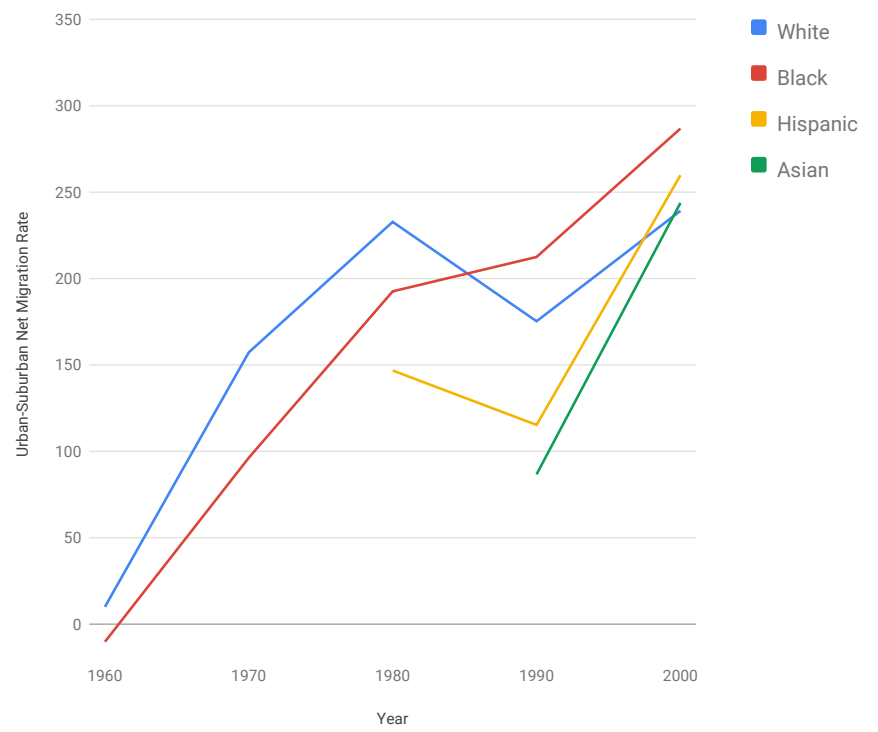

Low-Income Persons by Race
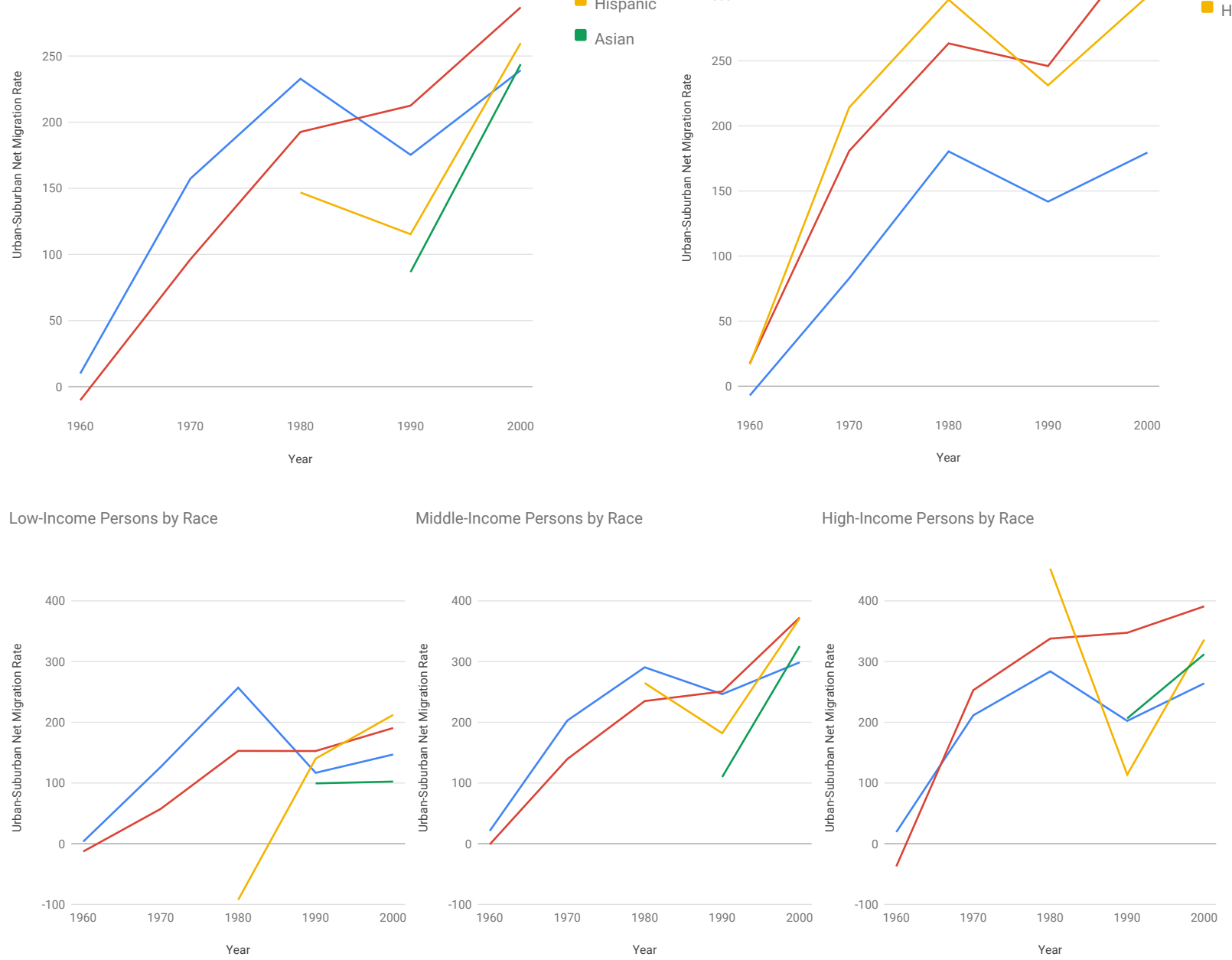

Low Income

Middle Income

High Income 
Urban and Suburban Residence, 1955-1995, and 5-year migration

\section{Columbus, $\mathrm{OH}$}

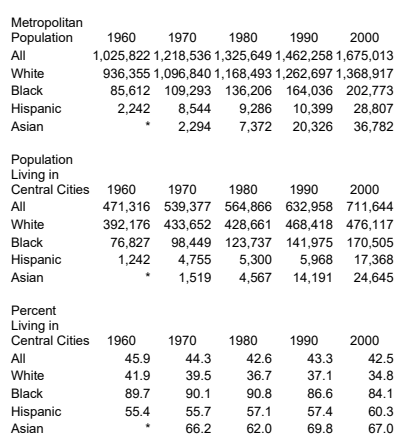

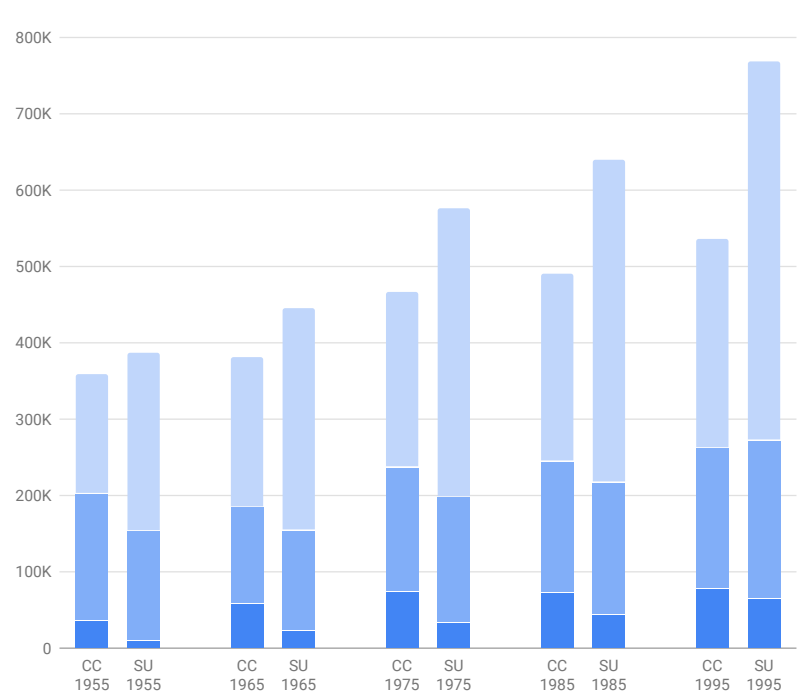

Migrants from central city to suburbs or suburbs to central city in next five years

Migrants within central city or within suburban area in next five years

Nonmovers (lived in the same house five years later)
Urban-Suburban Net Migration Rate by Race

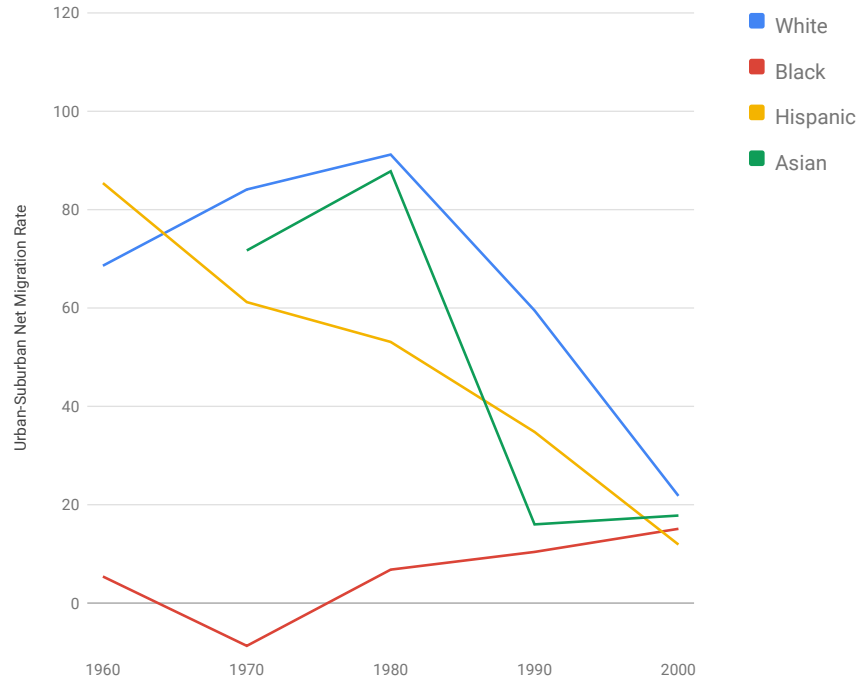

Year
Urban-Suburban Net Migration Rate by Income

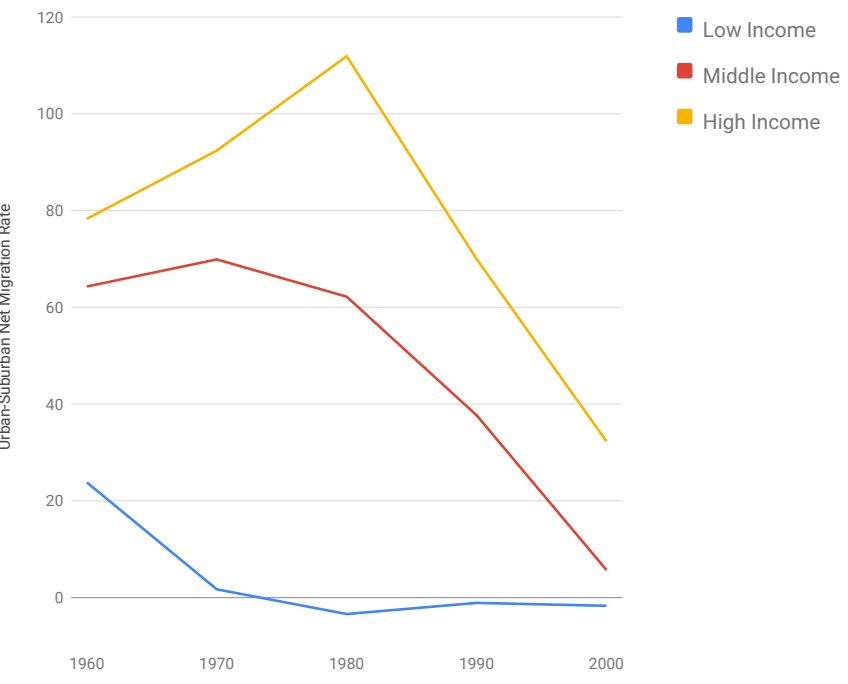

Low-Income Persons by Race

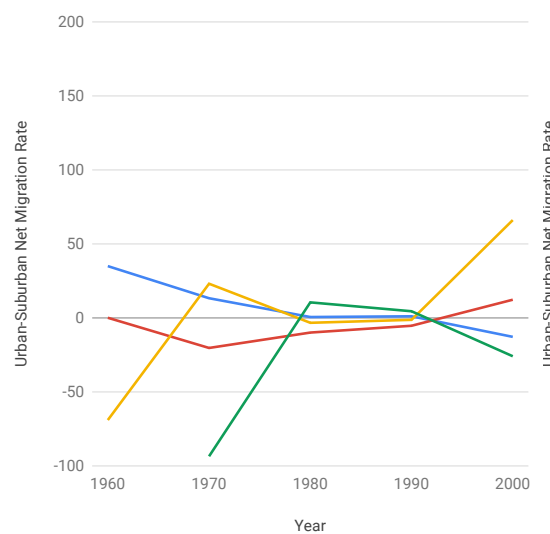

Middle-Income Persons by Race

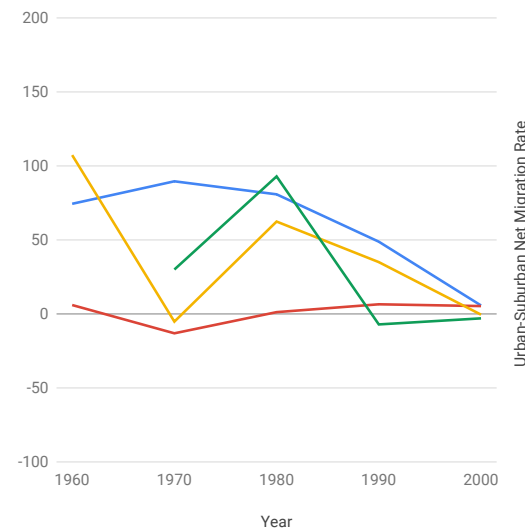

High-Income Persons by Race

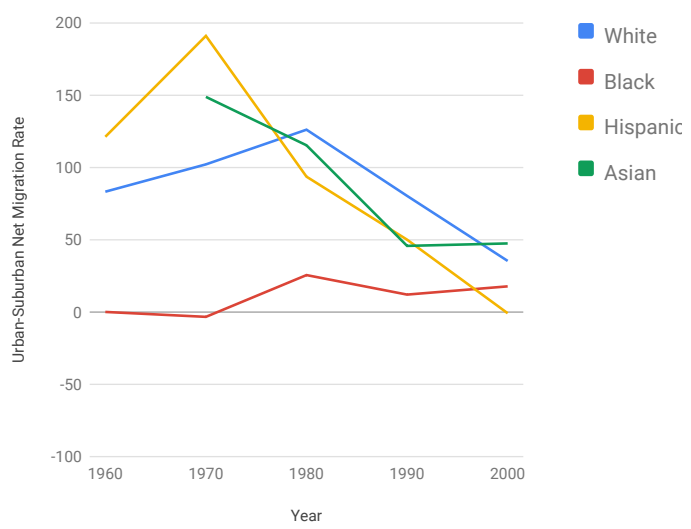


Urban and Suburban Residence, 1955-1995, and 5-year migration

\section{$2.5 \mathrm{~N}$}

\section{Dallas-Fort Worth, TX}

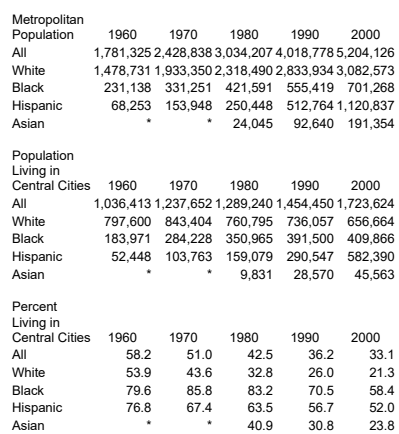

Urban-Suburban Net Migration Rate by Race

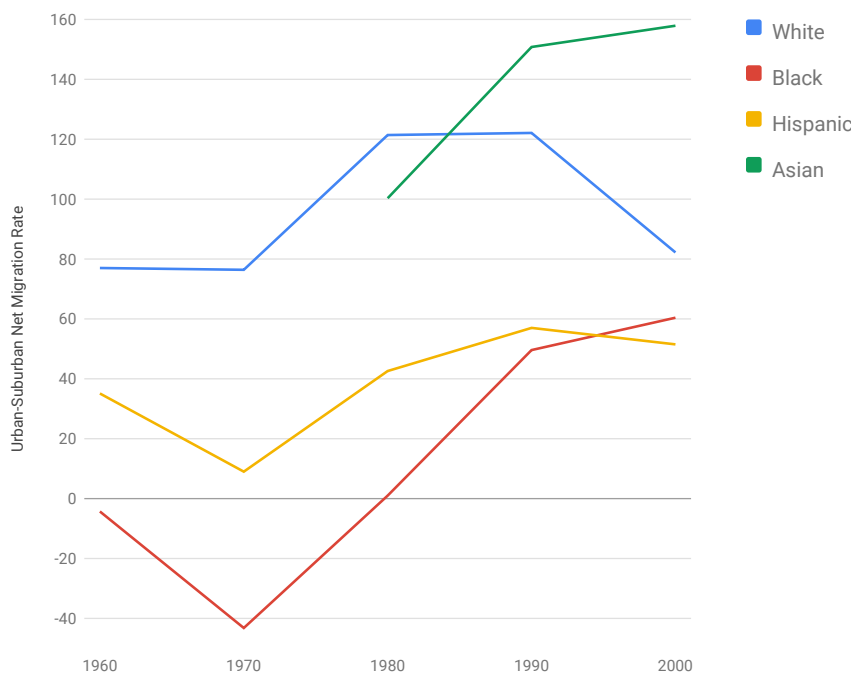

Year

Low-Income Persons by Race
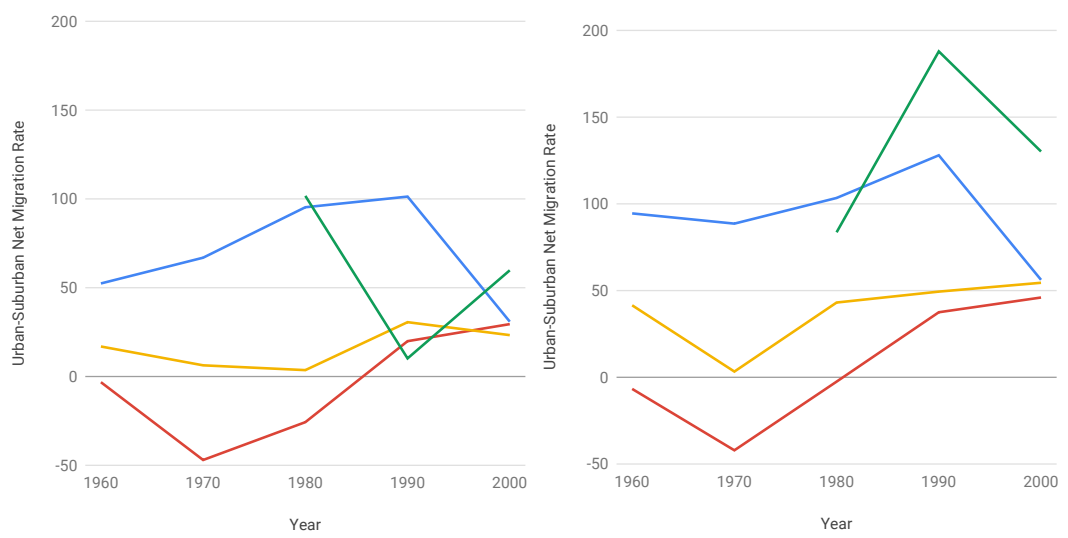

$1.5 \mathrm{M}$

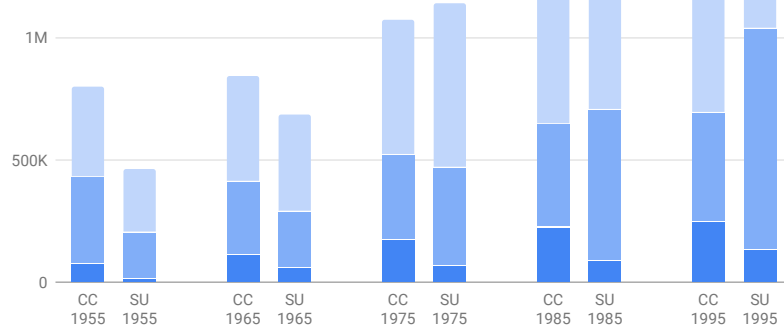

Central City (CC)/Suburban (SU) Residence in Yea
Migrants from central city to suburbs or suburbs to central city in next five years

Migrants within central city or within

suburban area in next five years

Nonmovers (lived in the same house five years later)
Urban-Suburban Net Migration Rate by Income

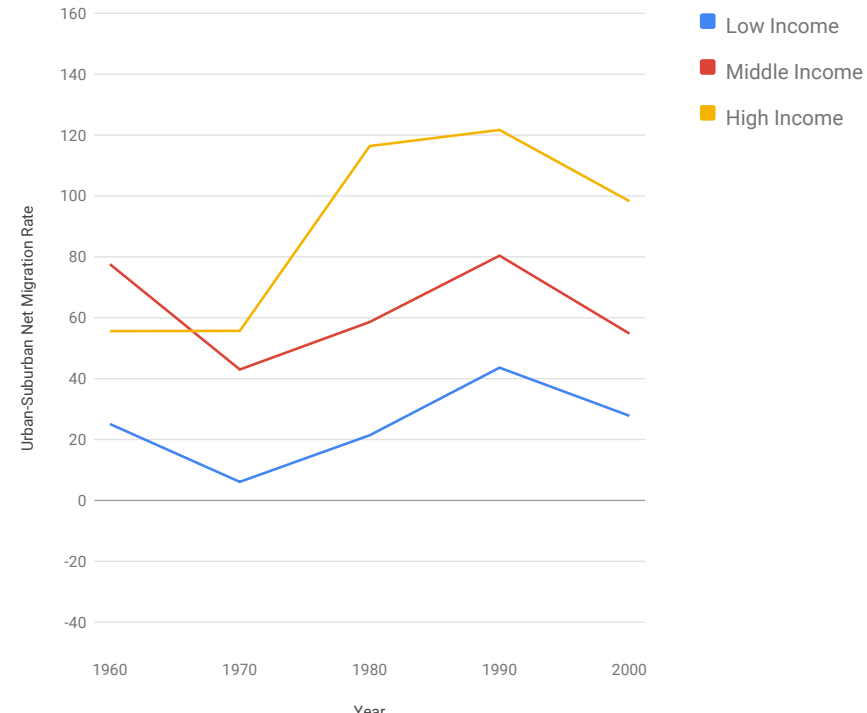

High-Income Persons by Race

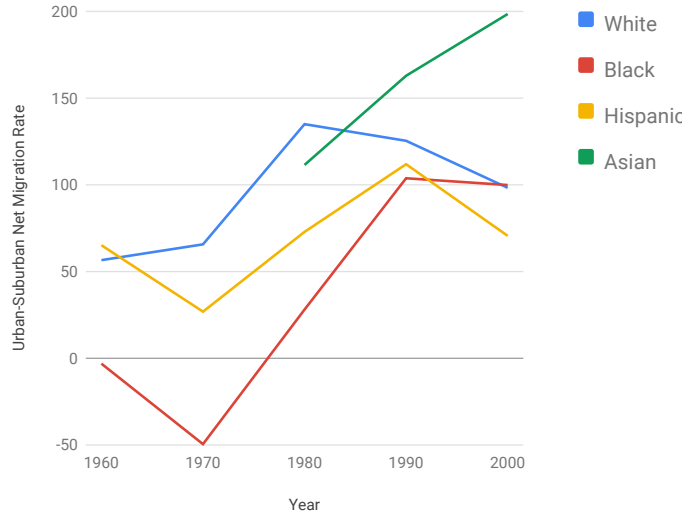




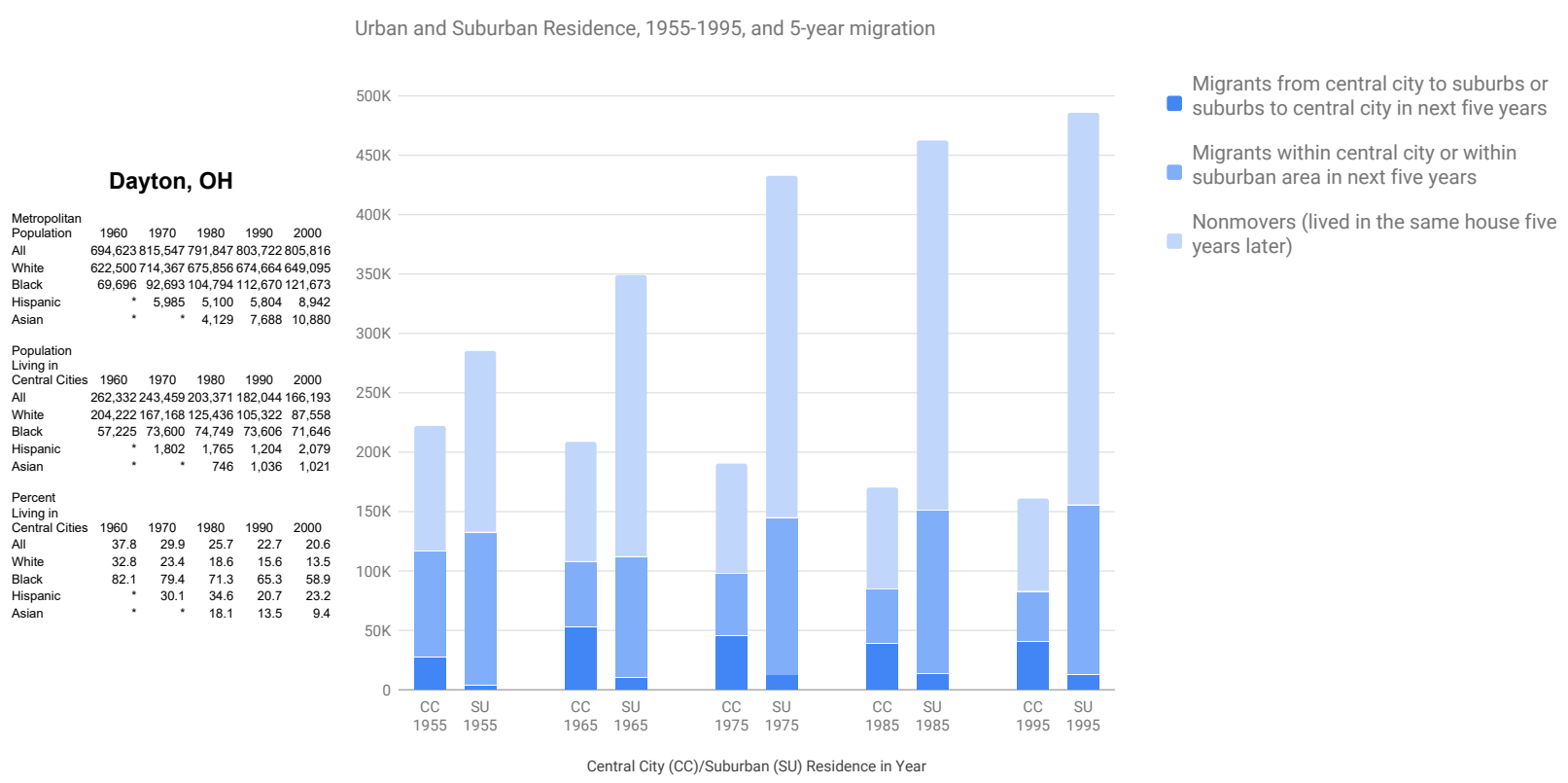

Urban-Suburban Net Migration Rate by Race

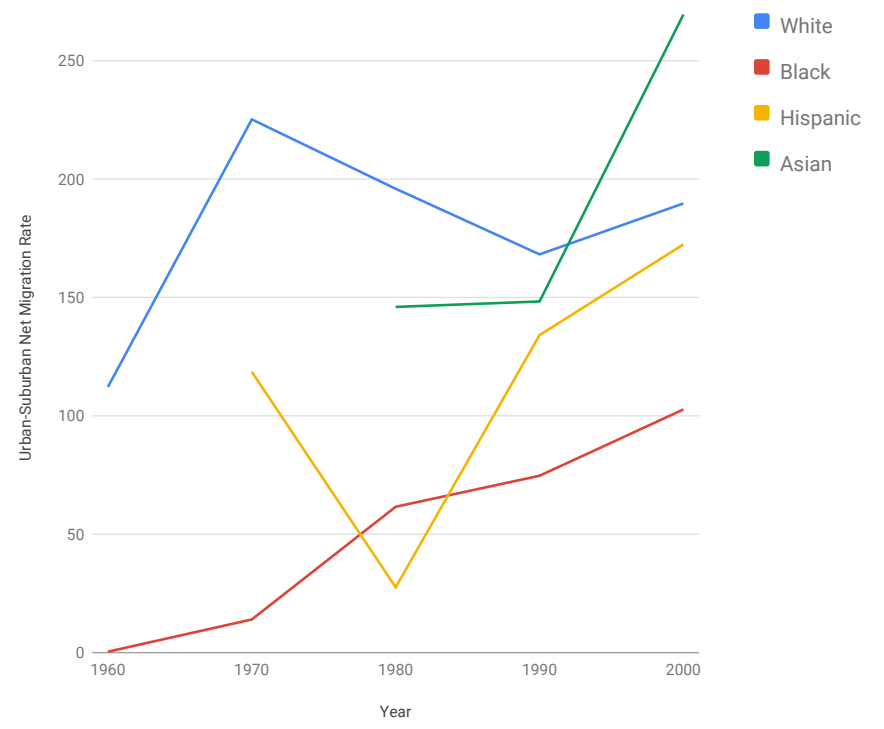

Low-Income Persons by Race

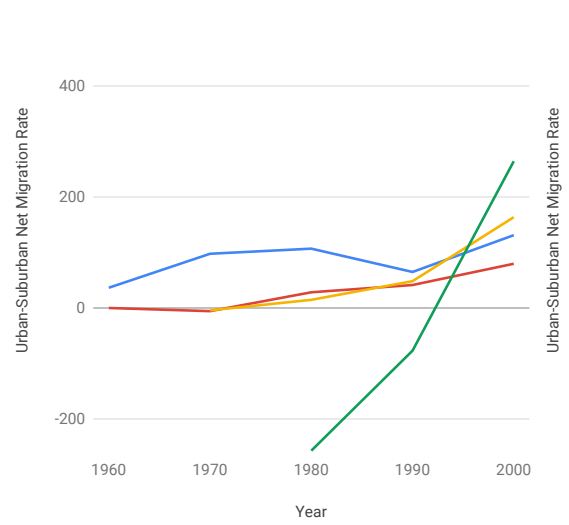

Middle-Income Persons by Race
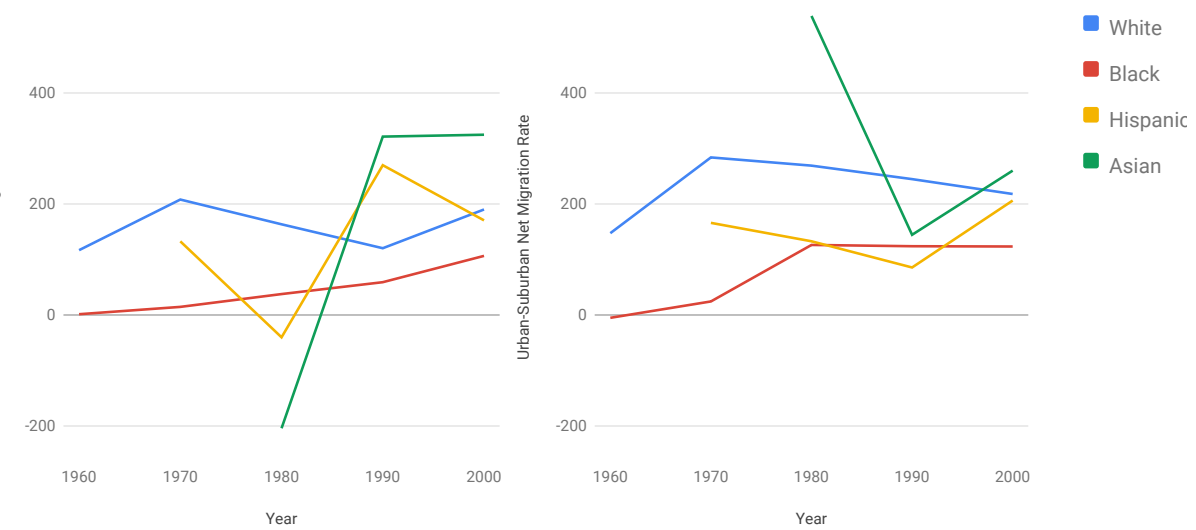

Urban-Suburban Net Migration Rate by Income

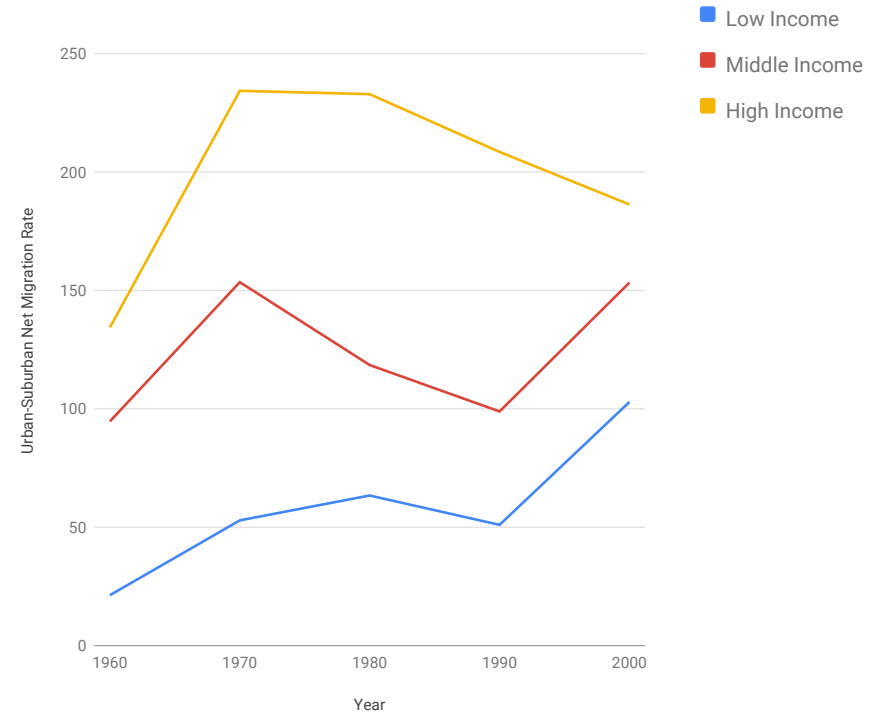

High-Income Persons by Race 
Urban and Suburban Residence, 1955-1995, and 5-year migration

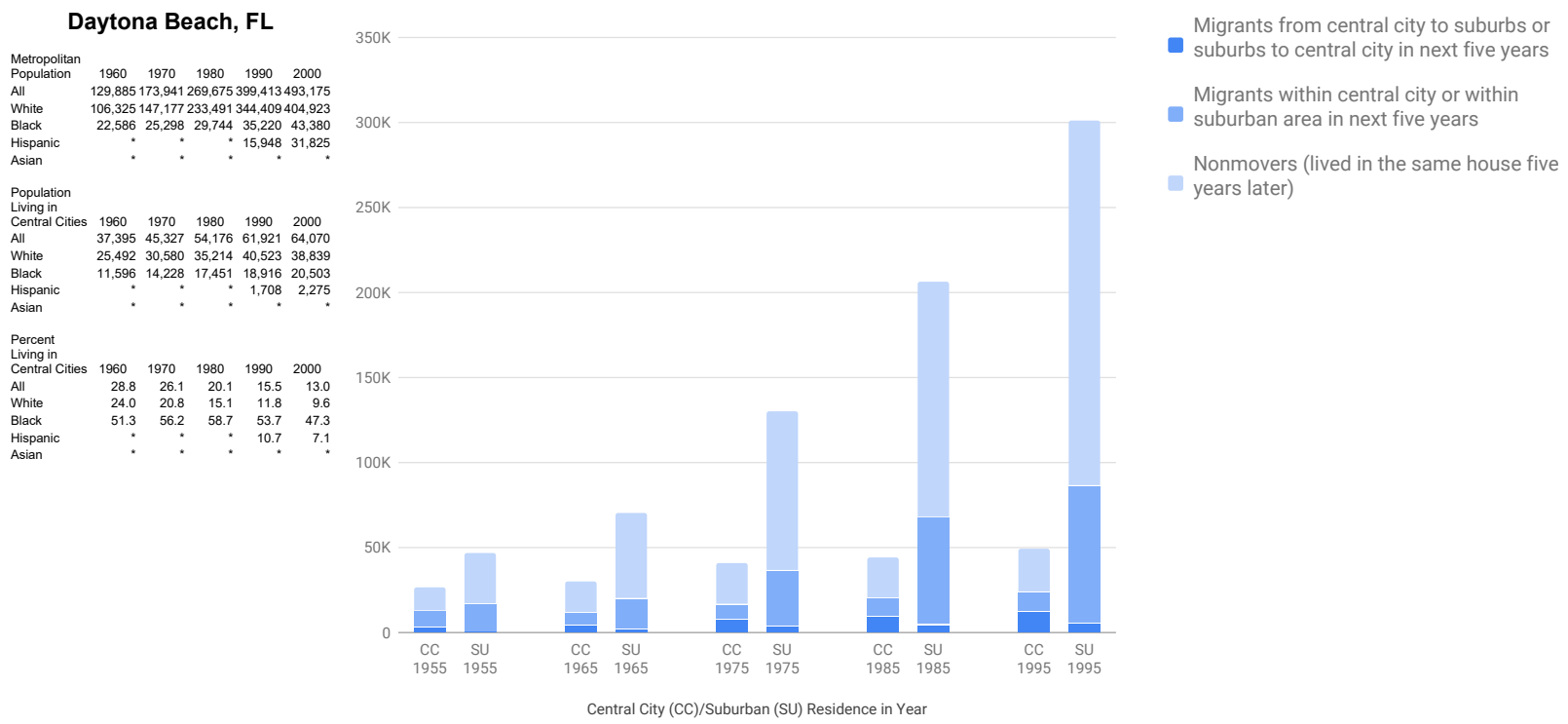

Urban-Suburban Net Migration Rate by Race

Urban-Suburban Net Migration Rate by Income

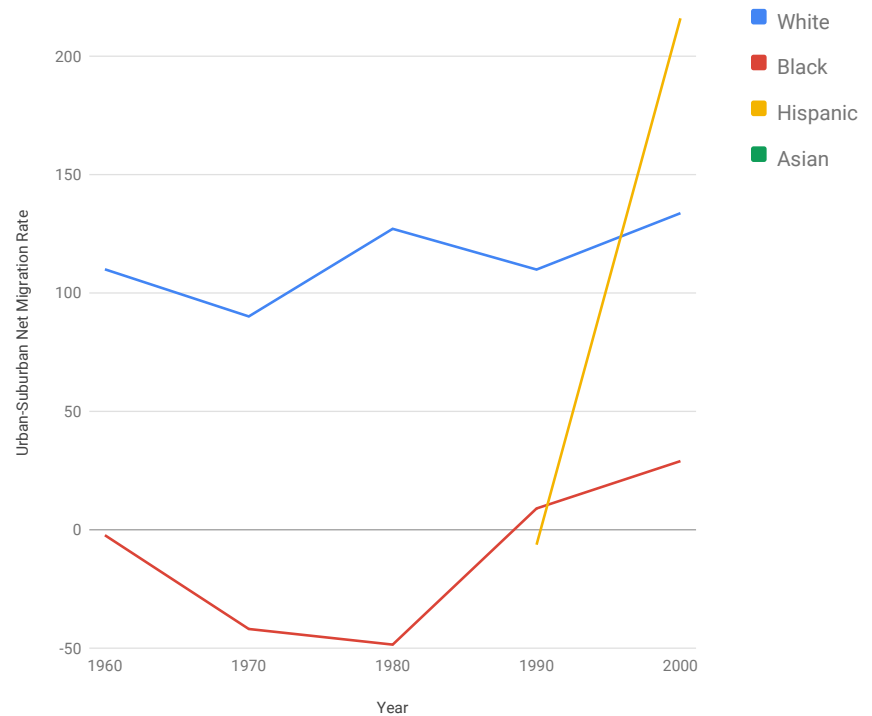

Low-Income Persons by Race

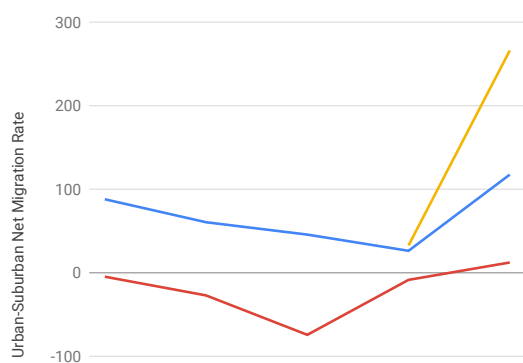

$-200$

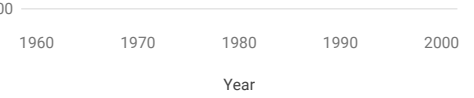

Middle-Income Persons by Race

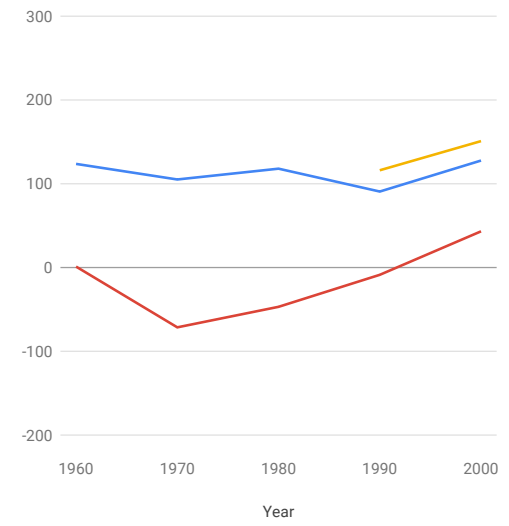

High-Income Persons by Race

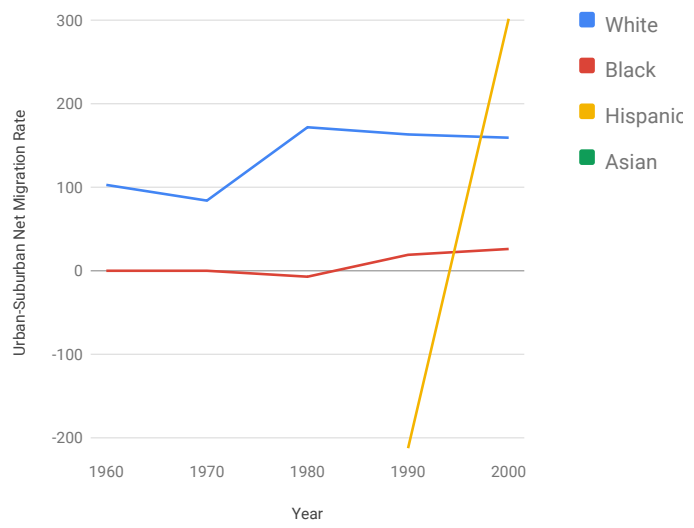


Urban and Suburban Residence, 1955-1995, and 5-year migration

\section{Denver, CO}

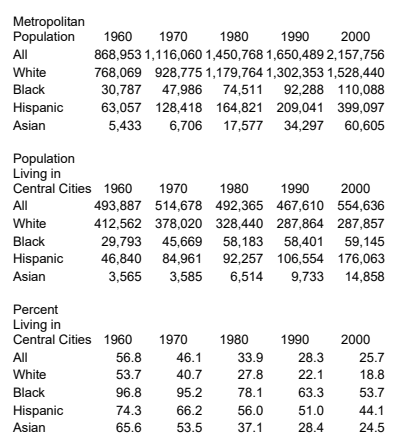

$1.2 \mathrm{M}$

$$
1 \mathrm{M}
$$$$
800 \mathrm{~K}
$$$$
600 \mathrm{~K}
$$$$
600 \mathrm{~K}
$$

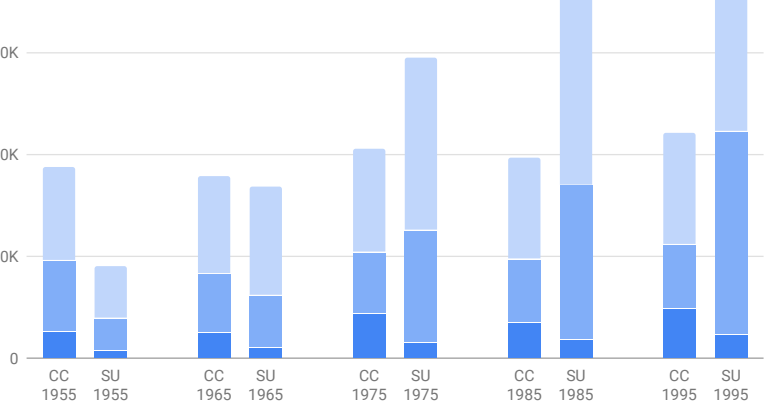

Central City (CC)/Suburban (SU) Residence in Year

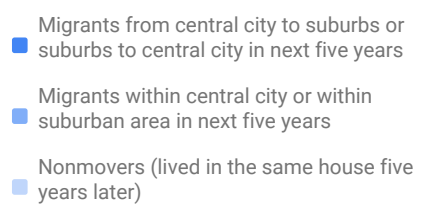

Migrants from central city to suburbs or suburbs to central city in next five years

years later)
Urban-Suburban Net Migration Rate by Race

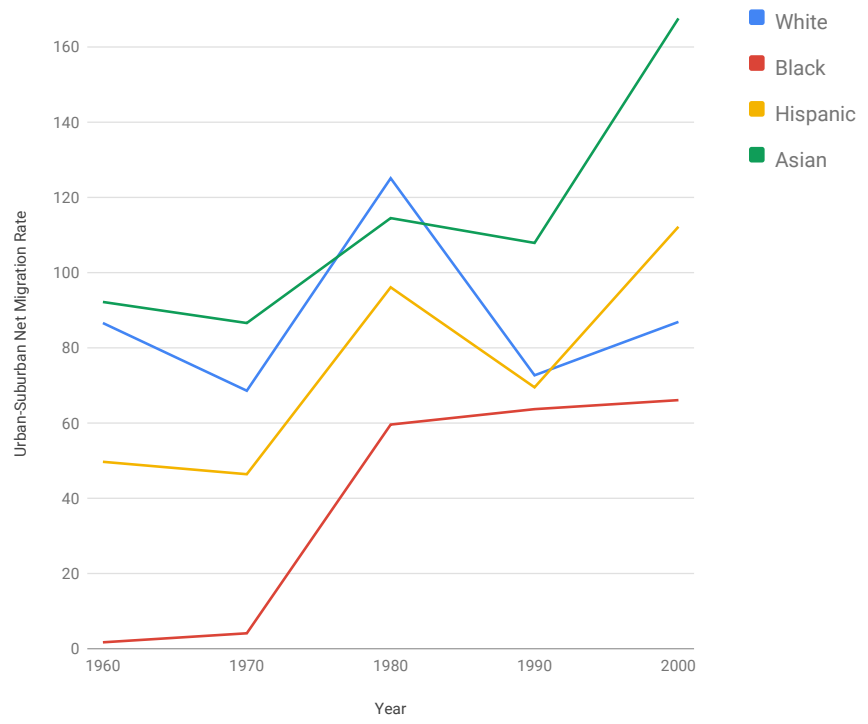

Low-Income Persons by Race
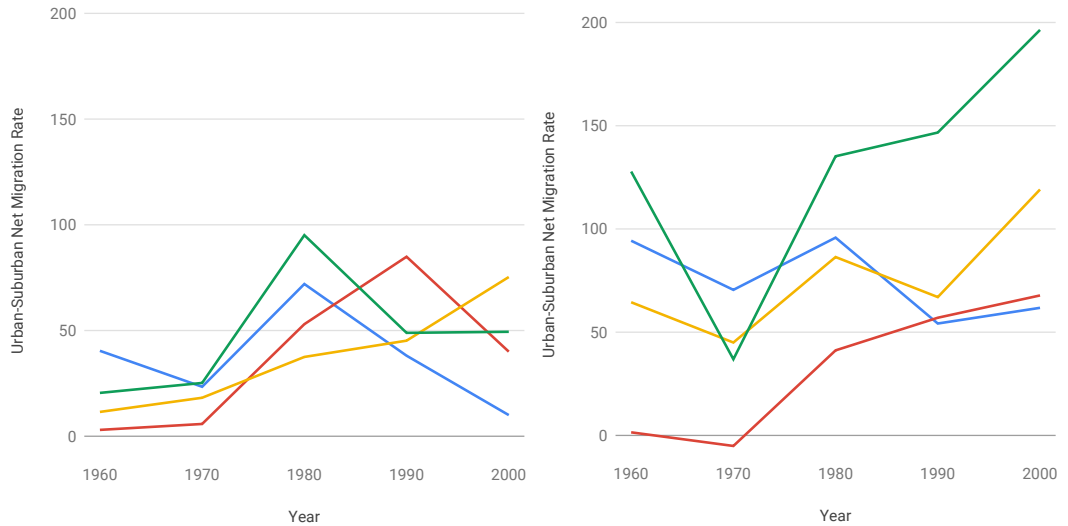

Year
Urban-Suburban Net Migration Rate by Income

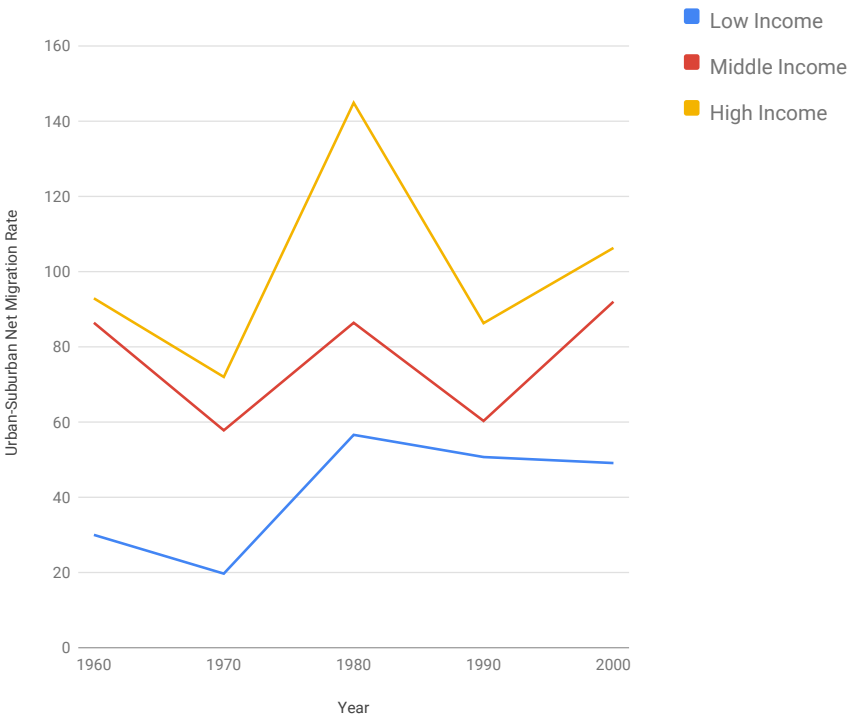

High-Income Persons by Race

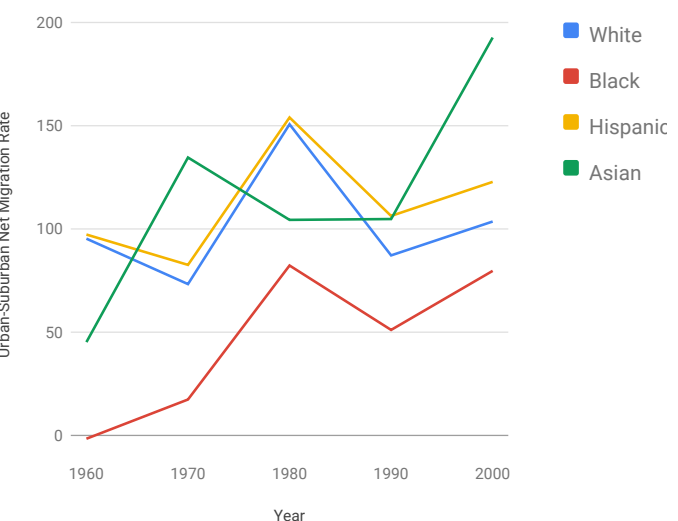


Urban and Suburban Residence, 1955-1995, and 5-year migration

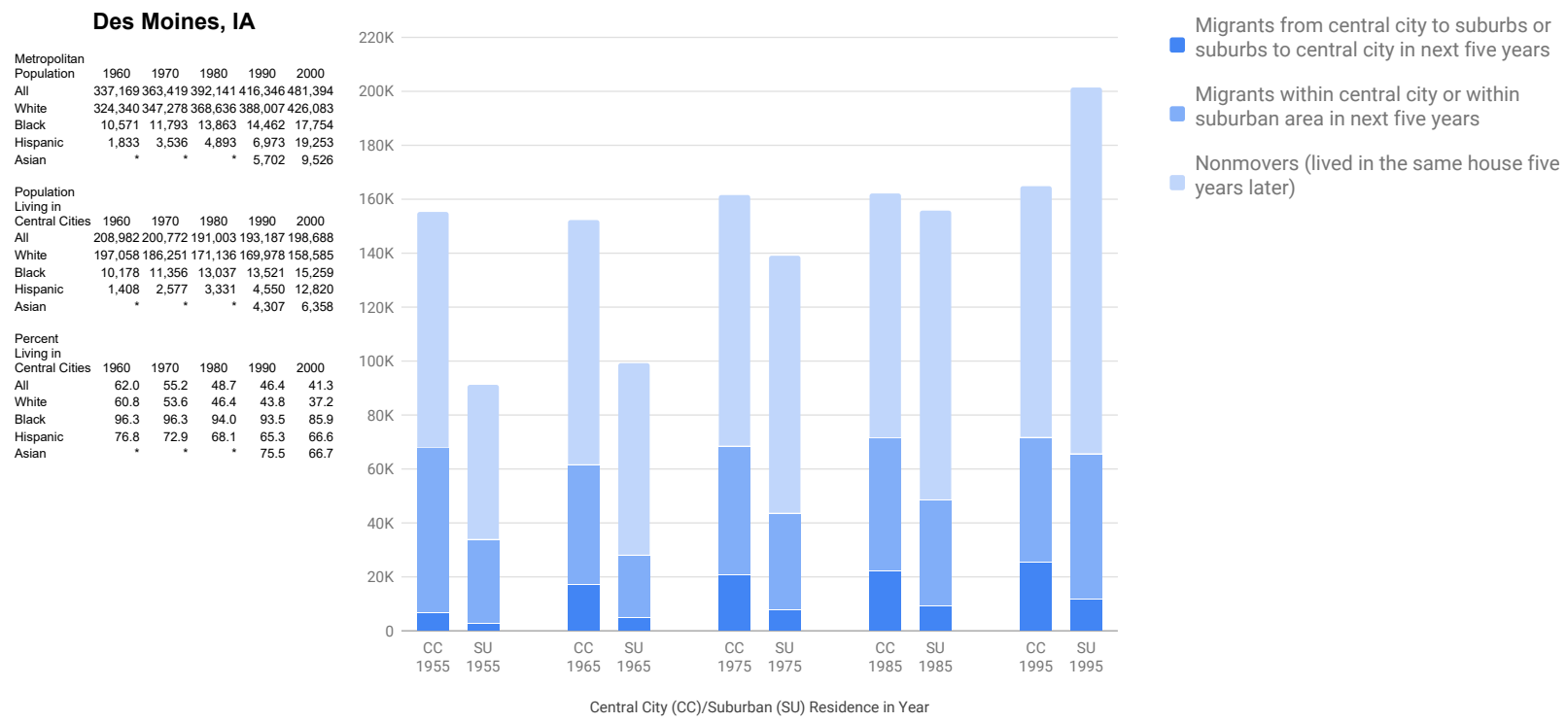

Urban-Suburban Net Migration Rate by Race

Urban-Suburban Net Migration Rate by Income

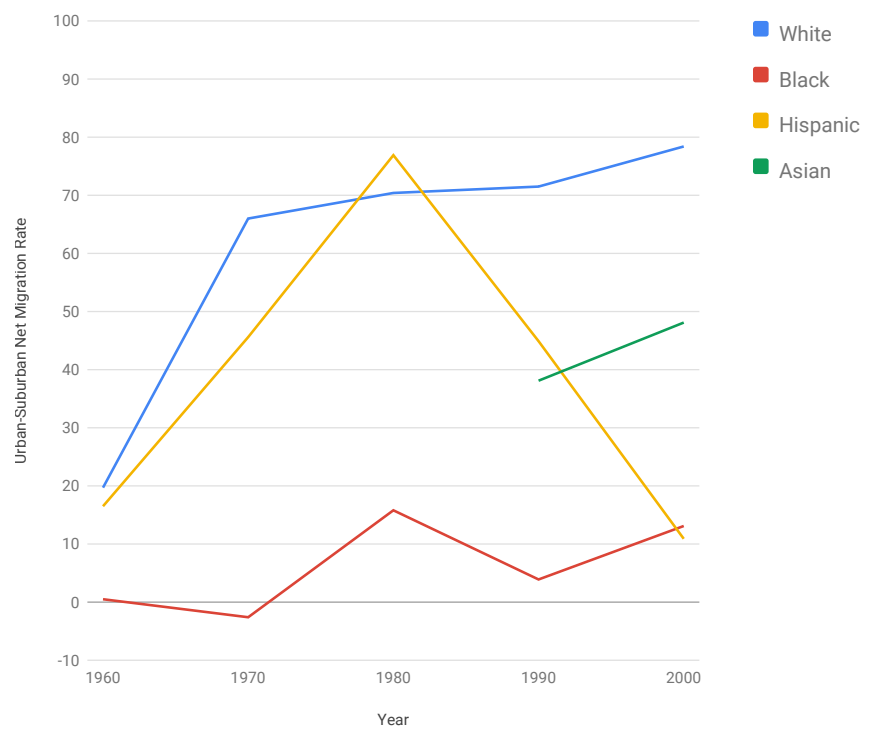

Low-Income Persons by Race

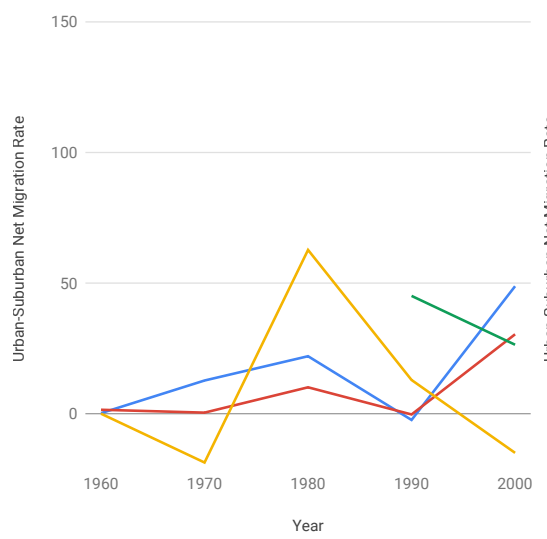

150

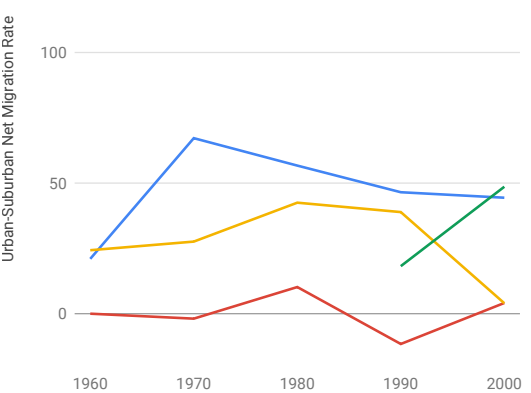

Year

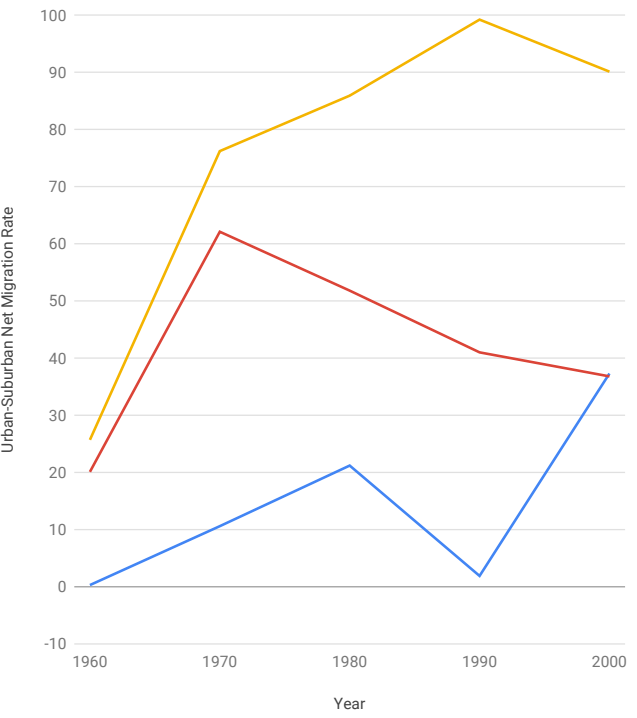

Low Income

Middle Income

- High Income 
Urban and Suburban Residence, 1955-1995, and 5-year migration

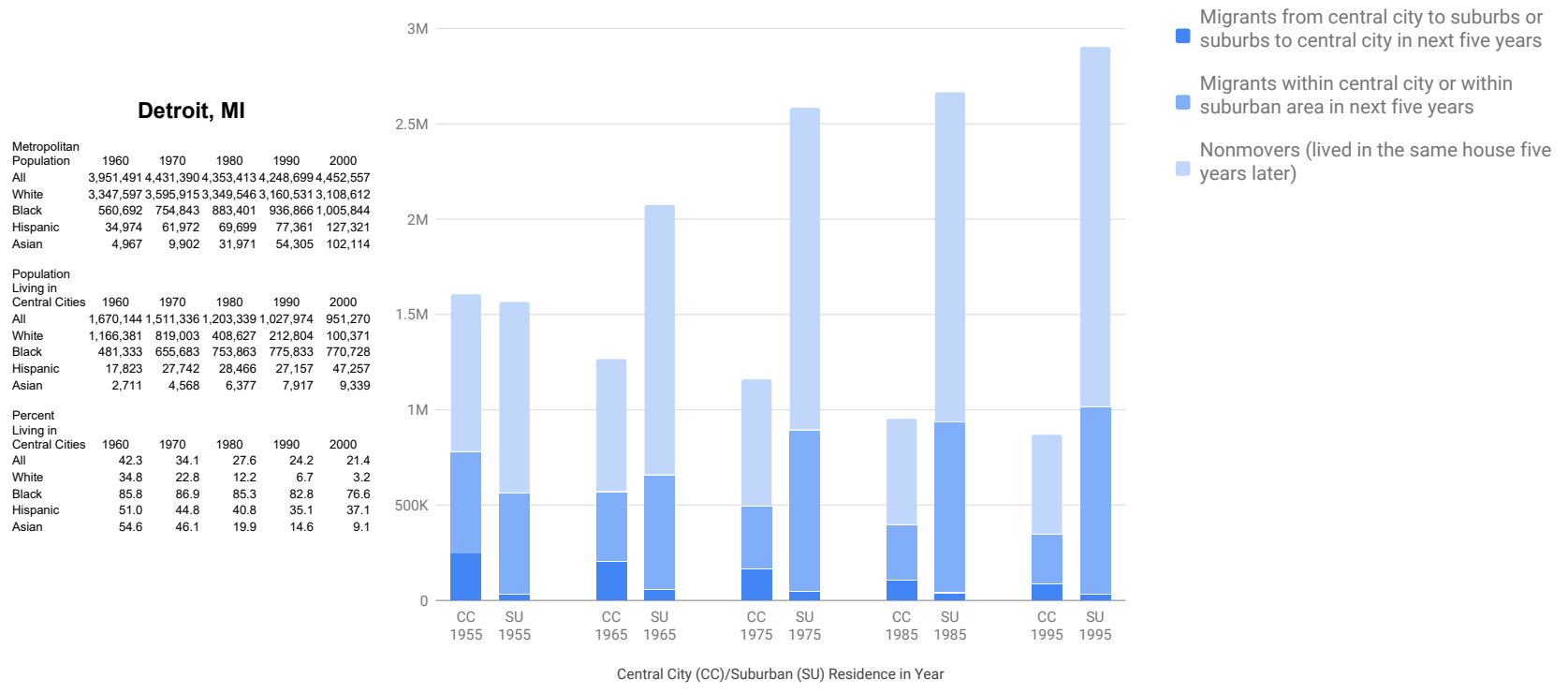

Urban-Suburban Net Migration Rate by Race

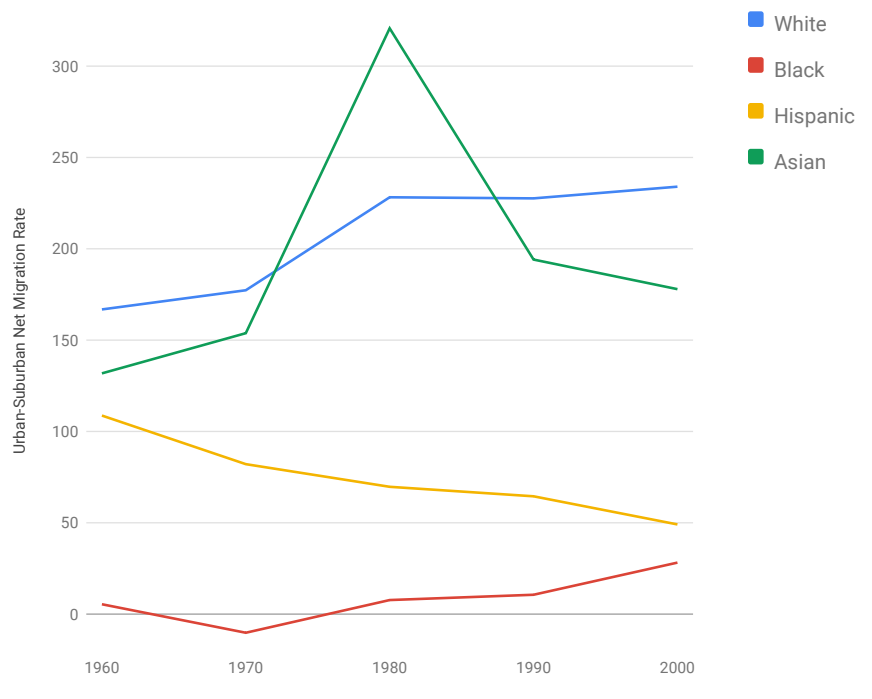

Low-Income Persons by Race

Urban-Suburban Net Migration Rate by Income
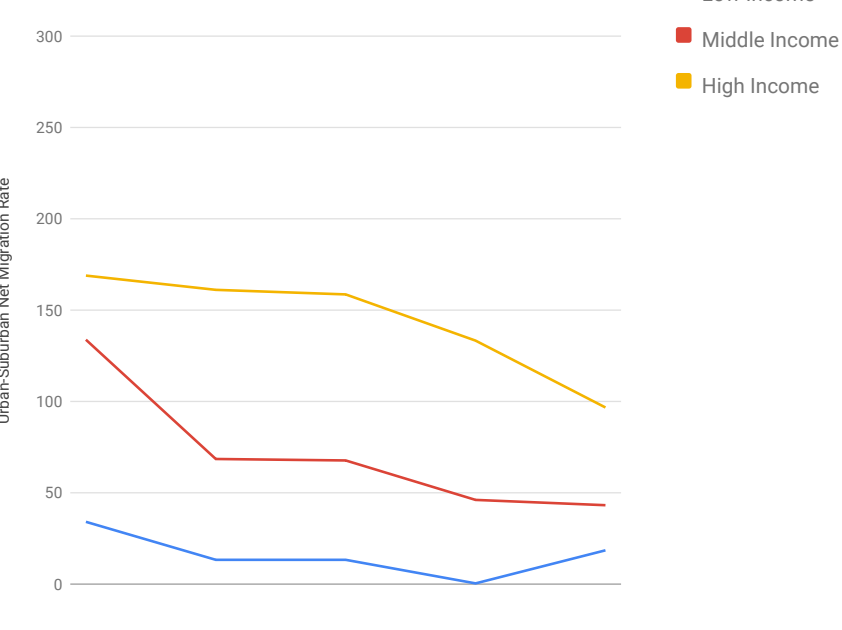

$\begin{array}{lllll}1960 & 1970 & 1980 & 1990 & 2000\end{array}$

Year

High-Income Persons by Race

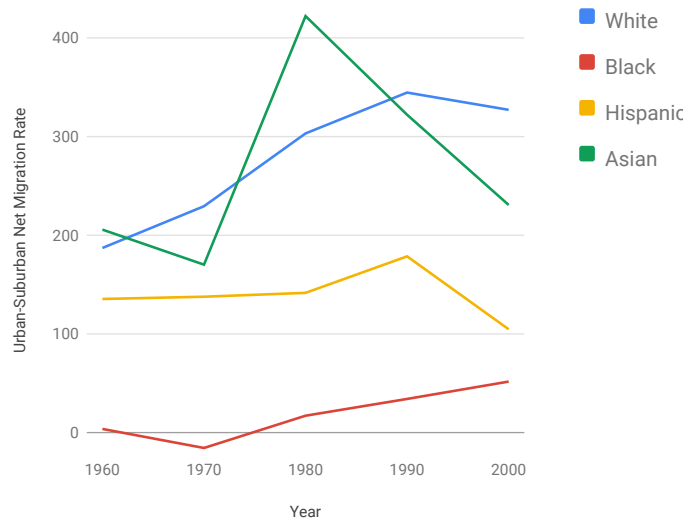




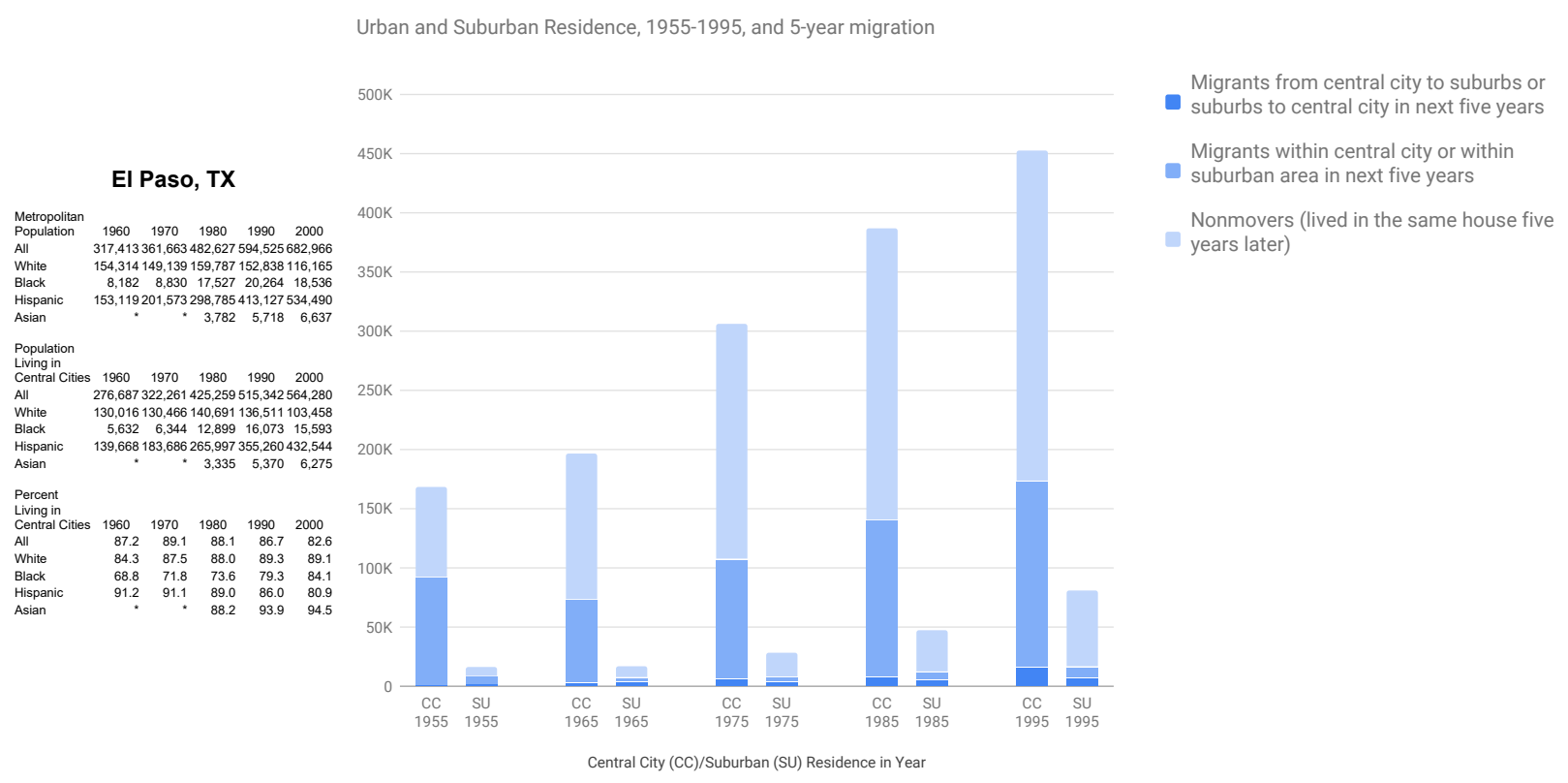

Urban-Suburban Net Migration Rate by Race

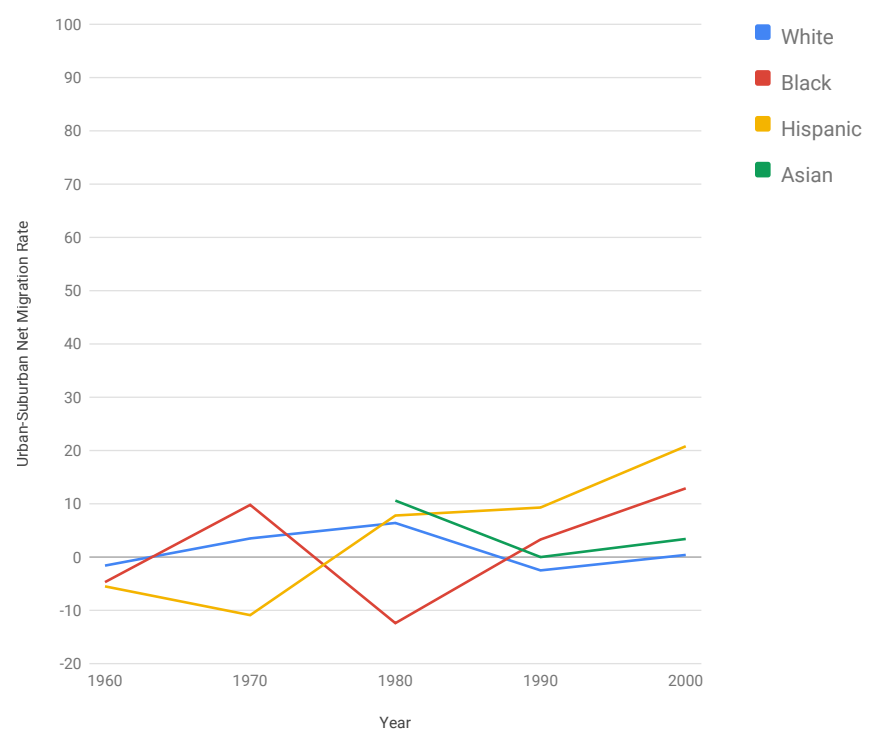

Low-Income Persons by Race

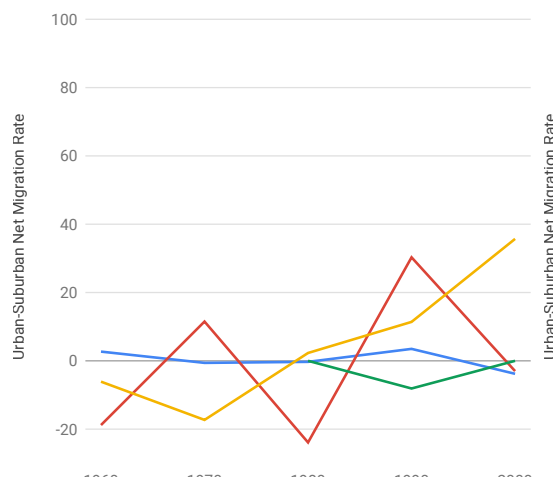

1960
Middle-Income Persons by Race

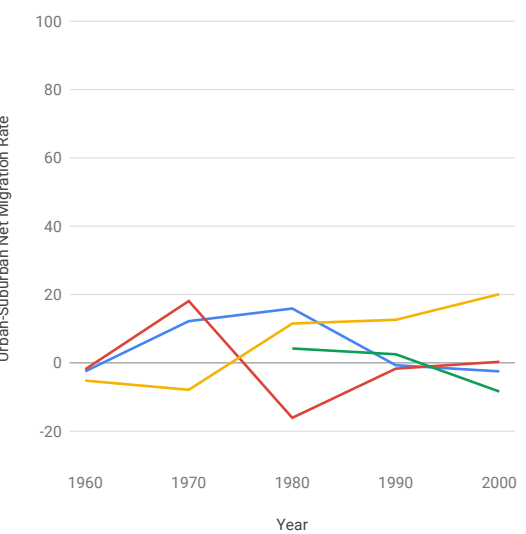

Urban-Suburban Net Migration Rate by Income

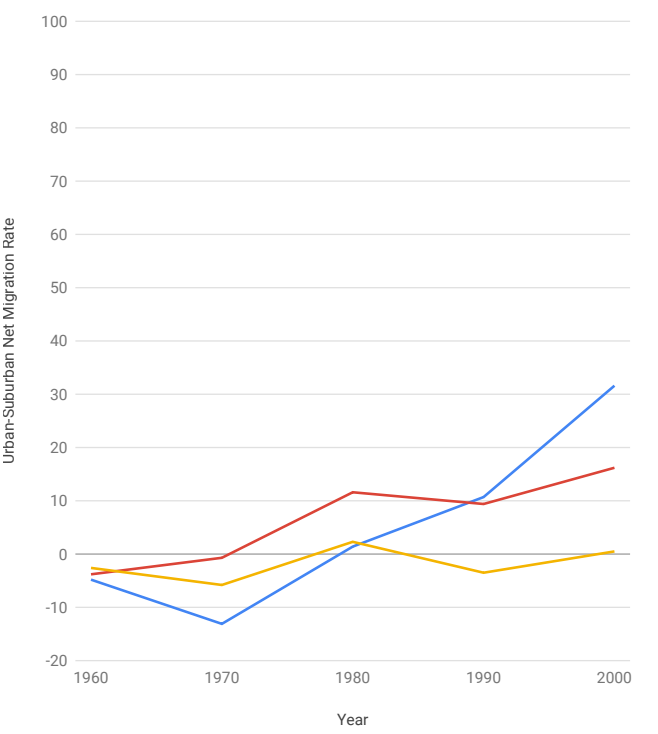

Low Income

Middle Income

High Income

High-Income Persons by Race

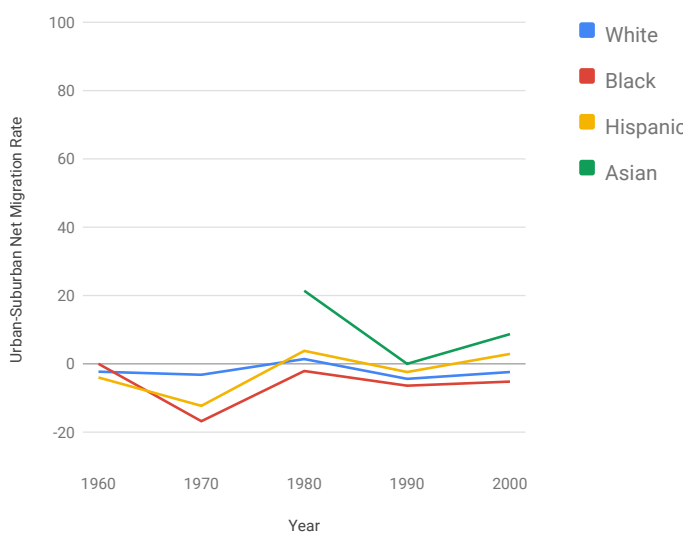


Urban and Suburban Residence, 1955-1995, and 5-year migration

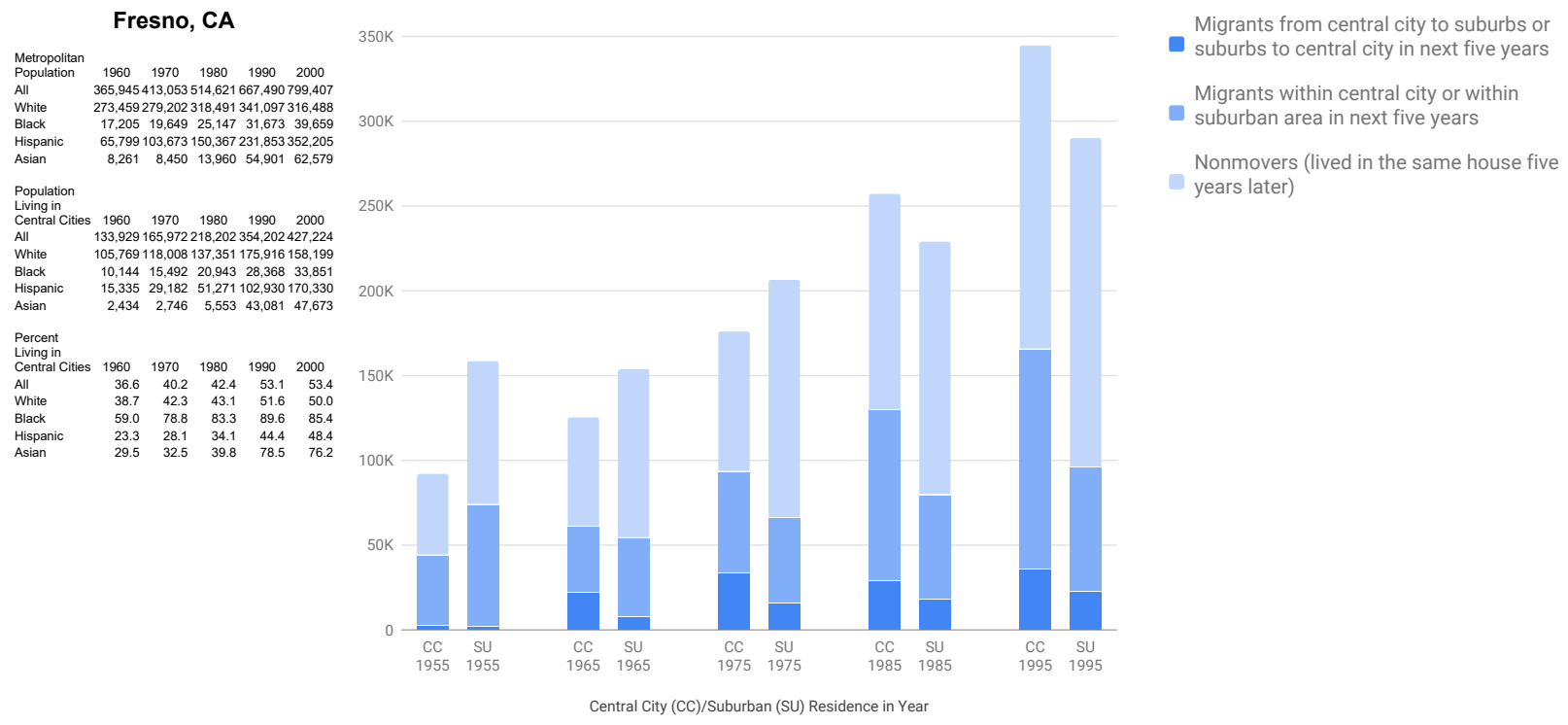

Urban-Suburban Net Migration Rate by Race

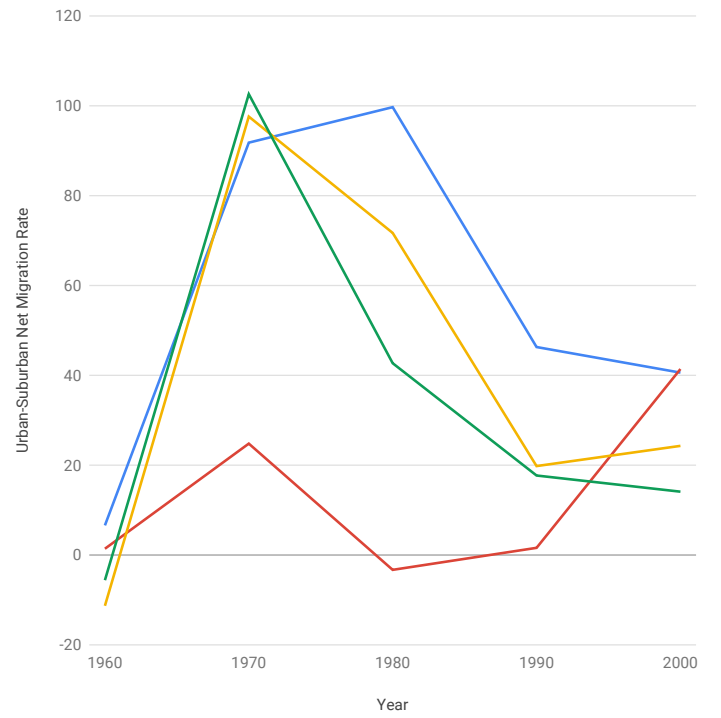

Low-Income Persons by Race

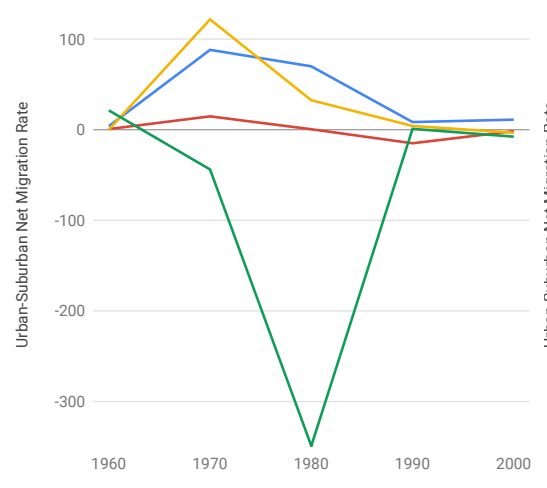

Year

\begin{abstract}
White
Black
\end{abstract}

Hispanic

- Asian
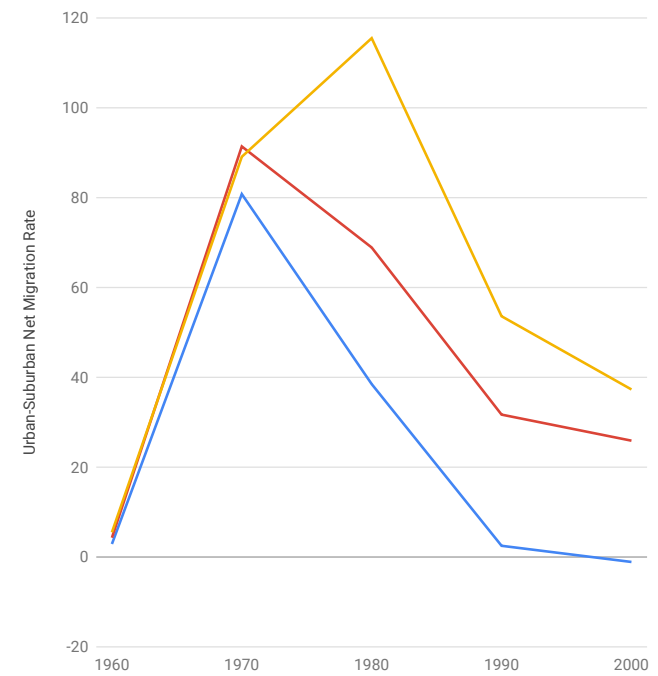

Year

High-Income Persons by Race

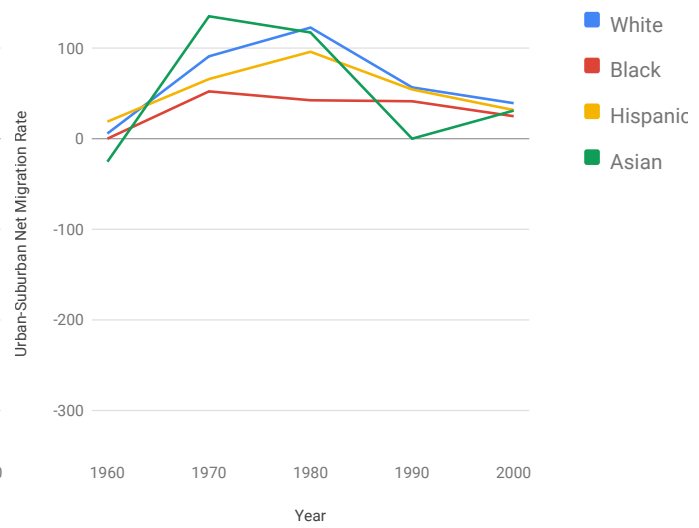

- Low Income

- Middle Income

High Income

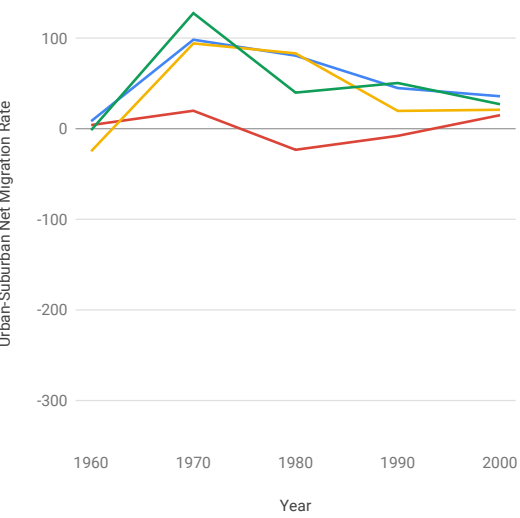

Year 
Urban and Suburban Residence, 1955-1995, and 5-year migration

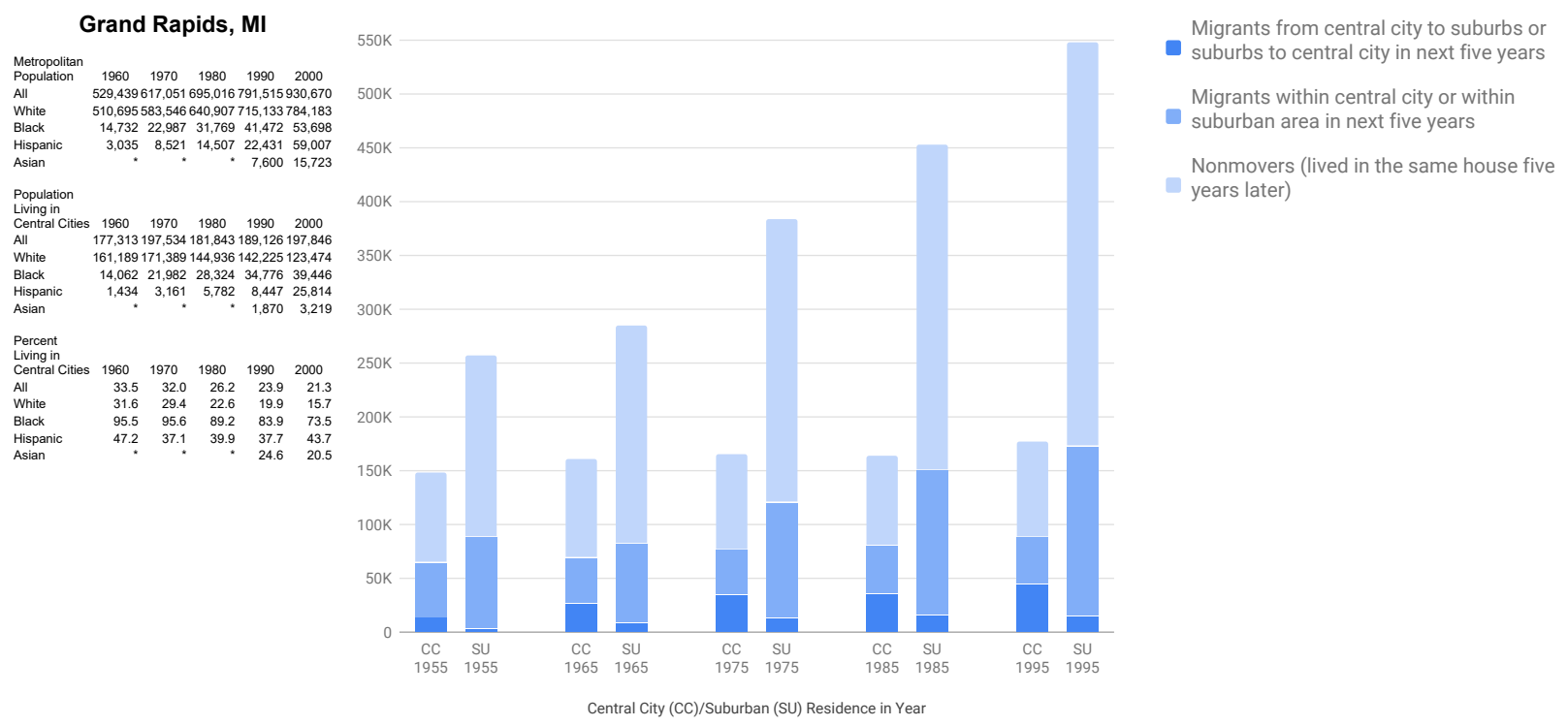

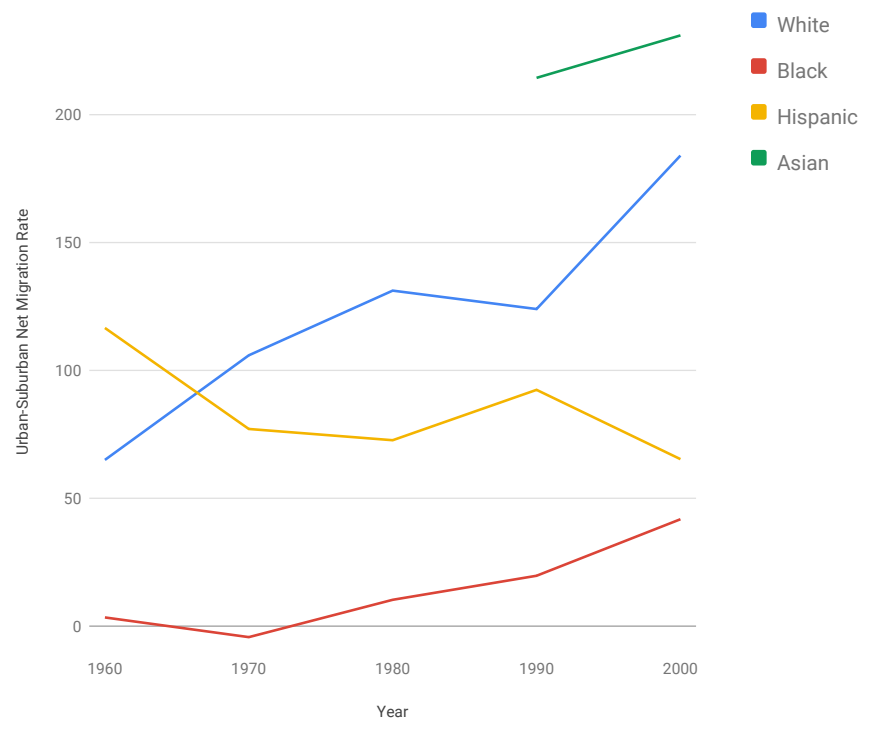

Low-Income Persons by Race

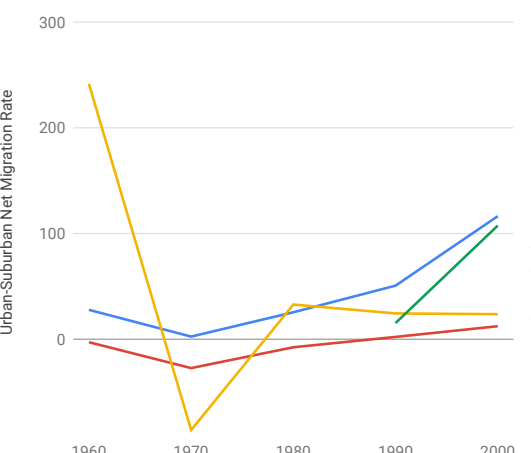

1960
Middle-Income Persons by Race

300

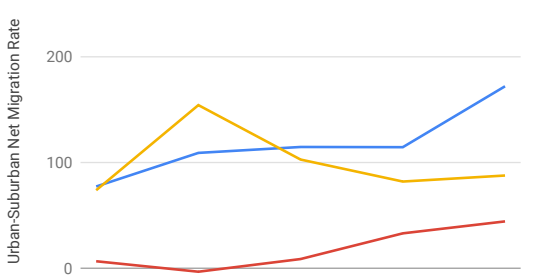

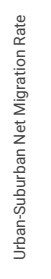

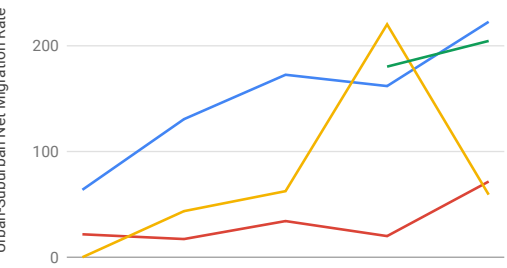

1980

High-Income Persons by Race

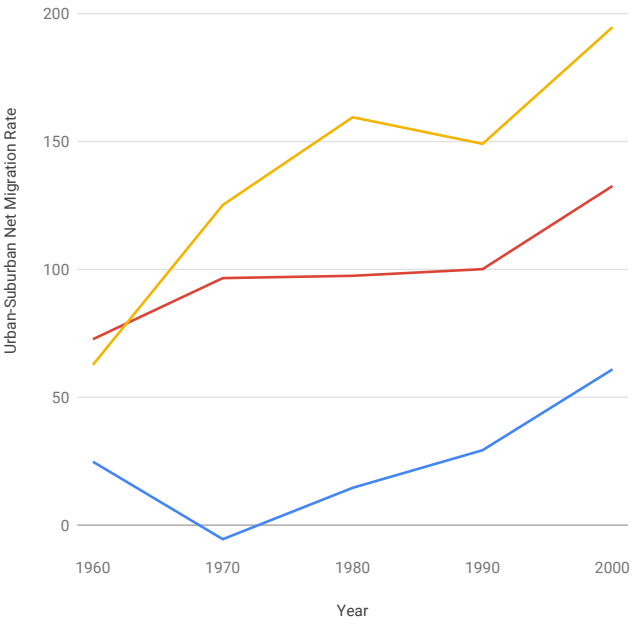

White

Black

- Hispanic

- Asian 
Urban and Suburban Residence, 1955-1995, and 5-year migration

\section{Greensboro-High Point, NC}

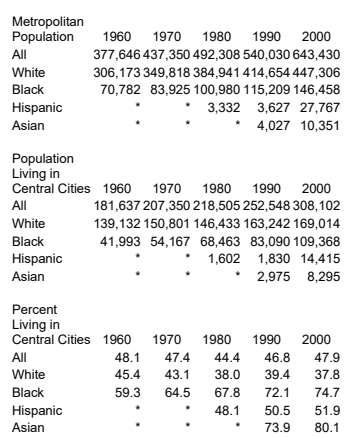

$300 \mathrm{~K}$
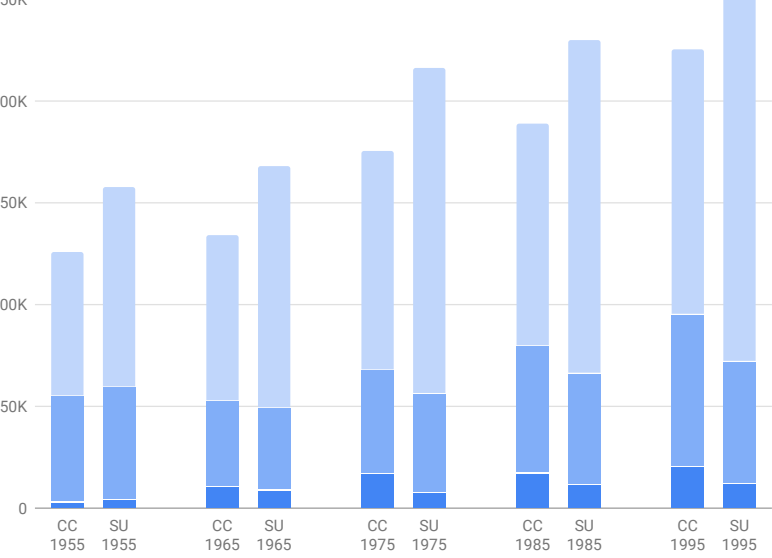

Central City (CC)/Suburban (SU) Residence in Year
Migrants from central city to suburbs or - suburbs to central city in next five years

Migrants within central city or within

suburban area in next five years

Nonmovers (lived in the same house five years later)
Urban-Suburban Net Migration Rate by Race

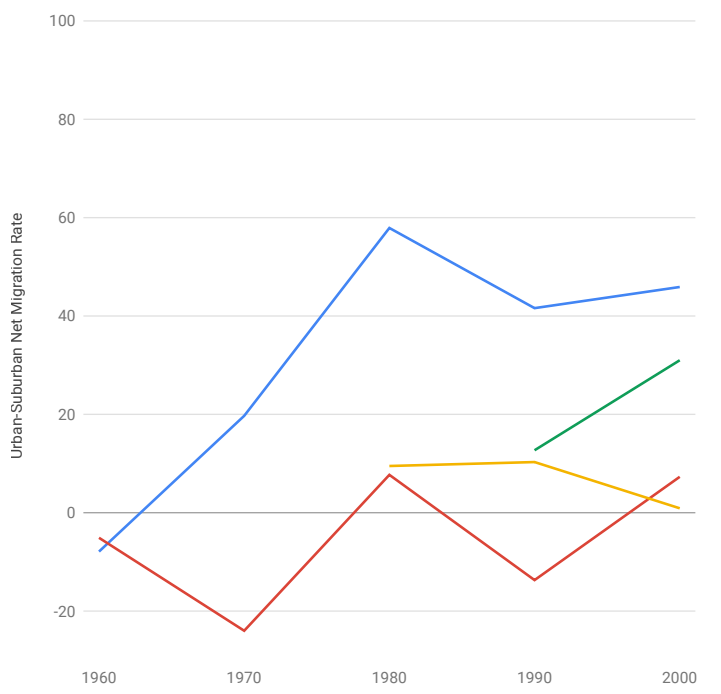

Year
Middle-Income Persons by Race

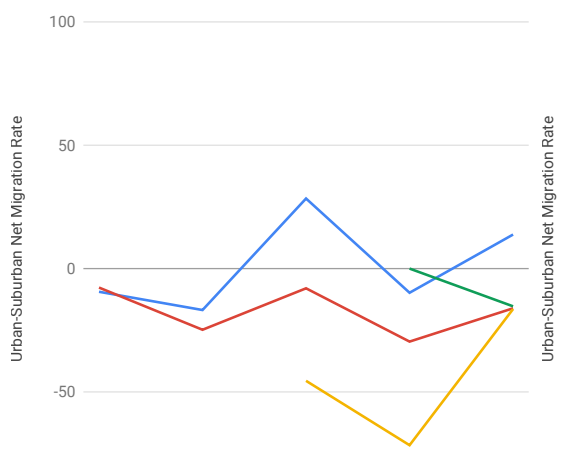

1960$$
100
$$

100

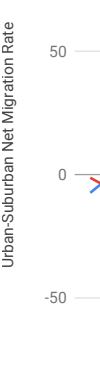

Year
White
- Black
Hispanic
- Asian

100

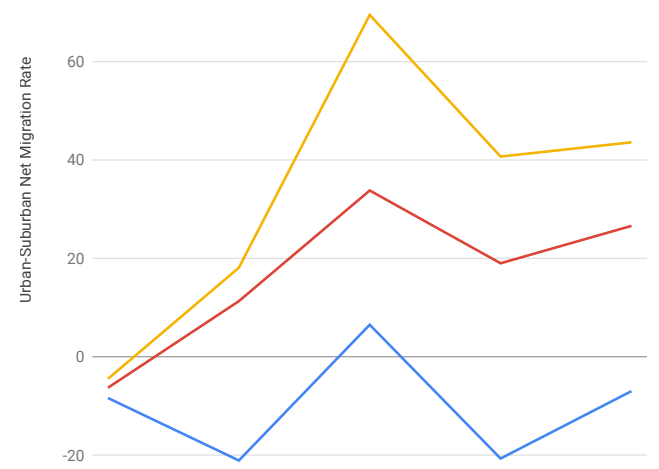

1960

1970

1980

Year
Low Income

- Middle Income

- High Income

High-Income Persons by Race

White

- Black

Hispanic

- Asian 


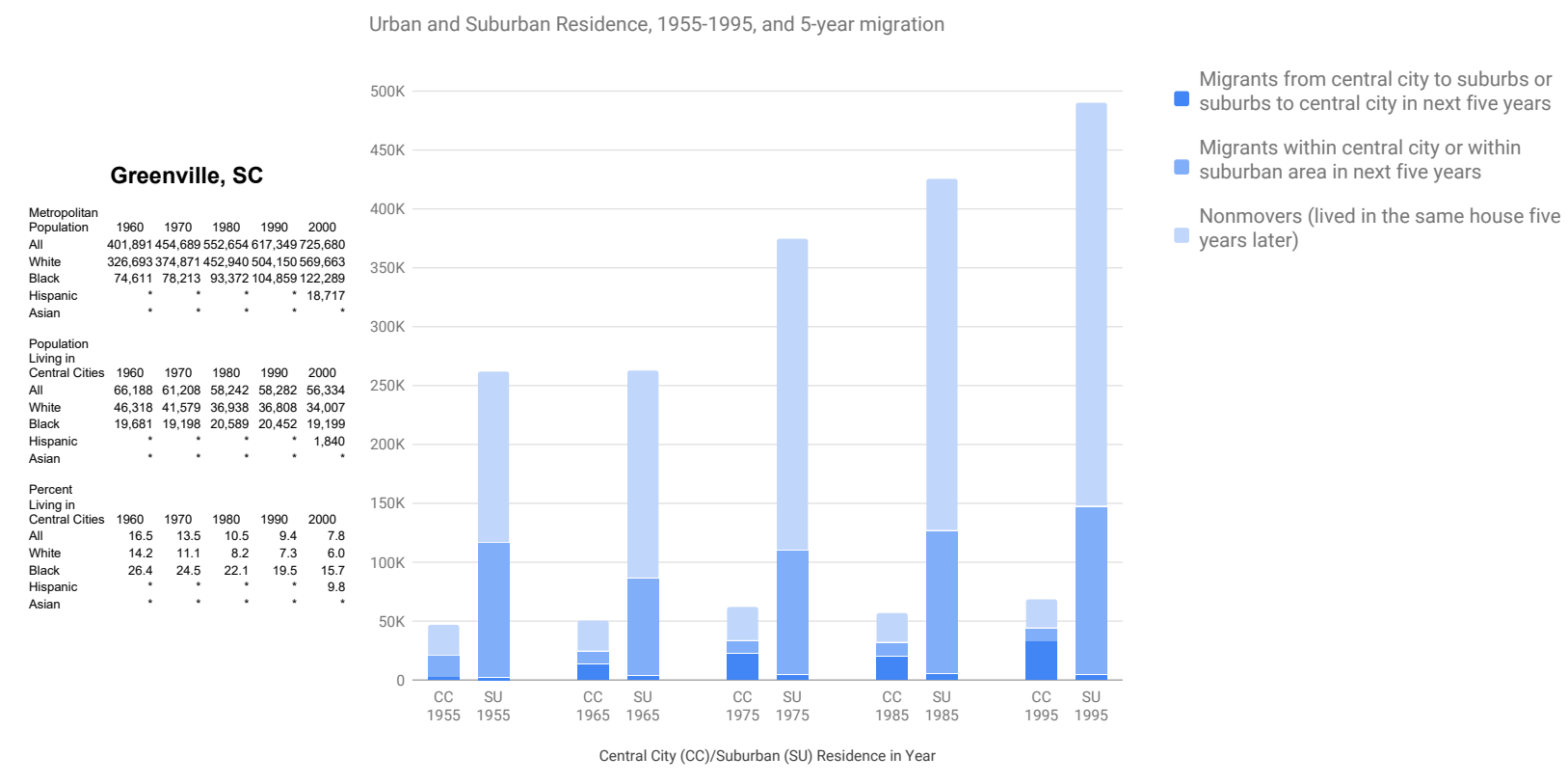

Urban-Suburban Net Migration Rate by Race

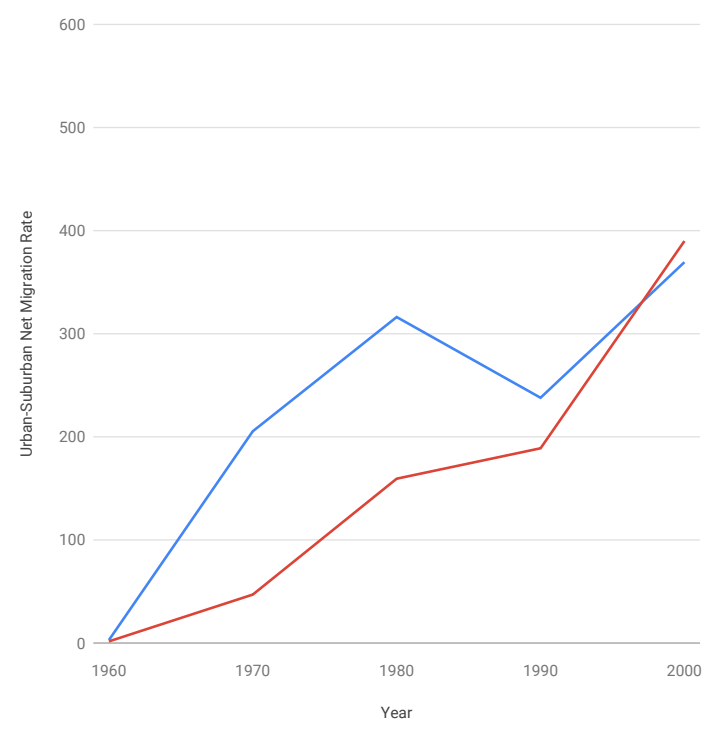

Low-Income Persons by Race

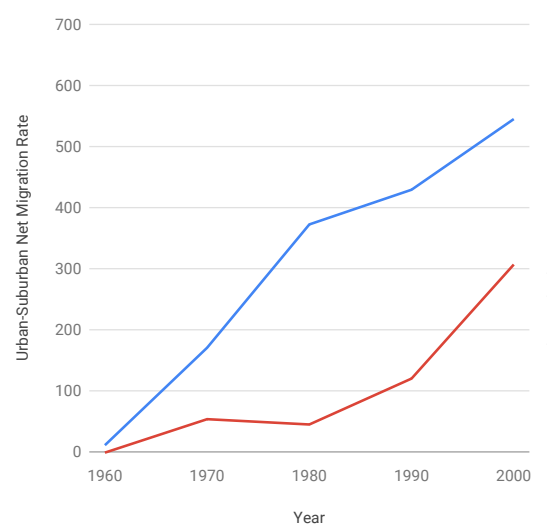

Middle-Income Persons by Race

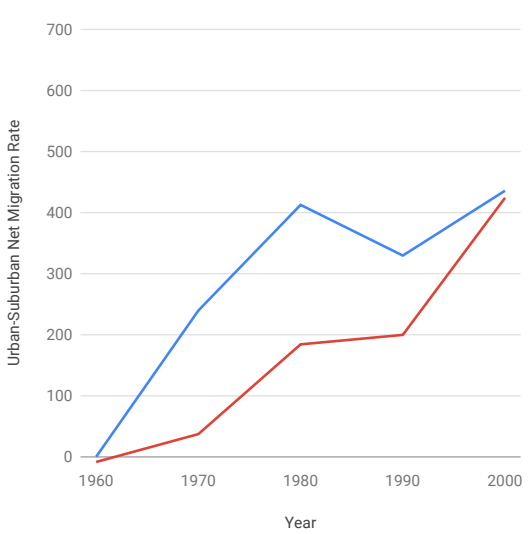

High-Income Persons by Race

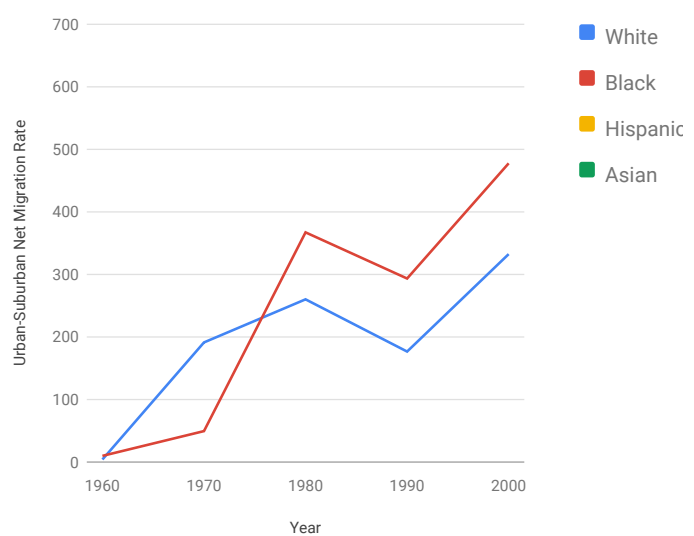


Urban and Suburban Residence, 1955-1995, and 5-year migration

$400 \mathrm{~K}$

Harrisburg, PA

$\begin{array}{lrrrrr}\text { Metropolitan } & 1960 & 1970 & 1980 & 1990 & 2000 \\ \text { Population } & 1960 & \\ \text { All } & 371,653410,626446,576474,242509.074 & 300 K\end{array}$

$\begin{array}{ll}\text { All } & 371,653410,626446,576474,242509,074 \\ \text { White } & 347,696379,322405,055423,371433043\end{array}$

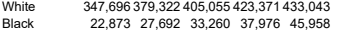

Hispanic $\quad * \quad 4,465 \quad 6,837 \quad 13,968$

Population

$\begin{array}{lllllll}\text { Living in } & & & & & & \\ \text { Central Cities } & 1960 & 1970 & 1980 & 1990 & 2000 & \\ \text { All } & 79697 & 67830 & 53264 & 52,376 & 49,100 & 200 K\end{array}$

$\begin{array}{llllll}\text { All } & 79,697 & 67,880 & 53,264 & 52,376 & 49,100 \\ \text { White } & 64,365 & 46,102 & 27,493 & 21,275 & 14,331\end{array}$

$\begin{array}{lllllll}\text { Black } & 15,036 & 20,664 & 23,007 & 26,205 & 25,841\end{array}$

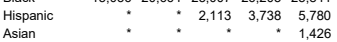

Percent
Living in

Living in
Central Cities $1960-1970 \quad 1900$
W

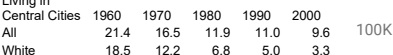

$\begin{array}{lrrrrr}\text { White } & 18.5 & 12.2 & 6.8 & 5.0 & 3.3 \\ \text { Black } & 65.7 & 74.6 & 69.2 & 69.0 & 56.2 \\ & & & & & \end{array}$

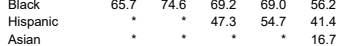

$150 \mathrm{~K}$
$50 \mathrm{~K}$

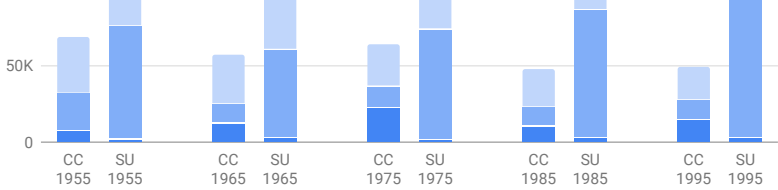

Central City (CC)/Suburban (SU) Residence in Year
Migrants from central city to suburbs or - suburbs to central city in next five years

Migrants within central city or within

suburban area in next five years

Nonmovers (lived in the same house five

years later)
Urban-Suburban Net Migration Rate by Race

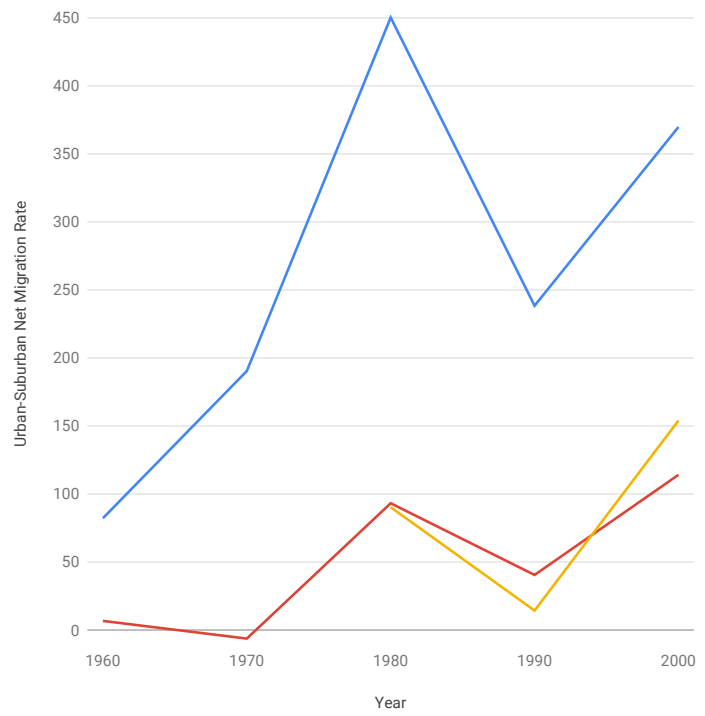

Low-Income Persons by Race
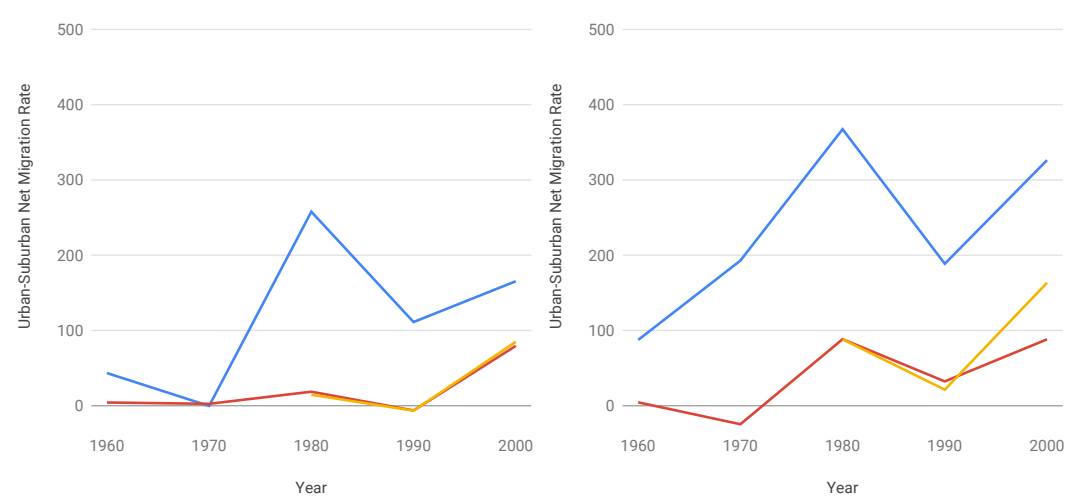

Urban-Suburban Net Migration Rate by Income
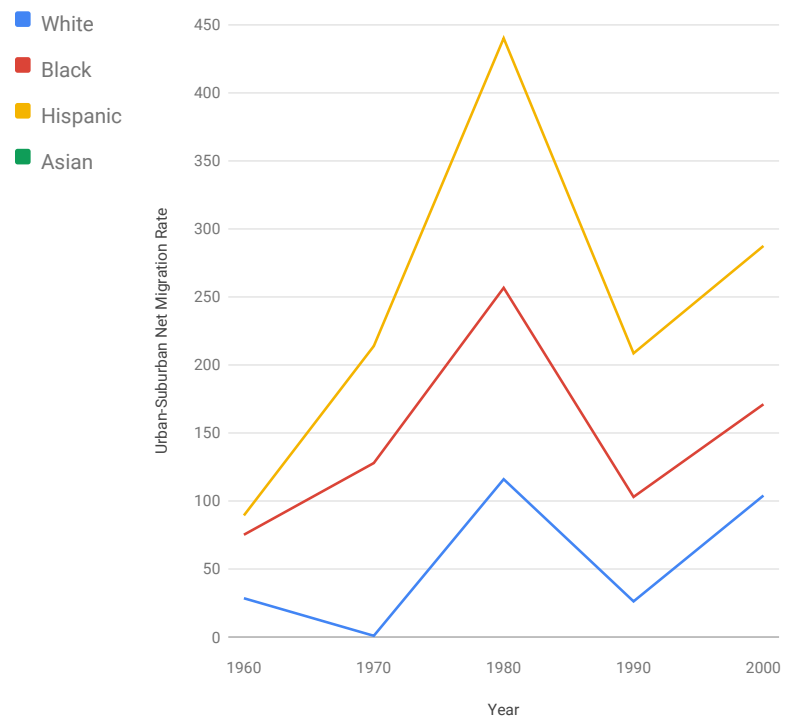

Low Income

- Middle Income

High Income

High-Income Persons by Race

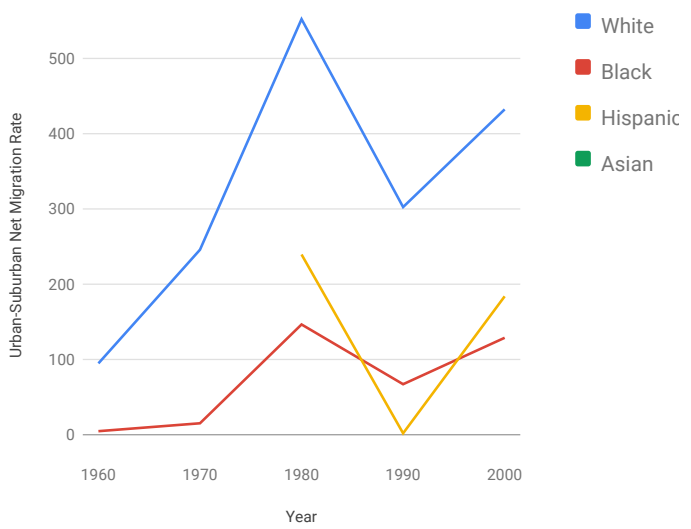


Urban and Suburban Residence, 1955-1995, and 5-year migration

\section{Hartford, CT}

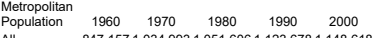

$\begin{array}{llllll}\text { All } & 847,1571,034,9931,051,6061,123,6781,148,618 \\ \text { White } & 804,106 & 950,772 & 926,523 & 939,238 & 888,658\end{array}$

\begin{tabular}{lrrrrr} 
White & 804,106 & 950,772 & 926,523 & 939,238 & 888,658 \\
Black & 33,343 & 58,641 & 71,840 & 90,493 & 103,599 \\
\hline
\end{tabular}

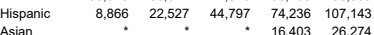

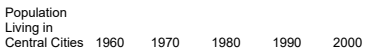

$\begin{array}{lllllll}\text { Central Cities } & 1960 & 1970 & 1980 & 1990 & 2000 \\ \text { All } & 162,178 & 158,017 & 136,392 & 139,739 & 121,578\end{array}$

$\begin{array}{lllllll}\text { White } & 133,787 & 101,089 & 61,784 & 42,867 & 21,513 \\ \text { Black } & 24,415 & 43,881 & 45,34 & 50,695 & 43,728\end{array}$

$\begin{array}{llllllll}\text { Hispanic } & 3,740 & 12,023 & 27,724 & 43,372 & 49,323\end{array}$

$\begin{array}{lllll}\text { Assian } & \text { * } & 1,891 & 1,914 & 500\end{array}$

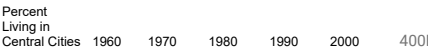

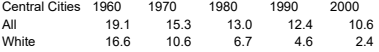

$\begin{array}{lrrrrr}\text { White } & 16.6 & 10.6 & 6.7 & 4.6 & 2.4 \\ \text { Black } & 73.2 & 74.8 & 63.2 & 56.0 & 42.2\end{array}$

Hispanic
Asian
$900 \mathrm{~K}$

$800 \mathrm{~K}$

$700 \mathrm{~K}$

$50 \mathrm{~K}$
$500 \mathrm{~K}$
$300 \mathrm{~K}$

$200 \mathrm{~K}$

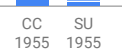

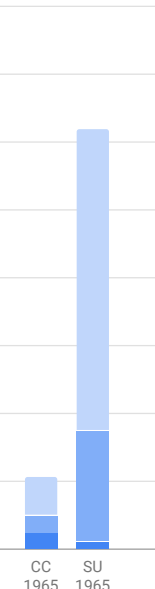

$\begin{array}{cc}\text { CC } & \text { SU } \\ 1965 & 1965\end{array}$

Central City (CC)/Suburban (SU) Residence in Year

Migrants from central city to suburbs or - suburbs to central city in next five years

Migrants within central city or within

suburban area in next five years

Nonmovers (lived in the same house five

years later)
Urban-Suburban Net Migration Rate by Race
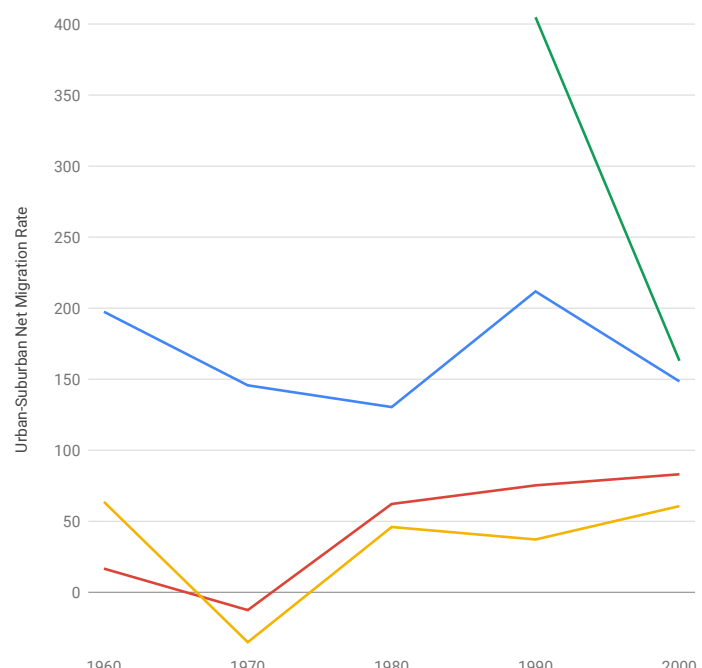

Year
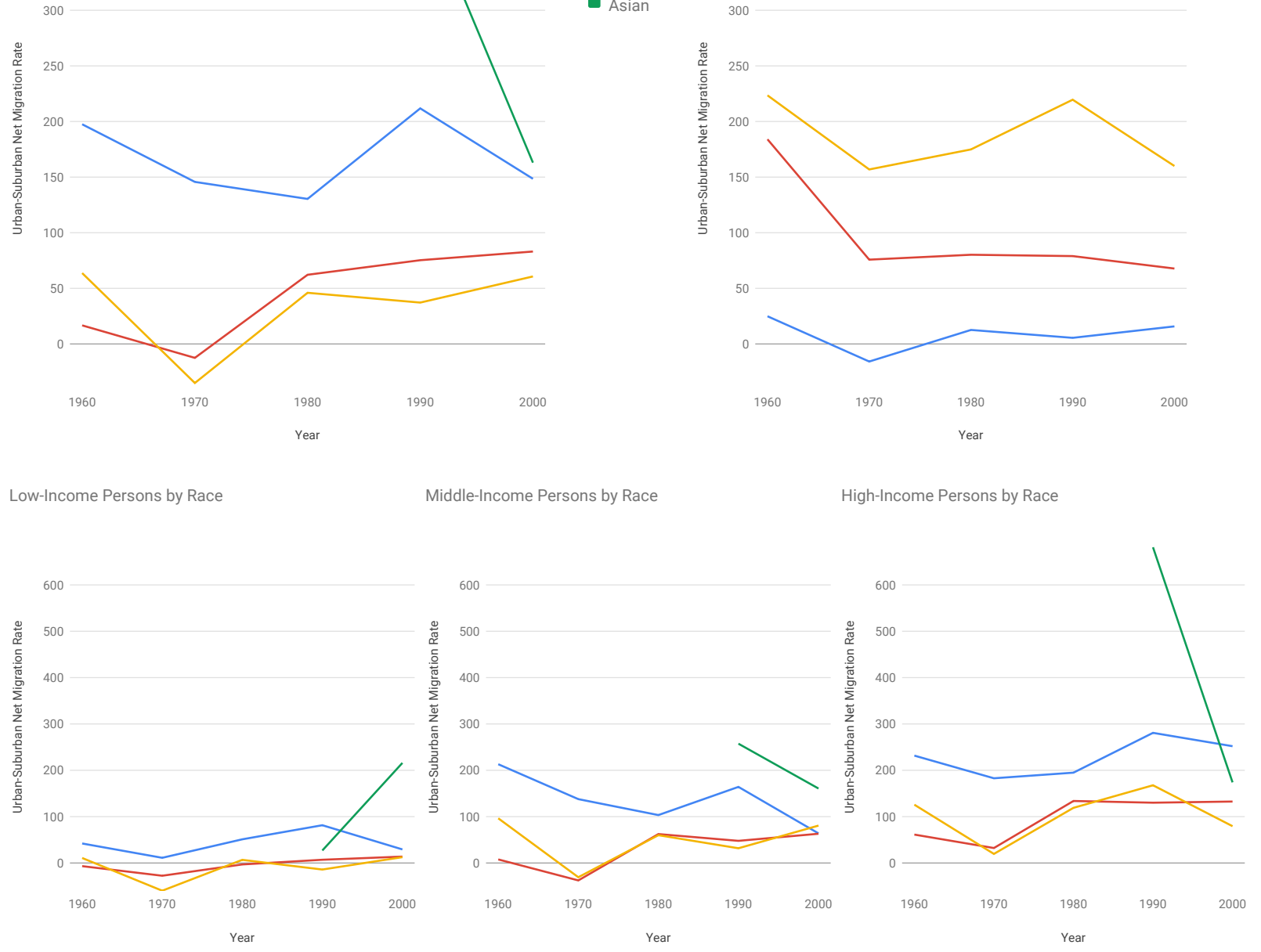

Year

High-Income Persons by Race
- Low Income

- Middle Income

- High Income
White

- Black

Hispanic

- Asian 
Urban and Suburban Residence, 1955-1995, and 5-year migration

\section{Houston, TX}

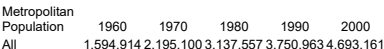

$\begin{array}{ll}\text { All } & 1,594,9142,195,1003,137,5573,750,9634,693,161 \\ \text { White } & 1,158,2711,533,2392,057,5682,186,3792,253,516\end{array}$

White $\quad 1,158,271,533,2592,057,5082,186,3792,253,510$

$\begin{array}{lllllll}\text { Hispanic } & 117,796 & 232,008 & 448,708 & 7628091,353294\end{array}$

$\begin{array}{llrllll}\text { Asian } & 2,787 & 6,349 & 51,345 & 126,576 & 222,029\end{array}$

Population

$\begin{array}{llllll}\text { Central Cities } & 1960 & 1970 & 1980 & 1990 & 2000\end{array}$

All $\quad 1,005,3941,294,2201,657,0691,689,7442,012,034$

$\begin{array}{lllllll}\text { Black } & 231,204 & 328,926 & 453,746 & 468,234 & 501,1365\end{array}$

$\begin{array}{lrrrrr}\text { Hispanic } & \quad 81,678 & 159,705 & 291,906 & 455,434 & 746,410 \\ \text { Asian } & 2283 & 4923 & 32,229 & 65562 & 103,10\end{array}$

Percent

$\begin{aligned} & \text { Percent } \\ & \text { Living in } \\ & \text { Central Cities }\end{aligned} 1960 \quad 1970 \quad 1980 \quad 1990 \quad 2000$
Whe

$\begin{array}{lrrrrr}\text { All } & 63.0 & 59.0 & 52.8 & 45.0 & 42.9 \\ \text { White } & 59.6 & 52.1 & 42.3 & 31.7 & 27.8\end{array}$

$\begin{array}{llllll}\text { White } & 59.6 & 52.1 & 42.3 & 31.7 & 27.8 \\ \text { Black } & 73.3 & 78.3 & 80.5 & 71.0 & 64.3 \\ \text { Hispanic } & 69.3 & 68.8 & 65.1 & 59.7 & 55.2\end{array}$

$\begin{array}{lllllll}\text { Hispanic } & 69.3 & 68.8 & 65.1 & 59.7 & 55.2 & \\ \text { Asian } & 81.9 & 77.5 & 62.8 & 51.8 & 46.5 & 600 K\end{array}$

$1.8 \mathrm{M}$

$1.6 \mathrm{M}$

$1.2 \mathrm{M}$

$1 \mathrm{M}$
$800 \mathrm{~K}$
$600 \mathrm{~K}$

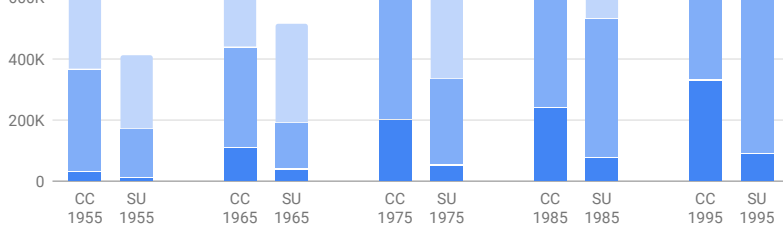

Central City (CC)/Suburban (SU) Residence in Year
Migrants from central city to suburbs or
suburbs to central city in next five years

Migrants within central city or within

suburban area in next five years

Nonmovers (lived in the same house five

years later)
Urban-Suburban Net Migration Rate by Race

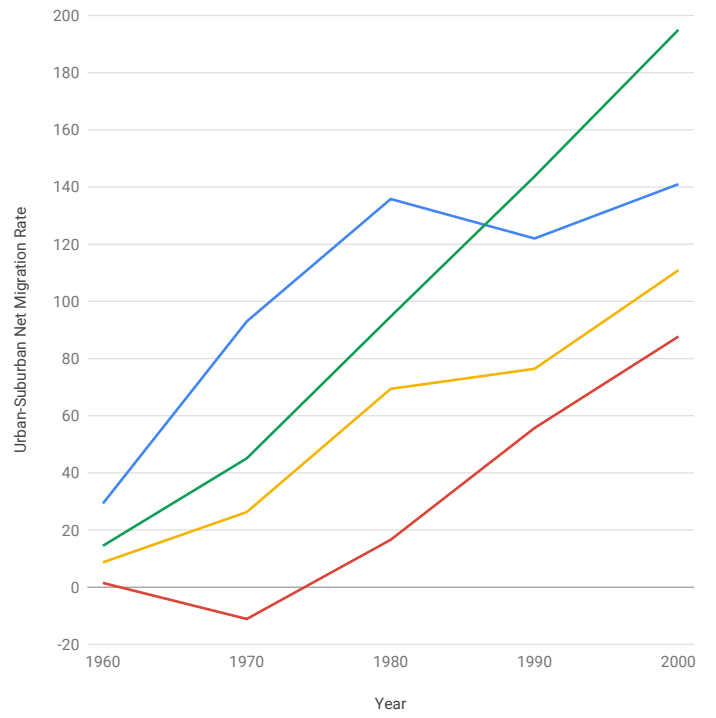

Low-Income Persons by Race

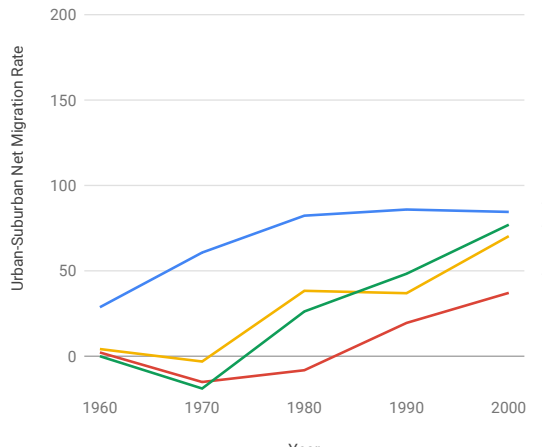

Year
Middle-Income Persons by Race

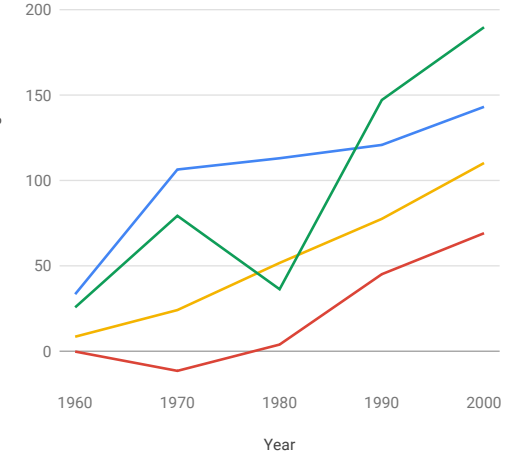

Year
Urban-Suburban Net Migration Rate by Income

$$
\begin{aligned}
& \text { White } \\
& \text { Black } \\
& \text { Hispanic } \\
& \text { Asian }
\end{aligned}
$$

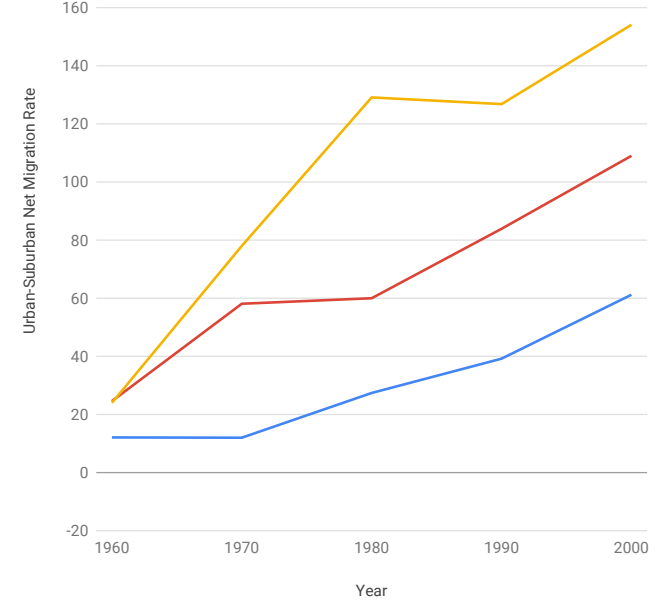

- Low Income

- Middle Income

High Income 
Urban and Suburban Residence, 1955-1995, and 5-year migration

Indianapolis, IN

$\begin{array}{llllll}\begin{array}{l}\text { Metropolitan } \\ \text { Population }\end{array} & 1960 & 1970 & 1980 & 1990 & 2000\end{array}$

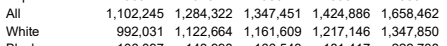

$\begin{array}{lrrrrr}\text { Black } & 106,097 & 143,998 & 166,543 & 181,417 & 222,738 \\ \text { Hispanic } & 2,745 & 14,788 & 9,965 & 12,224 & 41,651 \\ \text { Asian } & * & * & 5,933 & 10,024 & 18,817\end{array}$

Population

$\begin{array}{llllll}\begin{array}{l}\text { Population } \\ \text { Living in }\end{array} & & & & \\ \text { Central Cities } & 1960 & 1970 & 1980 & 1990 & 2000 \\ \text { All } & 476,258 & 744,570 & 700,719 & 731,321 & 782,414\end{array}$

$\begin{array}{llllll}\text { All } & 476,258 & 744,570 & 700,719 & 731,321 & 782,414 \\ \text { White } & 376,512 & 600,822 & 536,481 & 550,910 & 528,300 \\ \text { Black } & 97,681 & 133001 & 151,735 & 164,142 & 197,893\end{array}$

\begin{tabular}{lrrrrr} 
Black & 97,681 & 133,001 & 151,735 & 164,142 & 197,893 \\
Hispanic & 1,294 & 8,756 & 6,430 & 7,463 & 29,641 \\
\hline
\end{tabular}

$\begin{array}{lllllll}\begin{array}{l}\text { Percent } \\ \text { Livinin }\end{array} & & & & & & \\ \text { Central Cities } & 1960 & 1970 & 1980 & 1990 & 2000 & 300 \mathrm{~K}\end{array}$

\begin{tabular}{lrrrrr} 
Central Cities & \multicolumn{1}{c}{1960} & \multicolumn{1}{c}{1970} & \multicolumn{1}{c}{1980} & \multicolumn{1}{c}{1990} & \multicolumn{1}{l}{2000} \\
All & 43.2 & 58.0 & 52.0 & 51.3 & 47.2 \\
White & 38.0 & 53.5 & 46.2 & 45.3 & 39.2 \\
Black & 92.1 & 92.4 & 91.1 & 90.5 & 88.8
\end{tabular}

$\begin{array}{llllll}\text { Black } & 92.1 & 92.4 & 91.1 & 90.5 & 88.8 \\ \text { Hispanic } & 47.1 & 59.2 & 64.5 & 61.1 & 71.2 \\ \text { Asian } & . & . & 65.8 & & \end{array}$

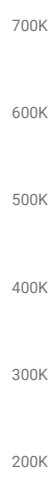

$700 \mathrm{~K}$

$00 \mathrm{~K}$

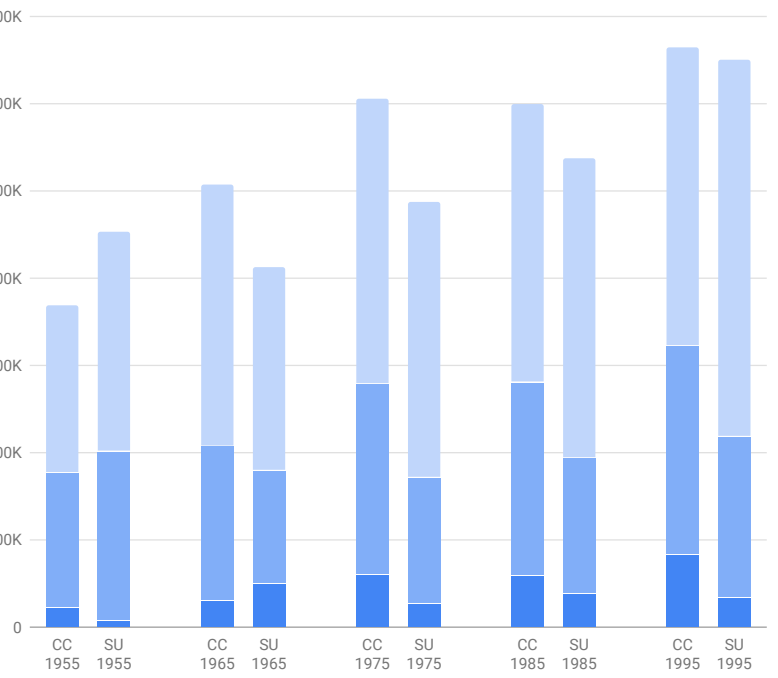

Migrants from central city to suburbs or suburbs to central city in next five years

Migrants within central city or within suburban area in next five years

Nonmovers (lived in the same house five years later)
Urban-Suburban Net Migration Rate by Race

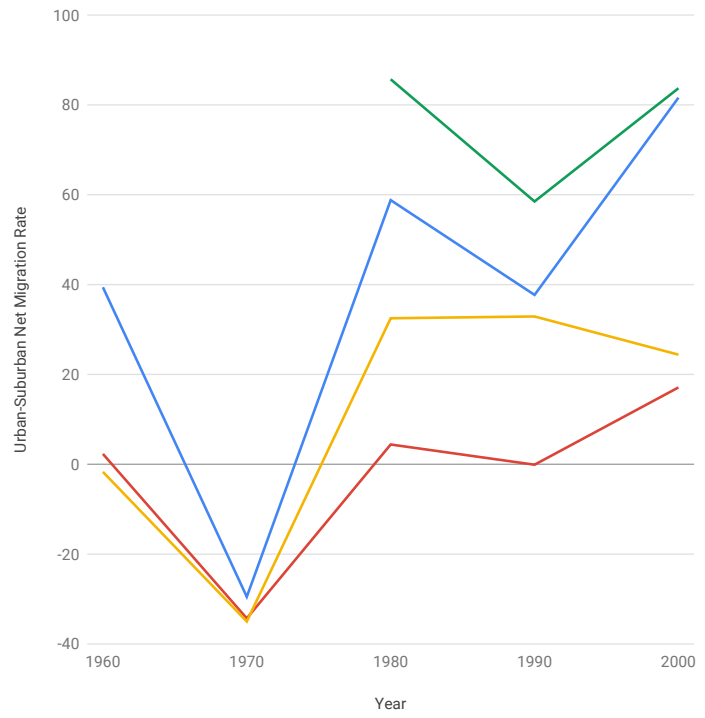

Low-Income Persons by Race

Middle-Income Persons by Race

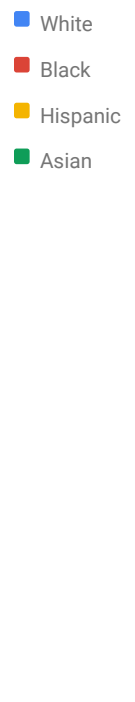

Urban-Suburban Net Migration Rate by Income

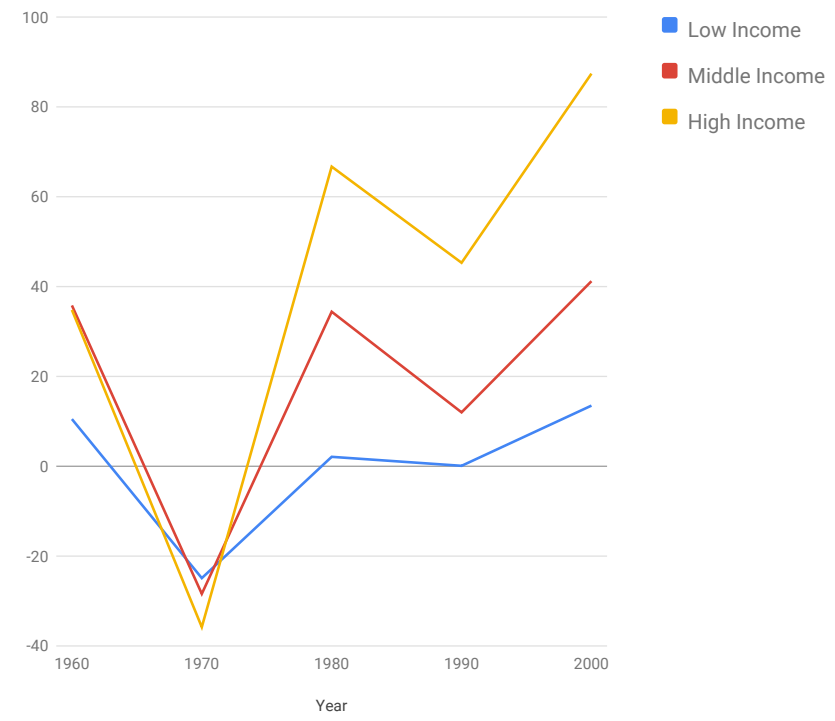

High-Income Persons by Race

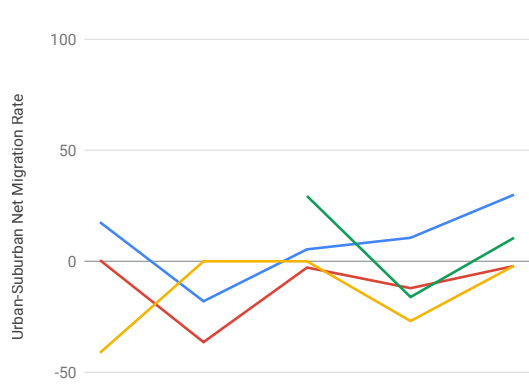

1960
100
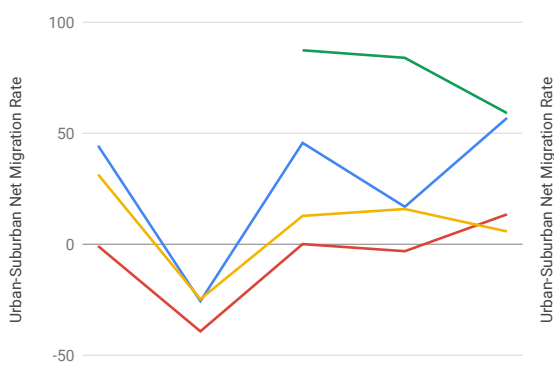

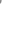

1980

Year

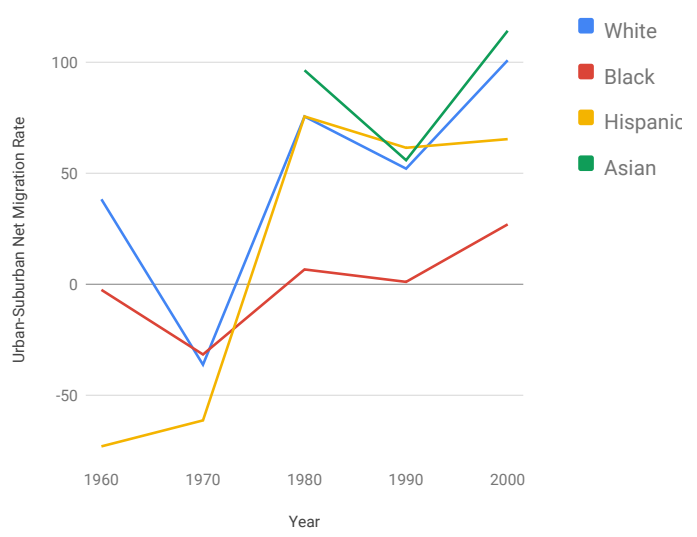


Urban and Suburban Residence, 1955-1995, and 5-year migration

$300 \mathrm{~K} \quad$ Migrants from central city to suburbs or

suburbs to central city in next five years

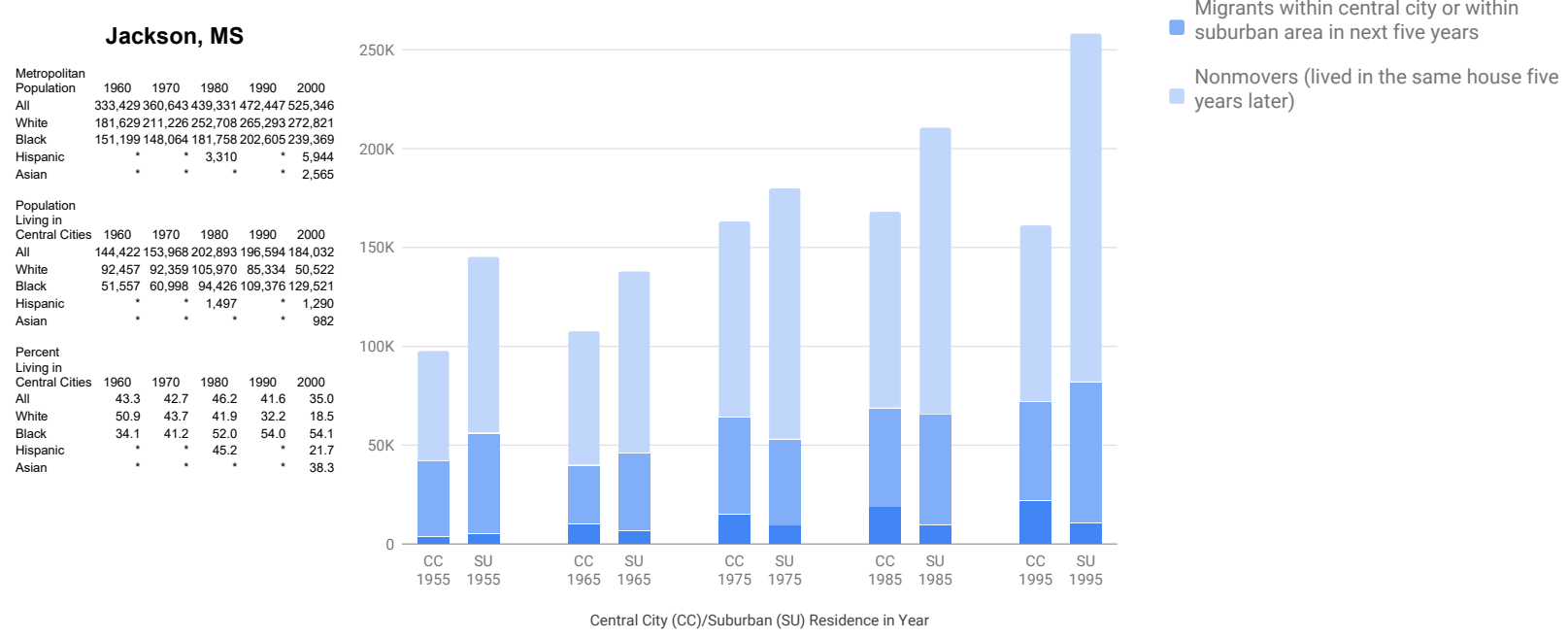

Urban-Suburban Net Migration Rate by Race

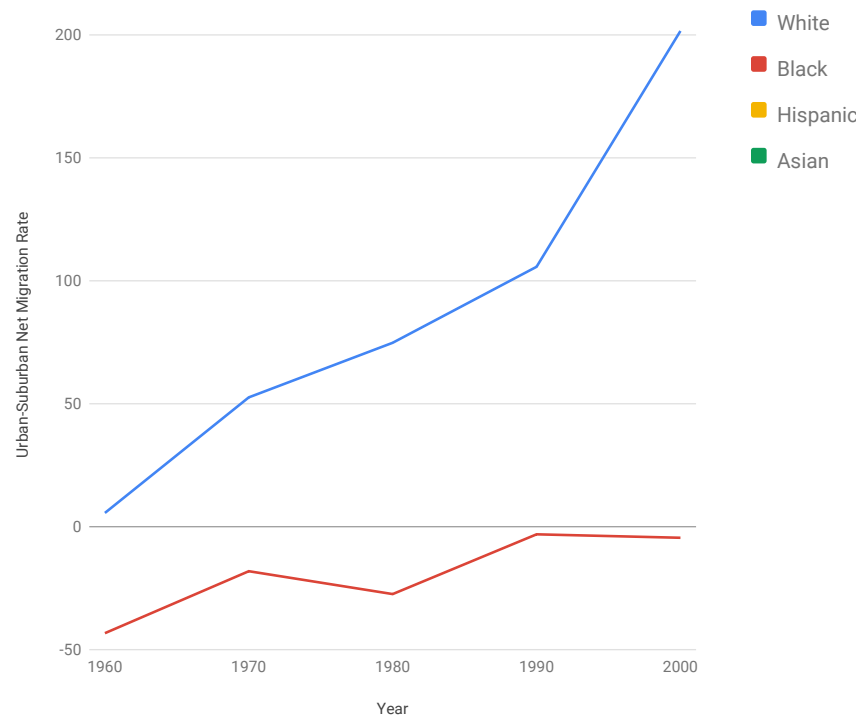

Low-Income Persons by Race

Middle-Income Persons by Race
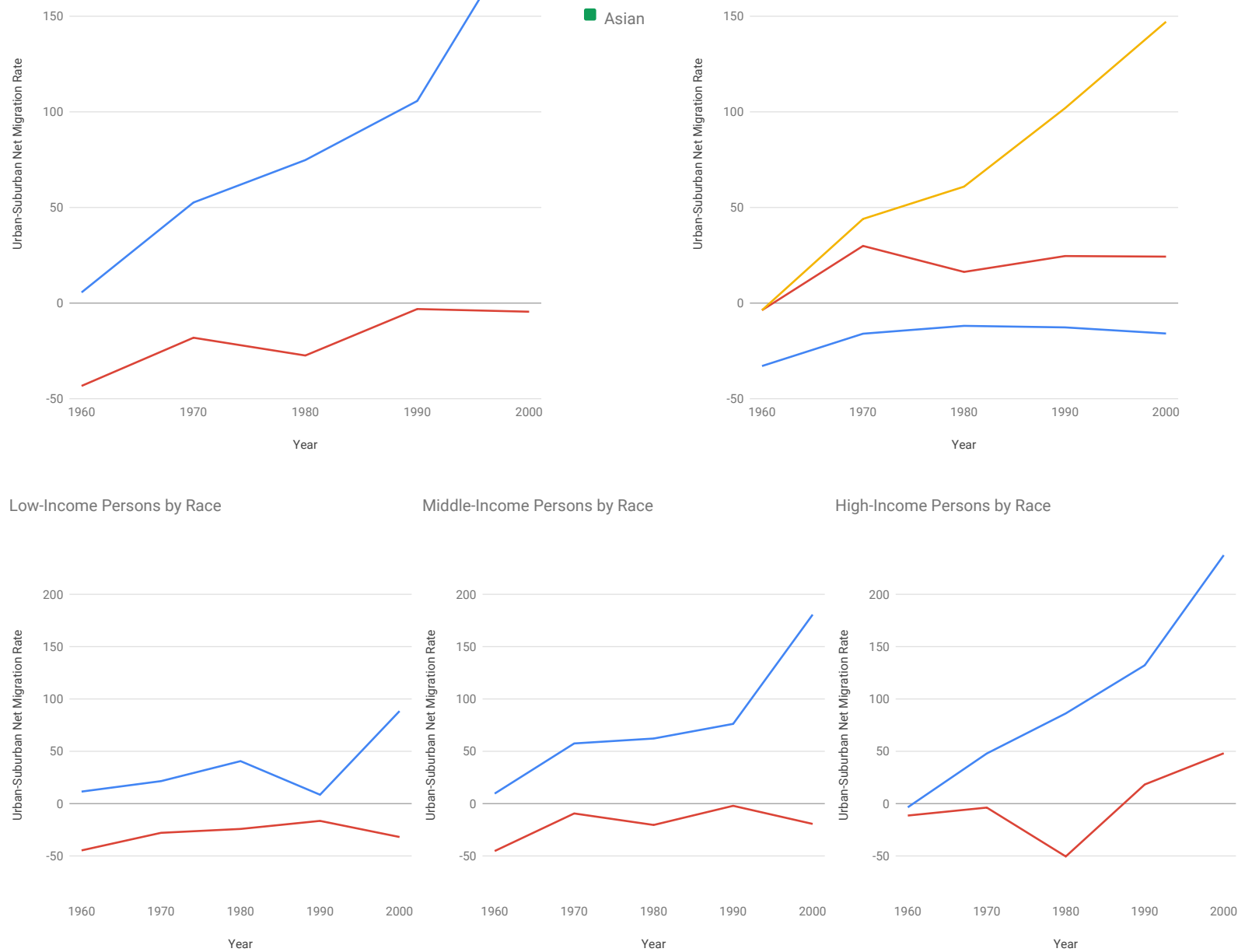

Year

High-Income Persons by Race

White

- Black

Hispanic

- Asian 


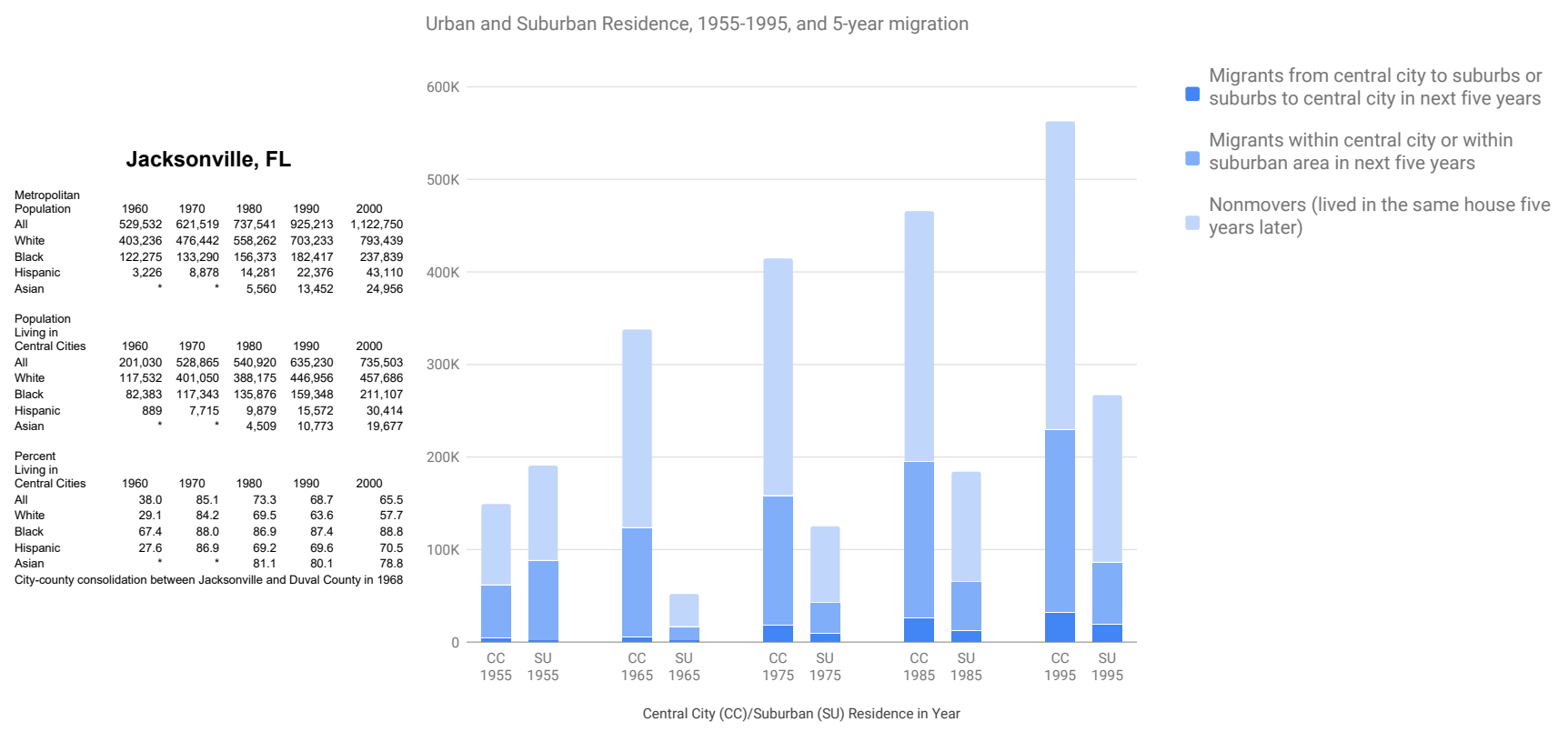

Urban-Suburban Net Migration Rate by Race

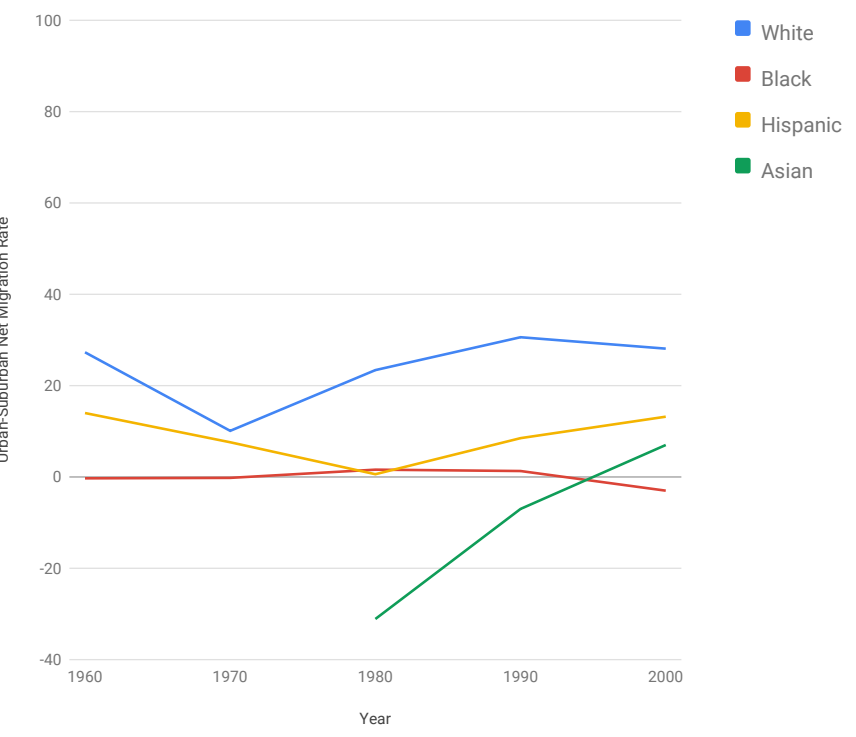

Low-Income Persons by Race

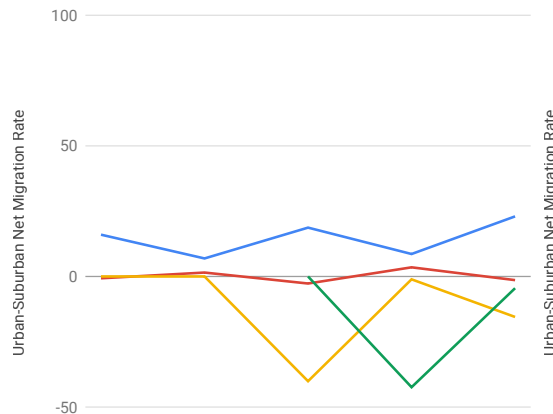

1960
Middle-Income Persons by Race

100
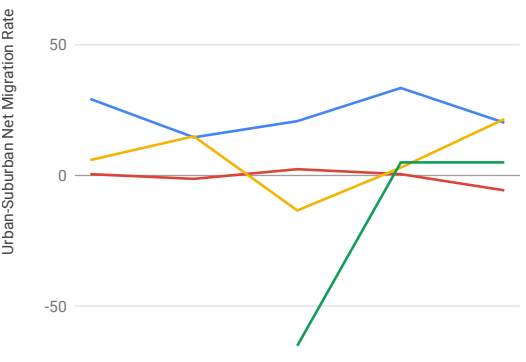

Year
Urban-Suburban Net Migration Rate by Income

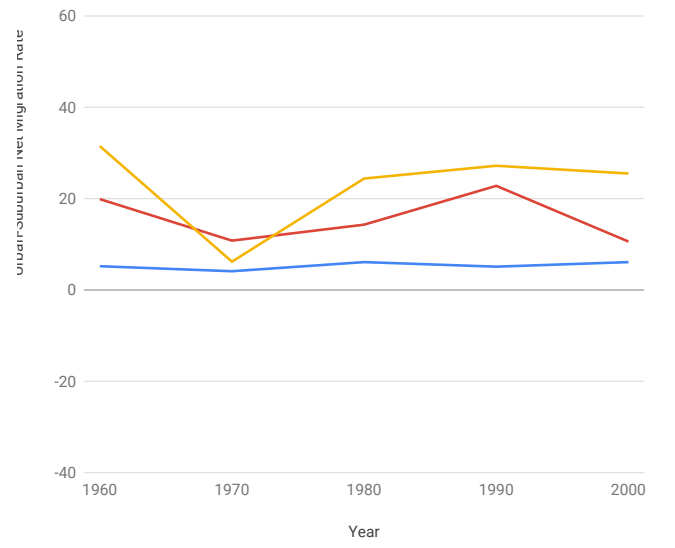

High-Income Persons by Race

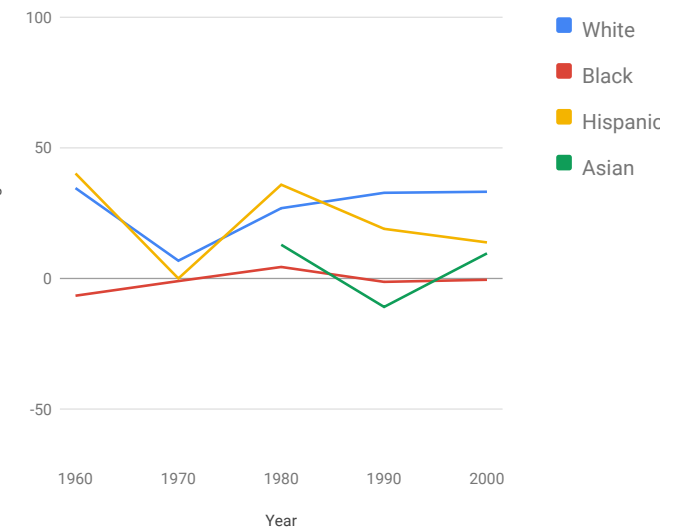


Urban and Suburban Residence, 1955-1995, and 5-year migration

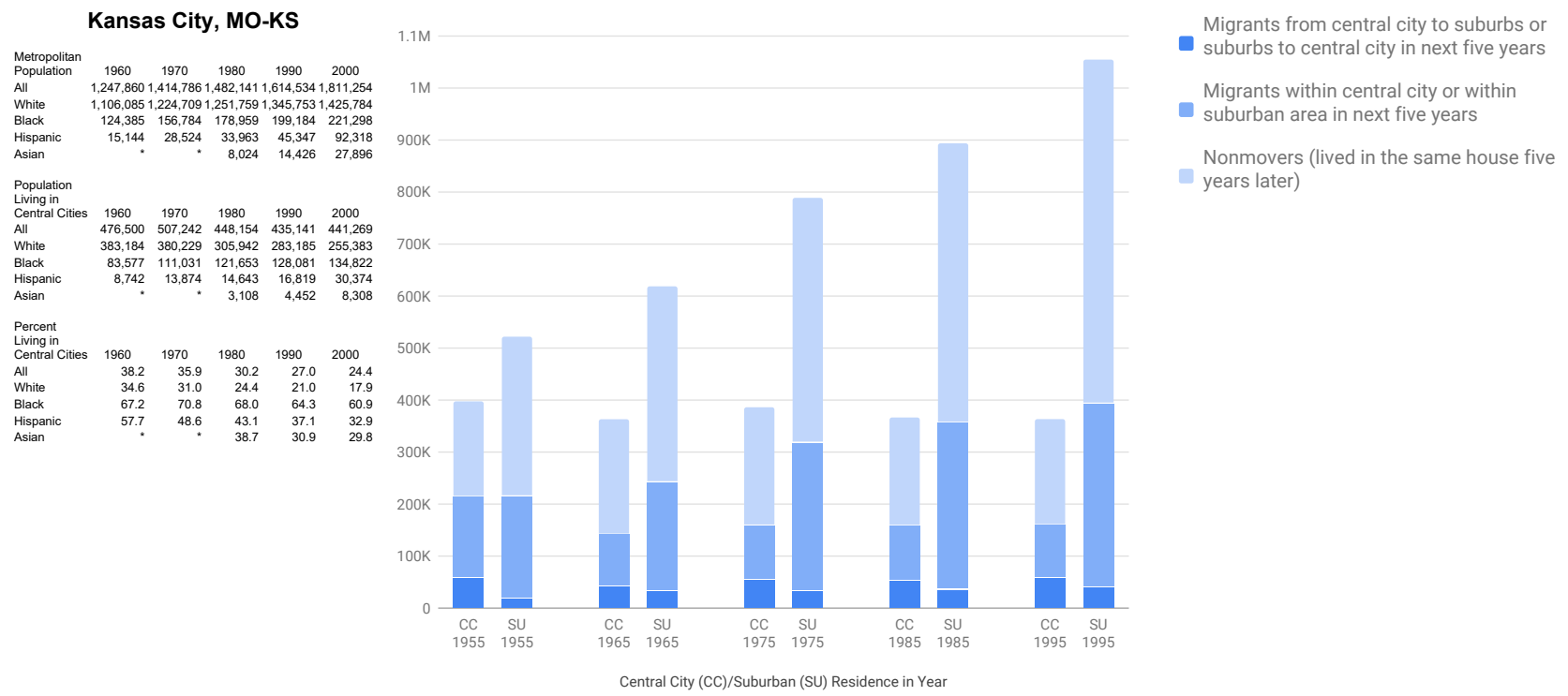

Urban-Suburban Net Migration Rate by Race

Urban-Suburban Net Migration Rate by Income

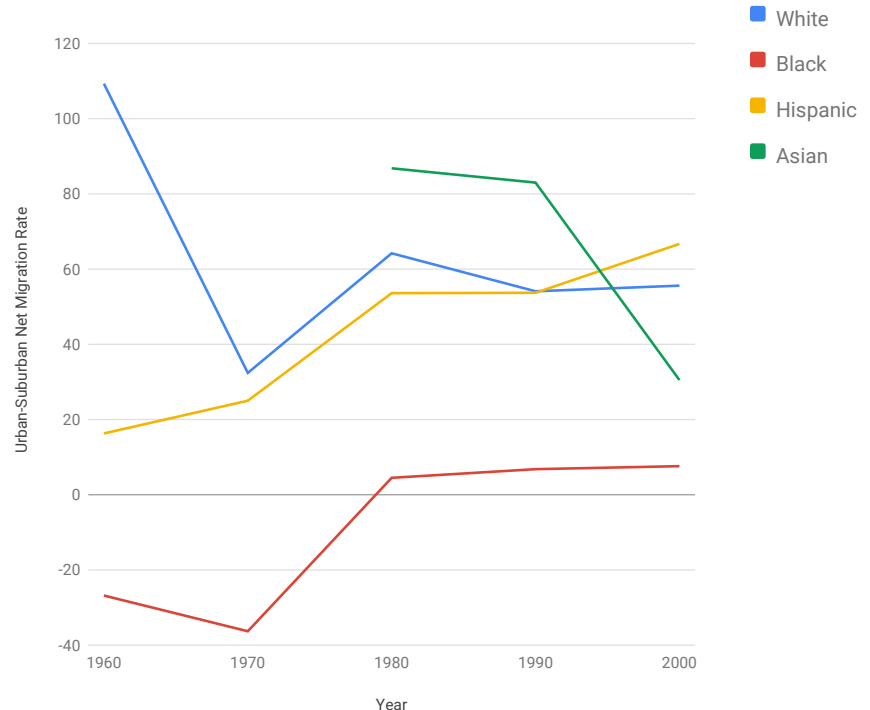

Low-Income Persons by Race

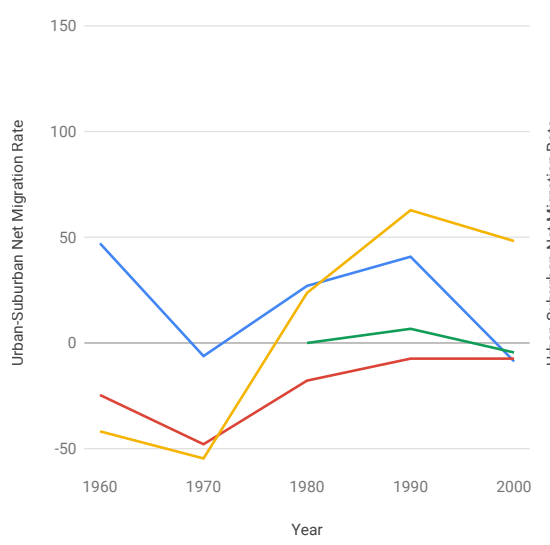

150

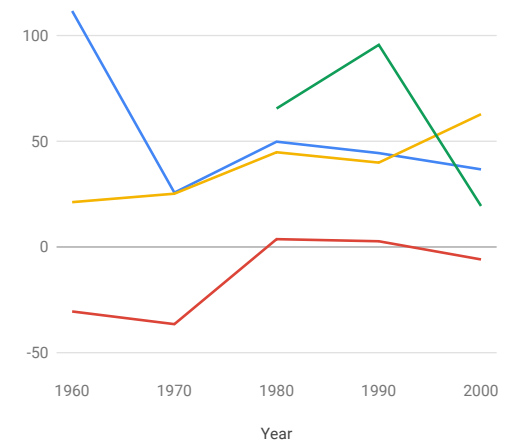

Year
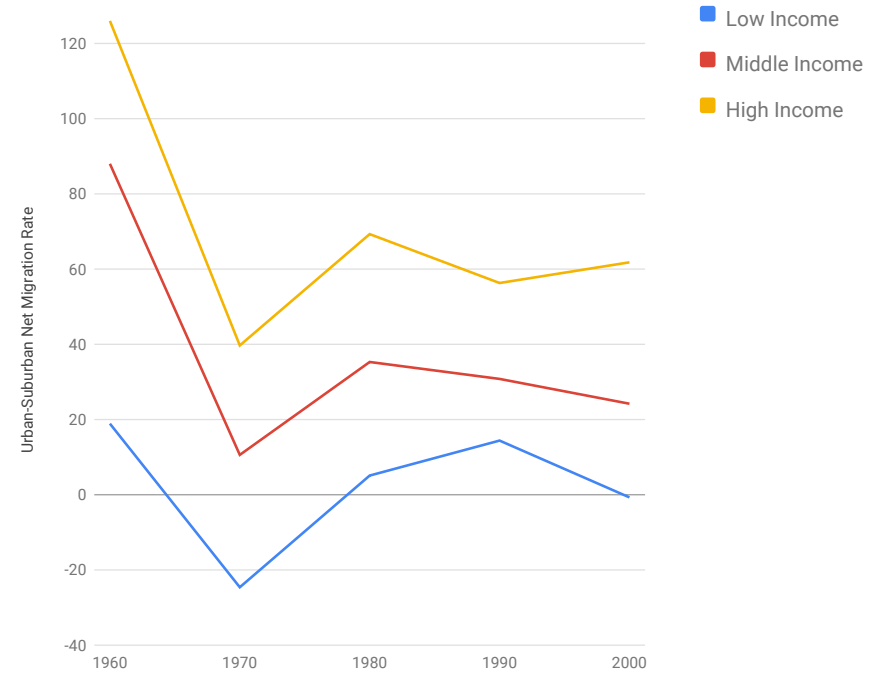

Year

High-Income Persons by Race

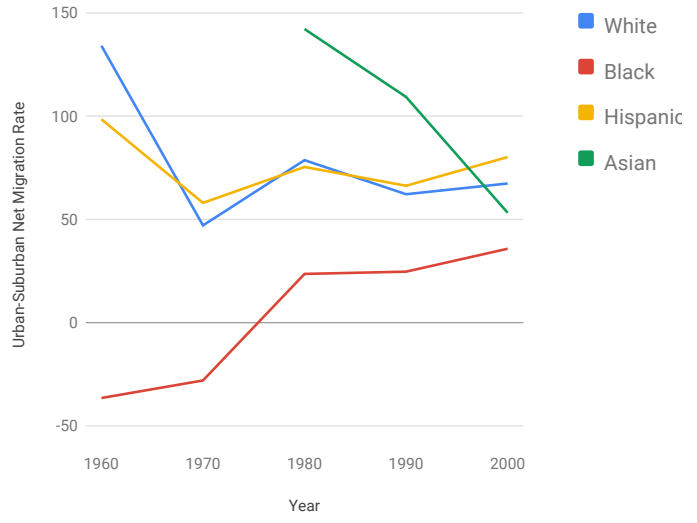


Urban and Suburban Residence, 1955-1995, and 5-year migration

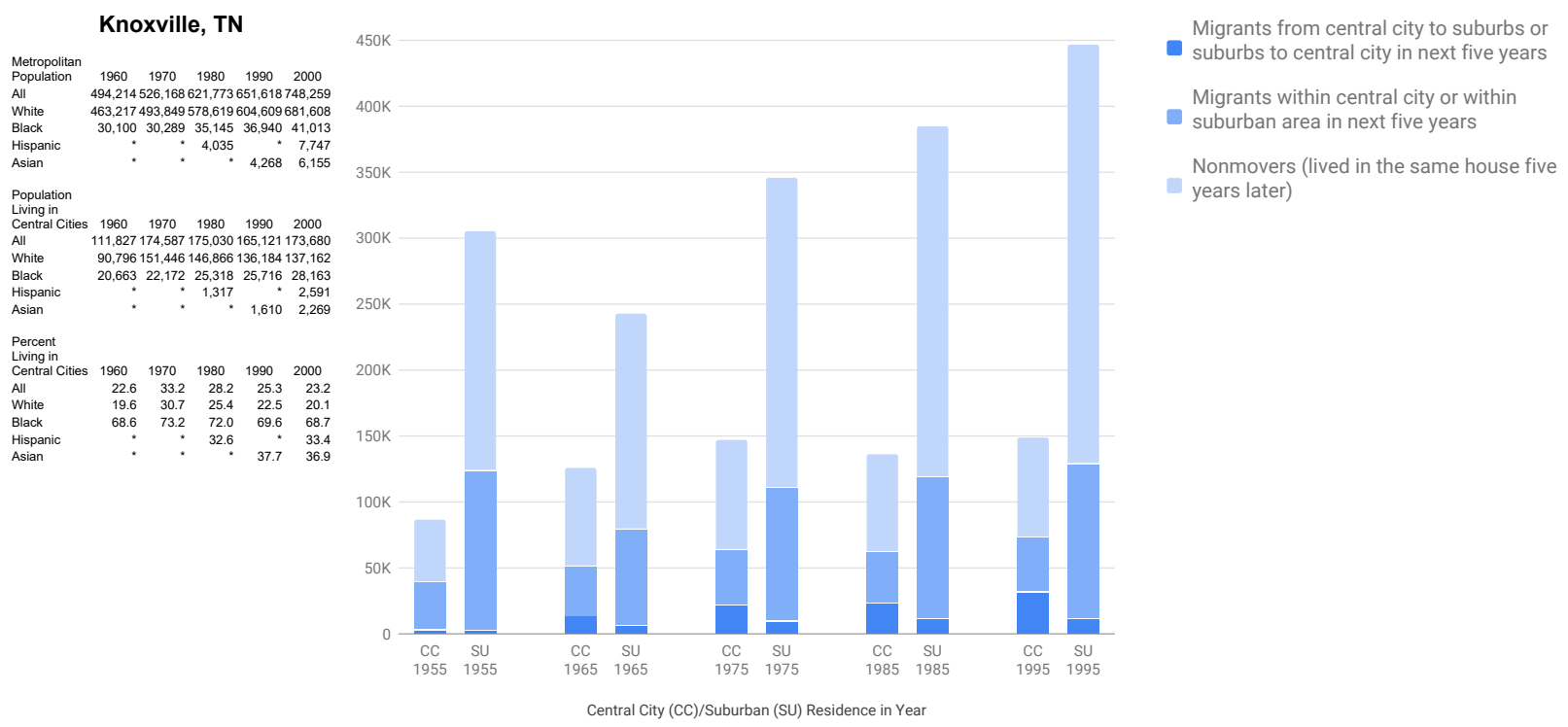

Urban-Suburban Net Migration Rate by Race

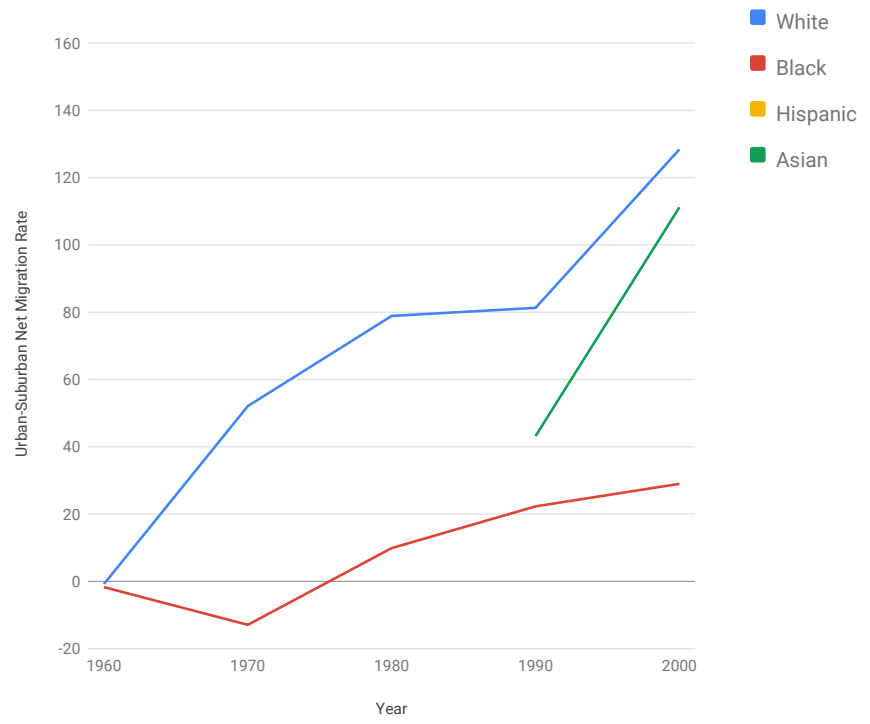

Low-Income Persons by Race
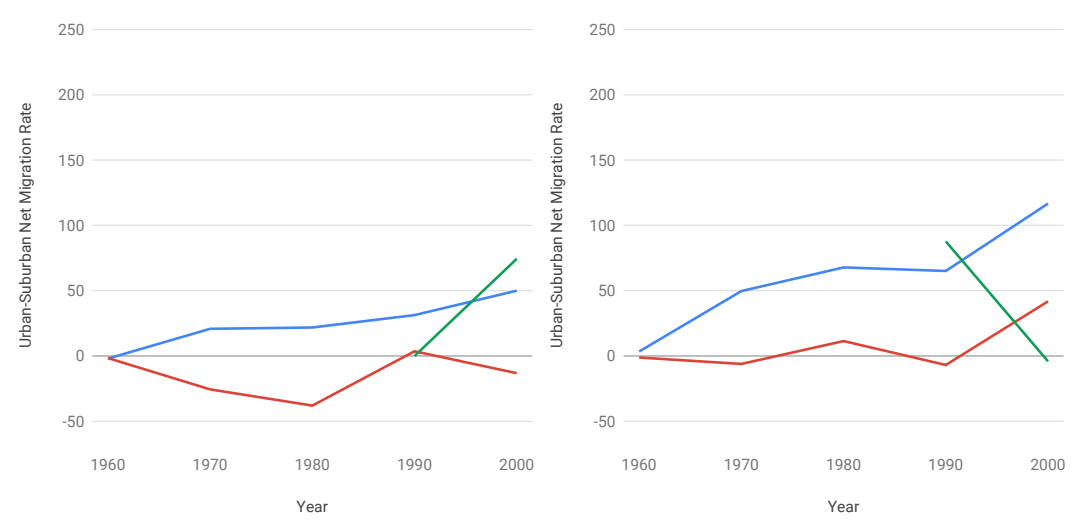

Urban-Suburban Net Migration Rate by Income

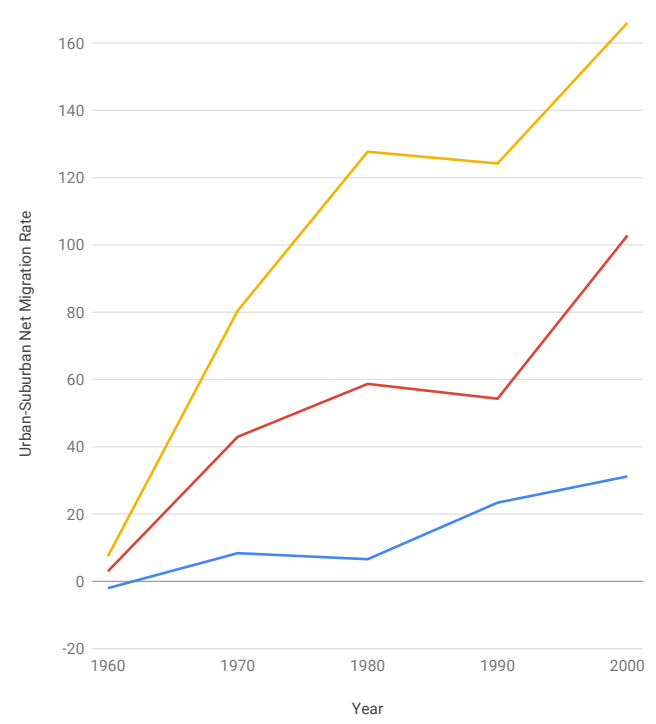

- Low Income

- Middle Income

High Income

High-Income Persons by Race

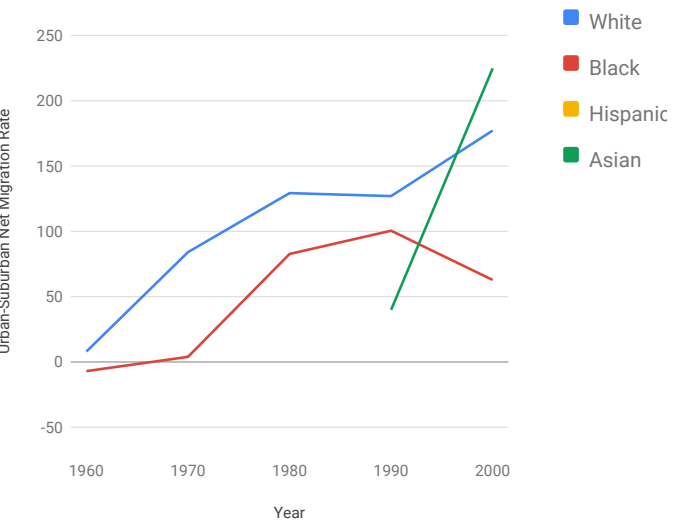


Urban and Suburban Residence, 1955-1995, and 5-year migration

\section{Lakeland-Winter Haven, FL}

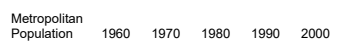

All $\quad 195,139227,222321,652405,382483,924$

White $158,32127,253261,666332,891361,079$

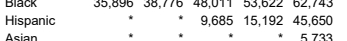

Population

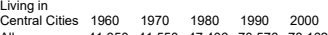

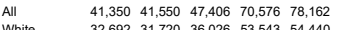

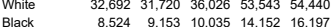

$\begin{array}{llll}\text { Hispanic } & \vdots \\ \text { Asian } & \text { : } 1,044 & 2,171 & 5,050 \\ & & \end{array}$

Percent $150 \mathrm{~K}$

$\begin{array}{lccccc}\text { Living in } & & & & & \\ \text { Central Cities } & 1960 & 1970 & 1980 & 1990 & 2000 \\ \text { All } & 21.2 & 18.3 & 14.7 & 17.4 & 16.2\end{array}$

$\begin{array}{llllll}\text { All } & 21.2 & 18.3 & 14.7 & 17.4 & 16.2 \\ \text { White } & 20.6 & 17.2 & 13.8 & 16.1 & 15.1 \\ \text { lack } & & & & & \end{array}$

$\begin{array}{lrrrrrr}\text { Black } & 23.7 & 23.6 & 20.9 & 26.4 & 25.8 & \\ \text { Hispanic } & : & : & 10.8 & 14.3 & 11.1 & 100 \mathrm{~K} \\ \text { Asian } & & & & & & \end{array}$
$300 \mathrm{~K}$

$250 \mathrm{~K}$

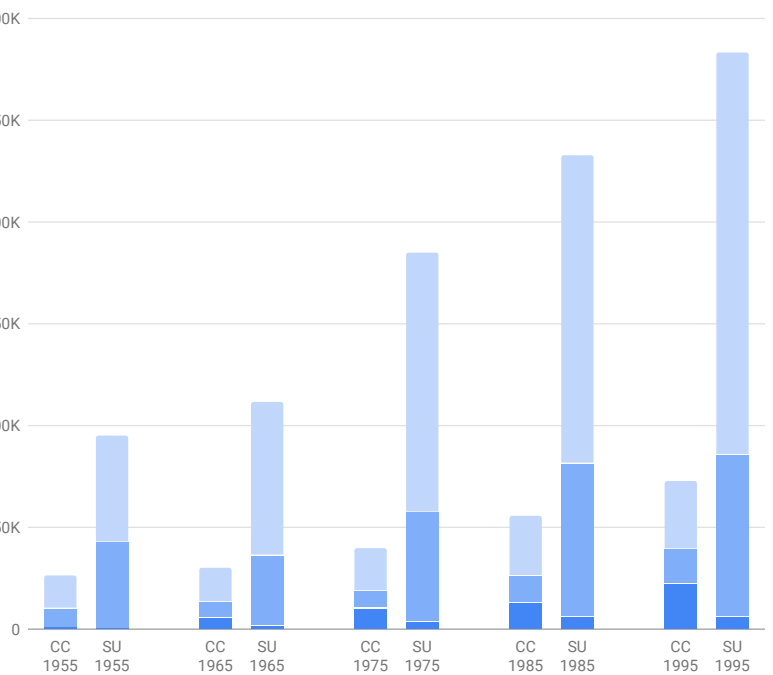

Migrants from central city to suburbs o suburbs to central city in next five years

Migrants within central city or within

suburban area in next five years

Nonmovers (lived in the same house five

years later)

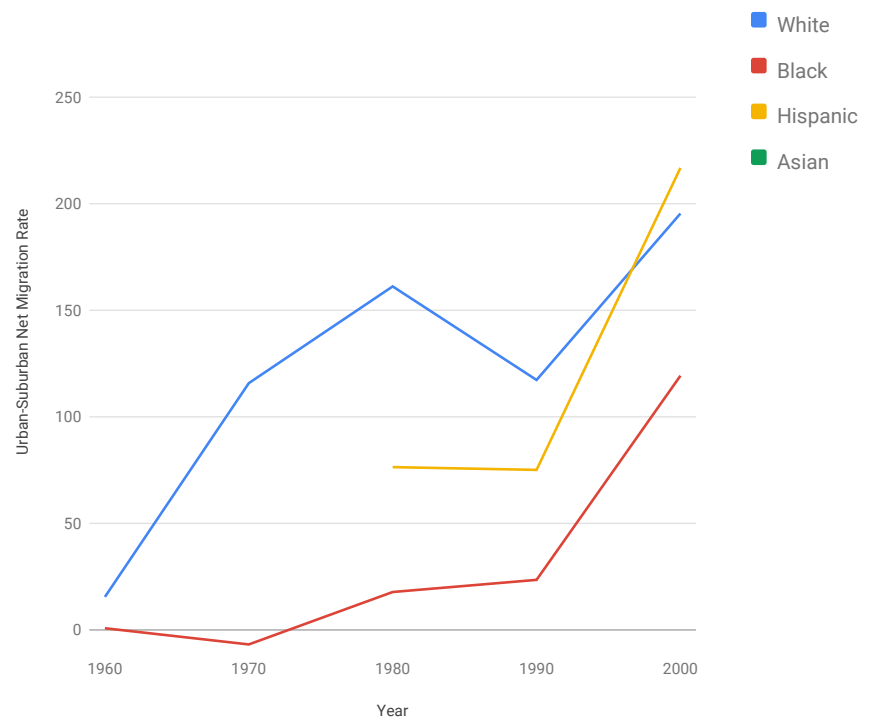

Low-Income Persons by Race

400

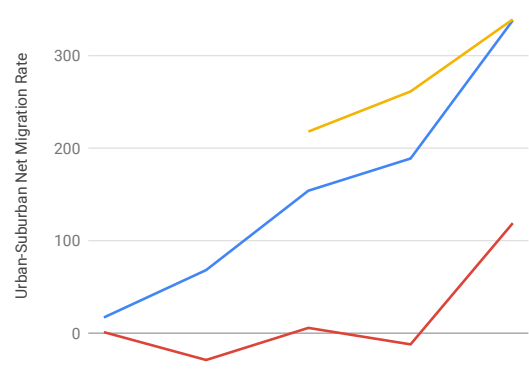

1960
Middle-Income Persons by Race

400

250

(20)

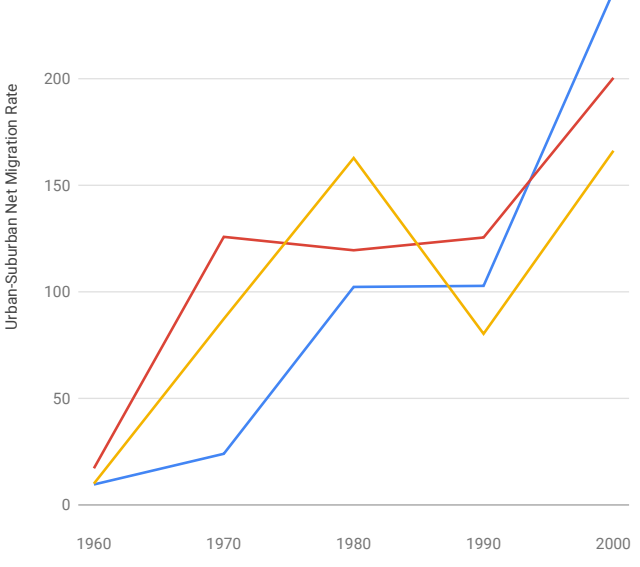

Year

High-Income Persons by Race
Low Income

- Middle Income

- High Income
$400 \div$ White

- Black

Hispanic

Asian 
Urban and Suburban Residence, 1955-1995, and 5-year migration
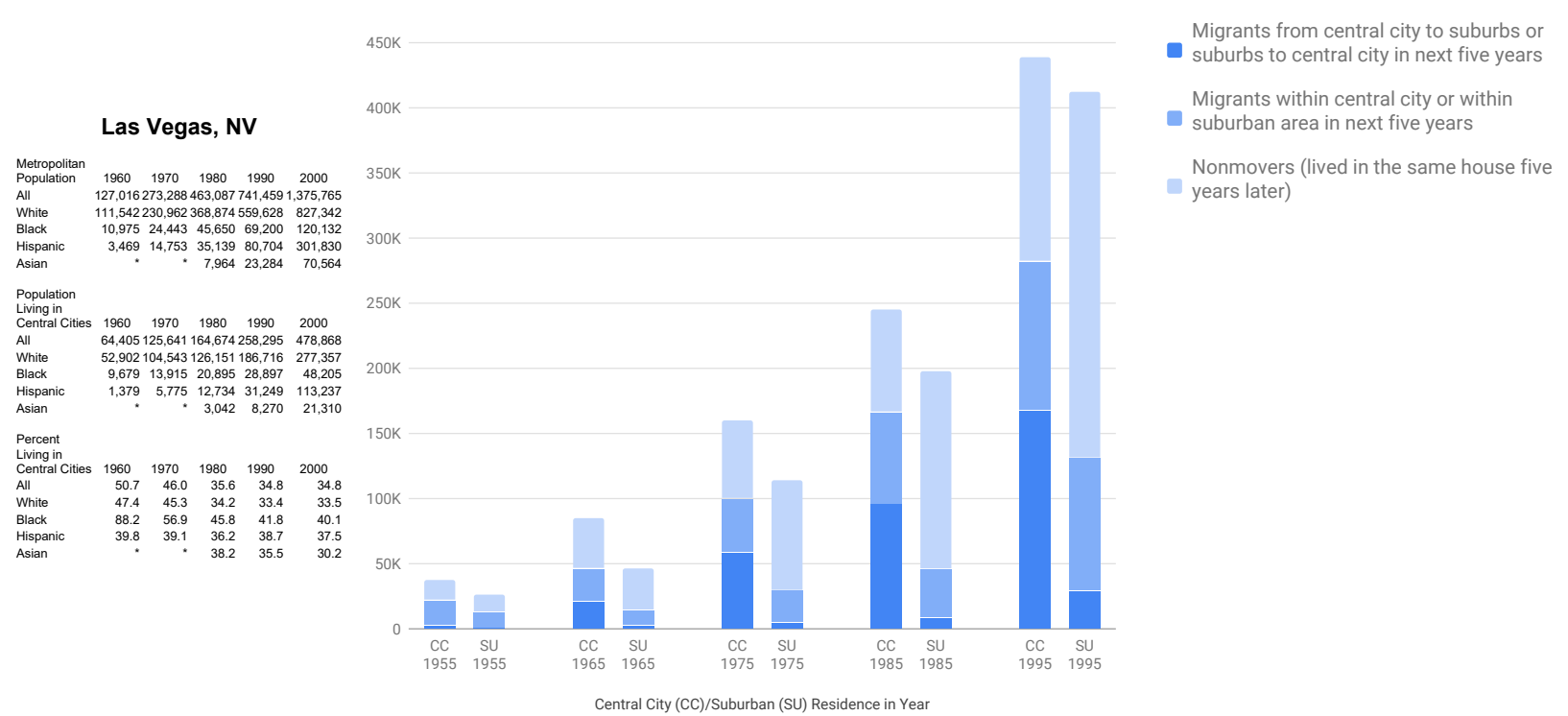

Urban-Suburban Net Migration Rate by Race

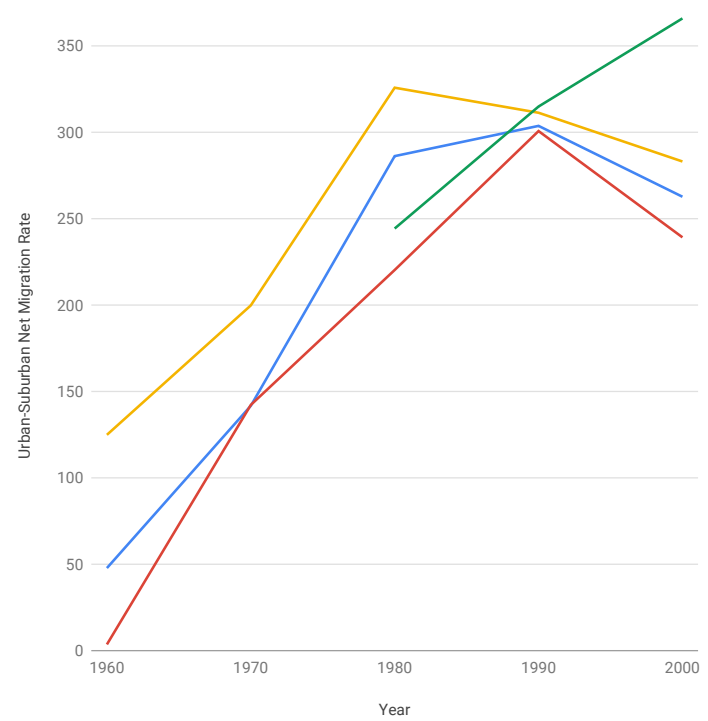

Low-Income Persons by Race
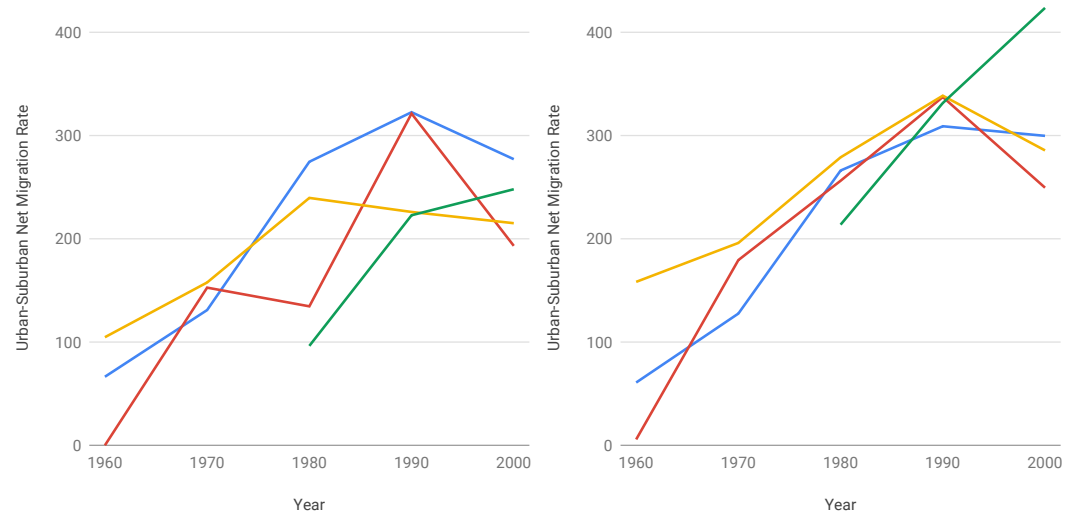

Year

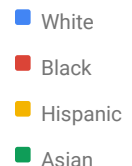

- Asian

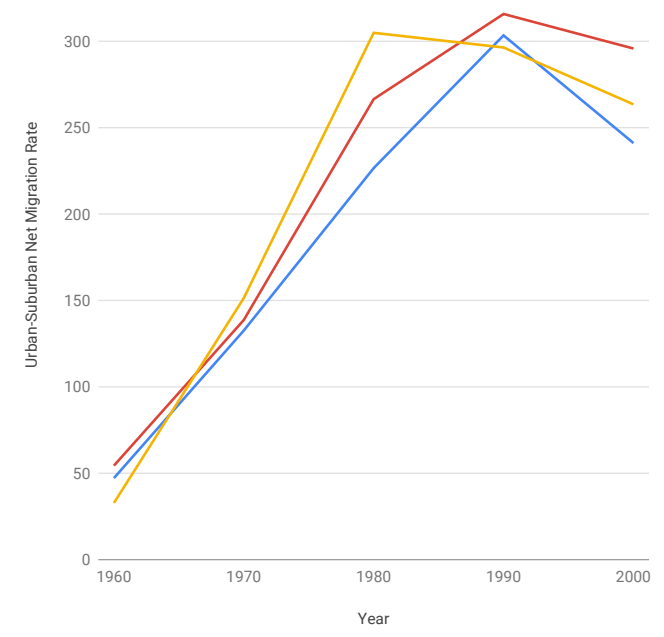

High-Income Persons by Race

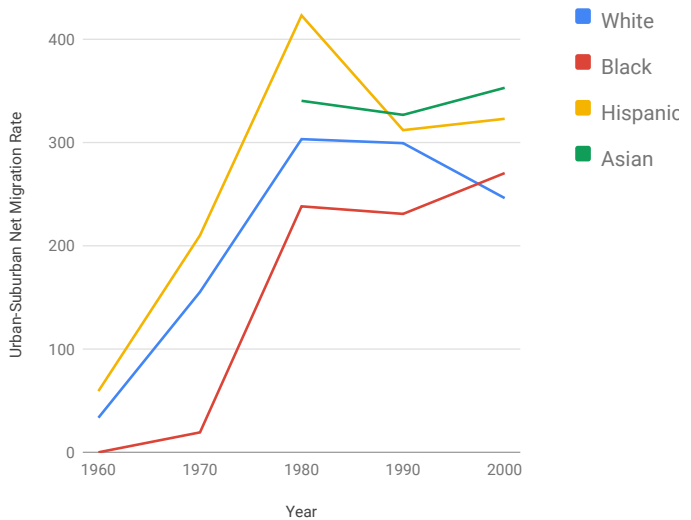


Urban and Suburban Residence, 1955-1995, and 5-year migration

$300 \mathrm{~K}$

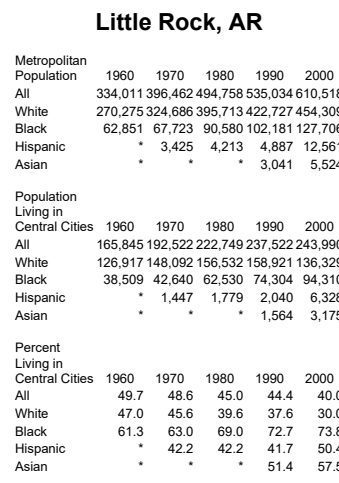

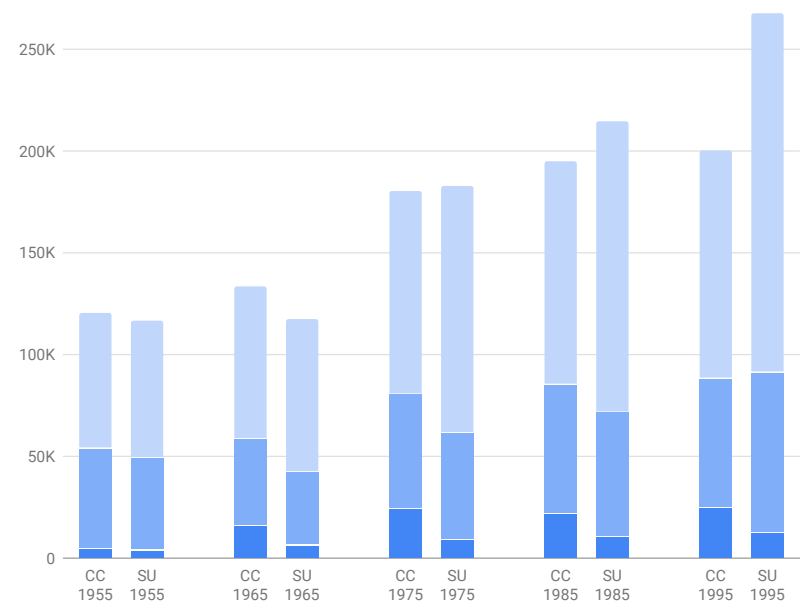

Central City (CC)/Suburban (SU) Residence in Year
Migrants from central city to suburbs or - suburbs to central city in next five years

Migrants within central city or within

suburban area in next five years

Nonmovers (lived in the same house five

years later)
Urban-Suburban Net Migration Rate by Race

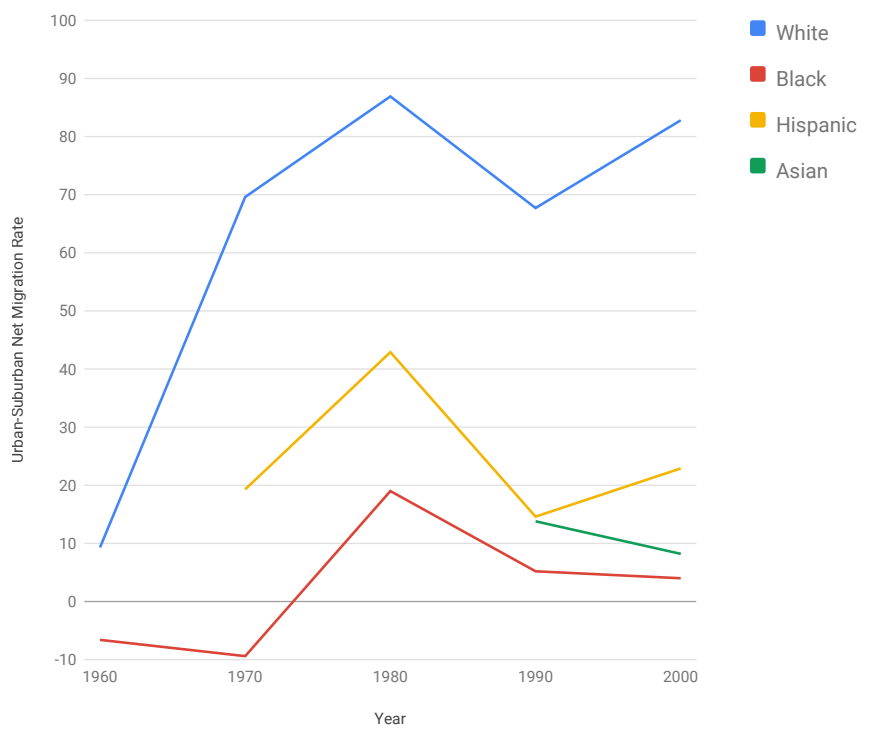

Low-Income Persons by Race

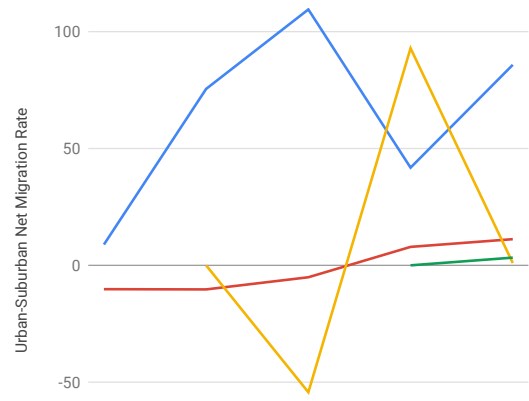

1960
Middle-Income Persons by Race

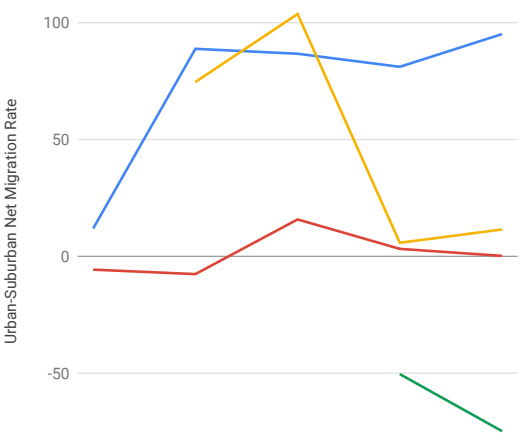

Urban-Suburban Net Migration Rate by Income

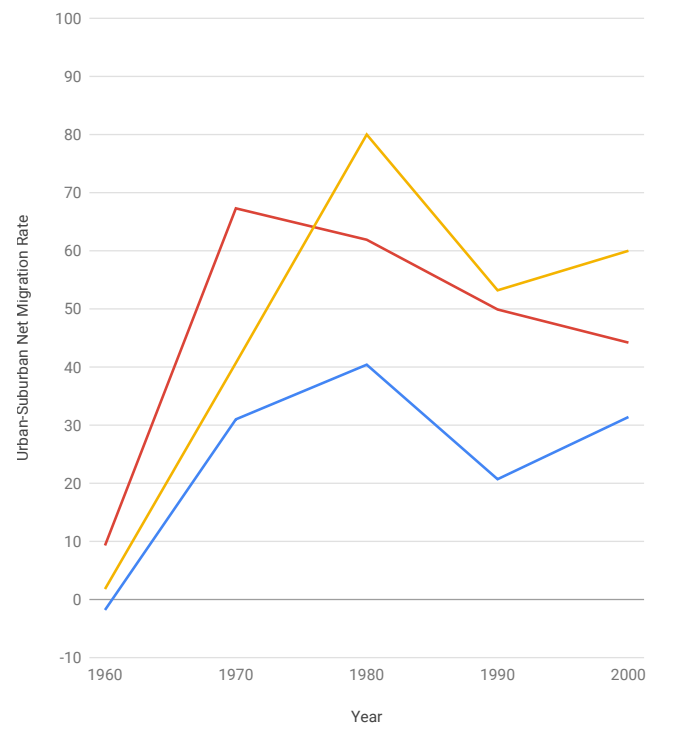

Low Income

Middle Income

- High Income

High-Income Persons by Race

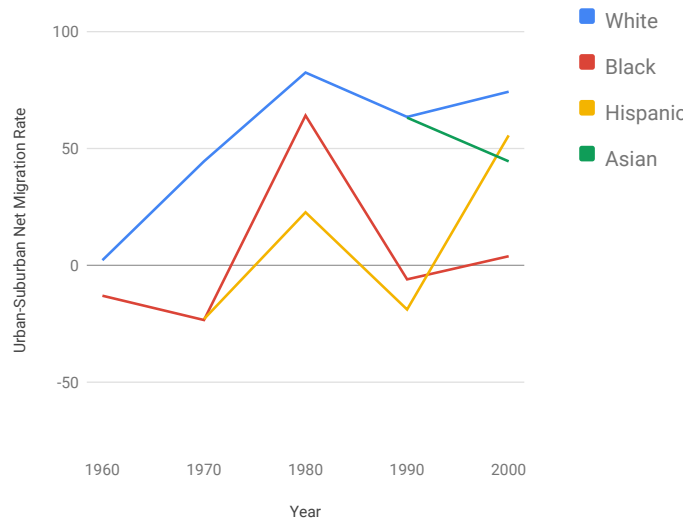




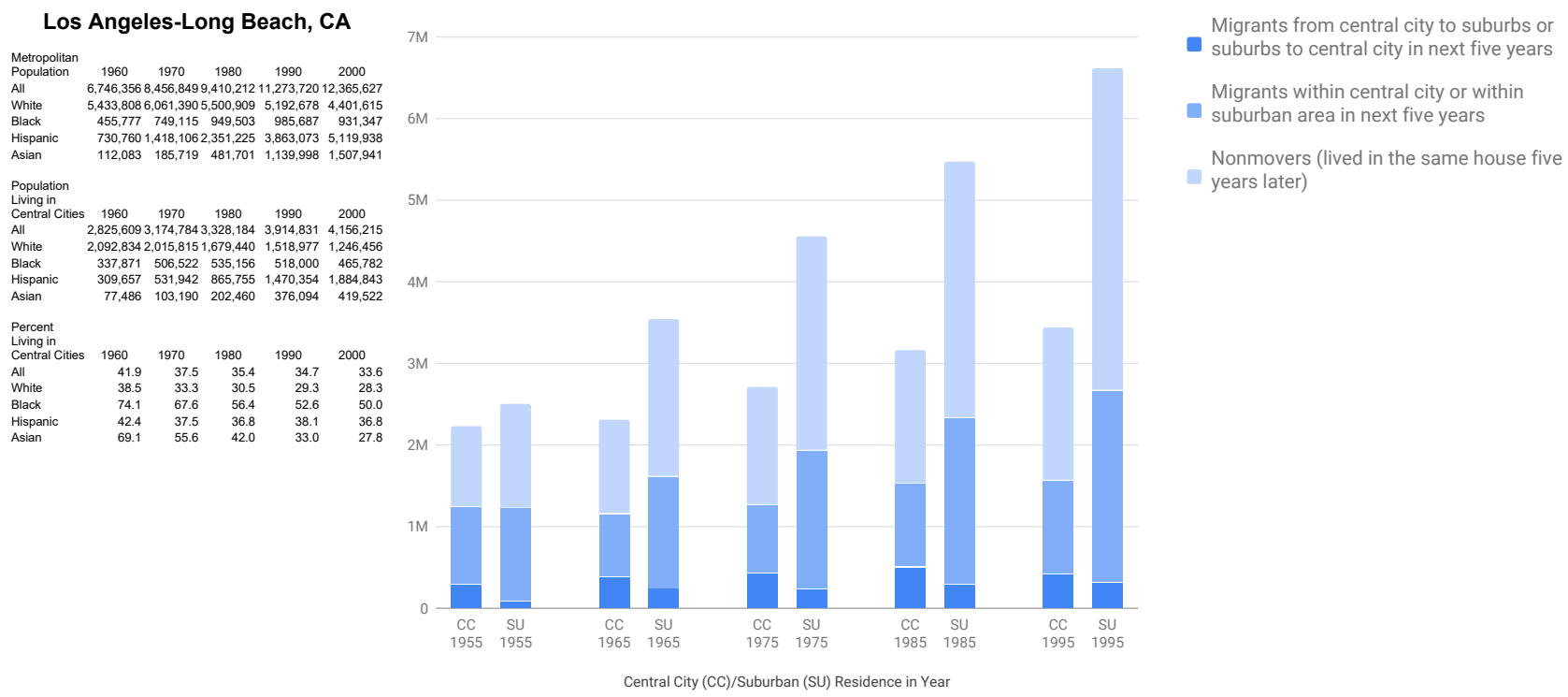

Urban-Suburban Net Migration Rate by Race

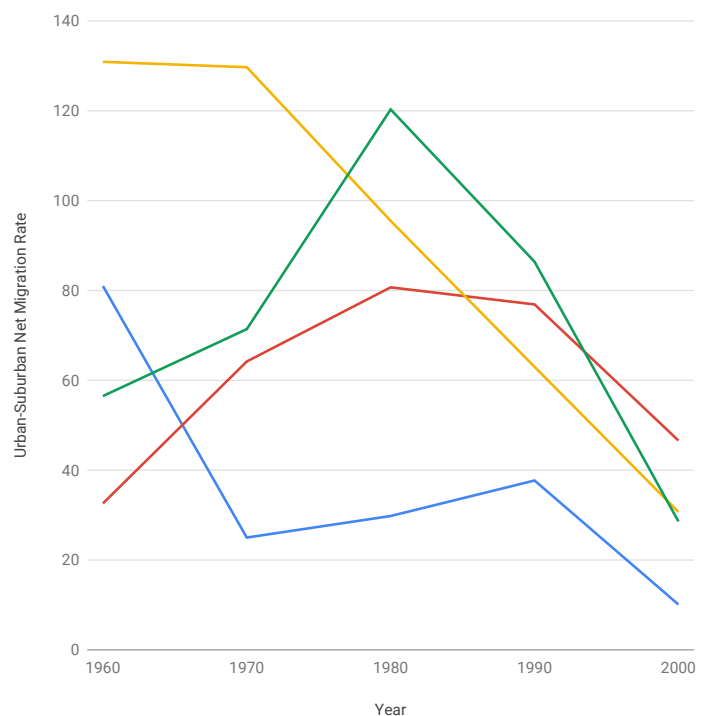

Low-Income Persons by Race

Middle-Income Persons by Race
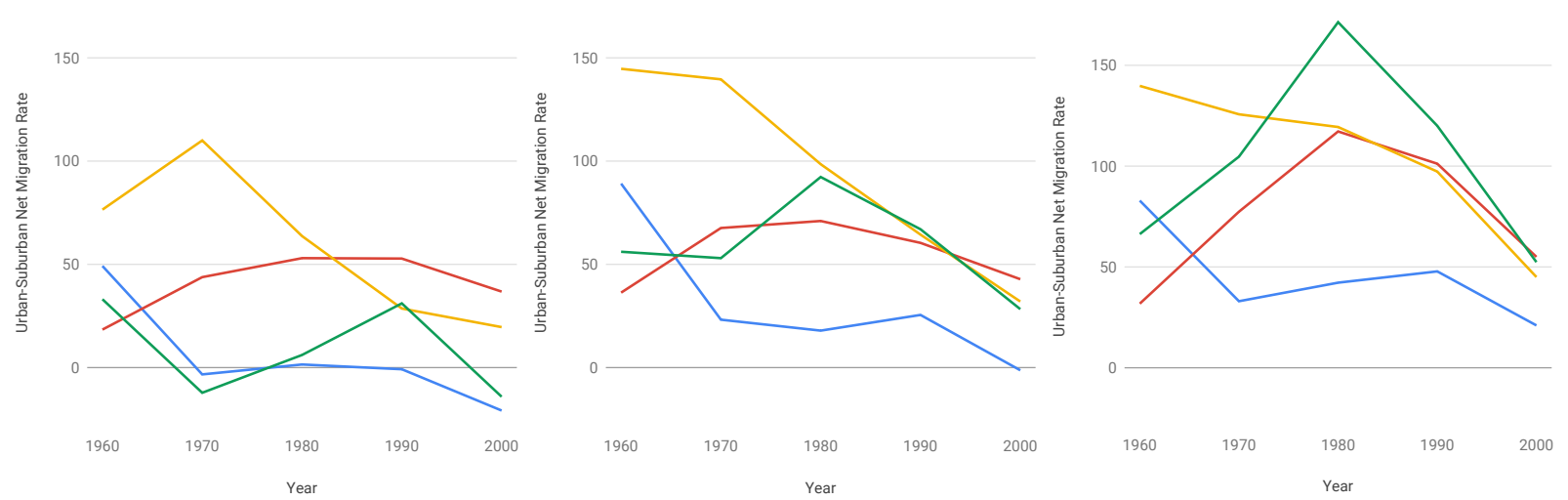

White

Black

Hispanic

Asian 
Urban and Suburban Residence, 1955-1995, and 5-year migration

Louisville, KY-IN

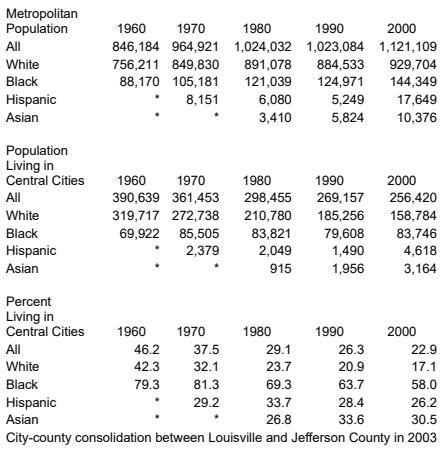

\section{$700 \mathrm{~K}$}

$600 \mathrm{~K}$

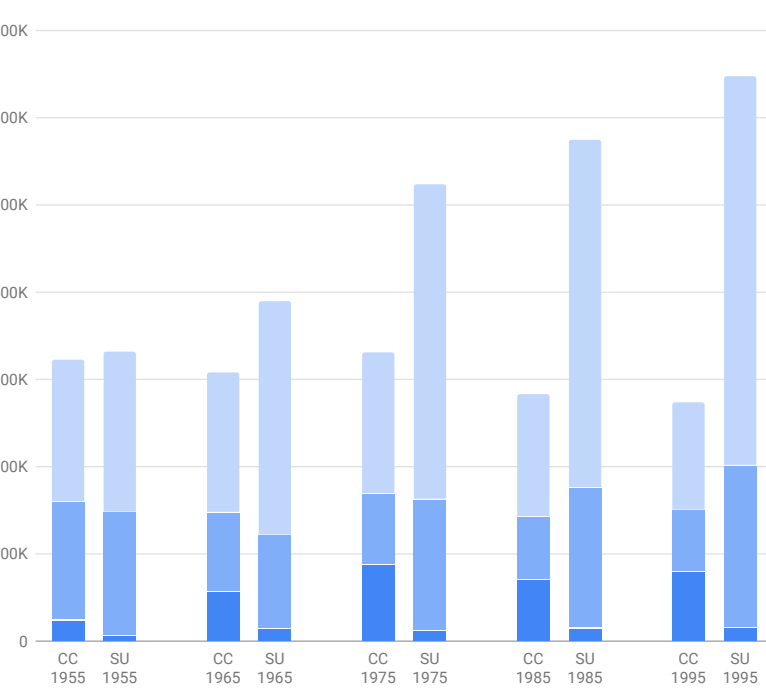

Migrants from central city to suburbs or suburbs to central city in next five years

Migrants within central city or within suburban area in next five years

Nonmovers (lived in the same house five years later)

Urban-Suburban Net Migration Rate by Race

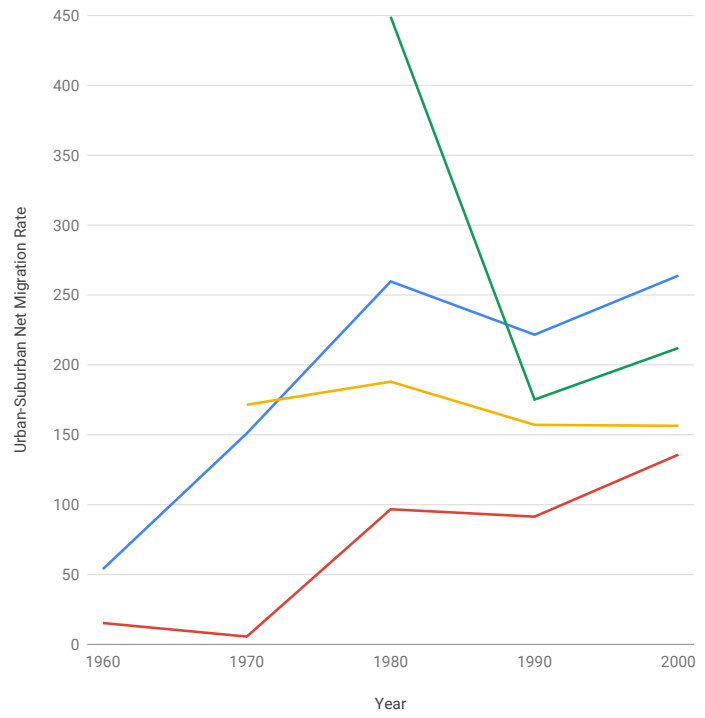

Low-Income Persons by Race

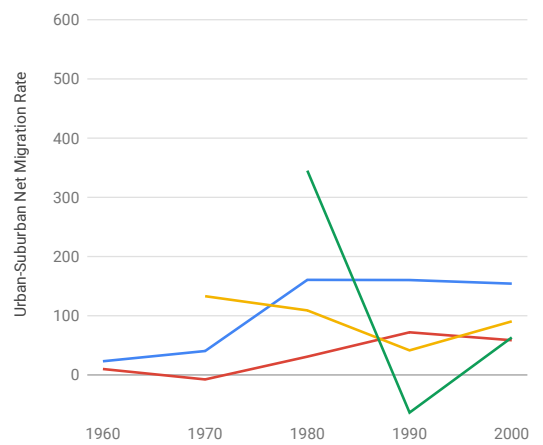

Year
Middle-Income Persons by Race

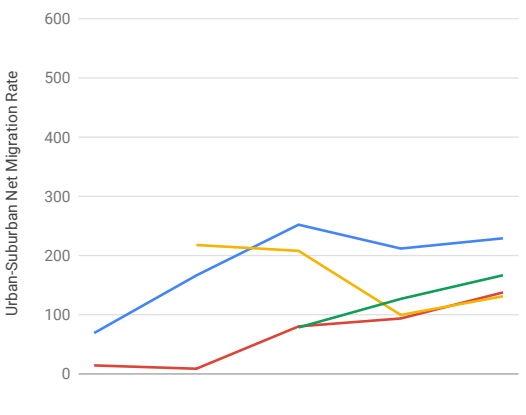

Year
Urban-Suburban Net Migration Rate by Income

$$
\begin{aligned}
& \text { White } \\
& \text { Black } \\
& \text { Hispanic } \\
& \text { Asian }
\end{aligned}
$$$$
350
$$

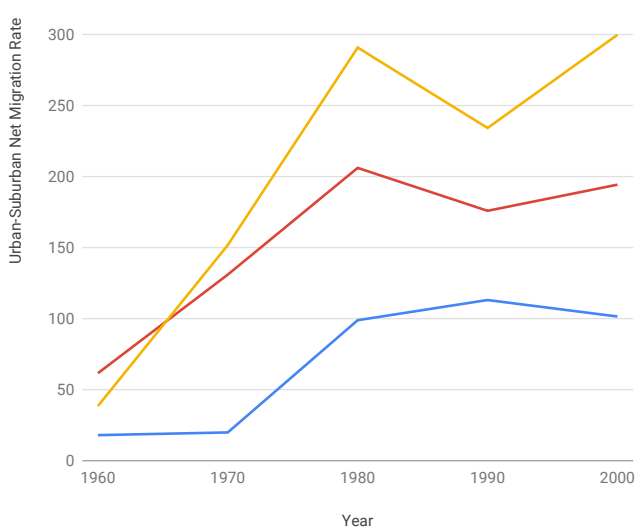

High-Income Persons by Race

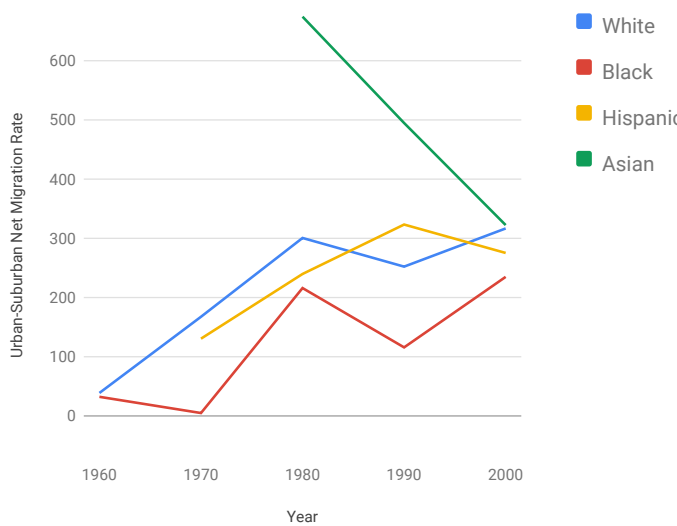


Urban and Suburban Residence, 1955-1995, and 5-year migration

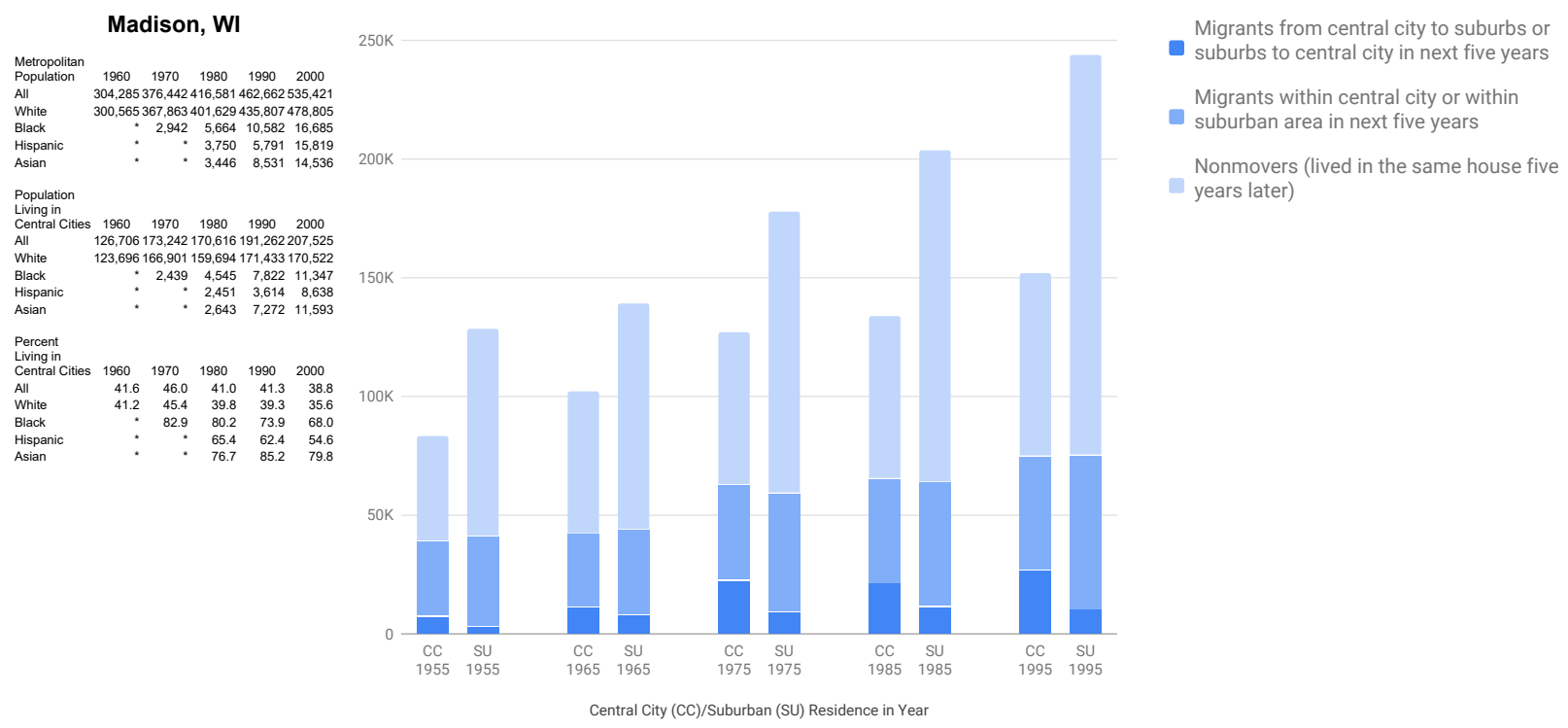

Urban-Suburban Net Migration Rate by Income

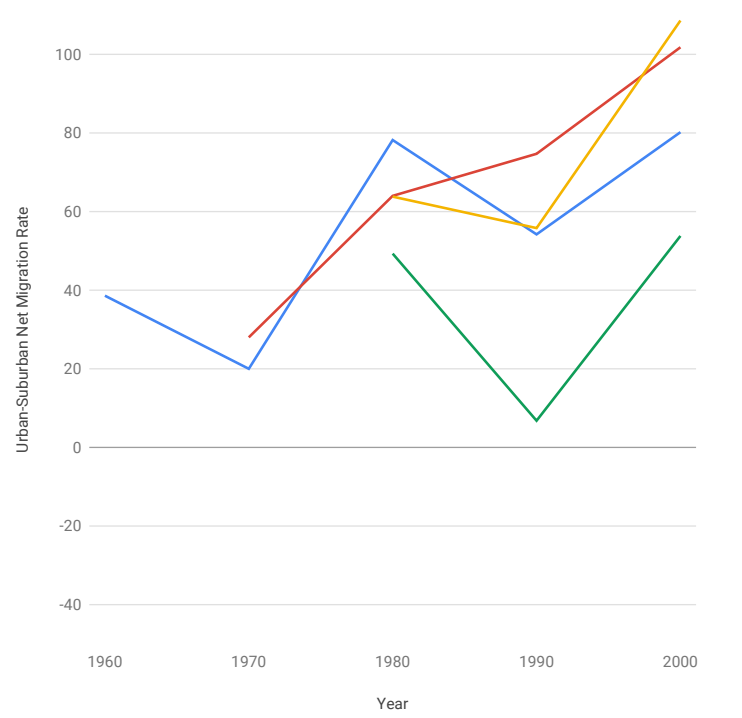

Low-Income Persons by Race

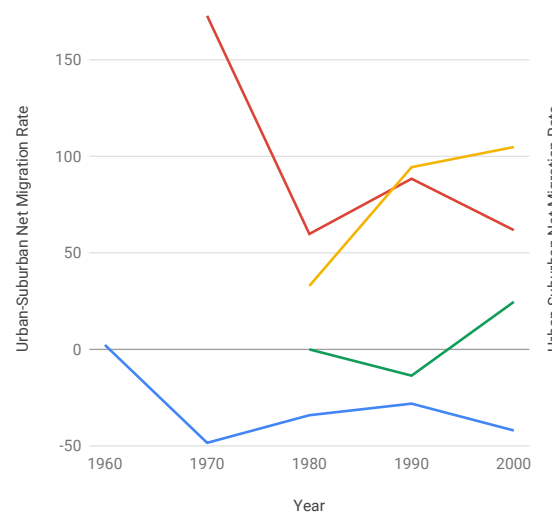

Middle-Income Persons by Race
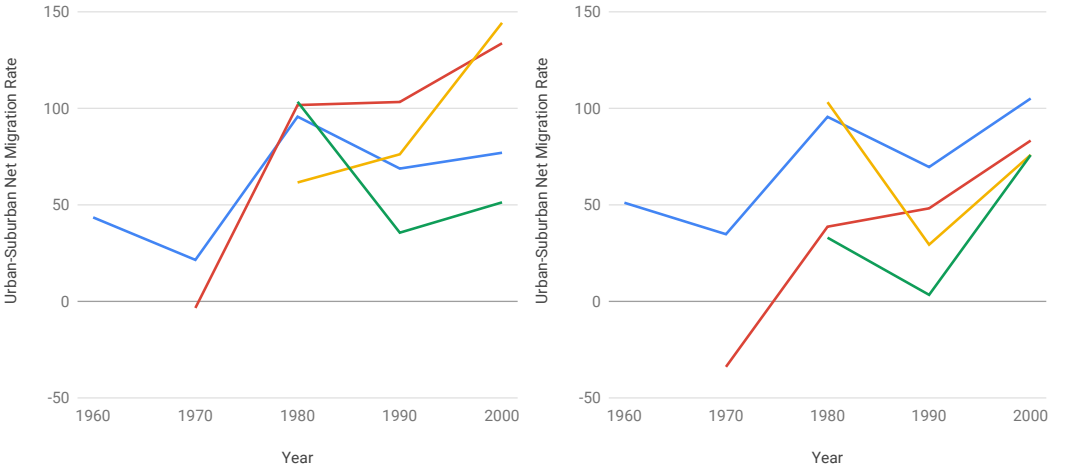

Hispanic

- Asian
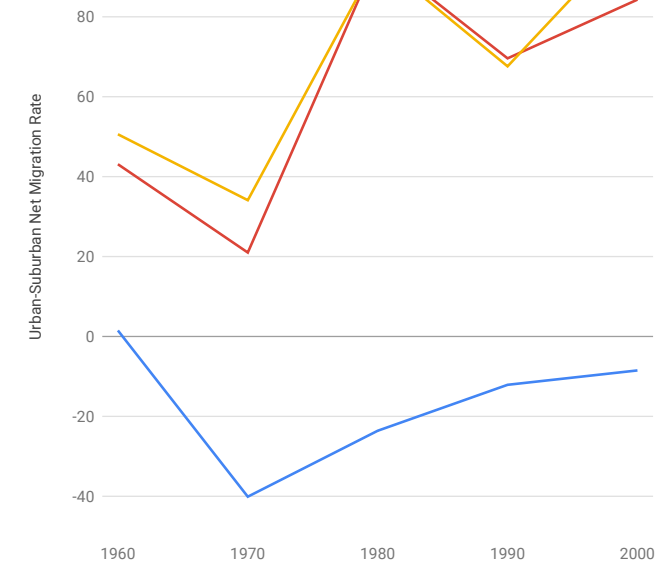

Year
High-Income Persons by Race
Low Income

Middle Income

High Income 
Urban and Suburban Residence, 1955-1995, and 5-year migration

$350 \mathrm{~K}$

McAllen-Edinburg, TX Metropolitan
Population 1960.18

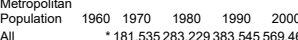

White :38,172 $51,82054,25959,009 \quad 250$

Hispanic " "143,017 230,287 $326,923503,526$

Population

$\begin{array}{lllll}\text { Lentral Cities } 1960 & 1970 & 1980 & 1990 & 2000\end{array}$

All $\quad * 54,79990,356113,906155,074$

White $\quad: 15,059,22,676,22,287,23,019$
Black

Hispanic : : 39,623 $67,145 \quad 90,191$ 128,322

Living in

$\begin{array}{lllllll}1970 & 1980 & 1990 & 2000 & 100 \mathrm{~K}\end{array}$

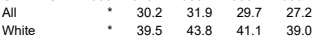

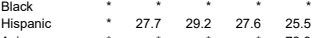

Urban-Suburban Net Migration Rate by Race

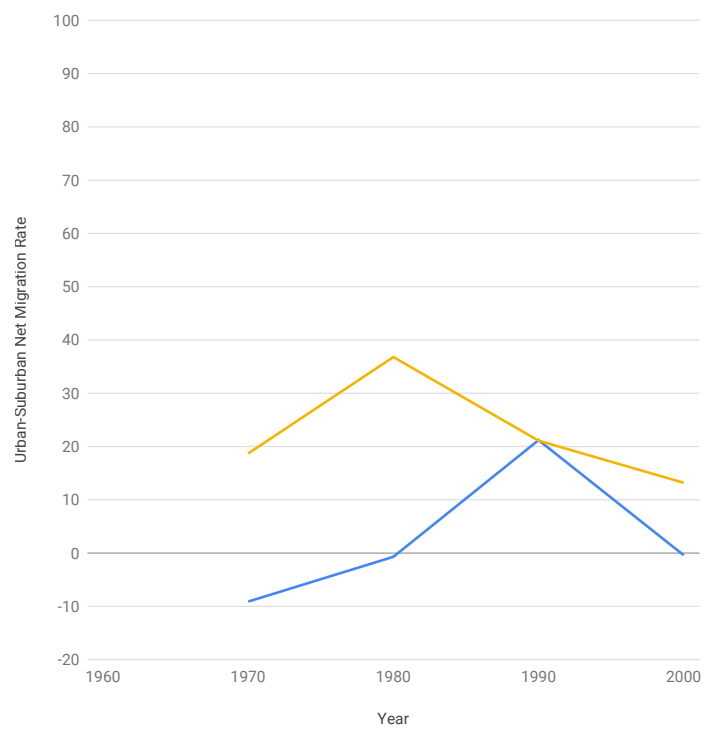

Low-Income Persons by Race

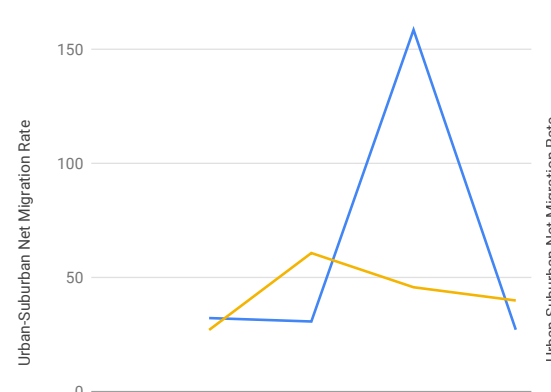

Middle-Income Persons by Race

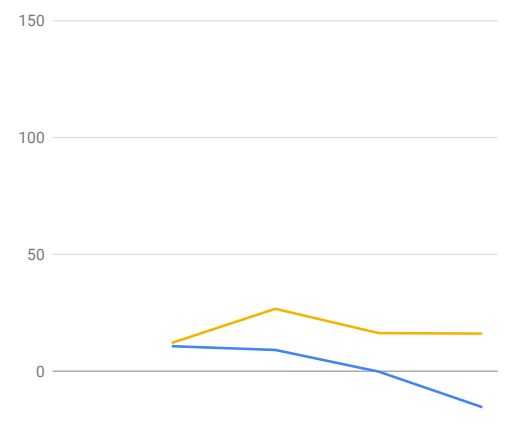

Urban-Suburban Net Migration Rate by Income

$$
\begin{aligned}
& \text { White } \\
& \text { Black } \\
& \text { Hispanic } \\
& \text { Asian }
\end{aligned}
$$

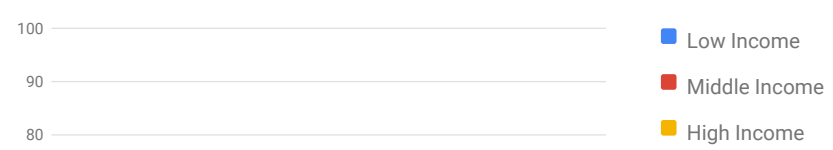

Migrants from central city to suburbs or suburbs to central city in next five years

Migrants within central city or within

- suburban area in next five years

Nonmovers (lived in the same house five

years later)

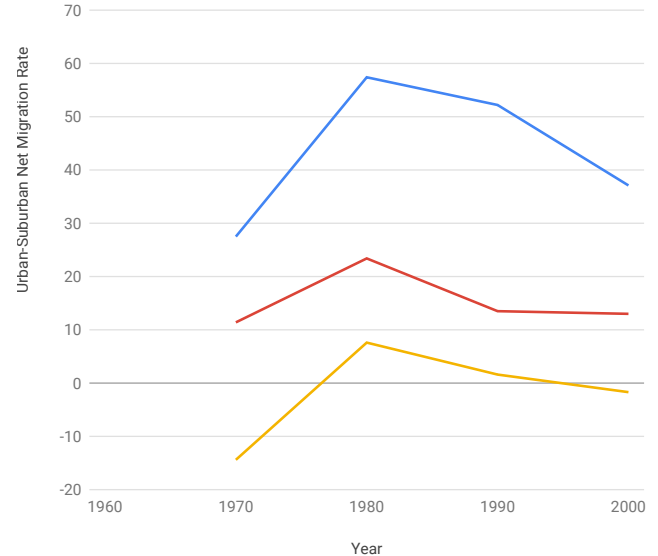

High-Income Persons by Race

White

Black

Hispanic

- Asian 
Urban and Suburban Residence, 1955-1995, and 5-year migration

$600 \mathrm{~K}$

Memphis, TN-MS-AR

$\begin{array}{lllll}\text { Metropolitan } & 1960 & 1970 & 1980 & 1990 \quad 2000\end{array}$

White $\quad 474,257551,843,584,380,615,681,2130,230$

$\begin{array}{lllll}\text { Black } \quad 342,424357,114 & 406,030 & 441,209 & 524,334\end{array}$

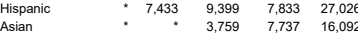

Population
Living in

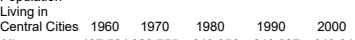

$\begin{array}{lllll}\text { All } & 497,524623,755 & 646,356 & 610,337 & 649,845 \\ \text { White } & 312,039376,559 & 331,999 & 266,547 & 216,447\end{array}$

Black $\quad 184,119240,360 \quad 304,708 \quad 333,935 \quad 396,809$

$\begin{array}{llllll}\text { Hispanic } \quad-\quad 5,261 & 5,730 & 4,011 & 18,75\end{array}$

Percent
Living in

$\begin{array}{lccccc}\text { Central Cities } & 1960 & 1970 & 1980 & 1990 & 2000 \\ \text { All } & 60.8 & 67.9 & 64.3 & 568 & 536\end{array}$

\begin{tabular}{lrrrrr} 
All & 60.8 & 67.9 & 64.3 & 56.8 & 53.6 \\
White & 65.8 & 68.2 & 56.8 & 43.3 & 34.3 \\
\hline
\end{tabular}

$\begin{array}{lrrrrrrr}\text { Black } & 53.8 & 67.3 & 75.0 & 75.7 & 75.7 & \\ \text { Hispanic } & & 70.8 & 61.0 & 512 & 69 . & 100 \mathrm{~K}\end{array}$

\section{$500 \mathrm{~K}$ \\ $200 \mathrm{~K}$}

Urban-Suburban Net Migration Rate by Race

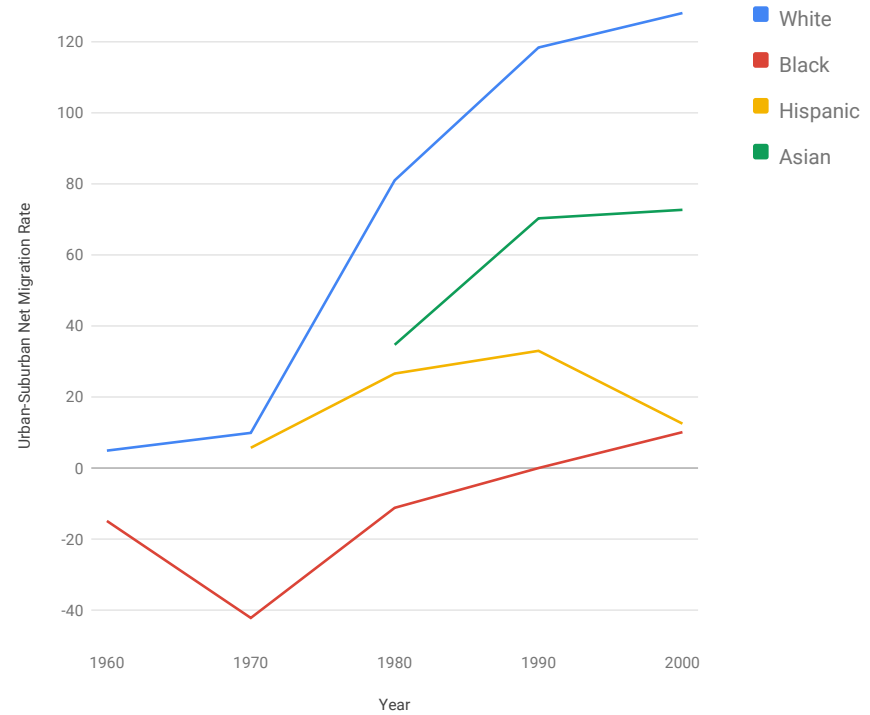

Low-Income Persons by Race

Middle-Income Persons by Race
Urban-Suburban Net Migration Rate by Income

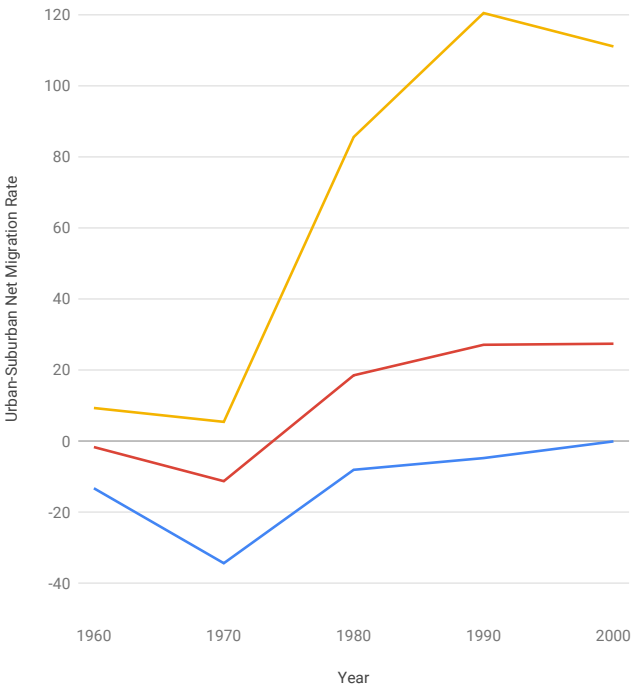

Low Income

- Middle Income

High Income

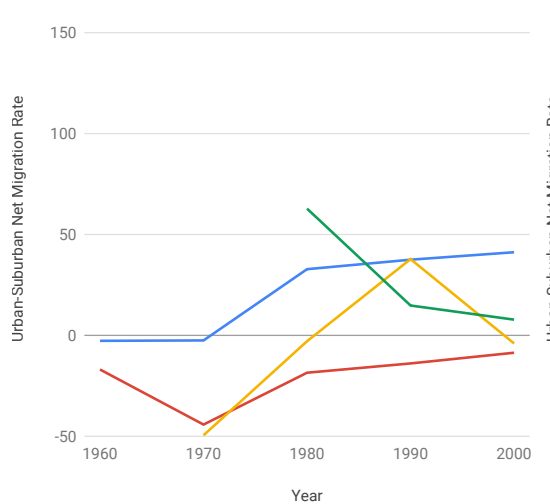

Year

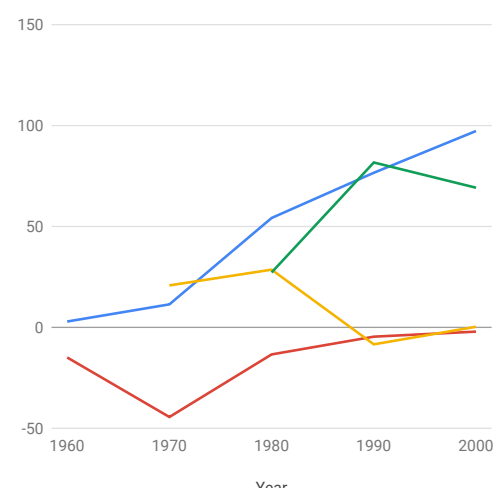

Year
High-Income Persons by Race

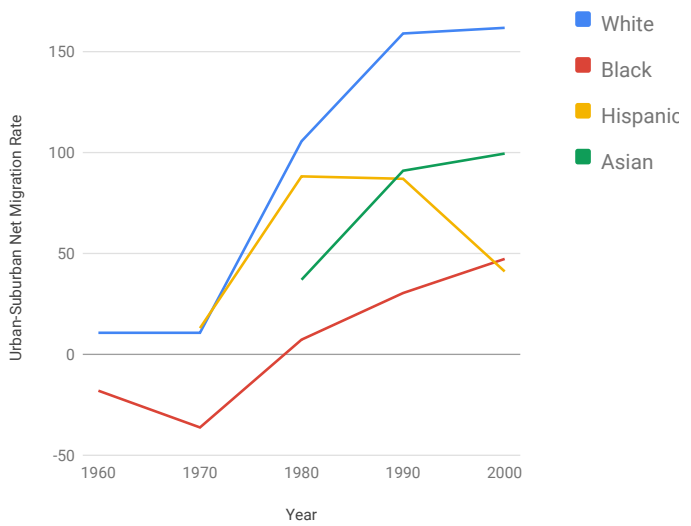


Urban and Suburban Residence, 1955-1995, and 5-year migration

\section{Miami, FL}

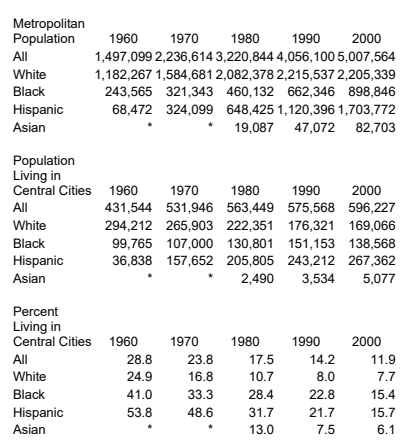

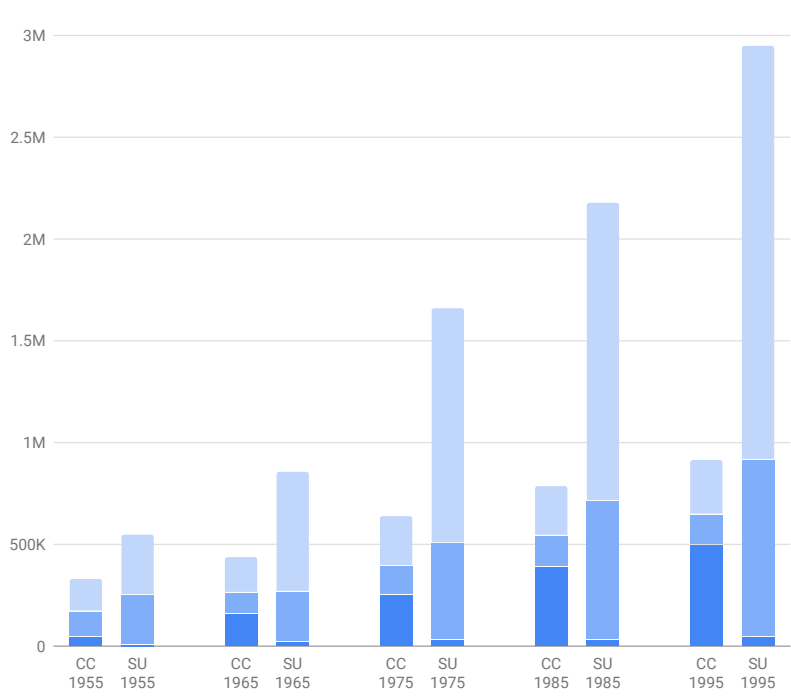

Central City (CC)/Suburban (SU) Residence in Year Migrants from central city to suburbs or
suburbs to central city in next five years

Migrants within central city or within

suburban area in next five years

Nonmovers (lived in the same house five

years later)

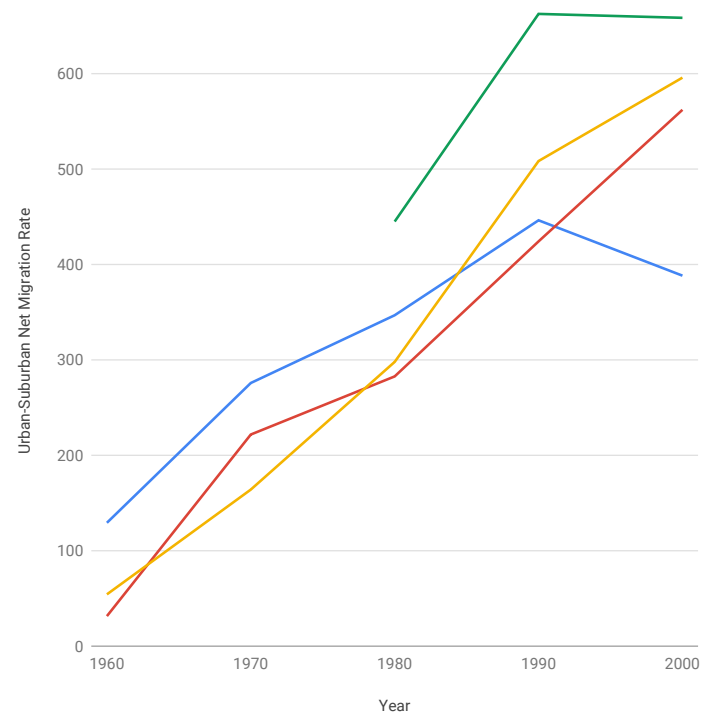

Low-Income Persons by Race
Middle-Income Persons by Race

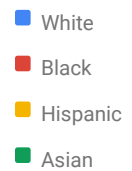

- Asian

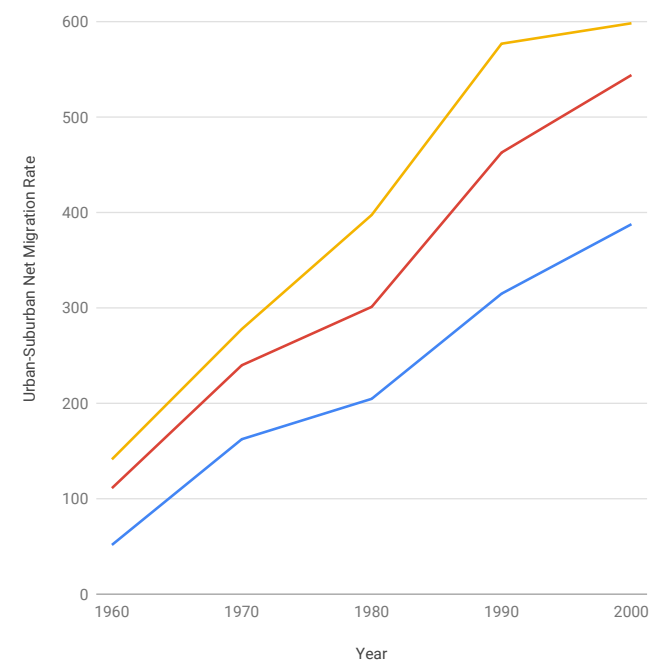

High-Income Persons by Race
- Low Income

- Middle Income

- High Income
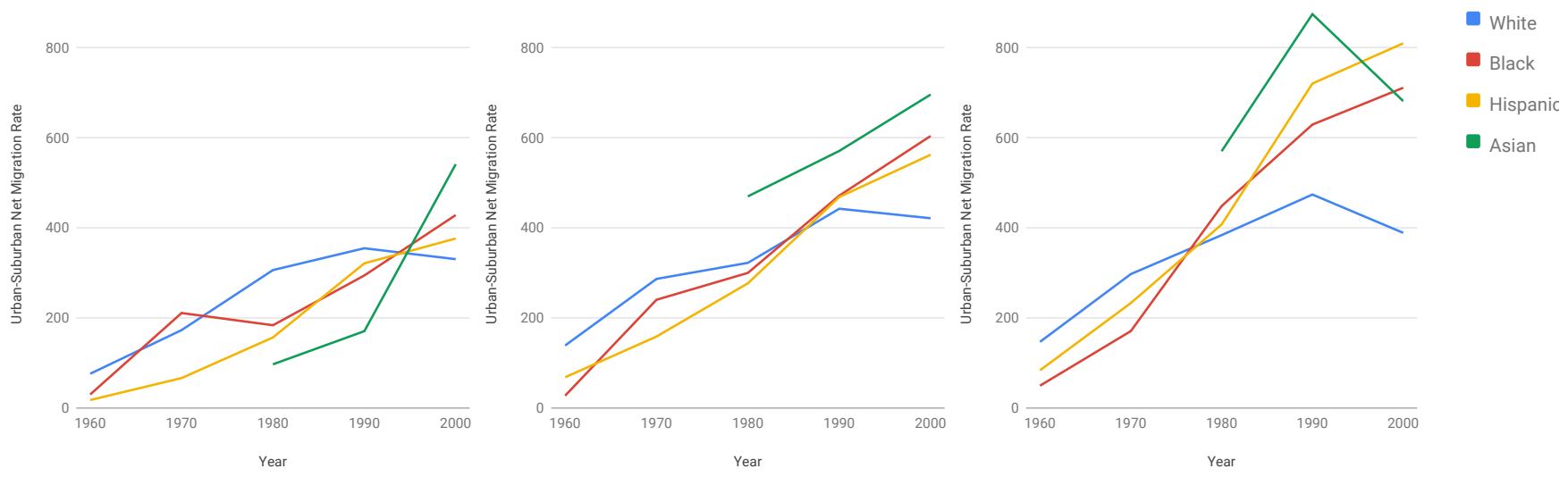
Urban and Suburban Residence, 1955-1995, and 5-year migration

\section{Milwaukee, WI}

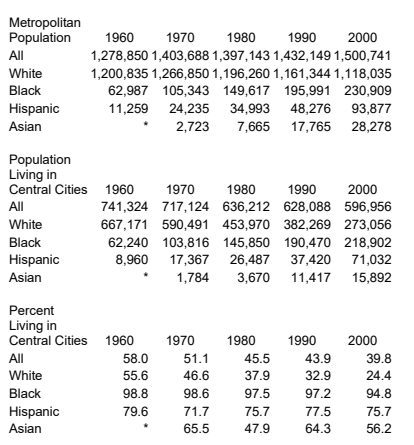

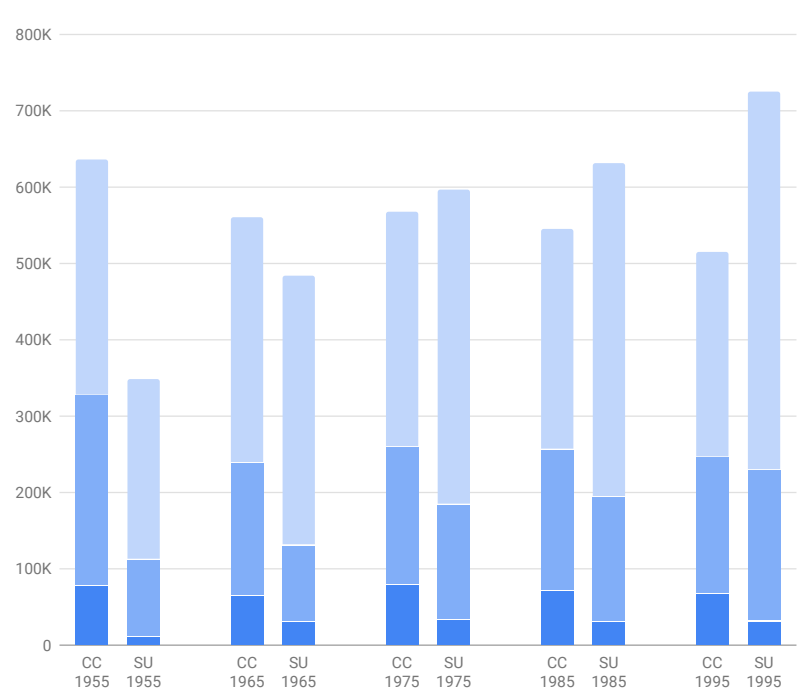

Migrants from central city to suburbs or suburbs to central city in next five years

Migrants within central city or within - suburban area in next five years

Nonmovers (lived in the same house five years later)
Urban-Suburban Net Migration Rate by Race

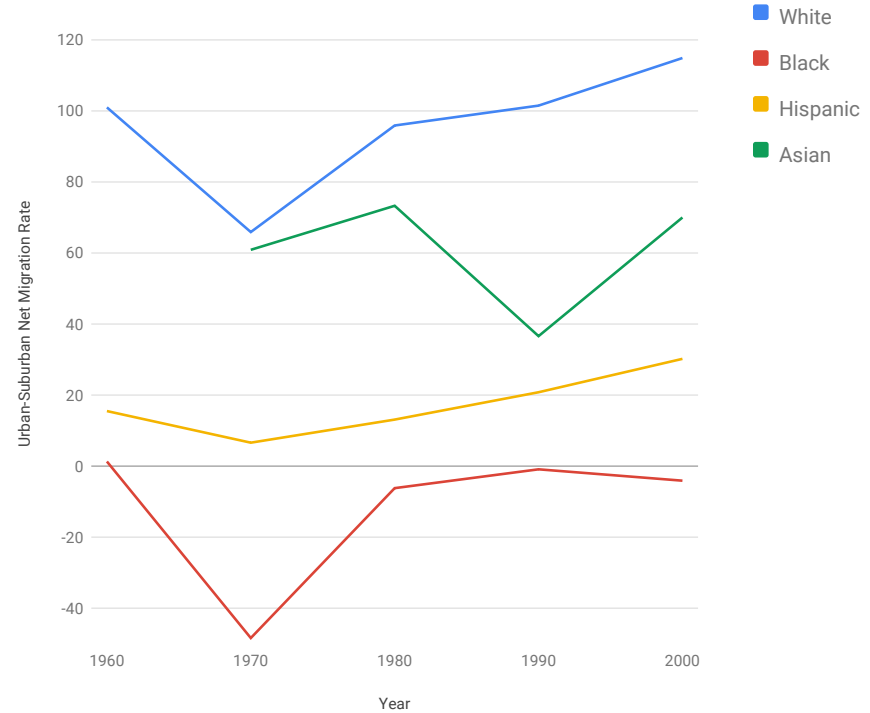

Low-Income Persons by Race

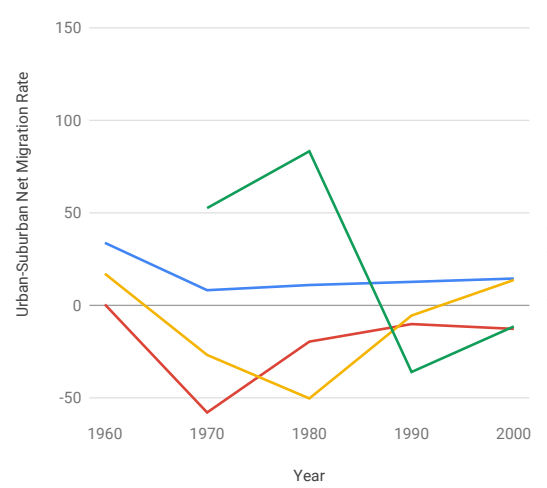

Year
Urban-Suburban Net Migration Rate by Income

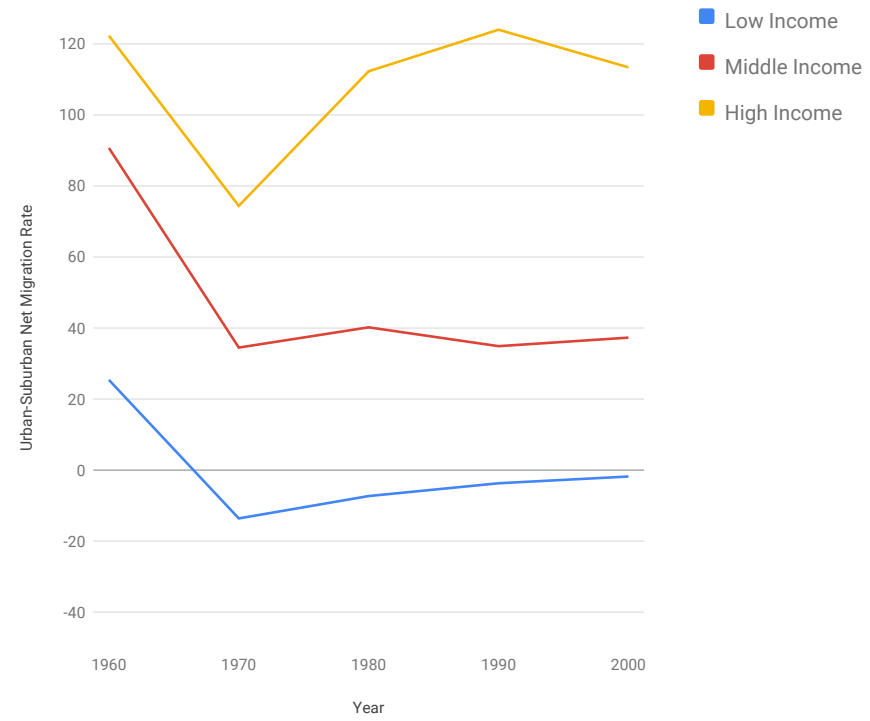

High-Income Persons by Race

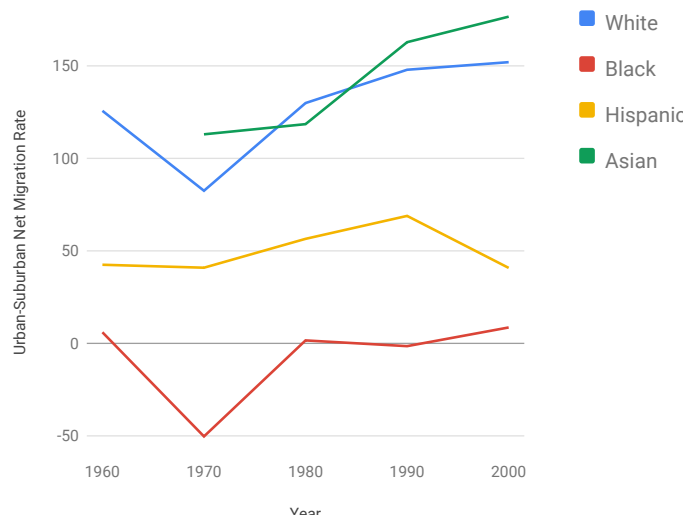


Urban and Suburban Residence, 1955-1995, and 5-year migration

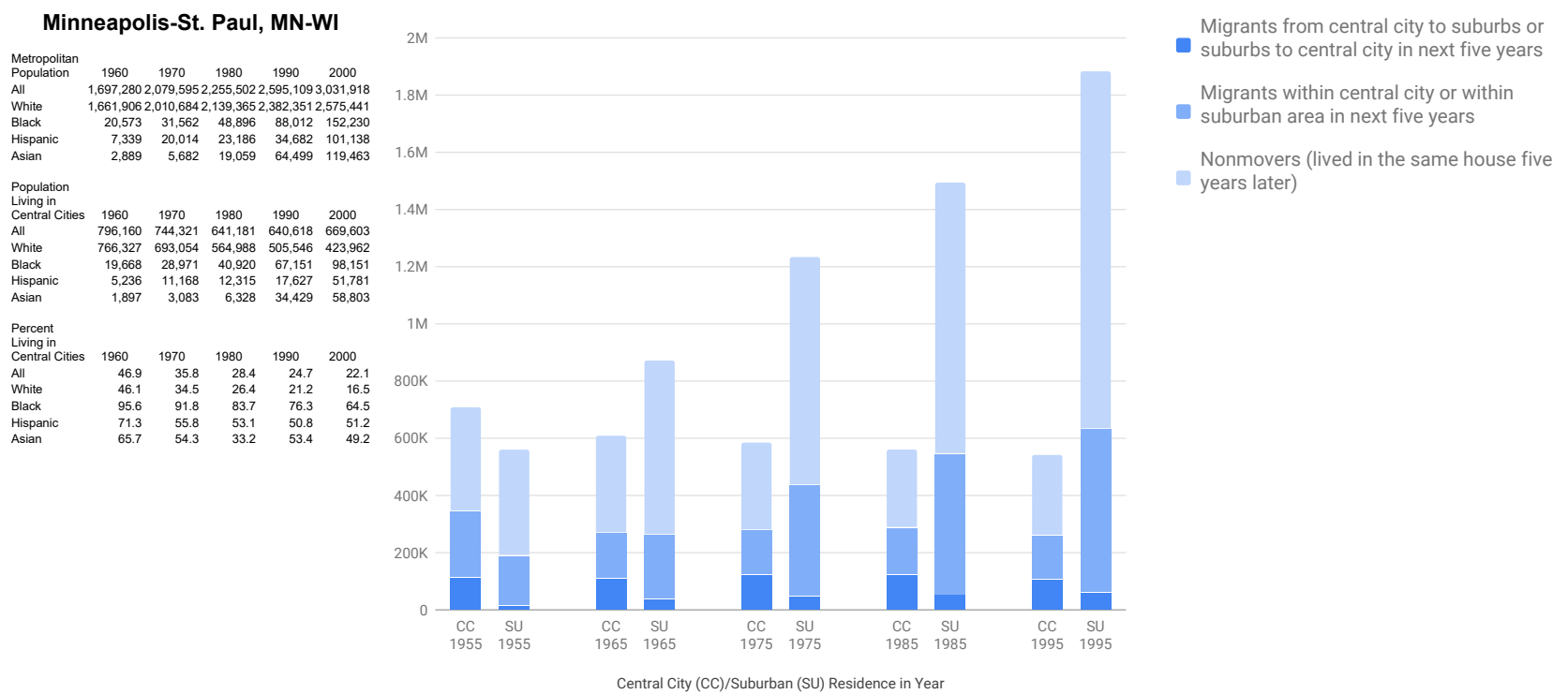

Urban-Suburban Net Migration Rate by Race

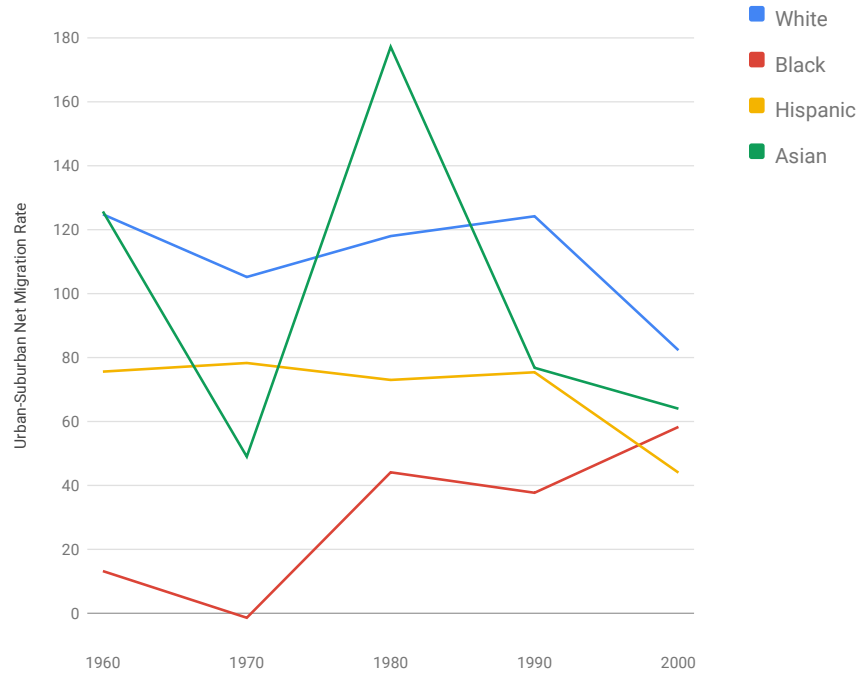

Year

Low-Income Persons by Race

Middle-Income Persons by Race
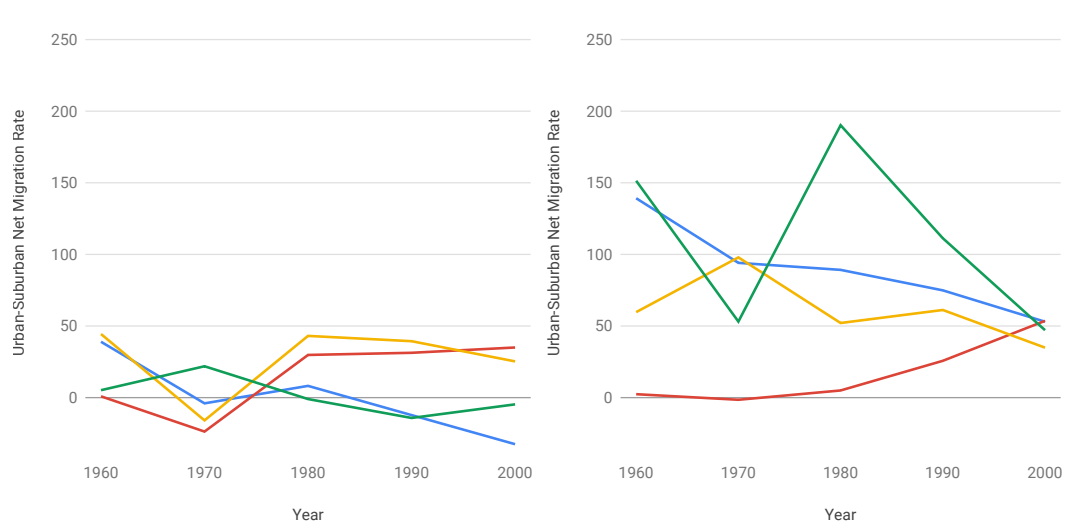

Year
Urban-Suburban Net Migration Rate by Income

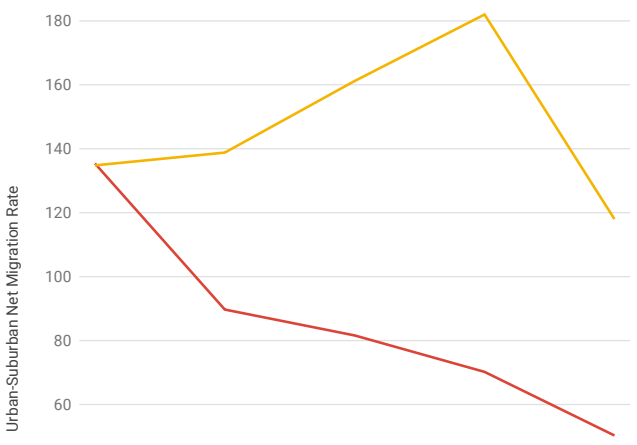

Low Income

Middle Income

- High Income

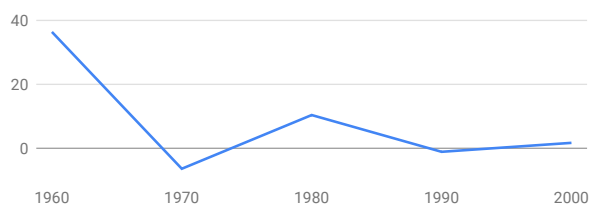

Year

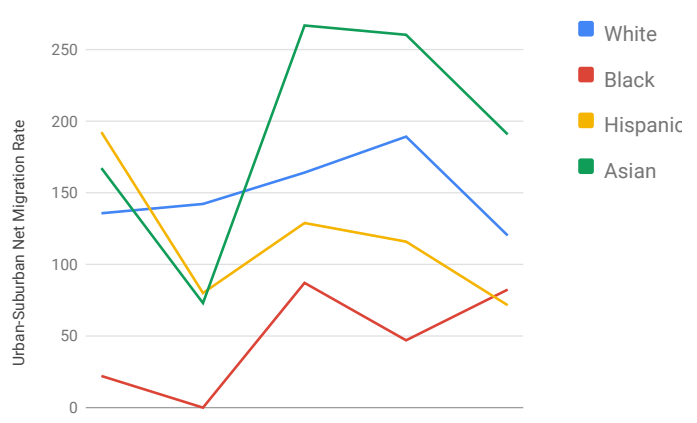


Urban and Suburban Residence, 1955-1995, and 5-year migration

$700 \mathrm{~K} \quad \begin{aligned} & \text { Migrants from central city to suburbs or } \\ & \text { suburbs to central city in next five years }\end{aligned}$

\section{Nashville, TN}

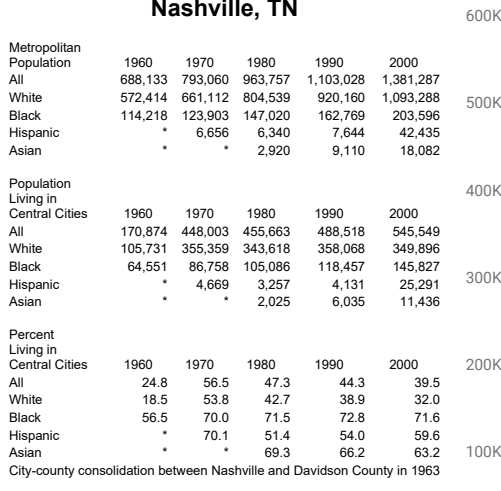

Urban-Suburban Net Migration Rate by Race

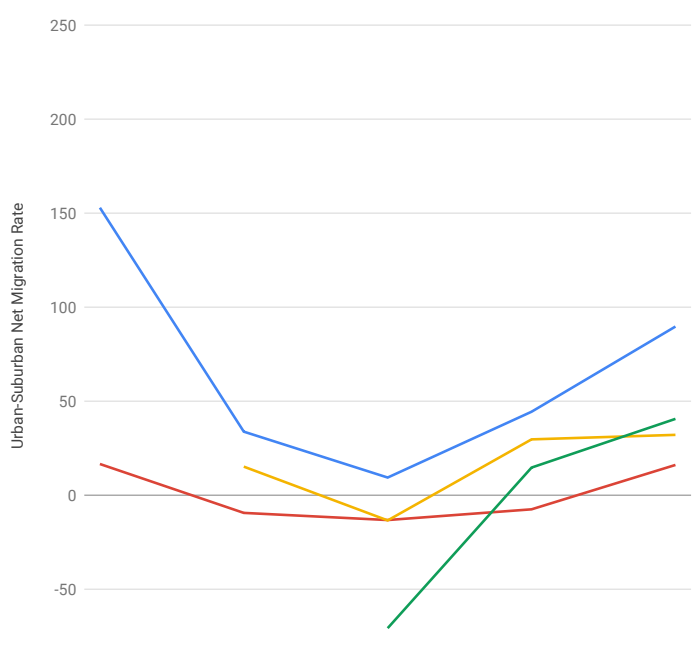

1960

1980

Low-Income Persons by Race

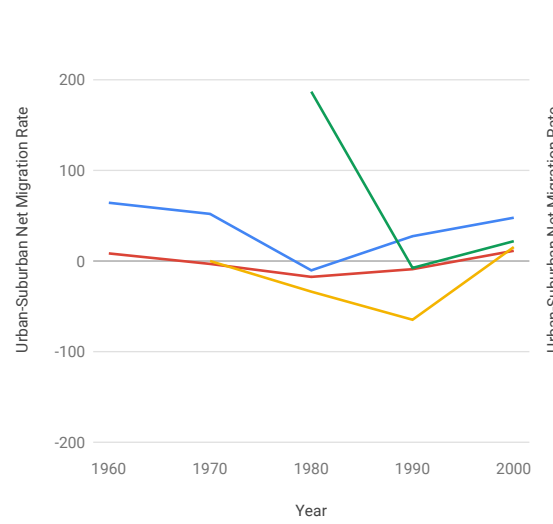

Year
Middle-Income Persons by Race

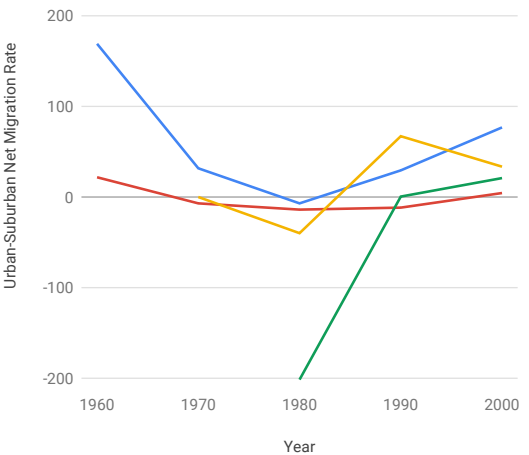

Year
Urban-Suburban Net Migration Rate by Income
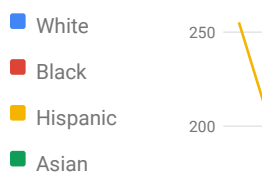

Migrants within central city or within

- suburban area in next five years

Nonmovers (lived in the same house five years later)

Central City (CC)/Suburban (SU) Residence in Year
- Low Income

- Middle Income

High Income

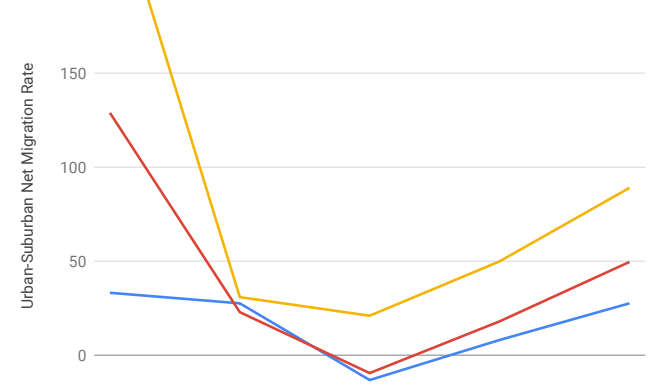

$-50$

$\begin{array}{lllll}1960 & 1970 & 1980 & 1990 & 2000\end{array}$

Year

High-Income Persons by Race

White

Black

Hispanic

- Asian

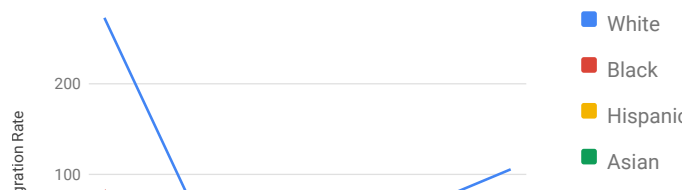

$-200$

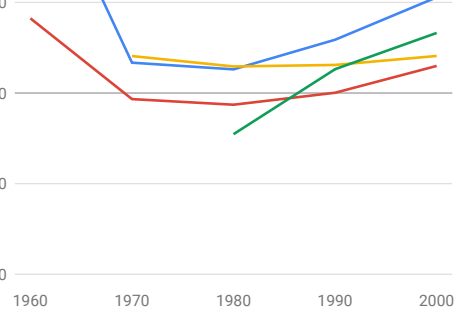

Year 
Urban and Suburban Residence, 1955-1995, and 5-year migration

$600 \mathrm{~K}$

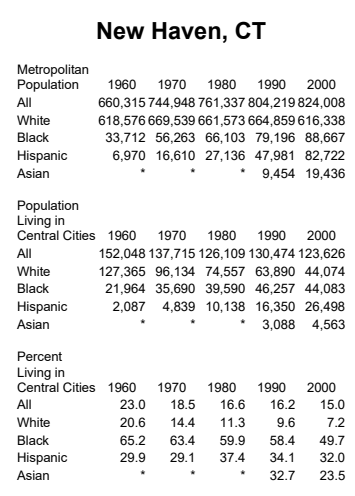

Migrants from central city to suburbs or - suburbs to central city in next five years Migrants within central city or within - suburban area in next five years

Nonmovers (lived in the same house five years later)
Urban-Suburban Net Migration Rate by Race

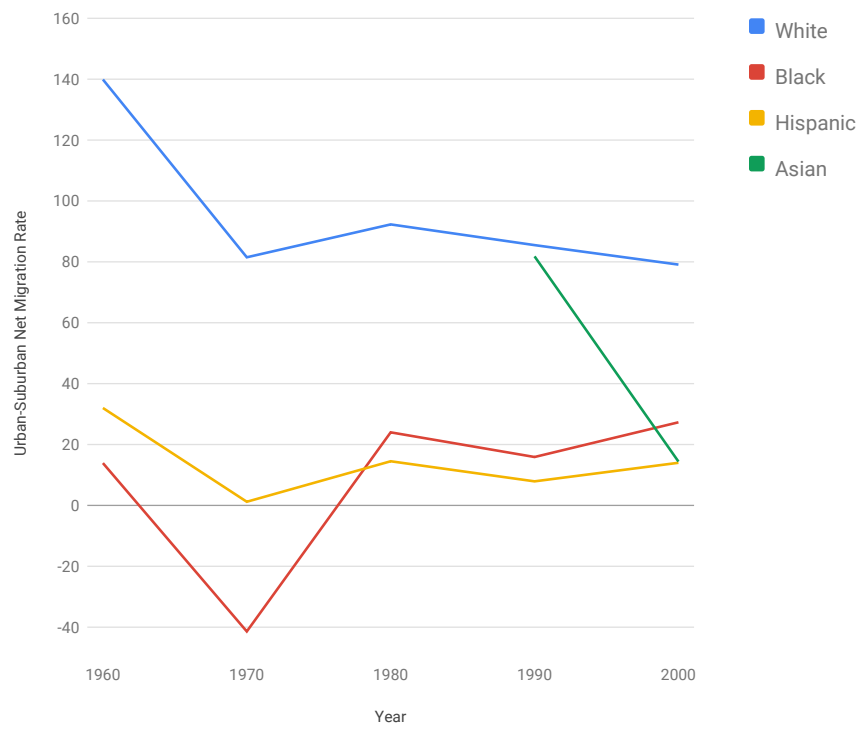

Low-Income Persons by Race

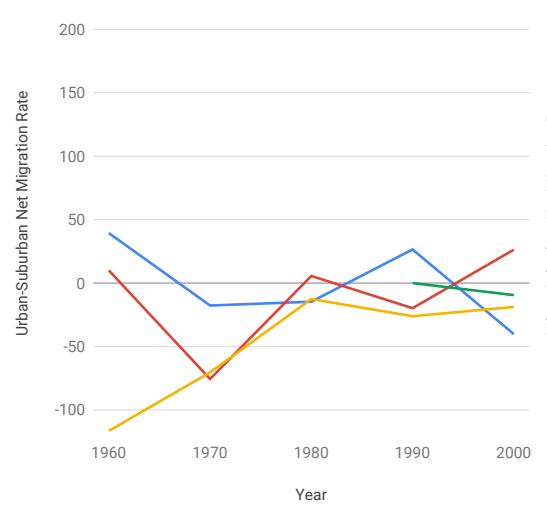

Middle-Income Persons by Race
Urban-Suburban Net Migration Rate by Income

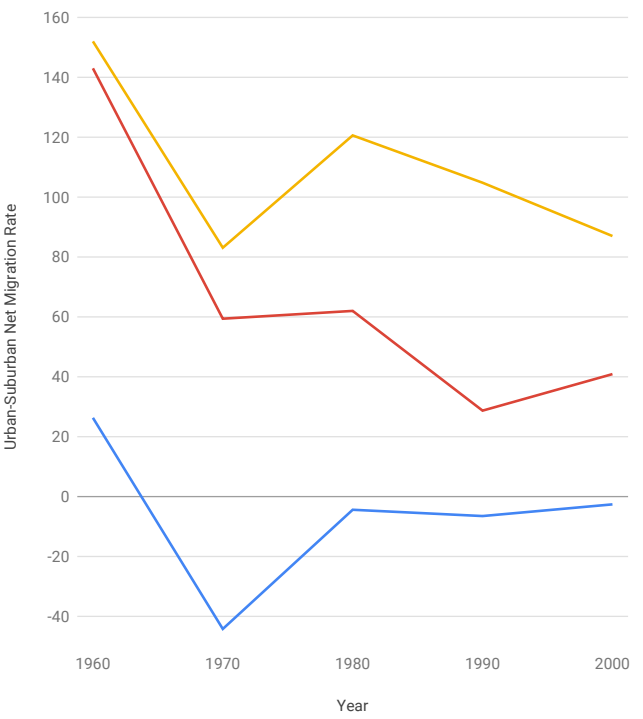

- Low Income

- Middle Income

High Income

High-Income Persons by Race
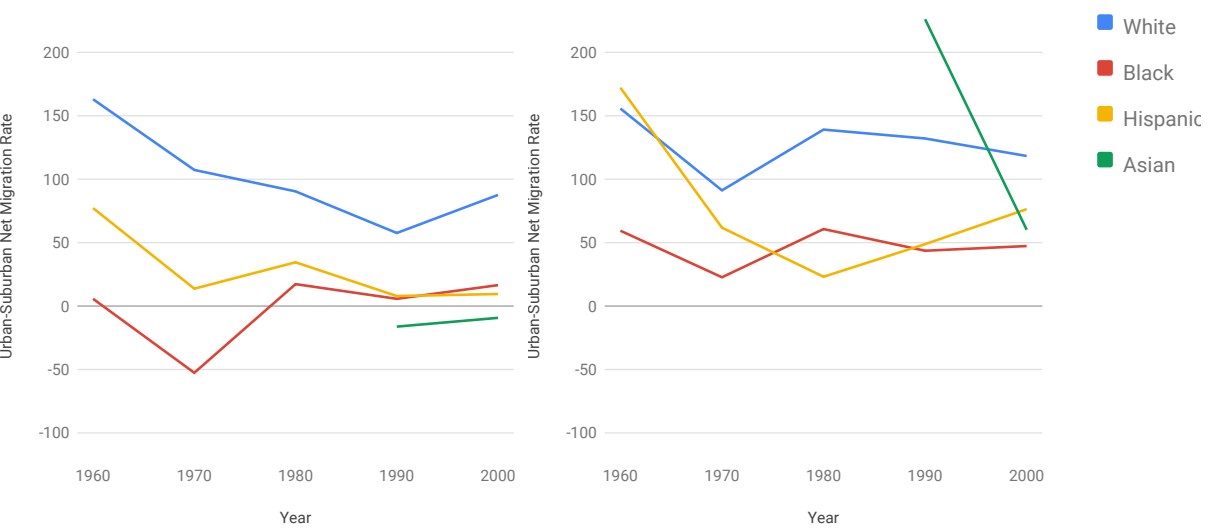
Urban and Suburban Residence, 1955-1995, and 5-year migration

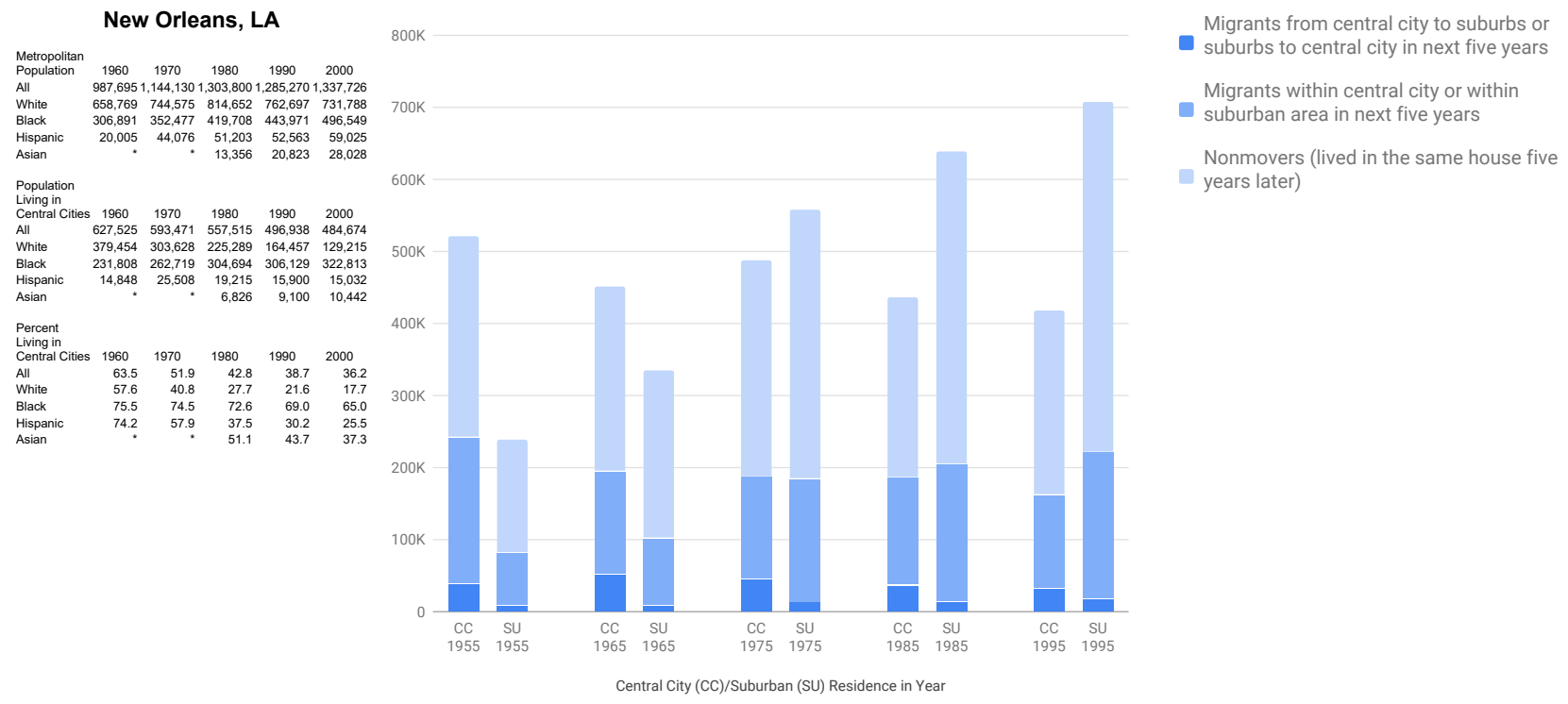

Urban-Suburban Net Migration Rate by Race

Urban-Suburban Net Migration Rate by Income

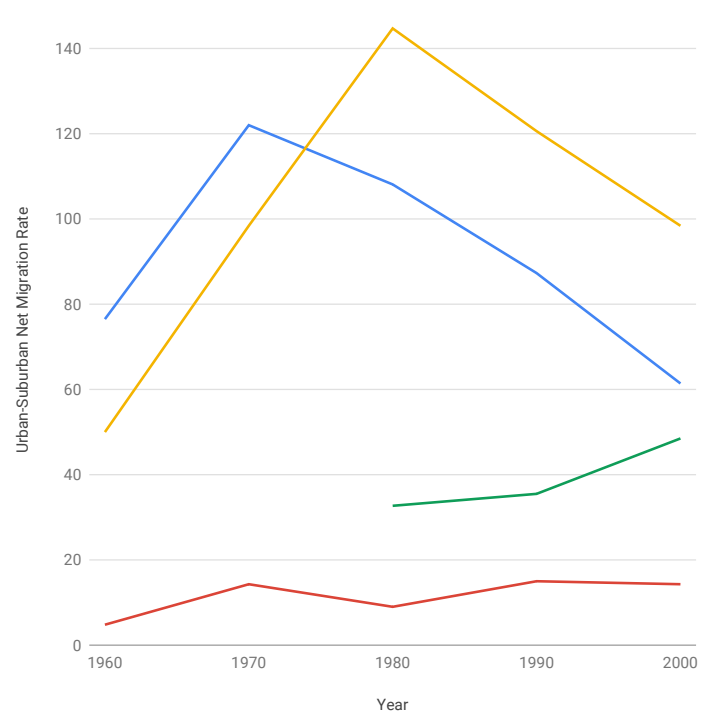

Low-Income Persons by Race
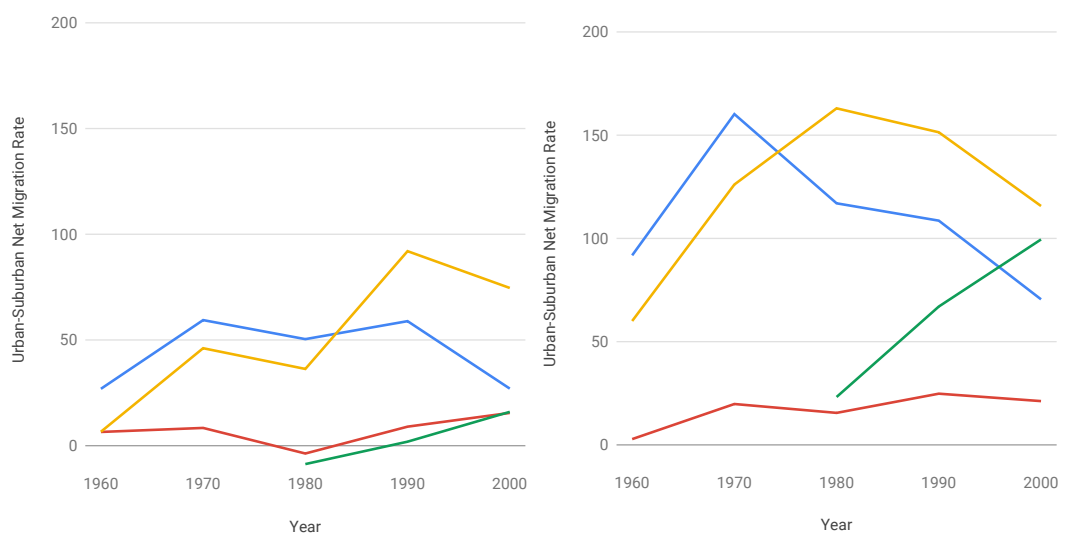

Middle-Income Persons by Race

White

Black

Hispanic

- Asian

120
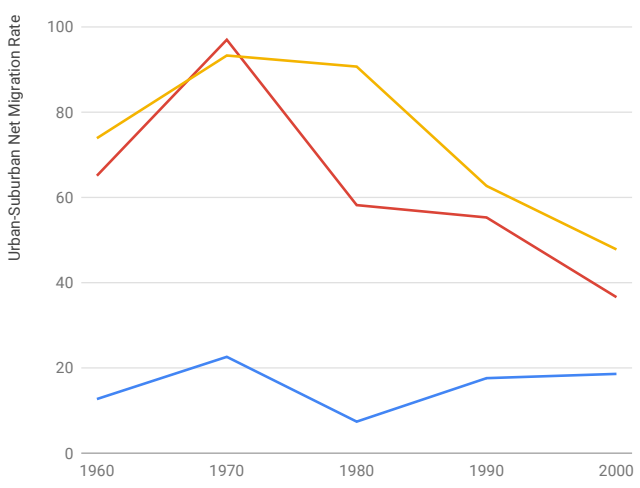

Year

High-Income Persons by Race
- Low Income

- Middle Income

- High Income

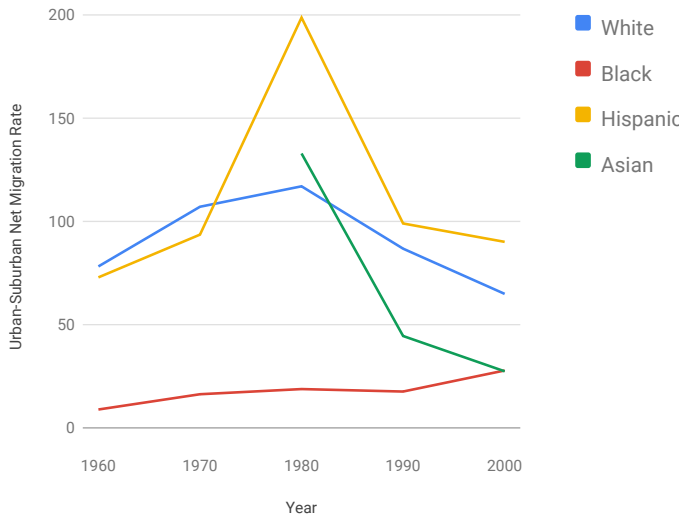


Urban and Suburban Residence, 1955-1995, and 5-year migration

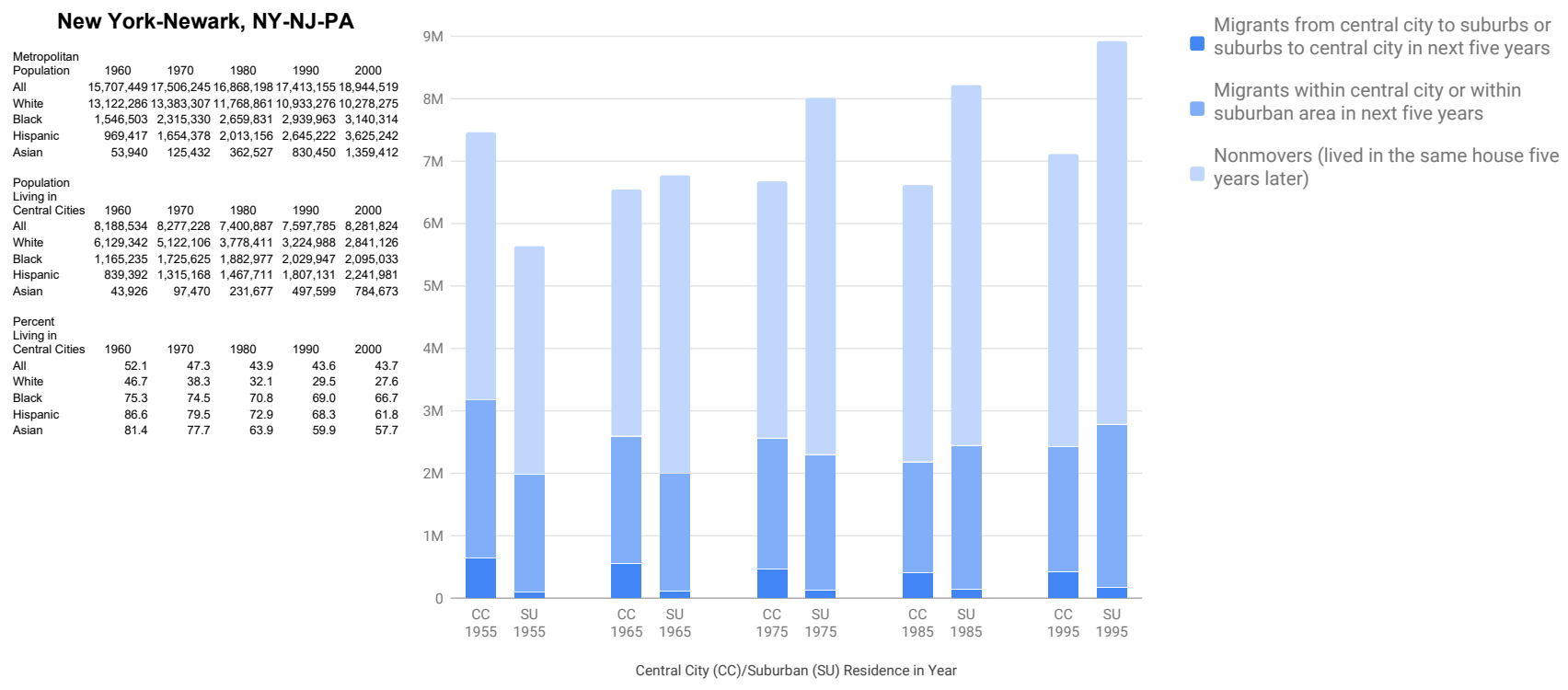

Urban-Suburban Net Migration Rate by Race

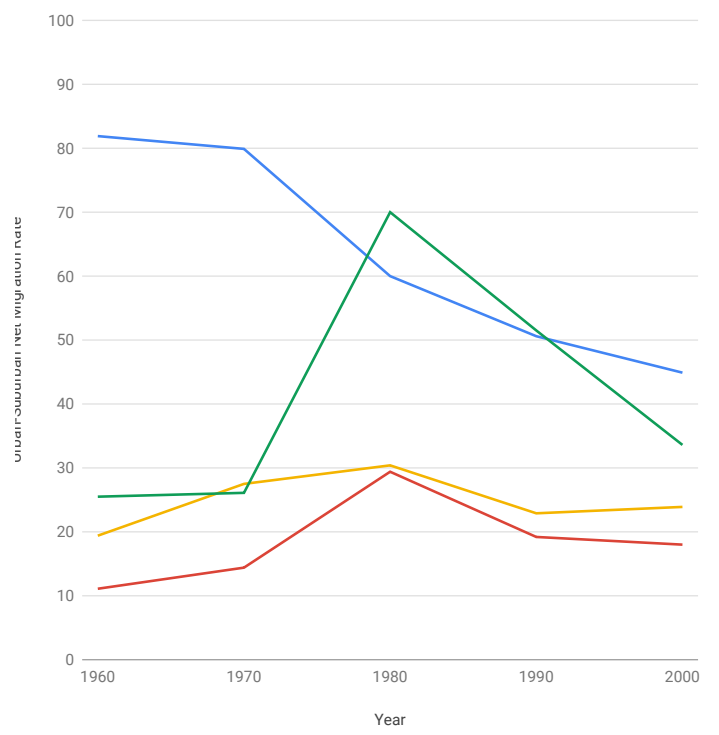

Low-Income Persons by Race

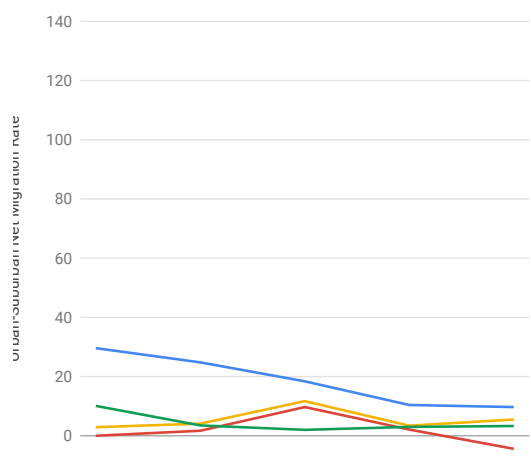

1960
Middle-Income Persons by Race

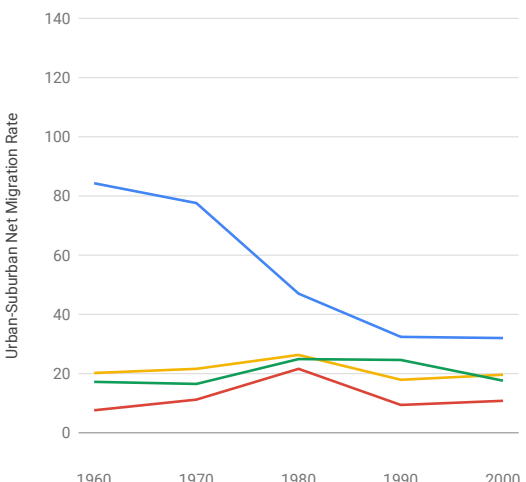

Urban-Suburban Net Migration Rate by Income
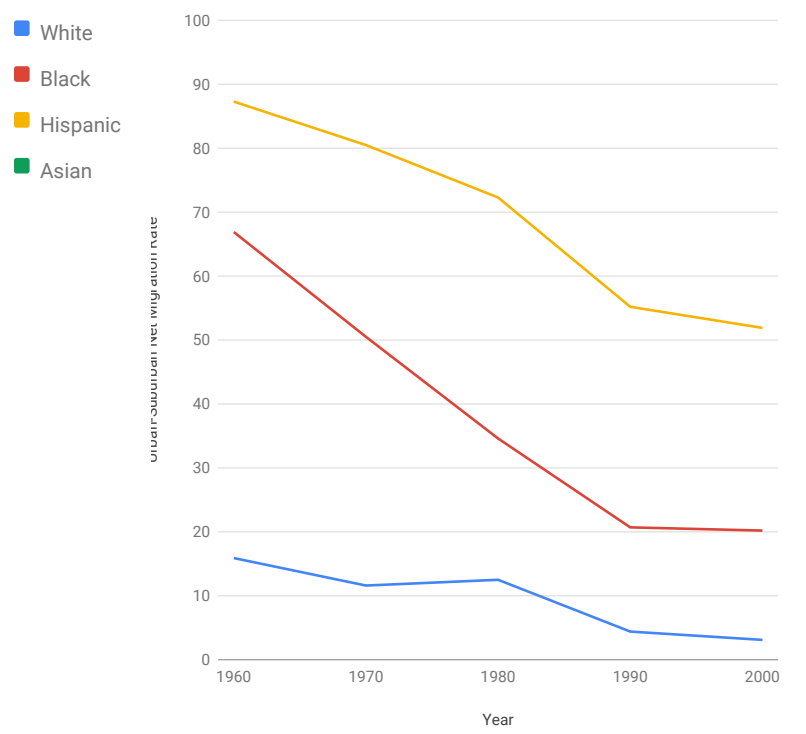

- Low Income

- Middle Income

High Income

High-Income Persons by Race

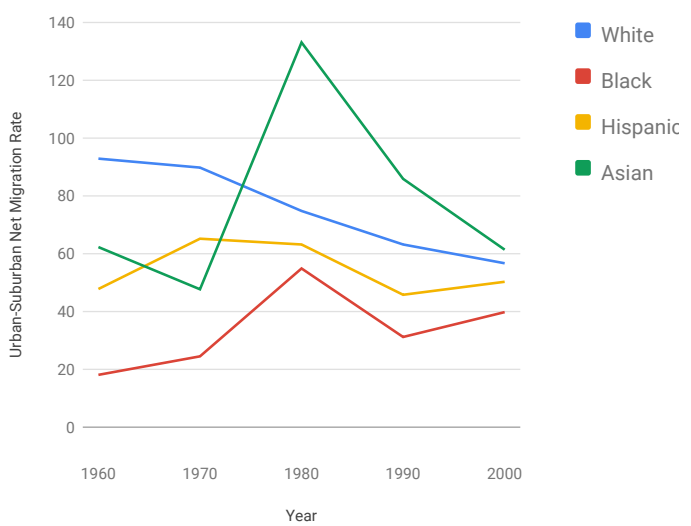


Urban and Suburban Residence, 1955-1995, and 5-year migration

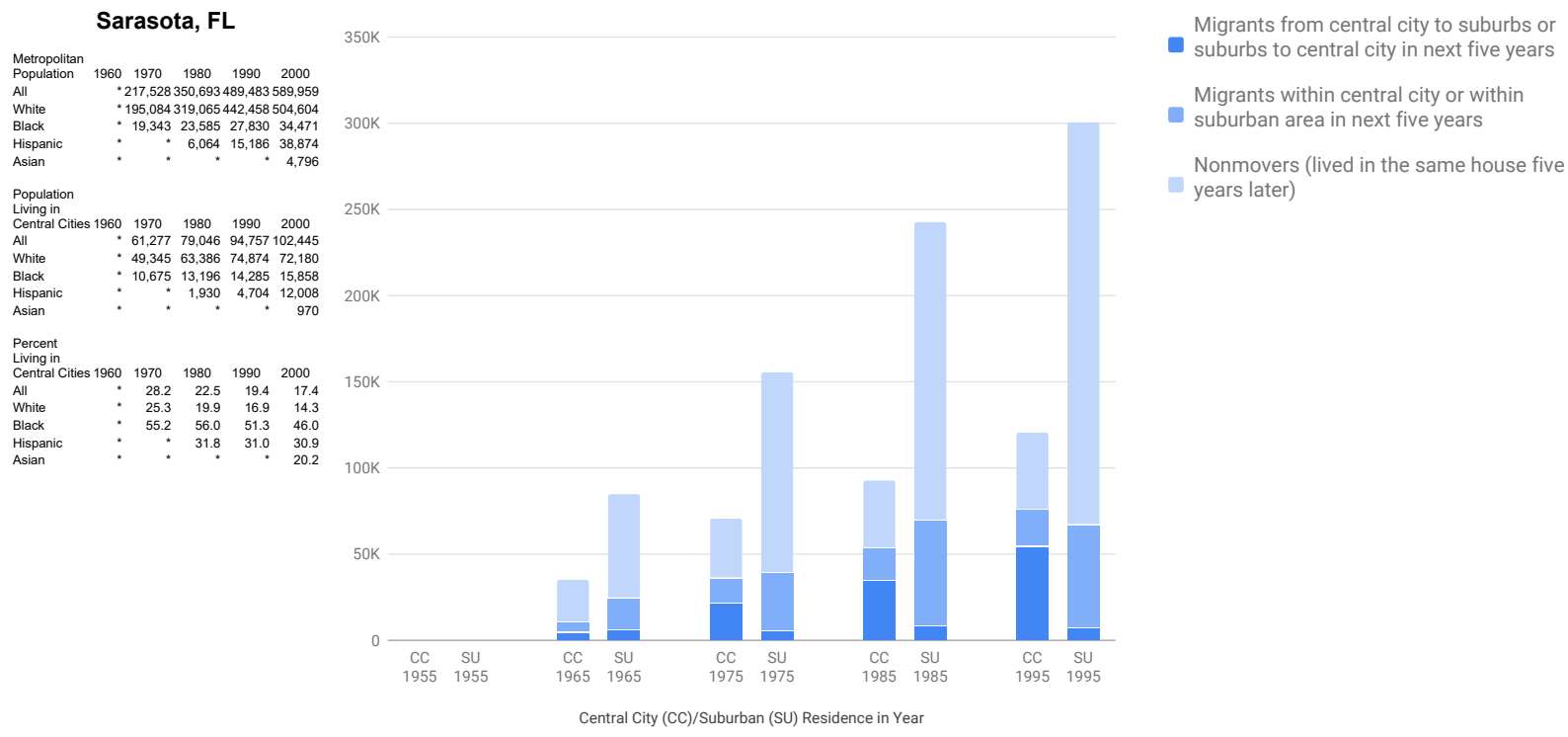

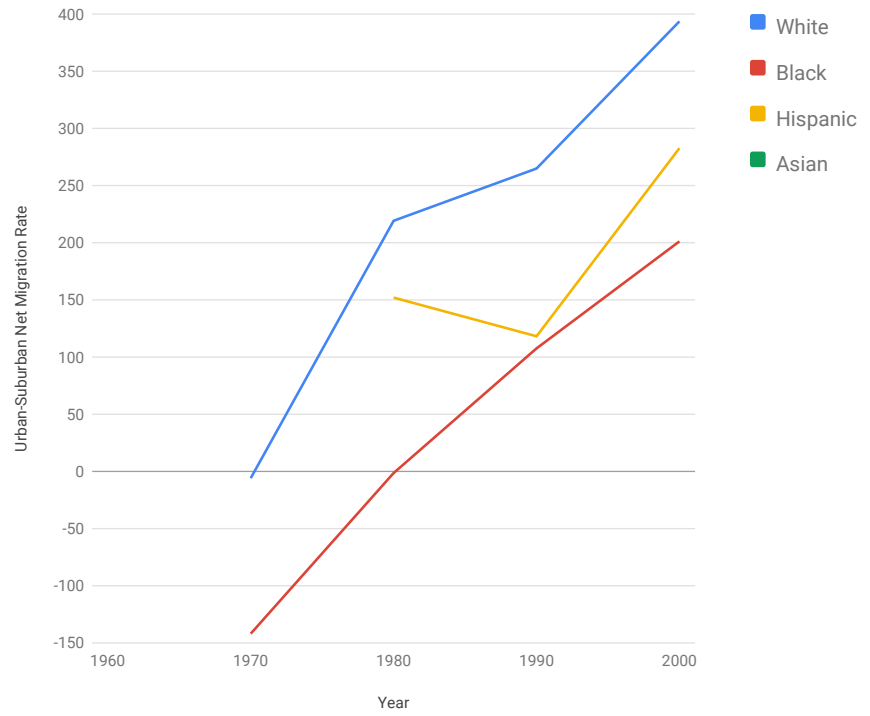

Low-Income Persons by Race
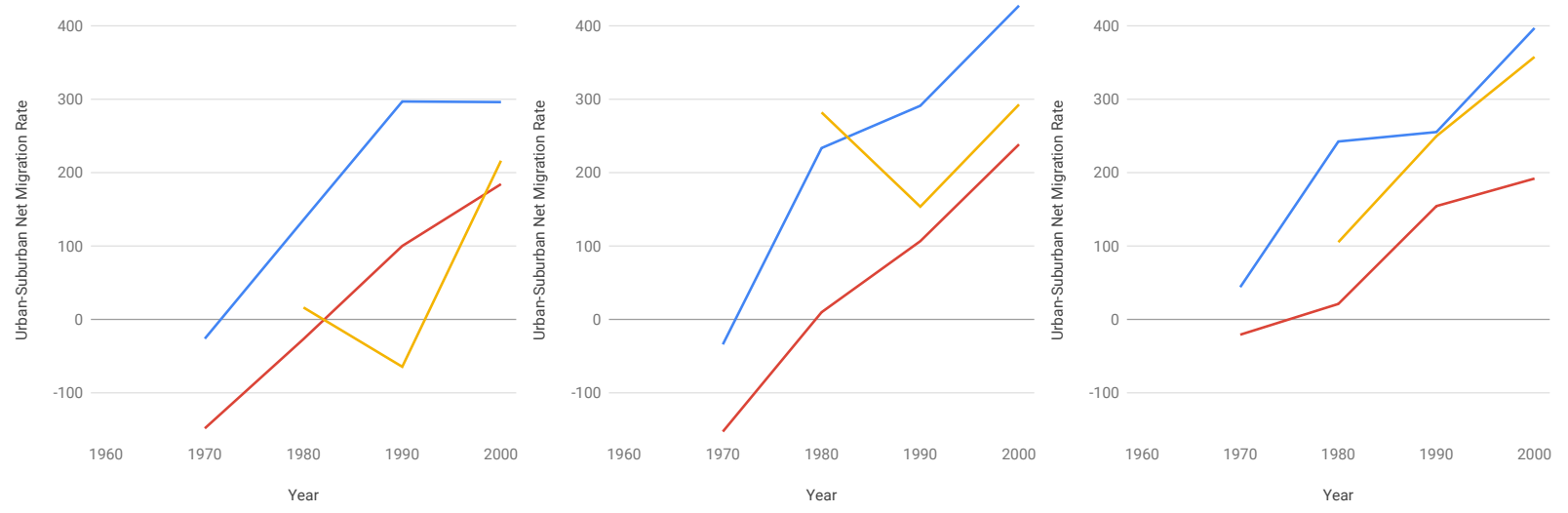

ear
Low Income

Middle Income

High Income 
Urban and Suburban Residence, 1955-1995, and 5-year migration

300k Migrants from central city to suburbs or suburbs to central city in next five years

Migrants within central city or within suburban area in next five years

Nonmovers (lived in the same house five
years later)

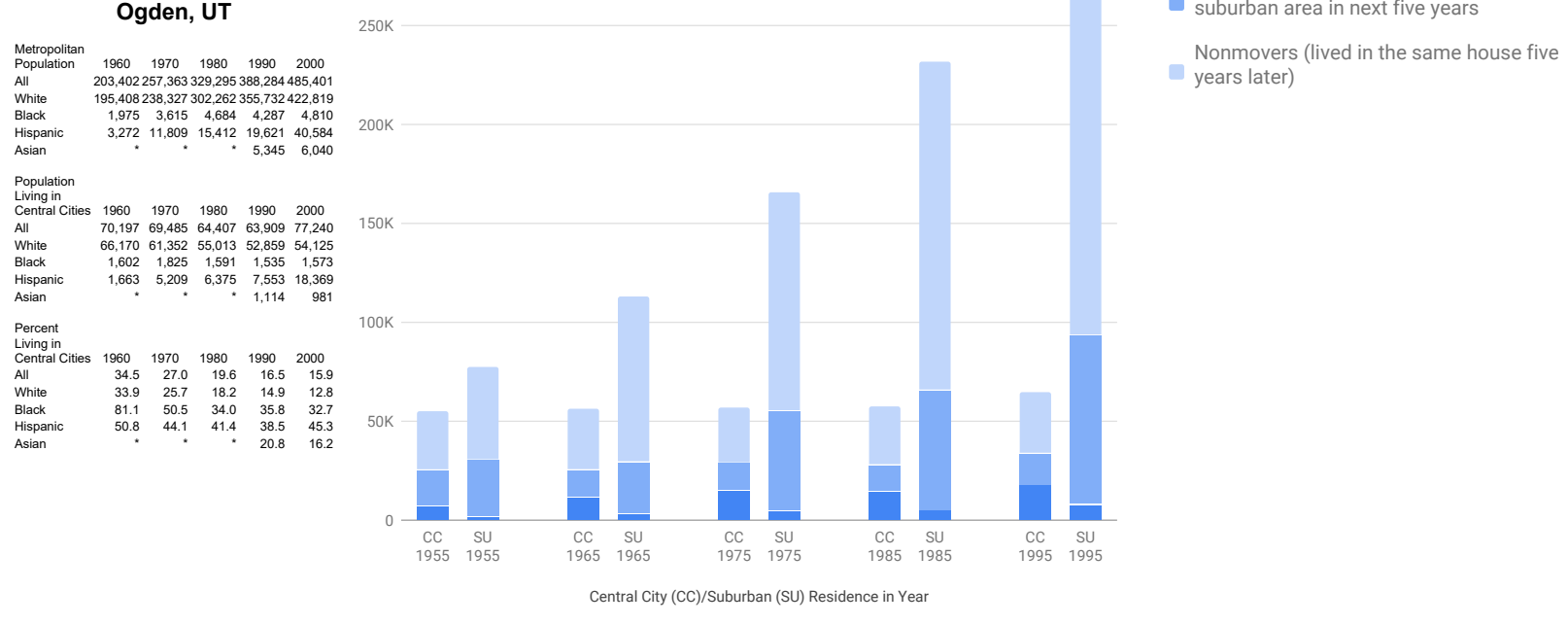

Urban-Suburban Net Migration Rate by Race

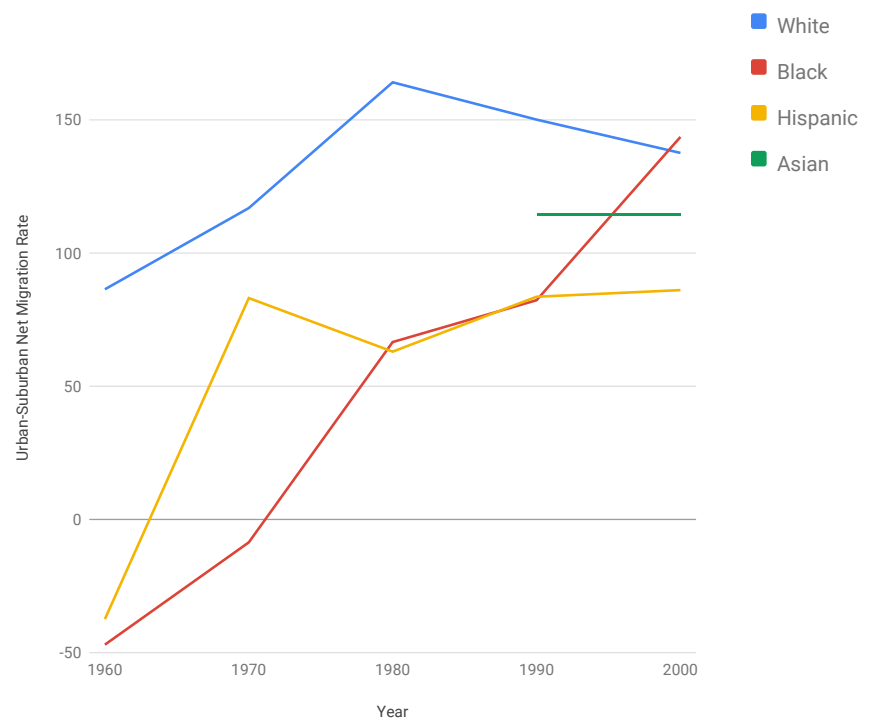

Low-Income Persons by Race

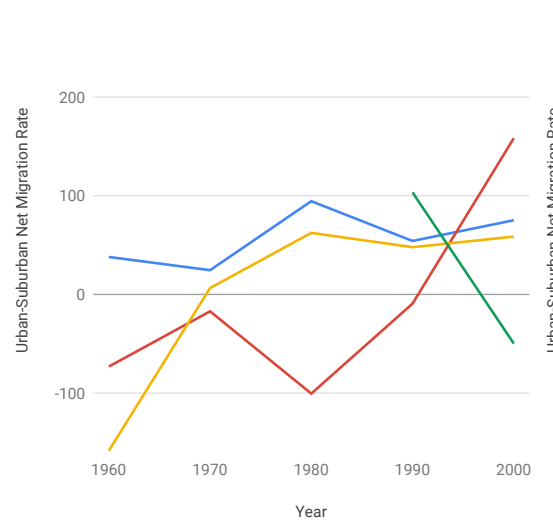

Year
Middle-Income Persons by Race

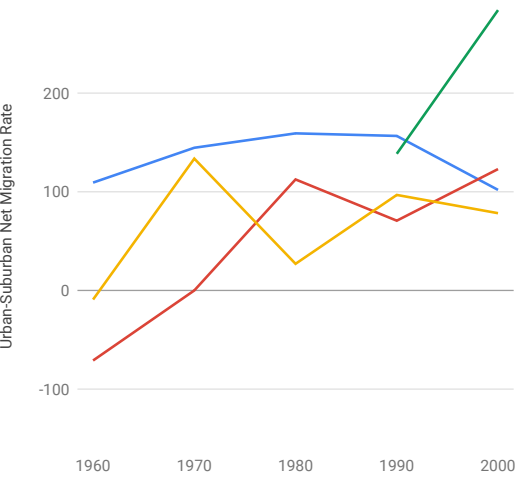

Year
Urban-Suburban Net Migration Rate by Income

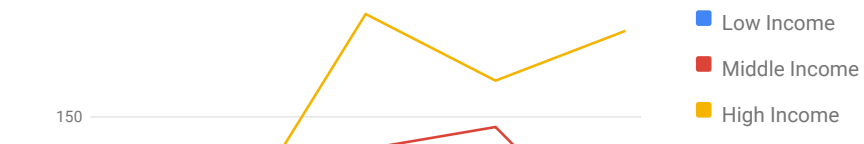

High-Income Persons by Race

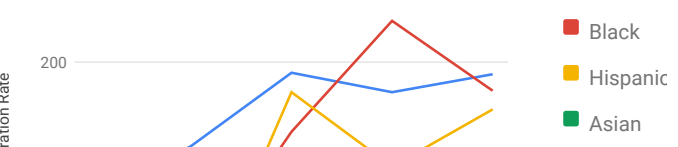


Urban and Suburban Residence, 1955-1995, and 5-year migration

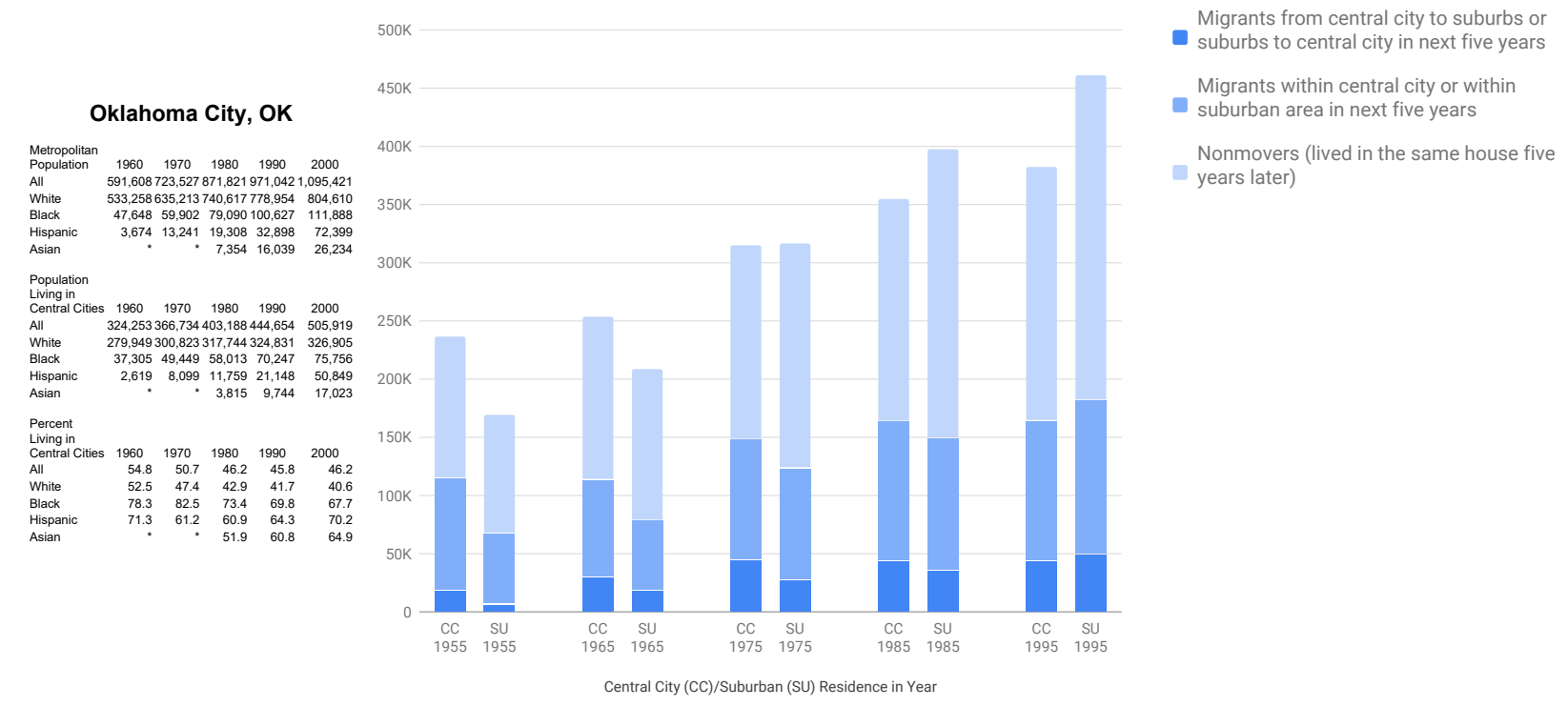

Urban-Suburban Net Migration Rate by Race
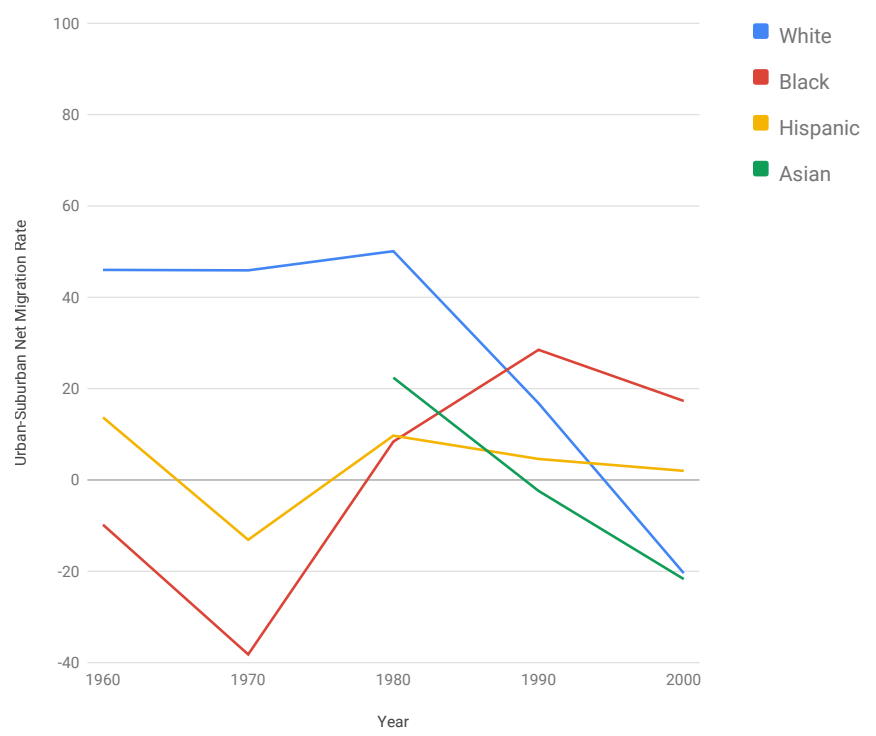

Low-Income Persons by Race

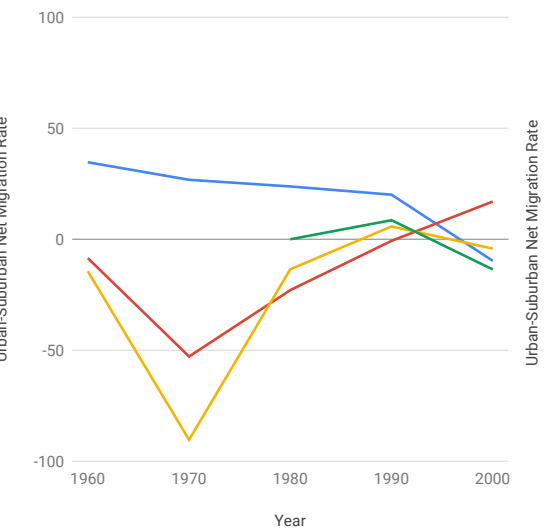

Year
Urban-Suburban Net Migration Rate by Income
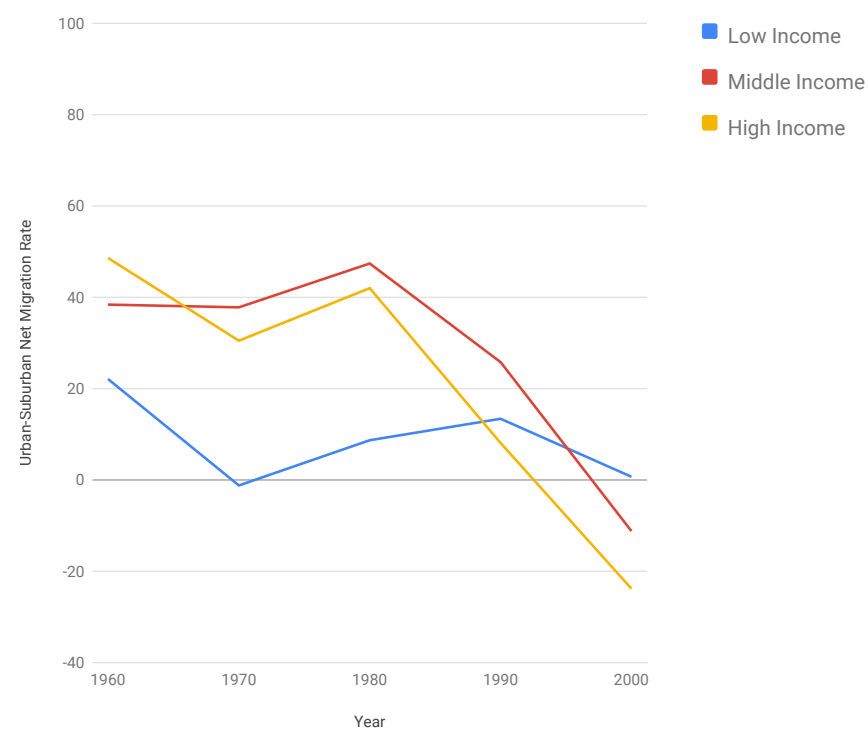

High-Income Persons by Race

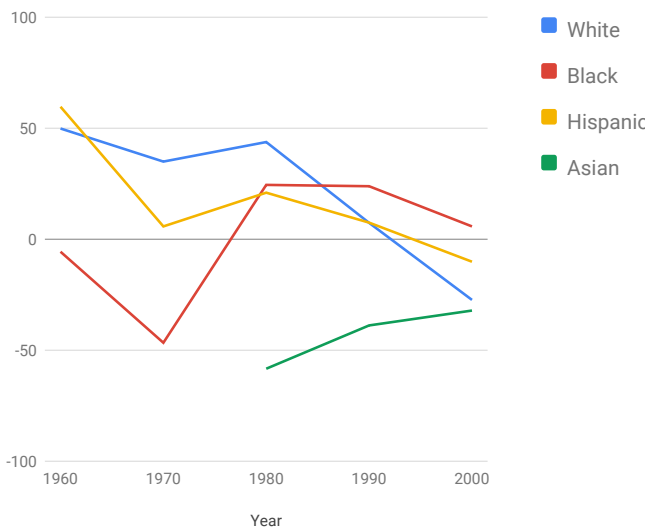

- High Income
100
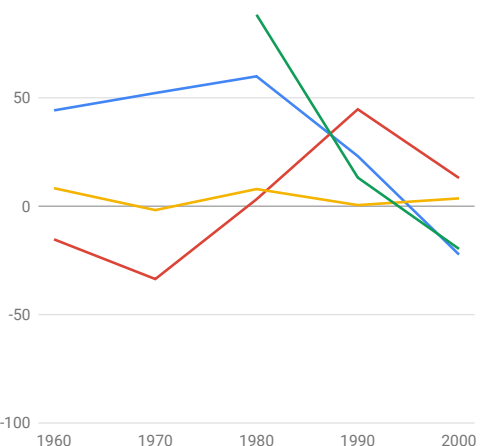
Urban and Suburban Residence, 1955-1995, and 5-year migration

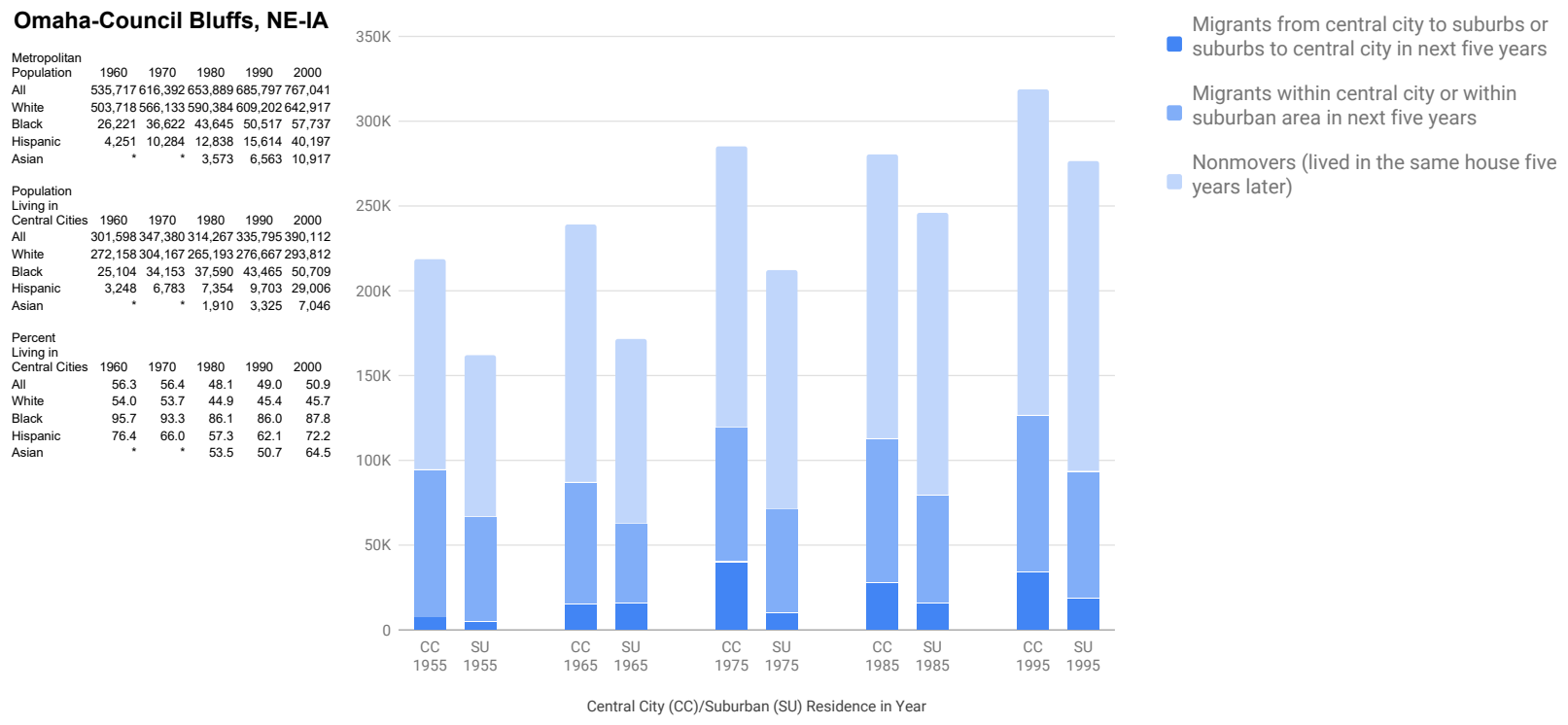

Urban-Suburban Net Migration Rate by Race

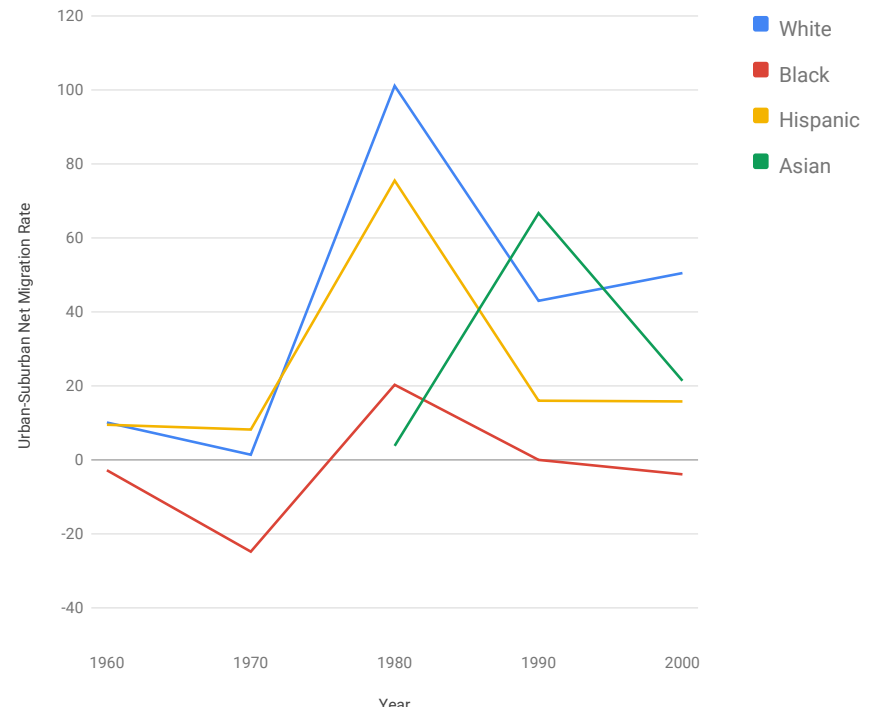

Low-Income Persons by Race
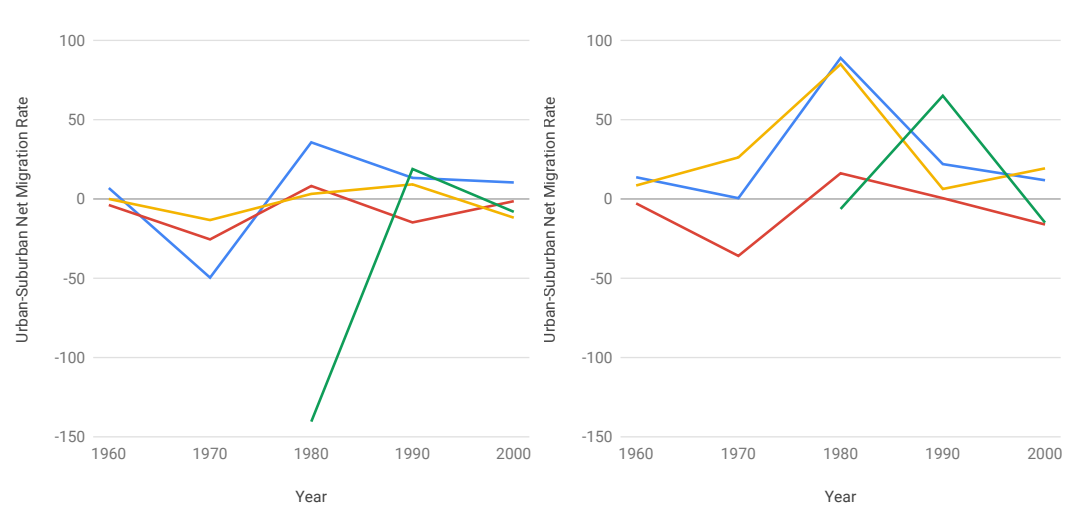

$\begin{array}{lllll}-150 & & & & \\ 1960 & 1970 & 1980 & 1990\end{array}$

Year
Urban-Suburban Net Migration Rate by Income

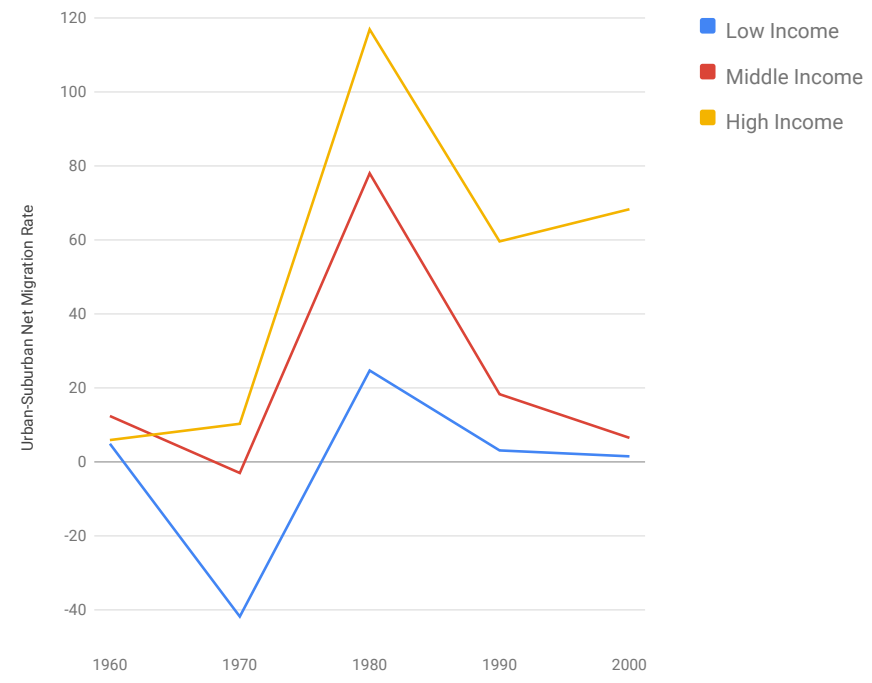

Year

High-Income Persons by Race

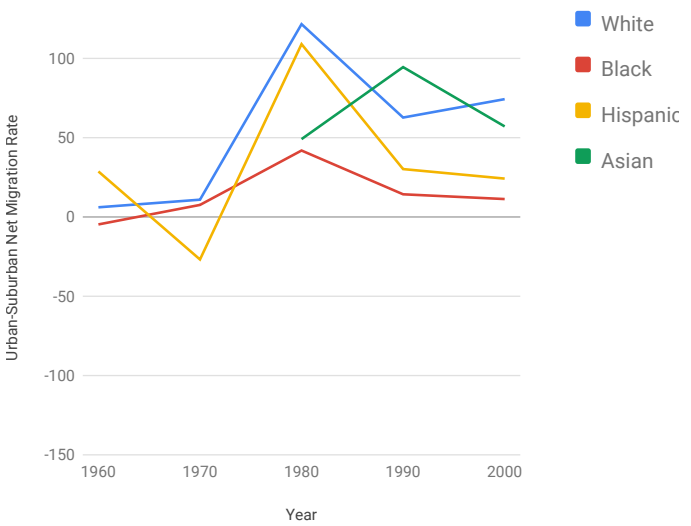


Urban and Suburban Residence, 1955-1995, and 5-year migration

\section{Orlando, FL}

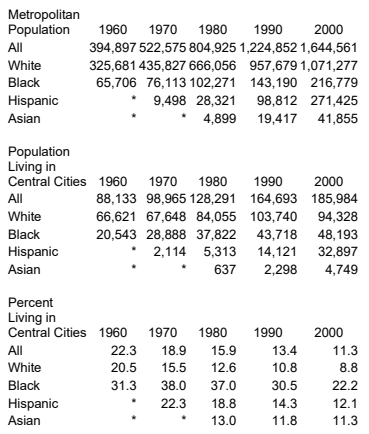

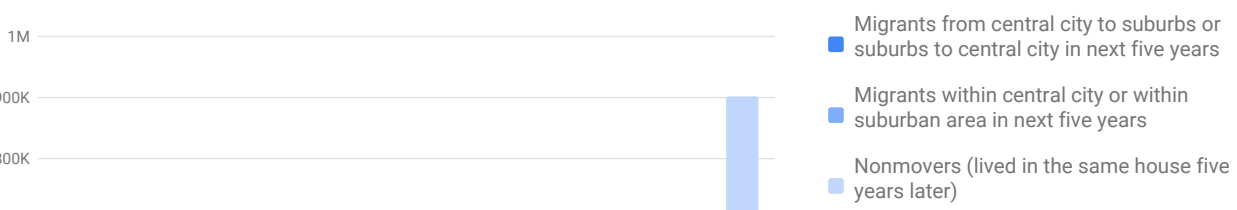

$1 \mathrm{M}$

$900 \mathrm{~K}$
Migrants from central city to suburbs or
- suburbs to central city in next five years
Migrants within central city or within
suburban area in next five years
Nonmovers (lived in the same house five
years later)

$800 \mathrm{~K}$

$700 \mathrm{~K}$

$600 \mathrm{~K}$

$500 \mathrm{~K}$

$400 \mathrm{~K}$

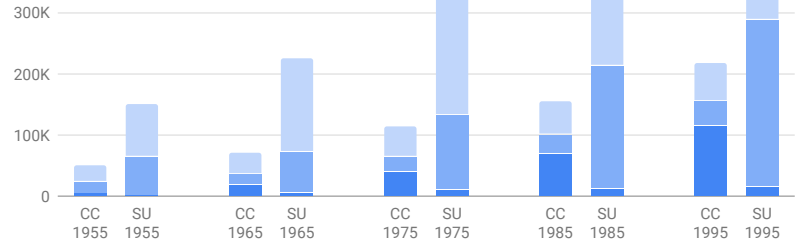

Urban-Suburban Net Migration Rate by Race

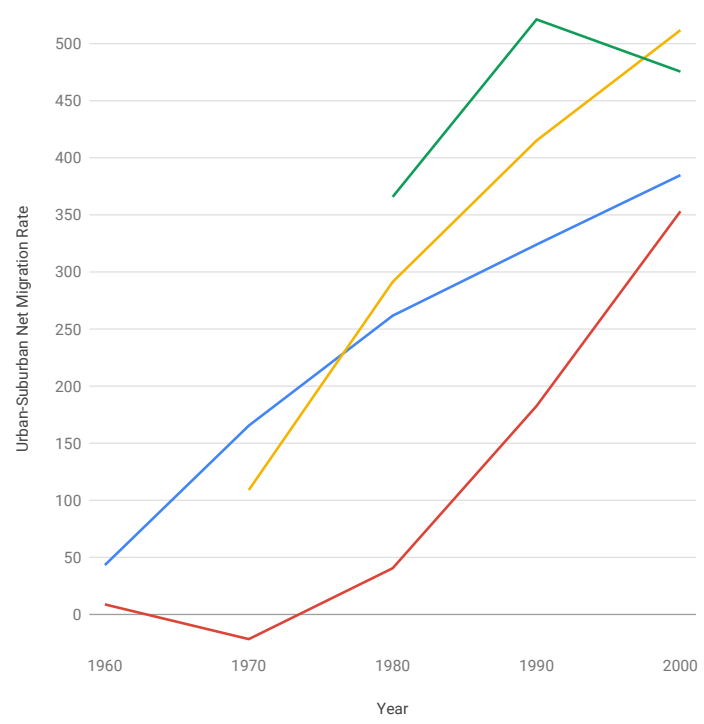

Low-Income Persons by Race
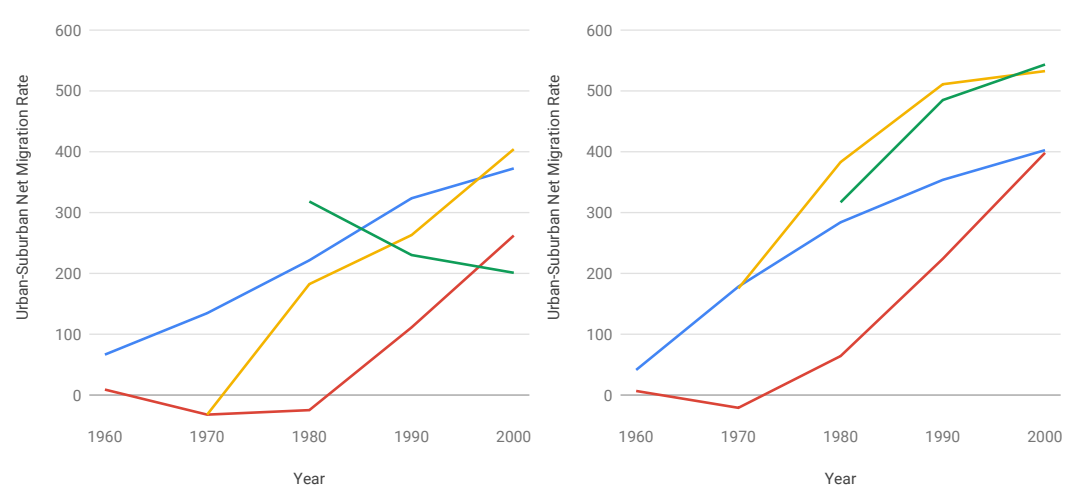

Urban-Suburban Net Migration Rate by Income
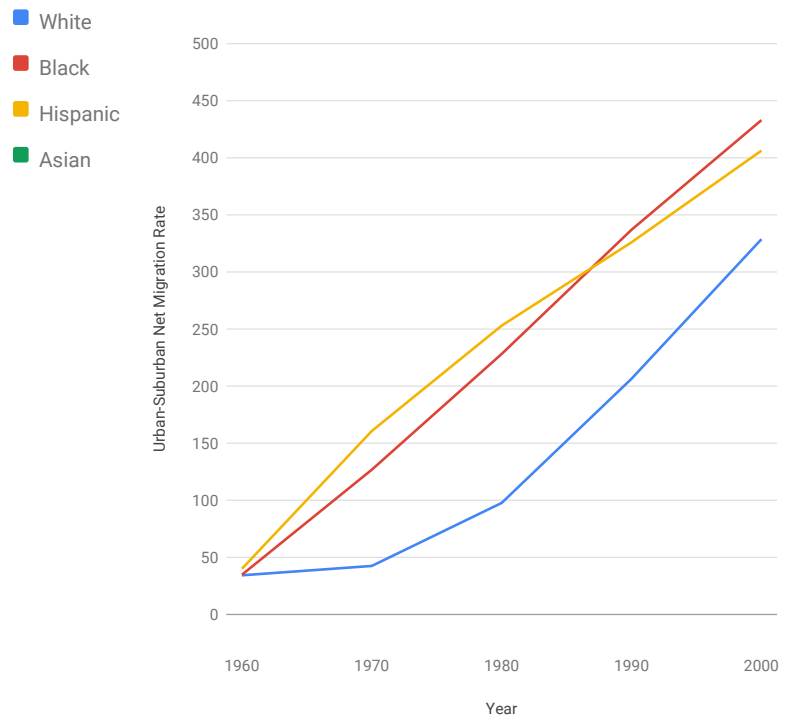

- Low Income

- Middle Income

High Income 
Urban and Suburban Residence, 1955-1995, and 5-year migration

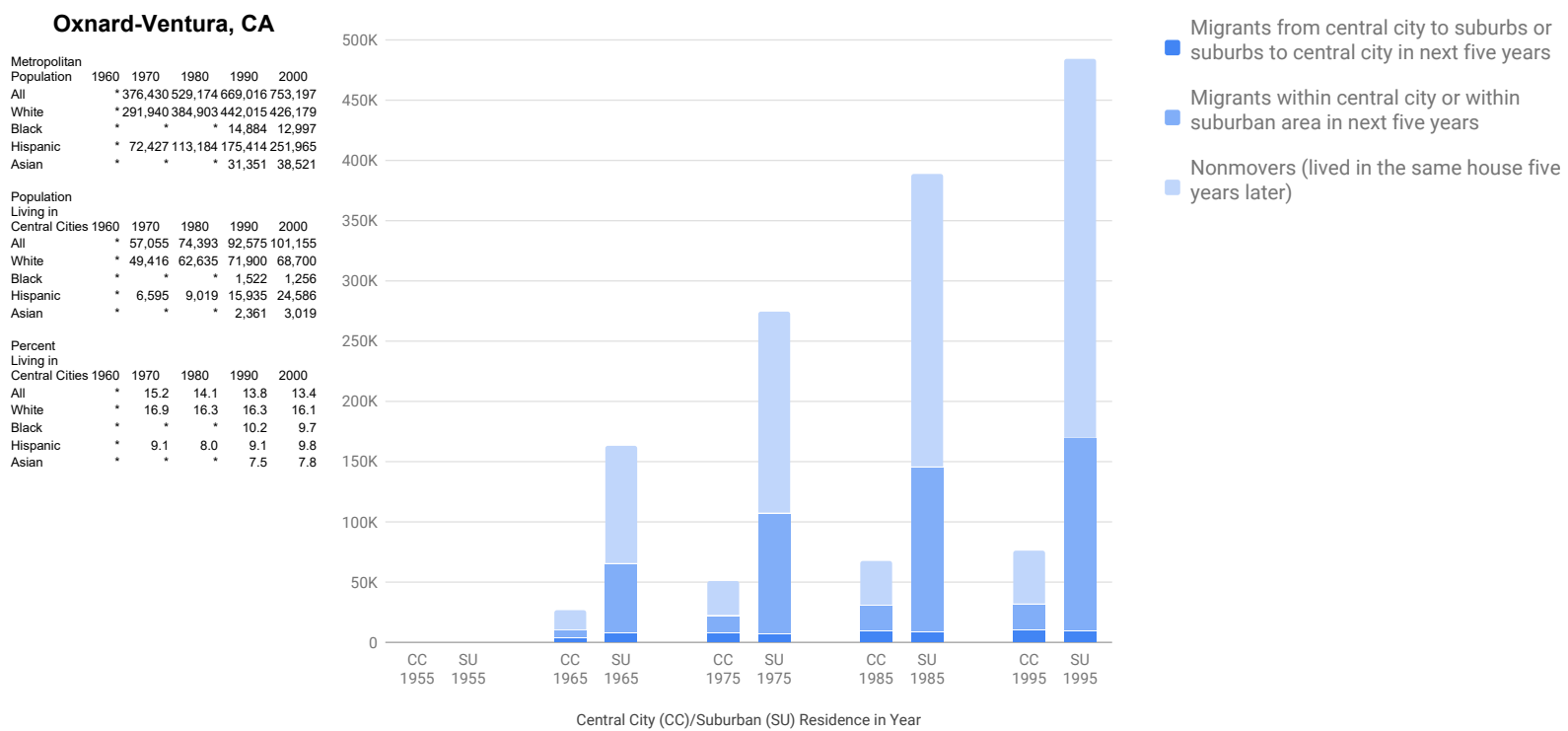

Urban-Suburban Net Migration Rate by Race

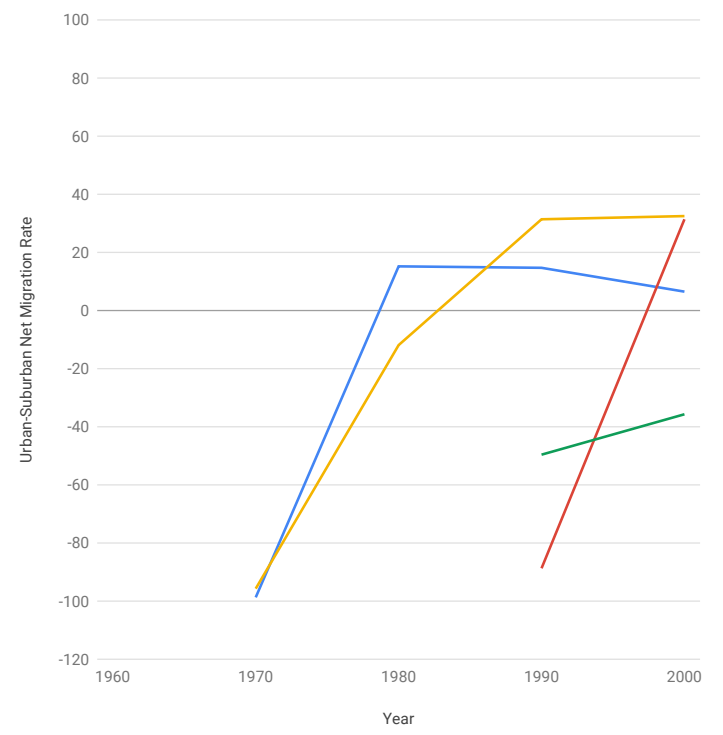

Low-Income Persons by Race

Middle-Income Persons by Race
Urban-Suburban Net Migration Rate by Income
White
Black
Hispanic
- Asian

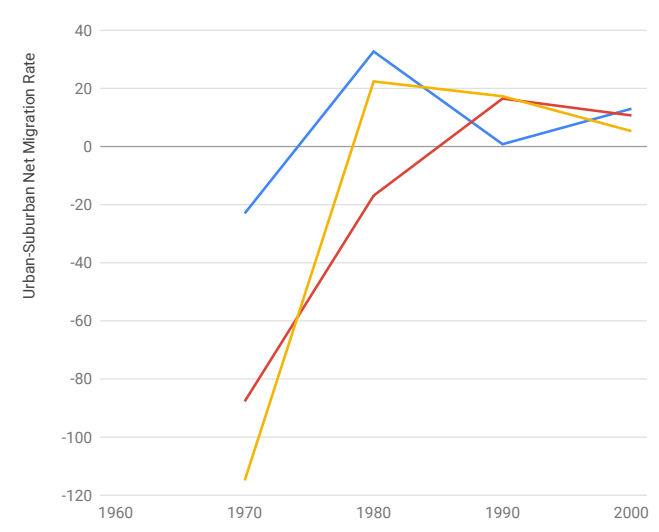

High-Income Persons by Race
100

80

60

Year

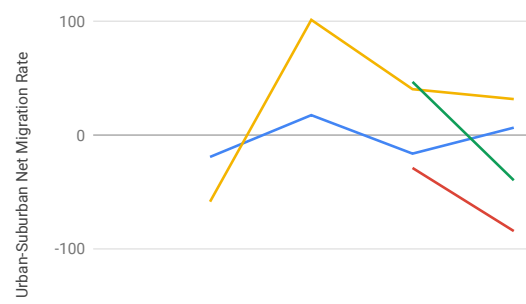

$-200$

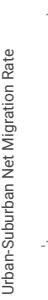

1960
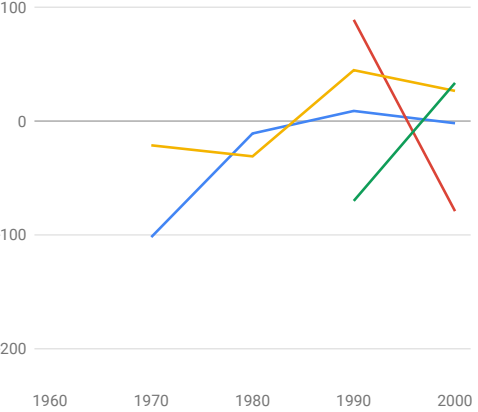

Low Income

Middle Income

- High Income

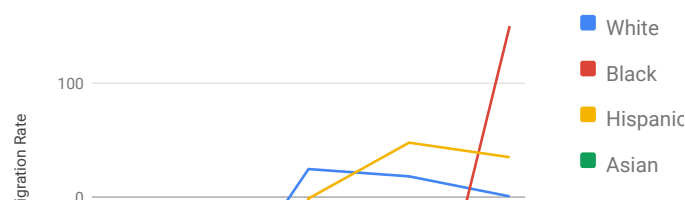


Urban and Suburban Residence, 1955-1995, and 5-year migration

$300 \mathrm{~K} \quad \begin{aligned} & \text { Migrants from central city to suburbs or } \\ & \text { suburbs to central city in next five years }\end{aligned}$

Palm Bay-Melbourne-Titusville, FL

$\begin{array}{llllll}\begin{array}{l}\text { Metropolitan } \\ \text { Population }\end{array} & 1960 & 1970 & 1980 & 1990 & 2000 \\ \text { All } & * & 230,006 & 272,959 & 398,978 & 476,230\end{array}$

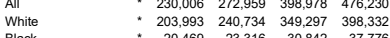

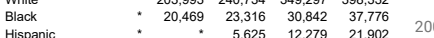

$\begin{array}{llrrr}\text { Hispanic } \quad: \quad: \quad 5,625 & 12,279 & 21,902 \\ \text { Asian } & \text { : } & & 4,789 & 7,024\end{array}$

Population
Living in

$\begin{array}{llllll}\text { Central Cities } & 1960 & 1970 & 1980 & 1990 & 2000 \\ \text { A } & * 0,827 & 78,407 & 99,040 & 112033\end{array}$

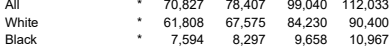

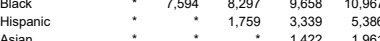

Percent $100 \mathrm{~K}$

$\begin{array}{lccccc}\text { Living in } & & & & & \\ \text { Central Cities } & 1960 & 1970 & 1980 & 1990 & 2000 \\ \text { All } & * & 30.8 & 28.7 & 24.8 & 23.5\end{array}$

$\begin{array}{lrrrrr}\text { All } & * & 30.8 & 28.7 & 24.8 & 23.5 \\ \text { White } & \vdots & 30.3 & 28.1 & 24.1 & 22.7 \\ \text { Black } & \vdots & 37.1 & 35.6 & 31.3 & 29.0 \\ & \vdots & & & 29.7 & 27.9\end{array}$

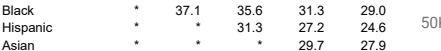

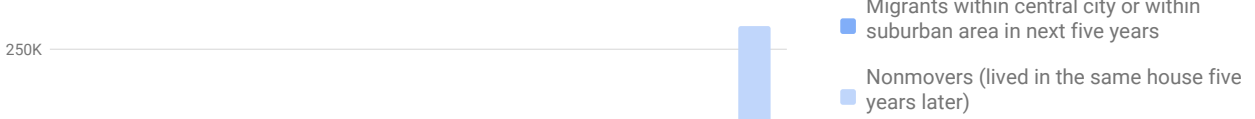

$150 \mathrm{~K}$

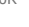

Urban-Suburban Net Migration Rate by Race

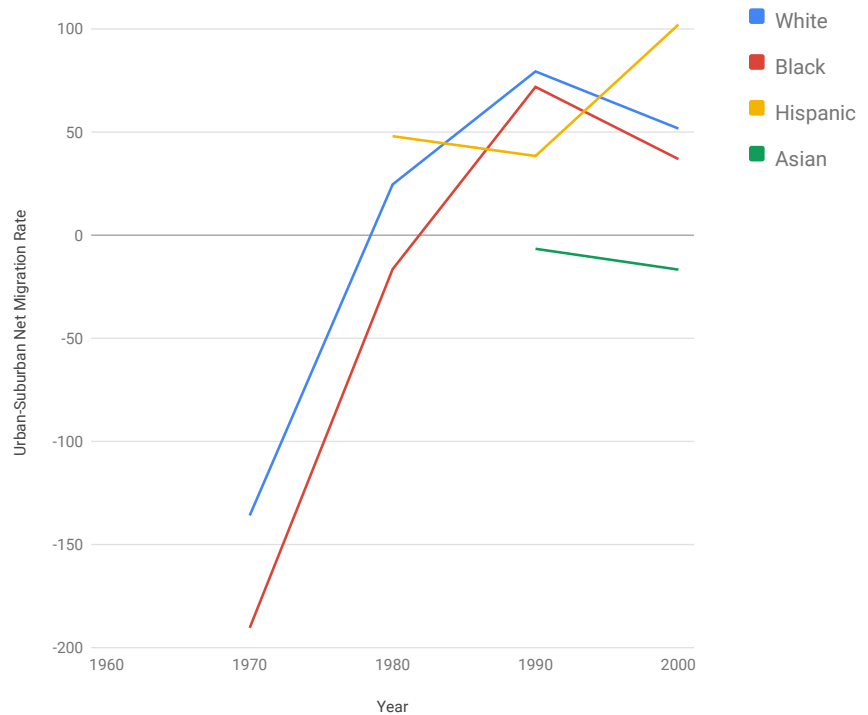

Low-Income Persons by Race
Middle-Income Persons by Race
Urban-Suburban Net Migration Rate by Income

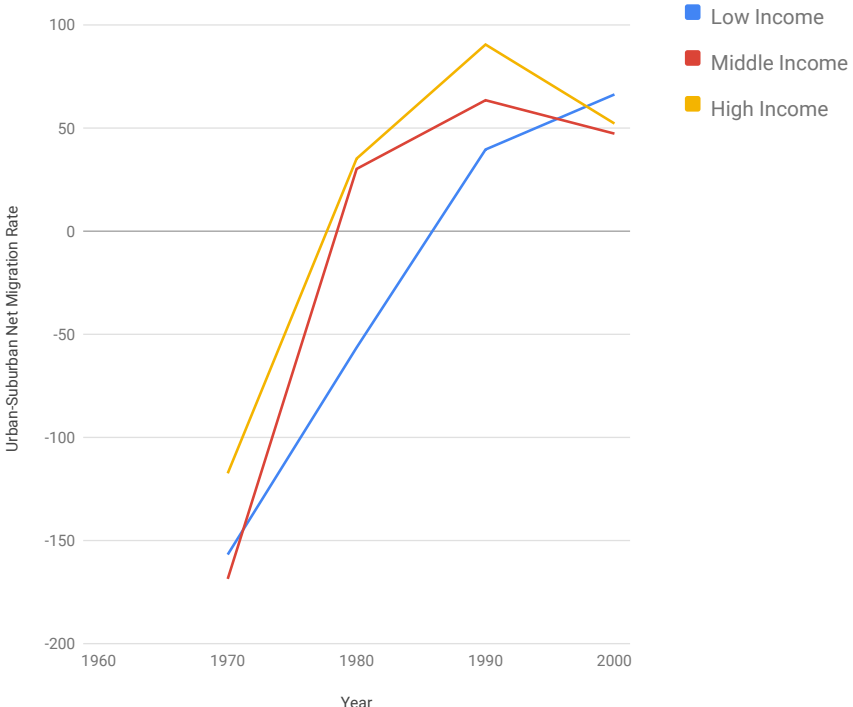

High-Income Persons by Race

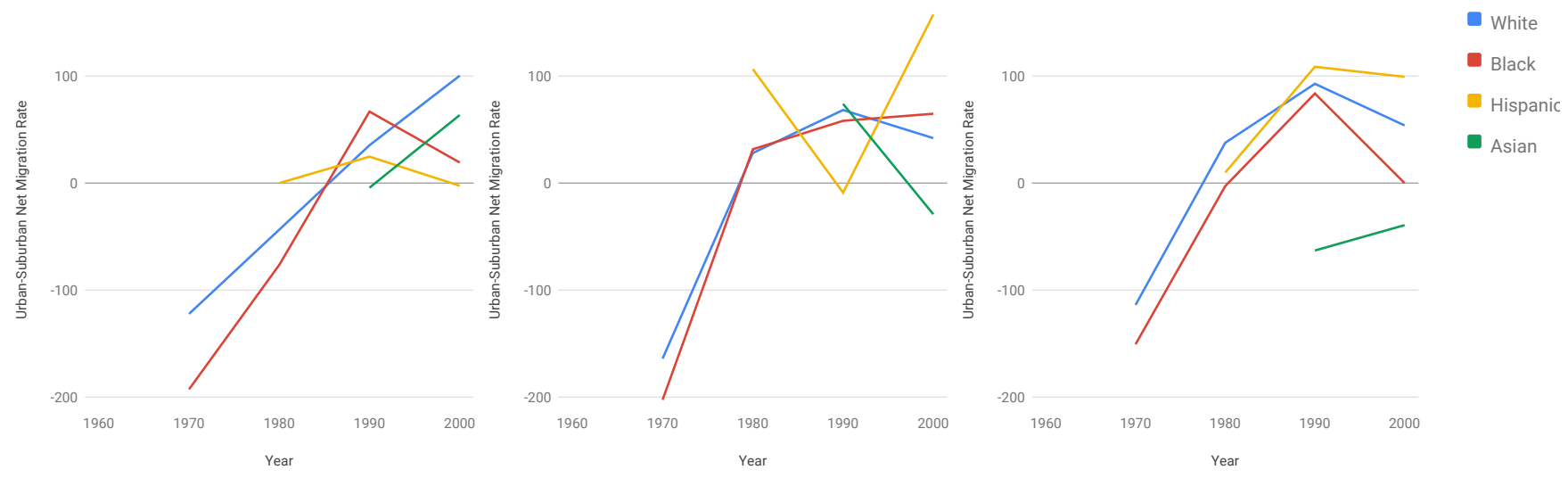


Urban and Suburban Residence, 1955-1995, and 5-year migration

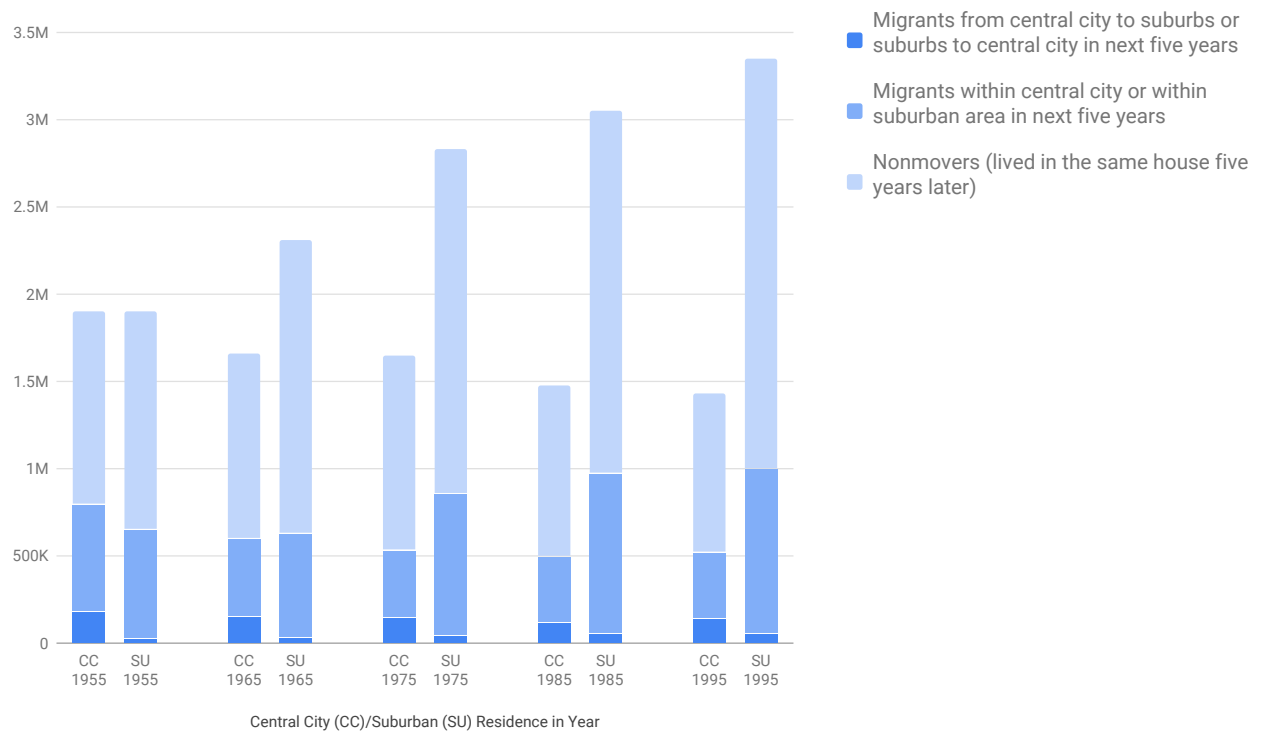

Urban-Suburban Net Migration Rate by Race

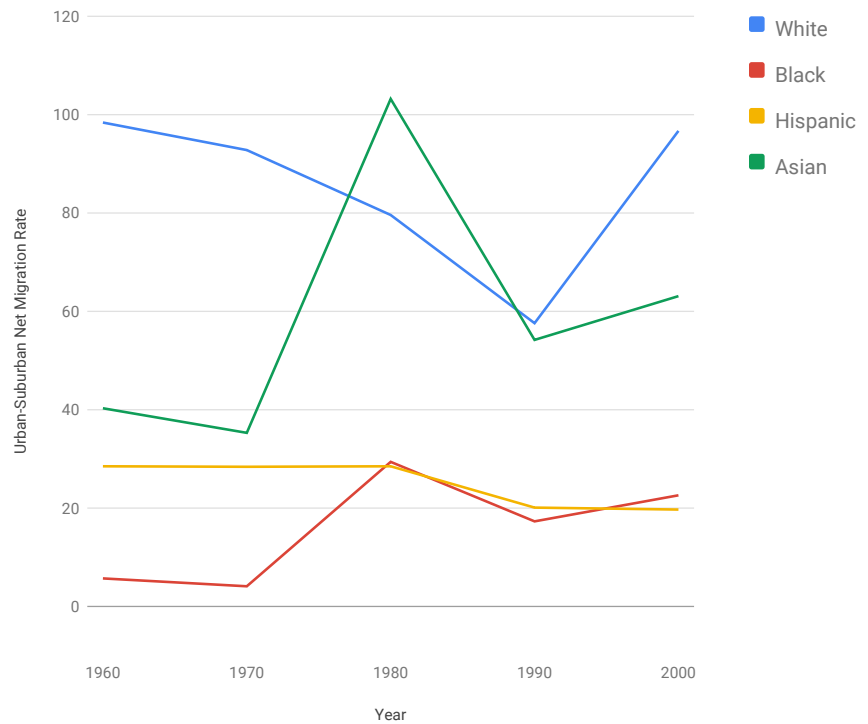

Low-Income Persons by Race

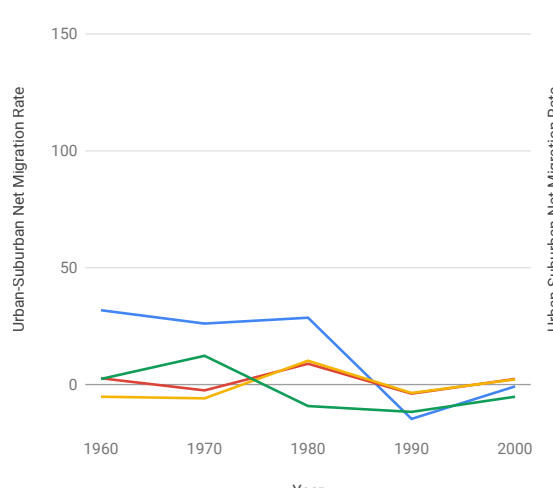

Year
Urban-Suburban Net Migration Rate by Income

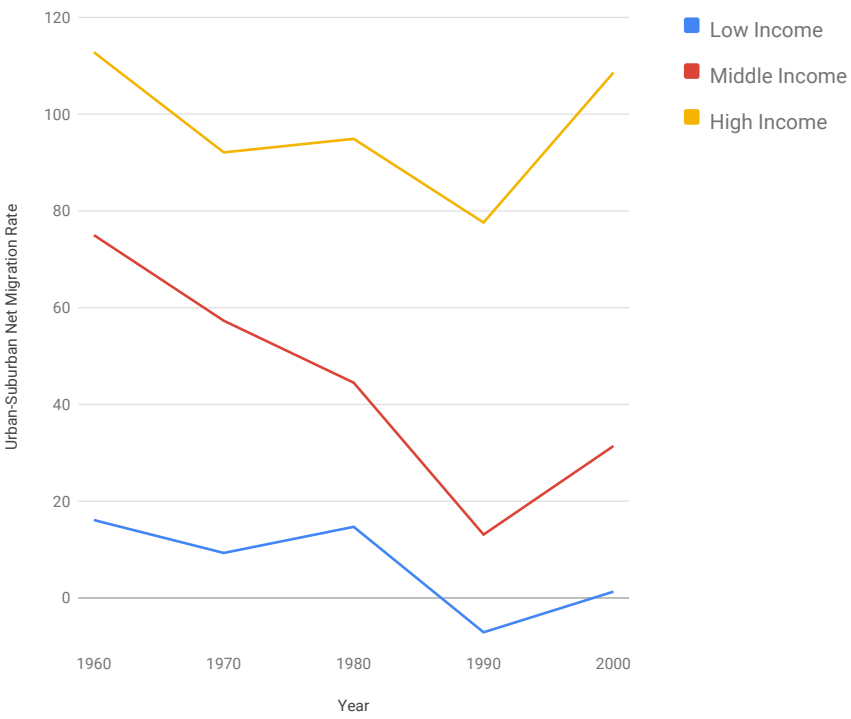

High-Income Persons by Race

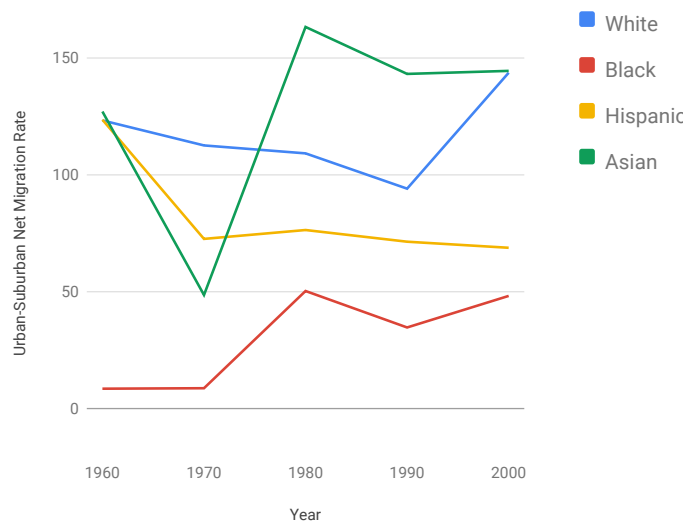


Urban and Suburban Residence, 1955-1995, and 5-year migration

\section{Phoenix, AZ}

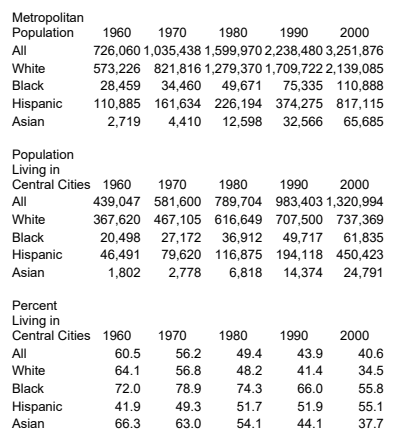

$1.4 \mathrm{M}$

\author{
$1.2 \mathrm{M}$
}

$1 \mathrm{M}$

$800 \mathrm{~K}$

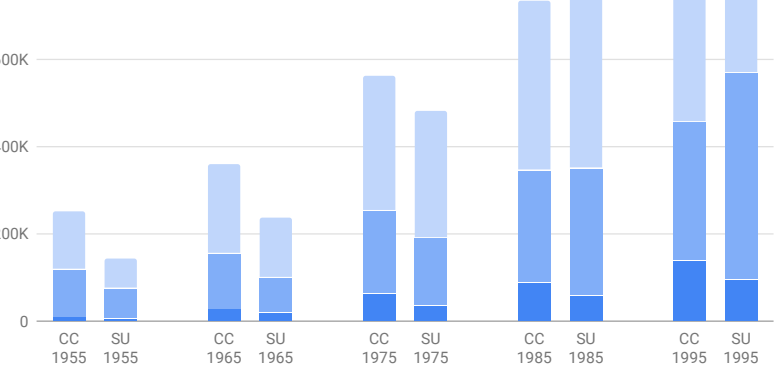

Migrants from central city to suburbs or - suburbs to central city in next five years

Migrants within central city or within

suburban area in next five years

Nonmovers (lived in the same house five

years later)
Urban-Suburban Net Migration Rate by Race

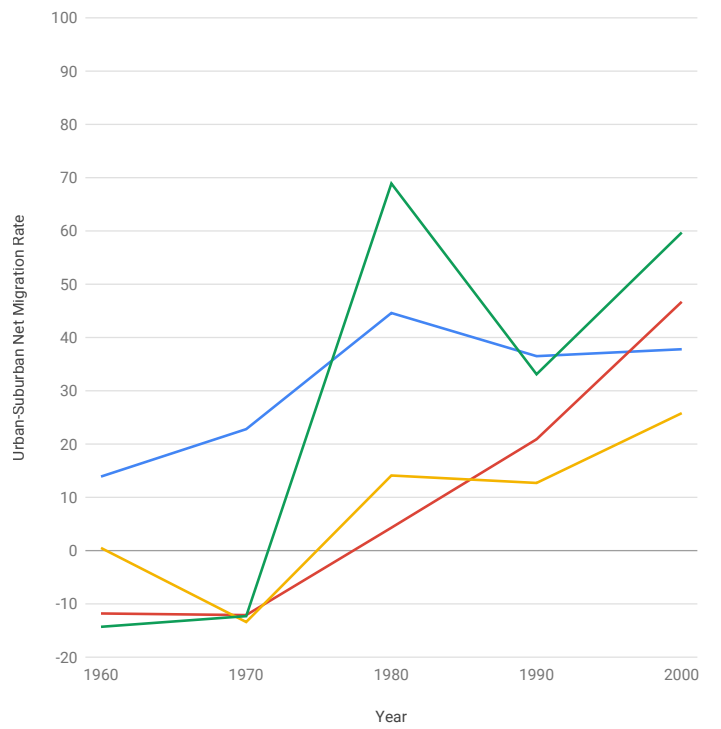

Low-Income Persons by Race

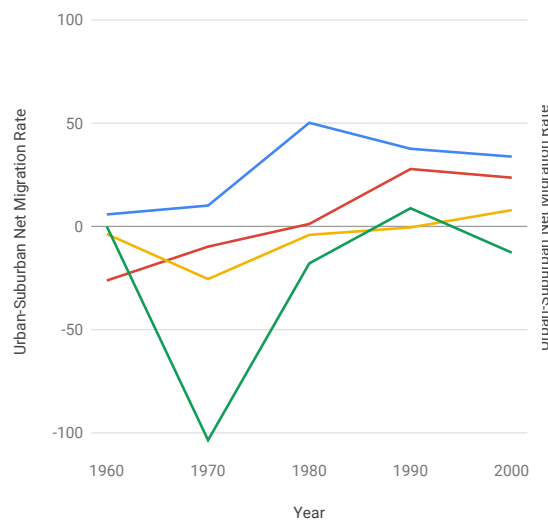

White

Black

Hispanic

- Asian

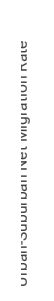

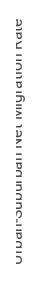

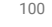

90

70

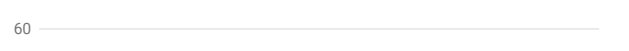

50

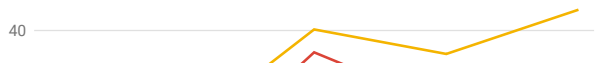

30

0

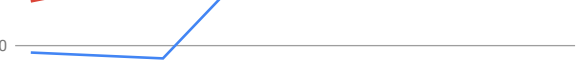

$-10$

$-20 \frac{}{1960}$

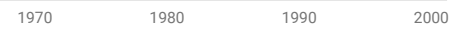

Year
100

Middle-Income Persons by Race

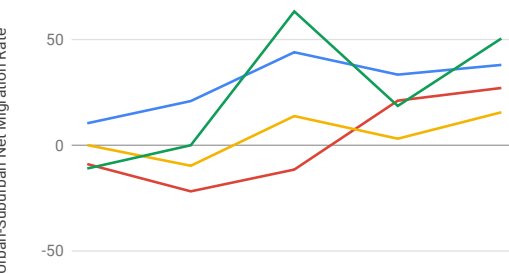

$-100$

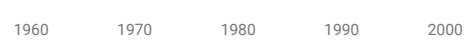

Year
Low Income

- Middle Income

High Income
High-Income Persons by Race

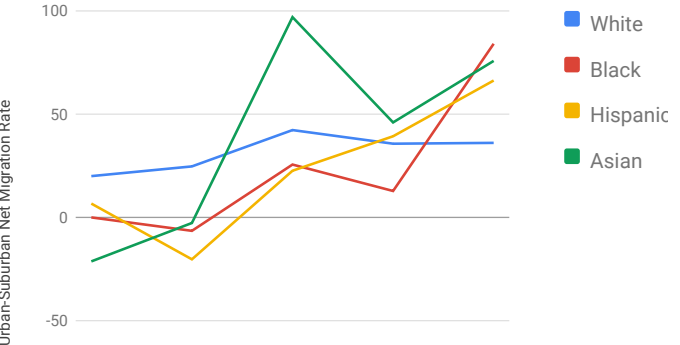

$-100$

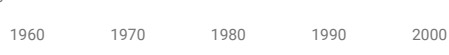


Urban and Suburban Residence, 1955-1995, and 5-year migration

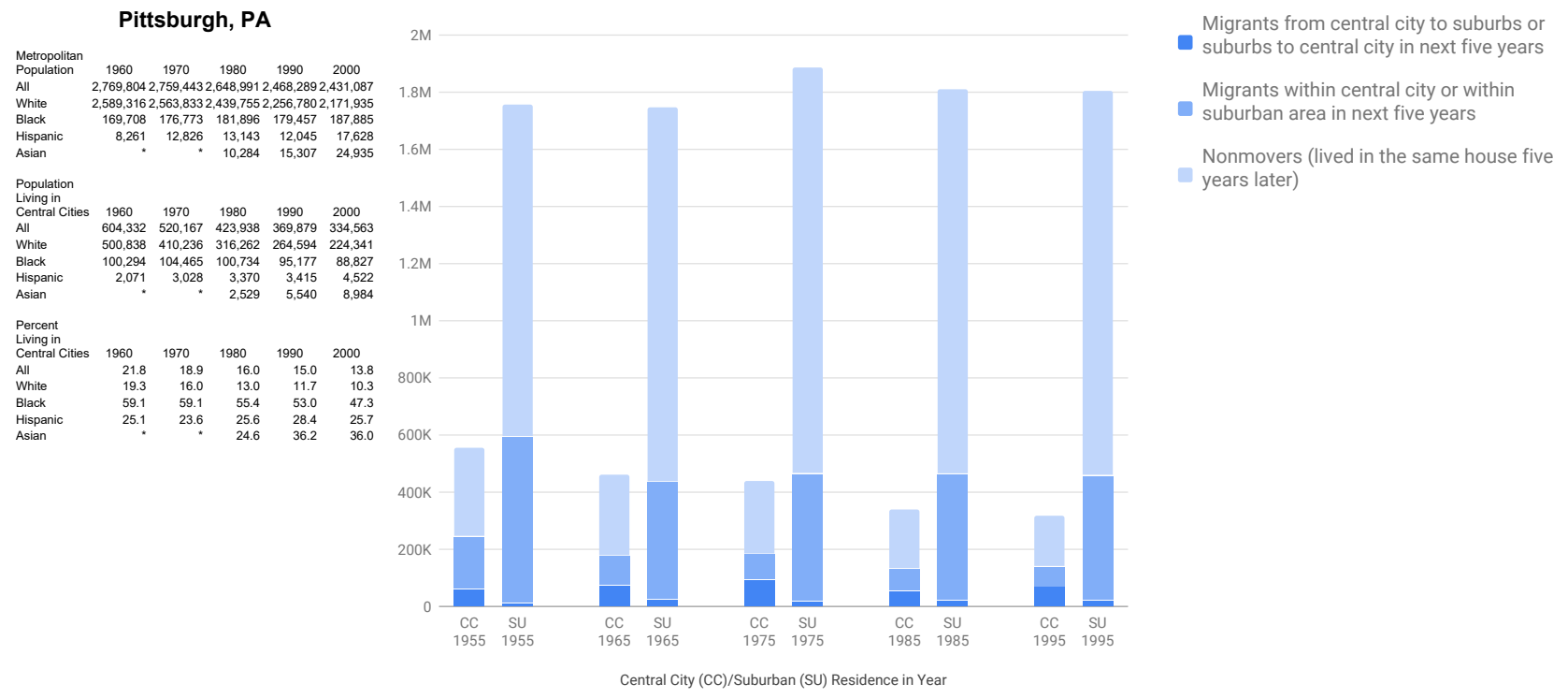

Urban-Suburban Net Migration Rate by Race

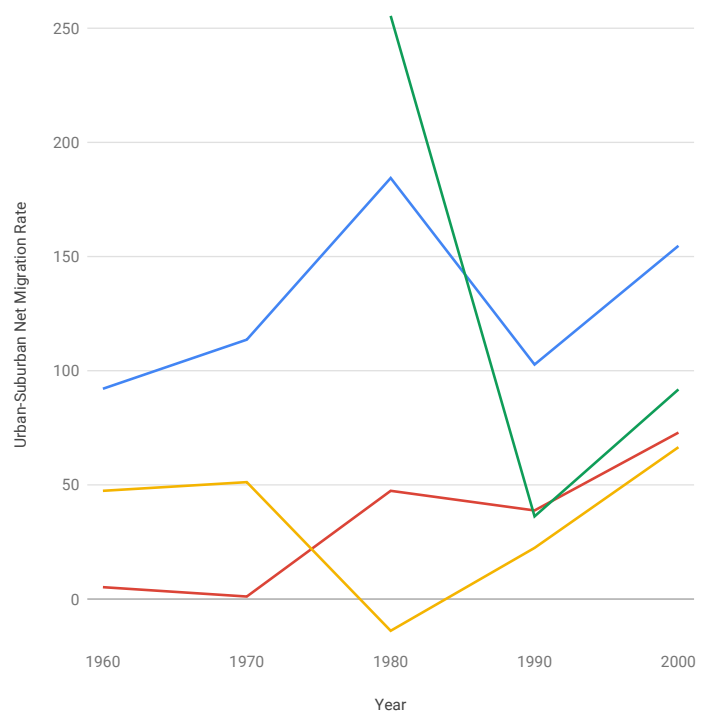

Low-Income Persons by Race

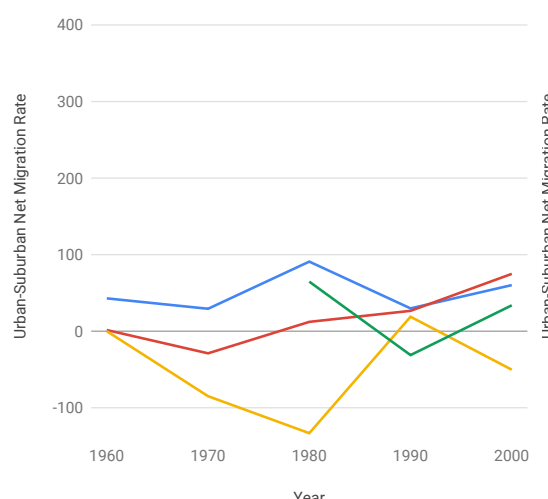

Year
Middle-Income Persons by Race

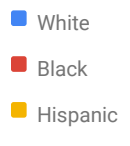

- Asian

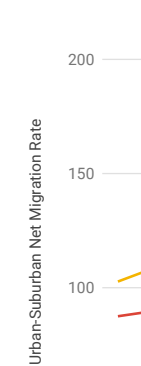

250

Low Income

- Middle Income

- High Income 
Urban and Suburban Residence, 1955-1995, and 5-year migration

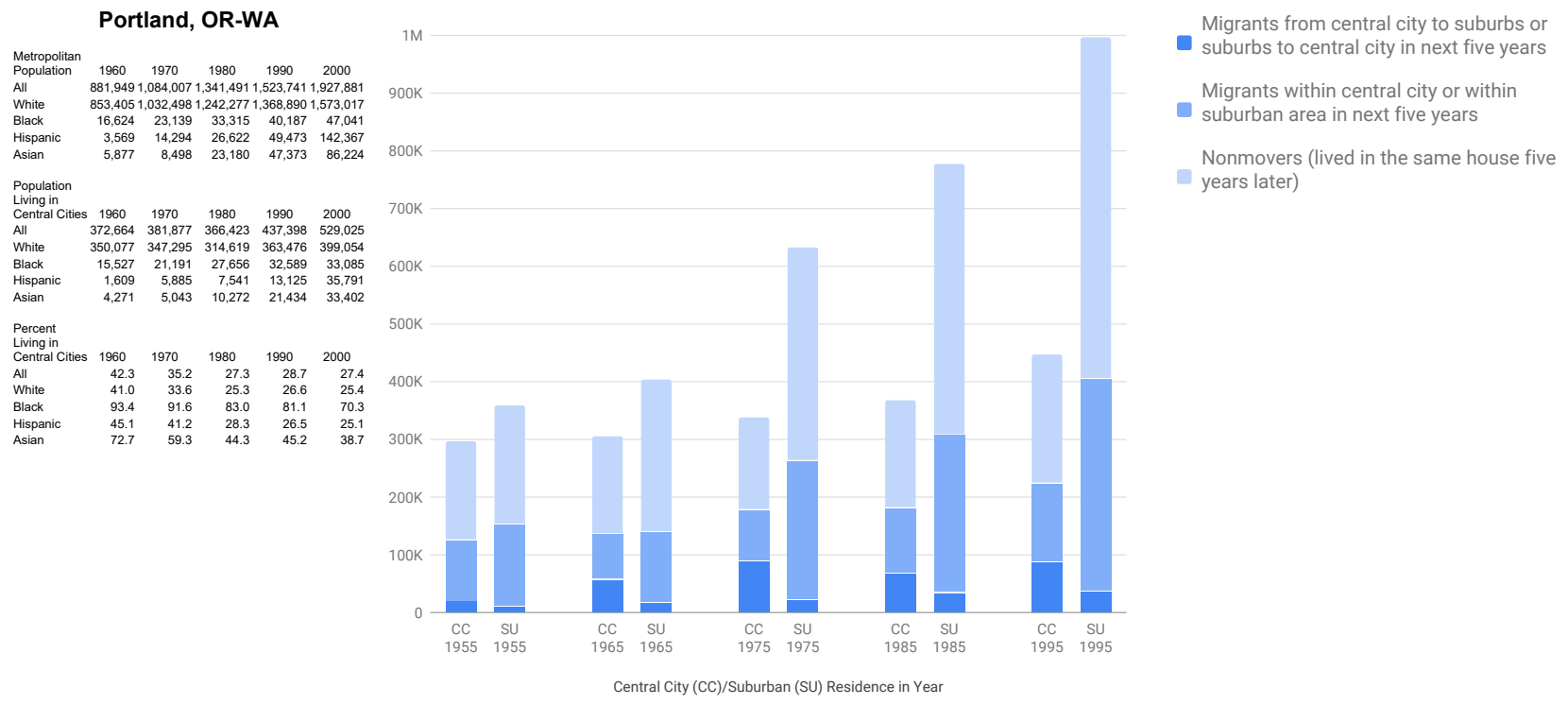

Urban-Suburban Net Migration Rate by Race

Urban-Suburban Net Migration Rate by Income
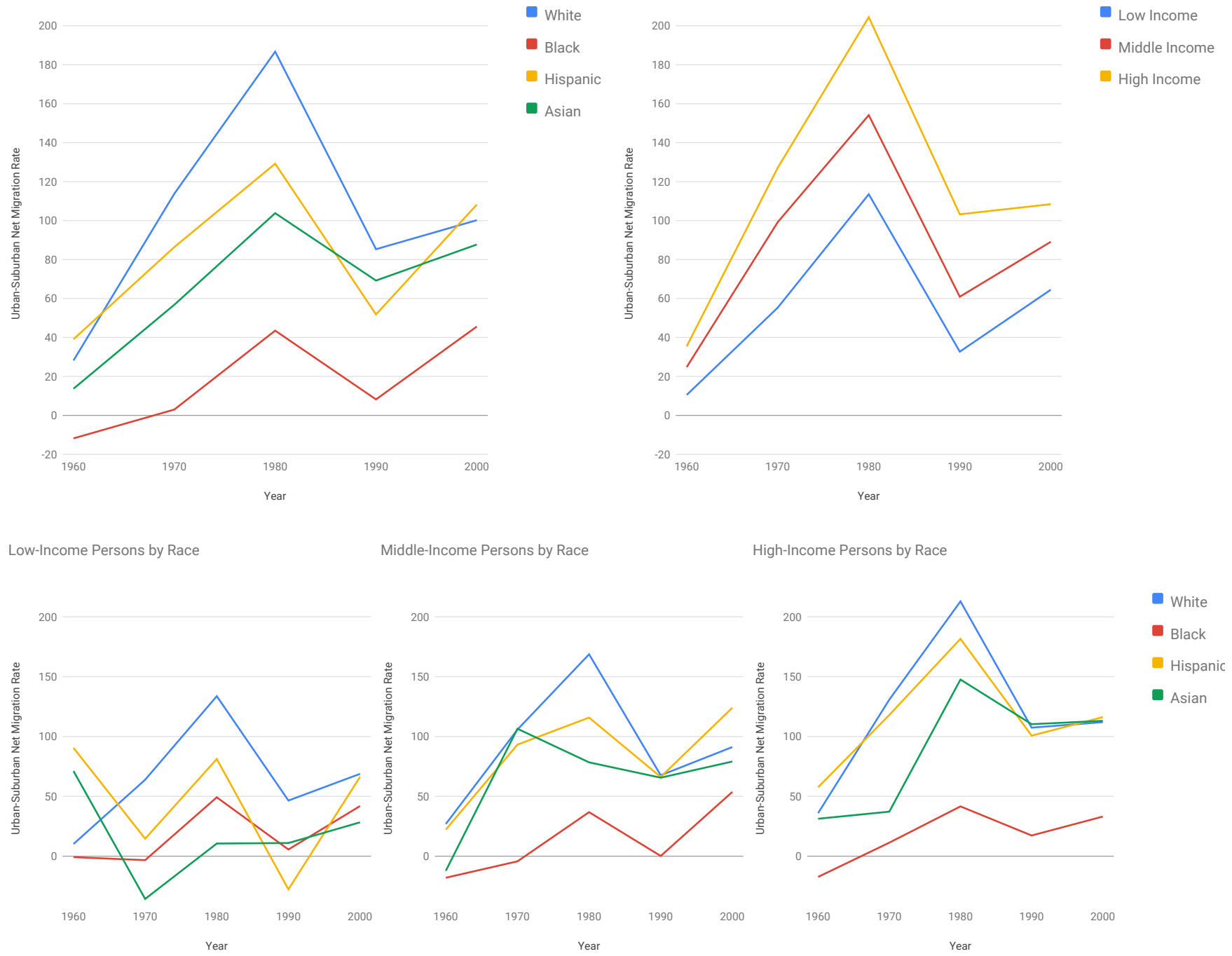
Urban and Suburban Residence, 1955-1995, and 5-year migration

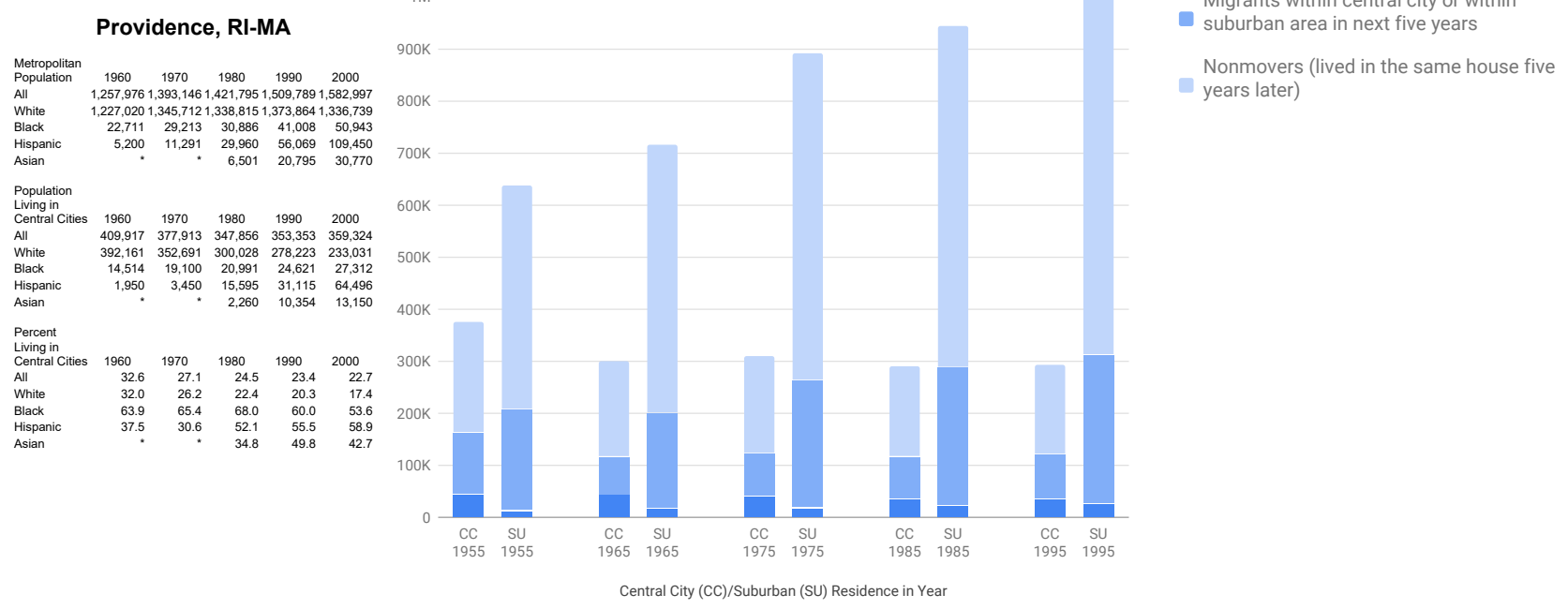

Urban-Suburban Net Migration Rate by Race

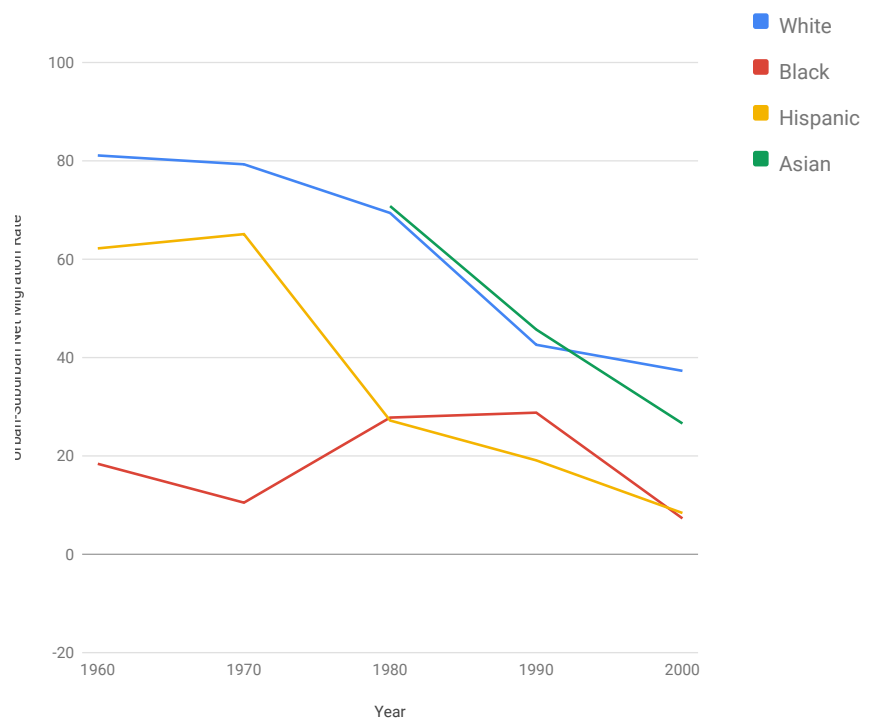

Low-Income Persons by Race
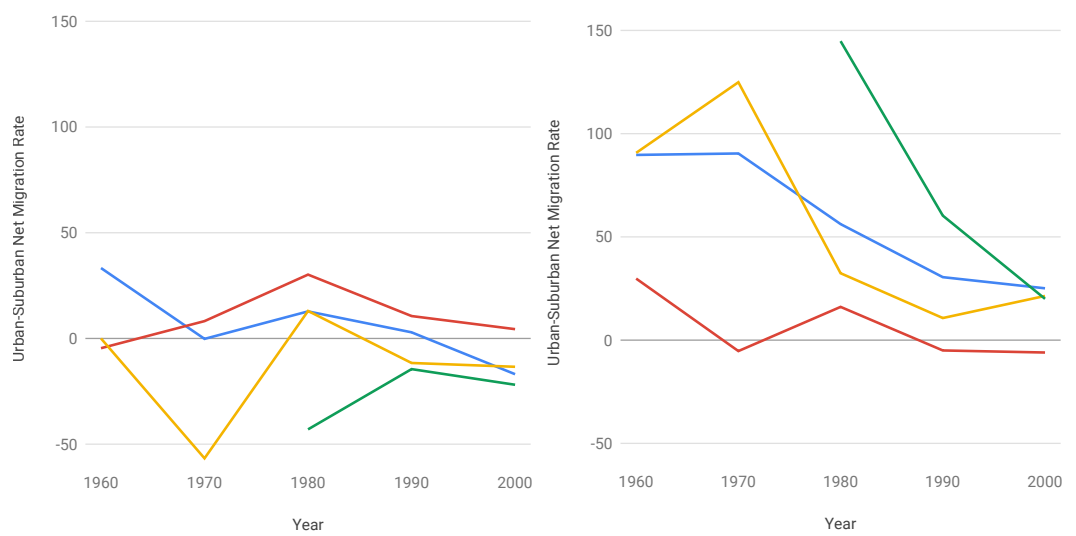

Urban-Suburban Net Migration Rate by Income

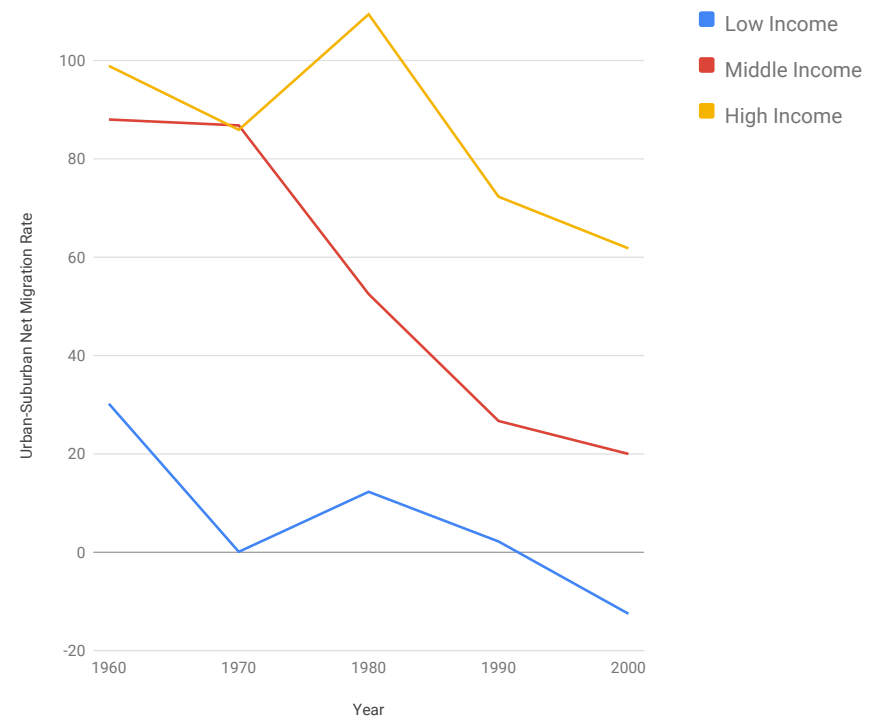

High-Income Persons by Race

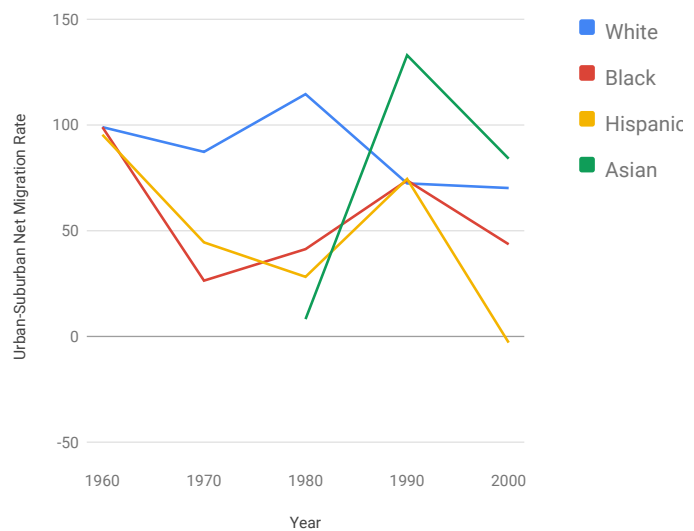


Urban and Suburban Residence, 1955-1995, and 5-year migration

140K $\begin{aligned} & \text { Migrants from central city to suburbs or } \\ & \text { suburbs to central city in next five years }\end{aligned}$

Migrants within central city or within

suburban area in next five years

Nonmovers (lived in the same house five

years later)

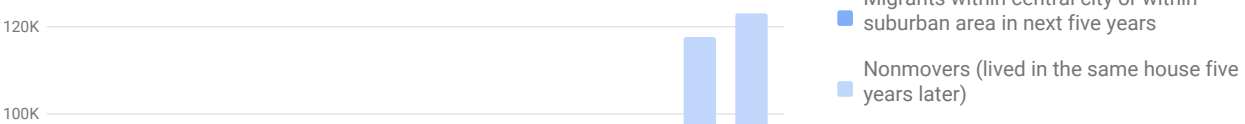

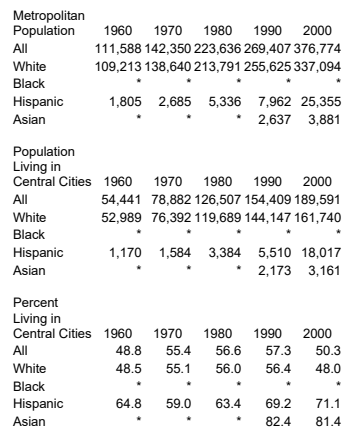

Urban-Suburban Net Migration Rate by Race

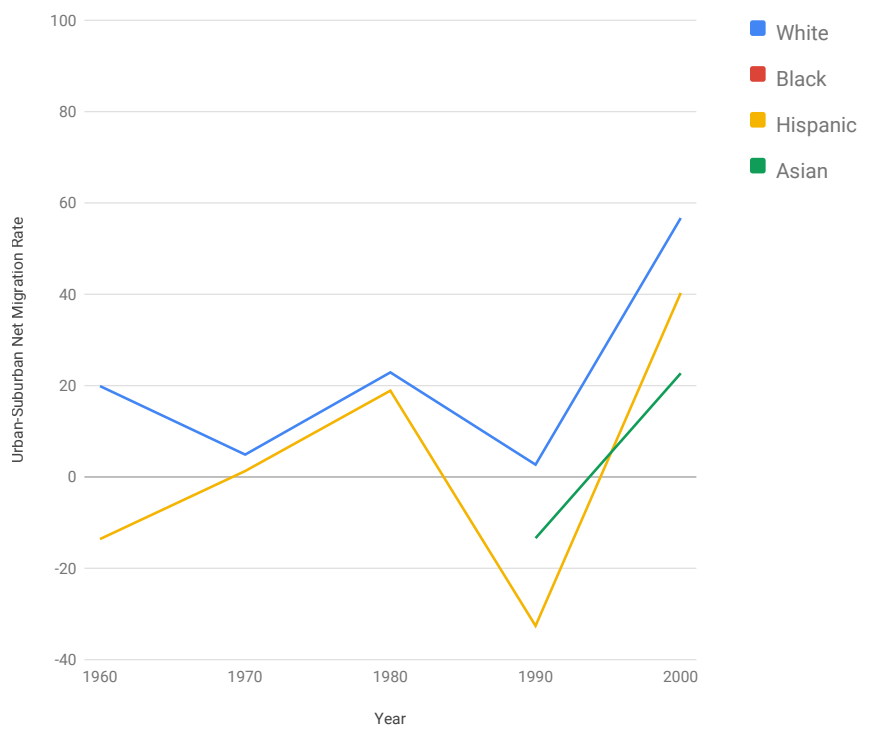

Low-Income Persons by Race

Middle-Income Persons by Race
Urban-Suburban Net Migration Rate by Income
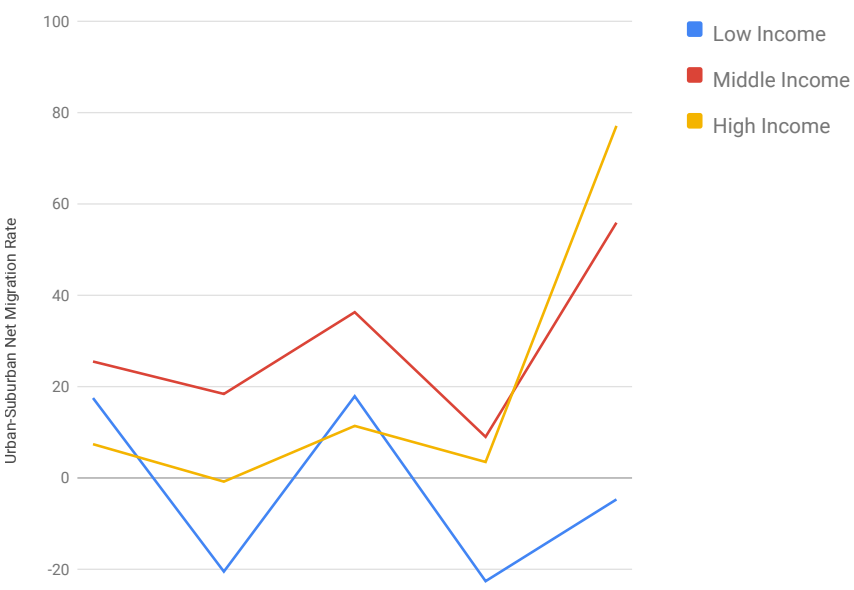

$\begin{array}{lllll}-40 & 1970 & 1980 & 1990 & 2000\end{array}$

Year

High-Income Persons by Race

High Income

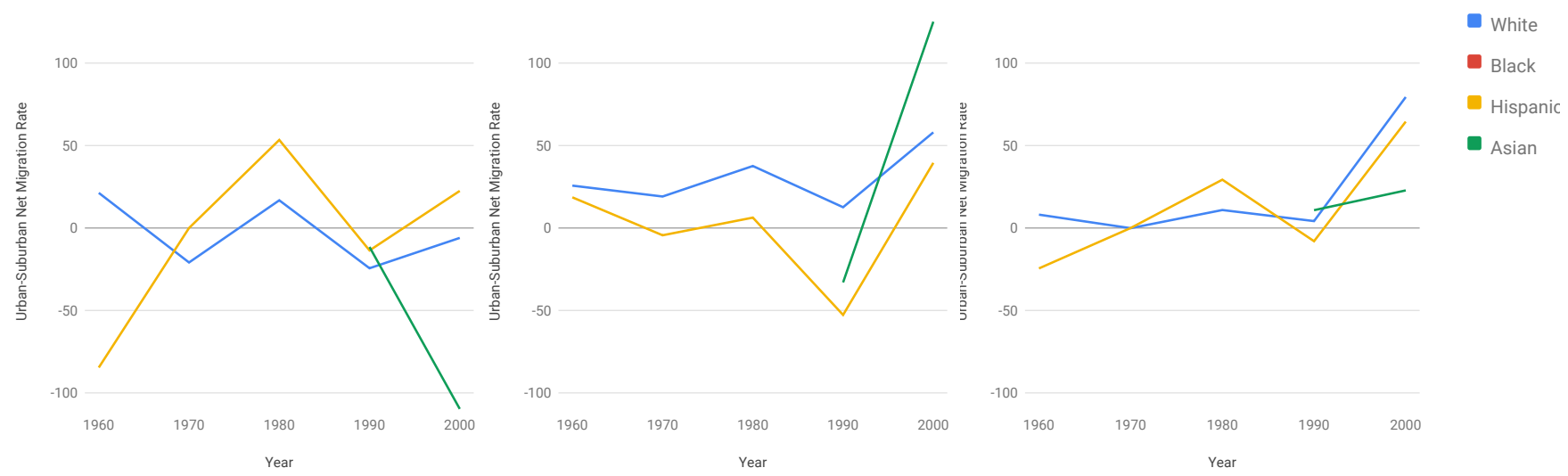


Urban and Suburban Residence, 1955-1995, and 5-year migration

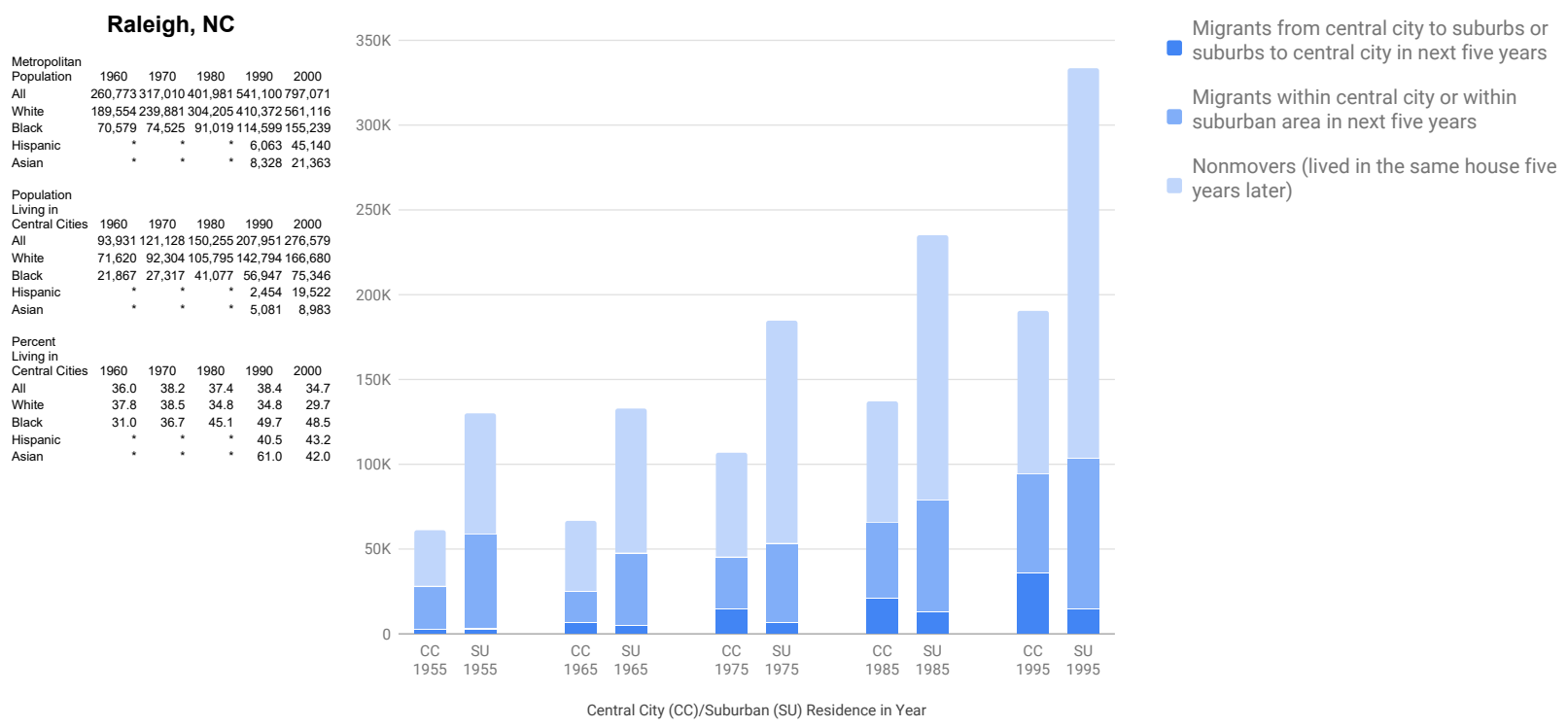

Urban-Suburban Net Migration Rate by Race

Urban-Suburban Net Migration Rate by Income
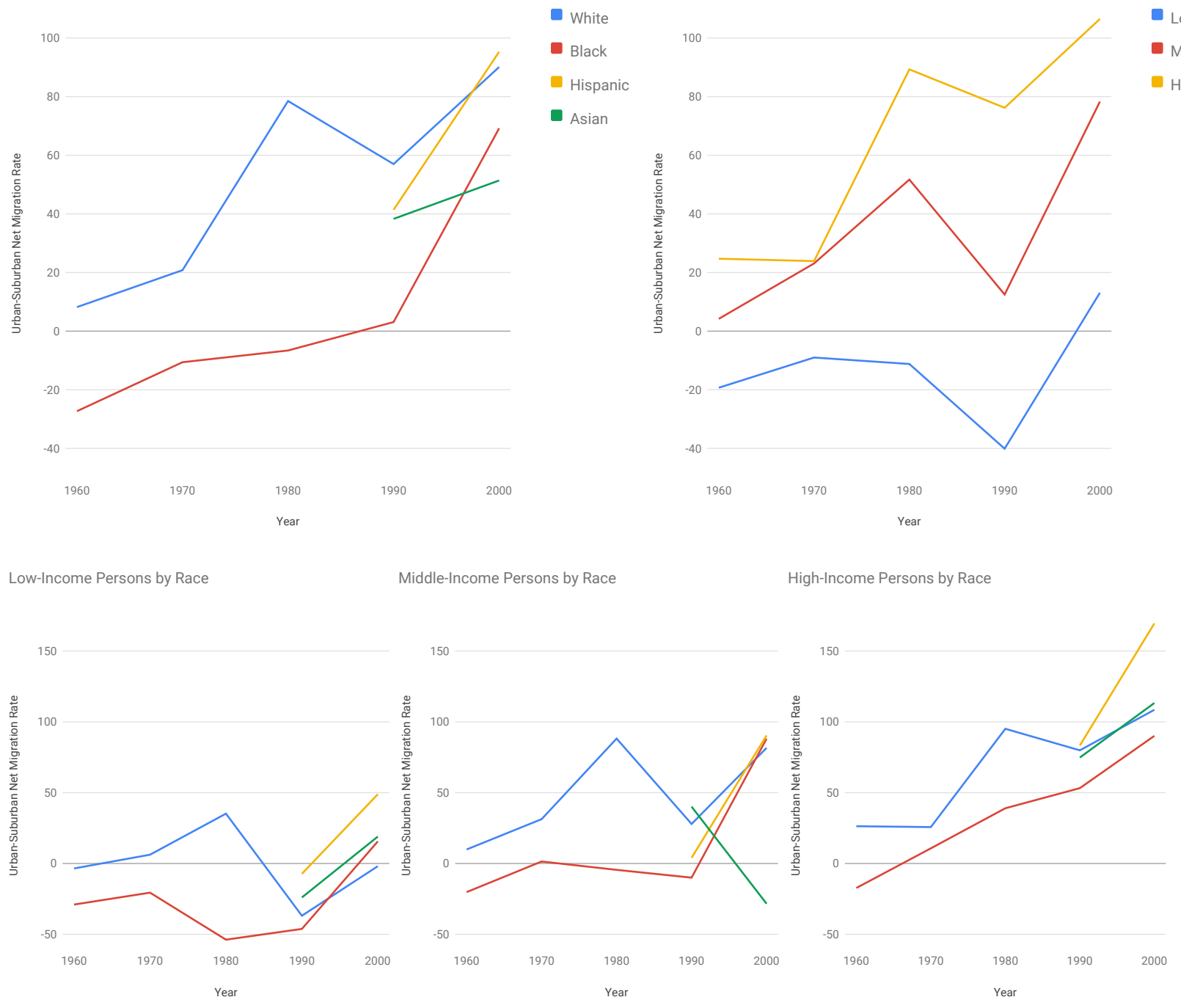
Urban and Suburban Residence, 1955-1995, and 5-year migration

\section{Richmond, VA}

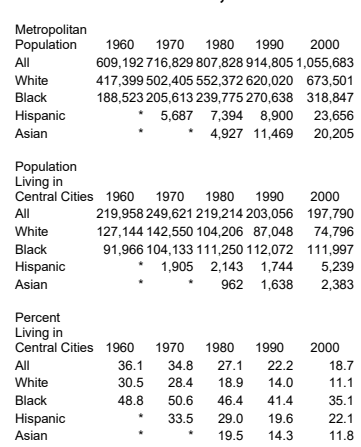

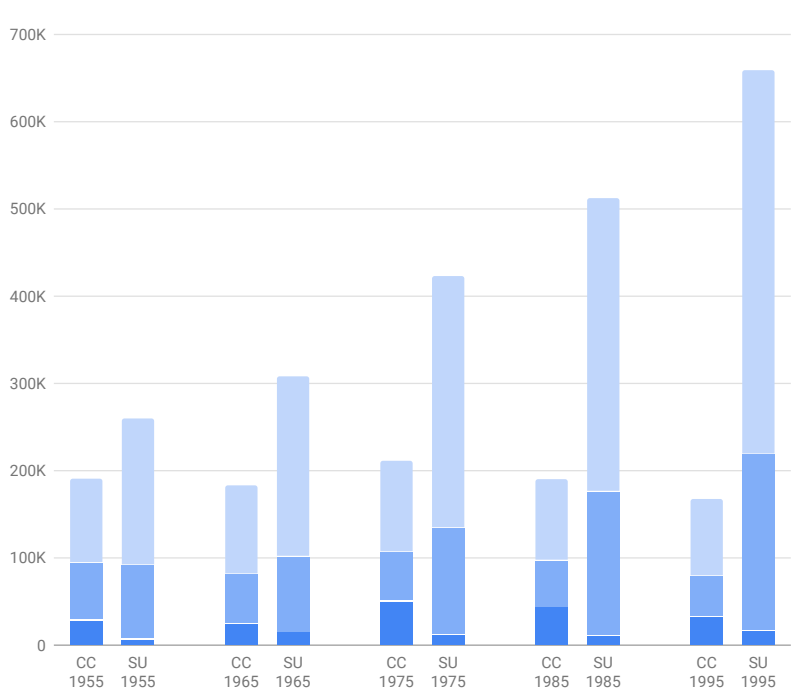

Migrants from central city to suburbs or suburbs to central city in next five years

Migrants within central city or within suburban area in next five years

Nonmovers (lived in the same house five

years later)
Urban-Suburban Net Migration Rate by Race

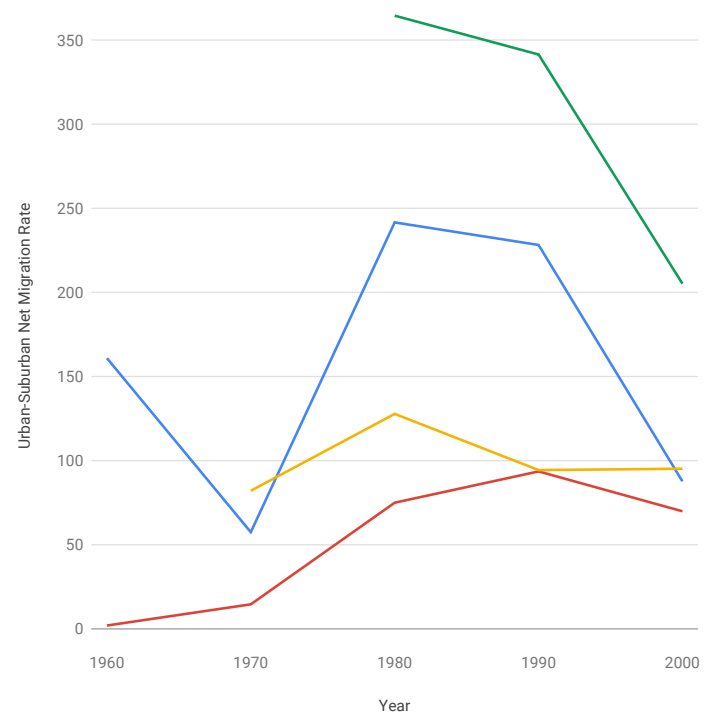

Urban-Suburban Net Migration Rate by Income
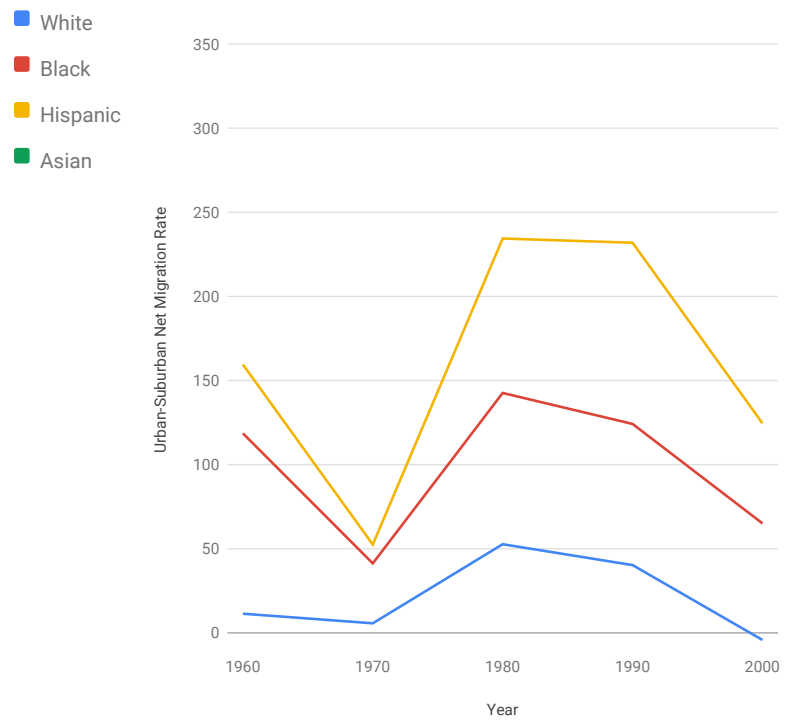

- Low Income

- Middle Income

- High Income
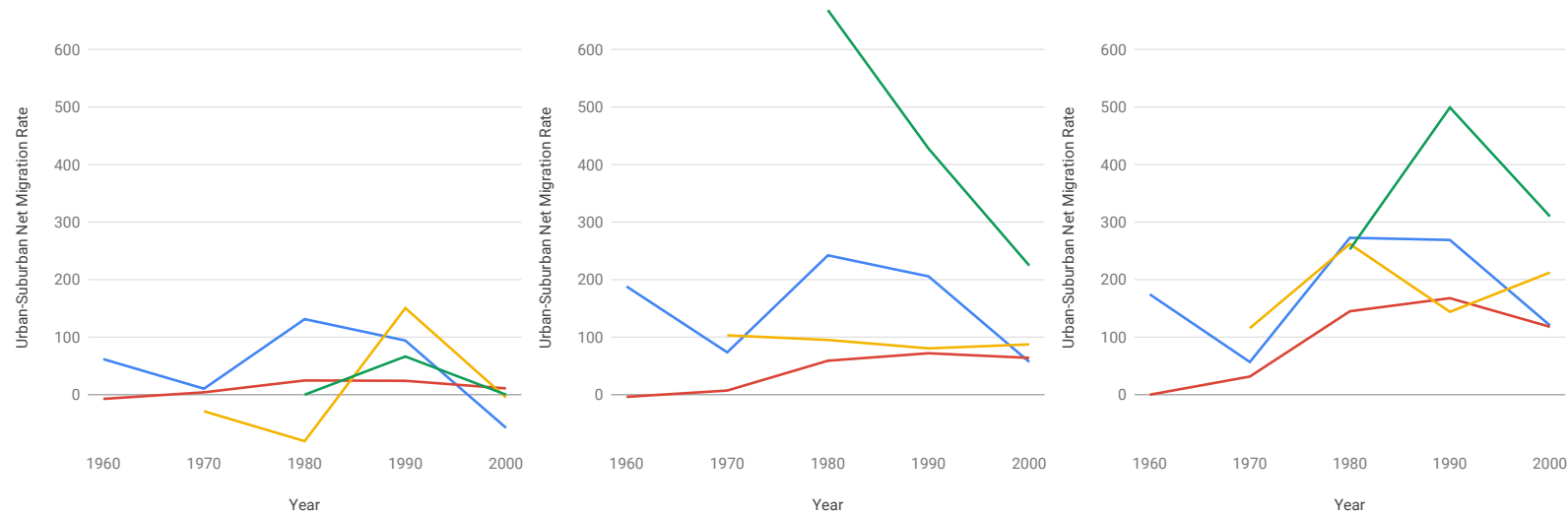
Urban and Suburban Residence, 1955-1995, and 5-year migration

Riverside-San Bernardino, CA

$\begin{array}{llllll}\begin{array}{l}\text { Metropolitan } \\ \text { Population }\end{array} & 1960 & 1970 & 1980 & 1990 & 2000\end{array}$

$\begin{array}{ll}\text { All } & 809,7821,140,1661,558,1822,588,7933,254,821 \\ \text { White } & 665,145 \quad 895,8251,146,8861,622,5391,536,542\end{array}$

$\begin{array}{lllllll}\text { Black } & 28,686 & 47,902 & 77,191 & 170,415 & 239,674\end{array}$

$\begin{array}{lrrrr}\text { Hispanic } & 108,384 & 185,455 & 289,803 & 675,9181,229,230 \\ \text { Asian } & 20,382 & 88,167 & 130,436\end{array}$

Population
Living in
Central Cities $1960 \quad 1970 \quad 1980 \quad 1990 \quad 2000$

$\begin{array}{llllll}\text { Central Cities } & 1960 & 1970 & 1980 & 1990 & 2000 \\ \text { All } & 176,254 & 244,163 & 288,366 & 390,669 & 440,481\end{array}$

$\begin{array}{llllll}\text { White } & 137,397 & 180,985 & 193,358 & 214,042 & 170,049 \\ \text { Black } & 11,608 & 20,914 & 28801 & 41248 & 46368\end{array}$

$\begin{array}{llllllll}\text { Hispanic } & 26,454 & 39,845 & 57,327 & 114,154 & 185,193\end{array}$

$\begin{array}{lllll}\text { Asian } & * & 4,297 & 16,198 & 21,251\end{array}$

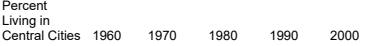

$\begin{array}{llllll}\text { All } & 21.8 & 21.4 & 18.5 & 15.1 & 13.5 \\ \text { White } & 20.7 & 202 & 169 & 132 & 11.1\end{array}$

$\begin{array}{lrrrrr}\text { White } & 20.7 & 20.2 & 16.9 & 13.2 & 11.1 \\ \text { Black } & 40.5 & 43.7 & 37.3 & 24.2 & 19.3 \\ \text { Hispanic } & 24.4 & 21.5 & 19.8 & 16.9 & 15.1 \\ \text { Asian } & . & . & 21.1 & 18.4 & 16.3\end{array}$

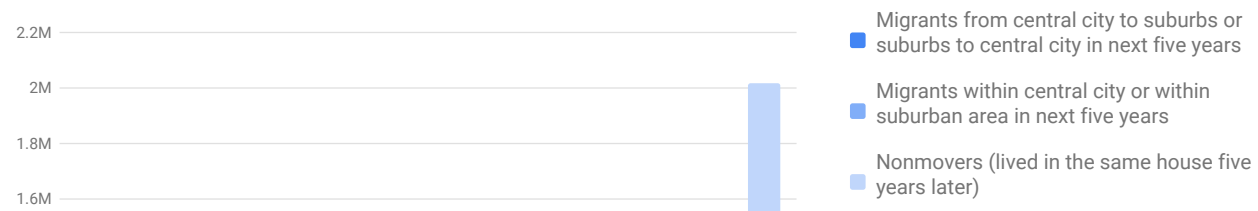

$1.2 \mathrm{M}$

$1 \mathrm{M}$
$800 \mathrm{~K}$

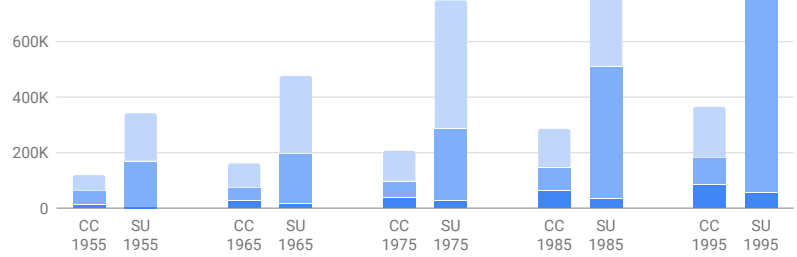

Central City (CC)/Suburban (SU) Residence in Year
Urban-Suburban Net Migration Rate by Race

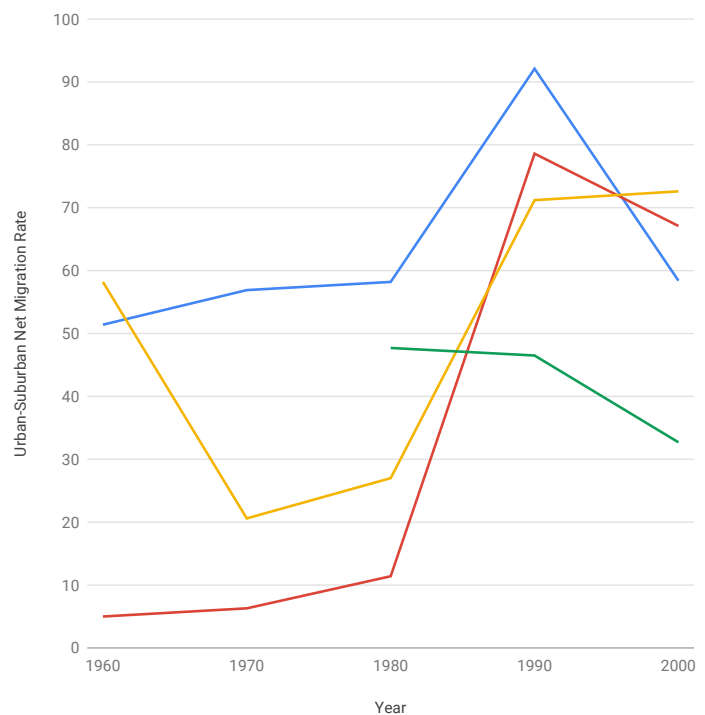

Low-Income Persons by Race

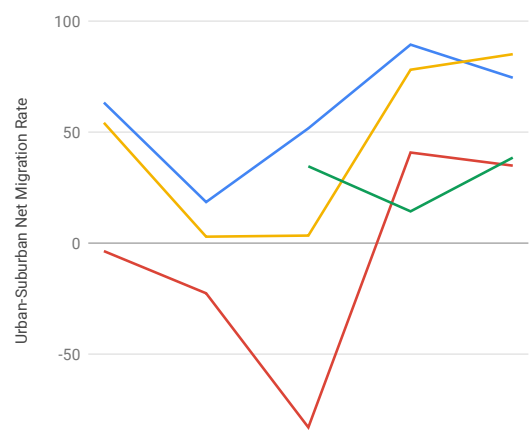

$1960 \quad 1970$
White

Black

Hispanic

- Asian

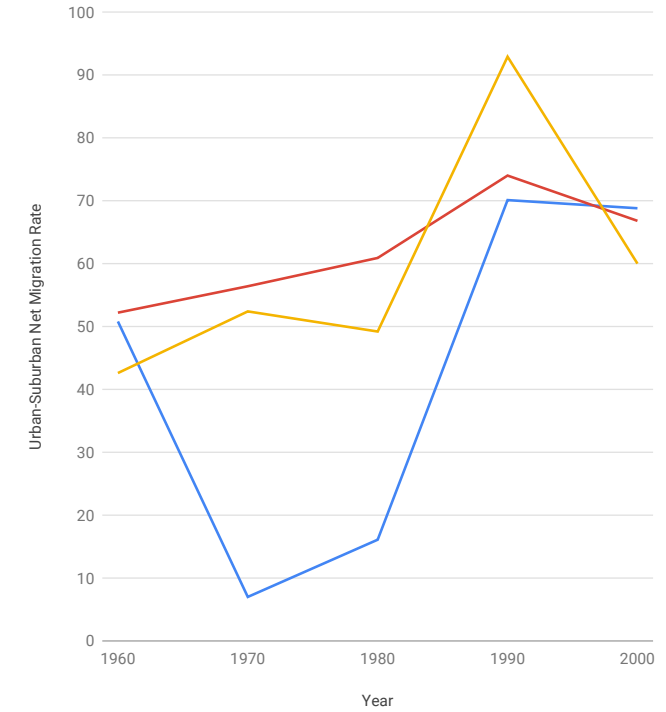

High-Income Persons by Race

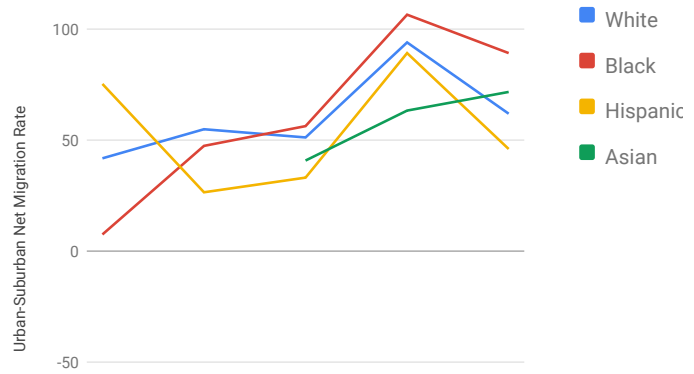

- Low Income

- Middle Income

High Income
100

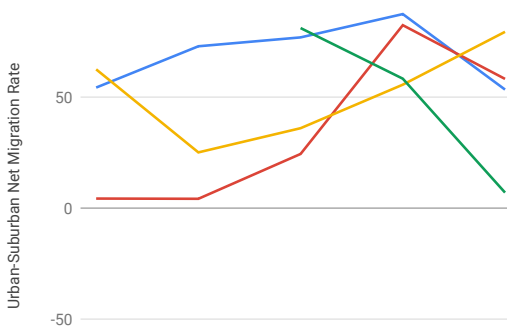

1960
1970

Year Year 
Urban and Suburban Residence, 1955-1995, and 5-year migration

$700 \mathrm{~K} \quad$ Migrants from central city to suburbs or suburbs to central city in next five years

Migrants within central city or within - suburban area in next five years

Nonmovers (lived in the same house five years later)
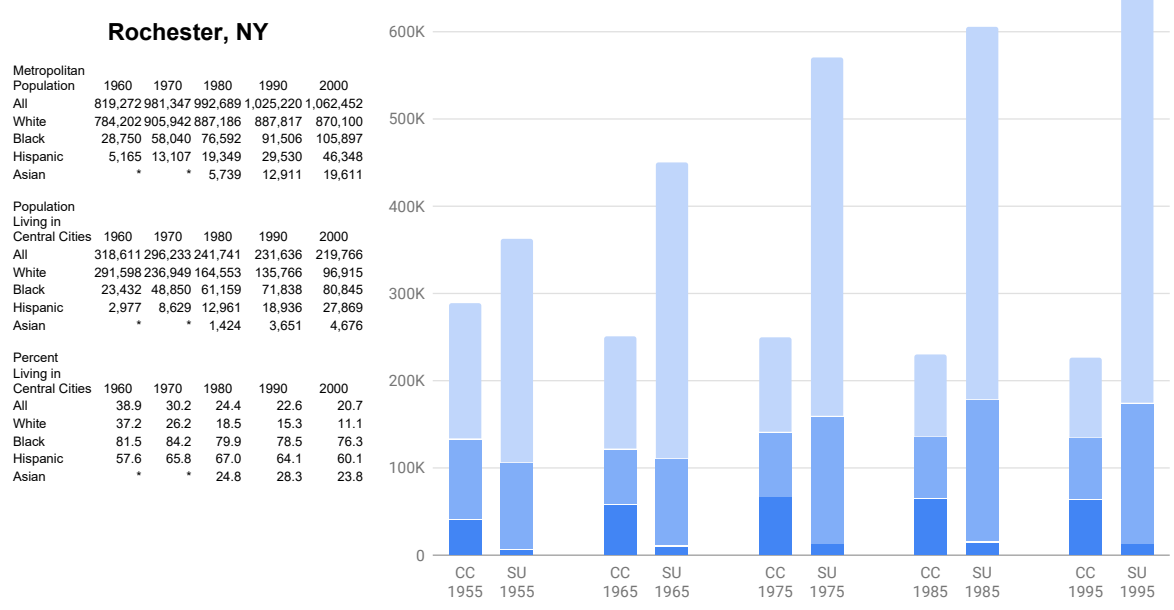

Urban-Suburban Net Migration Rate by Race
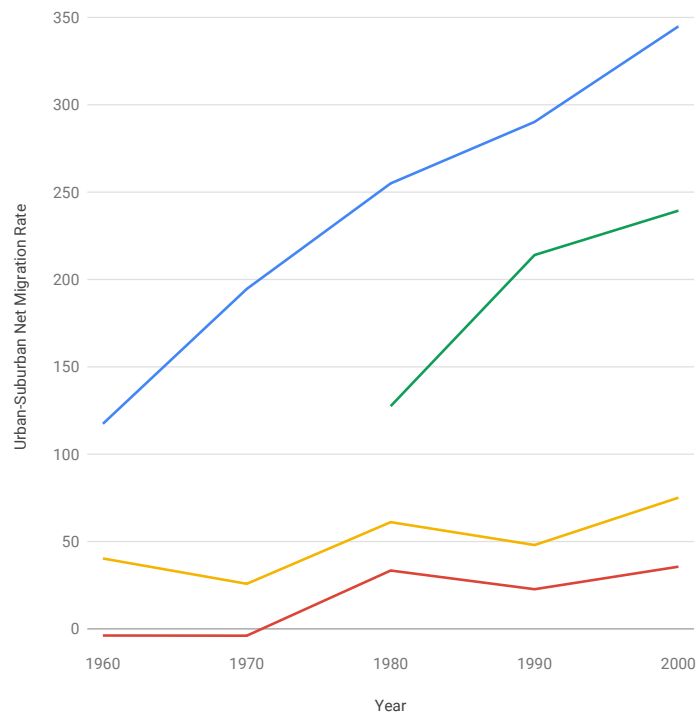

Low-Income Persons by Race

Middle-Income Persons by Race
Urban-Suburban Net Migration Rate by Income
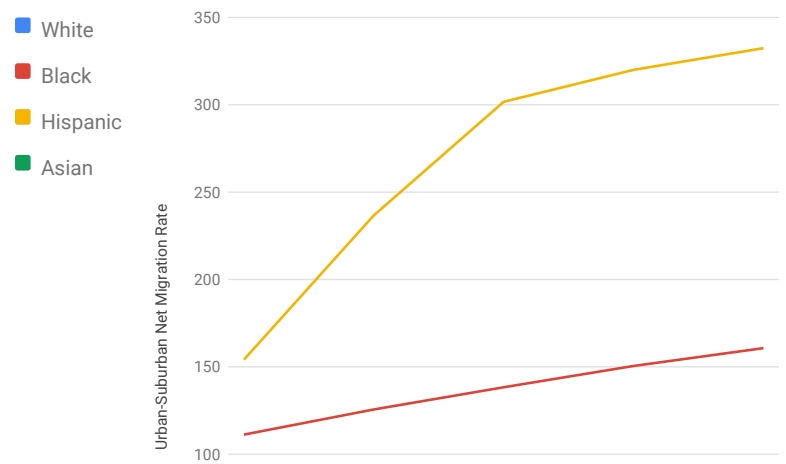

Low Income

- Middle Income

- High Income

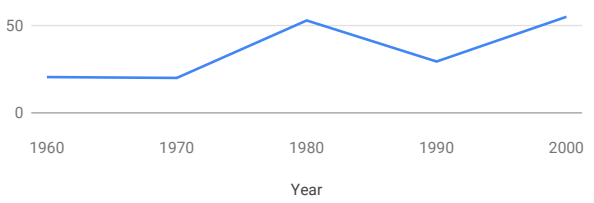

High-Income Persons by Race
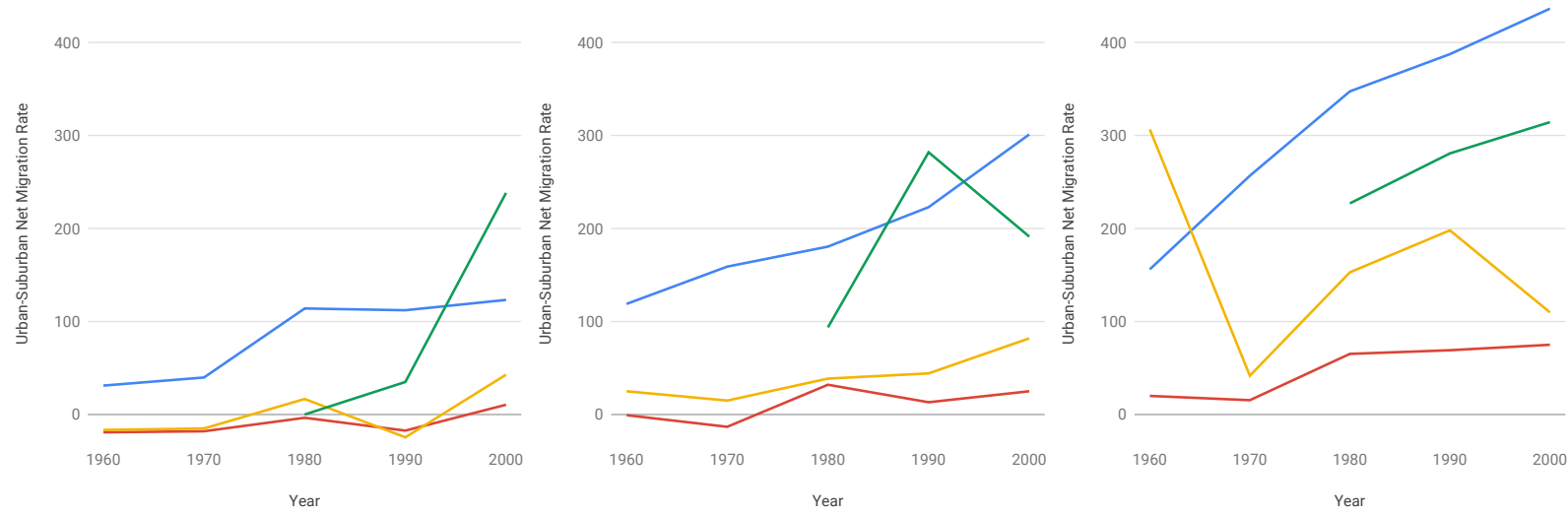

White

- Black

- Hispanic

- Asian 
Urban and Suburban Residence, 1955-1995, and 5-year migration

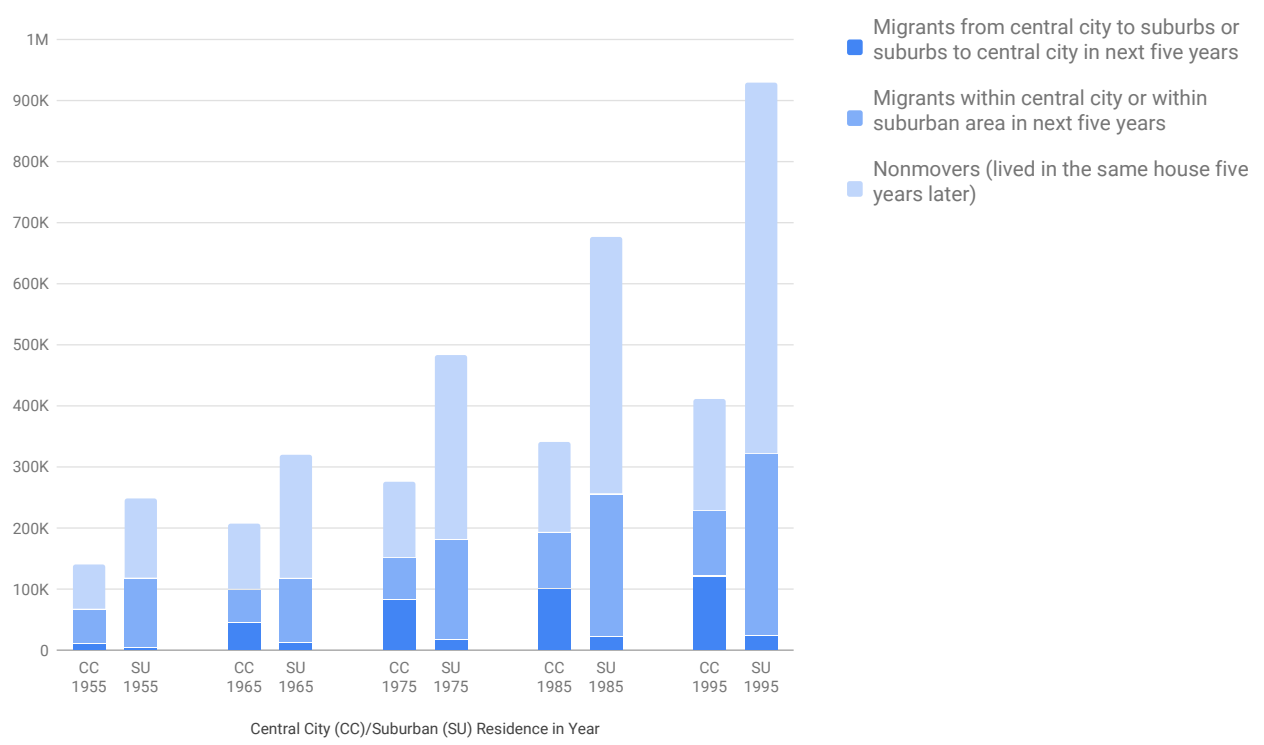

Urban-Suburban Net Migration Rate by Race

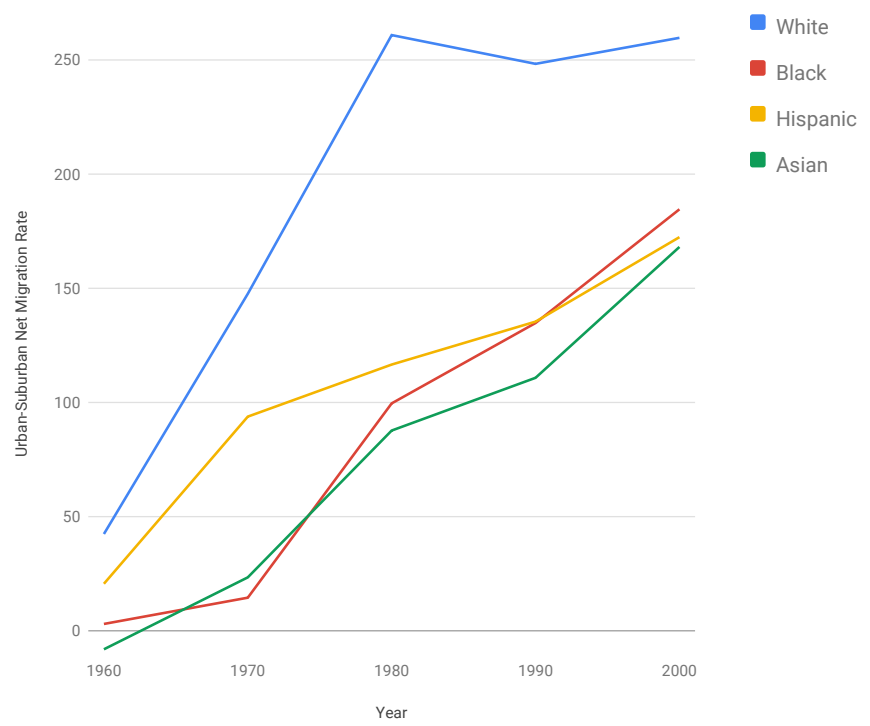

Low-Income Persons by Race

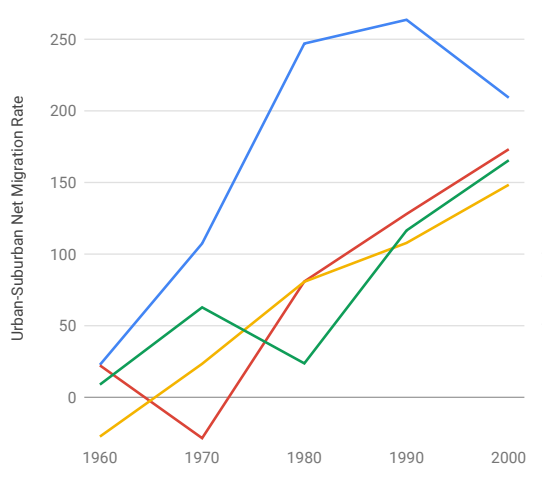

Year
Urban-Suburban Net Migration Rate by Income

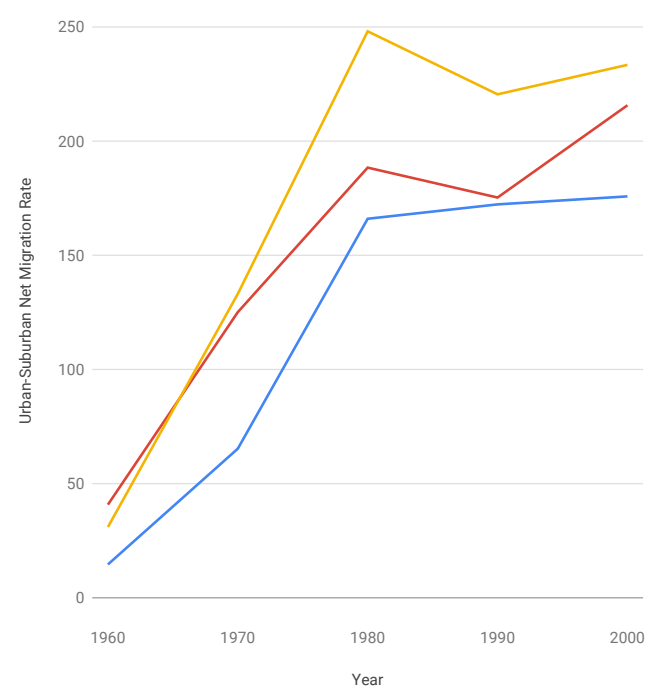

Low Income

- Middle Income

High Income
Middle-Income Persons by Race

High-Income Persons by Race

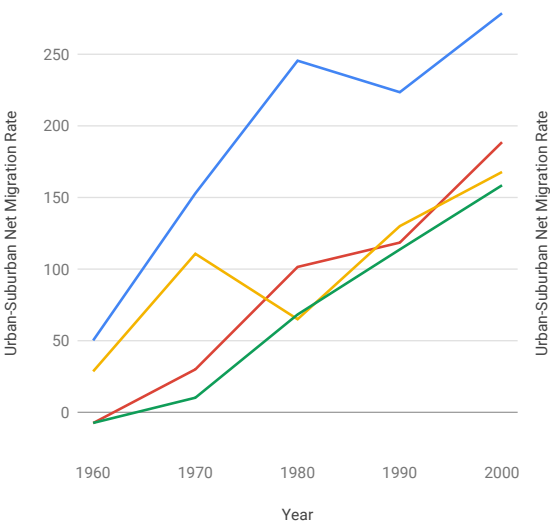

Year

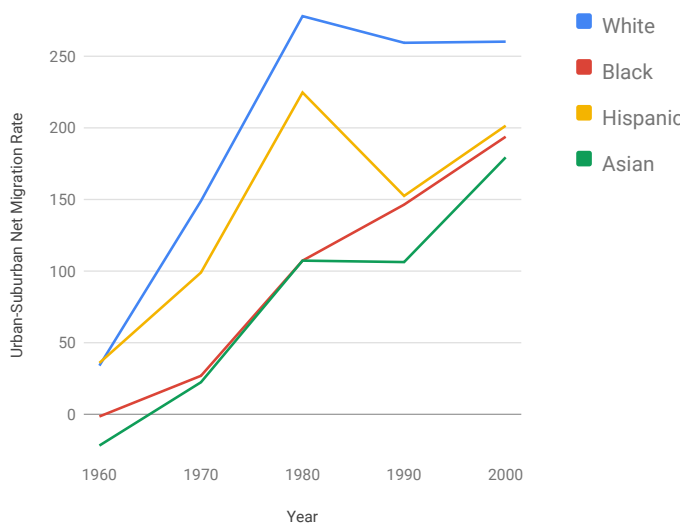


Urban and Suburban Residence, 1955-1995, and 5-year migration

\section{St. Louis, MO-IL}

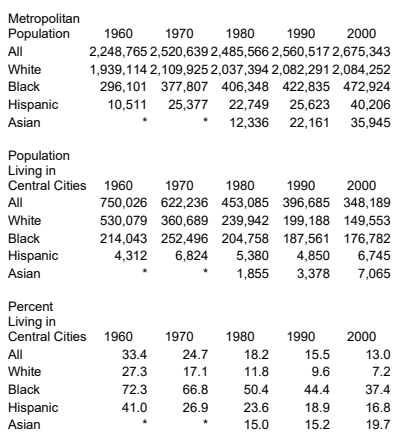

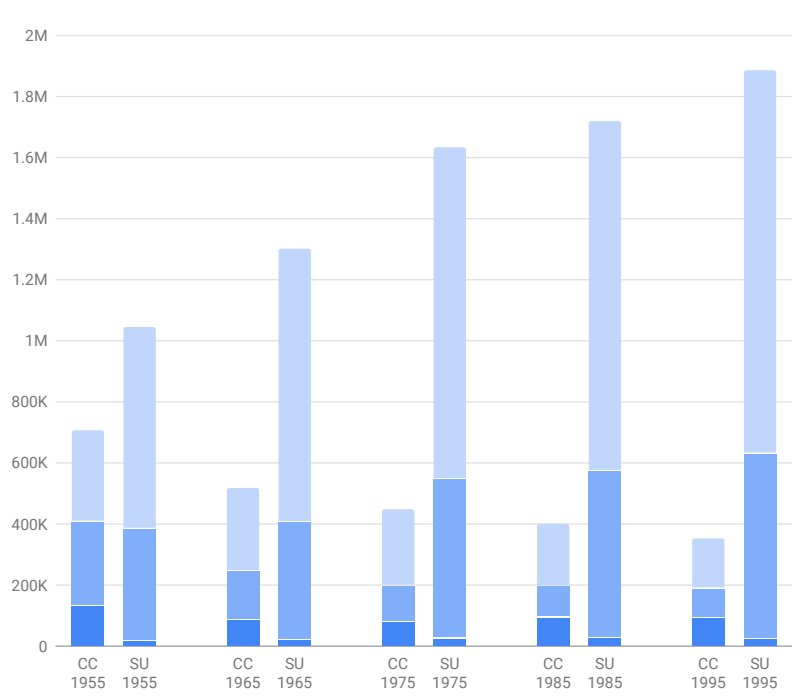

Migrants from central city to suburbs or suburbs to central city in next five years

Migrants within central city or within suburban area in next five years

Nonmovers (lived in the same house five

years later)
Urban-Suburban Net Migration Rate by Race

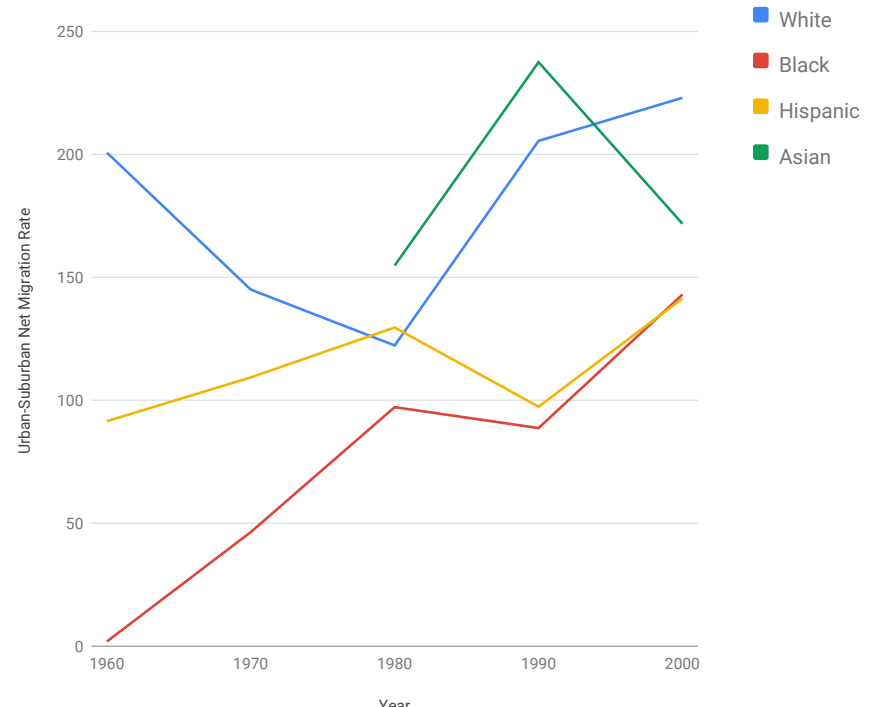

Low-Income Persons by Race

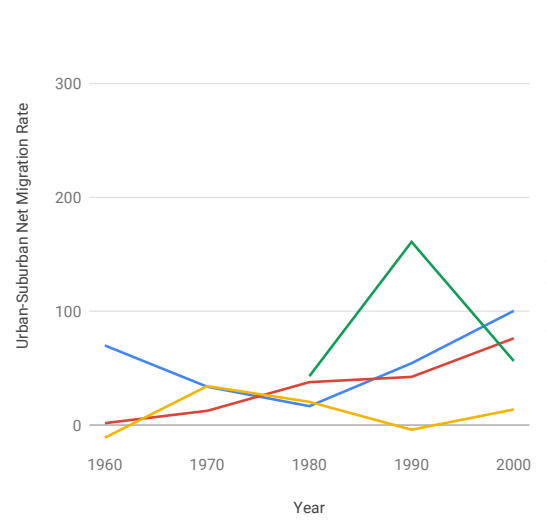

Middle-Income Persons by Race
Urban-Suburban Net Migration Rate by Income

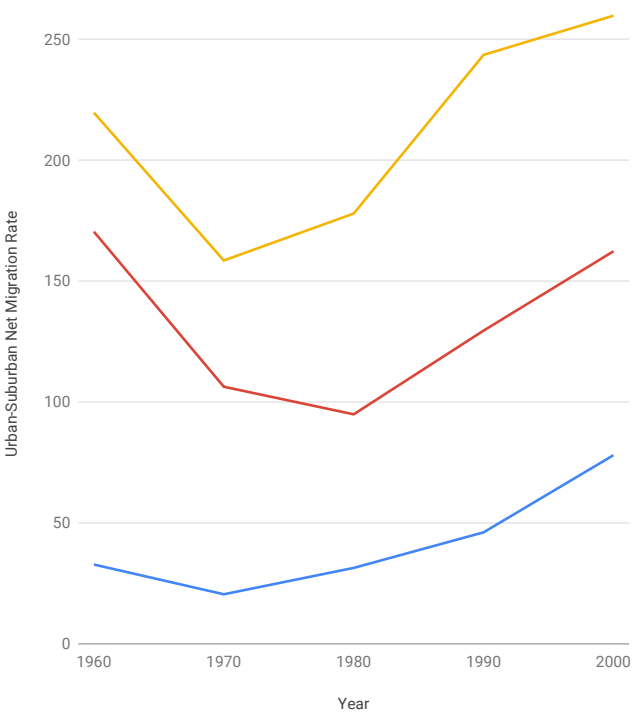

Low Income

- Middle Income

- High Income
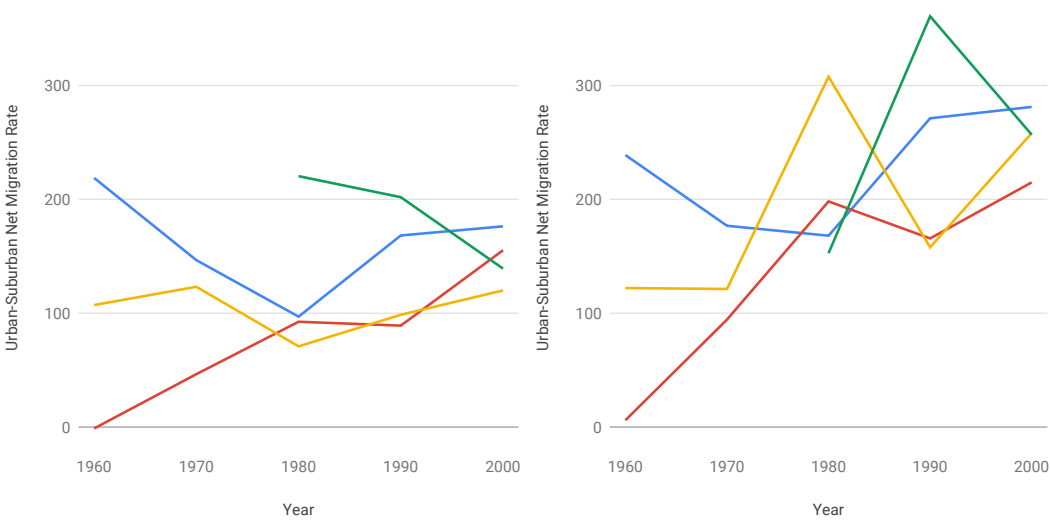

White

- Black

Hispanic

Asian 
Urban and Suburban Residence, 1955-1995, and 5-year migration

Salt Lake City, UT

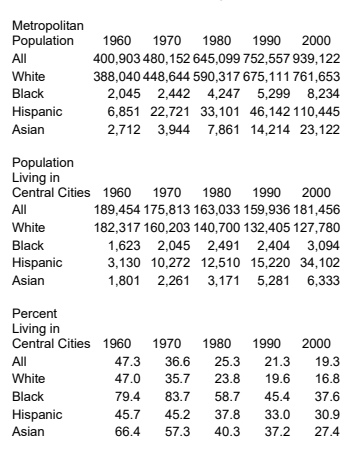

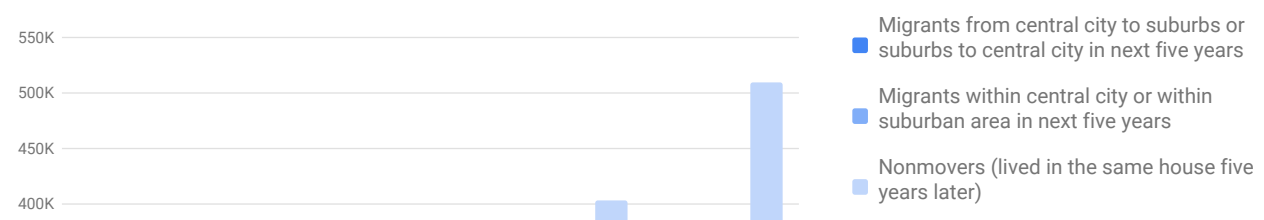

$350 \mathrm{~K}$

$300 \mathrm{~K}$

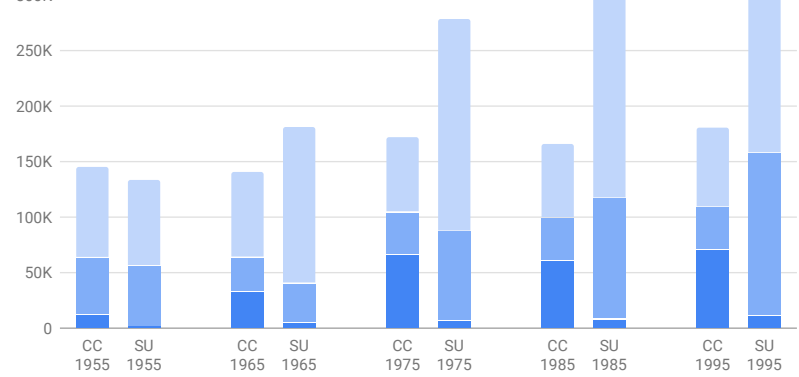

Central City (CC)/Suburban (SU) Residence in Year
Urban-Suburban Net Migration Rate by Race

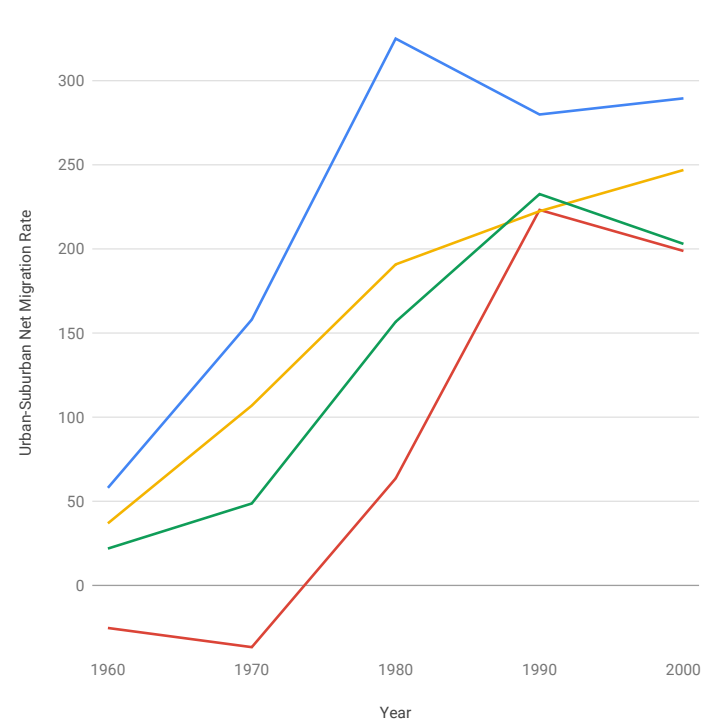

Low-Income Persons by Race
Middle-Income Persons by Race
Urban-Suburban Net Migration Rate by Income

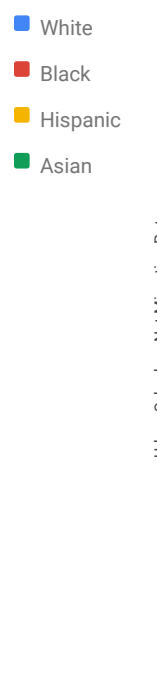

1960

1970

1980

Year

High-Income Persons by Race

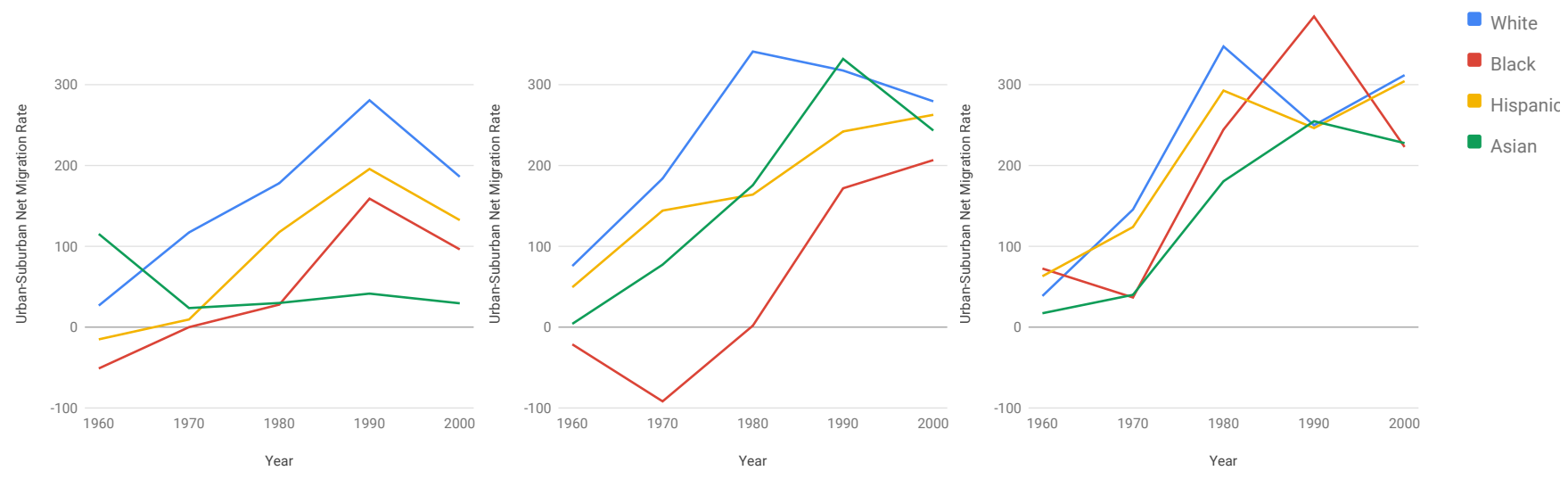


Urban and Suburban Residence, 1955-1995, and 5-year migration

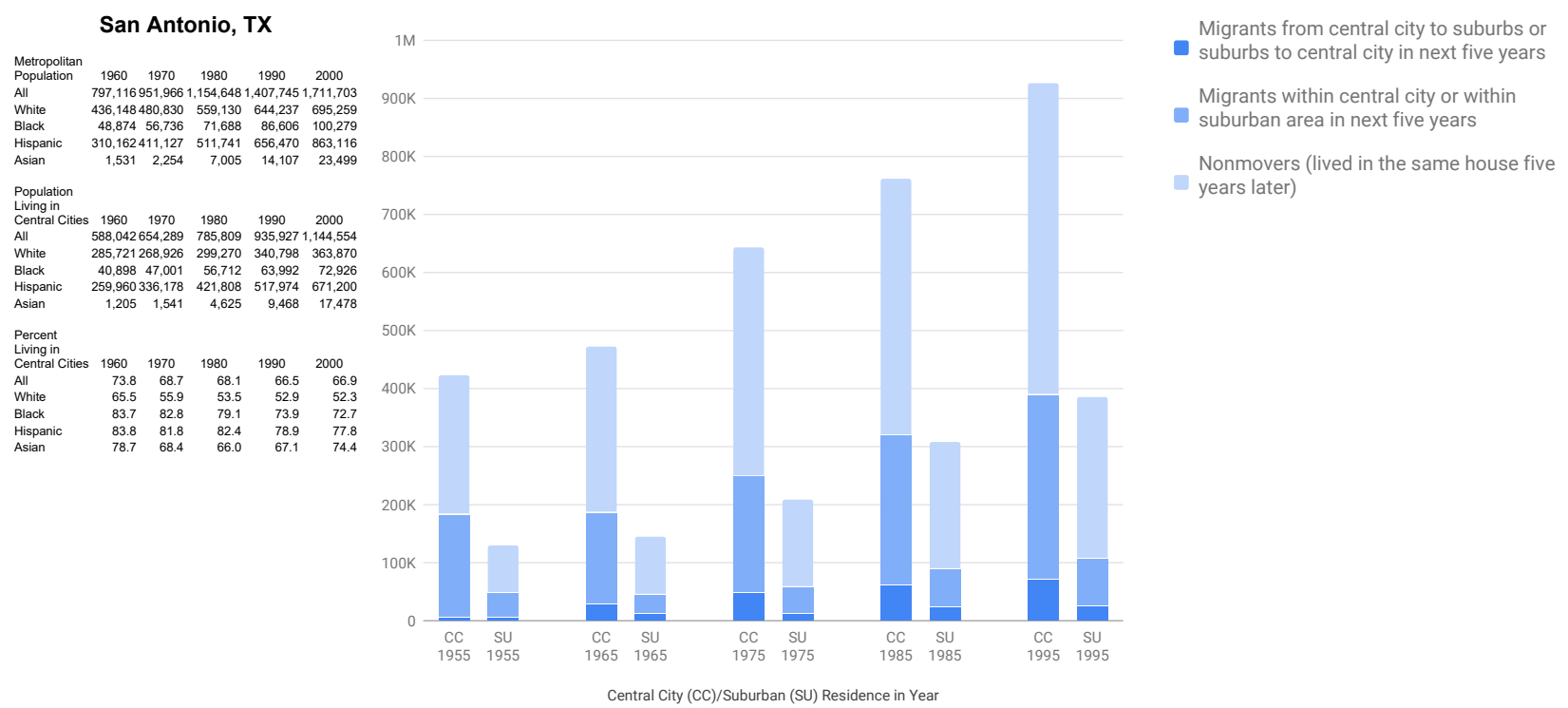

Urban-Suburban Net Migration Rate by Race

Urban-Suburban Net Migration Rate by Income
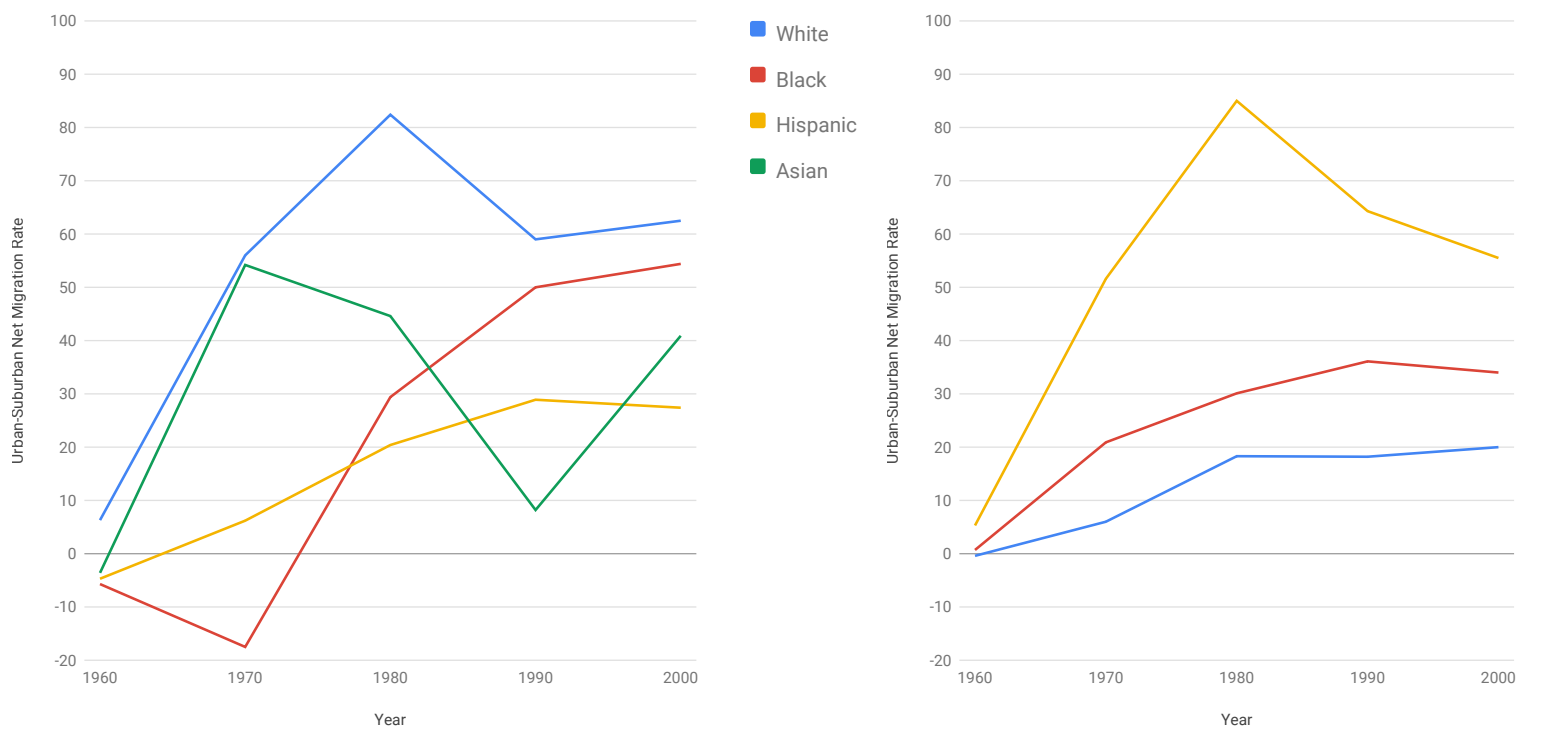

Low Income

Middle Income

High Income
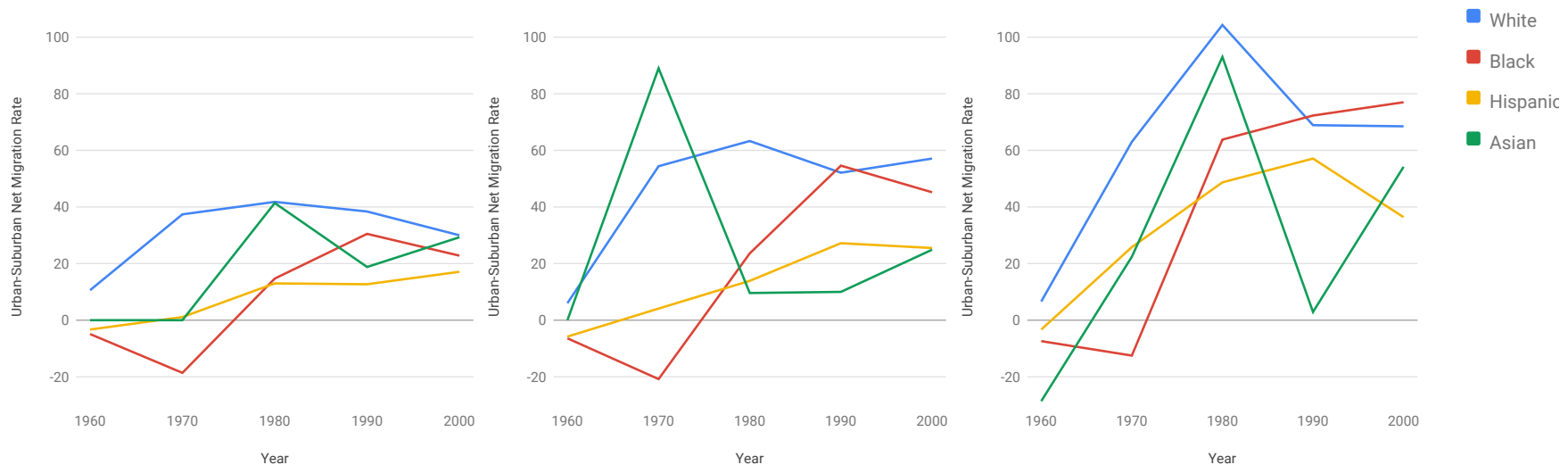
Urban and Suburban Residence, 1955-1995, and 5-year migration

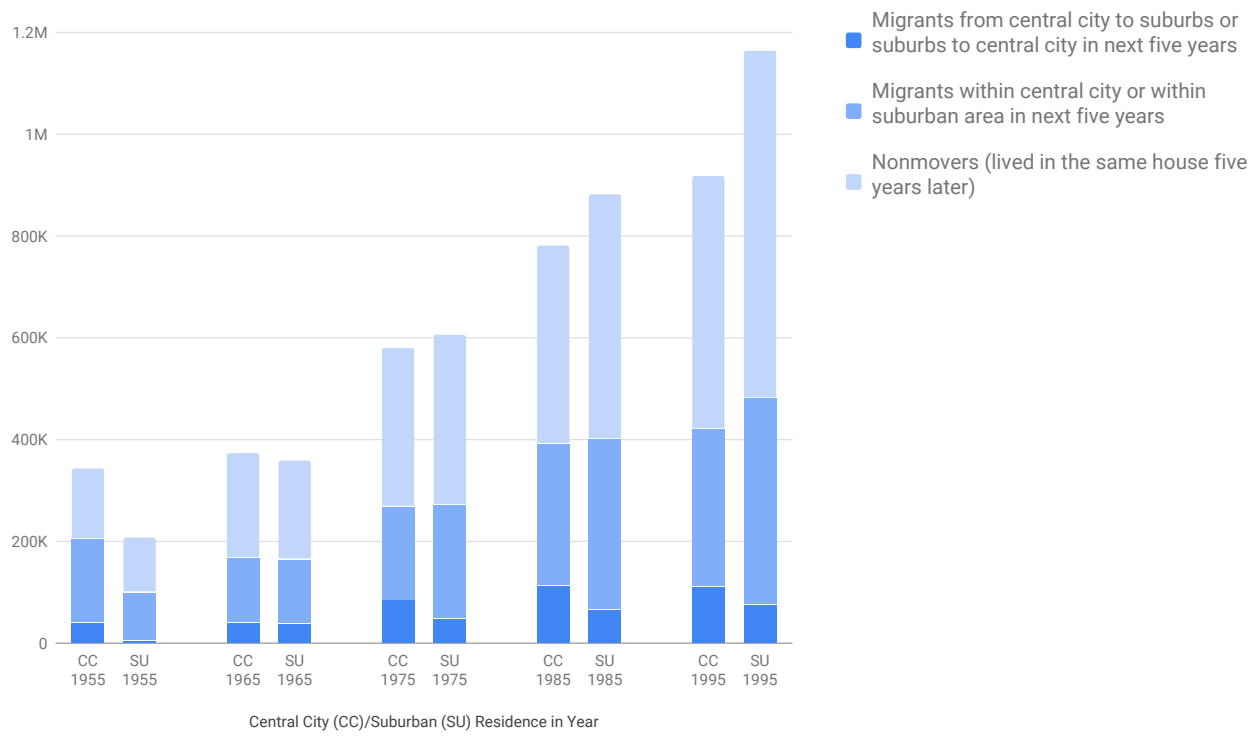

Urban-Suburban Net Migration Rate by Race

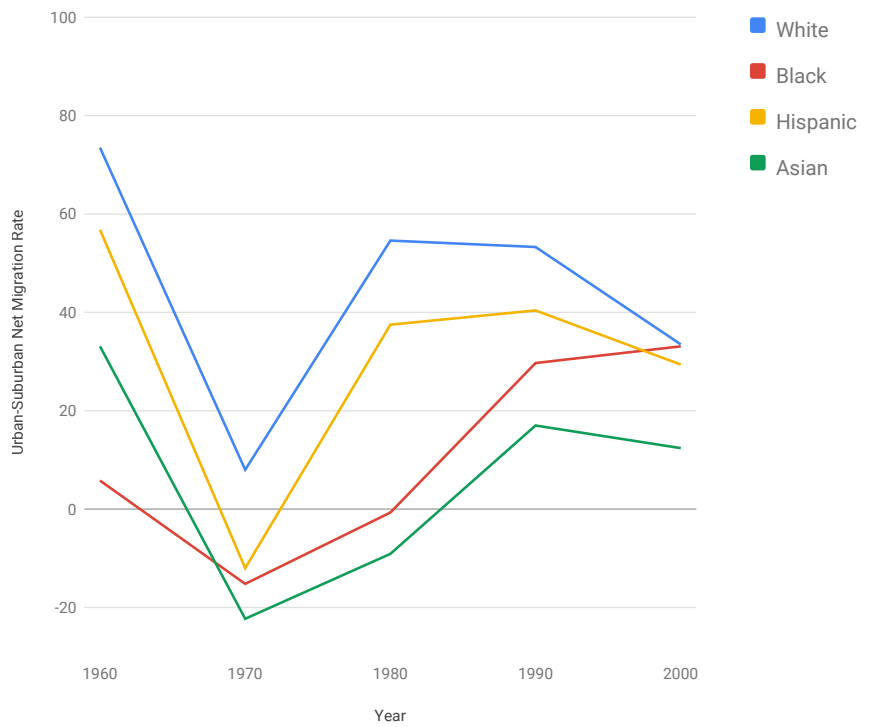

Low-Income Persons by Race

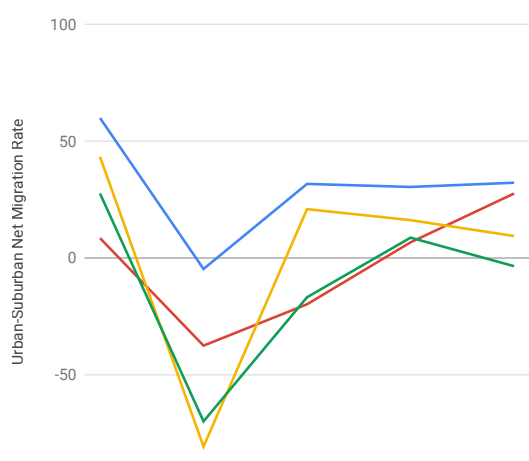

1960

Middle-Income Persons by Race

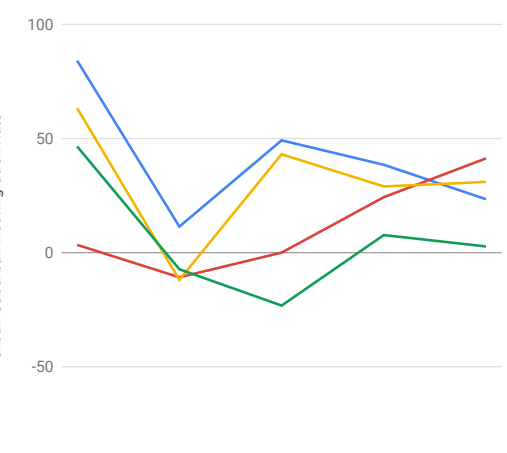

Year
San Diego, CA

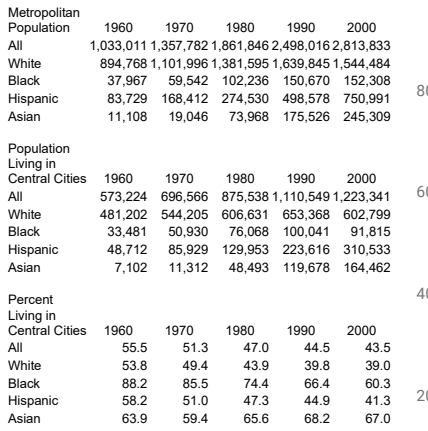

Urban-Suburban Net Migration Rate by Income
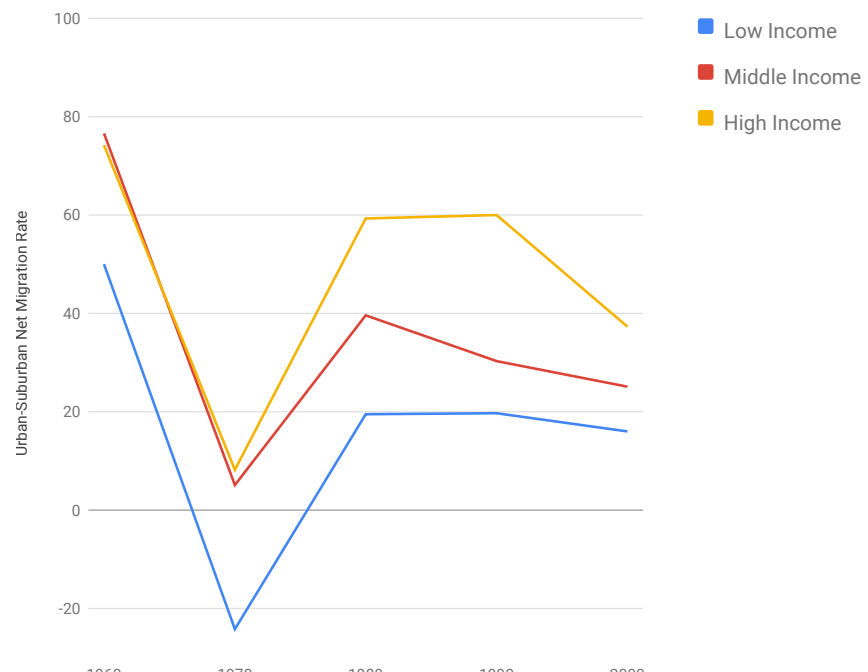

1960

Year

High-Income Persons by Race

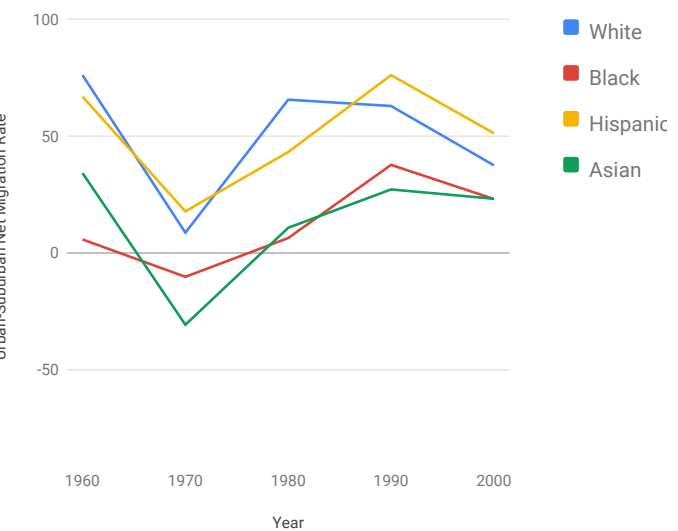




\section{$2.5 \mathrm{M}$}

\section{San Francisco-Oakland, CA}

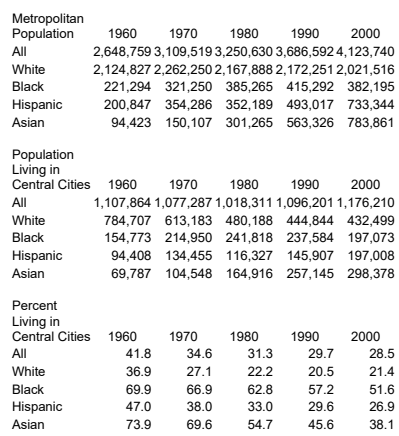

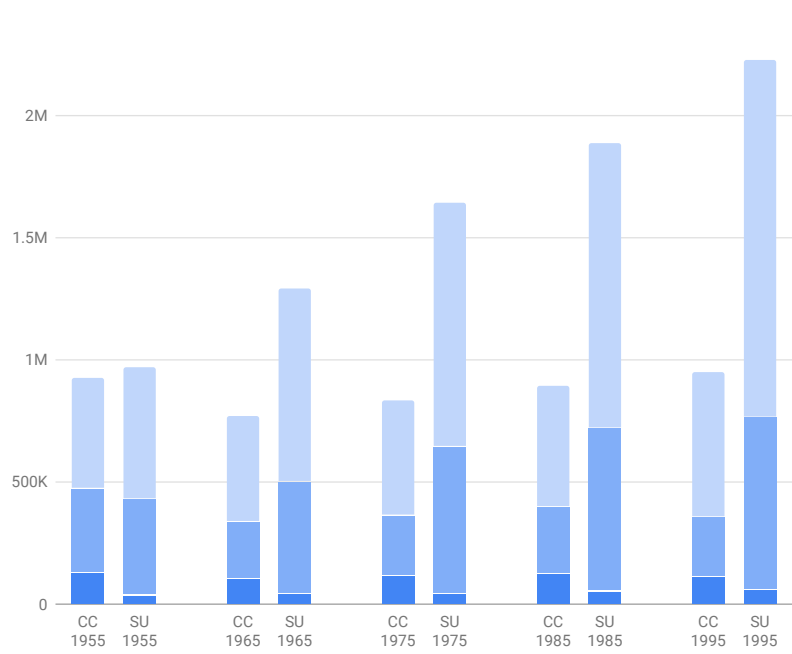

Central City (CC)/Suburban (SU) Residence in Year
Migrants from central city to suburbs or suburbs to central city in next five years

Migrants within central city or within - suburban area in next five years

Nonmovers (lived in the same house five years later)
Urban-Suburban Net Migration Rate by Race

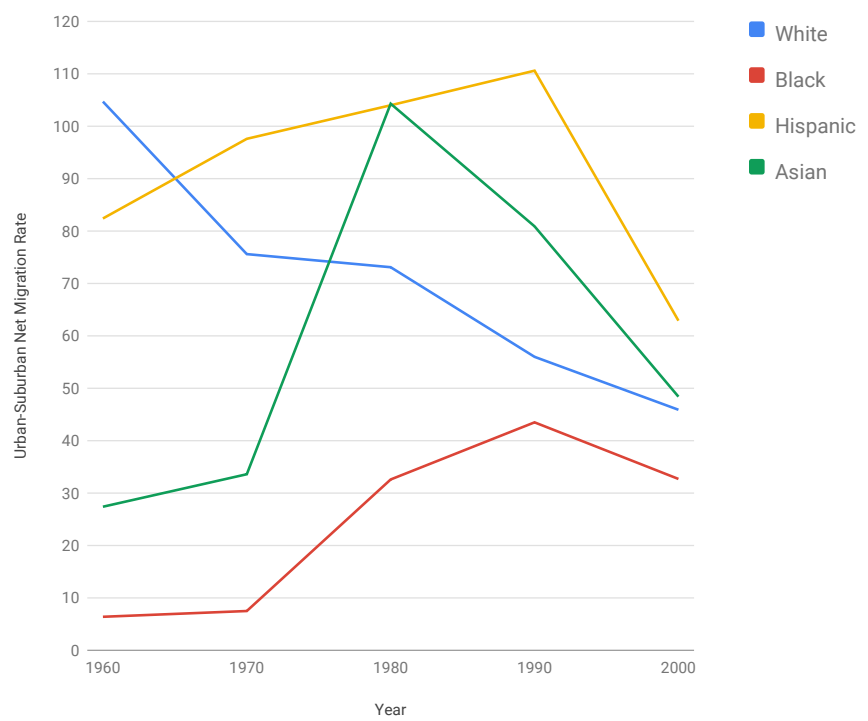

Low-Income Persons by Race

Middle-Income Persons by Race

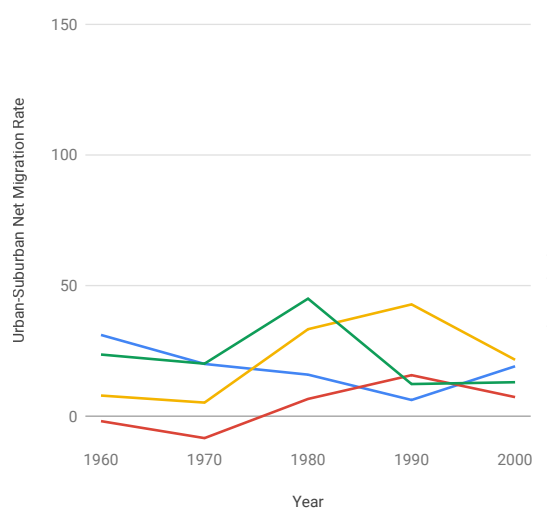

150

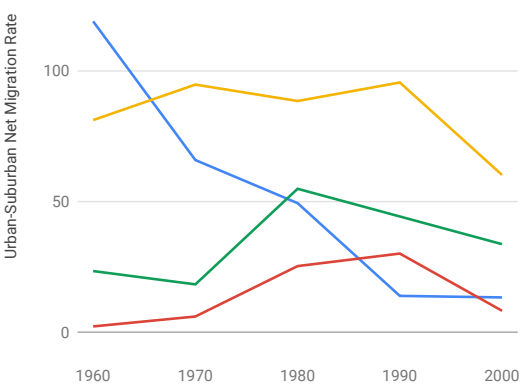

Year
Urban-Suburban Net Migration Rate by Income

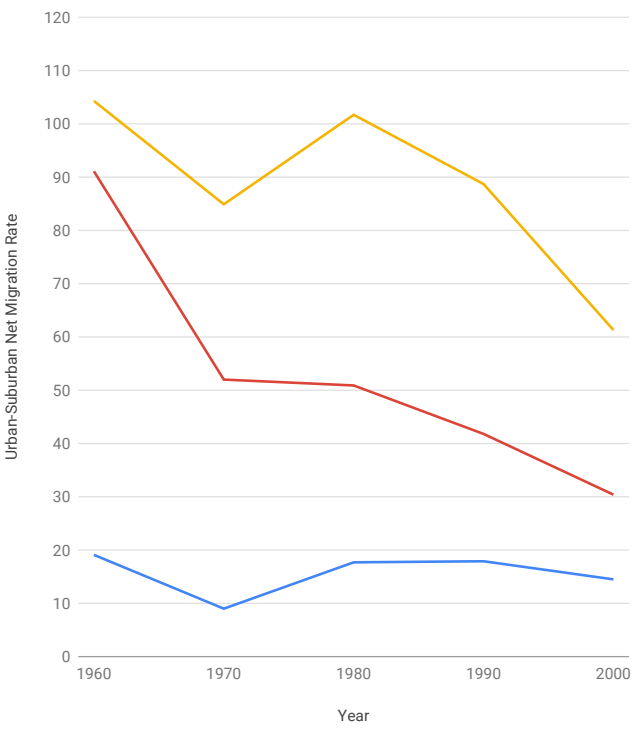

Low Income

- Middle Income

- High Income

High-Income Persons by Race

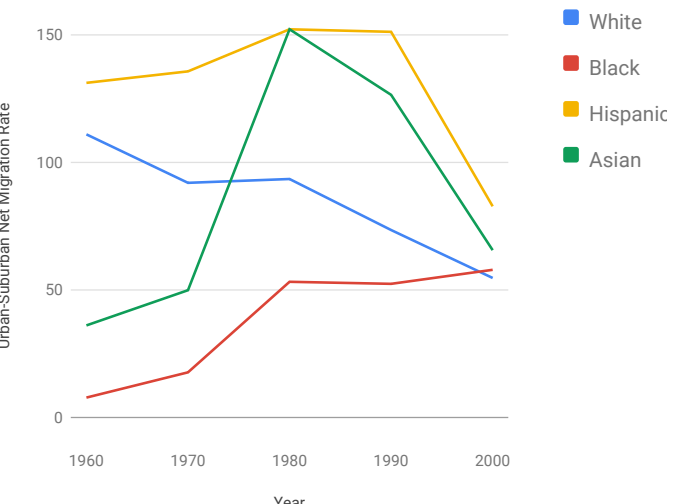


Urban and Suburban Residence, 1955-1995, and 5-year migration

\section{San Jose, CA}

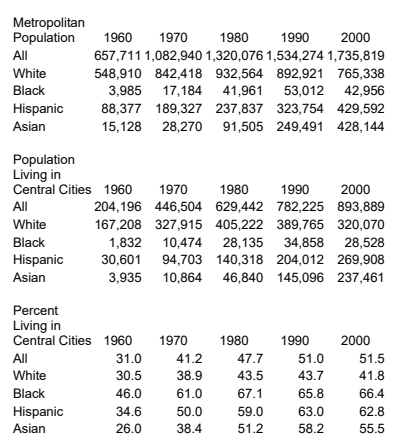

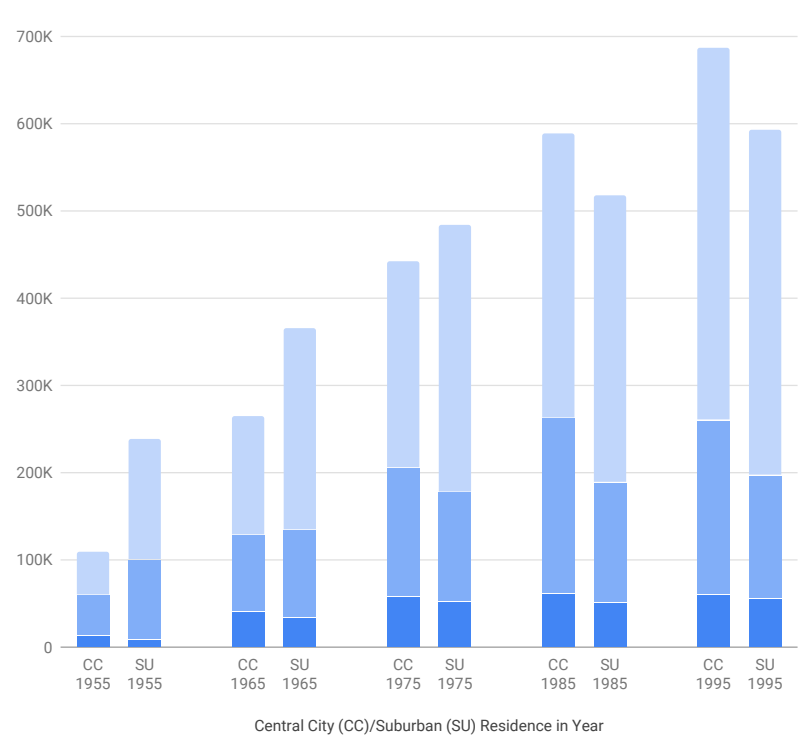

Migrants from central city to suburbs or - suburbs to central city in next five years

Migrants within central city or within suburban area in next five years

Nonmovers (lived in the same house five years later)
Urban-Suburban Net Migration Rate by Race

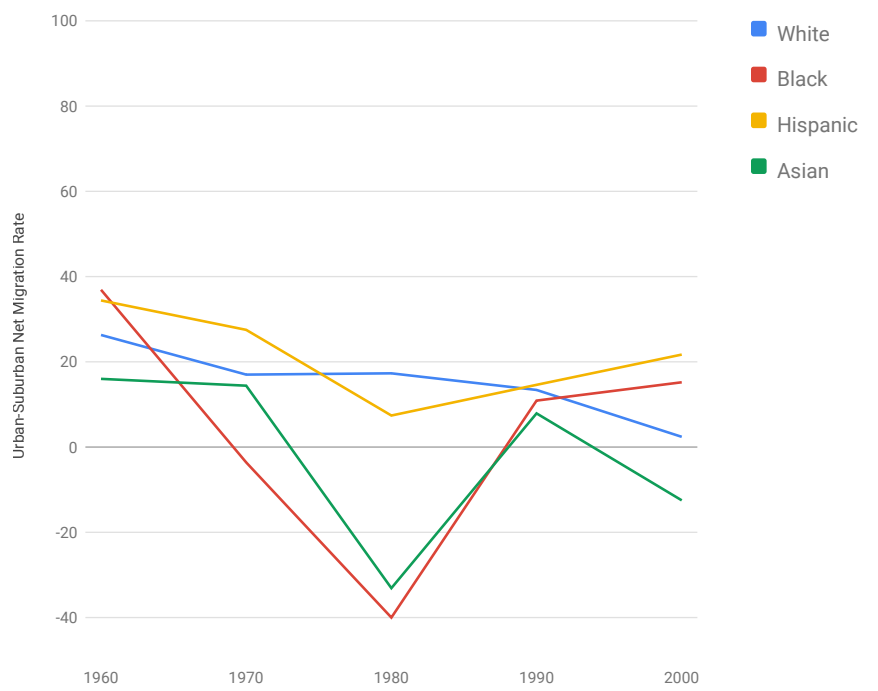

Year
Middle-Income Persons by Race

100

Urban-Suburban Net Migration Rate by Income
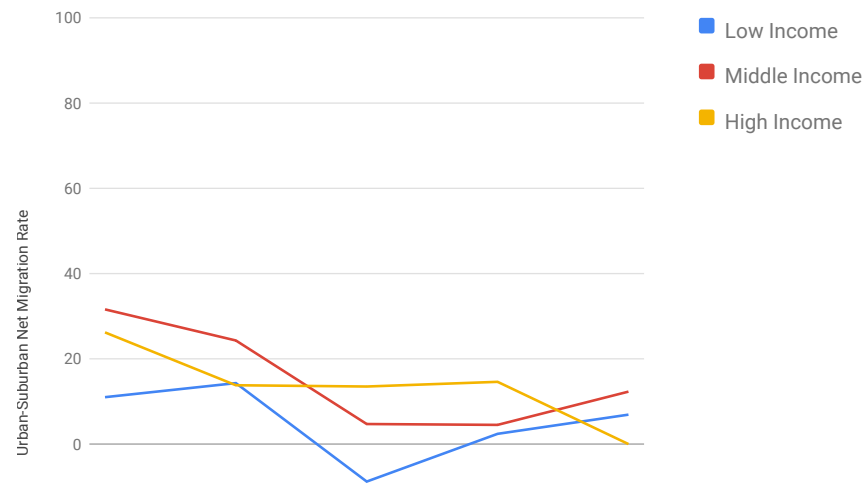

$-20$

40

1960

1970

1980

1990

Low-Income Persons by Race

100

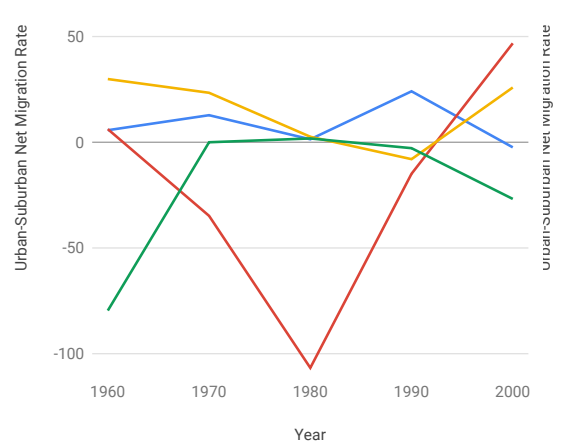

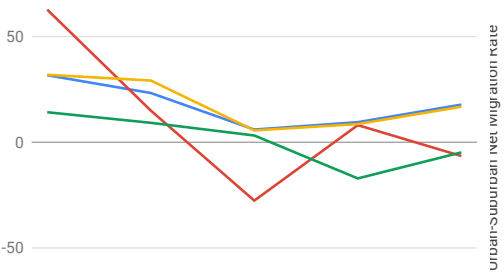

$-100$

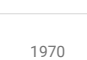

1970

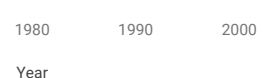

Year
High-Income Persons by Race

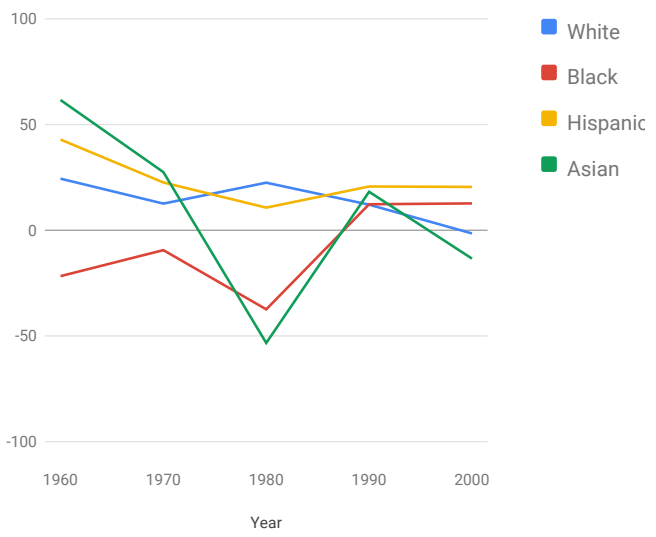


Urban and Suburban Residence, 1955-1995, and 5-year migration

\section{Scranton--Wilkes-Barre, PA}

$\begin{array}{llllll}\begin{array}{l}\text { Metropolitan } \\ \text { Population }\end{array} 1960 & 1970 & 1980 & 1990 \quad 2000\end{array}$

$\begin{array}{ll}\text { All } & 598,316595,490597,420575,264560,625 \\ \text { White } & 594974590,25589,791563668538610\end{array}$

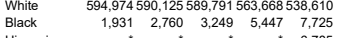

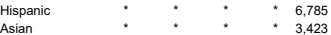

Population $300 \mathrm{~K}$

$\begin{array}{lllll}\text { Living in } & & & & \end{array}$

All $\quad 174,994162,311139,668$ 129,328 119,538

White $\quad 173,002159,672136,627124,721110,163 \quad 250$

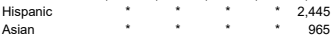

Percent $200 \mathrm{~K}$

$\begin{array}{lccccc}\text { Central Cities } & 1960 & 1970 & 1980 & 1990 & 2000 \\ \text { All } & 29.2 & 27.3 & 23.4 & 225 & 213\end{array}$

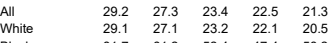

\begin{tabular}{lllllll} 
Black & 81.7 & 27.1 & 23.2 & 22.1 & 20.5 \\
Hispanic & & 61.2 & 52.4 & 47.4 & 58.2 \\
As & & & & & & \\
\hline
\end{tabular}

$\begin{array}{ll}\text { Hispanic } & \vdots \\ \text { Asian } & :\end{array}$
$400 \mathrm{~K}$

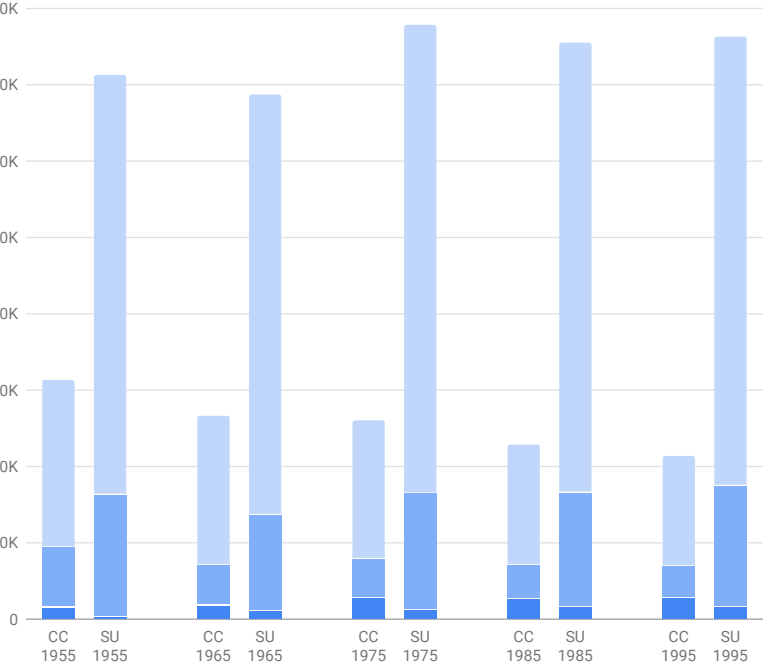

Migrants from central city to suburbs or - suburbs to central city in next five years

Migrants within central city or within

suburban area in next five years

Nonmovers (lived in the same house five

years later)
Urban-Suburban Net Migration Rate by Race

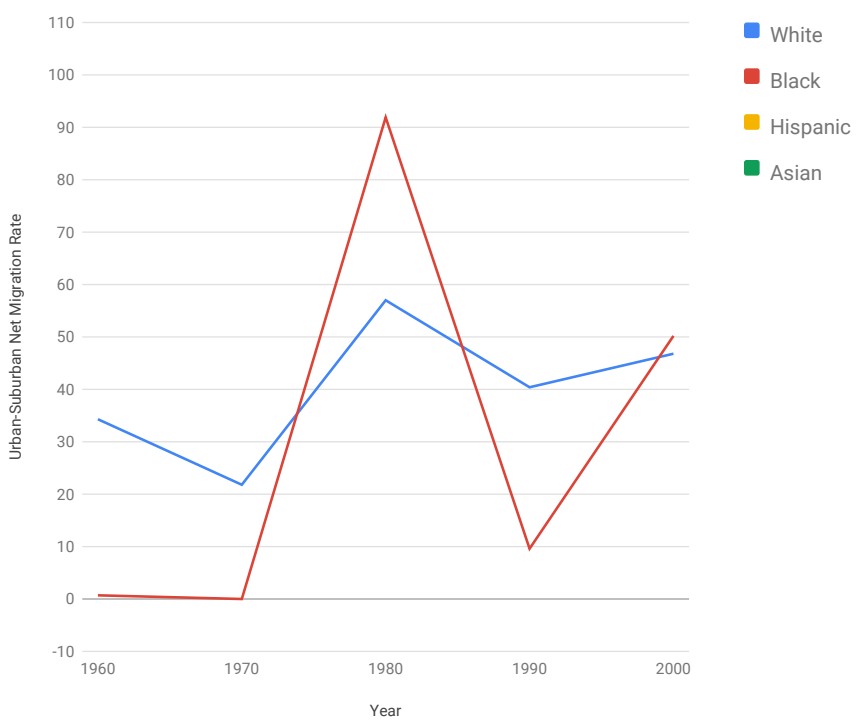

Low-Income Persons by Race
Middle-Income Persons by Race
Urban-Suburban Net Migration Rate by Income

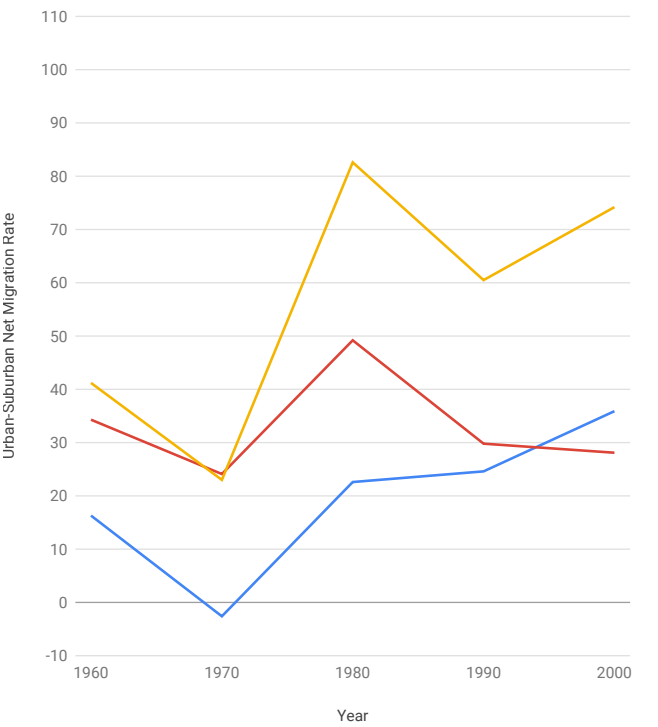

High-Income Persons by Race
Low Income

- Middle Income

High Income

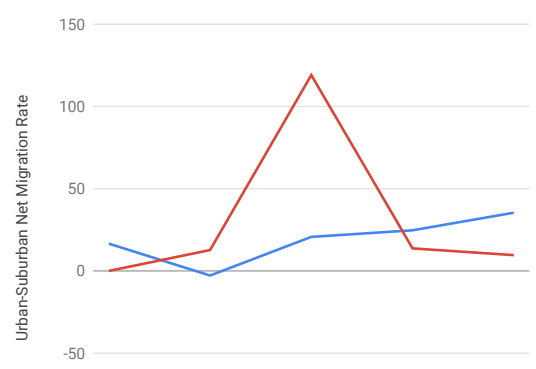

$-100$

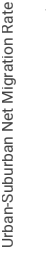

150

White

Black

Hispanic

- Asian 


\section{Urban and Suburban Residence, 1955-1995, and 5-year migration}

Seattle-Tacoma, WA

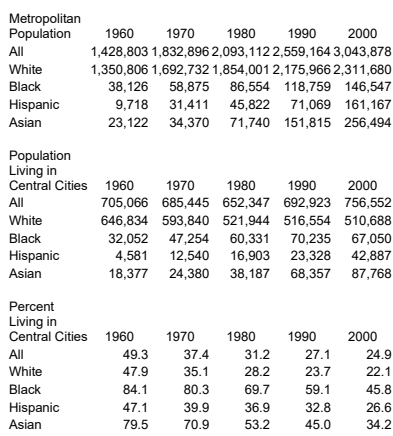

Migrants from central city to suburbs or suburbs to central city in next five years

Migrants within central city or within suburban area in next five years

Nonmovers (lived in the same house five

years later)
Urban-Suburban Net Migration Rate by Race

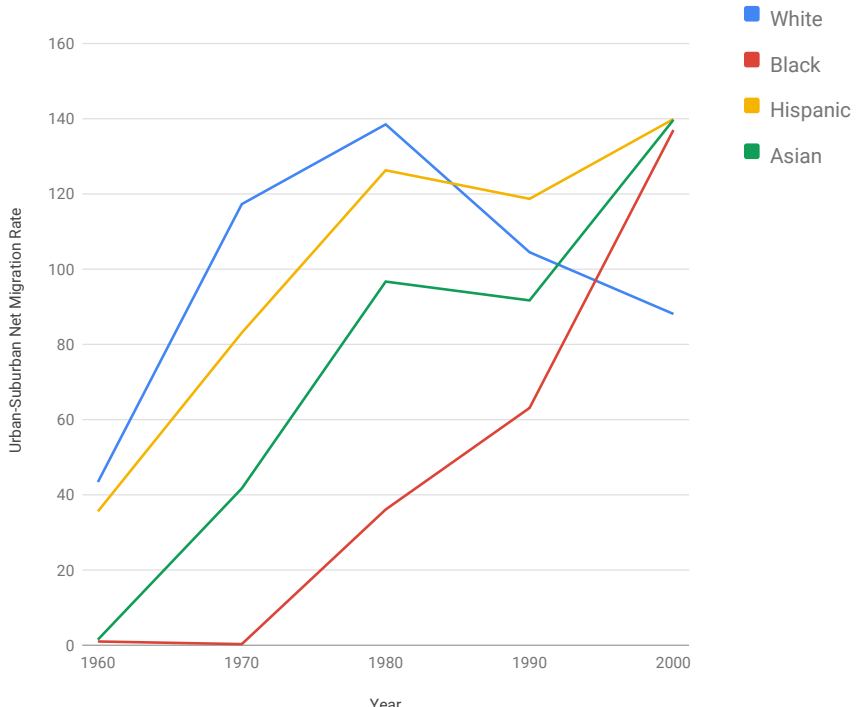

Low-Income Persons by Race

Middle-Income Persons by Race
Urban-Suburban Net Migration Rate by Income

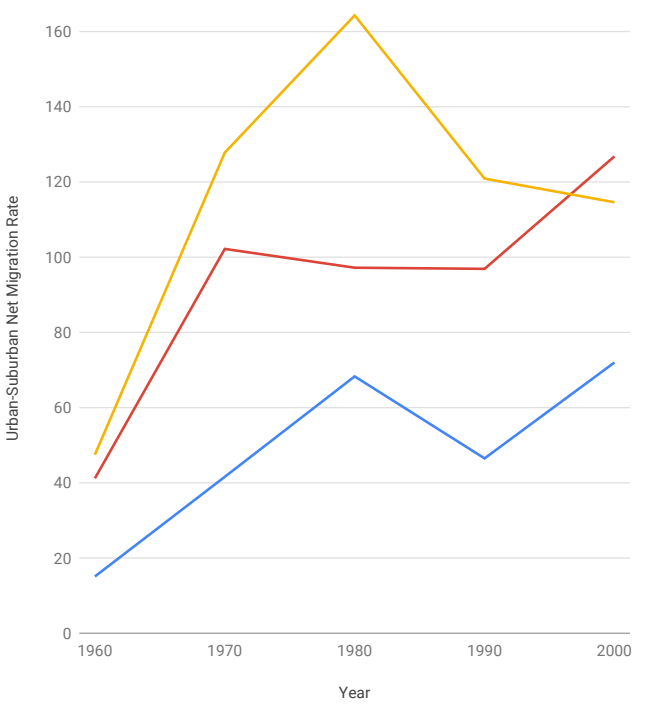

Low Income

Middle Income

High Income
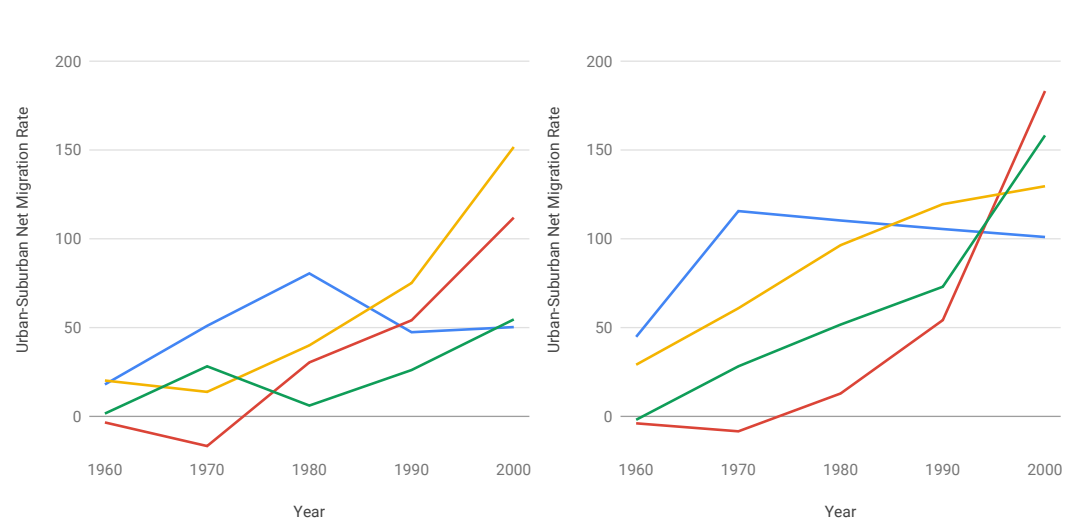

High-Income Persons by Race

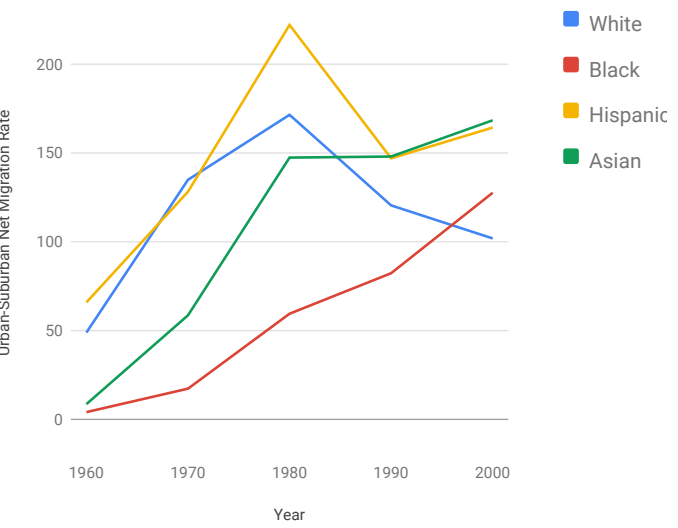




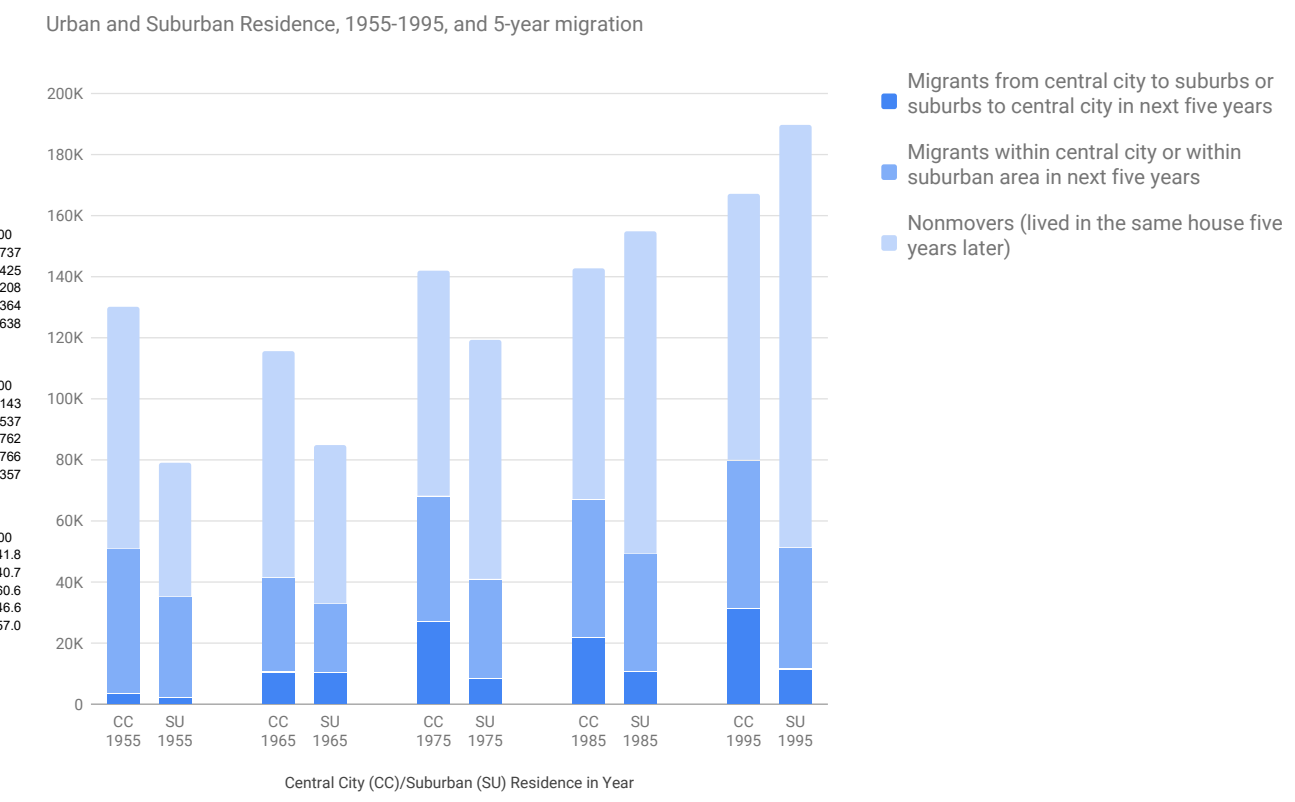

Urban-Suburban Net Migration Rate by Race

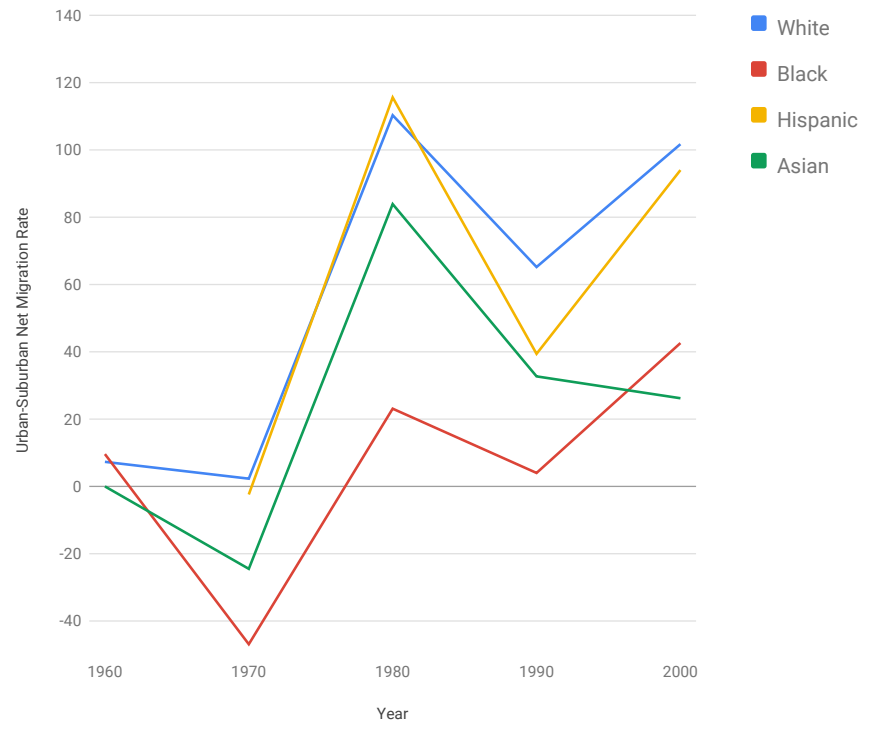

Low-Income Persons by Race
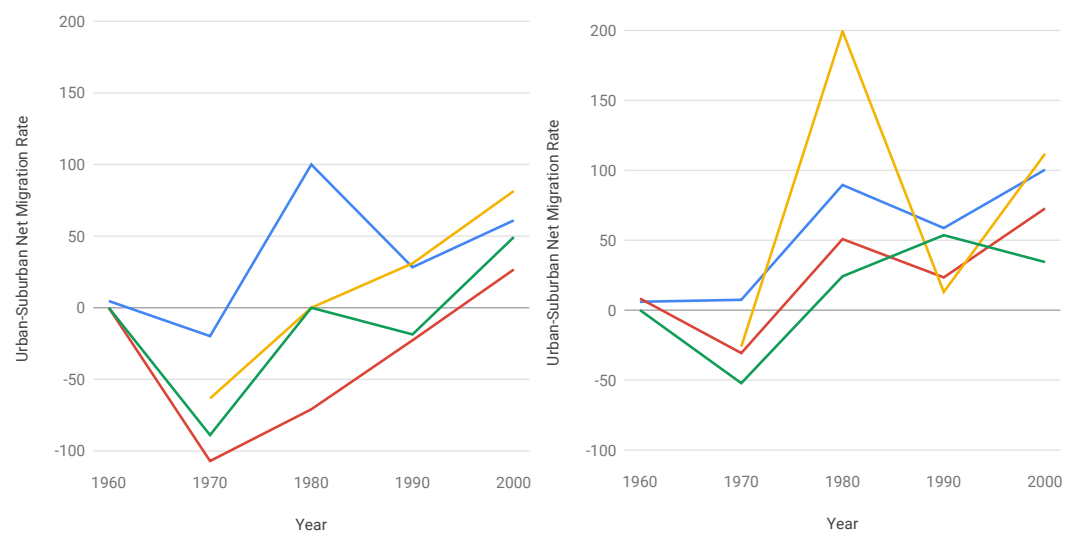

Urban-Suburban Net Migration Rate by Income

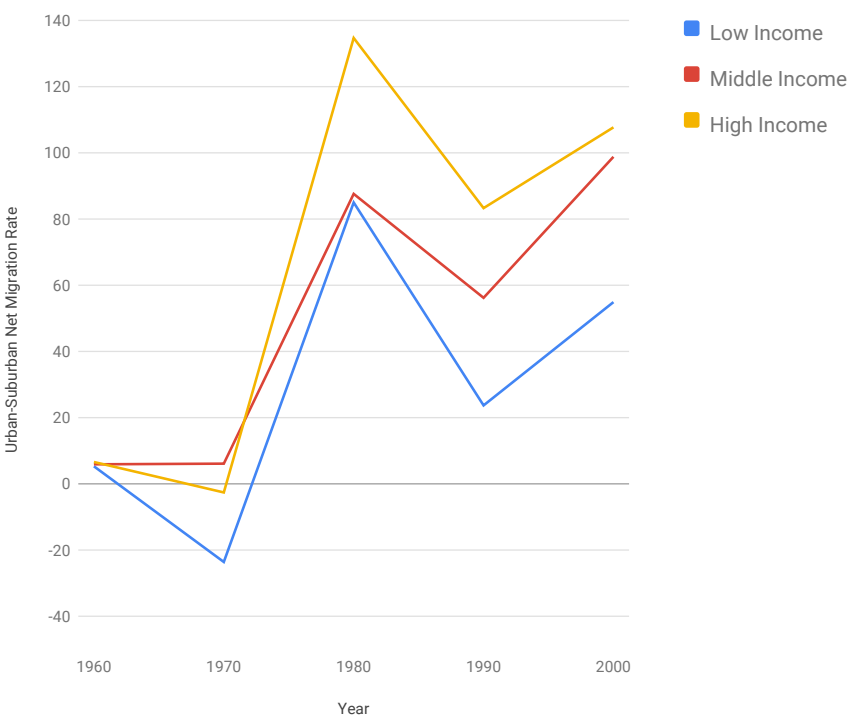

High-Income Persons by Race

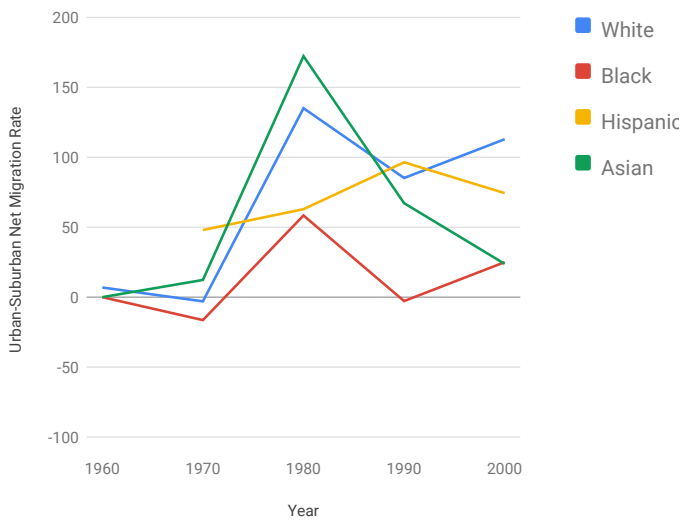


Urban and Suburban Residence, 1955-1995, and 5-year migration

$400 \mathrm{~K} \quad \begin{aligned} & \text { Migrants from central city to suburbs or } \\ & \text { suburbs to central city in next five years }\end{aligned}$

Migrants within central city or within

suburban area in next five years

Nonmovers (lived in the same house five

years later)
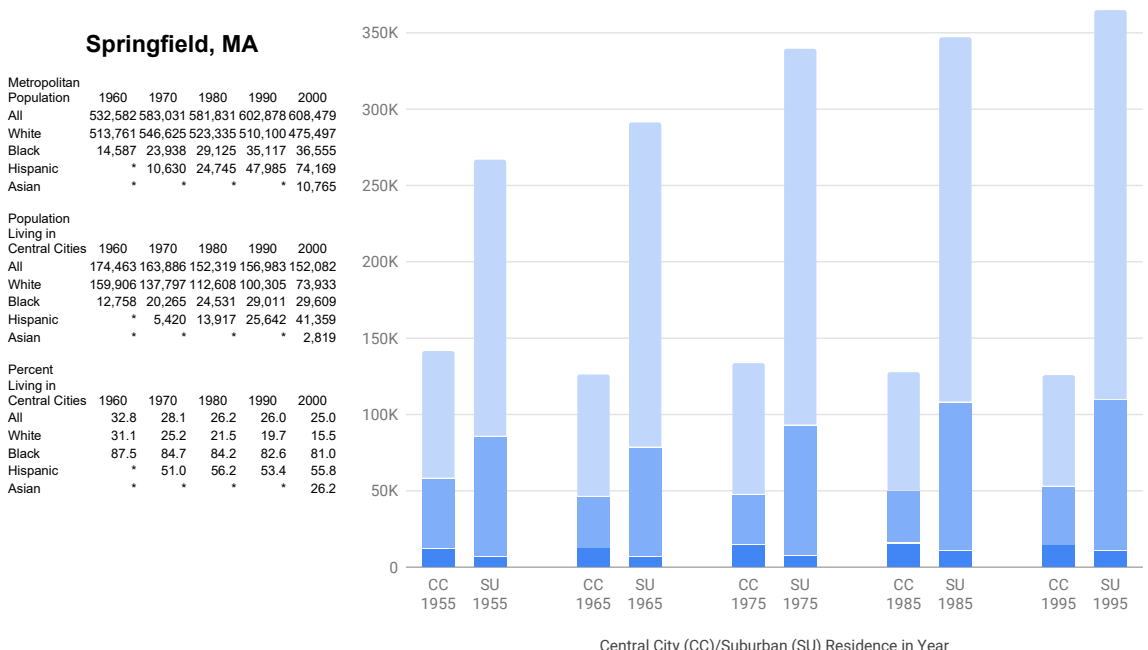

Urban-Suburban Net Migration Rate by Race

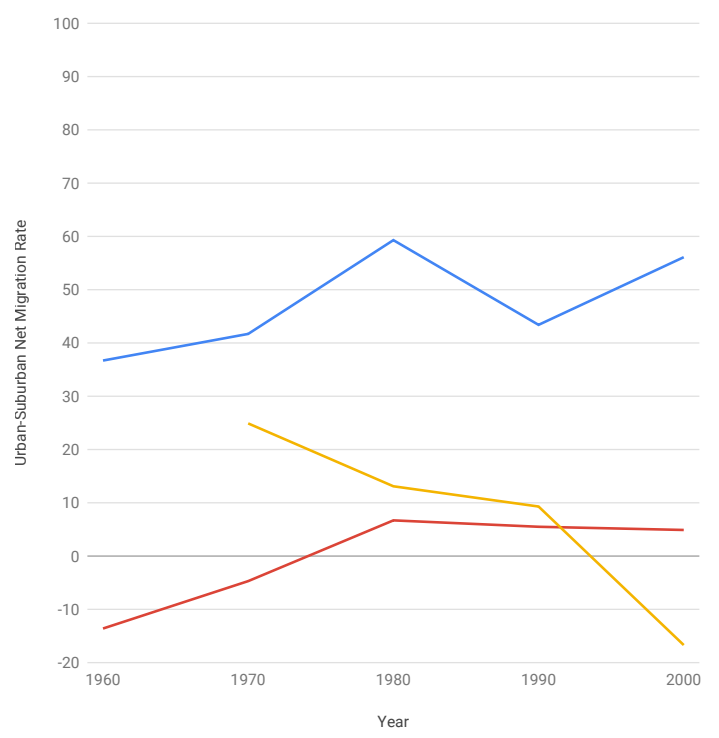

Low-Income Persons by Race

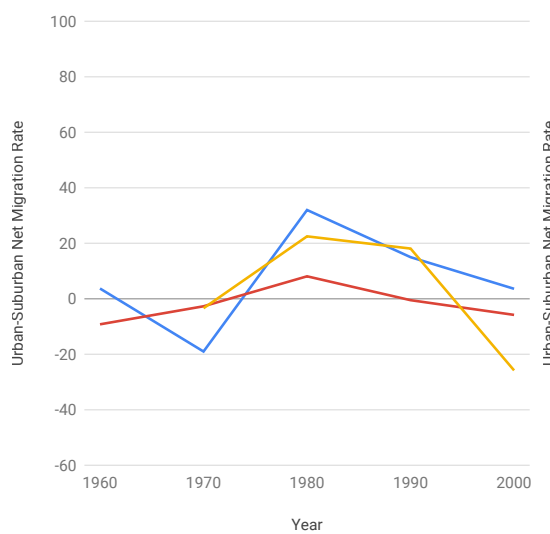

Middle-Income Persons by Race

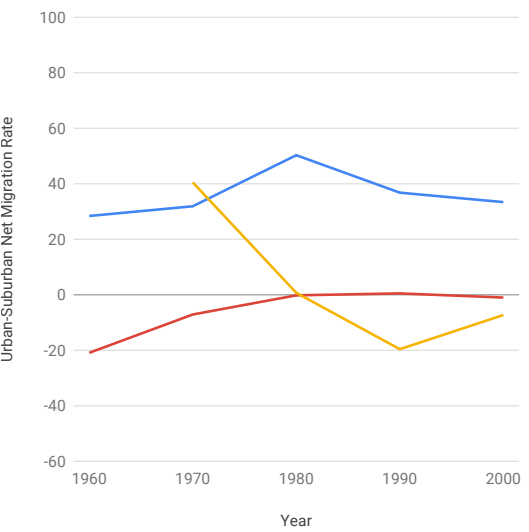

Year

Urban-Suburban Net Migration Rate by Income
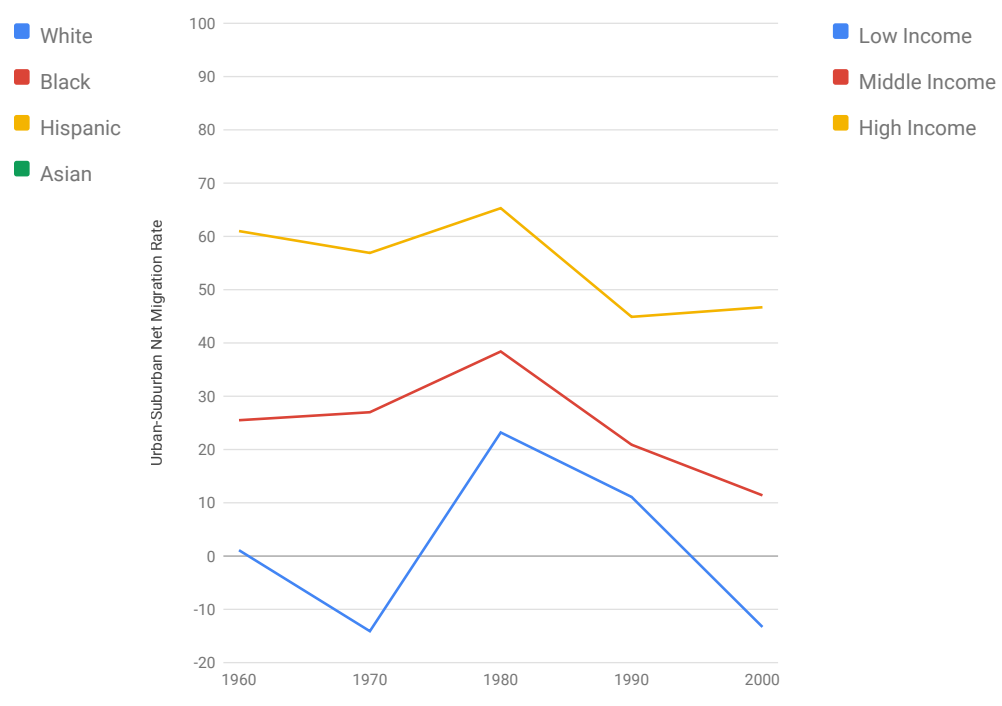

Year

High-Income Persons by Race

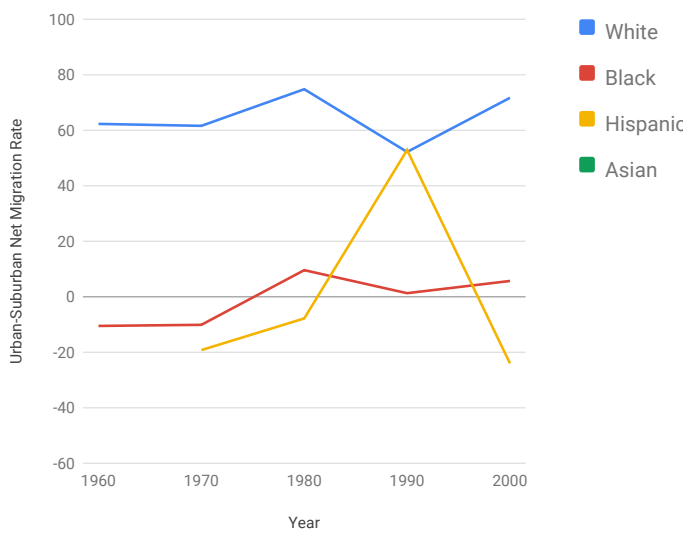


Urban and Suburban Residence, 1955-1995, and 5-year migration

\section{Stockton, CA}

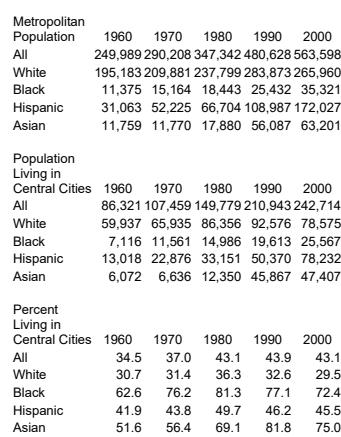

$250 \mathrm{~K}$

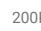

Migrants within central city or within
suburban area in next five years
Nonmovers (lived in the same house five
years later)

Central City (CC)/Suburban (SU) Residence in Year
Migrants from central city to suburbs or suburbs to central city in next five years
Urban-Suburban Net Migration Rate by Race

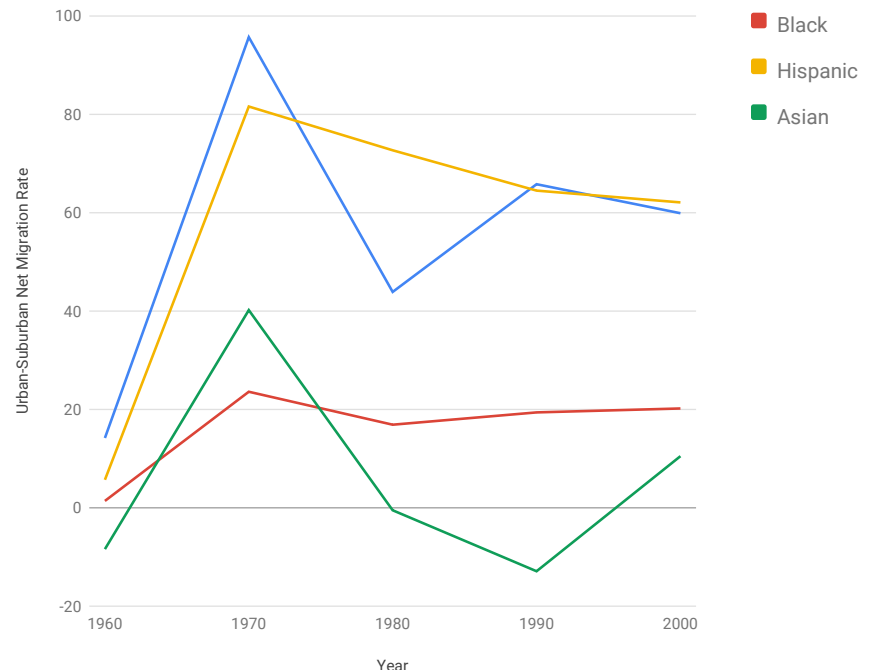

Low-Income Persons by Race

Middle-Income Persons by Race

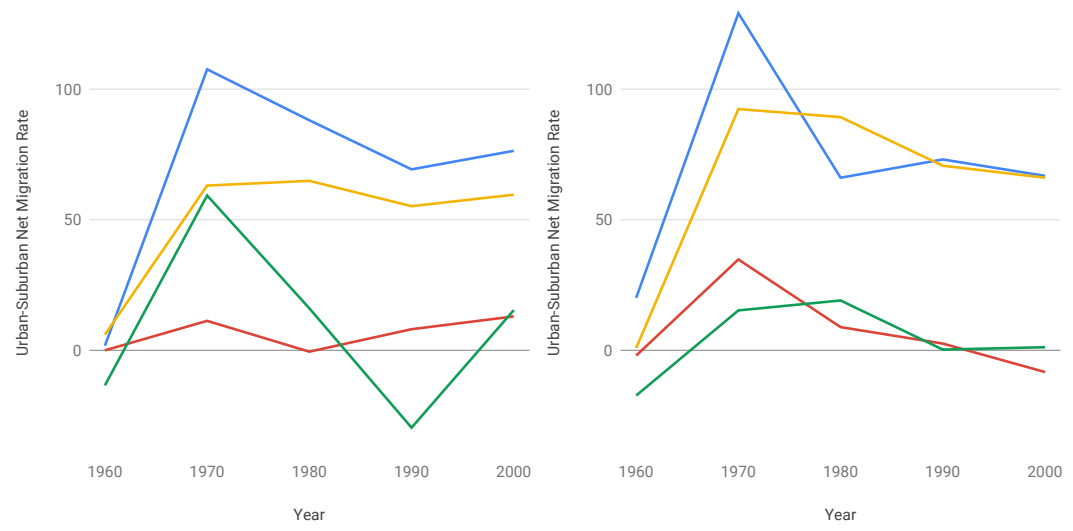

Urban-Suburban Net Migration Rate by Income

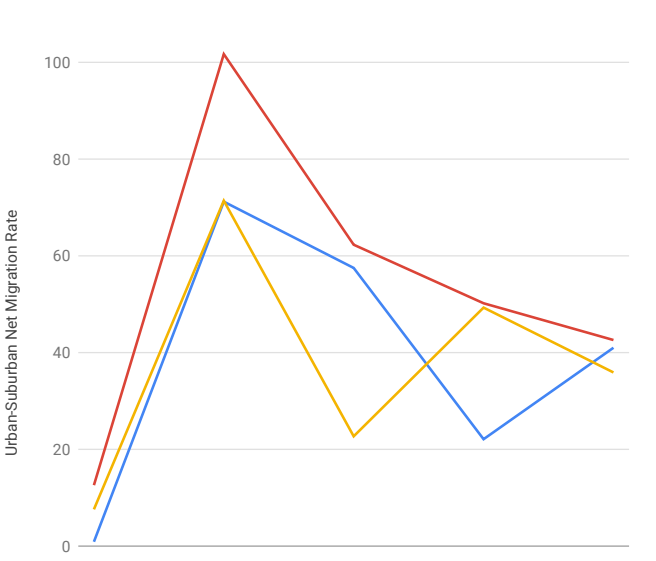

Low Income

Middle Income

- High Income

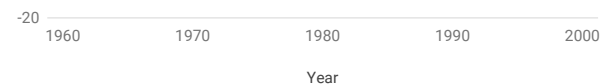

High-Income Persons by Race

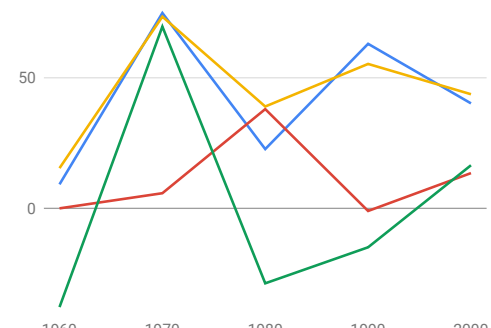


Urban and Suburban Residence, 1955-1995, and 5-year migration

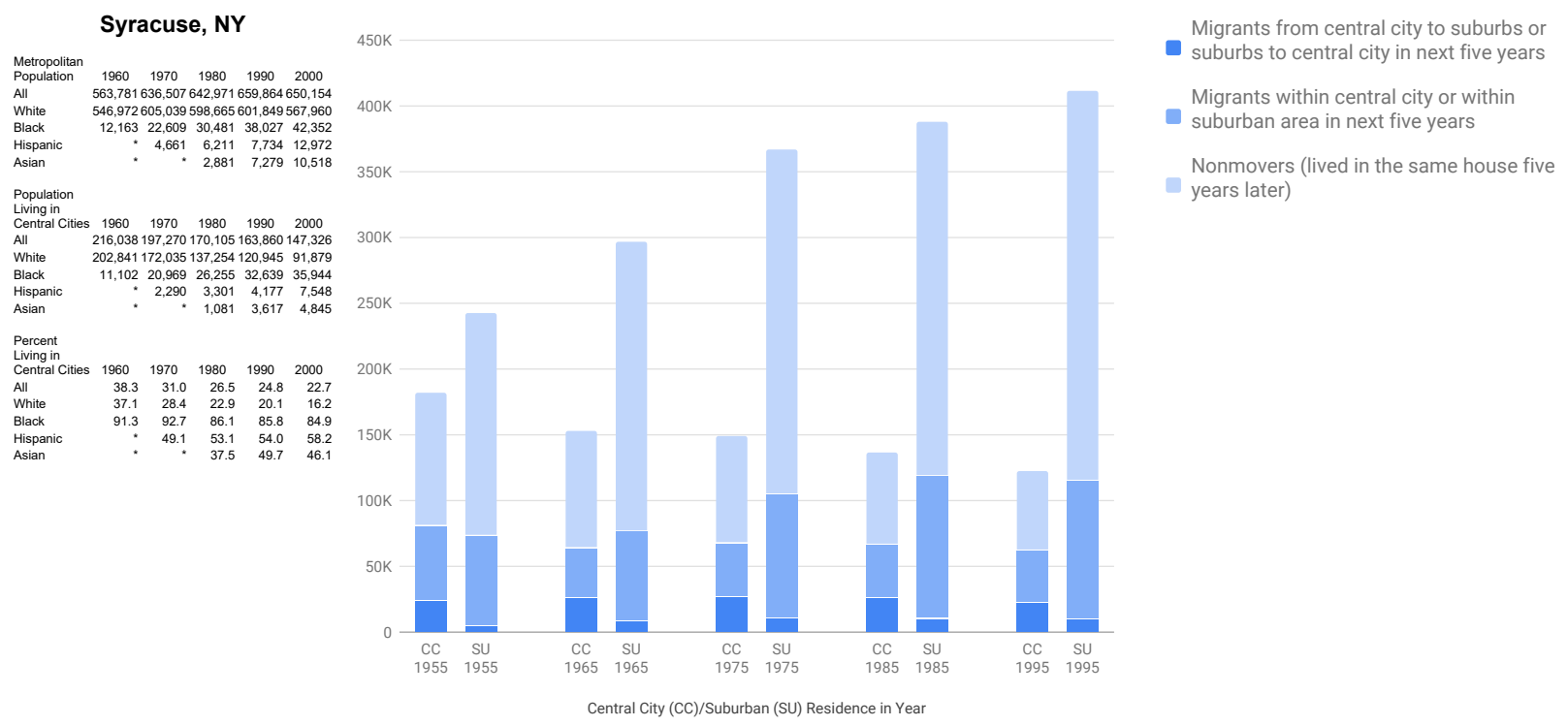

Urban-Suburban Net Migration Rate by Race

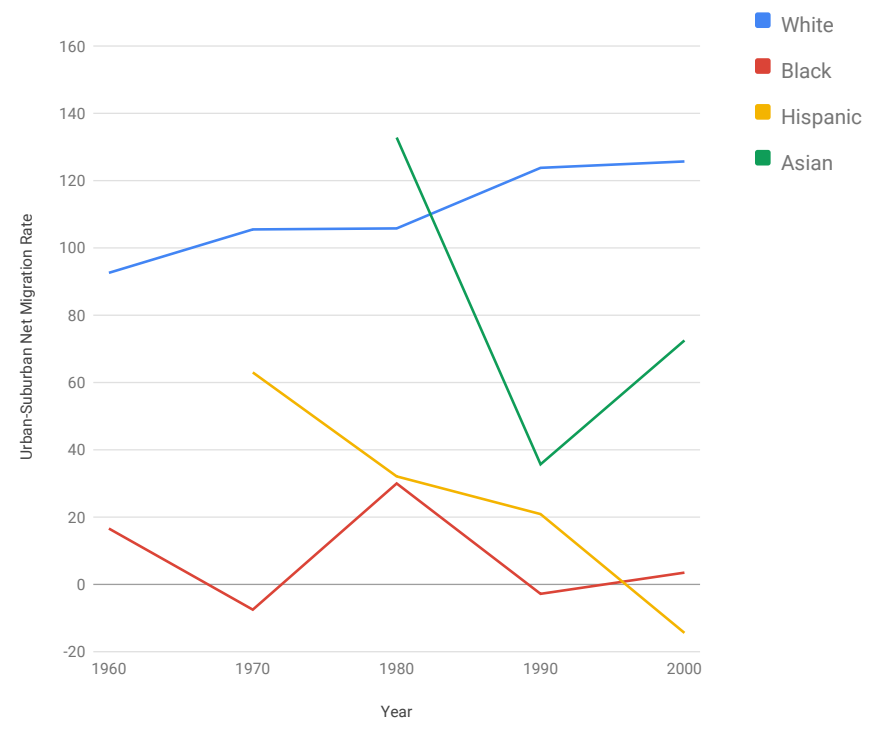

Low-Income Persons by Race
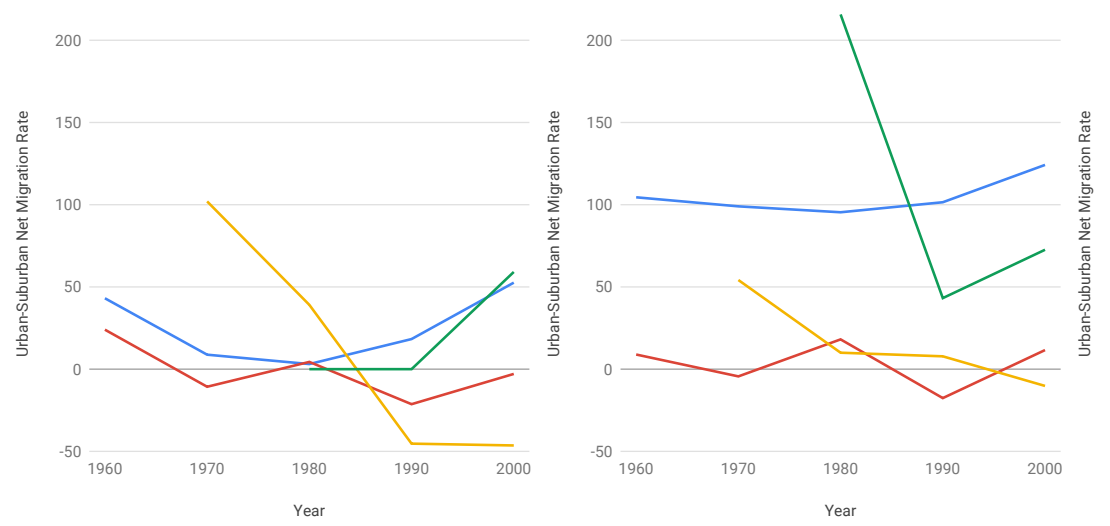

200

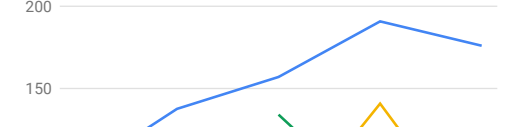

White

Black

Hispanic

- Asian 
Urban and Suburban Residence, 1955-1995, and 5-year migration

\section{Tampa-St. Petersburg, FL}

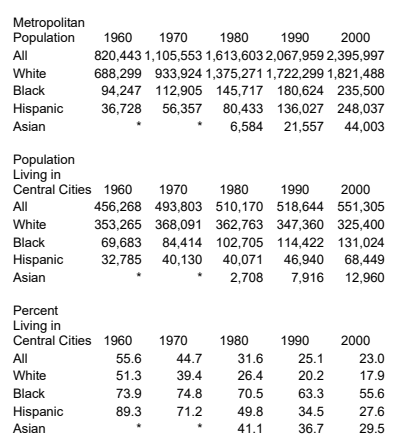

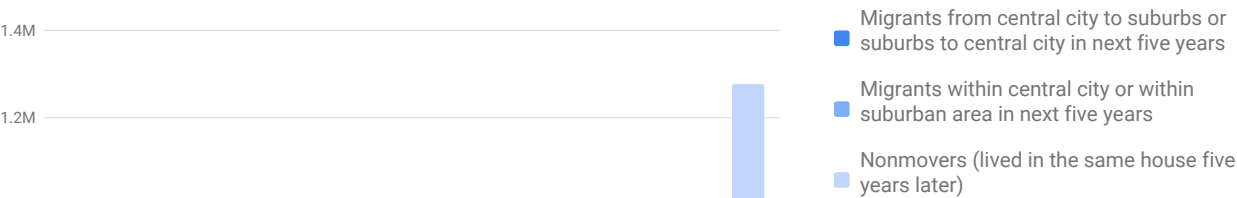

Migrants within central city or within

suburban area in next five years

Nonmovers (lived in the same house five years later)

Urban-Suburban Net Migration Rate by Race

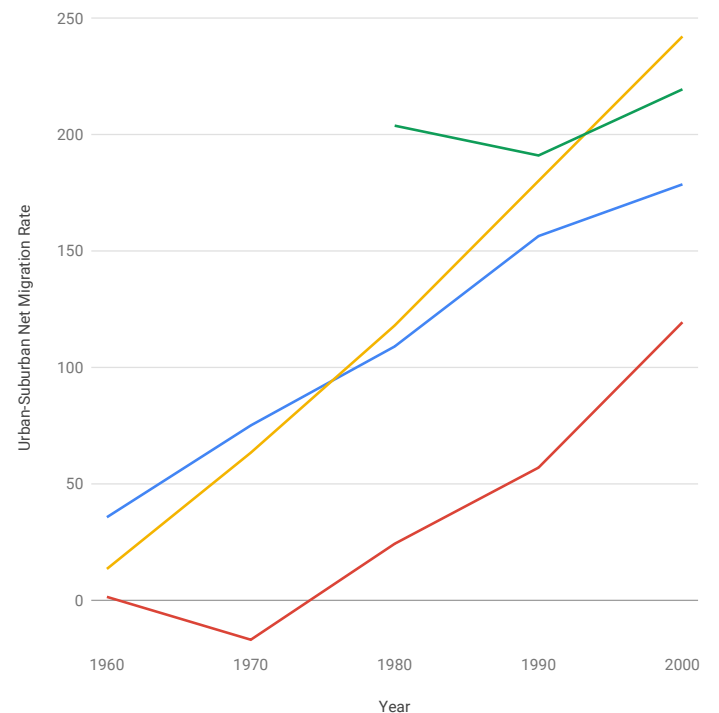

Low-Income Persons by Race

Middle-Income Persons by Race
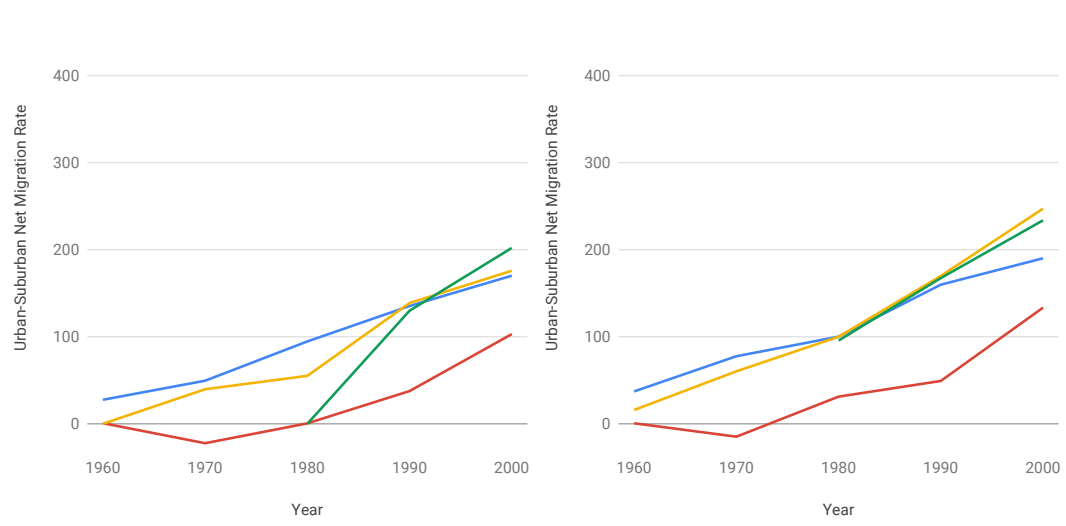

White

250

Black

Hispanic

200

- Asian

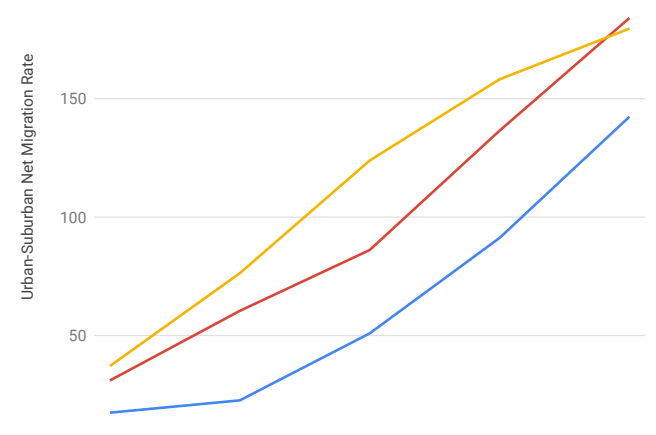

1960

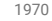

1980

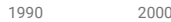

Year

High-Income Persons by Race
Low Income

- Middle Income

- High Income

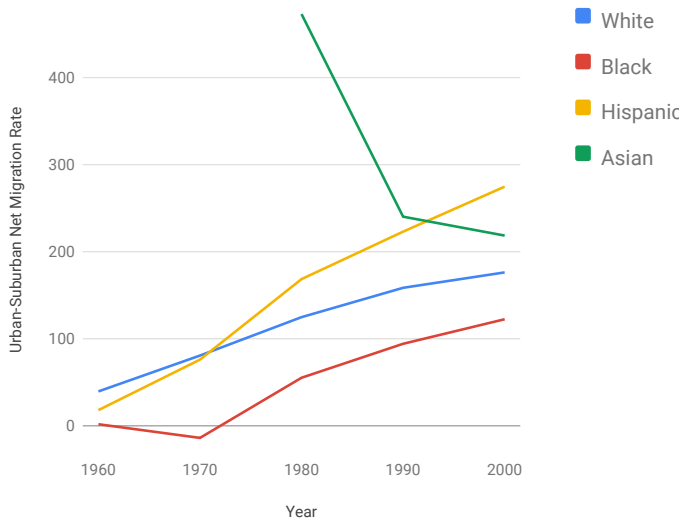


Urban and Suburban Residence, 1955-1995, and 5-year migration

$350 \mathrm{~K}$ suburbs to central city in next five years

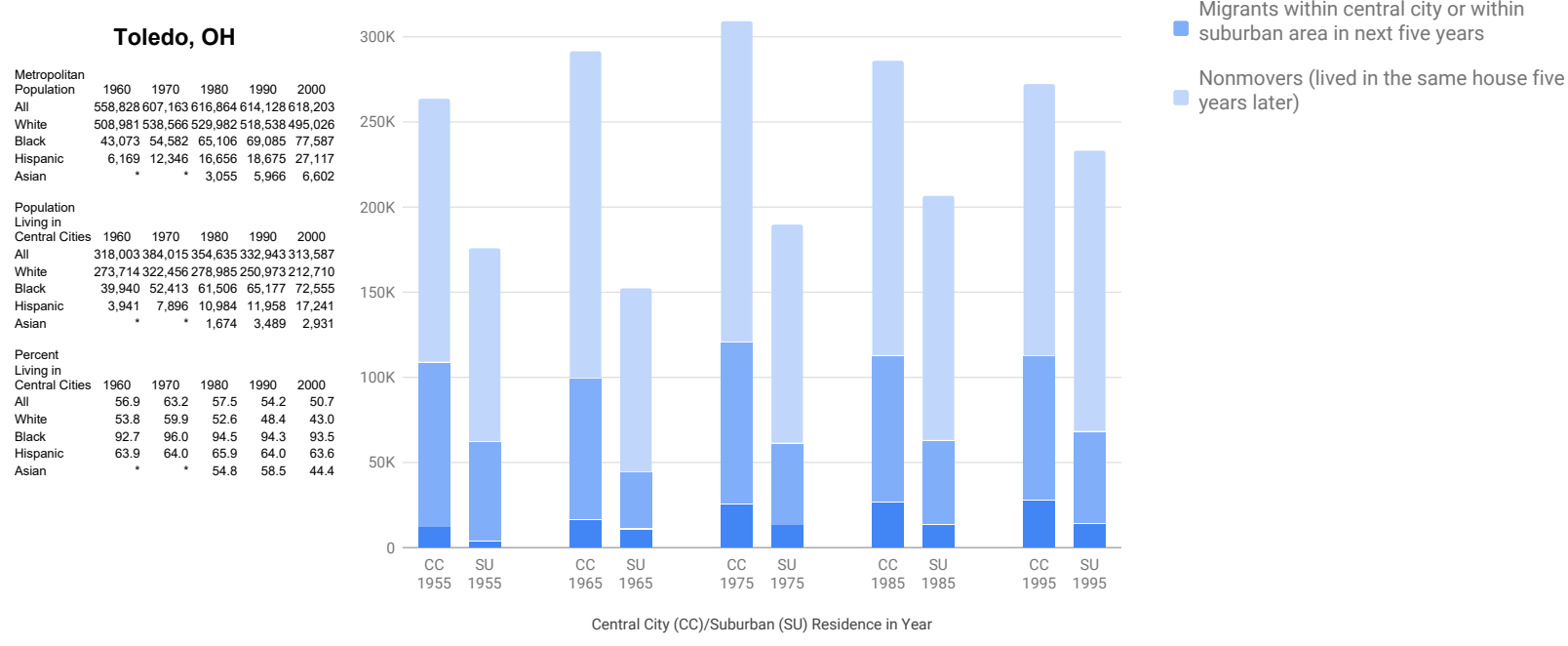

Urban-Suburban Net Migration Rate by Race

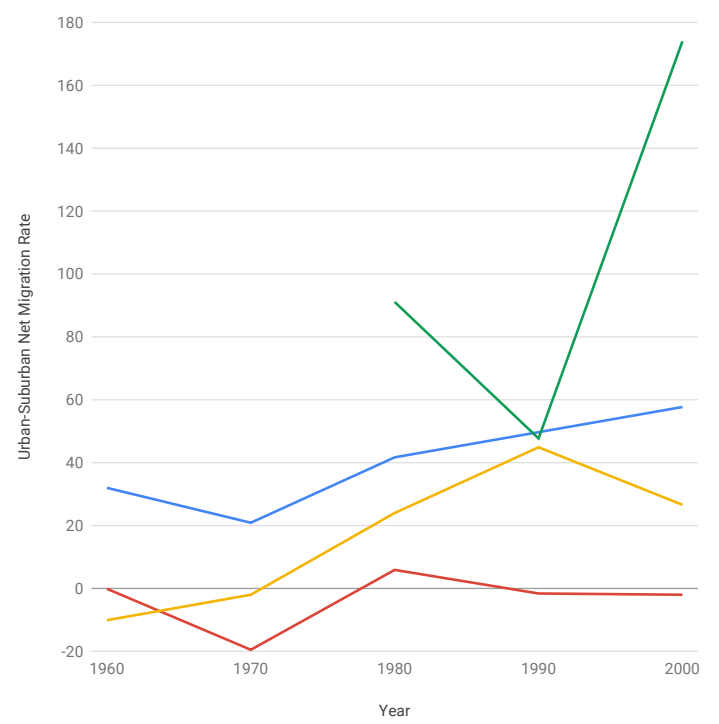

Low-Income Persons by Race

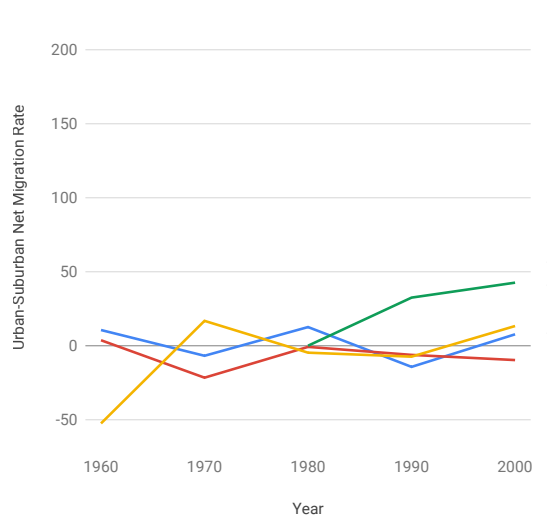

Year
Middle-Income Persons by Race

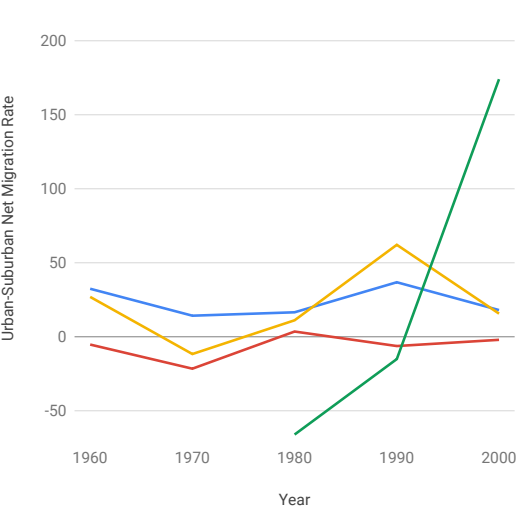

Year
Urban-Suburban Net Migration Rate by Income

$$
\begin{aligned}
& \text { White } \\
& \text { Black } \\
& \text { Hispanic } \\
& \text { Asian }
\end{aligned}
$$

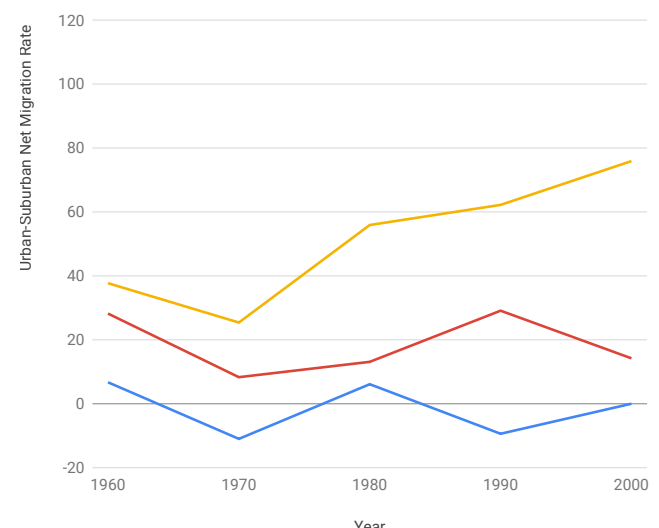

High-Income Persons by Race

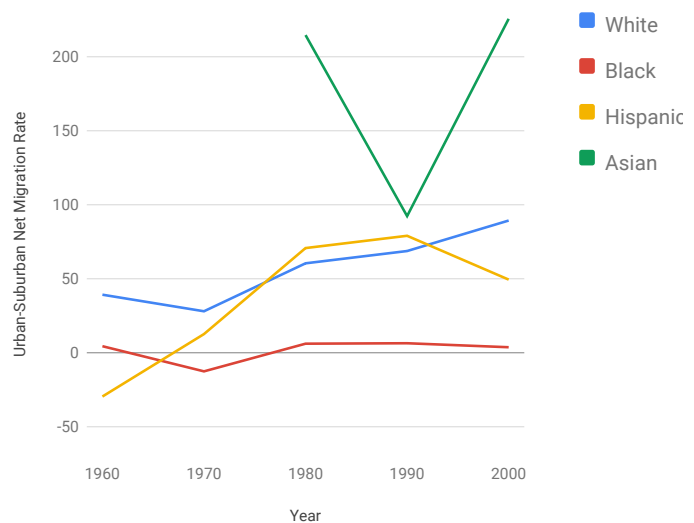


Urban and Suburban Residence, 1955-1995, and 5-year migration

$400 \mathrm{~K}-\begin{aligned} & \text { Migrants from central city to suburbs or } \\ & \text { suburbs to central city in next five years }\end{aligned}$

Migrants within central city or within

suburban area in next five years

Nonmovers (lived in the same house five

years later) $\begin{array}{lrrrrr}\begin{array}{l}\text { Metropolitan } \\ \text { Population }\end{array} & 1960 & 1970 & 1980 & 1990 & 2000 \\ \text { All } & 265.660351 .667531,443666,880843,746 & 300 \mathrm{~K}\end{array}$

$\begin{array}{ll}\text { All } & 265,660351,667531,443666,8808843,746 \\ \text { White } & 198,229256,550387,592456,630517,982 \\ \text { Black } & 7946,9647\end{array}$

$\begin{array}{lrrrrr}\text { Black } & 7,946 & 9,647 & 14,072 & 19,663 & 23,047 \\ \text { Hispanic } & 55,066 & 80,649111,378 & 161,053247,861\end{array}$

Asian $\quad 0,04,406 \quad 10,921 \quad 16,375 \quad 250 \mathrm{~K}$

Population
Living in

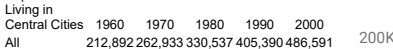

$\begin{array}{ll}\text { All } & 212,892262,933330,537405,390486,591 \\ \text { White } & 162,596191,350230,245258,036263,269\end{array}$

$\begin{array}{llllll}\text { Black } & 6,766 & 8,576 & 11,398 & 16,590 & 19,426\end{array}$

$\begin{array}{lrrrr}\text { Hispanic } & 41,939 . & 60,815 & 82,106 & 117,267174,354 \\ \text { Asian } & 2,909 & 7,837 & 11,268\end{array}$

Percent

Lercent

$\begin{array}{lcccccc}\text { Leving in } & & & & & & \\ \text { Central Cities } & 1960 & 1970 & 1980 & 1990 & 2000 & \\ \text { All } & 80.1 & 74.8 & 62.2 & 60.8 & 57.7 & 100 \mathrm{~K}\end{array}$

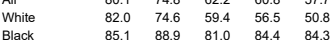

$\begin{array}{llllll}\text { Black } & 85.1 & 88.9 & 81.0 & 84.4 & 84.3 \\ \text { Hispanic } & 76.2 & 75.4 & 73.7 & 72.8 & 70.3 \\ \text { Asian } & . & . & 66.0 & 71.8 & 68.8\end{array}$

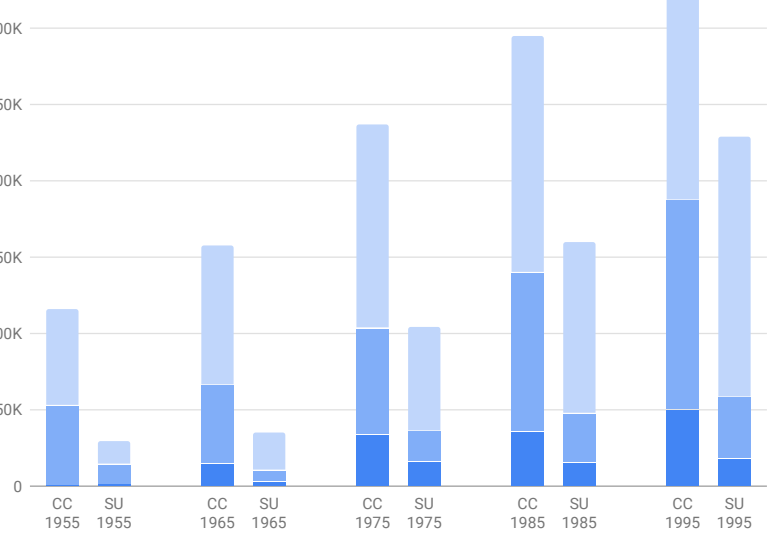

Urban-Suburban Net Migration Rate by Race

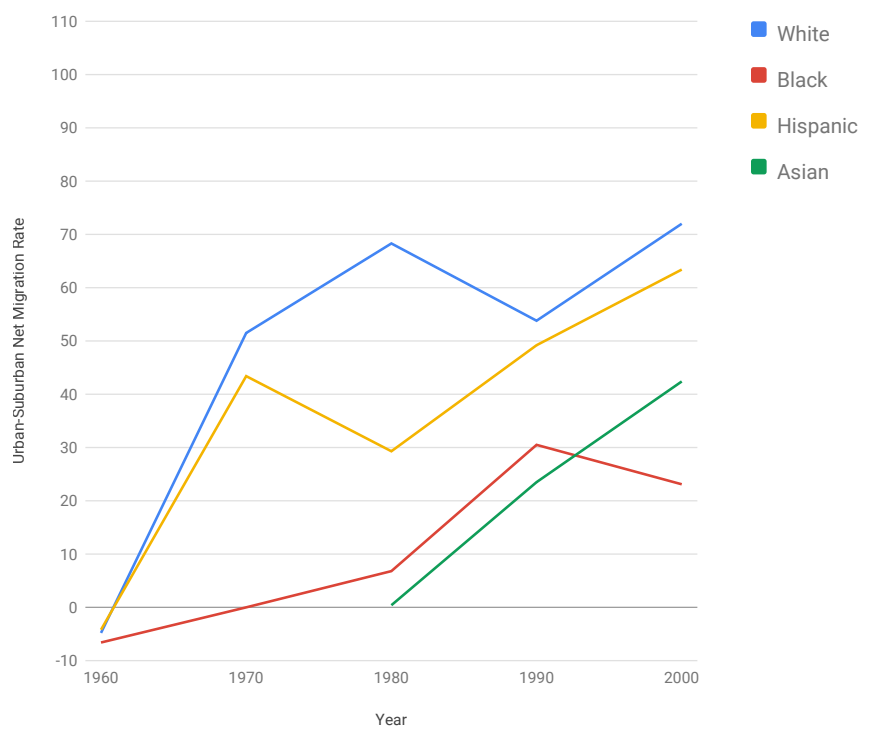

Low-Income Persons by Race

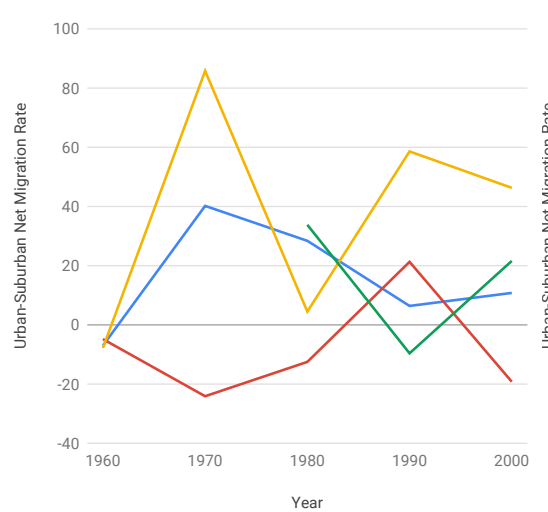

Middle-Income Persons by Race
Urban-Suburban Net Migration Rate by Income

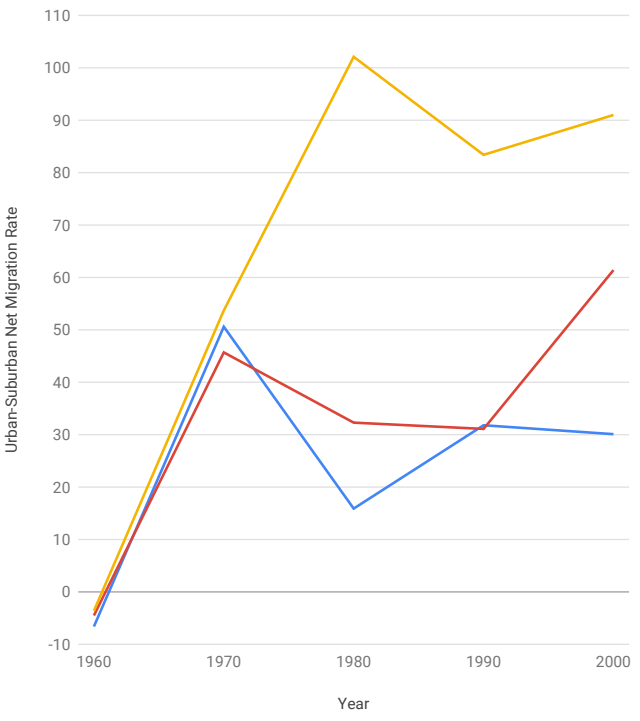

- Low Income

- Middle Income

High Income

High-Income Persons by Race

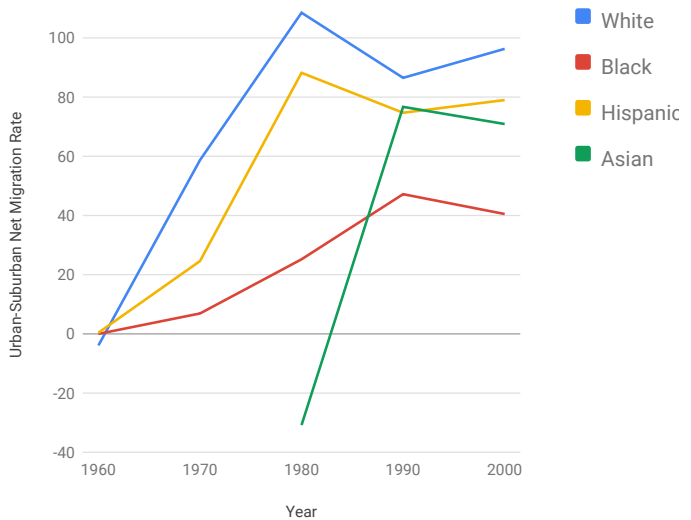


Urban and Suburban Residence, 1955-1995, and 5-year migration

Tulsa, OK

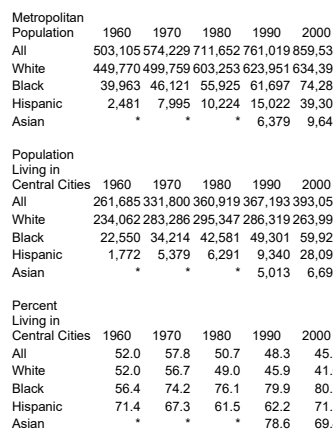

$400 \mathrm{~K}$

\author{
$350 \mathrm{~K}$
}

$300 \mathrm{~K}$

25

7

200

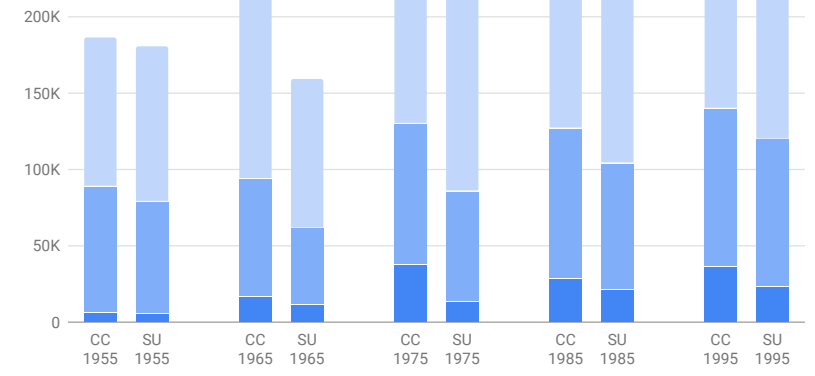

Migrants from central city to suburbs or - suburbs to central city in next five years

Migrants within central city or within

suburban area in next five years

Nonmovers (lived in the same house five

years later)
Urban-Suburban Net Migration Rate by Race

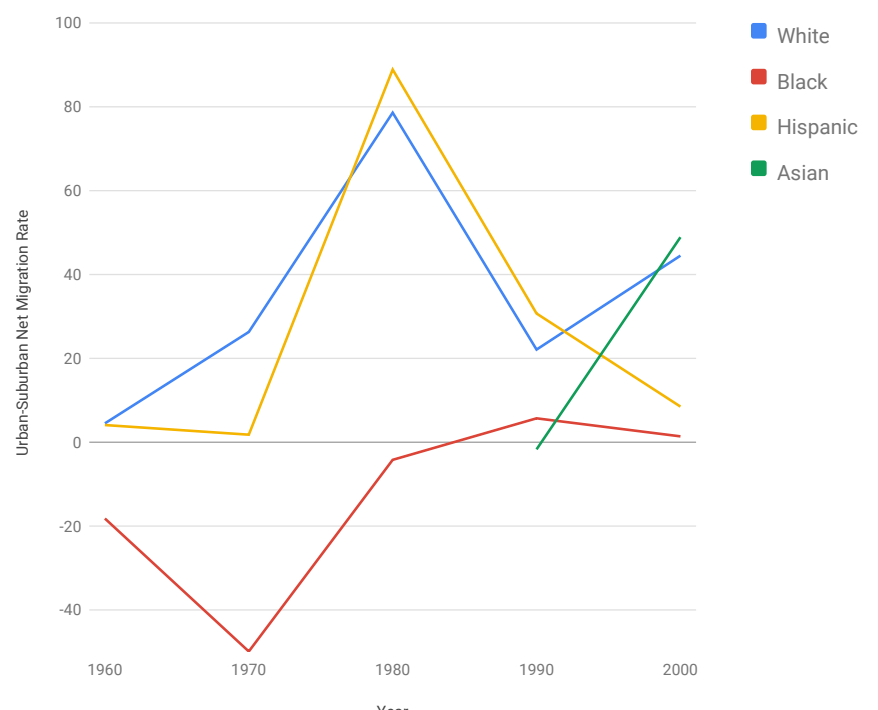

Low-Income Persons by Race

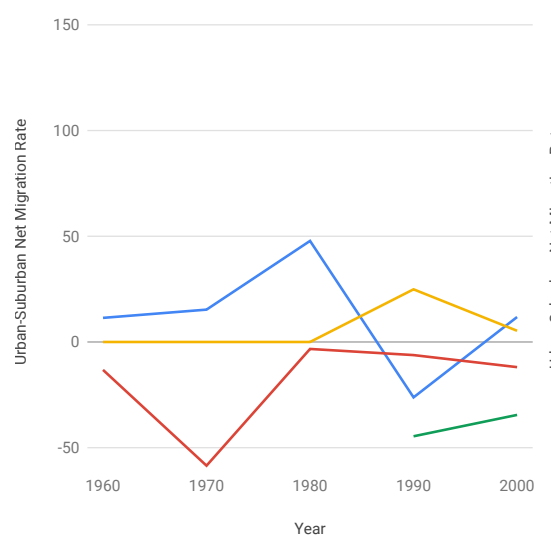

Middle-Income Persons by Race

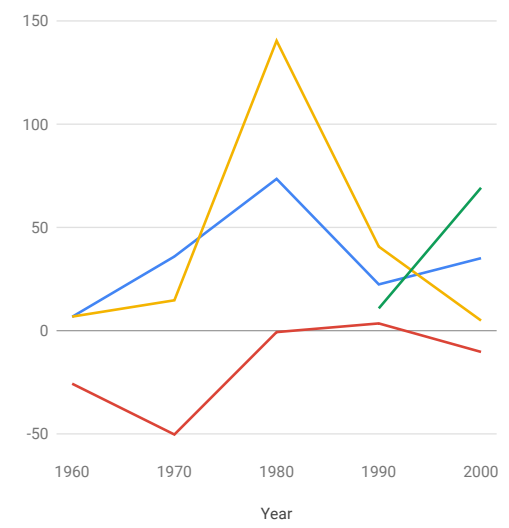

150

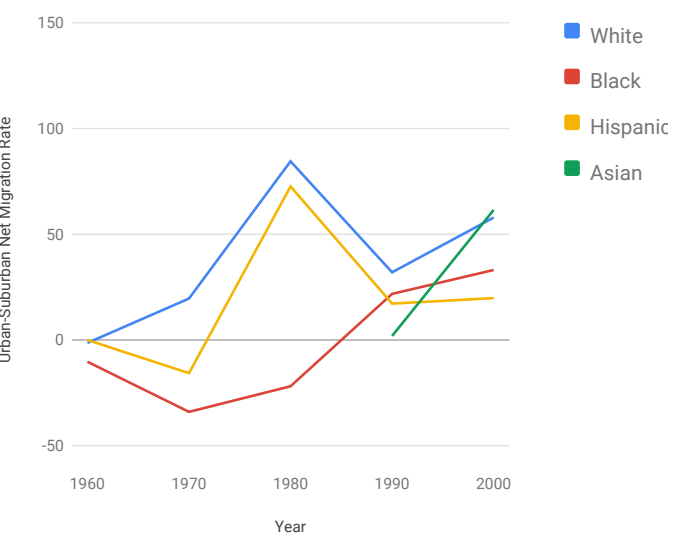

$-50$

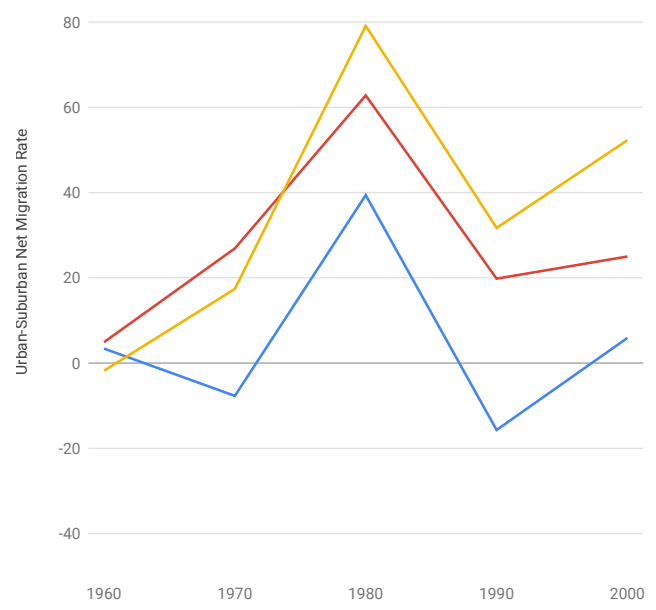

Year

High-Income Persons by Race 
Urban and Suburban Residence, 1955-1995, and 5-year migration

\section{(Urban) Honolulu, $\mathrm{HI}$}

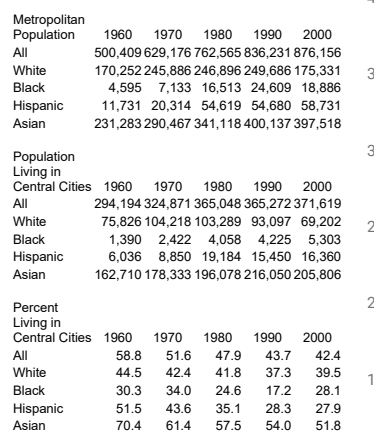

$400 \mathrm{~K}$

350

$300 \mathrm{~K}$

250

$200 \mathrm{~K}$

$150 \mathrm{~K}$

$100 \mathrm{~K}$

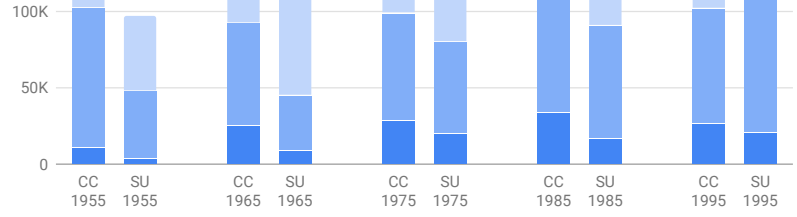

Migrants from central city to suburbs or - suburbs to central city in next five years

Migrants within central city or within

suburban area in next five years

Nonmovers (lived in the same house five

years later)

Central City (CC)/Suburban (SU) Residence in Year

Urban-Suburban Net Migration Rate by Race

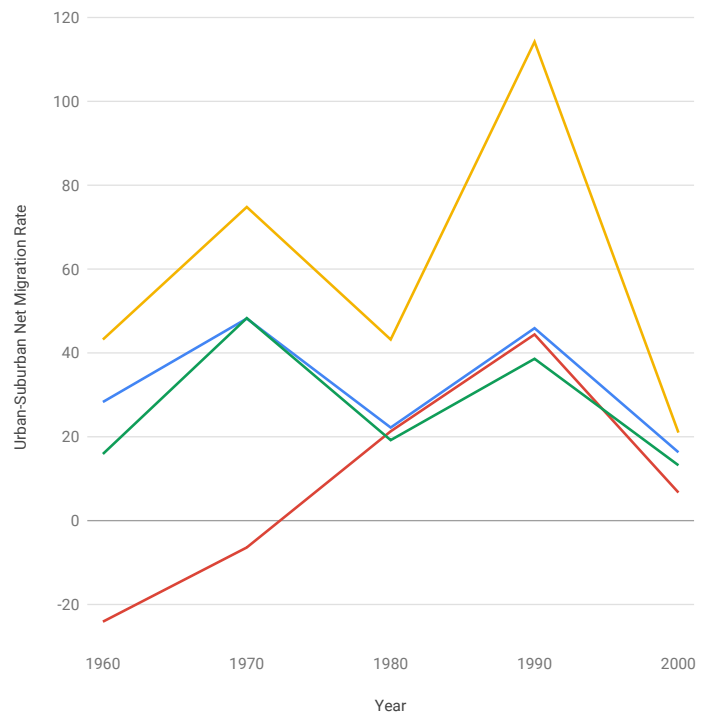

Low-Income Persons by Race

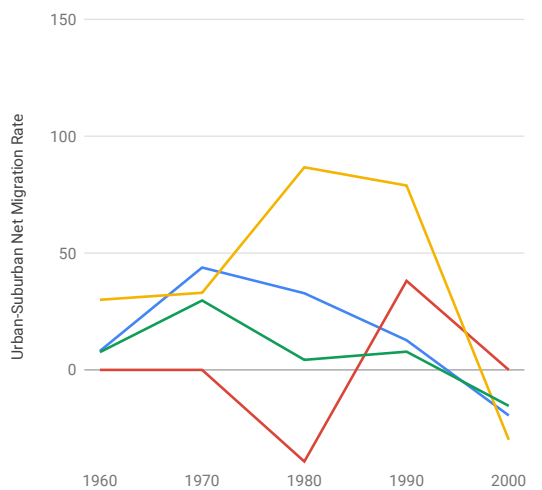

Year
Middle-Income Persons by Race

150

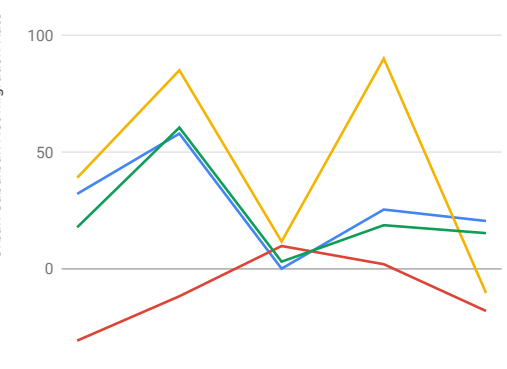

Year
Urban-Suburban Net Migration Rate by Income

$$
\begin{aligned}
& \text { White } \\
& \text { Black } \\
& \text { Hispanic } \\
& \text { Asian }
\end{aligned}
$$$$
100
$$

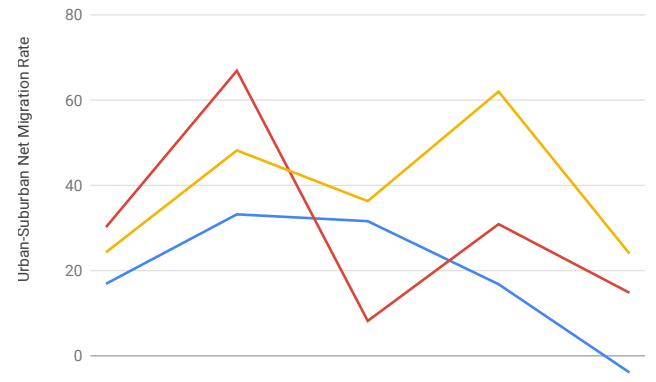

1960

1970

1980

Year
- Low Income

- Middle Income

- High Income 
Urban and Suburban Residence, 1955-1995, and 5-year migration

Virginia Beach-Norfolk-Newport News,

$$
\text { VA-NC }
$$

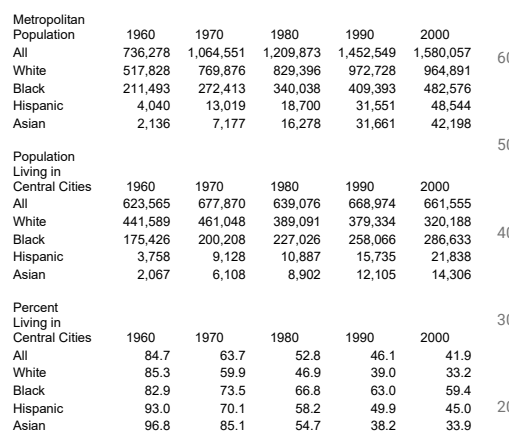

$700 \mathrm{~K}$

$600 \mathrm{~K}$$$
\text { K }
$$

Central City (CC)/Suburban (SU) Residence in Year

Urban-Suburban Net Migration Rate by Race

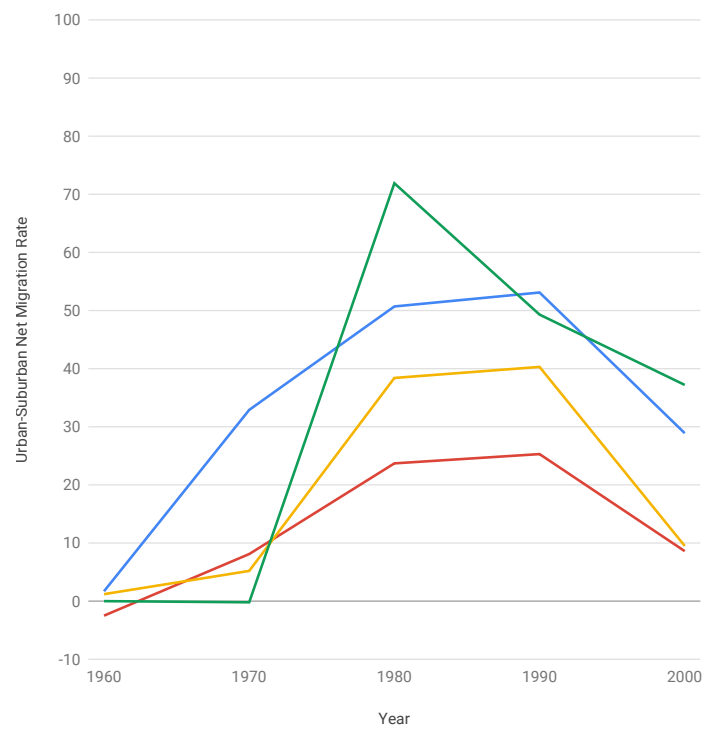

Low-Income Persons by Race

Middle-Income Persons by Race
Urban-Suburban Net Migration Rate by Income
White
Black
Hispanic
- Asian

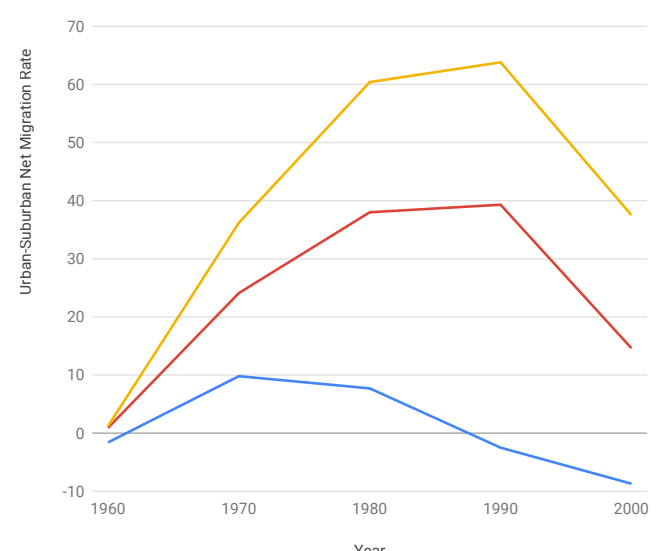

Year
Migrants from central city to suburbs or - suburbs to central city in next five years Migrants within central city or within suburban area in next five years

Nonmovers (lived in the same house five years later)

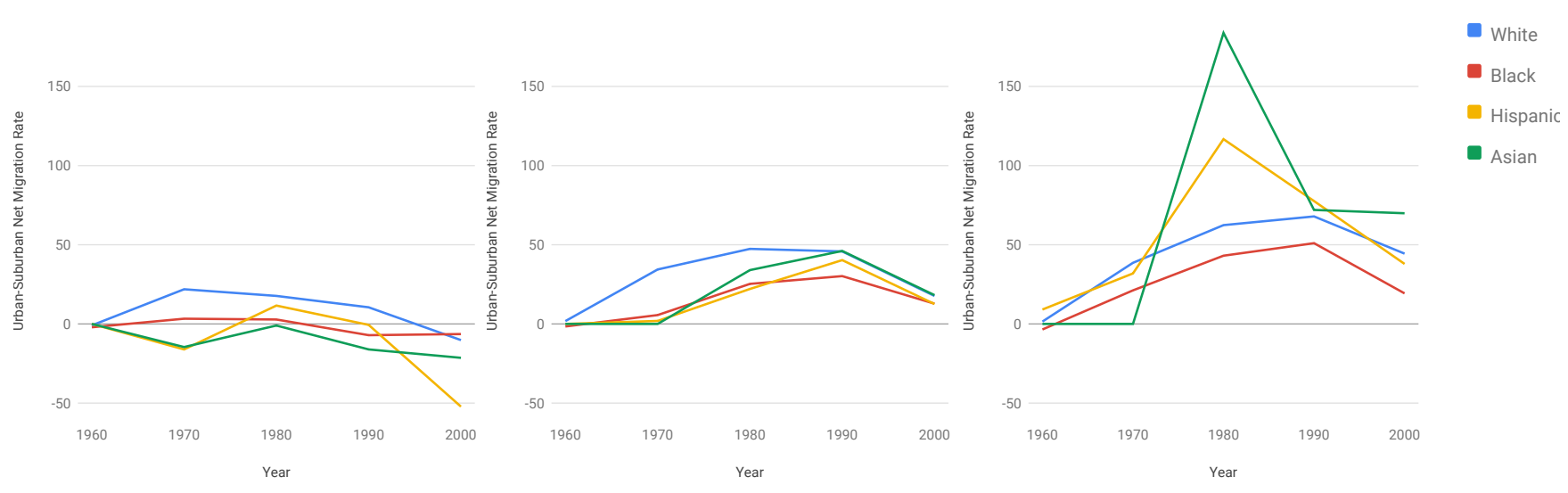


Urban and Suburban Residence, 1955-1995, and 5-year migration

$3.5 \mathrm{M} \quad \begin{aligned} & \text { Migrants from central city to suburbs or } \\ & \text { suburbs to central city in next five years }\end{aligned}$

Migrants within central city or within

- suburban area in next five years

Nonmovers (lived in the same house five

years later)

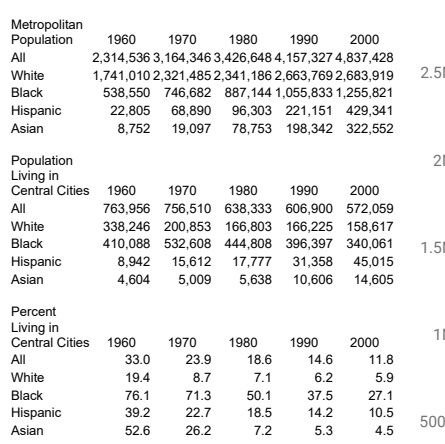

Urban-Suburban Net Migration Rate by Race

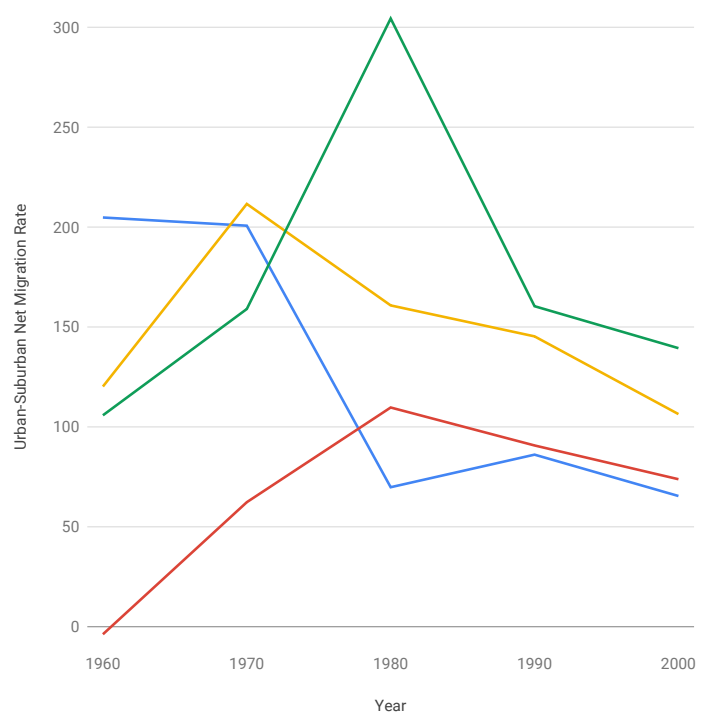

Low-Income Persons by Race

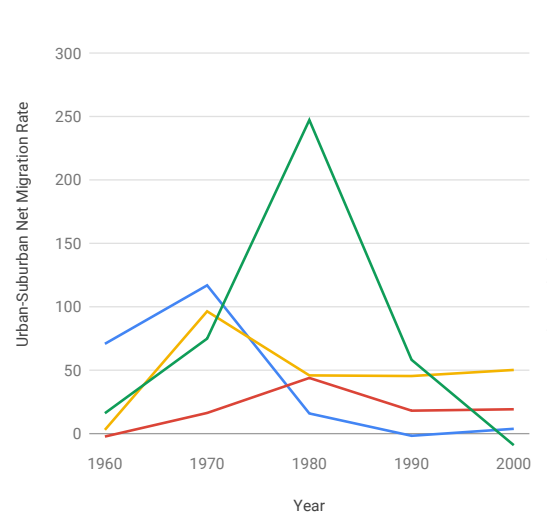

Middle-Income Persons by Race

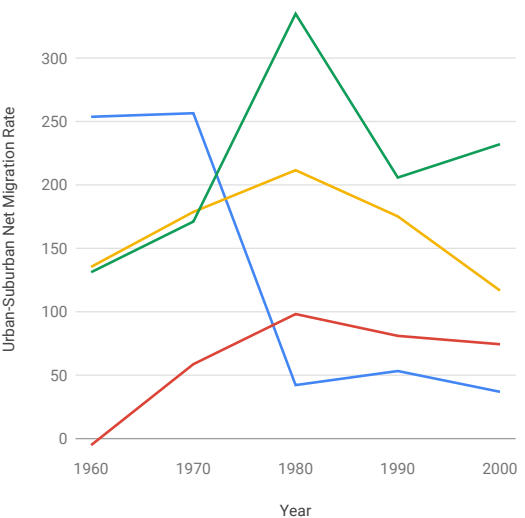

Urban-Suburban Net Migration Rate by Income

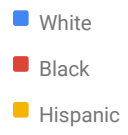

300

250

- Asian

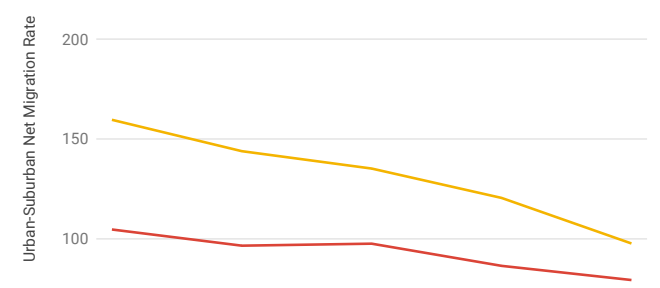

50

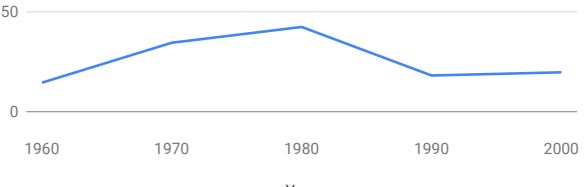

Year

High-Income Persons by Race

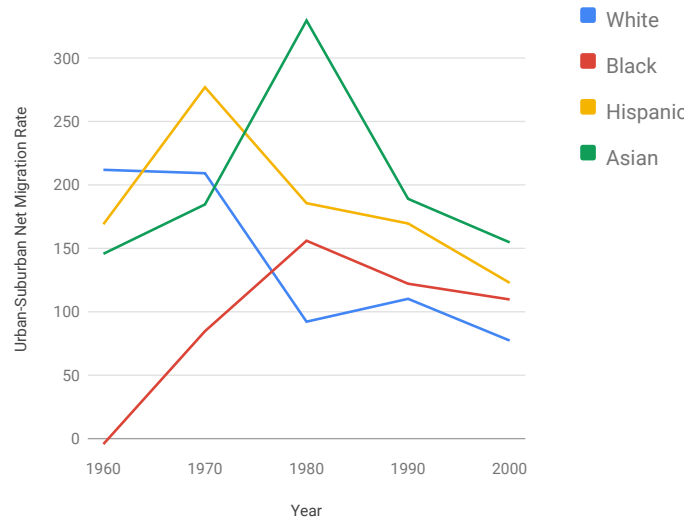


Urban and Suburban Residence, 1955-1995, and 5-year migration

$300 \mathrm{~K}$

Wichita, KS

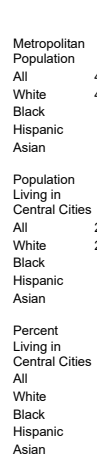

$1960 \quad 1970 \quad 1980 \quad 1990 \quad 2000$ $442,765449,027475,732519,403579,839$

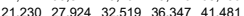
$\begin{array}{llllll}5,518 & 11,199 & 13,437 & 19,317 & 40,795\end{array}$ $\begin{array}{lllll}* & 4,531 & 8,623 & 14,568\end{array}$

Population

$\begin{array}{lllll}1960 \quad 1970 \quad 1980 \quad 1990 & 2000 \\ 25490876 & 150 \mathrm{~K}\end{array}$ $4,698276,699279,272304,011343,997$
$397240,369232,282244,801246,553$ $\begin{array}{lllll}19,706 & 26,238 & 30,127 & 33,826 & 38,253\end{array}$ $\begin{array}{llllll}3,296 & 7,799 & 9,455 & 14,314 & 33,002\end{array}$

$\begin{array}{lll}3,740 & 7,362 & 12,612\end{array}$

Ping in

$\begin{array}{ccccc}960 & 1970 & 1980 & 1990 & 2000 \\ 57.5 & 61.6 & 58.7 & 58.5 & 593\end{array}$

$\begin{array}{lllll}57.5 & 61.6 & 58.7 & 58.5 & 59.3 \\ 55.6 & 59.1 & 55.3 & 54.5 & 53.2\end{array}$

$\begin{array}{ccccc}92.8 & 94.0 & 92.6 & 93.1 & 92.2\end{array}$

$\begin{array}{lllll}59.7 & 69.6 & 70.4 & 74.1 & 80.9 \\ . & 82.5 & 85.4 & 86.6\end{array}$

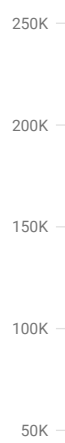

Urban-Suburban Net Migration Rate by Race

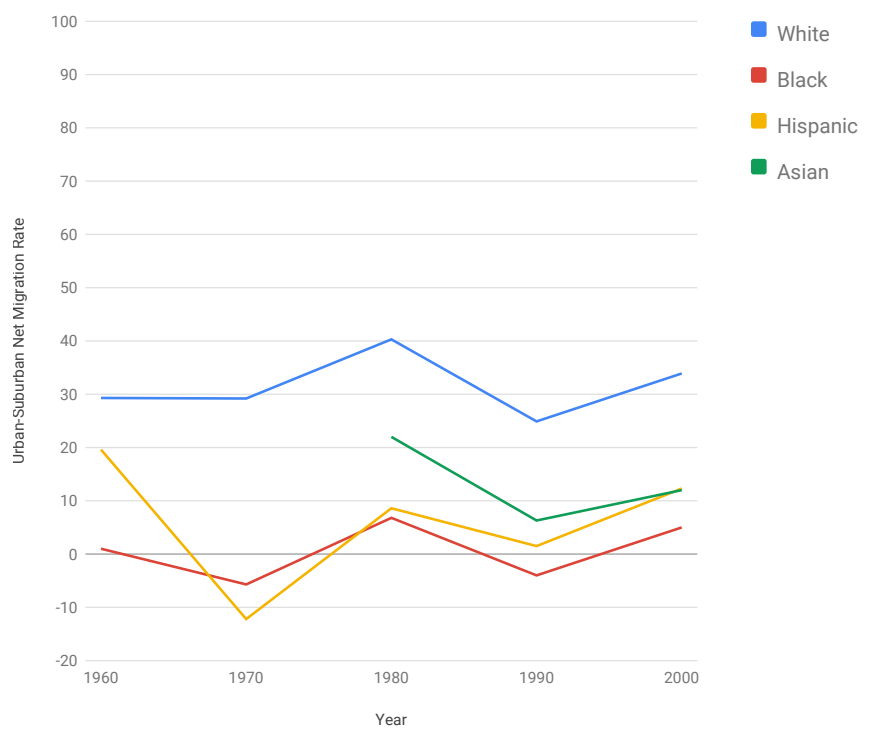

Low-Income Persons by Race

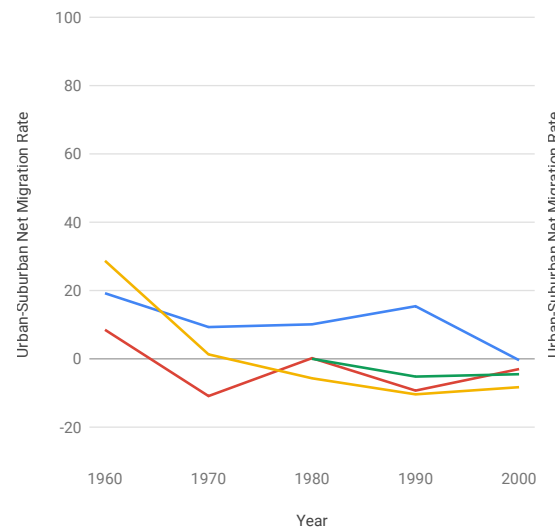

Urban-Suburban Net Migration Rate by Income

Migrants from central city to suburbs or - suburbs to central city in next five years

Migrants within central city or within

suburban area in next five years

Nonmovers (lived in the same house five

years later)
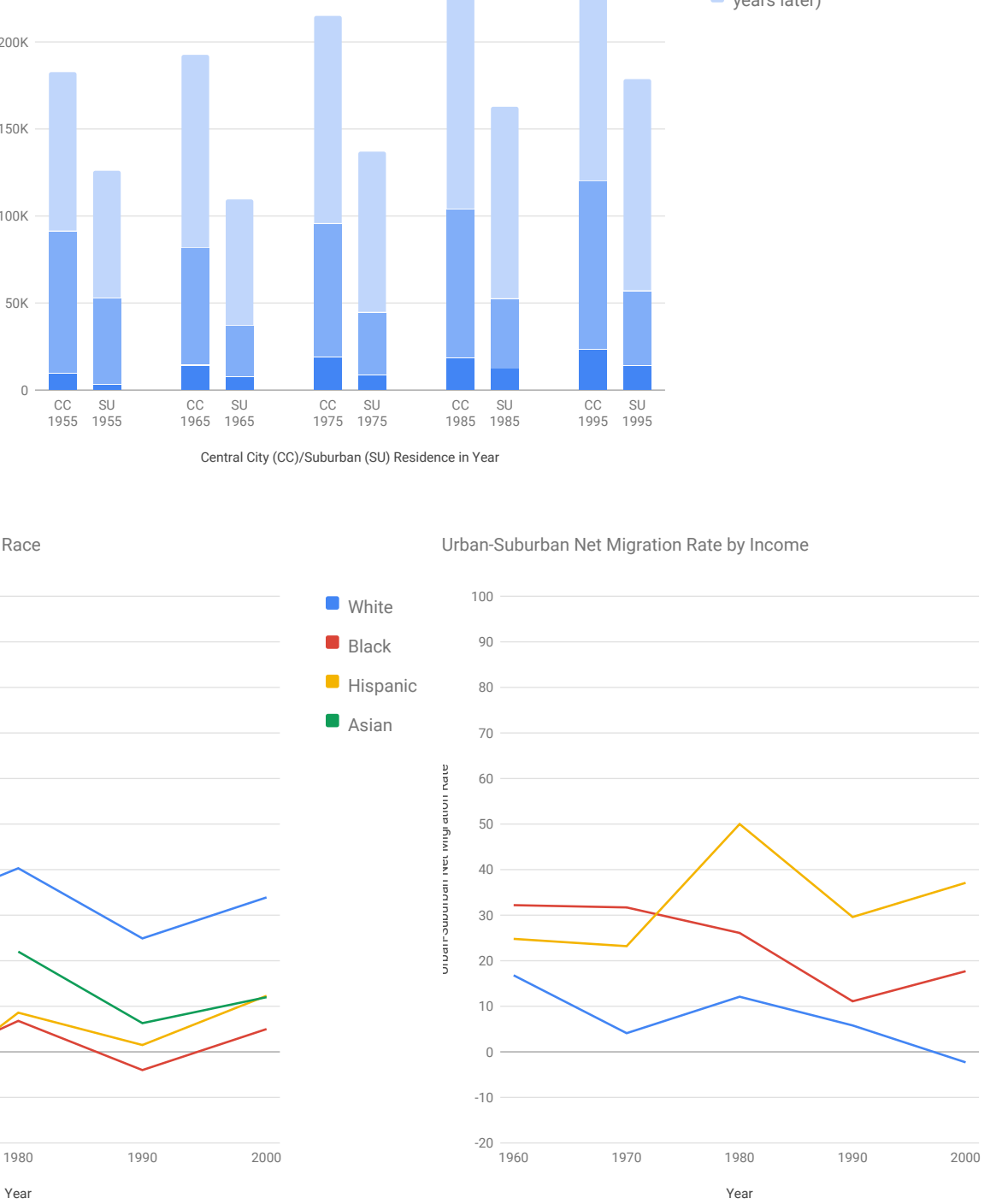

- Low Income

- Middle Income

- High Income

High-Income Persons by Race
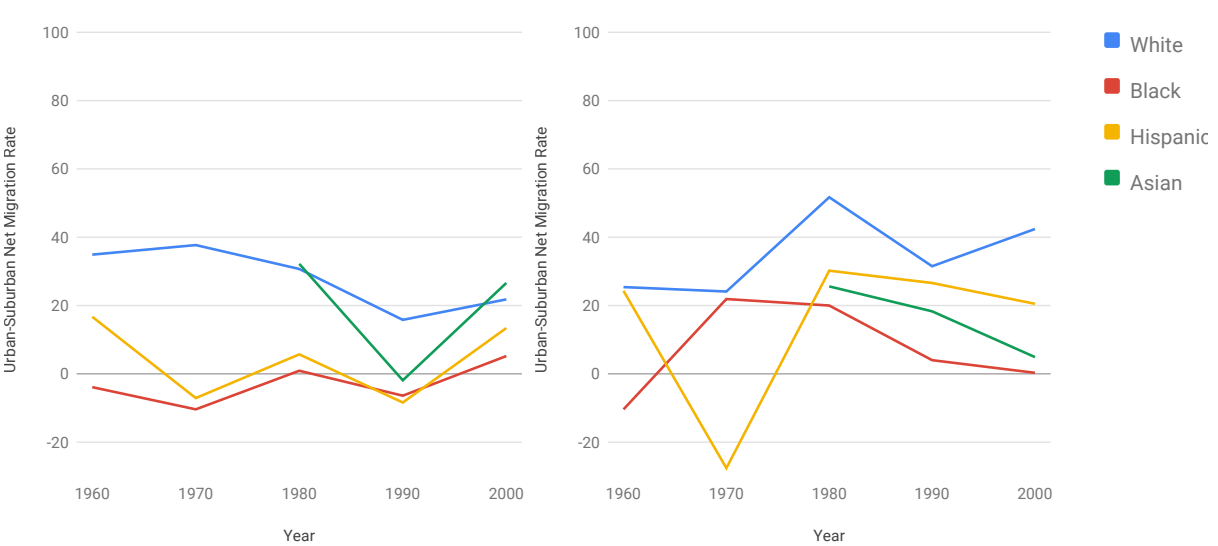
Urban and Suburban Residence, 1955-1995, and 5-year migration

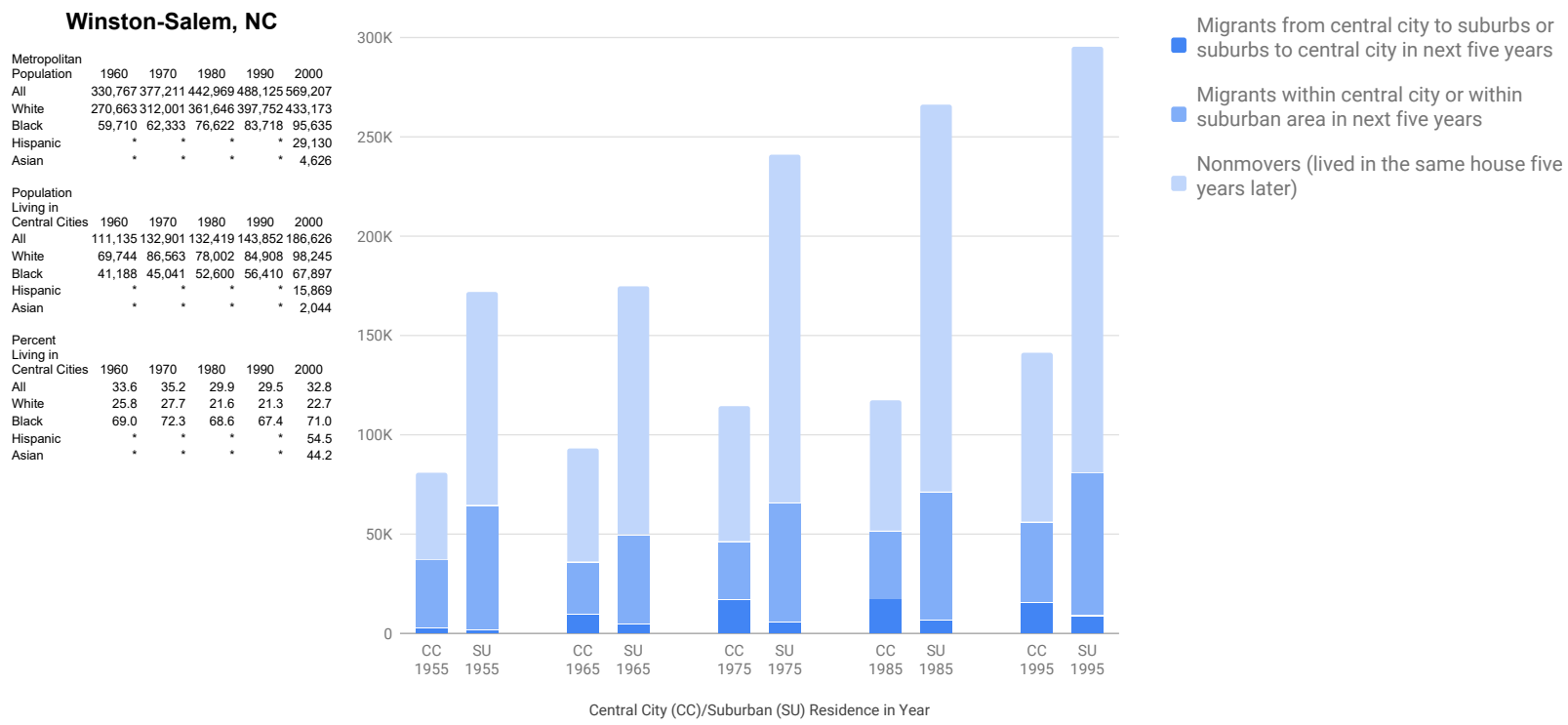

Urban-Suburban Net Migration Rate by Race

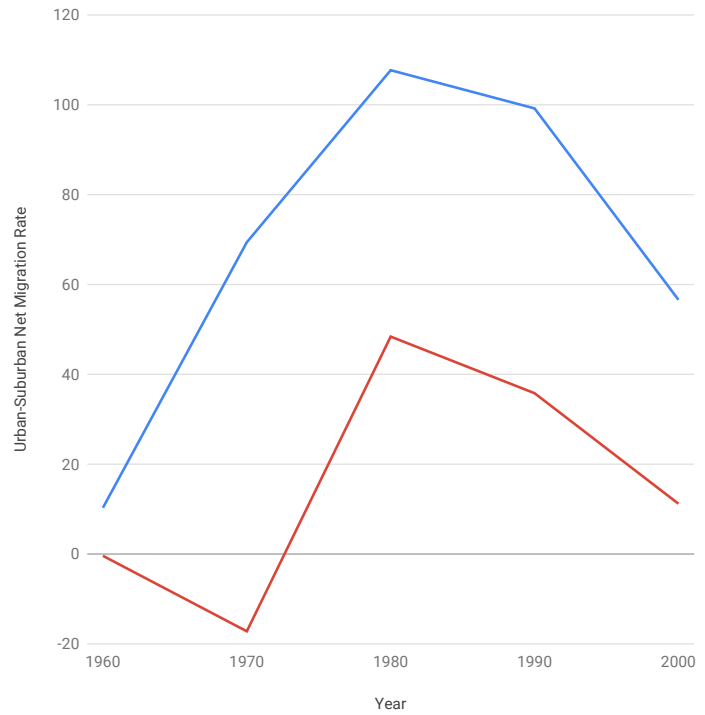

Low-Income Persons by Race

Middle-Income Persons by Race
Urban-Suburban Net Migration Rate by Income
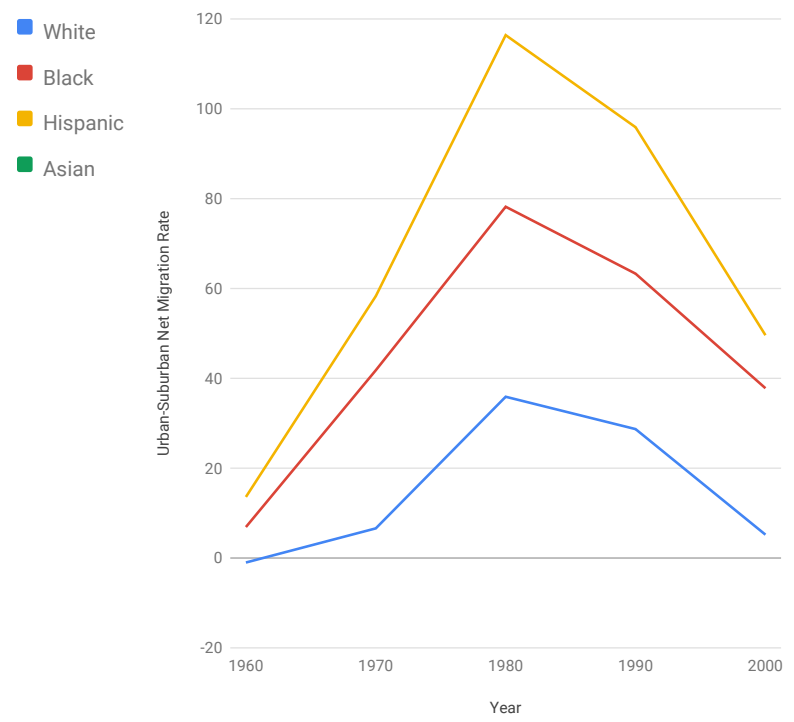

Low Income

Middle Income

- High Income

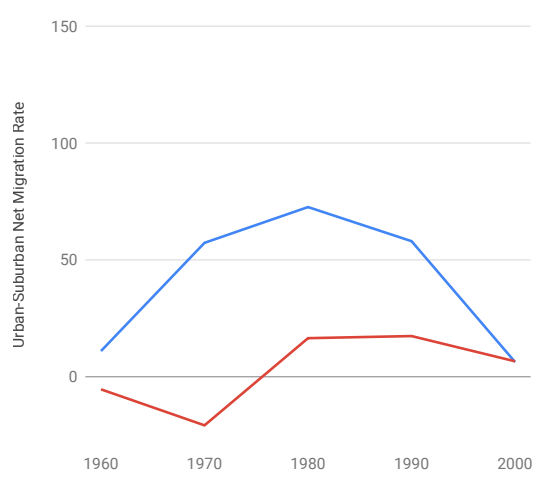

Year
150

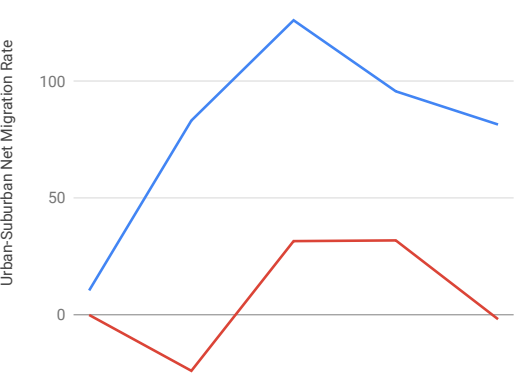

Year
High-Income Persons by Race

150

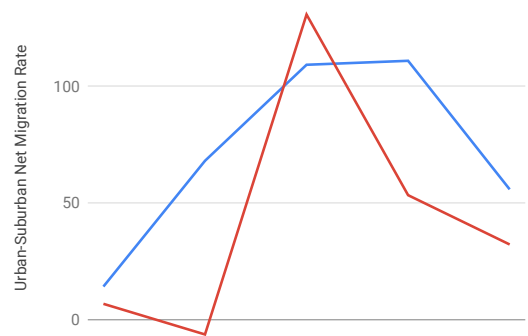

Hispanic

- Asian 
Urban and Suburban Residence, 1955-1995, and 5-year migration

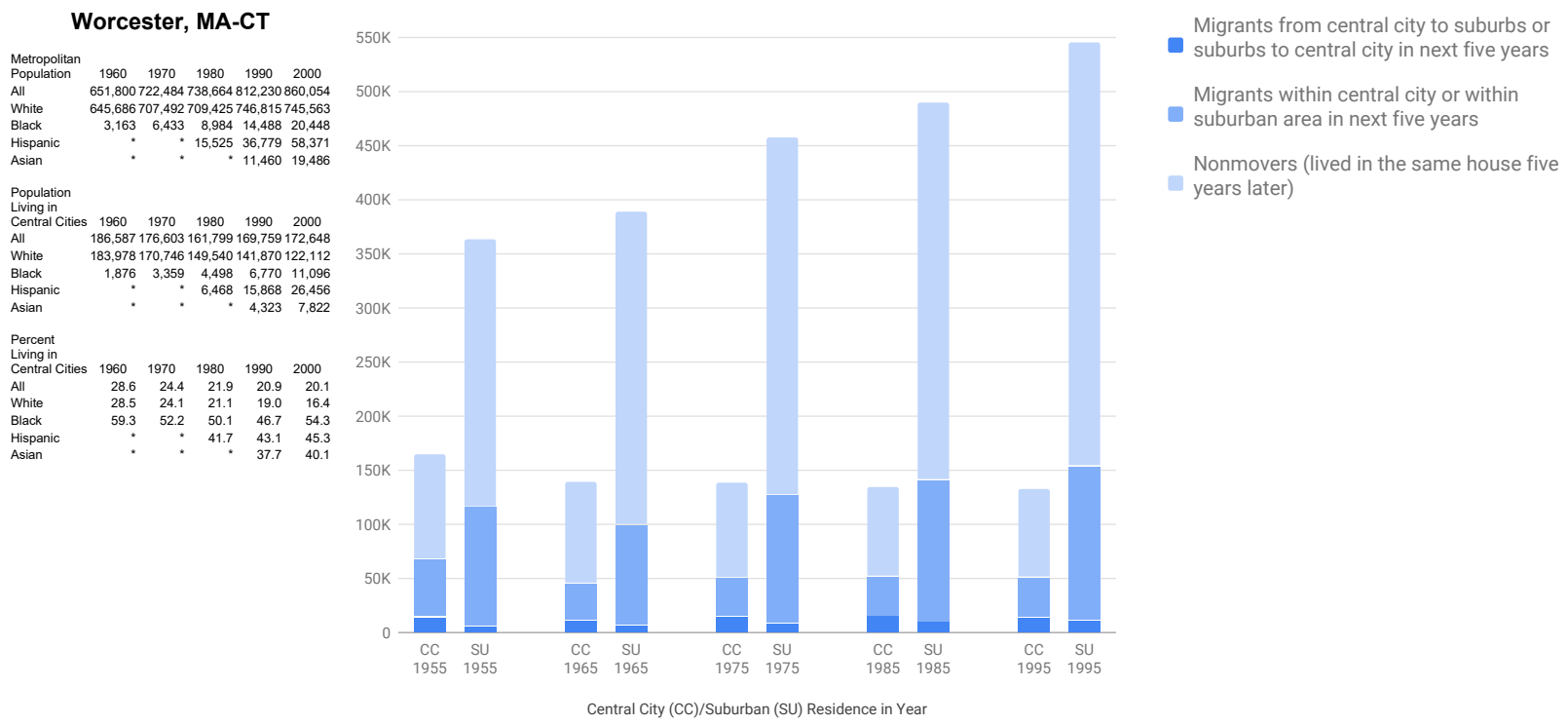

Urban-Suburban Net Migration Rate by Race

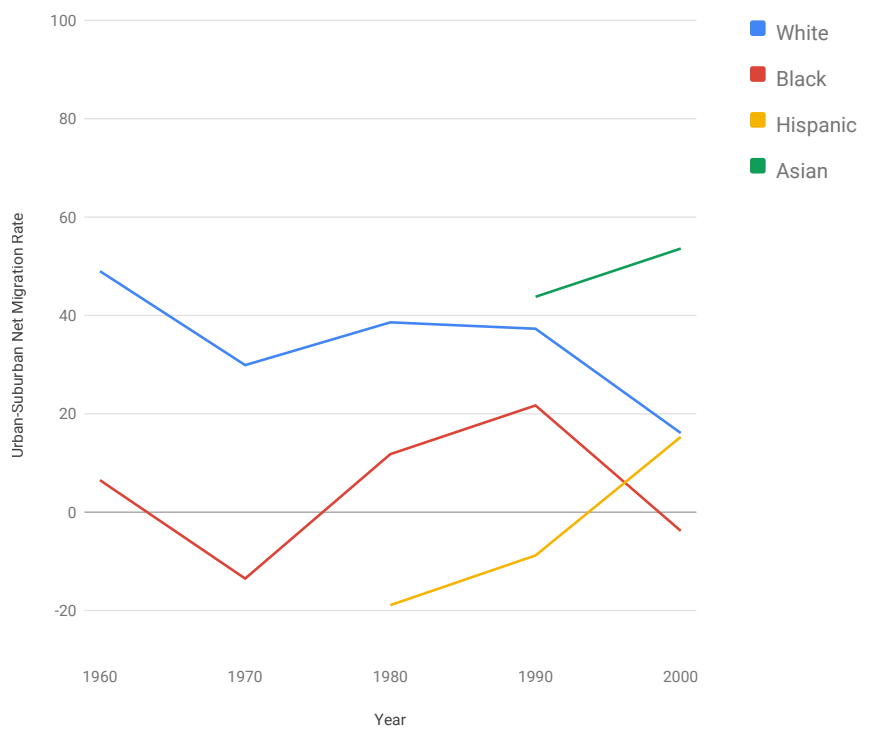

Low-Income Persons by Race

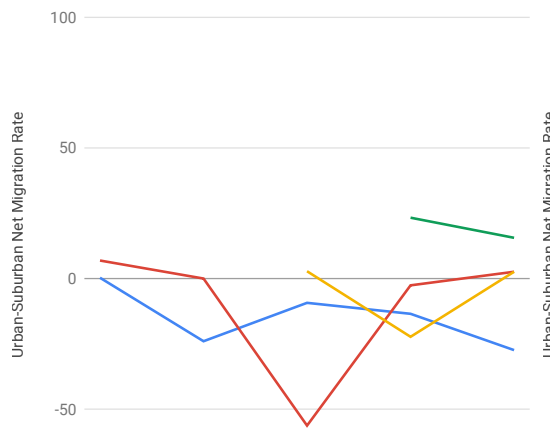

1960
Middle-Income Persons by Race

100

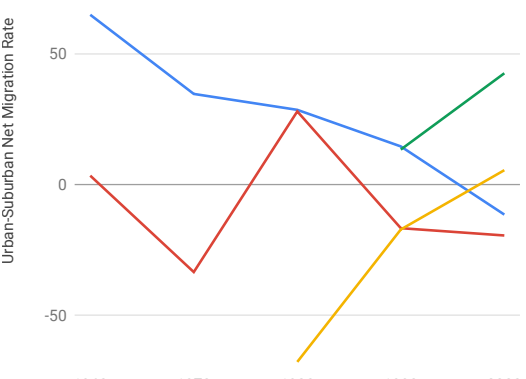

Year
Urban-Suburban Net Migration Rate by Income

100

80

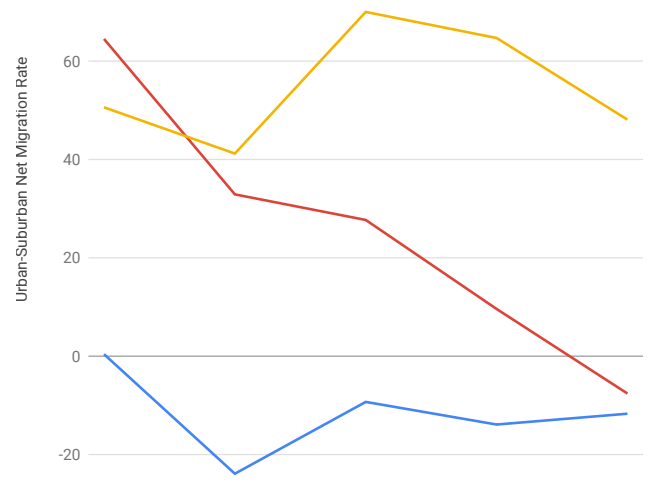

1960

1970

Year
- Low Income

- Middle Income

High Income

High-Income Persons by Race

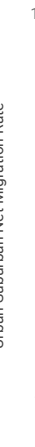

White

Black

Hispanic

- Asian 
Urban and Suburban Residence, 1955-1995, and 5-year migration

Youngstown-Warren, OH-PA

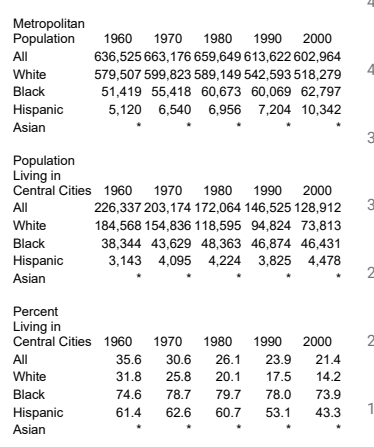

$450 \mathrm{~K}$

$400 \mathrm{~K}$

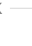

$350 \mathrm{~K}$

300 $250 \mathrm{~K}$

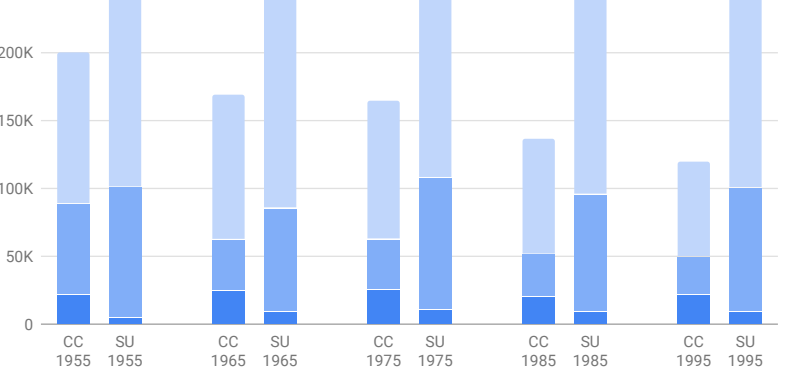

Migrants from central city to suburbs or - suburbs to central city in next five years Migrants within central city or within suburban area in next five years

Nonmovers (lived in the same house five years later)
Urban-Suburban Net Migration Rate by Race

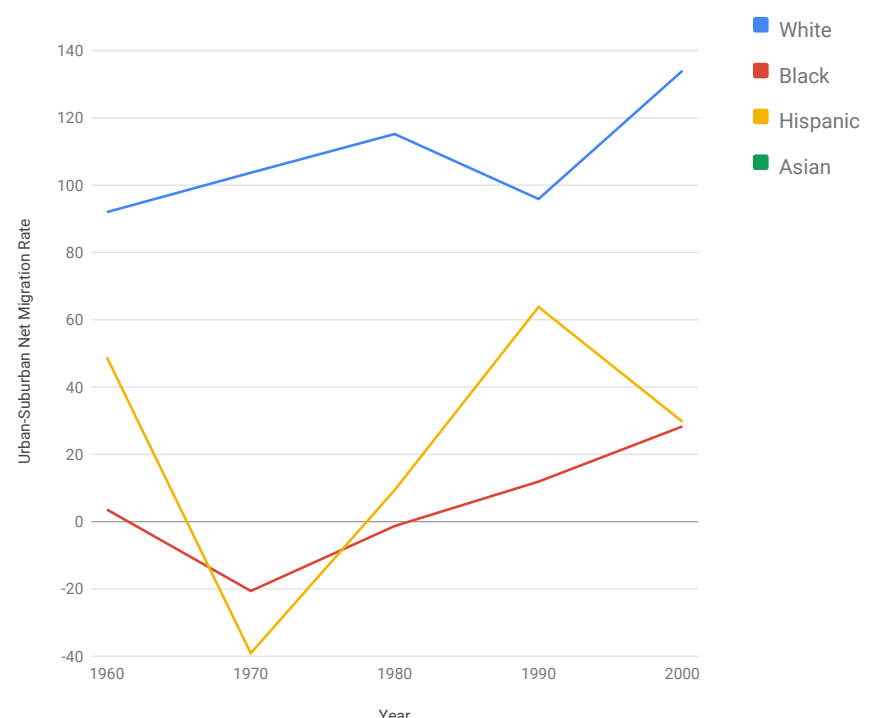

Low-Income Persons by Race

Middle-Income Persons by Race
Urban-Suburban Net Migration Rate by Income
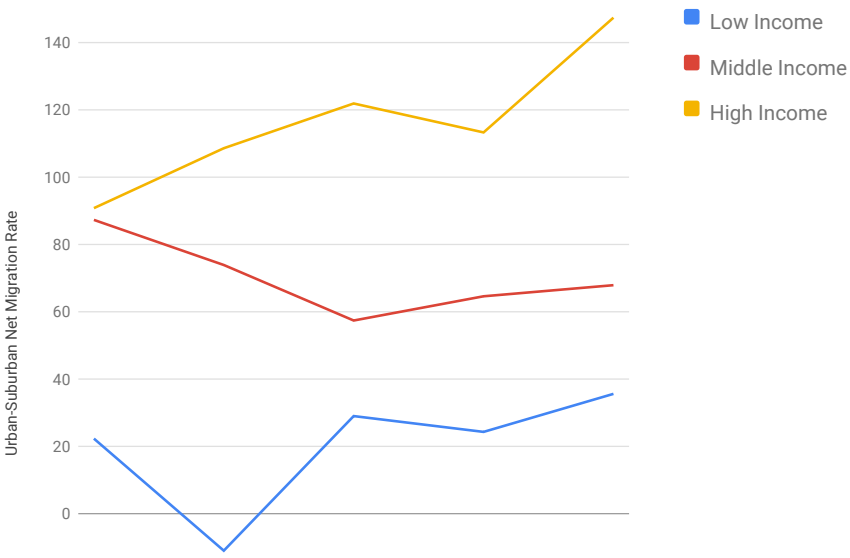

$-20$

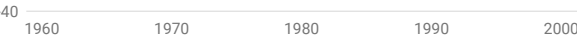

Year

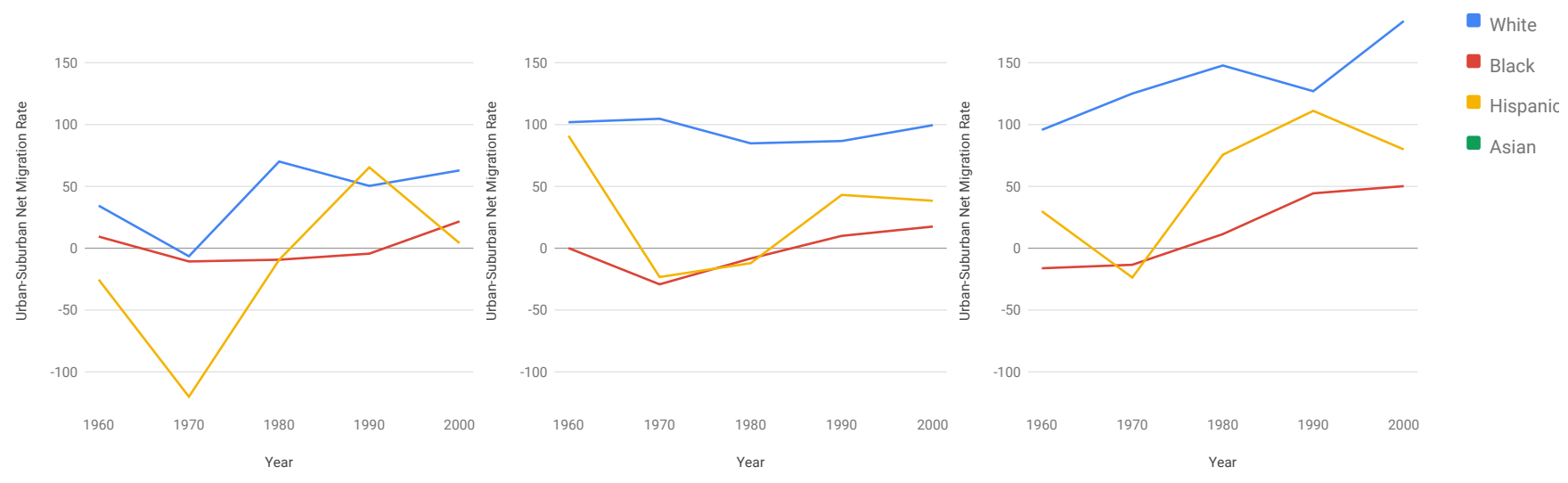

